\title{
Rates of Water Movement Through the Floors of Selected Stormwater Basins in Nassau County, Long Island, New York
}

By Henry. F.H. Ku and Donald B. Aaronson

U.S. GEOLOGICAL SURVEY

Water-Resources Investigations Report 91-4012

Prepared in cooperation with the

NASSAU COUNTY DEPARTMENT OF PUBLIC WORKS

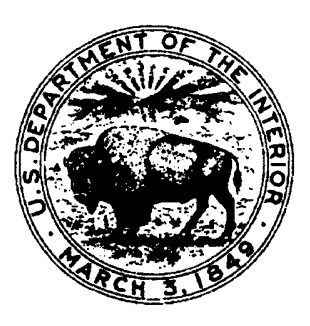

Syosset, New York 
U.S. DEPARTMENT OF THE INTERIOR

MANUEL LUJAN, JR., Secretary

\author{
U.S. Geological Survey \\ Dallas L. Peck, Director
}

For additional information write to:

U.S. Geological Survey

5 Aerial Way

Syosset, NY 11791
Copies of this report may be purchased from:

U.S. Geological Survey

Open-File Reports-ESIC

P.O. Box 25425

Denver, CO 80225 
Abstract . . . . . . . . . . . . . . . . . . . . . . . . . . . 1

Introduction . . . . . . . . . . . . . . . . . . . . . . . . . . 1

Purpose and scope . . . . . . . . . . . . . . . . . . . . . . 2

Previous investigations . . . . . . . . . . . . . . . . . . . . 3

Acknowledgments . . . . . . . . . . . . . . . . . . . . . 3

Description of study area. . . . . . . . . . . . . . . . . . . . 3

Surficial geology . . . . . . . . . . . . . . . . . . . . . 3

Soils . . . . . . . . . . . . . . . . . . . . . . . . . . 5

Recharge basins in Nassau County . . . . . . . . . . . . . . . . 8

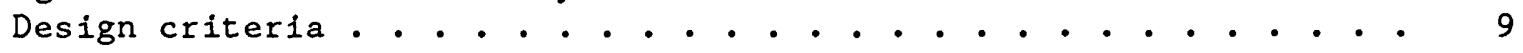

Distribution of clogged recharge basins and factors that affect

basin infiltration rates . . . . . . . . . . . . . . . . . 9

Permeability of basin materials. . . . . . . . . . . . . . 10

Particle-size analysis . . . . . . . . . . . . . . . . 12

Land use in the drainage areas of recharge basins. . . . . . .14

Intersection of basin bottom with local water table. $\cdot . \cdot$. .14

Age of basins. . . . . . . . . . . . . . . . . . • . . 14

Crest-stage-gage measurements. . . . . . . . . . . . . . . 14

Methods of determining infiltration rate and hydraulic conductivity. • . 19

Selection of test basins. . . . . . . . . . . . . . . . . . . 19

Double-ring infiltrometer . . . . . . . . . . . . . . . . . . . 21

Water-manometer tensiometer . . . . . . . . . . . . . . . . . 23

Rates of water movement . . . . . . . . . . . . . . . . . . . 25

Areal infiltration rates. . . . . . . . . . . . . . . . . . . 25

Change of infiltration rate during test period. . . . . . . . . . . 28

Vertical hydraulic conductivity . . . . . . . . . . . . . . . . . 29

One- and two-day infiltration tests . . . . . . . . . . . . . . . 31

Infiltration rate. . . . . . . . . . . . . . . . . . 31

Vertical hydraulic conductivity. . . . . . . . . . . . . 33

Movement of wetting front . . . . . . . . . . . . . . . . 33

Summary and conclusions. . . . . . . . . . . . . . . . . . . . . . 34

References cited . . . . . . . . . . . . . . . . . . . . . . . . 34

Appendix: Location and physical features of recharge basins on

Long Island, N.Y................... 37

\section{ILLUSTRATIONS}

Figure 1. Map showing location and surface features of Long Island. .

2. Generalized geologic section showing major hydrogeologic units of Long Island. . . . . . . . . . . . . . . .

3-6. Maps of Nassau County showing:

3. Extent of poorly drained solls. . . . . . . . . . . . 7

4. Extent of discontinuous zones of perched water. . . . . . 7

5. Locations of recharge basins in Nassau County, 1988 . . . 8

6. Locations of clogged recharge basins. . . . . . . . 10 


\section{ILLUSTRATIONS (continued)}

Page

Figure 7. Graphs showing grain-size distribution of basin soils in three physiographic areas of Nassau County. . . . . . . .

8. Graphs showing number of recharge basins in Nassau County through time: A. Basins built since 1930. B. Basins clogged by 1988.0.

9. Schematic drawing of crest-stage gage . . . . . . . .

10-11. Maps of Nassau County showing locations of recharge basins:

10. With crest-stage gages ............. 18

11. That contained 5 feet or more of stormwater at least once during the study..................

12. Graph showing number of times water depth in basins equaled or exceeded 5 feet during the study . . . . . . . . . .

13. Map of Nassau County showing locations of recharge basins tested for infiltration rate. . . . . . . . . . . . .

14-16. Diagrams showing:

14. Double-ring infiltrometer. . . . . . . . . . . 22

15. Field installation of water-manometer tensiometer. . . . 24

16. Details of coaxial wire preparation. . . . . . . 25

17. Map of Nassau County showing average infiltration rates at

51 selected recharge basins . . . . . . . . . . . 26

18-20. Graphs showing:

18. Change in infiltration rate through time at basin $\mathrm{N}$ 527. . . . . . . . . . . . . . . . . . .

19. Change in infiltration rate at East Meadow basin EM 2 during 24-hour test on April 28, 1986 and 48-hour tes $L$ on September 23,1986 . . . . . . . . . . . .

20. Soll-moisture logs from neutron-access tube in basin EM 2 during 24- and 48-hour tests with infiltration rings. . . . . . . . . . . . . . . . . . . 


\section{PLATE}

(in pocket)

Plate 1. Surficial geology of Nassau County.

\section{TABLES}

Page

Table 1. Physical characteristics of major hydrogeologic units on

Long Island, N.Y....................... 4

2. Description of equivalent soil units drained by recharge basins in Nassau and Suffolk Counties. . . . . . . . 6

3. Grain-size distribution of recharge-basin soils. . . . . . 13

4. Recharge basins with crest-stage gages . . . . . . . . 17

5. Recharge basins tested for infiltration rates. . . . . . 2 20

6. Individual and averaged infiltration rates at selected recharge basins, 1985-86 . . . . . . . . . . . . 27

7. Vertical hydraulic conductivity of basin-floor material at selected Nassau County recharge basins, 1986 . . . . . . .

8. Hydraulic data on selected depth intervals below floor of basins 2 and 3 during ponding tests at East Meadow recharge

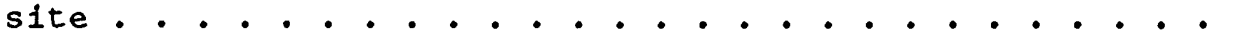

9. Infiltration rates and vertical hydraulic conductivity at East Meadow recharge site, basin EM 2, during 24- and 48hour tests....................... 31

10. Infiltration rates at East Meadow artificial-recharge site. 


\section{CONVERSION FACTORS, ABBREVIATIONS, AND VERTICAL DATUM}

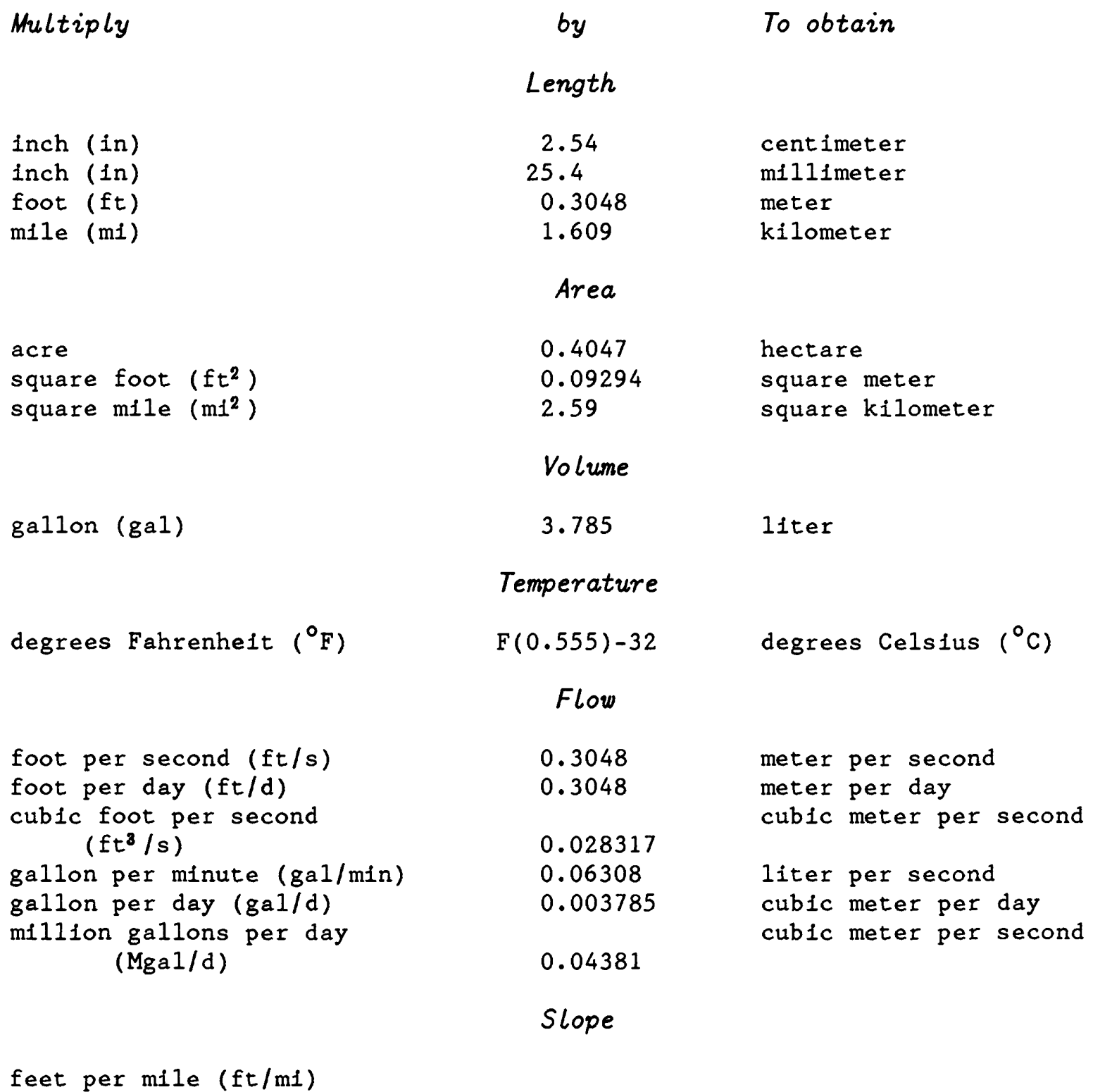

\section{Time}

hours ( $h$ )

minutes $(\mathrm{min})$

Sea level: In this report "sea level" refers to the National Geodetic Vertical Datum of 1929 (NGVD of 1929)-a geodetic datum derived from a general adjustment of the first-order level nets of both the United States and Canada, formerly called sea level datum of 1929. 


\title{
RATES OF WATER MOVEMENT THROUGH THE FLOORS OF SELECTED \\ STORM-WATER BASINS IN NASSAU COUNTY, NEW YORK
}

\author{
by Henry F. H. Ku and Donald B. Aaronson
}

\begin{abstract}
Recharge basins have been used extensively on Long Island, N.Y. since the 1930's to dispose of storm runoff; therefore, the location and performance of recharge basins affect the distribution and amount of recharge to the aquifer. Because ground water is the sole source of freshwater supply for 1.5 million people in Nassau County, the use of recharge basins not only helps to conserve storm runoff and augment the ground-water supply, it also eliminates the need for long, costly trunk storm sewers to route excess storm runoff to coastal waters to prevent local flooding.

Infiltration tests with double-ring infiltrometers at 51 recharge basins showed the median infiltration rate to be 1.83 feet per hour. The median vertical hydraulic conductivity of the upper foot of the unsaturated zone at the bottom of selected recharge basins is 1.63 feet per hour. Crest-stage gages were installed in 58 recharge basins to determine the maximum depth of water in the basins during storms; 20 had at least 5 feet of standing water at least once during the 24-month study period.
\end{abstract}

Surficial geology has a large effect on the infiltration rate of recharge basins. Between 19 and 36 percent of the recharge basins that are excavated in the morainal deposits are clogged, as compared to only 14 percent of those in the outwash plain, and more than half of the recharge basins built before 1950 are clogged. Clogged recharge basins are not likely to cause flooding at present because nearly all are able to discharge stormwater through the side walls, if not through the bottom, under most conditions.

Updated (1987) Nassau County recharge basin statistics (including locations, area, altitude, degree of clogging, land use, geologic unit, and soil unit) are included in an appendix with 1973 data on Suffolk County recharge basins.

\section{INTRODUCTION}

Recharge basins have been in use on Long Island, N.Y., since the 1930's (fig. 1). Nassau County, recognizing early that urbanization was causing a loss of natural recharge, began in 1935 to excavate recharge basins to impound and transmit stormwater to the ground-water system (Welsch, 1935). Because ground water is the sole source of freshwater supply for $1.5 \mathrm{million}$ people in Nassau County, the use of recharge basins not only helps to conserve storm runoff and augment the ground-water supply but also eliminates the need for 
long, costly trunk storm sewers to route excess storm runoff to coastal waters to prevent local flooding. Nassau County contains about 800 recharge basins, and new basins are being added with the construction of housing developments, shopping centers, offices, industrial complexes, and roads. The increasing demand for freshwater is a matter of vital concern to local government officials and planners; thus, an accurate accounting of all sources of freshwater to the hydrologic system is needed. In 1985, the U.S. Geological Survey, in cooperation with the Nassau County Department of Public Works, Division of Water Supply, began a 3-year study to:

1. depict in map form the surficial geology, soil characteristics, and changes in recharge patterns that have resulted from urbanization;

2. quantify the local infiltration rates and vertical hydraulic conductivity of recharge basins from current information and additional data collected at more than 50 recharge basins; and

3. update and revise a 1973 catalog of recharge basins and their characteristics (Seaburn and Aronson, 1973) to include all basins in Nassau County that were constructed through 1986 (data for Suffolk County recharge basins were included but not updated) and incorporate this information, together with field measurements of infiltration rate, into a computerized data base.

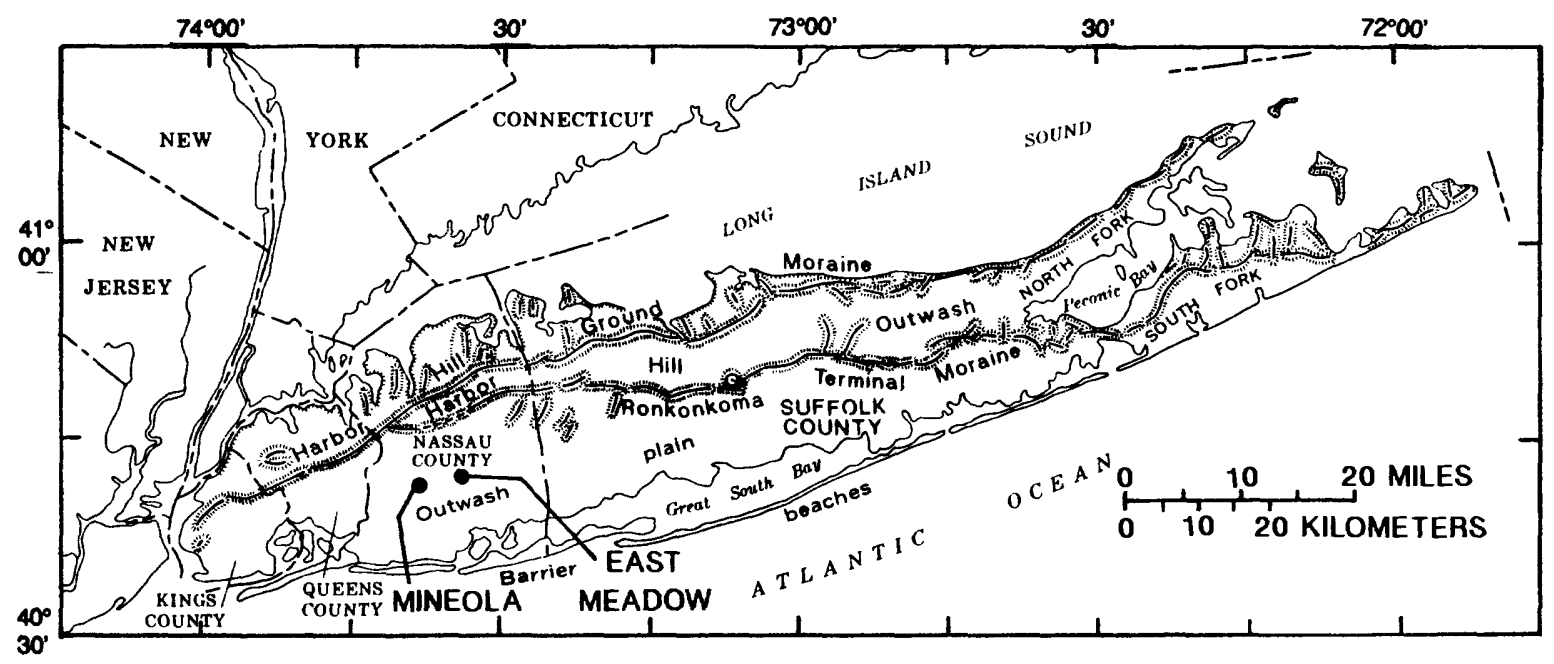

Figure 1.--Location and surface features of Long Island, N.Y. (Modified from McClymonds and Franke, 1972, fig. 2.)

\section{Purpose and Scope}

This report presents results of the 1985-88 study. It (1) quantifies the infiltration rates and vertical hydraulic conductivity of recharge basins from previous and new information, (2) shows the distribution of recharge basins and describes factors that affect their ability to transmit runoff, and (3) shows the areal distribution of infiltration rates in relation to surficial geology. The appendix is a table of data on Nassau County recharge basins. 


\section{Previous Investigations}

Artificial recharge has long been of interest to Long Island's local governments and scientific communities. Several studies (Brashears, 1946; Welsch, 1949; Brice and others, 1956; Holzmacher and others, 1970) have have addressed individual recharge basins, and Seaburn and Aronson (1973) cataloged all recharge basins on Long Island. More recent studies (Prill and Aaronson, 1973; Seaburn and Aronson, 1974; Aronson and Seaburn, 1974; Aronson and Prill 1977; Prill and Aronson, 1978; Ku and Simmons, 1986; Schneider, Ku, and Oaksford, 1987) have addressed the operating efficiency of recharge basins, the effect of stormwater quality on basin efficiency, and the effect of recharge basins on ground-water quality. Flow through the unsaturated zone beneath a recharge basin was analyzed by Prill and others (1979).

\section{Acknow ledgments}

The water companies in Nassau County allowed use of their hydrant water for infiltration tests. Herbert Rusinoff and Peter Barrere, Nassau County Department of Public Works, Highway and Engineering, furnished recharge-basin data and historical information. James Mulligan of Nassau County Department of Public Works, Water Supply, helped obtain the use of East Meadow Recharge Facility for infiltration tests and provided maps and data as well as assistance in many phases of fieldwork and particle-size analysis.

\section{DESCRIPTION OF STUDY AREA}

Long Island is underlain by a thick sequence of unconsolidated sediments consisting of gravel, sand, silt, and clay, which are in turn underlain by the southward dipping crystalline bedrock surface (fig. 2). The thickness of these sediments ranges from zero in northern Queens County, where bedrock is exposed, to more than 2,000 ft in south-central Suffolk County. The characteristics of the aquifers and intervening confining units that form the hydrologic system are summarized in table 1.

Surficial deposits on Long Island generally are the result of the Wisconsinan glaciation (fig. 1). Two terminal moraines form east-west-trending lines of hills of poorly sorted glacial deposits that reach a maximum altitude of $400 \mathrm{ft}$ (fig. 1). A gently sloping outwash plain composed of well-sorted and permeable sand and gravel of glaciofluvial origin extends southward from the line of moraines to the south shore, with a slope of about $20 \mathrm{ft} / \mathrm{mi}$ (Cohen and others, 1968). Small, scattered areas of till form localized zones of low permeability. The headlands along the north shore are mainly glacial deposits that were eroded by streams and waves to produce several embayments. The south shore is lined with swamp and marsh deposits. Barrier islands, composed mainly of sand that was transported and deposited by littoral currents, enclose the shallow saltwater bays along the south shore.

\section{Surficial Geology}

The surficial material throughout Nassau County consists of Pleistocene deposits, the distribution of which is shown on plate 1 . This map is a 
Table 1.--Physical characteristics of major hydrogeologic units, Long Island, N.Y.

[Modifled from Cohen and others, 1968]

\begin{tabular}{|c|c|c|c|}
\hline $\begin{array}{l}\text { Hydro- } \\
\text { geologic } \\
\text { unit } \\
\end{array}$ & $\begin{array}{l}\text { Geologic } \\
\text { name }\end{array}$ & $\begin{array}{l}\text { Approximate } \\
\text { maximum } \\
\text { thlckness } \\
\text { (feet) }\end{array}$ & Water-bearing character \\
\hline $\begin{array}{l}\text { Upper } \\
\text { glaclal } \\
\text { aquifer }\end{array}$ & $\begin{array}{l}\text { Upper } \\
\text { Plelstocene } \\
\text { deposits }\end{array}$ & 400 & $\begin{array}{l}\text { Mainly sand and gravel of moderate to high } \\
\text { permeability; also includes clayey } \\
\text { deposits of t1ll of low permeabllity. }\end{array}$ \\
\hline $\begin{array}{l}\text { Gardiners } \\
\text { Clay }\end{array}$ & $\begin{array}{l}\text { Gard1ners } \\
\text { Clay }\end{array}$ & 150 & $\begin{array}{l}\text { Clay, silty clay and some fine sand of low } \\
\text { to very low permeablifty. }\end{array}$ \\
\hline $\begin{array}{l}\text { Jameco } \\
\text { aquifer }\end{array}$ & $\begin{array}{l}\text { Jameco } \\
\text { Grave1 }\end{array}$ & 200 & $\begin{array}{l}\text { Malnly medlum to coarse sand and gravel of } \\
\text { moderate to high permeabllity. }\end{array}$ \\
\hline $\begin{array}{l}\text { Magothy } \\
\text { aquifer }\end{array}$ & $\begin{array}{l}\text { Magothy } \\
\text { Formation }\end{array}$ & 1,000 & $\begin{array}{l}\text { Coarse to fine sand of moderate permeabil- } \\
\text { ity; locally contains gravel of high per- } \\
\text { meabllity in basal section, and abundant } \\
\text { sllt and clay of low to very low perme- } \\
\text { abllity. }\end{array}$ \\
\hline $\begin{array}{l}\text { Raritan } \\
\text { clay }\end{array}$ & $\begin{array}{l}\text { Clay member } \\
\text { of the Raritan } \\
\text { Formation }\end{array}$ & 300 & $\begin{array}{l}\text { Clay of very low permeablifty; some silt } \\
\text { and fine sand of low permeablifty. }\end{array}$ \\
\hline $\begin{array}{l}\text { Lloyd } \\
\text { aquifer }\end{array}$ & $\begin{array}{l}\text { Lloyd Sand } \\
\text { Member of the } \\
\text { Raritan Formation }\end{array}$ & 300 & $\begin{array}{l}\text { Sand and gravel of moderate permeability; } \\
\text { some clayey material of low permeability. } \\
\text { of low permeability. }\end{array}$ \\
\hline
\end{tabular}

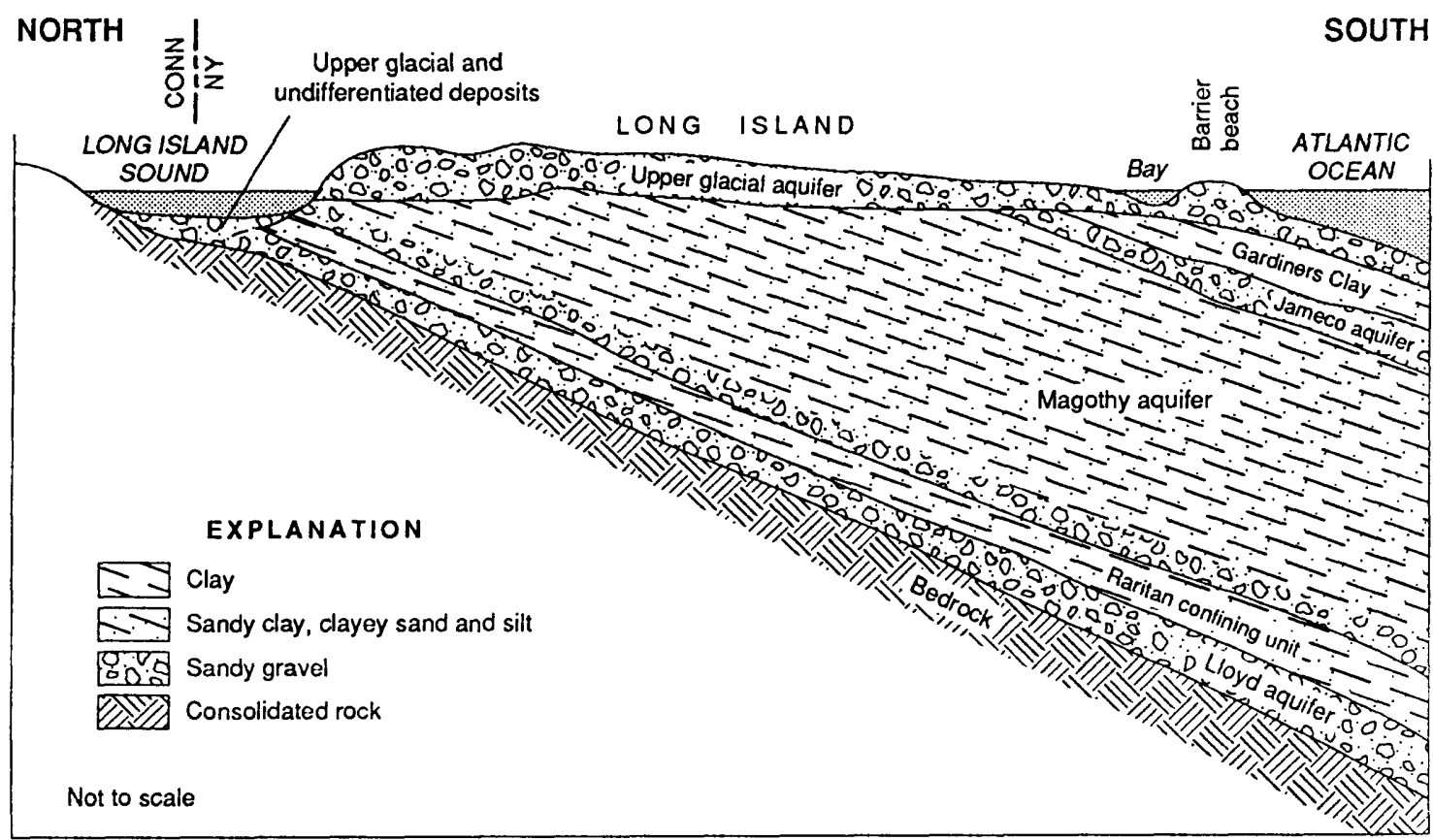

Figure 2.--Major hydrogeologic units of Long Island. (Modified from Cohen and others, 1968, fig. 2C.) 
composite made from geologic maps by Isbister (1966), who reported on northeastern Nassau County, Swarzenski (1963), who reported on northwestern Nassau County, and Perlmutter and Geraghty (1963), who reported on southern Nassau County.

Nassau County is divided by the Ronkonkoma terminal moraine (fig. 1), which forms the topographic high of Nassau County. The area south of the moraine is an outwash plain that contains stratified sand and gravel and is well sorted and permeable; the area to the north consists of moraines and isolated areas of stratified material.

Because the various Pleistocene (glacial) deposits have differing water-transmitting properties, the recharge basins excavated into them also have differing infiltration properties.

\section{Soils}

The earliest comprehensive soil survey of Nassau and Suffolk Counties was made by Lounsbury and others (1928). A new soil survey was prepared by the U.S. Soll Conservation Service (1982), in cooperation with the Nassau County Soil and Water Conservation District, to include urban developments. Seaburn and Aronson (1973) summarized and tabulated the major soil types in Nassau and Suffolk Counties on the basis of data presented in Lounsbury and others (1928). (See table 2.)

A map showing areas with poorly drained soils was prepared (fig. 3) in an effort to determine the effect of soil type on the performance of the recharge basins. The poorly drained soils represented in this simplified map include those classified by the U.S. Soil Conservation Service (1982) as "somewhat poorly," "poorly," and "very poorly" drained.

The area of poorly drained soils generally coincides with the terminal and ground moraines ( $\mathrm{pl}, 1$ ) and the perched water-table zones (fig. 4) as described by Swarzenski (1963) and Isbister (1966). The locations of recharge-basin sites need to be evaluated individually because the zones of poorly permeable soils are discontinuous. For example, the southeastern part of the perched-water zone (fig. 4) coincides with relatively impermeable ice-contact deposits ( $p 1.1)$, and several recharge basins in that area often contain standing water and may overflow during heavy storms.

The soil cover over the southern half of Nassau County consists mostly of sandy loam developed over permeable and well-drained outwash. The exceptions are the fine-grained, organic-rich, marsh soils near the south shore and along the stream valleys.

Surface-soil permeability does not necessarily reflect a recharge basin's infiltration rate because the basin bottoms generally are $10 \mathrm{ft}$ or more below land surface. Soll permeability does affect the runoff characteristics of the area surrounding the basin, however, as well as the sediment load in the stormwater. A particle-size analysis of basin-bottom soil was done for 54 recharge basins; results are presented in the section "Particle-Size Analysis." 


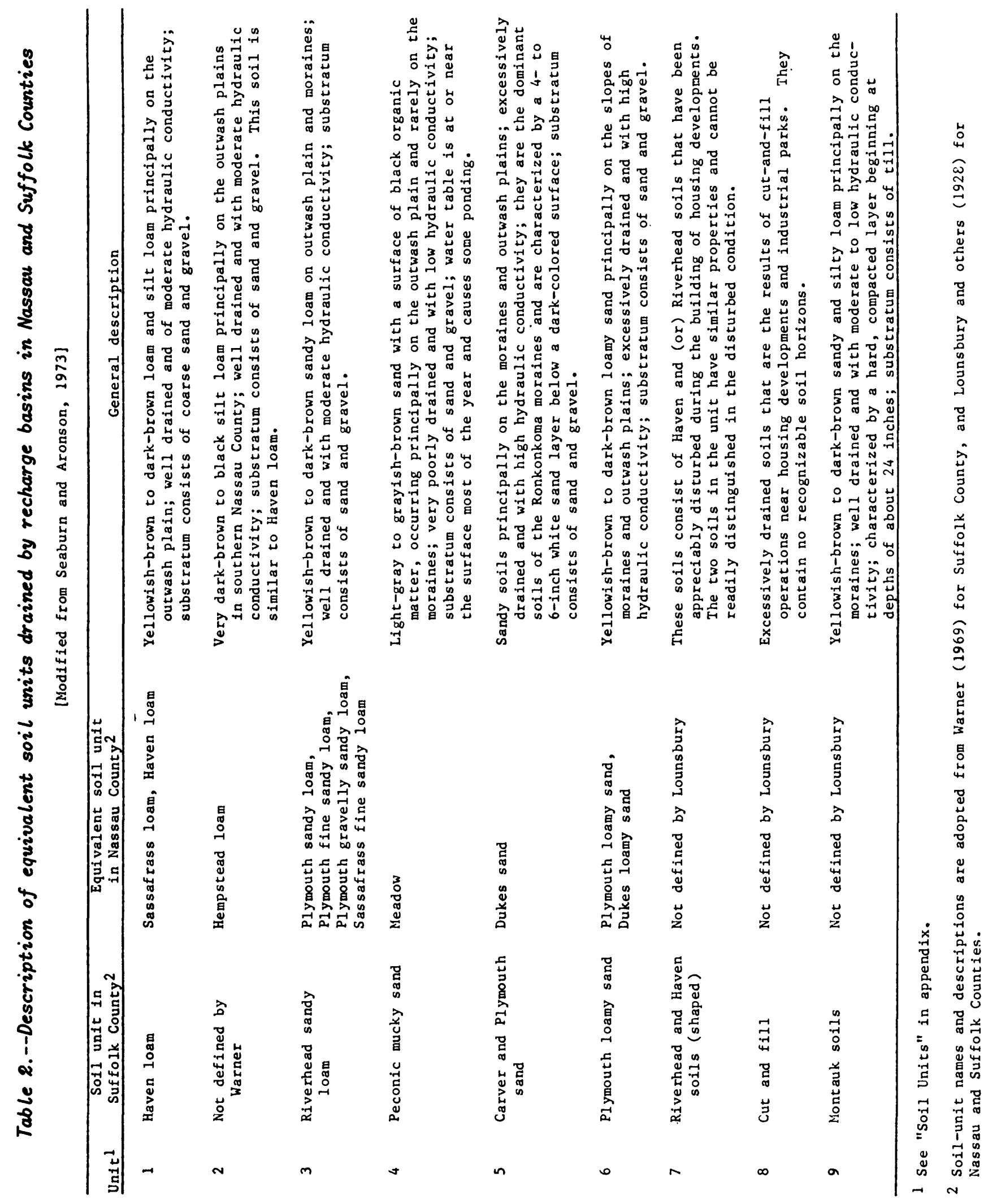



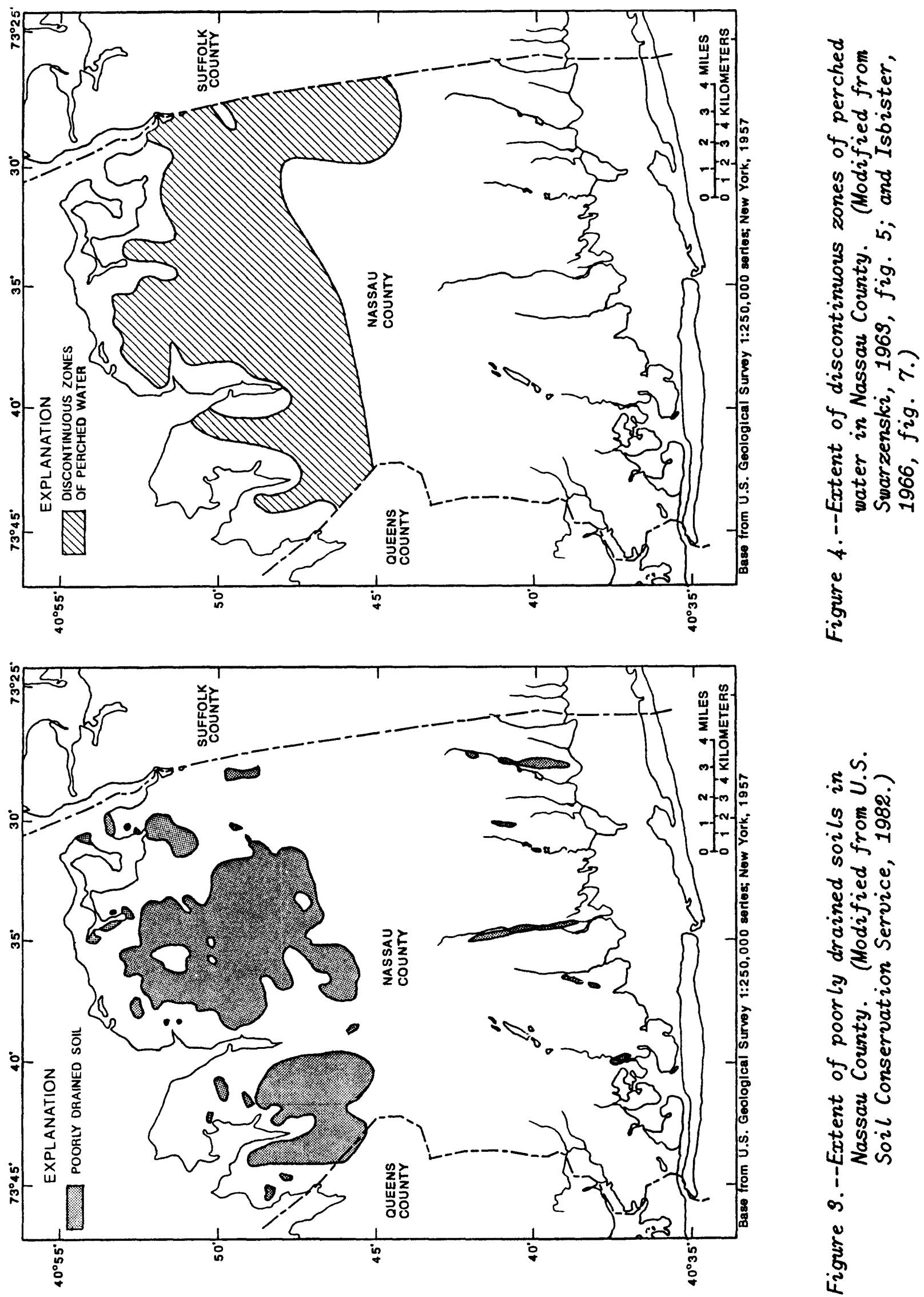


\section{RECHARGE BASINS IN NASSAU COUNTY}

Storm runoff in areas away from the island's shores or streams is disposed of through recharge basins. Most of the recharge basins on Long Island are unlined, open pits that receive storm runoff from residential, commercial, and industrial areas and from highways. The area of these basins ranges from 0.1 to 30 acres and averages 1.5 acres. Most recharge basins are closed and do not have overflow structures. The average depth of the basin bottom below land surface is $10 \mathrm{ft}$, but a few are as deep as $40 \mathrm{ft}$. Storm runoff reaches recharge basins by way of gutters that drain into street inlets, which in turn drain to sewers that are connected to the basins.

An updated list of data (including location, size, area, altitude, use, geologic unit, and soil unit) for Nassau County basins and previously published data on Suffolk County basins (Seaburn and Aronson, 1973) is given in the appendix. Many new basins have been constructed in Suffolk County since the publication of Seaburn and Aronson (1973), but this report deals only with Nassau County basins. No Suffolk County recharge basins were studied nor were the data on them updated.

The design and construction of recharge basins on Long Island is regulated and approved by the local governments. The two major government agencles in Nassau County are the Nassau County Department of Public Works and the New York State Department of Transportation.

Nassau County has more than 800 recharge basins (fig. 5), most of which are concentrated in a roughly trlangular area with the apex at the western County line. The shape reflects the increased use of recharge basins over other means of stormwater disposal as development progressed from west to east. With a few exceptions, the majority of recharge basins are individual basins without an overflow structure.

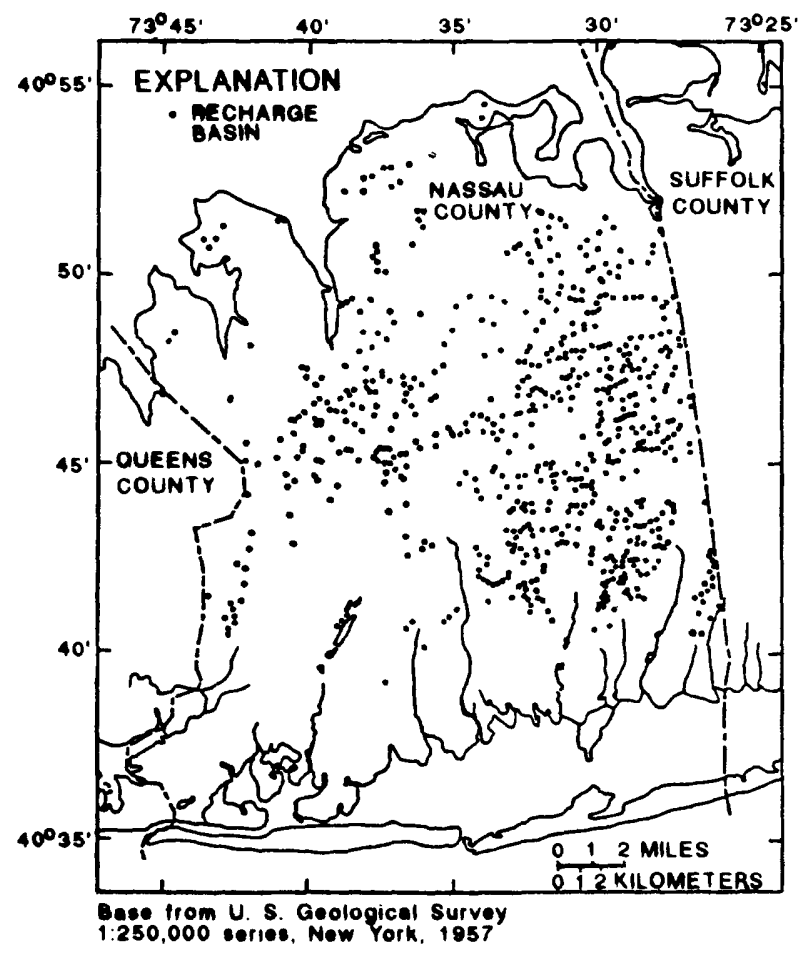

Figure 5 .

Locations of recharge basins in Nassau County, 1988. 


\section{Design Criteria}

The design criteria for stormwater basins on Long Island have been evolving for 50 years. These criteria are based primarily on engineering experience and include a generous safety factor for overflow. In Nassau County, the required capacity of a recharge basin is based on the following assumptions: The basin has no outlet, (2) 5 in. of rain falls per storm (such a storm has a recurrence interval of 10 years, Miller and Frederick, 1969 ), and (3) the area served by the basin's storm-sewer system is 40 percent impervious in residential areas, 60 percent impervious in business areas, and 90 percent impervious at shopping centers. Any area that drains to a recharge basin but is not served by any storm-sewer system is considered to be 20 percent impervious.

The capacity calculated by this method is known as "required volume" and is calculated as:

$$
V_{r}=3,630 P\left(0.4 A_{r}+0.6 A_{b}+0.9 A_{s}+0.2 A_{n}\right)
$$

where $A_{b}=$ contributing business area, in acres;

$A_{n}=$ contributing area not served by a storm-sewer system, in acres;

$A_{r}^{n}=$ contributing residential area, in acres;

$A_{s}^{r}=$ contributing shopping center area, in acres;

$\mathrm{P}^{\mathrm{S}}=$ precipitation, in inches ( $5 \mathrm{in}$. was used for Nassau County);

$\mathrm{V}_{\mathrm{r}}=$ required volume, in cubic feet.

The value 3,630 is a dimensionless constant used to convert acres to square feet and inches to feet.

For example, basin $N 594$ serves a residential area of 42.3 acres. The drainage area not served by a sewer system is 32.3 acres. The required volume is computed as follows:

$$
\begin{aligned}
v_{r} & =3,630(5 \text { in. })[0.4(42.3)+0.2(32.3)] \\
& =424,000 \mathrm{ft}^{3} .
\end{aligned}
$$

The rate of stormwater infiltration into the floor of the recharge basin is omitted from the design equation to provide an additional safety factor in basin construction. Similar design criteria are used by other government agencies on Long Island, but the values assigned to the design (rainfall and runoff) coefficients may differ slightly.

The New York State Department of Transportation issued a report that describes the theory, design procedure, and recommended engineering practices for basin construction and includes an empirical method for basin-size design and diffusion-wel1 (dry-wel1) design (Weaver, 1971).

\section{Distribution of Clogged Recharge Basins and Factors that Affect Basin Infiltration Rates}

Clogged recharge basins are defined as those that retain water for 5 days after a 1-in. rainstorm (Seaburn and Aronson, 1973). In 1969, Aronson and 
Seaburn (1974) found that about 9 percent of the recharge basins on Long Island were clogged. Examination of aerial photographs dated April 11, 1969, 6 days after a 1.5-in. rainfall, revealed that 62 basins owned by Nassau County Department of Public Works (approximately 12 percent of the total number of basins in 1969) still contained water. In 1986, 106 out of the 598 recharge basins then owned by the Nassau County Department of Public Works (18 percent) were considered to be clogged (fig. 6), as determined by field inspections during July and August with the criteria developed by Seaburn and Aronson (1973). This indicates a sizable increase in the number of clogged basins in Nassau County since 1969.

Clogged recharge basins on Long Island are determined by four principal factors: (1) the permeability of basin materials, (2) land use within the basin's drainage area, (3) the age of the recharge basin, and (4) intersection of the basin bottom with the local water table. Each of these factors is discussed in the following paragraphs. Despite the large number of clogged basins, few actually overflow or exceed their capacity during a storm. Nearly all basins are able to discharge all storm water through the walls, if not the bottom, under most conditions. Close monitoring of recharge-basin performance, nevertheless, will remain an important component of a complete maintenance program.

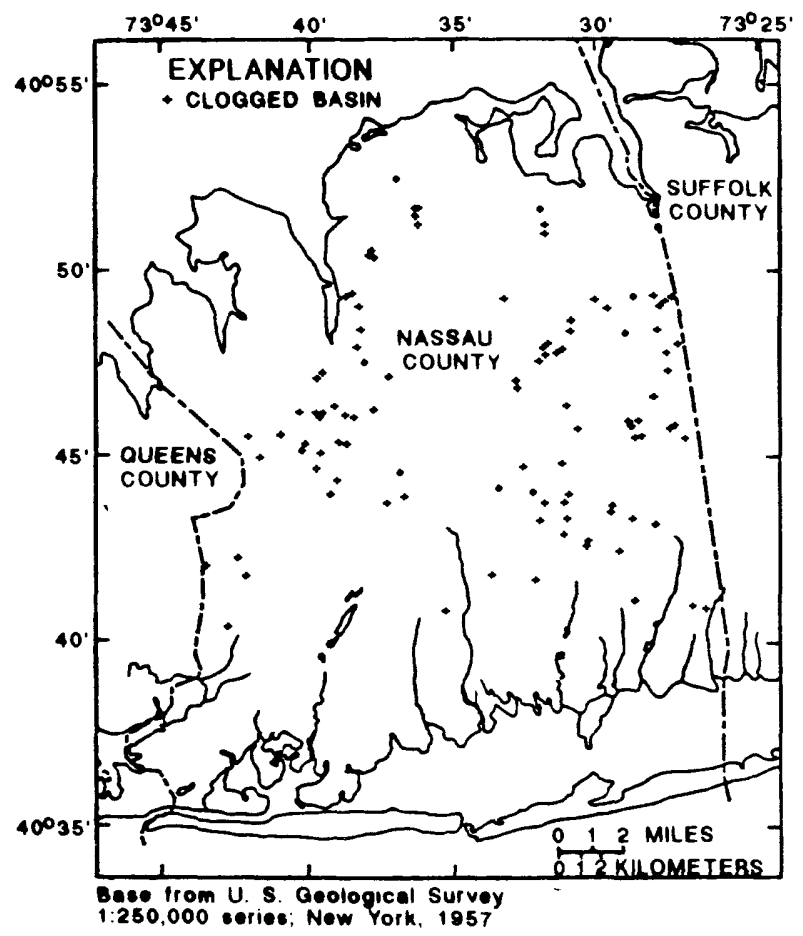

Figure 6.

Locations of clogged recharge basins.

\section{Permeability of Basin Materials}

Most of Long Island, especially the southern part, has highly permeable surficial outwash deposits; the central and northern parts are overlain by morainal deposits, which have lower infiltration rates. The area of surficial material with low hydraulic conductivity as determined by the U.S. Soil 
Conservation Service (1982) is shown in figure 3. The composition of the surficial material does not necessarily indicate the permeability of the basin bottom, however, because basins are generally at least $10 \mathrm{ft}$ deep.

The northern part of Nassau County is composed mainly of morainal and ice-contact deposits ( $\mathrm{pl}$. 1), which have a higher silt and clay content and lower permeability than the well-sorted outwash deposits of the southern part of the island. The geologic control over the permeability and therefore the infiltration rate of the recharge basin becomes evident when the surficial geology ( 1.1 ), the distribution of perched water (fig. 4 ), and the basin-soil grain size (fig. 7) are viewed together.
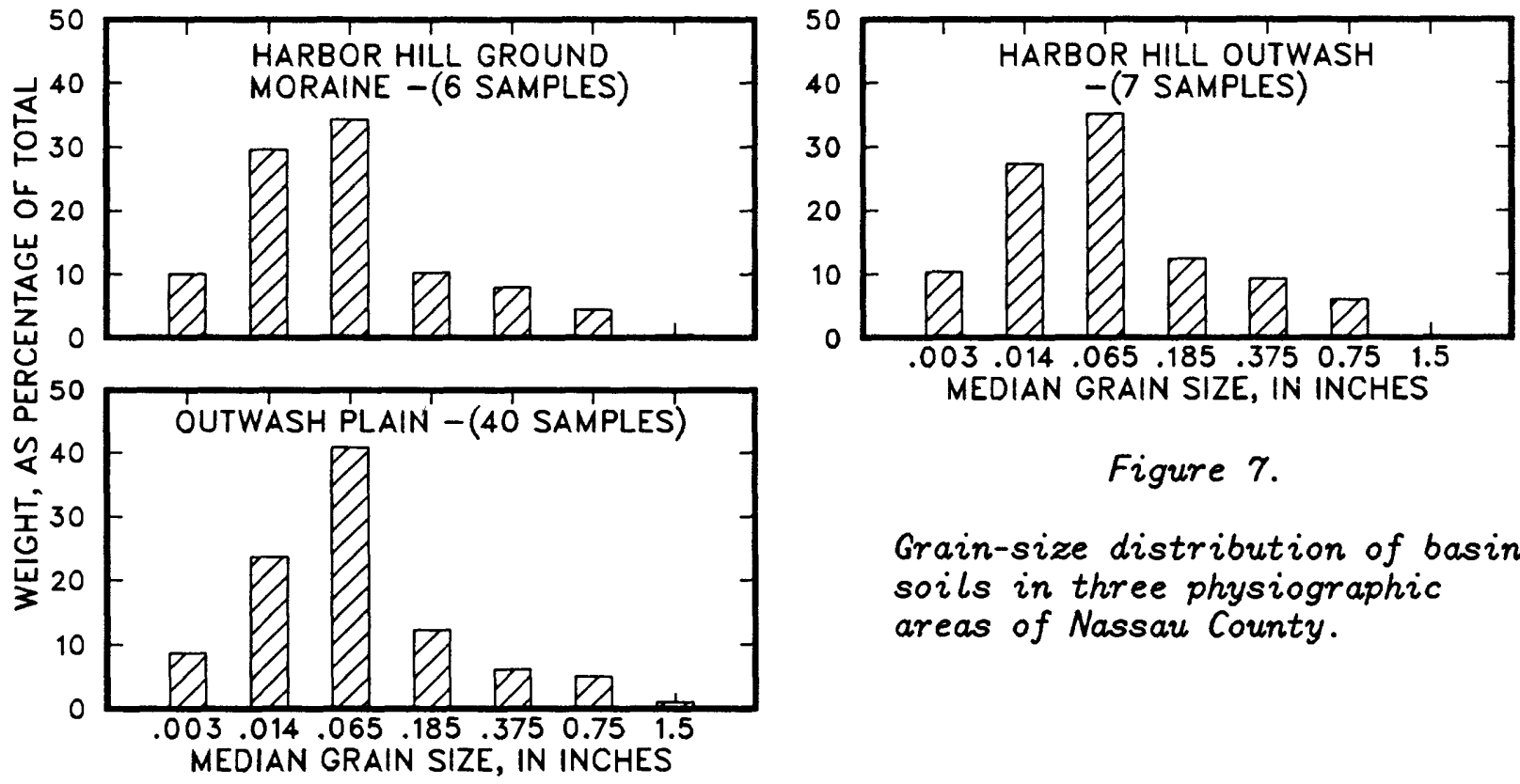

Figure 7 .

Grain-size distribution of basin soils in three physiographic areas of Nassau County.

To further ascertain the effect of surficlal geology on recharge-basin performance, the basins were grouped according to geologic character, and the number of clogged basins in each category was tabulated. The percentage of clogged Nassau County basins in each geologic untt (p1. 1) is 1isted below:

\section{Geologic unit}

Ice-contact deposits

Harbor Hill Drift

Harbor Hill end moraine

Harbor Hill ground moraine

Harbor Hill outwash deposits

Ronkonkoma Drift

Ronkonkoma terminal moraine

\section{Percentage clogged}

Total number of basins in unit 
The preceding list indicates that recharge basins on the outwash plain dispose of water more rapidly than those in morainal deposits. The glaciofluvial deposits, although stratified, are relatively poorly sorted and, therefore, do not have high infiltration rates. Outwash-plain deposits are well-sorted sand or sand and gravel and therefore have the highest infiltration rates, on average.

The potential performance of any recharge basin must be judged individually because the morainal and ice-contact deposits in which they are excavated may contain discontinuous clay units. Thus, test drilling and coring of subsurface material at each site can provide information on potential basin performance before the basin location is selected.

\section{Particle-Size Analysis}

Soll consists of particles of many sizes; the particle size and distribution both affect the soil's ability to transmit water. Three soil samples were collected from each of 54 recharge basins in Nassau County for particlesize analysis, and all were collected from an area in each basin near the inflow, where infiltration is likely to occur even after light precipitation. In general, sampling points formed a triangular pattern, with one near the inflow, and the other two about $20 \mathrm{ft}$ from the first and from each other. Samples were collected with a 4-in. hand-auger drill to a depth of 6 in.

The samples were analyzed by the Nassau County Department of Public Works Laboratory. The three samples from each basin were combined, dried, and split into composite subsamples before being sieved.

After the analyses were completed, the results from the 54 basins were divided into four units according to the surficial geology at each basin, as shown in table 3 (Isbister, 1966; Perlmutter and Geraghty, 1963; Swarzenski, 1963). These units are the Ronkonkoma terminal moraine, Harbor Hill ground moraine, Harbor Hill outwash, and outwash plain (pl. 1). Forty samples were collected from the outwash plain, one from the Ronkonkoma terminal moraine, six from the Harbor Hill ground moraine, and seven from the Harbor Hill outwash. All data from each of the three geologic units from which more than one sample was collected were combined to derive a median percentage for each particle size present in that deposit.

Median percentages of grain size for basin soils overlying the three geologic units are similar ( $f i g .7)$. Each unit has no more than 1 percent (by weight) of the 1.5-in. grain size (very large pebbles) (Krumbein and Sloss, 1963), and each consists mostly of the 0.065-in. grain size (very coarse sand). This grain size ranges from 34 percent of the soil samples, by weight, for basins on the Harbor Hill ground moraine to 40 percent for basins on the outwash plain. The second largest percentage is the 0.014-in. size (medium sand), which ranges from 23.5 percent to 29.5 percent of the soil, by weight.

Calculations of the sorting coefficient (Krumbein and Sloss, 1963) of each unft Indicates the outwash plain soils to be well sorted and the Harbor Hill ground moraine, Harbor Hill outwash, and Ronkonkoma terminal moraine basin solls to be moderately well sorted. Poorly sorted deposits are expected to have lower porosity than well-sorted deposits. 
Table 9.--Grain-size distribution of recharge-basin soils

[So11 locations are shown on p1. 1; values are in percent by we 1ght; $>$ less than; basin locations are given in appendix]

\begin{tabular}{|c|c|c|c|c|c|c|c|}
\hline \multirow{2}{*}{$\begin{array}{l}\text { Basin } \\
\text { number }\end{array}$} & \multicolumn{7}{|c|}{ Grain size, in inches } \\
\hline & 21.5 & $\begin{array}{r}1.5- \\
20.75 \\
\end{array}$ & $\begin{array}{r}0.75- \\
20.375 \\
\end{array}$ & $\begin{array}{l}0.375- \\
20.185\end{array}$ & $\begin{array}{l}0.185- \\
>0.065\end{array}$ & $\begin{array}{l}0.065- \\
20.014\end{array}$ & $\begin{array}{l}0.014- \\
>0.003\end{array}$ \\
\hline \multicolumn{8}{|c|}{ Ronkonkoma terminal moraine } \\
\hline N513 & 0 & 6 & 3 & 5 & 31 & 35 & 20 \\
\hline \multicolumn{8}{|c|}{ Harbor H111 ground moraine } \\
\hline N192 & 2 & 3 & 6 & 9 & 29 & 31 & 20 \\
\hline N206 & 0 & 2 & 4 & 12 & 46 & 33 & 3 \\
\hline N215 & 0 & 5 & 10 & 12 & 39 & 28 & 6 \\
\hline N469 & 0 & 4 & 6 & 11 & 43 & 30 & 6 \\
\hline N539 & 9 & 9 & 10 & 9 & 20 & 20 & 23 \\
\hline N563 & 1 & 8 & 10 & 10 & 28 & 29 & 14 \\
\hline
\end{tabular}

Harbor H111 outwash

$\begin{array}{lrrrrrrr}N 18 & 0 & 6 & 11 & 12 & 37 & 29 & 5 \\ N 143 & 0 & 7 & 9 & 9 & 35 & 27 & 13 \\ N 415 & 0 & 1 & 2 & 4 & 24 & 49 & 20 \\ N 425 & 0 & 0 & 2 & 6 & 32 & 33 & 27 \\ N 482 & 0 & 6 & 8 & 16 & 42 & 19 & 9 \\ N 527 & 2 & 5 & 13 & 15 & 29 & 27 & 9 \\ N 594 & 0 & 7 & 9 & 13 & 39 & 22 & 10\end{array}$

\section{Outwash plain}

$\begin{array}{lrrrrrrr}\text { N1 } & 0 & 0 & 4 & 10 & 44 & 34 & 8 \\ \text { N16 } & 7 & 11 & 10 & 12 & 36 & 20 & 4 \\ \text { N29 } & 0 & 2 & 6 & 16 & 37 & 20 & 19 \\ \text { N38 } & 0 & 3 & 2 & 6 & 47 & 30 & 12 \\ \text { N68 } & 5 & 5 & 6 & 8 & 37 & 27 & 12 \\ \text { N78 } & 0 & 0 & 1 & 6 & 46 & 38 & 9 \\ \text { N98 } & 6 & 3 & 5 & 15 & 47 & 20 & 4 \\ \text { N121 } & 2 & 2 & 5 & 12 & 45 & 29 & 5 \\ \text { N132 } & 0 & 3 & 5 & 8 & 43 & 35 & 6 \\ \text { N140 } & 7 & 8 & 10 & 13 & 38 & 16 & 8\end{array}$

N 150

N153

N155

N186

N187

$\mathrm{N} 202$

N213

N219

N236

N254

$0 \quad 5$

$8 \quad 11$

$\begin{array}{rrrr}11 & 41 & 24 & 11 \\ 18 & 36 & 13 & 4 \\ 11 & 46 & 23 & 6 \\ 5 & 36 & 29 & 14 \\ 12 & 45 & 24 & 11 \\ 14 & 45 & 25 & 8 \\ 9 & 50 & 27 & 8 \\ 11 & 39 & 26 & 6 \\ 16 & 39 & 26 & 10 \\ 7 & 35 & 25 & 25\end{array}$

N260

N261

N313

N315

N325

N328

N334

N343

N344

N352

N353

N361

N363

N365

N439

N444

N504

N540

N549

N556

$\begin{array}{rrrr}13 & 45 & 22 & 6 \\ 11 & 22 & 28 & 13 \\ 12 & 42 & 22 & 12 \\ 9 & 59 & 21 & 2 \\ 11 & 34 & 22 & 11 \\ 12 & 40 & 21 & 4 \\ 11 & 40 & 17 & 10 \\ 16 & 41 & 17 & 4 \\ 10 & 47 & 24 & 11 \\ 12 & 38 & 20 & 8\end{array}$

\begin{tabular}{rrrr}
12 & 45 & 21 & 5 \\
19 & 37 & 16 & 2 \\
12 & 43 & 27 & 7 \\
17 & 38 & 23 & 12 \\
11 & 40 & 28 & 6 \\
17 & 29 & 21 & 27 \\
11 & 27 & 19 & 9 \\
14 & 32 & 19 & 10 \\
18 & 45 & 24 & 10 \\
9 & 41 & 31 & 11 \\
\hline
\end{tabular}




\section{Land Use in the Drainage Areas of Recharge Basins}

The types of land use in the drainage area of a recharge basin is a major factor in the clogging of the basin. Aronson and Seaburn (1974) found that 28 percent of basins in commercial and industrial areas were clogged. As part of the U.S. Environmental Protection Agency's Nationwide Urban Runoff Program, Ku and Simmons (1986) studied one recharge basin that was used solely to collect stormwater from a shopping center and found it to be clogged and to contain water all the time. An adjacent recharge basin that collects storm runoff from a residential area is dry during fair weather. The high percentage of clogged recharge basins in commercial areas is probably due to the relatively large inflow of asphalt, grease, oil, tar, and rubber particles in runoff from the adjacent parking fields (Seaburn and Aronson, 1974).

\section{Intersection of Basin Bottom with Local Water Table}

A few basins that have been designated as clogged are not actually clogged but, rather, intersect the water table. Most of these are near the south shore, where the water table is just below land surface most of the year. Included in this category are a few basins that were built during the 1962-66 drought, when the water table was lower than normal. As the water table recovered, the basin floors became flooded (Aronson and Seaburn, 1974).

\section{Age of Basins}

The oldest recharge basins in Nassau County were constructed in the 1930's. The graph in figure 8A shows the number of recharge basins built since 1930, in 5-year increments. By 1988, the Nassau County Department of Public Works maintained 598 basins, of which about 70 percent were built during 1950-59. About 17 percent of these are clogged (fig. 8B), as are more than 50 percent of those built before 1950 ( $\mathrm{fig} .8 \mathrm{~B}$ ). These data suggest that the efficiency of recharge basins decreases with age. The cause of clogging with age is not clear but may be the accumulation of fine sediment and debris on the basin floor and microbial activity within the surface sediment. The increasing age of the basins, coupled with changing land use, may pose a potential for flooding in the future.

\section{Crest-Stage-Gage Measurements}

The granulated-cork crest-stage gage (fig. 9) is an effective and inexpensive way of determining the efficlency of recharge basins. The ability of stormwater to infiltrate recharge basins is difficult to estimate because many variables are involved, such as geology, basin size, type and size of contributing area, slope, and rainfall duration and intensity. Also, the maximum water stage in the recharge basin is difficult to observe because it is a short-lived event. A study of urban storm runoff on Long Island by Ku and Simmons (1986) showed that the flow into the recharge basin responds quickly to changes in rainfall intensity, and the high infiltration rate in many areas causes the water level to drop quickly once the rainfall intensity slackens. With crest-stage gages, one can determine which recharge basins are approaching capacity and which have excess storage capacity. 

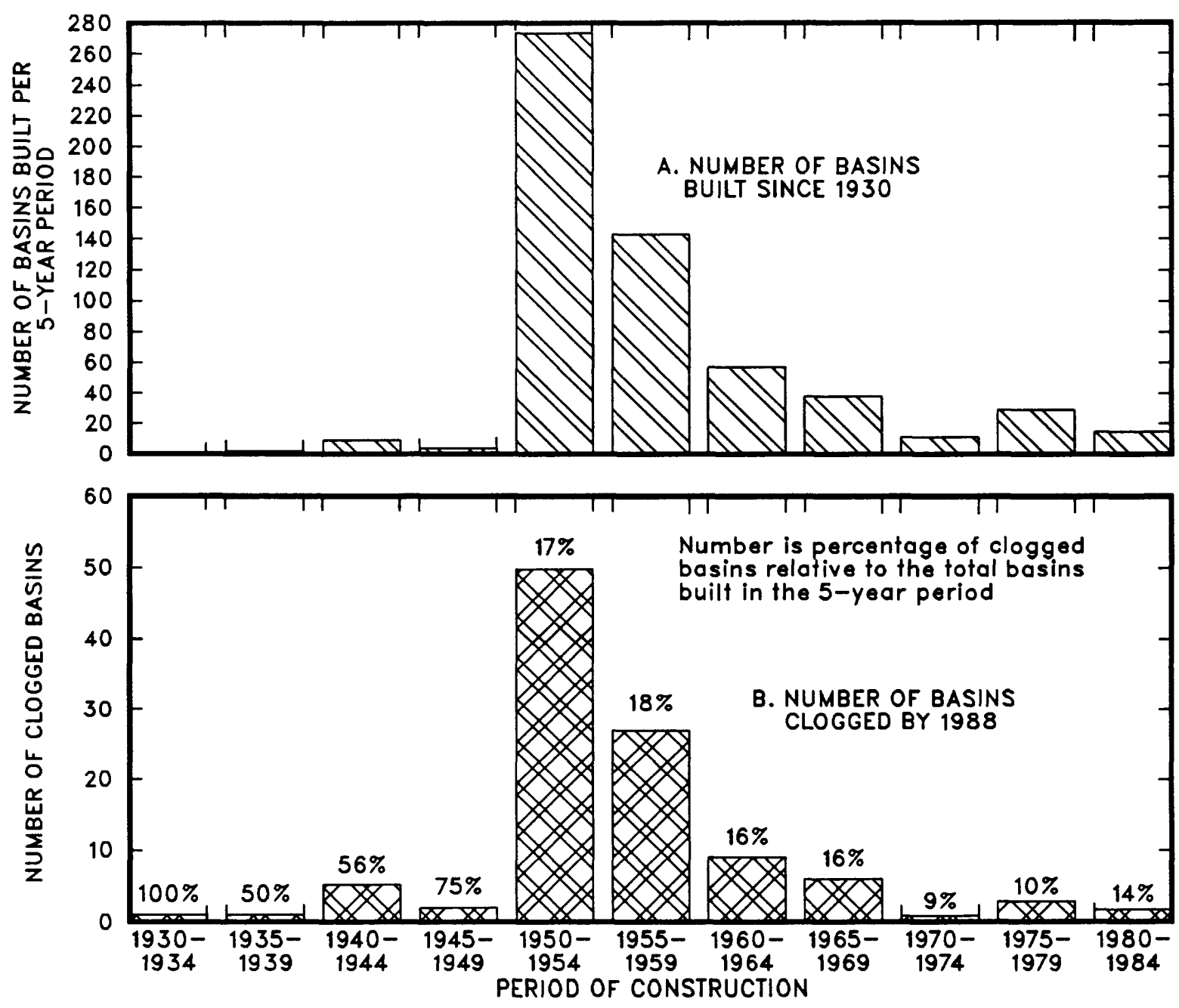

Figure 8.--Number of recharge basins in Nassau County through time: A. Basins built since 1980. B. Basins clogged by 1988.

Crest-stage gages were installed in 58 recharge basins in Nassau County. Most of the infiltration tests were run in these basins, although some tests were run in basins in which a crest-stage gage was not installed. The locations of basins with crest-stage gages are indicated in figure 10 (p. 18) and table 4. In this study, most of the gages were installed near the stormwater inlet at the bottom of the basin and were anchored to the wing wall of the culvert inlet. The base of the gage was placed as close to the ground as possible so that the water level indicated by the crest-stage gage would represent the maximum height of water in the basin.

Gages at the few basins that either contain water all the time or that could contain enough water to hinder access to the culvert were secured to a metal post that was driven into the basin floor. The base of the gage was placed as close to the floor as possible so that the water level indicated by the gage would represent the height of water in the basin. 
After all crest-stage gages were installed, the first reading was made on May 29, 1985. Approximately eight readings were made at each gage; the last was made on July $1,1987$.

Of the 58 recharge basins studied, 20 had $5 \mathrm{ft}$ or more of standing water at least once during the 3-year study. The locations of these basins are shown in figure 11 ( $p .18$ ); the number of times the water depth equaled and exceeded $5 \mathrm{ft}$ is shown in figure 12 ( $p .19$ ). In nine basins, the water depth equaled or exceeded $5 \mathrm{ft}$ once, and in one basin, it exceeded the 5 -ft depth

Note: Set nail at top of measuring stick for flush fit with cap.

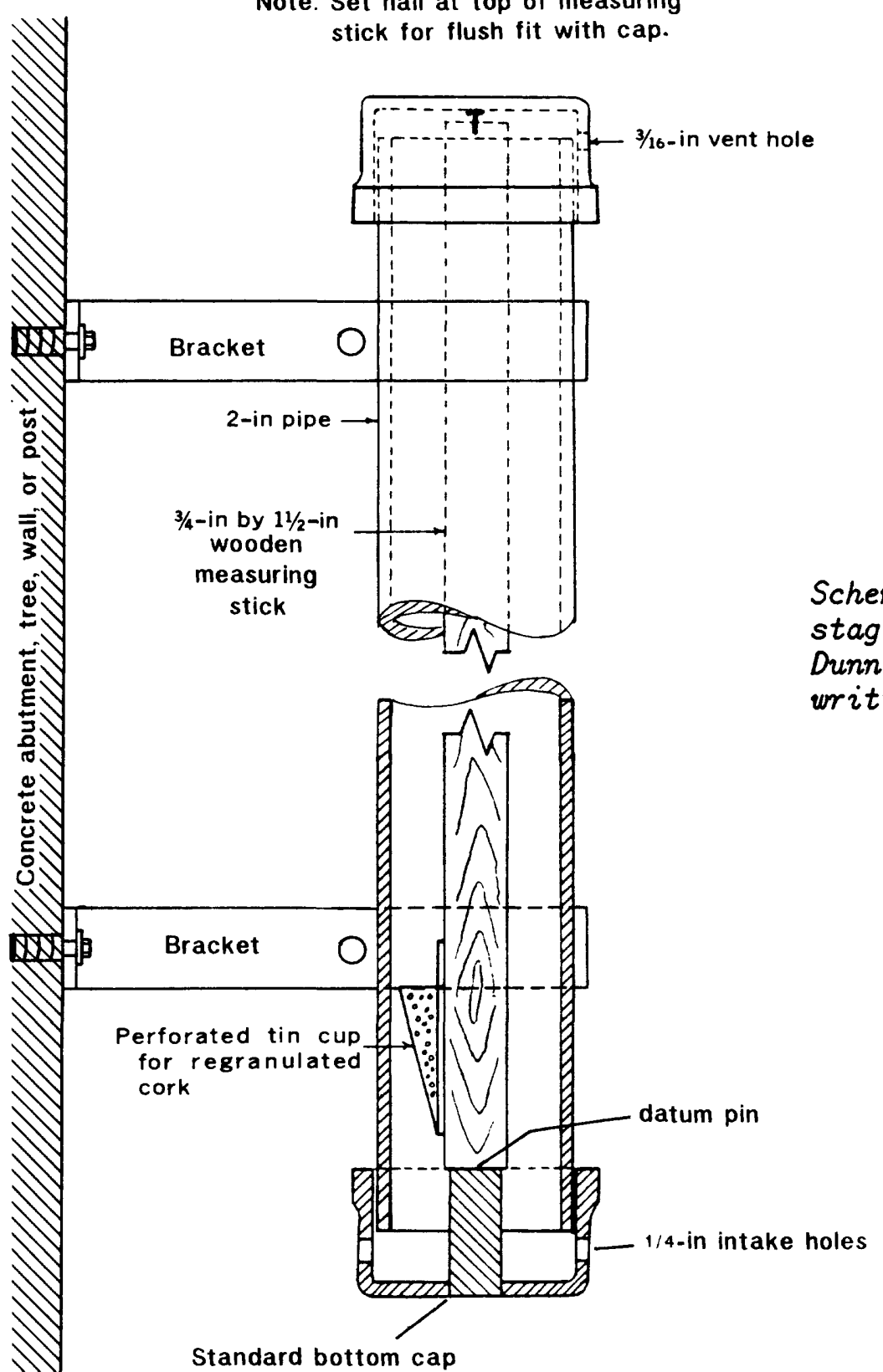

Figure 9.

Schematic drawing of creststage gage. (From Bernard Dunn, U.S. Geological Survey, written commun., 1979.)

Standard bottom cap 
Table 4.--Recharge basins with crest-stage gages

[Locations described in appendix]

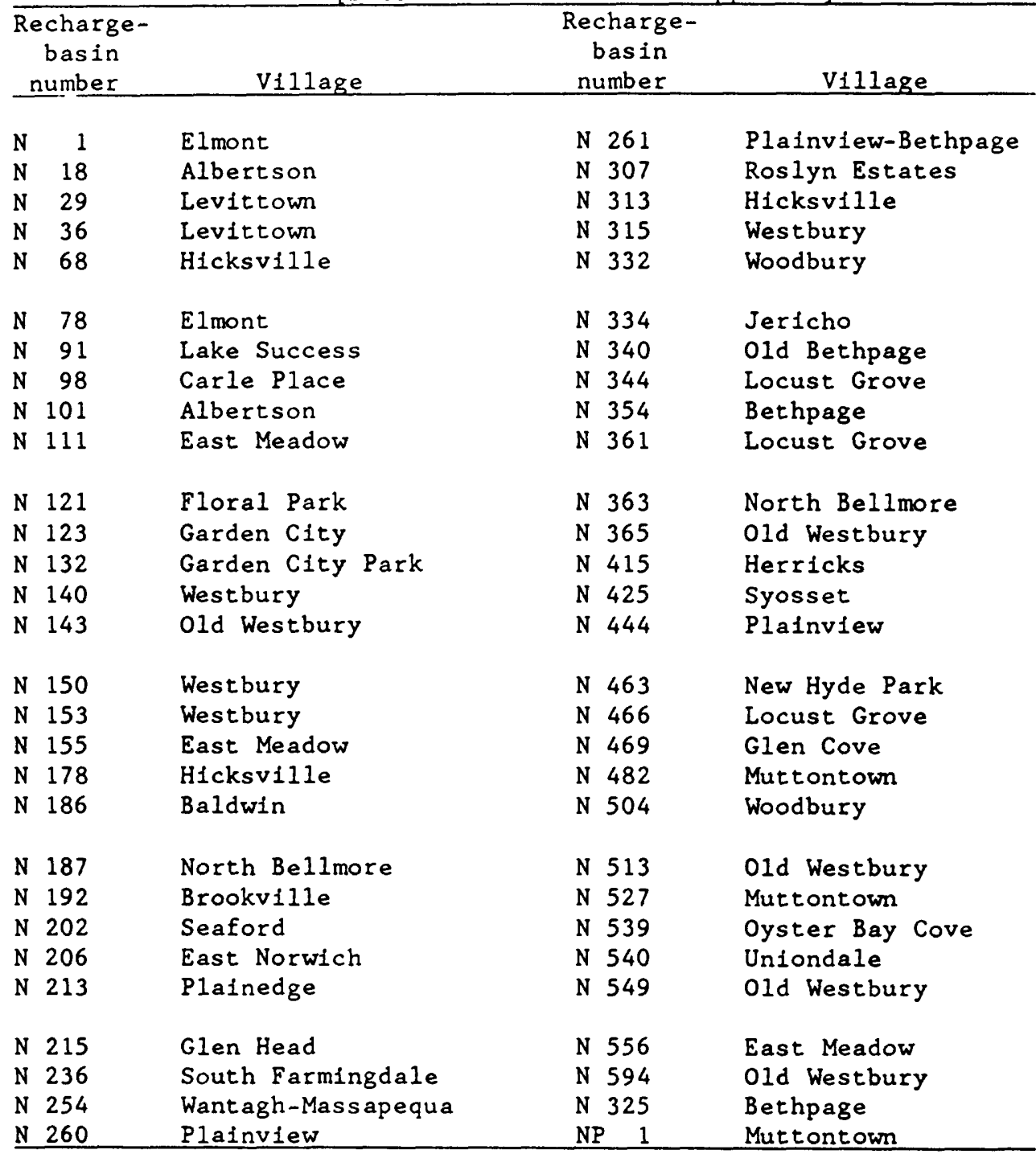

five times. Two basins have incomplete records as a result of vandalism. The largest storm during the study produced 2.9 in. of rain in 24 hours.

Although 20 basins contained $5 \mathrm{ft}$ or more of stormwater at least once, most of them contained no water a few days after the storm, and none indicated that overflow had occurred. Several of the basins that reached a 5 -ft water depth are considered to be clogged. These basins were not dry when visited after heavy rainfall ( 2.9 in. per $24 \mathrm{~h}$ ), but the water levels had returned to near normal (pre-storm) depth (less than $2 \mathrm{ft}$ ). Of the 58 recharge basins with crest-stage gages, $38 \mathrm{did}$ not become ponded to $5 \mathrm{ft}$ at any time. Most recharge basins are at least $10 \mathrm{ft}$ deep, and all are designed for 5-in. storms (in $24 \mathrm{~h}$ ) without infiltration and overflow. Therefore, the amount of rainfall during this study was inadequate to determine the excess storage capacity of the gaged basins. 

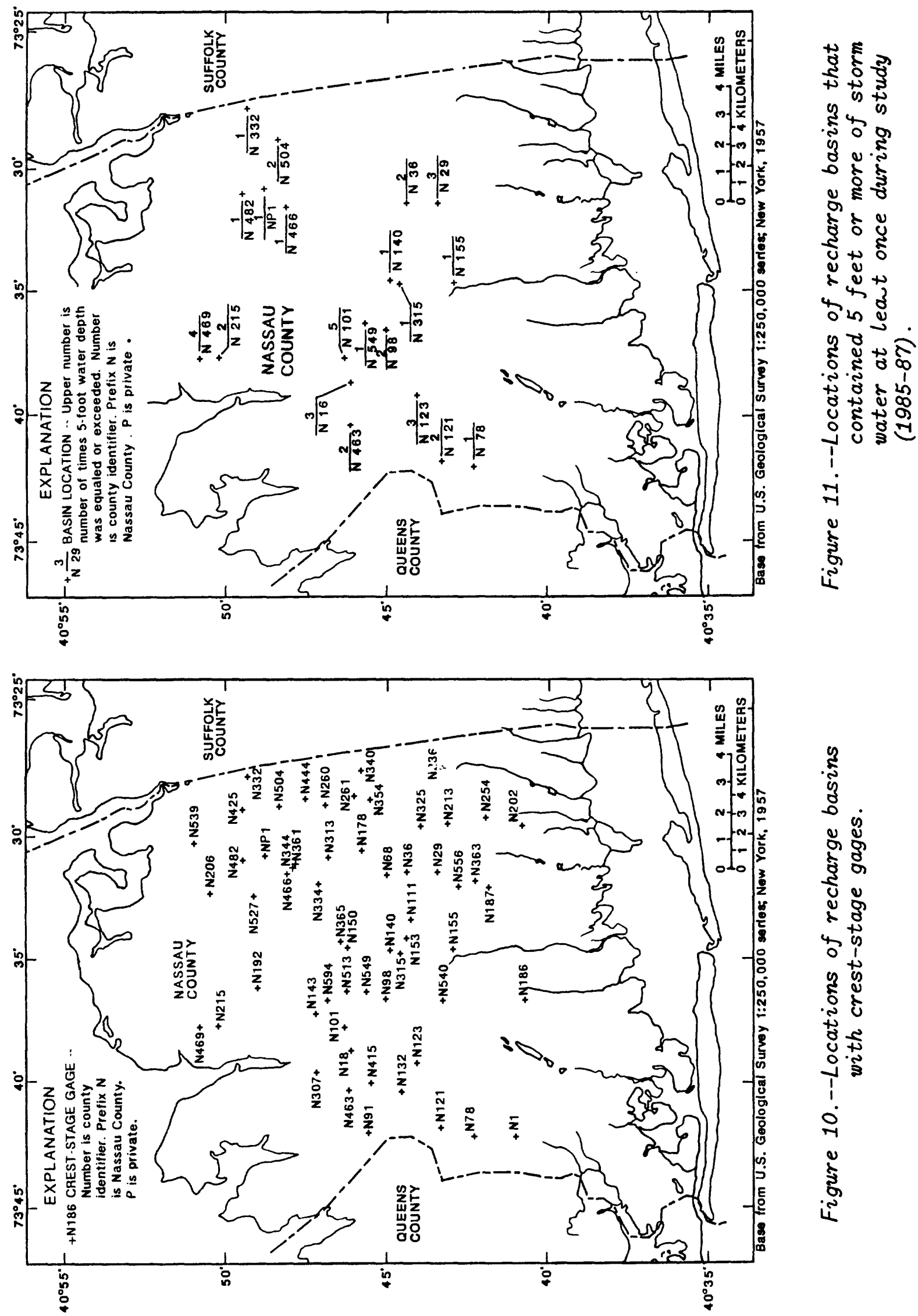


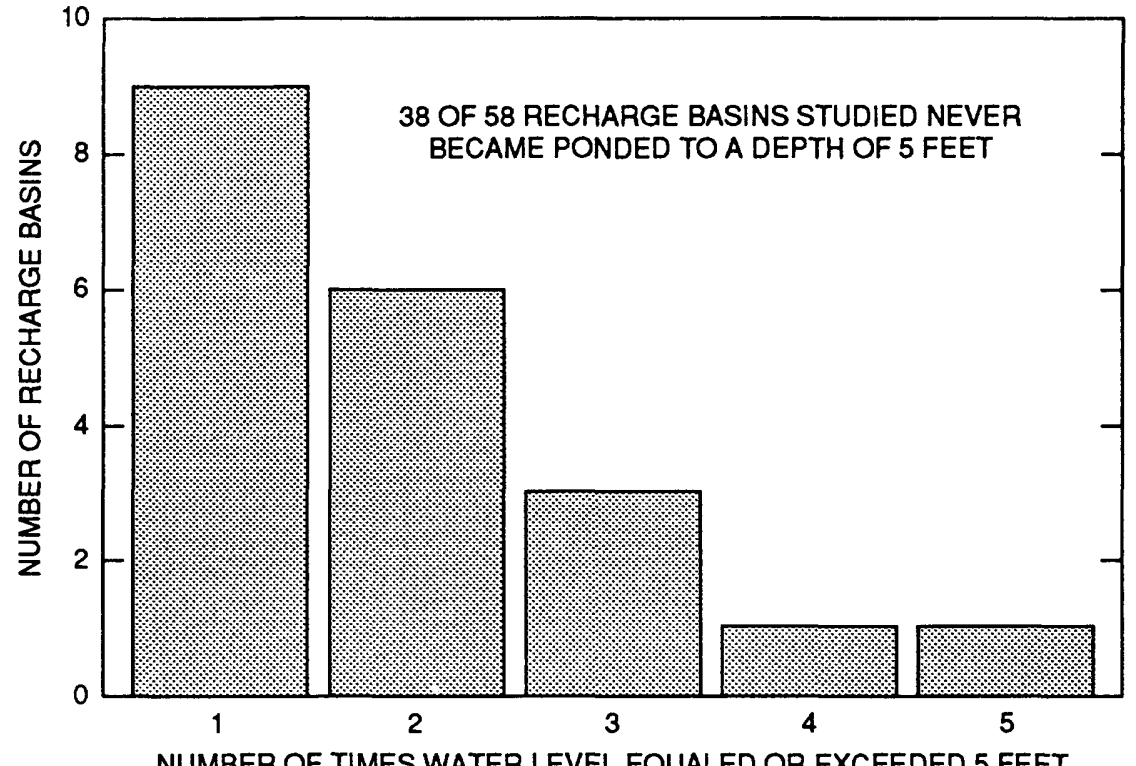

NUMBER OF TIMES WATER LEVEL EQUALED OR EXCEEDED 5 FEET
Figure 12.

Number of times water depth in basins equaled or exceeded 5 feet during study (1985-87).

\section{METHODS OF DETERMINING INFILTRATION RATE AND HYDRAULIC CONDUCTIVITY}

Concern as to the ability of recharge basins on Long Island to dispose of stormwater has prompted several studies of basin infiltration in the past. Prill and Aronson (1978) described a ponding test at a basin excavated in outwash. Aronson and Seaburn (1974) monitored three recharge basins. Ku and Simmons (1986) investigated the water quality and hydrologic properties of five recharge basins in Nassau and western Suffolk Counties. The 1985-87 study expanded on these previous studies to depict the areal distribu-tion of basin recharge rates.

\section{Selection of Test Basins}

Recharge basins in all parts of the county were selected as test sites for evaluation of their ability to transmit runoff and their average annual recharge. Selection of test sites was done on the basis of geographical distribution, surficial geology, and age of the basin. The selected sites represent the outwash plain, morainal deposits, and sandy or clayey soils. total of 51 test basins were selected for study (fig. 13). Several points (called sites A through F) were selected within each basin for infiltration tests. Table 5 lists the recharge basin number, the nearest village, the site of the infiltration test within the basin (sites A through $F$, all within $50 \mathrm{ft}$ of the stormwater inlet), and the starting date and time of the test.

Instruments used to conduct the tests included a double-ring infiltrometer, crest-stage gages, and tensiometers. Other considerations in the selection of test basins were the availability of a nearby fire hydrant (source of water) and a point within the basin that was relatively secure for the installation of the crest-stage gages, which were permanently mounted in most of the basins. 
Table 5.--Recharge basins tested for infiltration rates

[Basin locations shown in f1g. 13. Dashes indicate no test]

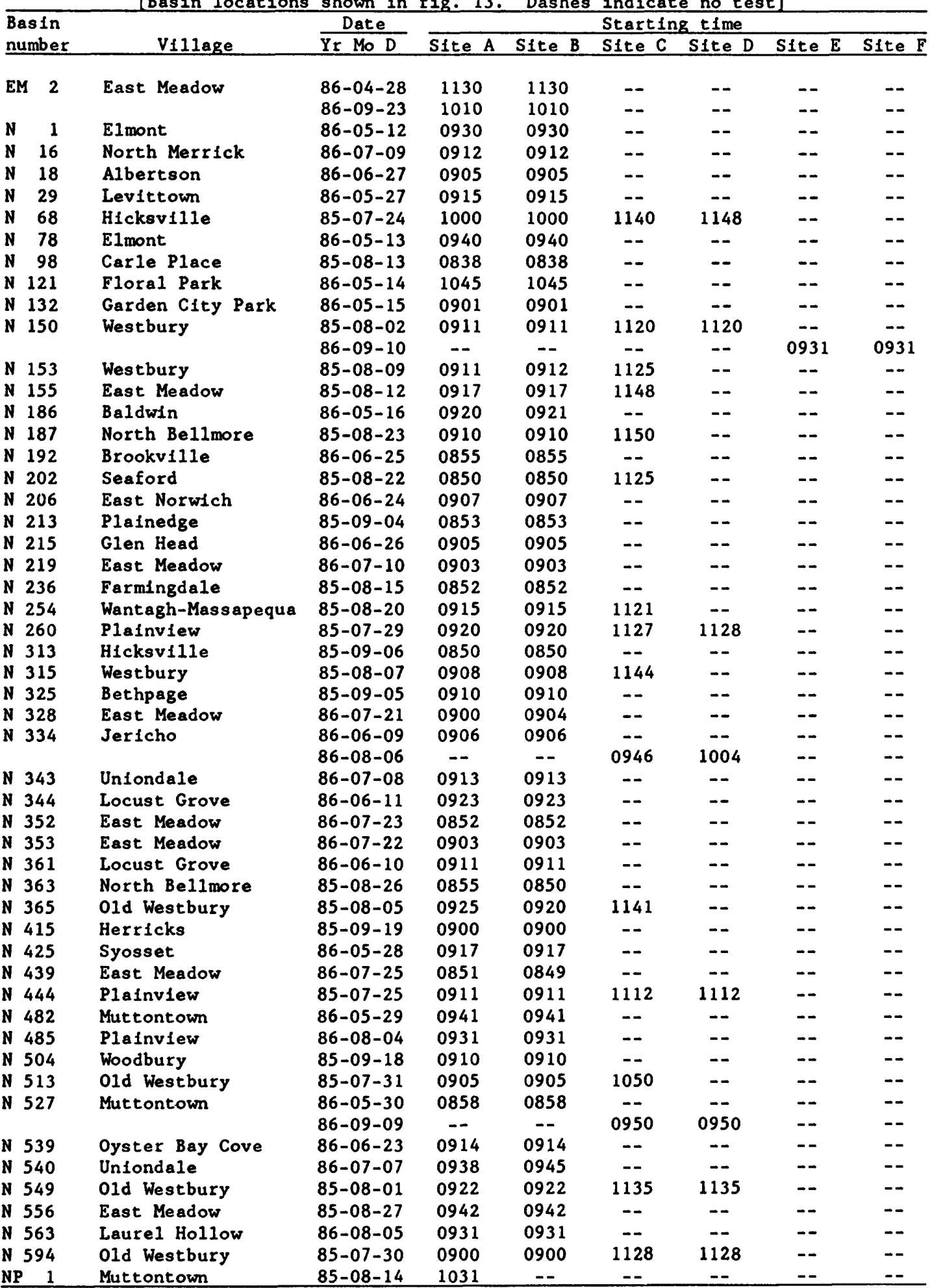




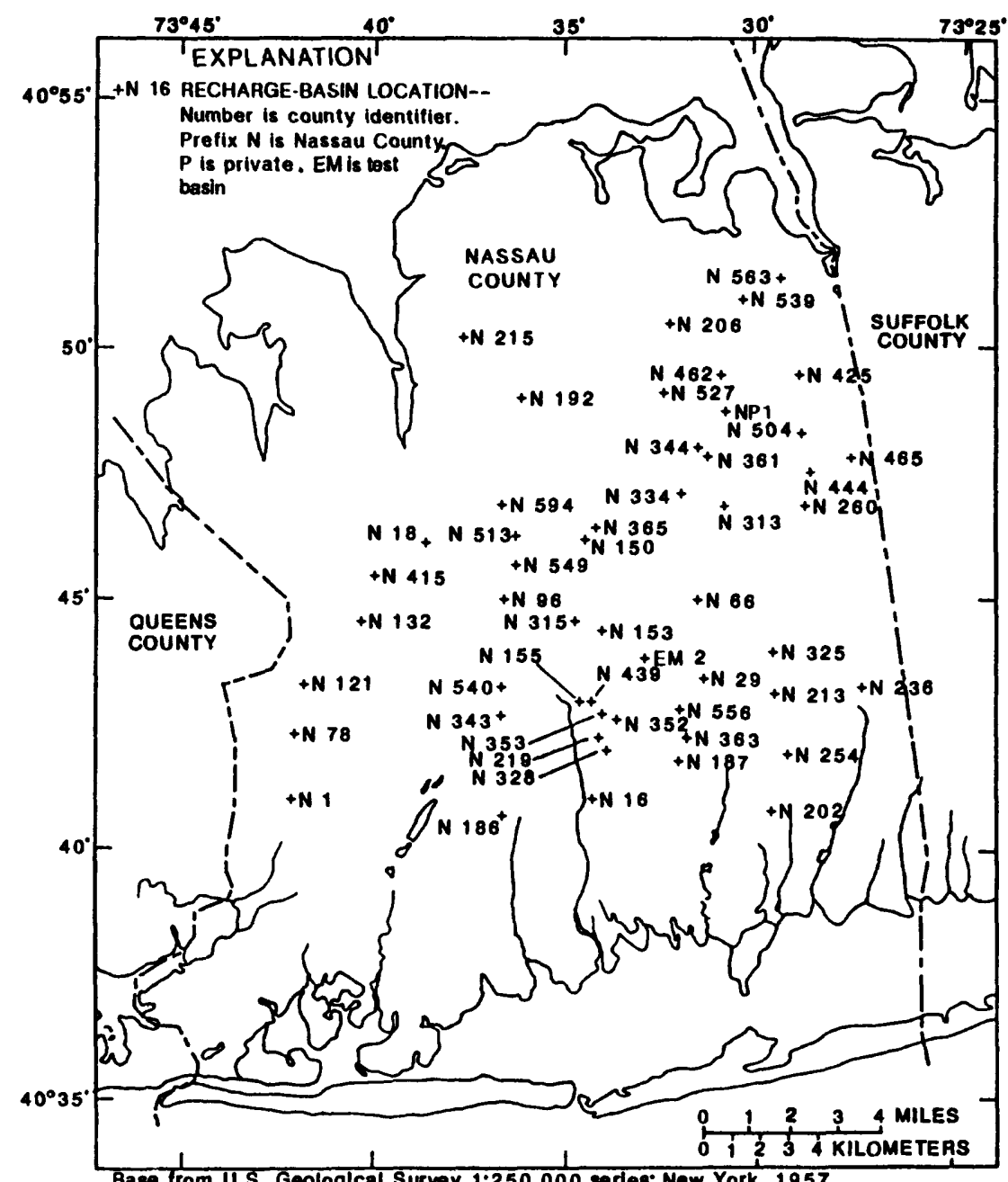

Figure 13.

Locations of recharge basins tested for infiltration rate. (Locations are listed in table 5.)

\section{Double-Ring Infiltrometer}

The double-ring infiltrometer was selected for this study because it is easy to assemble and set up in the field and gives consistent results (Musgrave and Holtan, 1964; Slater, 1957). The device consists of two concentric cylinders (fig. 14). Both are filled with water, and the rate of infiltration into the soil is calculated. The double-ring design minimizes nonvertical flow because the water in the outer ring forms a buffer area. The infiltration rate measured in the center ring was assumed to be indicative of the vertical component of flow. A 15-in. Inside diameter was selected because a ring diameters of at least $4 \mathrm{in}$. give considerably more consistent results than rings of smaller diameter (Slater, 1957; Aronovici, 1955). The outer ring was made of 1/4-in.-thick steel with a diameter of $30 \mathrm{in}$. and a height of 24 in. (fig. 14). Two bent 1/2-in. steel round bars were installed at the top of the ring to serve as handles. A vertical slot 6 in. long and $9 / 16$ in. wide was cut into the side $4 \mathrm{in}$. below the top to provide an overflow. The bottom edge of the ring was bevelled to facilitate twisting into the ground. The inner ring also was made of 1/4-in.-thick steel and was 24 in. high with an inside diameter of $15 \mathrm{in.} \mathrm{All} \mathrm{other} \mathrm{specifications} \mathrm{were} \mathrm{the} \mathrm{same} \mathrm{as} \mathrm{for} \mathrm{the}$ outer ring, except that the inner ring had no overflow slot. 

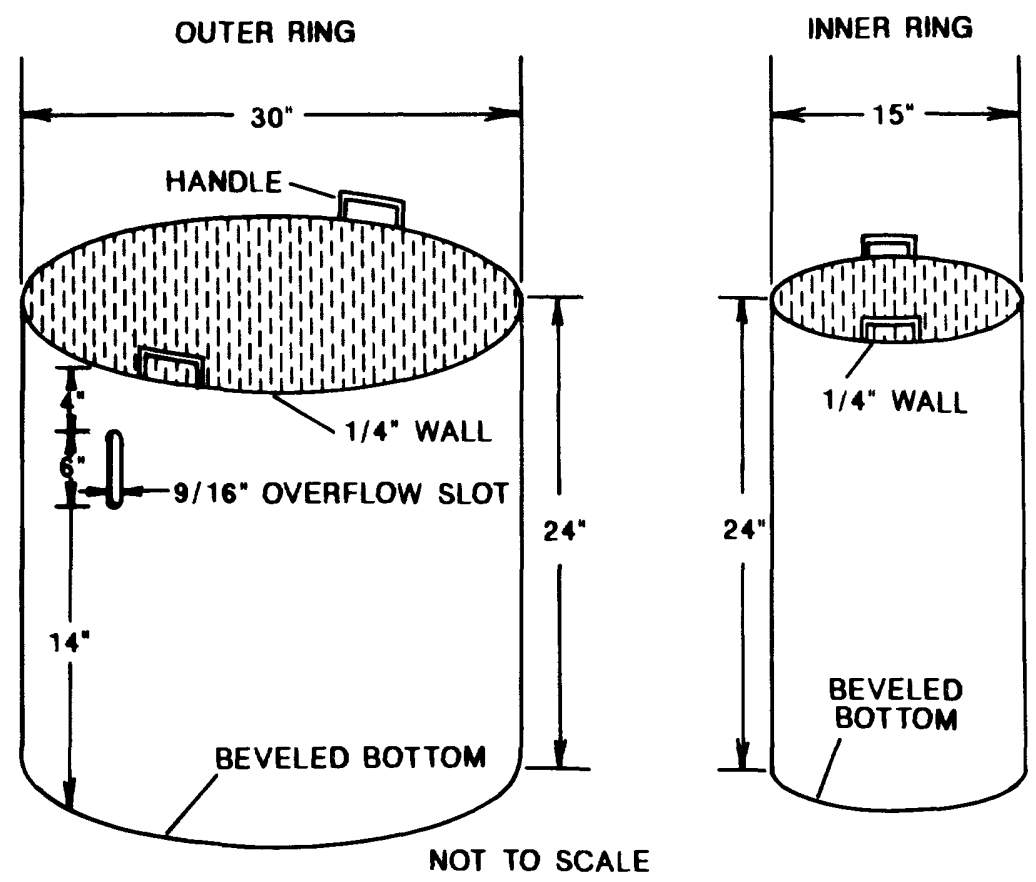

Figure 14 .

Double-ring

infiltrometer.

Recharge basins become only partly flooded during most storm-runoff periods. The runoff usually collects near the outfall apron and infiltrates into the ground within a few hours after the cessation of runoff. The extent of the ponded area is evident from the soll and vegetation; the flooded areas generally have dark, fine-grained topsoil with a luxuriant stand of vegetation, whereas the rest generally has sparse vegetation with exposed sandy soil. The areas near the outfall aprons were selected for installation of the double-ring infiltrometer. Generally these sites were no more than $50 \mathrm{ft}$ from the apron.

Each recharge basin was allowed to dry after a rainfall. A dry area near the inflow was chosen, and rocky and pebbly areas were avoided to prevent difficulty in sealing the bottom of the double-ring infiltrometer against leakage. In general, one site at the level of the bottom of the inflow pipe and one at a high elevation were chosen. First the outer ring, then the inner ring, were carefully driven 4 to 6 in. into the ground by rotating and pounding. Care was taken to minimize the disturbance of the soil and surrounding vegetation within the rings. The rings were seated into the soll, and additional soll was piled against the outside of the outer ring to reduce the likelihood of leakage.

After multiple sets of double-ring infiltrometers were installed (a maximum of two sets per basin at any given time), municipal water was brought in from the nearest fire hydrant or from storage tanks. The water supply was divided between two hoses near each infiltrometer. One line fed water into the outer ring, and the water level in this ring was maintained at 10 to 12 in. above ground surface by manual adjustment of the flow rate. Excess water was channeled out of the outer ring through the slot on its side. The other hose fed water into the inner ring. The amount of water flowing into the inner ring was monitored by a water meter that was accurate to \pm 1 percent. A float-andvalve system automatically maintained the water leve $\bar{l}$ in the inner ring at $1 \mathrm{ft}$ above land surface. 
The initial reading on the flow meter was recorded. Before any water was fed into the inner ring, the inner and outer rings were allowed to fill to the $1-\mathrm{ft}$ level. After the $1-\mathrm{ft}$ level was reached in the inner ring, the total number of gallons needed to maintain that level in the inner ring, and the water temperature, were recorded at regular intervals, usually every $15 \mathrm{~min}$.

At the end of the test, the infiltration rate was calculated for each 15-min. interval between readings. The number of gallons recorded by the flowmeter was then converted to cubic feet per hour, and this flow rate was then converted to infiltration rate by dividing by $1.23 \mathrm{ft}^{2}$ (the area of the bottom of the inner ring). The resulting infiltration rate is expressed in feet per hour.

\section{Water-Manometer Tensiometer}

To compute vertical hydraulic conductivity from the infiltration data collected with the double-ring infilircmeter, a water-manometer tensiometer was installed to measure head at depth. Clogging at the recharge basin floor generally forms a zone that controls the infiltration rate. During recharge, flow in this zone is typically saturated and rarely extends more than $1 \mathrm{ft}$ below land surface. Therefore, the tensiometer was installed at a depth of $1 \mathrm{ft}$ to monitor the head values needed to compute the saturated vertical hydraulic conductivity.

Water-manometer tensiometers were installed before constant-head infiltration tests with the double-ring infiltrometer at nine recharge basins and at the East Meadow recharge site (Schneider and others, 1987, fig. 1). Vertical hydraulic conductivity is computed from Darcy's equation expressed in terms of hydraulic conductivity (Prill and Aronson, 1978, p. 17) by

$$
k=\frac{q}{d h / d l+1} \frac{v_{2}}{v_{1}},
$$

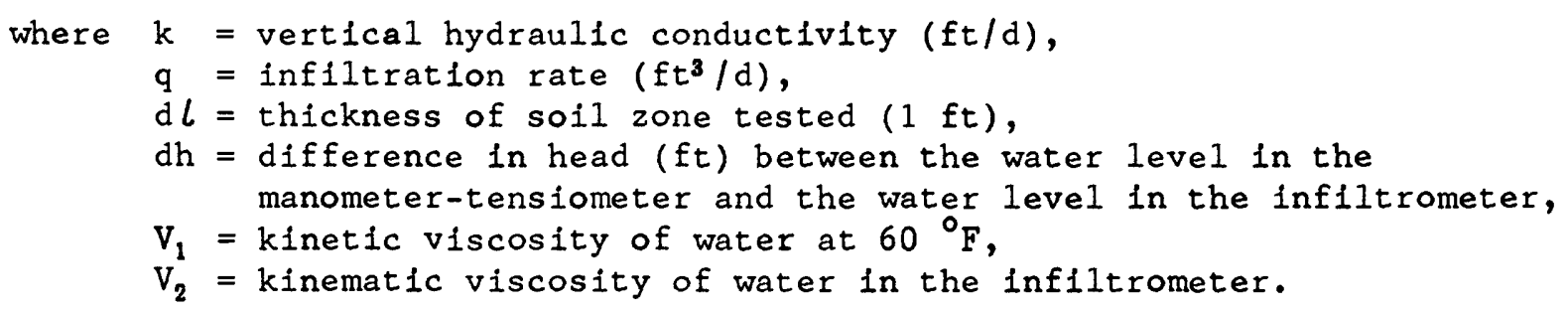

A generalized diagram of a water-manometer tensiometer, placed 1 ft below land surface $(l=1 \mathrm{ft})$ and showing the location of the measured properties for the above equations, is shown in figure 15 .

The operation and use of water manometers installed and read from land surface is described by 0aksford (1978). The modified tensiometer built for use in this study is made from $1 / 4-i n$. (inside diameter) plastic tubing that is bent into a $\mathrm{J}$ shape (fig. 15). The short arm of the $\mathrm{J}$ is exactly $1 \mathrm{ft}$ long and covered with porous membrane (0.015-mesh stainless-steel screen). The system is operated by filling the $J$ tube with gas-free water and placing it between the inner and outer rings in an augered hole $2 \mathrm{ft}$ deep. The hole is 


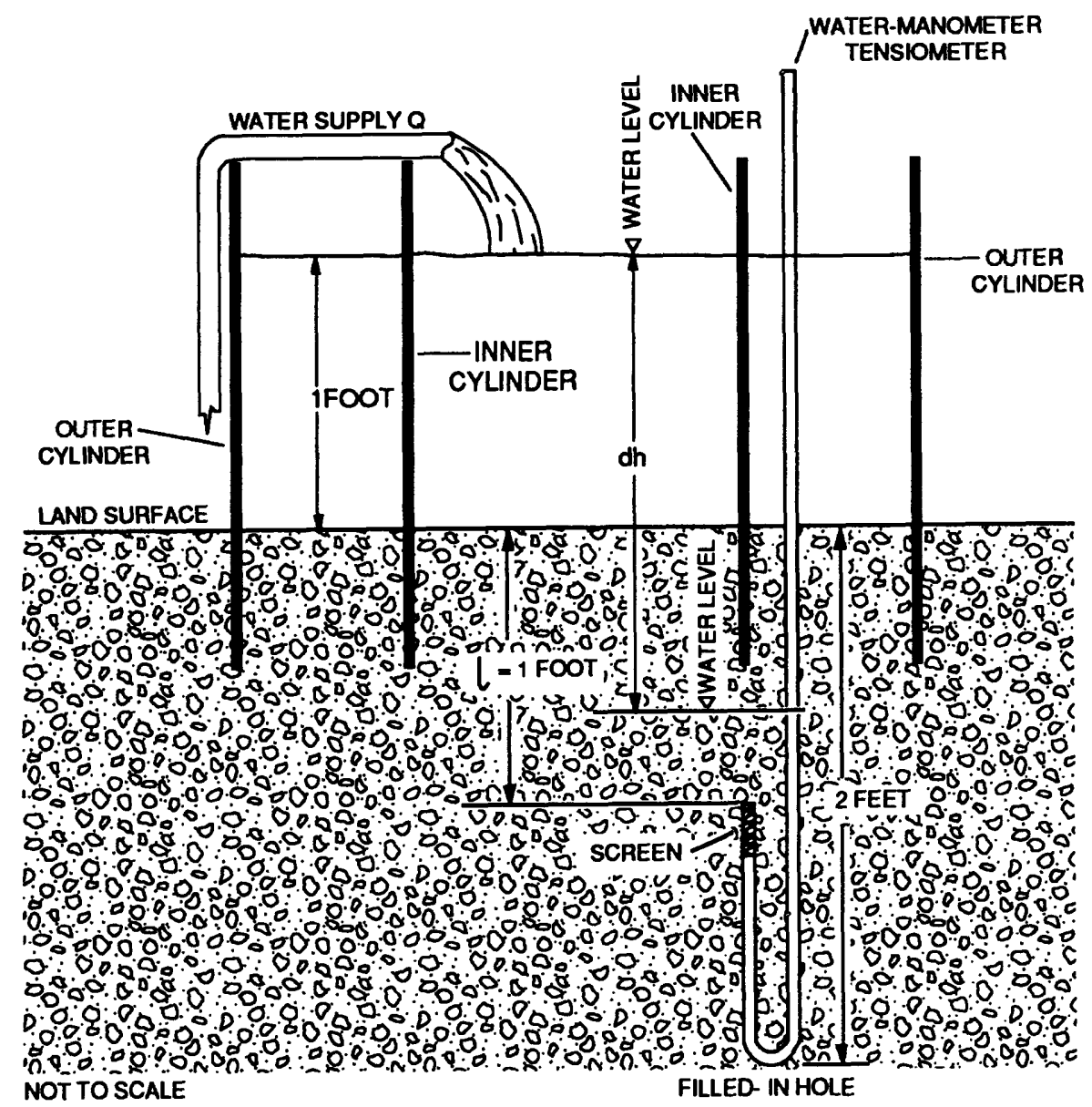

Figure 15.--Field installation of water-manometer tensiometer.

positioned as equidistant from the rings as possible without greatly disturbing the soll column. The tensiometer is then lowered into the hole with the screened end placed $1 \mathrm{ft}$ below the land surface $(l=1 \mathrm{ft})$. Voids between the unit and the soll are carefully backfilled with previously excavated material (fig. 15). The principle of tensiometer operation is straightforward. As the water content of the soll decreases, water moves into the soll through the porous membrane, which draws down the water in the manometer until the pressure outside the porous membrane is balanced by the height of the water in the manometer. The converse is true when the water content of the soil is increased, as is the case during the infiltration tests.

The water level in the manometer is measured with a coaxial wire and ohmmeter. One end of the coaxial wire is connected to the ohmmeter, and the other is lowered through the tube for contact with the water. The wire is stripped, as shown in figure 16, to avoid measurement interference by water droplets at the end of the wire. An index mark is placed on the wire such that when the wire is inserted into the manometer tube, the index mark will be at the zero point on the scale when the outer conductor is even with the top of the porous membrane. This calibrated wire is then lowered into the manometer tube until the outer conductor touches water. The point of contact is indicated by the sharp decrease in resistance measured in the ohmmeter. 
Figure 16.

Details of coaxial-wire preparation. (Modified from Oaksford, 1978, fig. 5.)

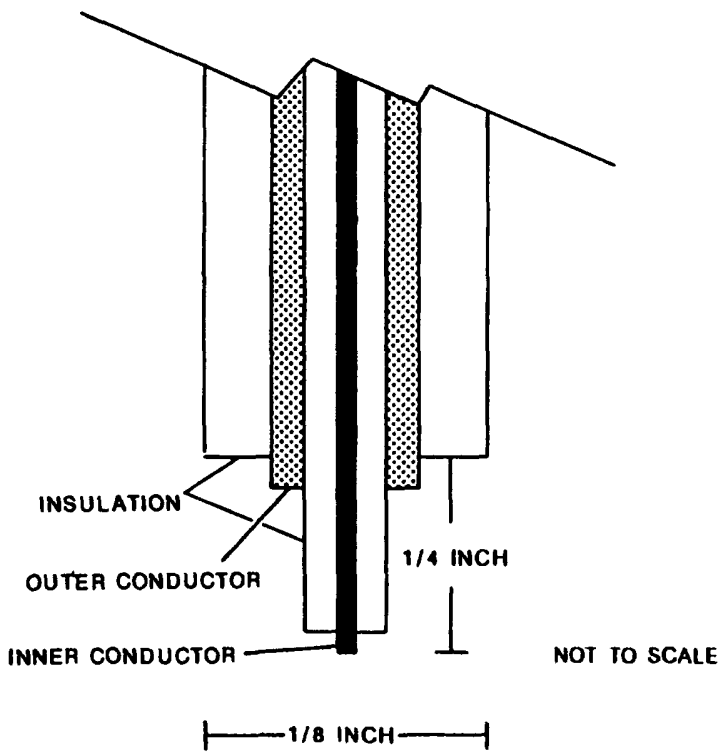

\section{RATES OF WATER MOVEMENT}

\section{Areal Infiltration Rates}

In addition to the one basin at the East Meadow recharge site, 51 recharge basins were tested for infiltration rates (table 6 and $\mathrm{flg} .17$ ). As indicated in table 6 , at least two infiltration tests were performed at all but two recharge basins. The infiltration tests were performed between July 24, 1985 and September 9, 1986. Most of the tests lasted about $4 \mathrm{~h}$, but a few lasted only about $2 \mathrm{~h}$. The last infiltration rate obtained during the test, calculated from the last reading, is used as the representative of the infiltration rate for that area. The last reading was used because the infiltration rate needs to be stabilized (fig. 18).

The lowest individual infiltration rate was $0 \mathrm{ft} / \mathrm{h}$, the median individual infiltration value was $1.72 \mathrm{ft} / \mathrm{h}$, and the maximum individual rate was 9.75 $\mathrm{ft} / \mathrm{h}$. The average infiltration rate for each recharge basin is shown in figure 17 and table 6 . The minimum, median, and maximum averaged values for the 51 test basins are $0.13 \mathrm{ft} / \mathrm{h}, 1.83 \mathrm{ft} / \mathrm{h}$, and $5.63 \mathrm{ft} / \mathrm{h}$, respectively. A summary of infiltration rates is shown below:

\begin{tabular}{lcc}
\hline & \multicolumn{2}{c}{$\begin{array}{c}\text { Infiltration rates } \\
\text { (1n feet per hour) }\end{array}$} \\
\cline { 2 - 3 } & $\begin{array}{c}\text { Ind1v1dual value } \\
\text { (126 tests) }\end{array}$ & $\begin{array}{c}\text { Averaged basin } \\
\text { value }(51 \text { basins) }\end{array}$ \\
\hline Minimum & 0 (approx.) & 0.13 \\
Median & 1.72 & 1.83 \\
Maximum & 9.75 & 5.63 \\
\hline
\end{tabular}
follows:

The infiltration rates obtained in previous studies are summarized as 
[Locations are indicated in the appendix; $\mathrm{ft} / \mathrm{h}$, feet per hour; a dash indicates no data.]

\begin{tabular}{|c|c|c|c|}
\hline Basin & $\begin{array}{l}\text { Average } \\
\text { inf } 11 \text { tration } \\
\text { rate }(\mathrm{ft} / \mathrm{h})\end{array}$ & $\begin{array}{c}\text { Range of } \\
\text { infiltration } \\
\text { rate }(\mathrm{ft} / \mathrm{h})\end{array}$ & Source \\
\hline $\begin{array}{l}\text { N156 } \\
\text { N377 } \\
\text { N123 } \\
\text { East Meadow } \\
\text { N197 }\end{array}$ & $\begin{array}{r}0.9 \\
.8 \\
-- \\
-- \\
.94 \\
\end{array}$ & $\begin{array}{l}0.3-1.7 \\
.3-1.8 \\
.11-2.23 \\
.44-1.5 \\
.84-2.21 \\
\end{array}$ & $\begin{array}{l}\text { Aronson and Seaburn, } 1974 \\
\text { Aronson and Seaburn, } 1974 \\
\text { Brice and others, } 1956 \\
\text { Schneider and others, } 1987 \\
\text { Pr111 and Aronson, } 1978\end{array}$ \\
\hline
\end{tabular}

The results of this and previous studies indicate that average infiltration rates, in general, are 1 to $2 \mathrm{ft} / \mathrm{h}$, although the individual values differed widely as a result of such factors as land use, geology, age of the basin, duration of the tests, and water temperature. (See section on "Change of infiltration rates during test period".) Among the 126 sites tested, the difference between the maximum and minimum value is almost an order of magnitude.

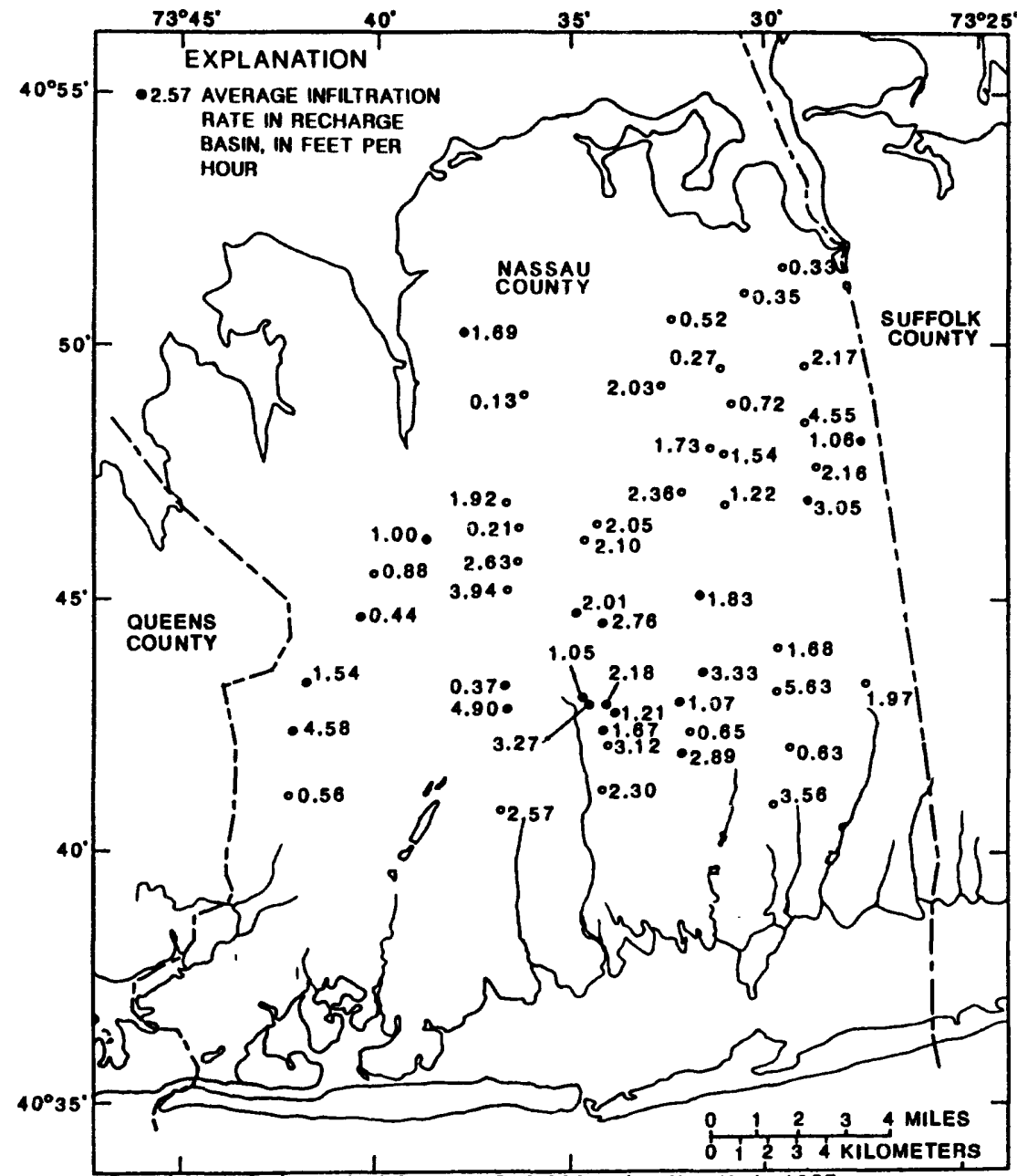

Figure 17.--Average infiltration rates at 51 selected recharge basins in Nassau County. 
Table 6.--Individual and averaged infiltration rates at selected recharge basins, $1985-86$

[Locations are shown on fig. 13. Measurements are in feet per hour;

average infiltration rates are shown in $\mathrm{fig}$. 17]

\begin{tabular}{|c|c|c|c|c|c|c|c|c|c|}
\hline \multirow{2}{*}{\multicolumn{2}{|c|}{$\begin{array}{l}\text { Basin } \\
\text { number }\end{array}$}} & \multirow{2}{*}{\multicolumn{2}{|c|}{$\frac{\text { Infiltration rate }}{\text { Individual }}$}} & \multirow{2}{*}{$\begin{array}{l}\text { Basin } \\
\text { number }\end{array}$} & \multicolumn{2}{|c|}{ Infiltration rate } & \multirow{2}{*}{$\begin{array}{l}\text { Basin } \\
\text { number }\end{array}$} & \multicolumn{2}{|c|}{ Infiltration rate } \\
\hline & & & Average & & Individual ${ }^{1}$ & Average & & Individual & Average \\
\hline $\mathrm{N}$ & 1 & $\begin{array}{r}0.62 \\
.53\end{array}$ & 0.58 & N 213 & $\begin{array}{l}1.50 \\
9.75\end{array}$ & 5.63 & $N 415$ & $\begin{array}{l}0.11 \\
1.65\end{array}$ & 0.88 \\
\hline N & 16 & $\begin{array}{l}2.30 \\
2.30\end{array}$ & 2.30 & N 215 & $\begin{array}{l}1.52 \\
1.85\end{array}$ & 1.69 & N 425 & $\begin{array}{l}3.03 \\
1.30\end{array}$ & 2.17 \\
\hline N & 18 & $\begin{array}{r}.95 \\
1.04\end{array}$ & 1.00 & N 219 & $\begin{array}{l}1.39 \\
1.95\end{array}$ & 1.67 & N 439 & $\begin{array}{l}2.86 \\
3.68\end{array}$ & 3.27 \\
\hline N & 29 & $\begin{array}{l}1.71 \\
4.94\end{array}$ & 3.33 & N 236 & $\begin{array}{r}.56 \\
3.38\end{array}$ & 1.97 & N 444 & $\begin{array}{l}2.34 \\
4.16 \\
1.49\end{array}$ & 2.16 \\
\hline N & 68 & $\begin{array}{l}1.36 \\
1.15 \\
3.15 \\
1.65\end{array}$ & 1.83 & N 254 & $\begin{array}{r}2.03 \\
.05 \\
.40\end{array}$ & .83 & N 482 & $\begin{array}{r}.65 \\
.91 \\
.14\end{array}$ & .27 \\
\hline $\mathrm{N}$ & 78 & $\begin{array}{l}5.00 \\
4.16\end{array}$ & 4.58 & N 260 & $\begin{array}{l}2.47 \\
2.14 \\
2.73 \\
4.87\end{array}$ & 3.05 & N 485 & $\begin{array}{r}.82 \\
1.34\end{array}$ & 1.08 \\
\hline $\mathrm{N}$ & 98 & $\begin{array}{l}2.81 \\
5.07\end{array}$ & 3.94 & N 313 & $\begin{array}{r}.30 \\
1.13\end{array}$ & 1.22 & $\begin{array}{l}\text { N } 504 \\
\text { N } 513\end{array}$ & $\begin{array}{l}4.55 \\
0\end{array}$ & $\begin{array}{r}4.55 \\
.21\end{array}$ \\
\hline $\mathbf{N}$ & 121 & $\begin{array}{l}1.73 \\
1.34\end{array}$ & 1.54 & N 315 & $\begin{array}{l}2.27 \\
3.47\end{array}$ & 2.01 & & $\begin{array}{r}.06 \\
.58\end{array}$ & \\
\hline $\mathbf{N}$ & 132 & $\begin{array}{l}.43 \\
.44\end{array}$ & .44 & & .29 & & N 527 & $\begin{array}{l}1.91 \\
2.34\end{array}$ & 2.03 \\
\hline $\mathrm{N}$ & 150 & $\begin{array}{l}3.12 \\
2.01\end{array}$ & 2.10 & 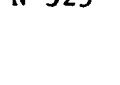 & 2.06 & 1.00 & & $\begin{array}{l}2.04 \\
1.82\end{array}$ & \\
\hline & & $\begin{array}{l}1.73 \\
1.86 \\
2.25\end{array}$ & & N 328 & $\begin{array}{l}3.16 \\
3.08\end{array}$ & 3.12 & N 539 & $\begin{array}{l}.26 \\
.43\end{array}$ & .35 \\
\hline & & 1.60 & & N 334 & $\begin{array}{l}4.77 \\
2.99\end{array}$ & 2.38 & N 540 & $\begin{array}{r}.35 \\
.39\end{array}$ & .37 \\
\hline $\mathbf{N}$ & 153 & $\begin{array}{l}2.50 \\
3.64 \\
2.15\end{array}$ & 2.76 & & $\begin{array}{r}.48 \\
1.26\end{array}$ & & N 549 & $\begin{array}{l}3.44 \\
3.08\end{array}$ & 2.63 \\
\hline $\mathrm{N}$ & 155 & 2.18 & 1.05 & N 343 & $\begin{array}{l}4.81 \\
4.98\end{array}$ & 4.90 & & $\begin{array}{r}3.13 \\
.87\end{array}$ & \\
\hline & & .05 & & N 344 & $\begin{array}{r}2.64 \\
.82\end{array}$ & 1.73 & N 556 & $\begin{array}{r}.74 \\
1.39\end{array}$ & 1.07 \\
\hline $\mathbf{N}$ & 186 & $\begin{array}{l}3.38 \\
1.76\end{array}$ & 2.57 & N 352 & $\begin{array}{r}.87 \\
1.54\end{array}$ & 1.21 & N 563 & $\begin{array}{l}.09 \\
.56\end{array}$ & .33 \\
\hline $\mathrm{N}$ & 187 & $\begin{array}{l}2.21 \\
3.07 \\
3.38\end{array}$ & 2.89 & N 353 & $\begin{array}{l}2.21 \\
2.14\end{array}$ & 2.18 & N 594 & $\begin{array}{r}.47 \\
2.06 \\
1.10\end{array}$ & 1.92 \\
\hline $\mathrm{N}$ & 192 & $\begin{array}{l}.22 \\
.04\end{array}$ & .13 & N 361 & $\begin{array}{l}1.56 \\
1.52\end{array}$ & 1.54 & & 4.03 & \\
\hline $\mathrm{N}$ & 202 & $\begin{array}{l}2.95 \\
3.30 \\
4.42\end{array}$ & 3.56 & N 363 & $\begin{array}{l}.39 \\
.91\end{array}$ & .65 & & 176 & 10 \\
\hline $\mathrm{N}$ & 206 & $\begin{array}{r}.35 \\
.69\end{array}$ & .52 & N 365 & $\begin{array}{l}1.30 \\
1.60 \\
3.25\end{array}$ & 2.05 & & & \\
\hline
\end{tabular}

1 Last reading during an infiltration test was used to calculate infiltration rate. 
The average infiltration rates of basins tested during this study (fig. 17) show that basins in the northern part of the county, which coincides with the area of poorly drained soil (fig. 3 ), perched water (fig. 4), and ground and terminal moraine deposits ( $p 1.1$ ), tend to have lower infiltration rates than those elsewhere. Basin N1, built in 1951 (see appendix) has an average infiltration rate of $0.58 \mathrm{ft} / \mathrm{h}$ and may be an example of a basin whose age is a factor in the decreased infiltration rate. (See section "Clogged Recharge Basins" and "Vertical Hydraulic Conductivity.")

\section{Change in Infiltration Rate During Test Period}

The trend of change in infiltration rate during tests was similar at all basins. During the initial minutes of a test, the infiltration rate was high but then declined and tended to stabilize after 80 to 120 minutes because water enters the soil by gravity and vertical matric potential gradient within the soll (soil suction). Although gravity remains constant, the matric potential gradient decreases over time as the soil becomes saturated. This observation is consistent with results of a study by Prill and Aronson (1978). The change in infiltration rate after the double-ring infiltrometer was filled to $1-f t$ stage is shown by graphs of infiltration rate as a function of ponding duration in figure 18. In many cases, half of the decline in infiltration rate occurred during the first 2 hours.

The infiltration rate obtained from a 2 - to 4-h test probably does not represent the long-term infiltration rate of the basin; however, the infiltration rate obtained probably reflects the natural operating efficiency of the recharge basin during a rainstorm of approximately a $4-h$ duration.

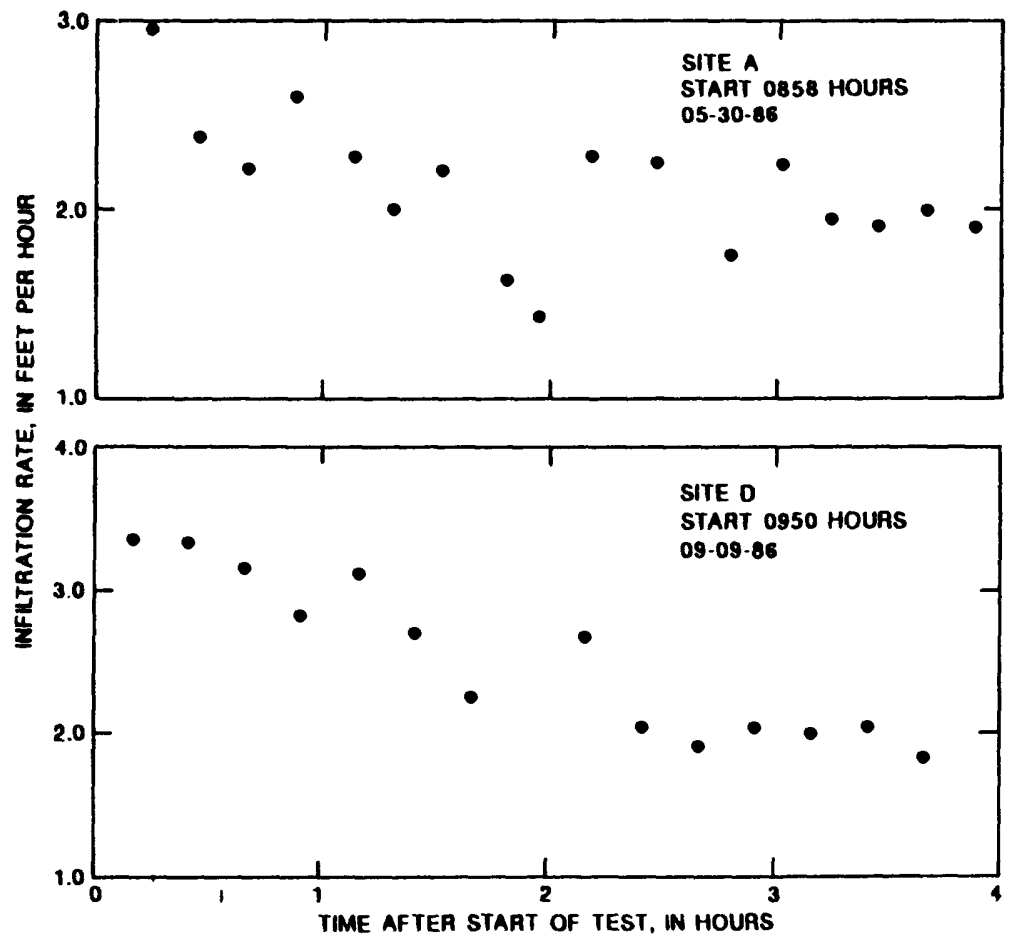

Figure 18.

Change of infiltration rate through time at basin N527. (Location is indicated in table 15 and fig. 19.) 


\section{Vertical Hydraulic Conductivity}

Vertical hydraulic conductivity was measured at 14 points in nine recharge basins. Data from infiltration tests and water-manometer tensiometer tests are combined to trace the movement of water under saturated and unsaturated conditions. During the tests, the water level in the infiltrometer and in the water-manometer tenslometer (fig. 15) was read every $15 \mathrm{~min}$ to obtain dh in eq. 1. The last reading of the day was used to calculate the vertical hydraulic conductivity; results of these tests are shown in table 7 . The hydraulic conductivity values represent the vertical flow rate at the end of each test (after about $4 \mathrm{~h}$ ). The median hydraulic conductivity was $1.63 \mathrm{ft} / \mathrm{h}$ with a range of 0.08 to $5.33 \mathrm{ft} / \mathrm{h}$ at a water-temperature range of $71.6{ }^{\circ} \mathrm{F}$ to $88.7^{\circ} \mathrm{F}$. With this temperature range, the hydraulic conductivity could vary by as much as \pm 20 percent.

In an earlier recharge study with tertiary-treated sewage (for water quality, see Schneider and others, 1987) at the East Meadow Recharge site (site EM 2, fig. 13), Schneider and others (1987) measured the vertical hydraulic conductivity of soil in the 0- to 5-ft and 4- to 5-ft depth intervals of basin 3 and the 0 - to 5 - ft and 0 - to 1 -ft depth intervals of basin 2 . (See table 8.) Both basins had impermeable vertical walls and 5,000 $\mathrm{ft}^{2}$ of basin floor specifically designed for artificial-recharge tests. The ranges

Table 7.--Vertical hydraulic conductivity of basin-floor, material at selected Nassau County recharge basins, 1 y86

\begin{tabular}{|c|c|c|c|}
\hline $\begin{array}{l}\text { Basin } \\
\text { number }\end{array}$ & Site & $\begin{array}{c}\text { Infileration } \\
\text { rate }\end{array}$ & $\begin{array}{c}\text { Vertical hydraulic } \\
\text { conductivity }\end{array}$ \\
\hline N150 & $\begin{array}{l}E \\
F\end{array}$ & $\begin{array}{l}2.25 \\
1.60\end{array}$ & $\begin{array}{l}1.73 \\
1.66\end{array}$ \\
\hline N328 & B & 3.0 & 2.54 \\
\hline N334 & $\begin{array}{l}\text { C } \\
\text { D }\end{array}$ & $\begin{array}{r}.48 \\
1.26\end{array}$ & $\begin{array}{r}.39 \\
1.06\end{array}$ \\
\hline N352 & B & 1.54 & 1.6 \\
\hline N353 & A & 2.21 & 2.43 \\
\hline N439 & B & 3.68 & 5.33 \\
\hline N485 & $\begin{array}{l}\text { A } \\
\text { B }\end{array}$ & $\begin{array}{r}.82 \\
1.34\end{array}$ & $\begin{array}{l}.89 \\
3.1\end{array}$ \\
\hline N527 & $\begin{array}{l}\text { C } \\
\text { D }\end{array}$ & $\begin{array}{l}2.04 \\
1.82\end{array}$ & $\begin{array}{l}1.49 \\
1.93\end{array}$ \\
\hline N563 & $\begin{array}{l}\text { A } \\
\text { B }\end{array}$ & $\begin{array}{l}.09 \\
.56\end{array}$ & $\begin{array}{l}.08 \\
.57\end{array}$ \\
\hline & & $\begin{array}{c}1.62 \\
0.09-3.68 \\
\end{array}$ & $\begin{array}{c}1.63 \\
0.08-5.33 \\
\end{array}$ \\
\hline
\end{tabular}


of vertical hydraulic conductivity for basin 2 at 0 to 5 -ft depth and 0 to 1 - ft depth were 6.1 to $47.7 \mathrm{ft} / \mathrm{d}(0.25$ to $1.98 \mathrm{ft} / \mathrm{h})$ and 2.1 to $28.0 \mathrm{ft} / \mathrm{d}$ $(0.09$ to $1.16 \mathrm{ft} / \mathrm{h})$, respectively (table $8 \mathrm{~B})$. The 0 to 1 - $\mathrm{ft}$ interval was found to be the controlling zone for infiltration rates. During the test, nearly 50 percent of the basin floor was exposed (unflooded); therefore, the average head (water depth) in the basin was not a uniform $1 \mathrm{ft}$. If an entire basin surface is flooded to $1-f t$ depth, the vertical hydraulic conductivity probably will be higher. A comparison of vertical hydraulic conductivities and infiltration rates at the two basins in the 1987 study (table 8) and this study, which maintain 1 -ft head (table 7), shows an order of magnitude variation within each study but comparable results between the two studies.

Table 8.--Hydraulic data on selected depth intervals below floor of basins 2 and $s$ during ponding tests at East Meadow recharge site

[Data from Schnelder, Ku, and Oaksford, 1987; ${ }^{\circ} \mathrm{F}$, degrees

Fahrenheit; ft/h, feet per hour; ft/d, feet per day]

\begin{tabular}{|c|c|c|c|c|c|}
\hline & $\begin{array}{l}\text { Time } \\
\text { of }\end{array}$ & $\begin{array}{l}\text { Time since } \\
\text { test began }\end{array}$ & Hydraul1c & $\begin{array}{c}\text { Infiltration } \\
\text { rate } \\
\text { adjusted } \\
\text { to } 60{ }^{\circ} \mathrm{F}\end{array}$ & $\begin{array}{c}\text { Vertical } \\
\text { hydraulic } \\
\text { conductivity } \\
\text { at } 600^{\circ} \mathrm{F} \\
\end{array}$ \\
\hline Date & day & (hours) & gradient & $(\mathrm{ft} / \mathrm{h})$ & $(\mathrm{ft} / \mathrm{d})$ \\
\hline
\end{tabular}

A. JUNE $20-23,1983$, BASIN 2

0 - to 5-ft interval (58.92-inch thickness)

$\begin{array}{rrrrrrr}6-20-83 & 1345 & 0.45 & 1.12 & 0.83 & 17.8 & 0.74 \\ 6-20-83 & 1445 & 1.45 & 1.10 & .93 & 20.2 & .84 \\ 6-20-83 & 1545 & 2.45 & 1.10 & 1.03 & 22.5 & .94 \\ 6-22-83 & 0700 & 42 & 1.09 & 1.12 & 24.6 & 1.02 \\ 6-23-83 & 0800 & 67 & 1.07 & 1.37 & 30.7 & 1.28\end{array}$

4- to 5-ft interval (12.36-1nch thickness)

$\begin{array}{lllllll}6-20-83 & 1445 & 1.45 & 1.80 & .93 & 12.4 & .52 \\ 6-20-83 & 1545 & 2.45 & 1.96 & 1.03 & 12.6 & .53 \\ 6-21-83 & 1300 & 24 & 2.54 & 1.14 & 10.7 & .45 \\ 6-22-83 & 0700 & 42 & 2.29 & 1.12 & 11.7 & .49 \\ 6-23-83 & 0800 & 67 & 2.23 & 1.37 & 14.7 & .61\end{array}$

B. JULY '25, 1983 TO JANUARY 9, 1984, BASIN 3

0- to 5-ft interval (58.8-inch thickness)

$\begin{array}{rr}7-25-83 & 1250 \\ 8-16-83 & 0100 \\ 10-10-83 & 2300 \\ 12-20-83 & 2400 \\ 1-9-84 & 1500\end{array}$

$7-25-83 \quad 1250$

8-16-83 0100

10-10-83 2300

12-20-83 2400

$1-9-84 \quad 1500$
1.05
1.11
1.40
1.57
1.74

2.09

.91

.90

.65

.44

0 - to 1-ft interval (11.4-1nch thickness)

\begin{tabular}{rrrrc}
7 & 1.79 & 2.09 & 28.0 & 1.17 \\
29 & 1.59 & .91 & 13.7 & .57 \\
84 & 2.98 & .90 & 7.2 & .3 \\
155 & 4.01 & .65 & 3.9 & .16 \\
176 & 4.95 & .44 & 2.1 & .09 \\
\hline
\end{tabular}




\section{One- and Two-Day Infiltration Tests}

Two infiltration tests, one $24 \mathrm{~h}$ long and one $48 \mathrm{~h}$ long, were conducted with the double-ring infiltrometers during April 28-29 and September 23-25, 1986, respectively. The tests were held in basin EM 2 at the East Meadow artificial-recharge site (fig. 13). The basin is $50 \times 100 \mathrm{ft}$ with vertical concrete sides. A detailed description of this basin is given by Schneider and others (1987). This basin, among others, was used to study the feasibility of large-scale artificial recharge with reclaimed water (tertiary-treated sewage) during 1982-84.

\section{Infiltration Rate}

The purpose of these tests was to record the changes in infiltration rate and vertical hydraulic conductivity over a 1 - and 2 -day period and to compare with results of earlier studies. Two sites ( $A$ and $B$ ) on the southern side of the recharge basin were chosen for these tests. The movement of the wetting front was observed with a neutron logger. Results obtained in these tests with the double-ring infiltrometer were compared with those obtained through long-term basin flooding as described by Schneider and others (1987).

The same field equipment and procedures were used in the 48-hour test as in the 24-hour test. The infiltration rates recorded during these tests are shown in figure 19. The final infiltration rates at the two measurement points in this basin were 1.30 and $3.31 \mathrm{ft} / \mathrm{h}$ for the 24 -hour test and 1.30 and $2.23 \mathrm{ft} / \mathrm{h}$ for the 48 -hour test (table 9 ). At the end of the first 4 hours, the infiltration rate was two to three times higher than at the end of the 48 hours (table 9).

Although the two measurement points (sites A and B) are only a few feet apart within basin EM2, the infiltration rates differ. For example, the lowest and highest infiltration rates during the 24 -hour test were 1.30 and $3.31 \mathrm{ft} / \mathrm{h}$, respectively. Local variations of this magnitude are probably common. For example, the infiltration rates of seven recharge basins at the East Meadow site, which occupies only 35 acres or $0.055 \mathrm{mi}^{2}$ on the outwash plain, ranges from 0.44 to $1.37 \mathrm{ft} / \mathrm{h}$ (table 10).

\section{Table 9.--Infiltration rates and vertical hydraulic conductivity at East Meadow artificial recharge site, basin EM 2, during 24- and 48-hour tests}

[Values are in feet per hour; dash indicates no data]

\begin{tabular}{|c|c|c|c|c|c|c|}
\hline \multirow[b]{2}{*}{ Test } & \multirow[b]{2}{*}{ Dates } & & \multicolumn{3}{|c|}{ Inf1ltration rate } & \multirow[b]{2}{*}{$\begin{array}{l}\text { Vertical hydraulic } \\
\text { conductivity }\end{array}$} \\
\hline & & & Site & $\begin{array}{l}\text { After } \\
4 \text { hours }\end{array}$ & $\begin{array}{l}\text { At end } \\
\text { of test }\end{array}$ & \\
\hline \multirow[t]{2}{*}{ 24-hour } & $\begin{array}{l}4 / 28 / 86 \\
4 / 29 / 86\end{array}$ & to & A & 3.20 & 1.30 & -- \\
\hline & & & B & 3.50 & 3.31 & -- \\
\hline \multirow[t]{2}{*}{ 48-hour } & $\begin{array}{l}9 / 23 / 86 \\
9 / 25 / 86\end{array}$ & to & A & 3.70 & 1.30 & 1.30 \\
\hline & & & B & 3.10 & 2.23 & 2.35 \\
\hline
\end{tabular}


Table 10.--Infiltration rates at East Meadow artificial-recharge site

[Data from Schneider and others, 1987; values are in feet per hour]

\begin{tabular}{ccccc}
\hline Basin number & Infiltration rate & Basin number & Inf11tration rate \\
1 & & & & \\
2 & 0.55 to 0.60 & 5 & 0.84 to 1.04 \\
3 & .44 to 1.1 & 6 & .56 to .62 \\
4 & .83 to 1.37 & 7 & .97 to 1.1 \\
\hline
\end{tabular}

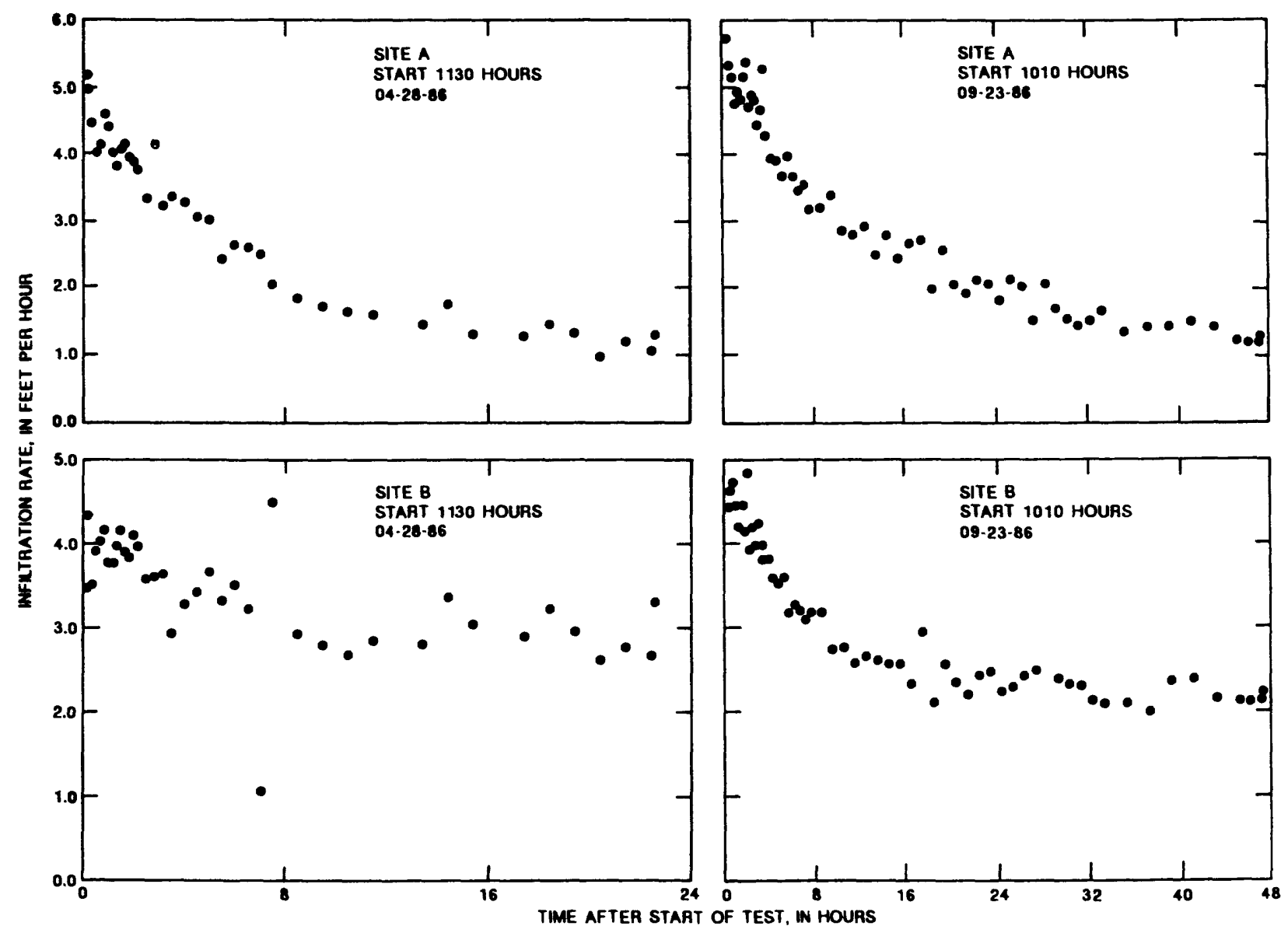

Figure 19.--Change in infiltration rate at East Meadow basin EM 2 during 24-hour test on April 28, 1986 and 48-hour test on September 23, 1986.

Results of the 24- and 48-hour infiltration tests at basin EM 2 indicate that the infiltration rate decreased at least 50 percent between 4 and 48 hours (fig. 19); thus, the ability of a recharge basin to dispose of stormwater decreases as the duration of the storms increase.

The double-ring infiltrometer tests and the earlier basin-flooding tests yielded results within the correct range; therefore, the double-ring infiltrometer, being portable and easy to install, is considered acceptable field equipment if properly designed and handled. 


\section{Vertical Hydraulic Conductivity}

During the 48-h test, a water-manometer tensiometer was placed in each of the two infiltration sites. The installation procedure was the same as described previously. At the end of the test, the vertical hydraulicconductivity values at the two sites were 1.30 and $2.35 \mathrm{ft} / \mathrm{h}$ (table 9), which are within the range of other tests performed during this study (table 7 ) and previous studies (table 8 ).

\section{Movement of Wetting Front}

To compare the movement of the wetting front between the infiltrometer and recharge basins, neutron geophysical logs were obtained within the infiltration rings at basin EM2 during the 24- and 48-hour infiltration tests. A series of neutron logs made during the two tests is shown in figure 20. The neutron access tube was installed and used by Schneider and others (1987). The wetting front reached the $20-\mathrm{ft}$ depth in about $5 \mathrm{~h}$. Below that depth, the soil moisture increased by only 10 percent, which suggests a controlling layer of less permeable material $20 \mathrm{ft}$ below the basin floor. The overall wettingfront profile however, is similar to wetting-front profiles during basinflooding tests by Schneider and others (1987, fig. 21). At the same basin, the wetting front reached the water table in just over $4 \mathrm{~h}$, and the water level in the basin continued to rise for the next $48 \mathrm{~h}$. The logs from neutron-logger access tubes surrounding the test basin (Schneider and others, 1987) show insignificant lateral movement of water within the unsaturated zone, even after 3 months of recharge.
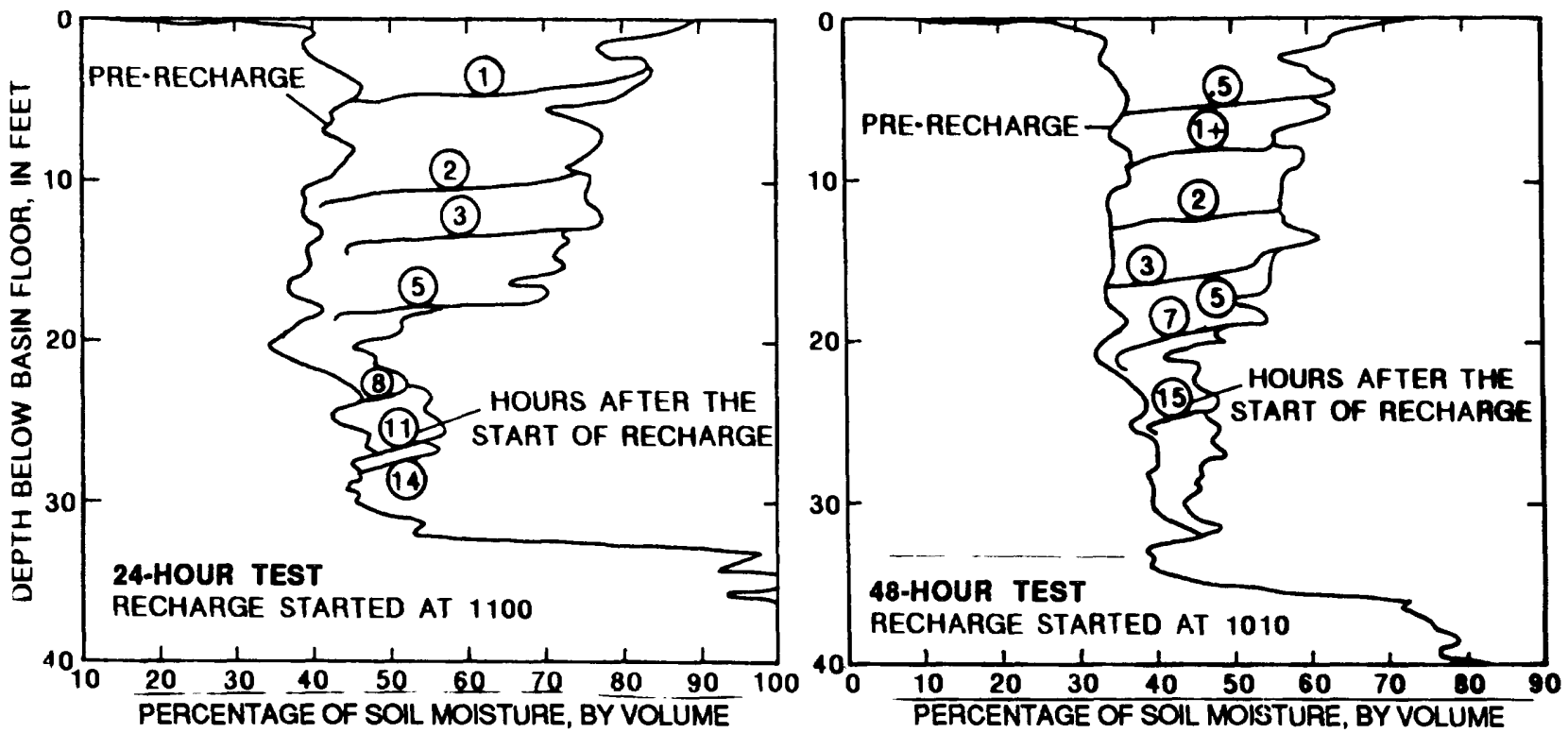

Figure 20.--Soil-moisture logs from neutron-access tube in basin EM 2 during 24- and 48-hour infiltration tests with infiltration rings. 


\section{SUMMARY AND CONCLUSIONS}

The aquifer system of Long Island receives natural recharge only from precipitation that infiltrates from the land surface to the water table. Therefore, the location and performance of recharge basins affect the distribution and amount of recharge.

The average infiltration rate from 51 selected recharge basins in Nassau County ranges from 0.13 to $5.63 \mathrm{ft} / \mathrm{h}$; the median value is $1.83 \mathrm{ft} / \mathrm{h}$. The recharge basins in the northern part of the county tend to have lower infiltration rates than basins in the southern part and generally coincide with areas of low soil permeability, zones of perched water, and morainal deposits.

Hydraulic-conductivity tests of the upper foot of soil at the bottom of the recharge basins indicate a median vertical hydraulic conductivity of 1.63 $\mathrm{ft} / \mathrm{h}$ with a range of 0.08 to $5.33 \mathrm{ft} / \mathrm{h}$. These values are consistent with results from an earlier study at the East Meadow recharge site in central Nassau County.

The distribution of clogged basins shows that surficial geology plays an important role in determining the infiltration rate of the basins. Between 19 and 36 percent of recharge basins excavated in morainal deposits are clogged, compared to only 14 percent of those on the outwash plain. More than half of the basins built before 1950 are considered clogged. At present, almost all basins are able to discharge stormwater, but with aging of the basins and increasing urbanization, the number of clogged basins is expected to increase.

Crest-stage gages were installed in 58 recharge basins to record the maximum depth of water in the basins during storms; ponded water in 20 basins attained a depth of $5 \mathrm{ft}$ or more at least once during the 3-year study. Water in the other 38 basins did not reach a $5-\mathrm{ft}$ depth at any time. Because almost all recharge basins are at least $10 \mathrm{ft}$ deep, many appear to have excess storage capacity under precipitation conditions that prevailed during the study.

\section{REFERENCES CITED}

Aronovici, V. S., 1955, Model study of ring infiltrometer performance under low Initial soil moisture, in Proceedings: Soil Science Society of America, v. 19, p. 1-6.

Aronson, D. A., and Prill, R. C., 1977, Analysis of the recharge potential of storm-water basins on Long Island, New York: U.S. Geological Survey Journal of Research, v. 5, no. 3, p. 307-318.

Aronson, D. A., and Seaburn, G. E., 1974, Appraisal of the operating efficiency of recharge basins on Long Island, New York, in 1969: U.S. Geological Survey Water-Supply Paper 2001-D, 22 p. 


\section{REFERENCES CITED (continued)}

Brashears, M. L., Jr., 1946, Artificial recharge of ground water on Long Island, New York: Economic Geology, v. 41, no. 5, p. 503-516.

Brice, H. D., Whitaker, C. L., and Sawyer, R. M., 1956, A progress report on the disposal of storm water at an experimental seepage basin near Mineola, New York: U.S. Geological Survey open-file report, 34 p.

Buchanan, T. J., and Somers, W. D., 1968, Stage measurement at gaging stations: U.S. Geological Survey Techniques of Water-Resources Investigations, Book 3, Chapter A7, 28 p.

Cohen, Philip, Franke, O. L., and Foxworthy, B. L., 1968, An at las of Long Island's water resources: New York State Water Resources Commission Bulletin 62, $117 \mathrm{p}$.

Holzmacher, McLendon, and Murrel1, Consulting Engineers, 1970, Report-Comprehensive public water supply study Suffolk County, New York: Melville, N.Y., v. 103, CPWS-24, 373 p.

Isbister, John, 1966, Geology and hydrology of northeastern Nassau County, Long Island, New York: U.S. Geological Survey Water-Supply Paper 1825, $89 \mathrm{p}$.

Krumbein, W. C., and Sloss, L. L., 1963, Stratigraphy and sedimentation: San Francisco, W. H. Freeman, p. 96-97.

Ku, H. F. H., and Simmons, D. L., 1986, Effect of urban stormwater runoff on ground water beneath recharge basins on Long Island, New York: U.S. Geological Survey Water Resources Investigations Report 85-4088, 67 p.

Lounsbury, Clarence, Howe, F. B., Zautner, R. E., Moran, W. J., and Beers, P. D., 1928, Soll Survey of Suffolk and Nassau Counties, New York: U.S. Department of Agriculture, Bureau of Chemistry and Solls, Ser. 1928, no. $28,46 \mathrm{p}$.

McClymonds, N.E. and Franke, O. L., 1972, Water-transmitting properties of aquifers on Long Island, N.Y.: U.S. Geological Survey Professional Paper $627-\mathrm{E}, 24 \mathrm{p}$.

Miller, J. F., and Frederick, R. H., 1969, The precipitation regime of Long Island, New York: U.S. Geological Survey Professional Paper 627-A, 21 p.

Musgrave, G. W., and Holtan, H. N., 1964, Infiltration, in Chow, V. T., ed., Handbook of applied hydrology: New York, McGraw-Hi11, p. 12-6, 12-7.

Oaksford, E. T., 1978, Water-manometer tensiometer installed and read from the land surface: Geotechnical Testing Journal, v. 1, no. 4, p. 119-202.

Perlmutter, N. M., and Geraghty, J. J., 1963, Geology and ground-water conditions in southern Nassau and southeastern Queens Counties, Long Island, N.Y.: U.S. Geological Survey Water-Supply Paper 1613-A, 205 p. 


\section{REFERENCES CITED (continued)}

Pri11, R. C., and Aaronson, D. B., 1973, Flow characteristics of a subsurface-controlled recharge basin on Long Island, New York: U.S. Geological Survey Journal of Research, v. 1, no. 6, p. 735-744.

Pril1, R. C., and Aronson, D. A., 1978, Ponding-test procedure for assessing the infiltration capacity of storm-water basins, Nassau County, New York: U.S. Geological Survey Water-Supply Paper 2049, 29 p.

Prill, R. C., Oaksford, E. T., and Potorti, J. E., 1979, A facility designed to monitor the unsaturated zone during infiltration of tertiary-treated sewage, Long Island, New York: U.S. Geological Survey Water-Resources Investigations Report 79-48, $14 \mathrm{p}$.

Schneider, B. J., Ku, H. F. H., and Oaksford, E. T., 1987, Hydrologic effects of artificial-recharge experiments with reclaimed water at East Meadow, Long Island, New York: U.S. Geological Survey Water-Resources Investigations Report 85-4323, $79 \mathrm{p}$.

Seaburn, G. E., and Aronson, D. A., 1973, Catalog of recharge basins on Long Island, New York in 1969: New York State Department of Environmental Conservation Bulletin 70, $80 \mathrm{p}$.

1974, Influence of recharge basins on the hydrology of Nassau and Suffolk Counties, Long Island, New York: U.S. Geological Survey Water-Supply Paper 2031, 66 p.

Slater, C. S., 1957, Cylinder infiltrometers for determining rates of irrigation, in Proceedings: Soil Science Society of America, v. 21, p. 457-460.

Swarzensk1, W. V., 1963, Hydrogeology of northwestern Nassau and northeastern Queens Counties, Long Island, New York: U.S. Geological Survey Water-Supply Paper $1657,90 \mathrm{p}$.

U.S. Soll Conservation Service, 1982, Interim soil survey of Nassau County, New York: U.S. Department of Agriculture, 17 p.

Warner, J. W., Jr., 1969, Soil interpretations--inventory and analysis, Suffolk County, New York: Prepared by Soil Conservation Service, U.S. Department of Agriculture, for the Nassau-Suffolk Regional Planning Board, $62 \mathrm{p}$.

Weaver, R. J., 1971, Recharge basin for disposal of highway storm drainage--Theory, design procedure, and recommended engineering practices: New York State Department of Transportation Research Report $69-2,64 \mathrm{p}$.

Welsch, W. F., 1935, Comprehensive drainage plan for Nassau County, New York-Summary report: Nassau County Sanitation Commission, 23 p.

1949, Conservation of ground-water resources, Nassau County, New York: Water Works Engineering, v. 102, no. 8, p. 708-710, 741-746. 


\section{EXPLANATION}

The underlined headings correspond to the column headings.

\section{Basin Number}

The letter(s) preceding each number refers to the ownership of the basin.

$B$ and MB - Town of Babylon, Highway Department

$B K$ and MBK - Town of Brookhaven, Highway Department

$E$ and ME - Town of East Hampton, Engineering Department

$H$ and $M H$ - Town of Huntington, Engineering Department

$I$ and MI - Town of Islip, Engineering Department

$N$, MN and EM - Nassau County Department of Public Works

NP - Private

$R$ and MR - Town of Riverhead, Engineering Department

$S$ and MS - Town of Smithtown, Engineering Department

SD and MSD - Town of Southold, Engineering Department

SN and MSN - Town of Southampton, Engineering Department

SP and MSP - New York State Parkway Commission

ST and MST - New York State Department of Transportation

The prefix $M$ indicates that aerial photographs were used to verify basin location and that engineering drawings from which to obtain design data were unavallable.

The number is used solely to reference data in this report and is not related to basin numbers assigned by other agencles.

\section{Nearest Intersection}

Names of the two intersecting streets nearest each recharge basin were determined.

\section{Lat itude and Longitude}

Latitude and longitude of each basin were determined from base maps.

\section{Date Built}

Many basins have no data on date of basin construction. The only avallable information on others was (1) the date on which engineering drawings were approved or (2) the date on which basin construction was finished and approved by the governing agency. The date given may be either one of these and Indicates, within about 1 year, the time of basin construction.

\section{Community}

The community name is that of the village closest to the recharge basin. The basin may or may not lie within the legal boundaries of the village 11sted. The number that appears under the column heading "community" corresponds to the code number indicated for that comminity in the table on p. 38-39. 


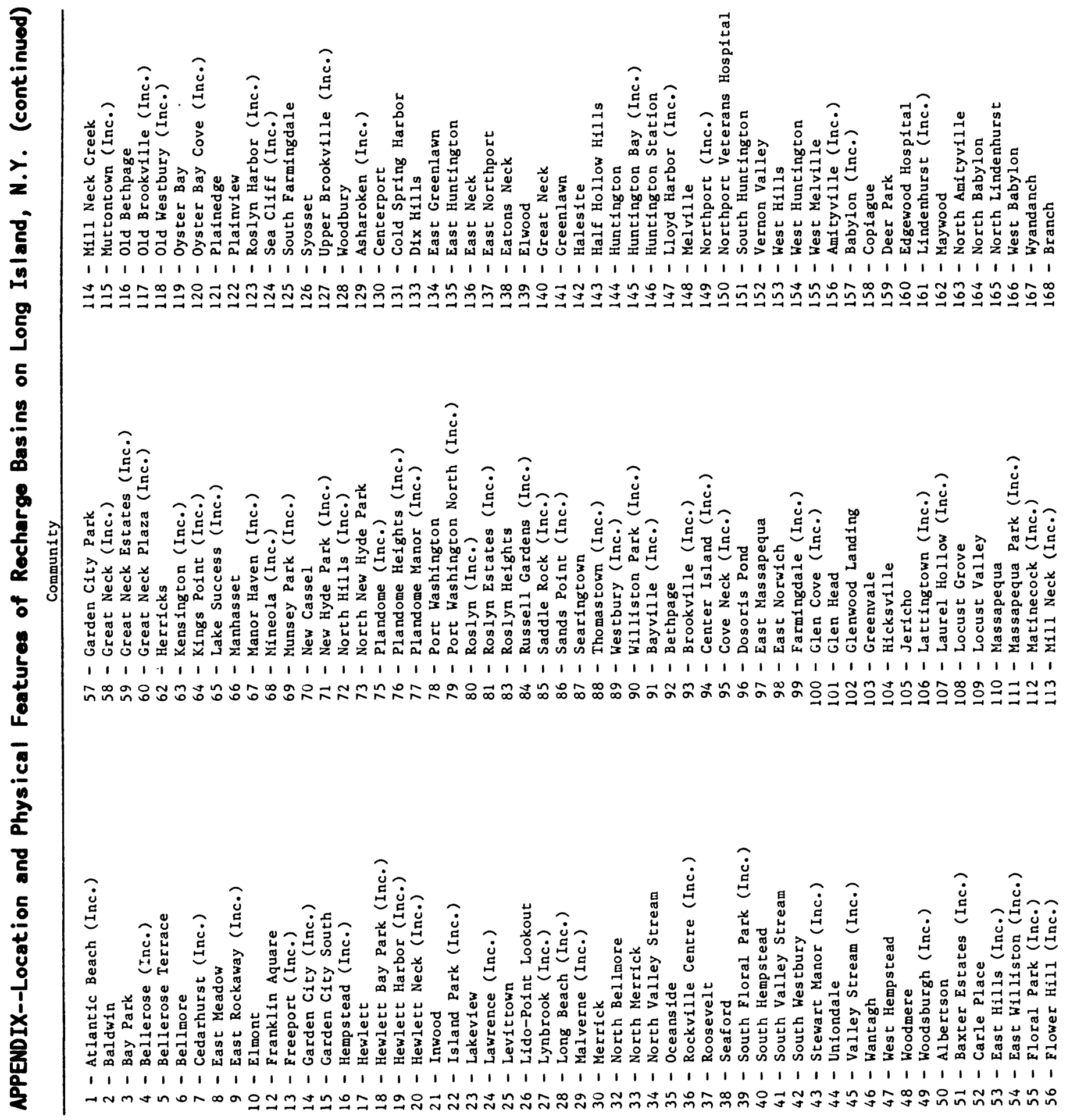




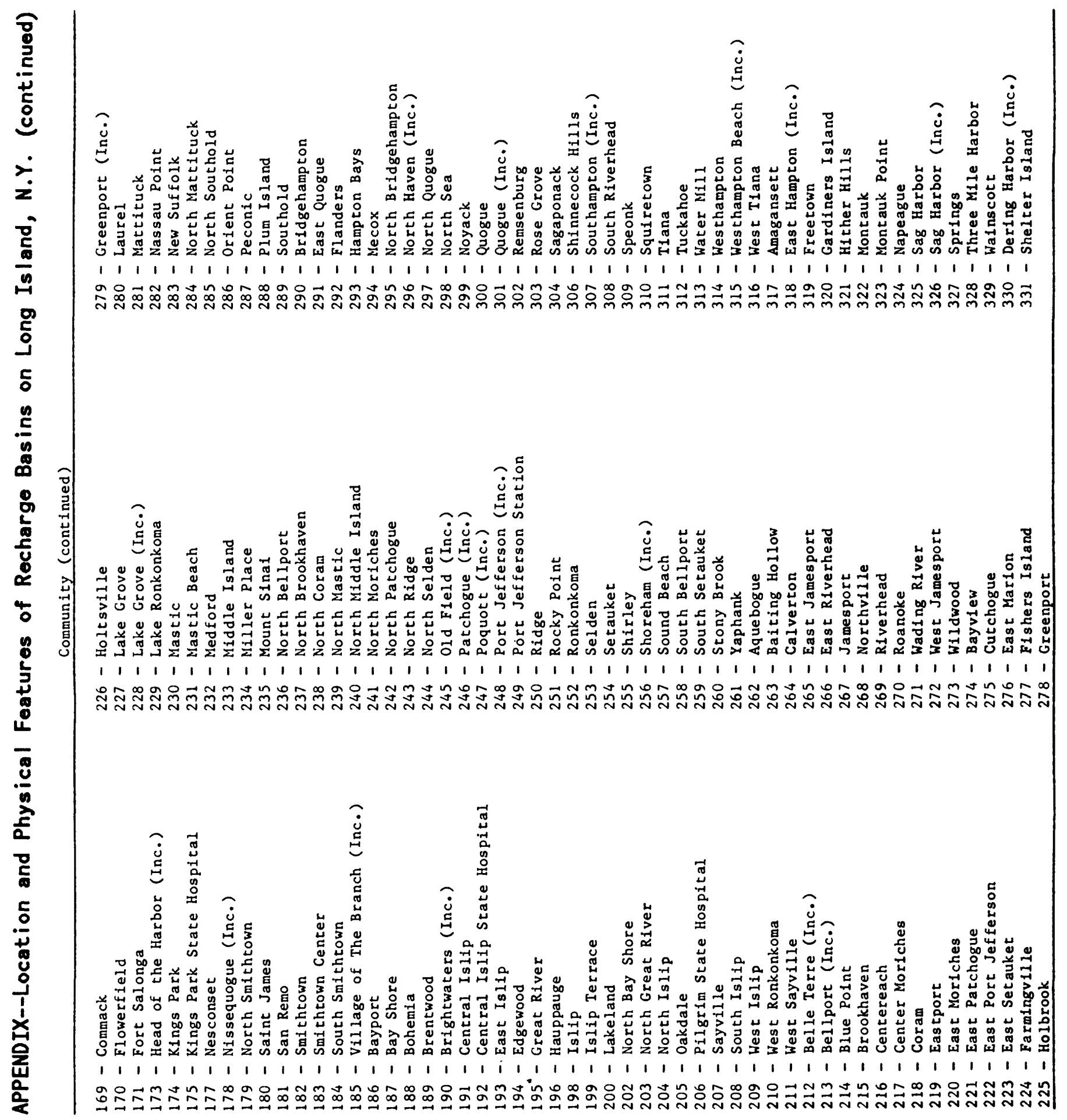




\section{APPENOIX.--Location and Physical Features of Recharge Basins \\ on Long Island, N.Y. (continued)}

\section{Design Capacity}

Design capacity, in cubic feet, is the computed volume of water that the recharge basin can hold without infiltration or overflow. For most basins, the design engineer computed this volume as the volume of water associated with a storm of 5 inches of rain falling on the contributing drainage area miltiplied by the percentage of impervious area within the drainage area. This value is related to physical conditions such as land slope and amount of impervious areas, which affect runoff in the drainage area.

\section{Actual Capacity}

Actual capacity, in cublc feet, is the volume of water the basin will hold without infiltration or overflow after construction.

\section{Maximum Infiltration Area (max. area)}

Maximum infiltration area, in square feet, is the profected average horizontal area computed by dividing the actual capacity by the difference between the overflow altitude and the bottom altitude of a basin. For basins where the overflow or bottom altitudes were unknown, the difference in altitude between the point of overflow and the bottom of the basin was assumed to be 10 feet.

\section{Basin Area}

Basin area, in square feet, is the projected horizontal area of the recharge basin, calculated by multiplying dimensions taken from the engineering drawings. It includes side slopes as well as planting and fencing areas bordering the basin.

\section{Altitude}

Rim.--is the lowest altitude of the land surface immediately bordering the recharge basin, in feet above sea level. This was obtained from engineering drawings or topographic maps.

Overflow.--the altitude, in feet above sea level at, which the basin overflows to another basin or to a nearby stream was obtained from engineering drawlings.

Bottom.--the basin-floor altitude, in feet above sea level, obtained from engineering drawings.

Water table.--ground-water level, in feet above sea level.

\section{Drainage Area (Drain. area)}

Drainage area, in acres, is the topographic and (or) the sewered area contributing storm inflow to the recharge basin. This was obtained by one of three methods:

1. Obtained from engineering drawings;

2. Computed from the basin's design capacity by the following rearrangement of the design equation:

$$
D A=D C \times \frac{1}{R / 12 \times 43,560 \times F}
$$


APPENDIX.--Location and Physical Features of Recharge Basins on Long Island, N.Y. (continued)

where: $D A=d r a 1 n a g e$ area, in acres;

$D C=$ design capacity (actual capacity was used when design capacity was not given), In cublc feet;

$R=$ magnitude of the design storm, In Inches; and

$F=$ factor reflecting conditions such as land slope and percent impervious area in the drainage basin.

A design storm (R) of 5 inches was assumed for all basins. A value of 0.35 was assigned to $F$ for basins draining residential areas, and a value of 0.90 for all other types of basins; or

3. Provided by Legette, Brashears and Graham (written commun., 1987).

\section{Basin Status}

The authors considered a recharge basin clogged if it held water 5 to 6 days after a 1 -inch rainfall. Aerial photographs were used to locate clogged basins, and each basin was inspected in the fleld to verify that it was clogged.

The following designations Indicate whether a basin is clogged:

Clogged $0 \quad$ Not clogged 1

\section{Basin Use}

Basin use was classified according to the predominant land use in the storm-runoff-contributing area:

\begin{tabular}{clcl} 
Code & Use & Code & \multicolumn{1}{c}{ Use } \\
1 & Res1dent1al & 3 & Highway \\
2 & Industrial & 4 & Commerc1al
\end{tabular}

Many basins drain more than one type of area, but only the predominant land use is indicated.

\section{Geologic Unit (Geo. unit)}

The surficlal geology in the dralnage area of each recharge basin was determined from geolog1c maps complled by Perlmutter and Geraghty (1963), Swarzensk1 (1963), and Isbister (1966). Brief descriptions of the geologic units drained by recharge basins are given on plate 1.

\section{Soll Unit}

The type of soll in the drainage area of each basin was determined from so11-unit maps complled by Lounsbury and others (1928) and Warner (1969). The Lounsbury soll survey delineated 22 soll units in Nassau and parts of Suffolk County. Equivalent soll units were designated by different names in these two studies; therefore, the authors have prepared a list of names of equivalent soll units and a general description of the equivalent soll units. (See table 2.) 
Appendix.--Location and basic physical features of recharge basins on Long Island,

\begin{tabular}{|c|c|c|c|c|c|c|c|c|}
\hline \multicolumn{2}{|c|}{$\begin{array}{l}\text { BASIN } \\
\text { NUMBER }\end{array}$} & \multicolumn{2}{|c|}{ NEAREST INTERSECTION } & $\begin{array}{l}\text { LATITUDE } \\
\text { LONGITUDE } \\
0, *\end{array}$ & $\begin{array}{l}\text { DATE } \\
\text { BUILT } \\
\text { YrMOD }\end{array}$ & COMMUNITY & $\begin{array}{l}\text { DESIGN } \\
\text { CAPACITY } \\
\text { (cubic }\end{array}$ & $\begin{array}{l}\text { ACTUAL } \\
\text { CAPACITY } \\
\text { feot) }\end{array}$ \\
\hline $\begin{array}{l}B \\
B \\
B \\
B \\
B\end{array}$ & $\begin{array}{l}1 \\
2 \\
3 \\
4 \\
6\end{array}$ & $\begin{array}{l}\text { RIDGE RD. } \\
\text { COLONIAL SPRINGS } \\
\text { BRIARWOOD ROAD } \\
\text { PERRY LANE } \\
\text { 23RD. STREET }\end{array}$ & $\begin{array}{l}\text { COLONIAL SPRINGS } \\
\text { CIRCLE DRIVE } \\
\text { BENNINGTON PLACE } \\
\text { WILLOW STREET } \\
\text { WASHINGTON AVE. }\end{array}$ & $\begin{array}{l}4645390732328 \\
4045260732252 \\
4645220732311 \\
4045420732156 \\
4046220732207\end{array}$ & $\begin{array}{l}- \\
- \\
-\end{array}$ & $\begin{array}{l}- \\
- \\
-\end{array}$ & $\begin{array}{l}- \\
- \\
- \\
-\end{array}$ & $\begin{array}{l}- \\
- \\
-\end{array}$ \\
\hline $\begin{array}{l}B \\
B \\
B \\
B\end{array}$ & $\begin{array}{r}6 \\
7 \\
8 \\
9 \\
10\end{array}$ & $\begin{array}{l}\text { BURNS LANE } \\
\text { BROOKLYN AVE. } \\
\text { STATE STREET } \\
\text { CUMBERBACH ST. } \\
\text { SEAMANS ROAD }\end{array}$ & $\begin{array}{l}\text { CHELMSFORD DRIVE } \\
\text { 23RD. STREET } \\
\text { RUSSELL STREET } \\
\text { TROY AVE. } \\
\text { STRAIGHT ROAD }\end{array}$ & $\begin{array}{l}4046080732121 \\
4044420732218 \\
4044370732144 \\
4044170732154 \\
4046440732126\end{array}$ & $\begin{array}{l}- \\
- \\
-\end{array}$ & $\begin{array}{l}- \\
- \\
- \\
-\end{array}$ & $\begin{array}{l}- \\
- \\
- \\
-\end{array}$ & $\begin{array}{l}- \\
- \\
-\end{array}$ \\
\hline $\begin{array}{l}B \\
B \\
B \\
B \\
B\end{array}$ & $\begin{array}{l}11 \\
12 \\
13 \\
14 \\
15\end{array}$ & $\begin{array}{l}\text { MOTOR PARKWAY } \\
\text { N. 16TH STREET } \\
\text { COPAIGUE ROAD } \\
\text { CATSKILL AVENUE } \\
\text { HEATHCOTE ROAD }\end{array}$ & $\begin{array}{l}\text { LEE AVENUE } \\
\text { NICOLLS ROAD } \\
\text { 5IST. STREET } \\
\text { 48TH STREET } \\
\text { MADISON DRIVE }\end{array}$ & $\begin{array}{l}4045230732224 \\
4045290732157 \\
4042120732338 \\
4041540732368 \\
4042220732259\end{array}$ & $\begin{array}{l}- \\
- \\
-\end{array}$ & $\begin{array}{l}- \\
- \\
-\end{array}$ & $\begin{array}{l}- \\
- \\
- \\
-\end{array}$ & $\begin{array}{l}- \\
- \\
-\end{array}$ \\
\hline $\begin{array}{l}B \\
B \\
B \\
B \\
B\end{array}$ & $\begin{array}{l}18 \\
17 \\
18 \\
19 \\
29\end{array}$ & $\begin{array}{l}\text { CENTER STREET } \\
\text { ALLEN BLVD. } \\
\text { SIDNEY CT. } \\
\text { SMITH STREET } \\
\text { SMITH STREET }\end{array}$ & $\begin{array}{l}\text { PINE STREET } \\
\text { ADAMS BLVD. } \\
\text { ALBANY AVENUE } \\
\text { REPUBLIC ROAD } \\
\text { AUGUSTA AVENUE }\end{array}$ & $\begin{array}{l}404253 \varnothing 732438 \\
4043 \varnothing 10732448 \\
4042580732460 \\
484517 \varnothing 732446 \\
4045230732424\end{array}$ & $\begin{array}{l}- \\
- \\
-\end{array}$ & $\begin{array}{l}- \\
- \\
- \\
-\end{array}$ & $\begin{array}{l}- \\
- \\
-\end{array}$ & $\begin{array}{l}- \\
- \\
- \\
-\end{array}$ \\
\hline $\begin{array}{l}B \\
B \\
B \\
B \\
B\end{array}$ & $\begin{array}{l}21 \\
22 \\
23 \\
24 \\
25\end{array}$ & $\begin{array}{l}\text { GARDEN STREET } \\
\text { SCHLEIG BLVD. } \\
\text { LINCOLN AVENUE } \\
\text { GOLD STREET } \\
\text { ERLANGER BLVD. }\end{array}$ & $\begin{array}{l}\text { WILSON AVENUE } \\
\text { MADISON AVENE } \\
\text { VAN BUREN STREET } \\
\text { FINN COURT } \\
\text { VANCOTT ROAD }\end{array}$ & $\begin{array}{l}4042390732532 \\
4642320732516 \\
4642410732586 \\
404528073242 \emptyset \\
4044560731941\end{array}$ & $\begin{array}{l}- \\
- \\
-\end{array}$ & $\begin{array}{l}- \\
- \\
-\end{array}$ & $\begin{array}{l}- \\
- \\
-\end{array}$ & $\begin{array}{l}- \\
- \\
- \\
-\end{array}$ \\
\hline $\begin{array}{l}B \\
B \\
B \\
B \\
B\end{array}$ & $\begin{array}{l}26 \\
27 \\
28 \\
29 \\
3 \varnothing\end{array}$ & $\begin{array}{l}\text { FLOYD STREET } \\
\text { NEW AVENUE } \\
\text { GROVE STREET } \\
\text { PRAIRIE DRIVE } \\
\text { AUCUST ROAD }\end{array}$ & $\begin{array}{l}\text { LEADER STREET } \\
\text { GROVE STREET } \\
\text { WRIGHT STREET } \\
\text { WOODS ROAD } \\
\text { IDA LANE }\end{array}$ & $\begin{array}{l}4044400731962 \\
4044310732125 \\
4044120732112 \\
4044460731949 \\
4044270732015\end{array}$ & $\begin{array}{l}\text { - } \\
\text { - }\end{array}$ & $\begin{array}{l}- \\
- \\
-\end{array}$ & $\begin{array}{l}- \\
- \\
- \\
-\end{array}$ & $\begin{array}{l}- \\
- \\
-\end{array}$ \\
\hline $\begin{array}{l}\mathbf{B} \\
\mathbf{B} \\
\mathrm{B} \\
\mathrm{B} \\
\mathrm{B}\end{array}$ & $\begin{array}{l}31 \\
32 \\
33 \\
34 \\
36\end{array}$ & $\begin{array}{l}\text { VICTORIA DRIVE } \\
\text { WILSHIRE DR. E. } \\
\text { CATALPA STREET } \\
\text { YOUNG STREET } \\
\text { HAMPTON AVENUE }\end{array}$ & $\begin{array}{l}\text { WOODS ROAD } \\
\text { WILSHIRE COURT } \\
\text { FORSYTHIA LANE } \\
\text { LEWTS AVENE } \\
\text { CLARK STREET }\end{array}$ & $\begin{array}{l}4044246731958 \\
4044280731961 \\
4644310731843 \\
4643480732101 \\
4643360732133\end{array}$ & $\begin{array}{l}- \\
- \\
- \\
-\end{array}$ & $\begin{array}{l}- \\
- \\
- \\
-\end{array}$ & $\begin{array}{l}- \\
- \\
- \\
-\end{array}$ & $\begin{array}{l}- \\
- \\
-\end{array}$ \\
\hline $\begin{array}{l}B \\
B \\
B \\
B \\
B\end{array}$ & $\begin{array}{l}36 \\
37 \\
38 \\
39 \\
40\end{array}$ & $\begin{array}{l}\text { COOPER STREET } \\
\text { ROCKAWAY AVENUE } \\
\text { ARTHUR ROAD } \\
\text { EDMNTON DRIVE } \\
\text { CANTERBURY LANE }\end{array}$ & $\begin{array}{l}\text { GLEN PLACE } \\
\text { DEER PARK ROAD } \\
\text { LARAMIE ROAD } \\
\text { HITHERDELL LANE } \\
\text { HITHERDELL LANE }\end{array}$ & $\begin{array}{l}4043280731919 \\
4043260731968 \\
4042510732047 \\
4043076731915 \\
4043090731911\end{array}$ & $\begin{array}{l}- \\
- \\
- \\
-\end{array}$ & $\begin{array}{l}- \\
- \\
- \\
-\end{array}$ & $\begin{array}{l}- \\
- \\
- \\
-\end{array}$ & $\begin{array}{l}- \\
- \\
- \\
-\end{array}$ \\
\hline $\begin{array}{l}B \\
B \\
B \\
B \\
B\end{array}$ & $\begin{array}{l}41 \\
42 \\
43 \\
44 \\
46\end{array}$ & $\begin{array}{l}\text { FULTON STREET } \\
\text { HAMPTON ROAD } \\
\text { FARRAGUT ROAD } \\
\text { BROADWAY } \\
\text { DEER PARK ROAD }\end{array}$ & $\begin{array}{l}\text { LAMONT PLACE } \\
\text { CLAREMONT AVENUE } \\
\text { AMSTEROAM AVENUE } \\
\text { BISHOP ROAD } \\
\text { MOHAYK DRIVE }\end{array}$ & $\begin{array}{l}4044020732118 \\
4043280732108 \\
4043130732115 \\
4042598732057 \\
4044150731907\end{array}$ & $\begin{array}{l}- \\
- \\
-\end{array}$ & $\begin{array}{l}- \\
- \\
- \\
-\end{array}$ & $\begin{array}{l}- \\
- \\
- \\
-\end{array}$ & $\begin{array}{l}- \\
- \\
- \\
-\end{array}$ \\
\hline $\begin{array}{l}\mathbf{B} \\
\mathbf{B} \\
\mathbf{B} \\
\mathbf{B} \\
\mathbf{B}\end{array}$ & $\begin{array}{l}46 \\
47 \\
48 \\
49 \\
50\end{array}$ & $\begin{array}{l}\text { BROADWAY } \\
\text { ELLENSUE DRIVE } \\
\text { DEER PARK LANE } \\
\text { ROLAN DRIVE } \\
\text { WYAN DOTTE ST. }\end{array}$ & $\begin{array}{l}\text { LENOX ROAD } \\
\text { KATHLEEN LANE } \\
\text { LEON PLACE } \\
\text { OLD COUNTRY ROAD } \\
\text { HOMER AVENUE }\end{array}$ & $\begin{array}{l}4042680732039 \\
4044460732054 \\
4046190732016 \\
4046370731929 \\
4048330731848\end{array}$ & $\begin{array}{l}- \\
\overline{-} \\
-\end{array}$ & $\begin{array}{l}- \\
- \\
- \\
-\end{array}$ & $\begin{array}{l}- \\
- \\
- \\
-\end{array}$ & $\begin{array}{l}- \\
- \\
- \\
-\end{array}$ \\
\hline $\begin{array}{l}\mathbf{B} \\
\mathbf{B} \\
\mathbf{B}\end{array}$ & $\begin{array}{l}51 \\
52 \\
63\end{array}$ & $\begin{array}{l}\text { VILLA AVENUE } \\
\text { BURLINGTON AVE. } \\
\text { ADAMS STREET }\end{array}$ & $\begin{array}{l}\text { LAPEER STREET } \\
\text { ANGELICA COURT } \\
\text { ATLANTIC AVENUE }\end{array}$ & $\begin{array}{l}4046220731851 \\
4046066731854 \\
4046350732022\end{array}$ & $\begin{array}{l}- \\
-\end{array}$ & $\begin{array}{l}- \\
-\end{array}$ & $\begin{array}{l}- \\
-\end{array}$ & $\begin{array}{l}- \\
-\end{array}$ \\
\hline
\end{tabular}


N.Y. [Dash indicates no data. Altitudes are in feot above sea level.]

\begin{tabular}{|c|c|c|c|c|c|c|c|c|c|c|c|c|}
\hline \multicolumn{2}{|c|}{$\begin{array}{l}\text { BASIN } \\
\text { NUMBER }\end{array}$} & $\begin{array}{l}\text { MAX. } \\
\text { AREA } \\
\text { (square }\end{array}$ & $\begin{array}{c}\text { BASIN } \\
\text { AREA } \\
\text { feøt) }\end{array}$ & RIM & $\begin{array}{l}\text { OVER- } \\
\text { FLOW }\end{array}$ & BOT- & $\begin{array}{l}\text { WATER } \\
\text { TABLE }\end{array}$ & $\begin{array}{c}\text { DRAIN. } \\
\text { AREA } \\
\text { (acres) }\end{array}$ & \multicolumn{2}{|c|}{$\begin{array}{l}---B A S I N-- \\
\text { STATUS USE }\end{array}$} & $\begin{array}{l}\text { GEO. } \\
\text { UNIT }\end{array}$ & $\begin{array}{l}\text { SOIL } \\
\text { UNIT }\end{array}$ \\
\hline $\begin{array}{l}B \\
B \\
B \\
B \\
B\end{array}$ & $\begin{array}{l}1 \\
2 \\
3 \\
4 \\
5\end{array}$ & $\begin{array}{l}- \\
- \\
- \\
-\end{array}$ & $\begin{array}{l}30000 \\
75000 \\
51000 \\
48800 \\
78000\end{array}$ & $\begin{array}{l}80.0 \\
75.0 \\
90.0 \\
80.0 \\
70.0\end{array}$ & $\begin{array}{l}- \\
- \\
- \\
-\end{array}$ & $\begin{array}{l}- \\
- \\
- \\
-\end{array}$ & $\begin{array}{l}57.0 \\
54.0 \\
54.0 \\
51.0 \\
50.0\end{array}$ & $\begin{array}{l}- \\
- \\
- \\
-\end{array}$ & $\begin{array}{l}1 \\
1 \\
1 \\
1 \\
1\end{array}$ & $\begin{array}{l}1 \\
1 \\
1 \\
1 \\
1\end{array}$ & $\begin{array}{l}1 \\
1 \\
1 \\
1 \\
1\end{array}$ & $\begin{array}{l}7 \\
7 \\
7 \\
7 \\
3\end{array}$ \\
\hline $\begin{array}{l}B \\
B \\
B \\
B \\
B\end{array}$ & $\begin{array}{r}6 \\
7 \\
8 \\
9 \\
10\end{array}$ & $\begin{array}{l}- \\
- \\
- \\
-\end{array}$ & $\begin{array}{l}51000 \\
1000 \emptyset \\
3400 \emptyset \\
1500 \emptyset \\
3000 \emptyset\end{array}$ & $\begin{array}{l}10.0 \\
70.0 \\
60.0 \\
60.0 \\
70.0\end{array}$ & $\begin{array}{l}- \\
- \\
- \\
-\end{array}$ & $\begin{array}{l}- \\
- \\
-\end{array}$ & $\begin{array}{l}52.0 \\
48 . \varnothing \\
48.0 \\
48 . \emptyset \\
50 . \varnothing\end{array}$ & $\begin{array}{l}- \\
- \\
-\end{array}$ & $\begin{array}{l}1 \\
1 \\
1 \\
1 \\
1\end{array}$ & $\begin{array}{l}1 \\
1 \\
1 \\
1 \\
1\end{array}$ & $\begin{array}{l}1 \\
1 \\
1 \\
1 \\
1\end{array}$ & $\begin{array}{l}5 \\
3 \\
7 \\
1 \\
3\end{array}$ \\
\hline $\begin{array}{l}B \\
B \\
B \\
B \\
B\end{array}$ & $\begin{array}{l}11 \\
12 \\
13 \\
14 \\
15\end{array}$ & $\begin{array}{l}- \\
- \\
- \\
-\end{array}$ & $\begin{array}{l}50000 \\
15000 \\
43800 \\
10000 \\
32000\end{array}$ & $\begin{array}{l}75.0 \\
70.0 \\
45.0 \\
35.0 \\
40.0\end{array}$ & $\begin{array}{l}- \\
- \\
- \\
-\end{array}$ & $\begin{array}{l}- \\
- \\
- \\
-\end{array}$ & $\begin{array}{l}52.0 \\
50.0 \\
29.0 \\
22.0 \\
28.0\end{array}$ & $\begin{array}{l}- \\
- \\
- \\
-\end{array}$ & $\begin{array}{l}1 \\
1 \\
1 \\
1 \\
1\end{array}$ & $\begin{array}{l}1 \\
1 \\
1 \\
1 \\
1\end{array}$ & $\begin{array}{l}1 \\
1 \\
1 \\
1 \\
1\end{array}$ & $\begin{array}{l}3 \\
3 \\
7 \\
7 \\
7\end{array}$ \\
\hline $\begin{array}{l}B \\
B \\
B \\
B \\
B\end{array}$ & $\begin{array}{l}16 \\
17 \\
18 \\
19 \\
20\end{array}$ & $\begin{array}{l}- \\
- \\
- \\
-\end{array}$ & $\begin{array}{l}250 \varnothing \varnothing \\
4 \varnothing \varnothing \varnothing \varnothing \\
18 \varnothing \varnothing \varnothing \\
250 \varnothing \varnothing \\
2 \varnothing \varnothing \varnothing \varnothing\end{array}$ & $\begin{array}{r}50.0 \\
55.0 \\
50.0 \\
95.8 \\
185.8\end{array}$ & $\begin{array}{l}- \\
- \\
- \\
-\end{array}$ & $\begin{array}{l}- \\
- \\
- \\
-\end{array}$ & $\begin{array}{l}43.0 \\
45.0 \\
41.0 \\
58.0 \\
57.0\end{array}$ & $\begin{array}{l}- \\
- \\
-\end{array}$ & $\begin{array}{l}1 \\
1 \\
1 \\
1 \\
1\end{array}$ & $\begin{array}{l}1 \\
1 \\
1 \\
3 \\
3\end{array}$ & $\begin{array}{l}1 \\
1 \\
1 \\
1 \\
1\end{array}$ & $\begin{array}{l}1 \\
1 \\
1 \\
1 \\
1\end{array}$ \\
\hline $\begin{array}{l}B \\
B \\
B \\
B \\
B\end{array}$ & $\begin{array}{l}21 \\
22 \\
23 \\
24 \\
25\end{array}$ & $\begin{array}{l}- \\
- \\
-\end{array}$ & $\begin{array}{r}30000 \\
20000 \\
45000 \\
500 \varnothing \\
39500\end{array}$ & $\begin{array}{r}50.0 \\
45.0 \\
50.0 \\
105.0 \\
50.0\end{array}$ & $\begin{array}{l}- \\
- \\
- \\
-\end{array}$ & $\begin{array}{l}- \\
- \\
- \\
-\end{array}$ & $\begin{array}{l}42.0 \\
38.0 \\
42.0 \\
58.0 \\
42.0\end{array}$ & $\begin{array}{l}- \\
- \\
-\end{array}$ & $\begin{array}{l}1 \\
1 \\
1 \\
1 \\
1\end{array}$ & $\begin{array}{l}1 \\
1 \\
1 \\
1 \\
1\end{array}$ & $\begin{array}{l}1 \\
1 \\
1 \\
1 \\
1\end{array}$ & $\begin{array}{l}1 \\
1 \\
7 \\
1 \\
7\end{array}$ \\
\hline $\begin{array}{l}B \\
B \\
B \\
B \\
B\end{array}$ & $\begin{array}{l}26 \\
27 \\
28 \\
29 \\
30\end{array}$ & $\begin{array}{l}- \\
- \\
- \\
-\end{array}$ & $\begin{array}{l}12000 \\
22000 \\
5 \varnothing 0 \varnothing 0 \\
15000 \\
15000\end{array}$ & $\begin{array}{l}50.0 \\
55.0 \\
50.0 \\
50.0 \\
45.0\end{array}$ & $\begin{array}{l}- \\
- \\
- \\
-\end{array}$ & $\begin{array}{l}- \\
- \\
- \\
-\end{array}$ & $\begin{array}{l}42.0 \\
45.0 \\
38.1 \\
41.0 \\
34.0\end{array}$ & $\begin{array}{l}- \\
- \\
- \\
-\end{array}$ & $\begin{array}{l}1 \\
1 \\
1 \\
1 \\
1\end{array}$ & $\begin{array}{l}1 \\
1 \\
1 \\
1 \\
1\end{array}$ & $\begin{array}{l}1 \\
3 \\
1 \\
1 \\
1\end{array}$ & $\begin{array}{l}7 \\
5 \\
3 \\
7 \\
7\end{array}$ \\
\hline $\begin{array}{l}B \\
B \\
B \\
B \\
B\end{array}$ & $\begin{array}{l}31 \\
32 \\
33 \\
34 \\
35\end{array}$ & $\begin{array}{l}- \\
- \\
- \\
-\end{array}$ & $\begin{array}{l}14000 \\
2460 \varnothing \\
1800 \emptyset \\
10 \varnothing \varnothing \varnothing \\
31500\end{array}$ & $\begin{array}{l}40.0 \\
50.0 \\
50.0 \\
45.0 \\
45.0\end{array}$ & $\begin{array}{l}- \\
- \\
- \\
-\end{array}$ & $\begin{array}{l}- \\
- \\
- \\
-\end{array}$ & $\begin{array}{l}34.0 \\
40.0 \\
46.0 \\
37.0 \\
37.0\end{array}$ & $\begin{array}{l}- \\
- \\
- \\
-\end{array}$ & $\begin{array}{l}1 \\
1 \\
1 \\
1 \\
1\end{array}$ & $\begin{array}{l}1 \\
1 \\
1 \\
1 \\
1\end{array}$ & $\begin{array}{l}1 \\
1 \\
1 \\
1 \\
1\end{array}$ & $\begin{array}{l}7 \\
7 \\
7 \\
7 \\
7\end{array}$ \\
\hline $\begin{array}{l}B \\
B \\
B \\
B \\
B\end{array}$ & $\begin{array}{l}38 \\
37 \\
38 \\
39 \\
40\end{array}$ & $\begin{array}{l}- \\
- \\
- \\
-\end{array}$ & $\begin{array}{r}22500 \\
1690 \varnothing \\
1500 \emptyset \\
80 \varnothing \emptyset \\
17 \varnothing \varnothing \emptyset\end{array}$ & $\begin{array}{l}42.0 \\
45.0 \\
35.0 \\
45.0 \\
45.0\end{array}$ & $\begin{array}{l}- \\
- \\
- \\
-\end{array}$ & $\begin{array}{l}- \\
- \\
- \\
-\end{array}$ & $\begin{array}{l}25 . \varnothing \\
25 . \varnothing \\
25.0 \\
19 . \varnothing \\
19 . \varnothing\end{array}$ & $\begin{array}{l}- \\
- \\
- \\
-\end{array}$ & $\begin{array}{l}1 \\
1 \\
1 \\
1 \\
1\end{array}$ & $\begin{array}{l}1 \\
1 \\
1 \\
1 \\
1\end{array}$ & $\begin{array}{l}1 \\
1 \\
1 \\
1 \\
1\end{array}$ & $\begin{array}{l}7 \\
7 \\
7 \\
7 \\
7\end{array}$ \\
\hline $\begin{array}{l}B \\
B \\
B \\
B \\
B\end{array}$ & $\begin{array}{l}41 \\
42 \\
43 \\
44 \\
45\end{array}$ & $\begin{array}{l}- \\
- \\
- \\
-\end{array}$ & $\begin{array}{l}25000 \\
12000 \\
20000 \\
20000 \\
43800\end{array}$ & $\begin{array}{l}50.0 \\
40.0 \\
45.0 \\
40.0 \\
45.0\end{array}$ & $\begin{array}{l}- \\
- \\
- \\
-\end{array}$ & $\begin{array}{l}- \\
- \\
- \\
-\end{array}$ & $\begin{array}{l}37.0 \\
33.0 \\
32.0 \\
31.0 \\
38.0\end{array}$ & $\begin{array}{l}- \\
- \\
- \\
-\end{array}$ & $\begin{array}{l}1 \\
1 \\
1 \\
0 \\
1\end{array}$ & $\begin{array}{l}1 \\
1 \\
1 \\
1 \\
4\end{array}$ & $\begin{array}{l}1 \\
1 \\
1 \\
1 \\
1\end{array}$ & $\begin{array}{l}7 \\
7 \\
7 \\
7 \\
7\end{array}$ \\
\hline $\begin{array}{l}B \\
B \\
B \\
B \\
B\end{array}$ & $\begin{array}{l}46 \\
47 \\
48 \\
49 \\
50\end{array}$ & $\begin{array}{l}- \\
- \\
- \\
-\end{array}$ & $\begin{array}{r}12000 \\
132000 \\
5800 \\
15000 \\
32500\end{array}$ & $\begin{array}{r}35.0 \\
70.0 \\
85.0 \\
100.0 \\
95.0\end{array}$ & $\begin{array}{l}- \\
- \\
-\end{array}$ & $\begin{array}{l}- \\
- \\
- \\
-\end{array}$ & $\begin{array}{l}30.0 \\
52.0 \\
54.0 \\
48.0 \\
52.0\end{array}$ & $\begin{array}{l}- \\
- \\
- \\
-\end{array}$ & $\begin{array}{l}1 \\
1 \\
1 \\
1 \\
1\end{array}$ & $\begin{array}{l}1 \\
1 \\
1 \\
1 \\
1\end{array}$ & $\begin{array}{l}1 \\
1 \\
1 \\
1 \\
1\end{array}$ & $\begin{array}{l}7 \\
7 \\
7 \\
7 \\
7\end{array}$ \\
\hline $\begin{array}{l}B \\
B \\
B\end{array}$ & $\begin{array}{l}51 \\
52 \\
53\end{array}$ & $\begin{array}{l}- \\
-\end{array}$ & $\begin{array}{l}32 \varnothing \varnothing \emptyset \\
4410 \varnothing \\
2 \varnothing \varnothing \varnothing \emptyset\end{array}$ & $\begin{array}{l}90.0 \\
85.0 \\
65.0\end{array}$ & - & - & $\begin{array}{l}51.0 \\
50 . \varnothing \\
45 . \varnothing\end{array}$ & - & $\begin{array}{l}1 \\
1 \\
1\end{array}$ & $\begin{array}{l}1 \\
1 \\
1\end{array}$ & $\begin{array}{l}1 \\
1 \\
1\end{array}$ & $\begin{array}{l}7 \\
7 \\
3\end{array}$ \\
\hline
\end{tabular}


Appendix.--Location and basic physical features of recharge basins on Long Island,

\begin{tabular}{|c|c|c|c|c|c|c|c|c|}
\hline \multicolumn{2}{|c|}{$\begin{array}{l}\text { BASIN } \\
\text { NUMBER }\end{array}$} & \multicolumn{2}{|c|}{ NEAREST INTERSECTION } & $\begin{array}{l}\text { LATITUDE } \\
\text { LONGITUDE } \\
0_{*},\end{array}$ & $\begin{array}{l}\text { DATE } \\
\text { BUILT } \\
\text { YrMOD }\end{array}$ & COMMNITY & $\begin{array}{l}\text { DESIGN } \\
\text { CAPACITY } \\
\text { (cubic fe }\end{array}$ & $\begin{array}{l}\text { ACTUAL } \\
\text { CAPACITY } \\
\text { et) }\end{array}$ \\
\hline $\begin{array}{l}\mathbf{B} \\
\mathbf{B} \\
\mathbf{B} \\
\mathbf{B} \\
\mathbf{B}\end{array}$ & $\begin{array}{l}54 \\
56 \\
58 \\
57 \\
58\end{array}$ & $\begin{array}{l}\text { LUCILLE LANE } \\
\text { IRVINO AVENUE } \\
\text { N. 2ND STREET } \\
\text { CARLLS PATH } \\
\text { MARCUS BLVD. }\end{array}$ & $\begin{array}{l}\text { KELLY PLACE } \\
\text { LONG ISLAND AVE. } \\
\text { HALF HOLLOW RD. } \\
\text { NICOLLS ROAD } \\
\text { GRAND BLVD. }\end{array}$ & $\begin{array}{l}4045470731934 \\
4045490731919 \\
4045390732004 \\
4046000731914 \\
4045410731853\end{array}$ & $\begin{array}{l}- \\
- \\
-\end{array}$ & $\begin{array}{l}- \\
- \\
-\end{array}$ & $\begin{array}{l}- \\
- \\
- \\
-\end{array}$ & $\begin{array}{l}- \\
- \\
- \\
-\end{array}$ \\
\hline $\begin{array}{l}\mathbf{B} \\
\mathbf{B} \\
\mathbf{B} \\
\mathbf{B} \\
\mathbf{B}\end{array}$ & $\begin{array}{l}69 \\
68 \\
61 \\
62 \\
63\end{array}$ & $\begin{array}{l}\text { SHARON LANE } \\
\text { REED DRIVE } \\
\text { W. 2OTH STREET } \\
\text { W. 9TH STREET } \\
\text { ELMWOOD ROAD }\end{array}$ & $\begin{array}{l}\text { GRAND BLVD. } \\
\text { COMMACK ROAD } \\
\text { WESTVIEW AVENUE } \\
\text { ERLANGER BLVD. } \\
\text { COMMACK ROAD }\end{array}$ & $\begin{array}{l}4046300731910 \\
4046150731901 \\
4 \varnothing 44330732025 \\
4 \varnothing 44480731953 \\
4046070731905\end{array}$ & $\begin{array}{l}- \\
- \\
- \\
-\end{array}$ & $\begin{array}{l}- \\
- \\
- \\
-\end{array}$ & $\begin{array}{l}- \\
- \\
- \\
-\end{array}$ & $\begin{array}{l}- \\
- \\
- \\
-\end{array}$ \\
\hline $\begin{array}{l}\mathbf{B} \\
\mathbf{B} \\
\mathbf{B} \\
\mathbf{B} \\
\mathbf{B}\end{array}$ & $\begin{array}{l}64 \\
65 \\
66 \\
67 \\
68\end{array}$ & $\begin{array}{l}\text { PARK AVENUE } \\
\text { W. 3RD. STREET } \\
\text { E. 8TH. STREET } \\
\text { CARDO CIRCLE } \\
\text { TOTTEN AVENUE }\end{array}$ & $\begin{array}{l}\text { 4TH STREET } \\
\text { CENTRAL AVENUE } \\
\text { LAKE AVENUE } \\
\text { COMMACK ROAD } \\
\text { OAK STREET }\end{array}$ & $\begin{array}{l}4046146731923 \\
4046050731946 \\
4 \varnothing 46380731923 \\
4046290731856 \\
4046040732017\end{array}$ & $\begin{array}{l}- \\
- \\
- \\
-\end{array}$ & $\begin{array}{l}- \\
- \\
- \\
-\end{array}$ & $\begin{array}{l}- \\
- \\
- \\
-\end{array}$ & $\begin{array}{l}- \\
- \\
- \\
-\end{array}$ \\
\hline $\begin{array}{l}\mathbf{B} \\
\mathbf{B} \\
\mathbf{B} \\
\mathbf{B} \\
\mathbf{B}\end{array}$ & $\begin{array}{l}89 \\
70 \\
71 \\
72 \\
73\end{array}$ & $\begin{array}{l}\text { IRVING AVENUE } \\
\text { NICOLLS ROAD } \\
\text { INDUSTRY COURT } \\
\text { E. 1ST. ST. } \\
\text { BOMLING LANE }\end{array}$ & $\begin{array}{l}\text { LIBERTY STREET } \\
\text { WEST WOOD AVE. } \\
\text { GRAND BLVD. } \\
\text { OAK LAND AVENUE } \\
\text { COMMACK ROAD }\end{array}$ & $\begin{array}{l}4046140731922 \\
4 \emptyset 46000731936 \\
4046460731814 \\
404506073193 \emptyset \\
4 \varnothing 46110731901\end{array}$ & $\begin{array}{l}- \\
- \\
- \\
-\end{array}$ & $\begin{array}{l}- \\
- \\
- \\
-\end{array}$ & $\begin{array}{l}- \\
- \\
- \\
-\end{array}$ & $\begin{array}{l}- \\
- \\
- \\
-\end{array}$ \\
\hline $\begin{array}{l}\mathbf{B} \\
\mathbf{B} \\
\mathbf{B} \\
\mathbf{B} \\
\mathbf{B}\end{array}$ & $\begin{array}{l}74 \\
75 \\
76 \\
77 \\
78\end{array}$ & $\begin{array}{l}\text { BOWLING LANE } \\
\text { SUBURBAN AVE. } \\
\text { WILDWOOD AVENUE } \\
\text { ARCADIA DRIVE } \\
\text { GREAT NECK ROAD }\end{array}$ & $\begin{array}{l}\text { LISA DRIVE } \\
\text { COMMACK ROAD } \\
\text { STEVENSON PL. E. } \\
\text { STRAIGHT PATH } \\
\text { DUNBAR ROAD }\end{array}$ & $\begin{array}{l}4045130731837 \\
4045090731859 \\
4046220731948 \\
4046220732031 \\
4042140732506\end{array}$ & $\begin{array}{l}- \\
- \\
- \\
-\end{array}$ & $\begin{array}{l}- \\
- \\
- \\
-\end{array}$ & $\begin{array}{l}- \\
- \\
- \\
-\end{array}$ & $\begin{array}{l}- \\
- \\
- \\
-\end{array}$ \\
\hline $\begin{array}{l}\mathbf{B} \\
\mathbf{B} \\
\mathbf{B} \\
\mathrm{B} \\
\mathrm{B}\end{array}$ & $\begin{array}{l}79 \\
80 \\
81 \\
82 \\
83\end{array}$ & $\begin{array}{l}\text { CORRAL DRIVE } \\
\text { JEFFERSON AVE. } \\
\text { HARDING AVENUE } \\
\text { AVON DRIVE } \\
\text { DITOMAS COURT }\end{array}$ & $\begin{array}{l}\text { FLORAL DRIVE } \\
\text { CAHILL STREET } \\
\text { WASHINGTON AVE. } \\
\text { GREAT NECK ROAD } \\
\text { BAYVIEW AVENUE }\end{array}$ & $\begin{array}{l}4842010732437 \\
4041470732513 \\
4841478732448 \\
4841478732437 \\
4841100732423\end{array}$ & $\begin{array}{l}- \\
- \\
- \\
-\end{array}$ & $\begin{array}{l}- \\
- \\
- \\
-\end{array}$ & $\begin{array}{l}- \\
- \\
- \\
-\end{array}$ & $\begin{array}{l}- \\
- \\
- \\
-\end{array}$ \\
\hline $\begin{array}{l}\mathbf{B} \\
\mathbf{B} \\
\mathbf{B} \\
\mathbf{B} \\
\mathbf{B}\end{array}$ & $\begin{array}{l}84 \\
85 \\
86 \\
87 \\
88\end{array}$ & $\begin{array}{l}\text { BEVERLY AVENUE } \\
\text { LAFAYETTE STREET } \\
15 T H . \text { STREET } \\
\text { HAZEL BLVD. } \\
\text { ECKES AVENUE }\end{array}$ & $\begin{array}{l}\text { BEACHVIEW STREET } \\
\text { KENMORE AVENUE } \\
\text { 4TH. AVENUE } \\
\text { 4TH. STREET } \\
\text { LITTLE E.NECK RD }\end{array}$ & $\begin{array}{l}404 \varnothing 280732411 \\
404 \varnothing 15 \varnothing 732402 \\
4043090732315 \\
404256 \varnothing 7323 \varnothing 6 \\
40424 \varnothing 0732110\end{array}$ & $\begin{array}{l}- \\
- \\
- \\
-\end{array}$ & $\begin{array}{l}- \\
- \\
- \\
-\end{array}$ & $\begin{array}{l}- \\
- \\
- \\
-\end{array}$ & $\begin{array}{l}- \\
- \\
- \\
-\end{array}$ \\
\hline $\begin{array}{l}B \\
B \\
B \\
9 \\
B K\end{array}$ & $\begin{array}{r}89 \\
90 \\
91 \\
92 \\
1\end{array}$ & $\begin{array}{l}\text { NIMS AVENUE } \\
\text { ROSELLA COURT } \\
\text { HENRY STREET } \\
\text { LARSEN LANE } \\
\text { GASLIGHT ROAD }\end{array}$ & $\begin{array}{l}\text { LITTLE E.NECK RD } \\
\text { PURITANA COURT } \\
\text { MNCY AVENUE } \\
\text { KAHAN LANE } \\
\text { CARRIAGE COURT }\end{array}$ & $\begin{array}{l}4 \emptyset 42420732106 \\
4 \emptyset 4216 \varnothing 732114 \\
404155 \emptyset 732057 \\
4 \emptyset 42110732102 \\
4 \varnothing 55180730823\end{array}$ & $\begin{array}{c}- \\
- \\
- \\
- \\
640814\end{array}$ & $\begin{array}{l}- \\
- \\
- \\
-\end{array}$ & $\begin{array}{l}- \\
- \\
\overline{87000}\end{array}$ & $\begin{array}{l}- \\
- \\
- \\
67800\end{array}$ \\
\hline $\begin{array}{l}\text { BK } \\
\text { BK } \\
\text { BK } \\
\text { BK } \\
\text { BK }\end{array}$ & $\begin{array}{l}2 \\
3 \\
4 \\
5 \\
6\end{array}$ & $\begin{array}{l}\text { SKY VIEW LANE } \\
\text { BLINKERLIGHT RD. } \\
\text { STONY BROOK RD. } \\
\text { SOPHMORE LANE } \\
\text { CYTHIA COURT }\end{array}$ & $\begin{array}{l}\text { BEACON HILL DR. } \\
\text { GLEN COURT } \\
\text { UNIVERSITY HTS. } \\
\text { COLLEGE DRIVE } \\
\text { SHERRY DRIVE }\end{array}$ & 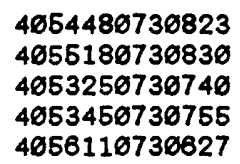 & $\begin{array}{l}6386 \\
661121 \\
6489 \\
6581 \\
640304\end{array}$ & $\begin{array}{l}- \\
- \\
- \\
-\end{array}$ & $\begin{array}{l}134000 \\
- \\
664000 \\
328000 \\
287100\end{array}$ & $\begin{array}{r}145080 \\
46800 \\
730080 \\
352060 \\
296498\end{array}$ \\
\hline $\begin{array}{l}B K \\
B K \\
B K \\
B K \\
B K\end{array}$ & $\begin{array}{r}7 \\
8 \\
9 \\
10 \\
11\end{array}$ & $\begin{array}{l}\text { MUSKET PLACE } \\
\text { FOX HOLLOW ROAD } \\
\text { TALLMADGE GATE } \\
\text { FOX HOLLOW ROAD } \\
\text { THREE VILLAGE LA }\end{array}$ & $\begin{array}{l}\text { GNARLED OAK DR. } \\
\text { THORNWOOD } \\
\text { RIDGEWAY AVENUE } \\
\text { THORNWOOD } \\
\text { SETALCUTT PL. }\end{array}$ & 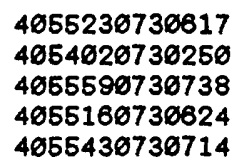 & $\begin{array}{l}670126 \\
6302 \\
651207 \\
679302 \\
618611\end{array}$ & $\begin{array}{l}- \\
- \\
- \\
-\end{array}$ & $\begin{array}{r}114000 \\
682000 \\
158100 \\
163000 \\
77900\end{array}$ & $\begin{array}{r}129200 \\
690690 \\
173800 \\
173600 \\
79300\end{array}$ \\
\hline $\begin{array}{l}\text { BK } \\
\text { BK } \\
\text { BK } \\
\text { BK } \\
\text { BK }\end{array}$ & $\begin{array}{l}12 \\
13 \\
14 \\
15 \\
16\end{array}$ & $\begin{array}{l}\text { CANTERBERRY BLVD } \\
\text { FOX DALE LANE } \\
\text { POSSUM LANE } \\
\text { COTTONTAIL LANE } \\
\text { HAWKINS ROAD }\end{array}$ & $\begin{array}{l}\text { OLD POST ROAD } \\
\text { MAIN STREET } \\
\text { WOODCHUCK LANE } \\
\text { OLD TOWN ROAD } \\
\text { LYNN STREET }\end{array}$ & $\begin{array}{l}4 \varnothing 5614 \varnothing 730443 \\
4 \varnothing 56360730449 \\
4 \varnothing 55290730436 \\
4 \varnothing 55230736452 \\
4 \varnothing 53010730454\end{array}$ & $\begin{array}{c}6112 \\
551216 \\
- \\
- \\
630305\end{array}$ & $\begin{array}{l}- \\
- \\
-\end{array}$ & $\begin{array}{l}70000 \\
295800 \\
148200 \\
306500 \\
621100\end{array}$ & $\begin{array}{l}78800 \\
351000 \\
155080 \\
312080 \\
643080\end{array}$ \\
\hline
\end{tabular}


N.Y. [Dash indicates no data. Altitudes are in feet above sea level.] (cont.)

\begin{tabular}{|c|c|c|c|c|c|c|c|c|c|c|c|c|}
\hline \multicolumn{2}{|c|}{$\begin{array}{l}\text { BASIN } \\
\text { NUMBER }\end{array}$} & \multirow{2}{*}{$\begin{array}{l}\text { MAX. } \\
\text { AREA } \\
\text { (square } \\
- \\
- \\
- \\
- \\
-\end{array}$} & \multirow{2}{*}{$\begin{array}{c}\text { BASIN } \\
\text { AREA } \\
\text { foet) } \\
28000 \\
12000 \\
14000 \\
11900 \\
72000\end{array}$} & \multicolumn{2}{|c|}{$\begin{array}{ll} & \text { OVERI } \\
\text { RIM } & \text { FLOW }\end{array}$} & $\begin{array}{l}\text { TUDE- } \\
\text { BOT- } \\
\text { TOM } \\
\end{array}$ & \multirow{2}{*}{$\begin{array}{l}\text { WATER } \\
\text { TABLE } \\
49 . \varnothing \\
49 . \varnothing \\
45 . \varnothing \\
49 . \varnothing \\
48 . \varnothing\end{array}$} & \multirow{2}{*}{$\begin{array}{c}\text { DRAIN. } \\
\text { AREA } \\
\text { (acres) } \\
- \\
- \\
- \\
-\end{array}$} & \multicolumn{2}{|c|}{$\begin{array}{l}-- \text { BASIN-- } \\
\text { STATUS USE }\end{array}$} & $\begin{array}{l}\text { GEO. } \\
\text { UNIT }\end{array}$ & \multirow{2}{*}{$\begin{array}{l}\text { SOIL } \\
\text { UNIT } \\
7 \\
7 \\
7 \\
7 \\
7\end{array}$} \\
\hline $\begin{array}{l}B \\
B \\
B \\
B \\
B\end{array}$ & $\begin{array}{l}54 \\
55 \\
56 \\
57 \\
58\end{array}$ & & & $\begin{array}{l}75.0 \\
75.0 \\
70.0 \\
80.0 \\
75.0\end{array}$ & $\begin{array}{l}- \\
- \\
- \\
-\end{array}$ & $\begin{array}{l}- \\
- \\
-\end{array}$ & & & $\begin{array}{l}1 \\
1 \\
1 \\
1 \\
1\end{array}$ & $\begin{array}{l}1 \\
1 \\
1 \\
1 \\
1\end{array}$ & $\begin{array}{l}1 \\
1 \\
1 \\
1 \\
1\end{array}$ & \\
\hline $\begin{array}{l}B \\
B \\
B \\
B \\
B\end{array}$ & $\begin{array}{l}59 \\
60 \\
61 \\
62 \\
63\end{array}$ & $\begin{array}{l}- \\
- \\
- \\
-\end{array}$ & $\begin{array}{l}37500 \\
10000 \\
40000 \\
60000 \\
20000\end{array}$ & $\begin{array}{l}70.0 \\
65.0 \\
45.0 \\
50.0 \\
60.0\end{array}$ & $\begin{array}{l}- \\
- \\
- \\
-\end{array}$ & $\begin{array}{l}- \\
- \\
- \\
-\end{array}$ & $\begin{array}{l}47.0 \\
45.0 \\
38.0 \\
42.0 \\
43.0\end{array}$ & $\begin{array}{l}- \\
- \\
- \\
-\end{array}$ & $\begin{array}{l}1 \\
1 \\
1 \\
1 \\
1\end{array}$ & $\begin{array}{l}1 \\
1 \\
1 \\
1 \\
1\end{array}$ & $\begin{array}{l}1 \\
1 \\
1 \\
1 \\
1\end{array}$ & $\begin{array}{l}7 \\
7 \\
7 \\
7 \\
1\end{array}$ \\
\hline $\begin{array}{l}B \\
B \\
B \\
B \\
B\end{array}$ & $\begin{array}{l}64 \\
65 \\
66 \\
67 \\
68\end{array}$ & $\begin{array}{l}- \\
- \\
- \\
-\end{array}$ & $\begin{array}{l}30000 \\
12500 \\
10000 \\
20000 \\
39100\end{array}$ & $\begin{array}{l}65.0 \\
60.0 \\
75.0 \\
70.0 \\
80.0\end{array}$ & $\begin{array}{l}- \\
- \\
- \\
-\end{array}$ & $\begin{array}{l}- \\
- \\
- \\
-\end{array}$ & $\begin{array}{l}48.0 \\
47.0 \\
46.0 \\
46.0 \\
53.0\end{array}$ & $\begin{array}{l}- \\
- \\
- \\
-\end{array}$ & $\begin{array}{l}1 \\
1 \\
1 \\
1 \\
1\end{array}$ & $\begin{array}{l}1 \\
1 \\
1 \\
1 \\
1\end{array}$ & $\begin{array}{l}1 \\
1 \\
1 \\
1 \\
1\end{array}$ & $\begin{array}{l}7 \\
7 \\
7 \\
7 \\
7\end{array}$ \\
\hline $\begin{array}{l}8 \\
8 \\
8 \\
8 \\
B\end{array}$ & $\begin{array}{l}69 \\
79 \\
71 \\
72 \\
73\end{array}$ & $\begin{array}{l}- \\
- \\
- \\
-\end{array}$ & $\begin{array}{l}32400 \\
68000 \\
40000 \\
42000 \\
40000\end{array}$ & $\begin{array}{l}90.0 \\
80.0 \\
75.0 \\
60.0 \\
60.0\end{array}$ & $\begin{array}{l}- \\
- \\
- \\
-\end{array}$ & $\begin{array}{l}- \\
- \\
- \\
-\end{array}$ & $\begin{array}{l}49.0 \\
47.0 \\
47.0 \\
43.0 \\
43 . \varnothing\end{array}$ & $\begin{array}{l}- \\
- \\
- \\
-\end{array}$ & $\begin{array}{l}1 \\
1 \\
1 \\
1 \\
1\end{array}$ & $\begin{array}{l}1 \\
1 \\
2 \\
1 \\
1\end{array}$ & $\begin{array}{l}1 \\
1 \\
1 \\
1 \\
1\end{array}$ & $\begin{array}{l}7 \\
7 \\
3 \\
7 \\
7\end{array}$ \\
\hline $\begin{array}{l}B \\
B \\
B \\
B \\
B\end{array}$ & $\begin{array}{l}74 \\
75 \\
76 \\
77 \\
78\end{array}$ & $\begin{array}{l}- \\
- \\
- \\
-\end{array}$ & $\begin{array}{l}48000 \\
18000 \\
22500 \\
30000 \\
10000\end{array}$ & $\begin{array}{l}75.0 \\
60.0 \\
90.0 \\
80.0 \\
45.0\end{array}$ & $\begin{array}{l}- \\
- \\
- \\
-\end{array}$ & $\begin{array}{l}- \\
- \\
- \\
-\end{array}$ & $\begin{array}{l}44.0 \\
44.0 \\
53.0 \\
53.0 \\
25.0\end{array}$ & $\begin{array}{l}- \\
- \\
- \\
-\end{array}$ & $\begin{array}{l}1 \\
1 \\
1 \\
1 \\
1\end{array}$ & $\begin{array}{l}1 \\
1 \\
1 \\
1 \\
1\end{array}$ & $\begin{array}{l}1 \\
1 \\
1 \\
1 \\
1\end{array}$ & $\begin{array}{l}7 \\
1 \\
7 \\
7 \\
7\end{array}$ \\
\hline $\begin{array}{l}8 \\
8 \\
8 \\
8 \\
8\end{array}$ & $\begin{array}{l}79 \\
80 \\
81 \\
82 \\
83\end{array}$ & $\begin{array}{l}- \\
- \\
- \\
-\end{array}$ & $\begin{array}{l}400 \varnothing \emptyset \\
2400 \emptyset \\
4800 \emptyset \\
4600 \emptyset \\
15000\end{array}$ & $\begin{array}{l}40.0 \\
40.0 \\
40.0 \\
40.0 \\
25.0\end{array}$ & $\begin{array}{l}\overline{-} \\
\bar{z} \\
\overline{-}\end{array}$ & $\begin{array}{l}- \\
- \\
- \\
-\end{array}$ & $\begin{array}{l}31.0 \\
27.0 \\
27.0 \\
27.0 \\
19.0\end{array}$ & $\begin{array}{l}- \\
- \\
- \\
-\end{array}$ & $\begin{array}{l}1 \\
1 \\
1 \\
1 \\
1\end{array}$ & $\begin{array}{l}1 \\
1 \\
1 \\
1 \\
1\end{array}$ & $\begin{array}{l}1 \\
1 \\
1 \\
1 \\
1\end{array}$ & $\begin{array}{l}7 \\
7 \\
7 \\
7 \\
7\end{array}$ \\
\hline $\begin{array}{l}B \\
B \\
B \\
B \\
B\end{array}$ & $\begin{array}{l}84 \\
85 \\
86 \\
87 \\
88\end{array}$ & $\begin{array}{l}- \\
- \\
- \\
-\end{array}$ & $\begin{array}{l}18000 \\
35000 \\
47300 \\
14000 \\
18000\end{array}$ & $\begin{array}{l}15.0 \\
10.0 \\
50.0 \\
45.0 \\
35.0\end{array}$ & $\begin{array}{l}- \\
- \\
- \\
-\end{array}$ & $\begin{array}{l}- \\
- \\
- \\
-\end{array}$ & $\begin{array}{r}9.9 \\
5.0 \\
42.0 \\
42.0 \\
23.0\end{array}$ & $\begin{array}{l}- \\
- \\
- \\
-\end{array}$ & $\begin{array}{l}1 \\
1 \\
1 \\
1 \\
1\end{array}$ & $\begin{array}{l}1 \\
1 \\
1 \\
1 \\
1\end{array}$ & $\begin{array}{l}1 \\
1 \\
1 \\
1 \\
1\end{array}$ & $\begin{array}{l}7 \\
7 \\
7 \\
7 \\
7\end{array}$ \\
\hline $\begin{array}{l}8 \\
B \\
8 \\
B \\
8 K\end{array}$ & $\begin{array}{r}89 \\
90 \\
91 \\
92 \\
1\end{array}$ & $\begin{array}{l}- \\
- \\
- \\
\overline{3800}\end{array}$ & $\begin{array}{r}5900 \\
20000 \\
7500 \\
17500 \\
27600\end{array}$ & $\begin{array}{r}35.0 \\
30.0 \\
25.0 \\
30.0 \\
116.0\end{array}$ & $\begin{array}{r}- \\
- \\
- \\
118 . \overline{0}\end{array}$ & $\begin{array}{r}\overline{-} \\
\overline{-} \\
\overline{-}\end{array}$ & $\begin{array}{l}23.0 \\
18.0 \\
13.0 \\
17.0 \\
25.0\end{array}$ & $\begin{array}{r}- \\
- \\
\overline{-} \\
10.5\end{array}$ & $\begin{array}{l}1 \\
1 \\
1 \\
1 \\
1\end{array}$ & $\begin{array}{l}1 \\
1 \\
1 \\
1 \\
1\end{array}$ & $\begin{array}{l}1 \\
1 \\
1 \\
1 \\
3\end{array}$ & $\begin{array}{l}7 \\
7 \\
3 \\
7 \\
6\end{array}$ \\
\hline $\begin{array}{l}\text { BK } \\
\text { BK } \\
\text { BK } \\
\text { BK } \\
\text { BK }\end{array}$ & $\begin{array}{l}2 \\
3 \\
4 \\
5 \\
6\end{array}$ & $\begin{array}{r}14500 \\
3600 \\
73000 \\
35200 \\
22800\end{array}$ & $\begin{array}{l}41400 \\
15400 \\
80000 \\
64000 \\
40000\end{array}$ & $\begin{array}{r}52.0 \\
94.0 \\
140.0 \\
151.0 \\
92.0\end{array}$ & $\begin{array}{r}92 . \bar{\emptyset} \\
\overline{-} \\
91 . \bar{\emptyset}\end{array}$ & $\begin{array}{r}41.0 \\
80.0 \\
125.0 \\
135.0 \\
78.0\end{array}$ & $\begin{array}{l}31 . \emptyset \\
25 . \emptyset \\
41 . \emptyset \\
45 . \emptyset \\
28 . \emptyset\end{array}$ & $\begin{array}{r}23.0 \\
3.0 \\
104.6 \\
37.4 \\
30.9\end{array}$ & $\begin{array}{l}1 \\
1 \\
1 \\
6 \\
1\end{array}$ & $\begin{array}{l}1 \\
1 \\
1 \\
1 \\
1\end{array}$ & $\begin{array}{l}3 \\
3 \\
1 \\
4 \\
3\end{array}$ & $\begin{array}{l}5 \\
6 \\
7 \\
7 \\
3\end{array}$ \\
\hline $\begin{array}{l}\text { BK } \\
\text { BK } \\
\text { BK } \\
\text { BK } \\
\text { BK }\end{array}$ & $\begin{array}{r}7 \\
8 \\
9 \\
10 \\
11\end{array}$ & $\begin{array}{r}10800 \\
69100 \\
17300 \\
15700 \\
6600\end{array}$ & $\begin{array}{r}27000 \\
36000 \\
40500 \\
22400\end{array}$ & $\begin{array}{r}92.0 \\
141.0 \\
103.6 \\
64.0 \\
109.0\end{array}$ & $\begin{array}{r}90.0 \\
- \\
62.0 \\
108.0\end{array}$ & $\begin{array}{r}78.0 \\
124.0 \\
92.0 \\
51.0 \\
96.0\end{array}$ & $\begin{array}{l}38.0 \\
51.0 \\
27.0 \\
37.0 \\
32.0\end{array}$ & $\begin{array}{r}12.5 \\
107.4 \\
27.0 \\
17.5 \\
14.3\end{array}$ & $\begin{array}{l}1 \\
1 \\
1 \\
1 \\
1\end{array}$ & $\begin{array}{l}1 \\
1 \\
1 \\
1 \\
1\end{array}$ & $\begin{array}{l}3 \\
1 \\
3 \\
3 \\
3\end{array}$ & $\begin{array}{l}6 \\
7 \\
6 \\
3 \\
7\end{array}$ \\
\hline $\begin{array}{l}\text { BK } \\
B K \\
B K \\
B K \\
B K\end{array}$ & $\begin{array}{l}12 \\
13 \\
14 \\
15 \\
16\end{array}$ & $\begin{array}{r}7900 \\
35100 \\
15500 \\
31200 \\
58500\end{array}$ & $\begin{array}{r}16800 \\
63600 \\
52000 \\
61400\end{array}$ & $\begin{array}{l}165.0 \\
118.5 \\
148.0 \\
169.0 \\
107.7\end{array}$ & $\begin{array}{r}- \\
- \\
- \\
103 . \overline{5}\end{array}$ & $\begin{array}{r}148.0 \\
95.0 \\
- \\
146.0 \\
92.5\end{array}$ & $\begin{array}{l}30.0 \\
20.0 \\
41.0 \\
41.0 \\
52\end{array}$ & $\begin{array}{l}11.0 \\
35.0 \\
27.0 \\
35.0 \\
97.8\end{array}$ & $\begin{array}{l}1 \\
0 \\
1 \\
1 \\
1\end{array}$ & $\begin{array}{l}1 \\
1 \\
1 \\
1 \\
1\end{array}$ & $\begin{array}{l}3 \\
3 \\
4 \\
3 \\
1\end{array}$ & $\begin{array}{l}7 \\
7 \\
5 \\
5 \\
5\end{array}$ \\
\hline
\end{tabular}


Appendix.--Location and basic physical features of recharge basins on Long Island,

\begin{tabular}{|c|c|c|c|c|c|c|c|c|}
\hline \multicolumn{2}{|c|}{$\begin{array}{l}\text { BASIN } \\
\text { NUMBER }\end{array}$} & \multicolumn{2}{|c|}{ NEAREST INTERSECTION } & \multirow{2}{*}{$\begin{array}{l}\text { LATITUDE } \\
\text { LONGITUDE } \\
\circ, " \text {, } \\
4953410730457 \\
4653370730651 \\
4652420736644 \\
4653350736468 \\
4653580739411\end{array}$} & \multirow{2}{*}{$\begin{array}{l}\text { DATE } \\
\text { BUILT } \\
\text { YrMOD } \\
636305 \\
6310 \\
638627 \\
6267 \\
6267\end{array}$} & \multirow{2}{*}{$\begin{array}{r}\text { COMMNITY } \\
- \\
- \\
- \\
-\end{array}$} & \multicolumn{2}{|c|}{$\begin{array}{l}\text { DESIGN ACTUAL } \\
\text { CAPACITY CAPACITY } \\
\text { (cubic feet) }\end{array}$} \\
\hline $\begin{array}{l}B K \\
B K \\
B K \\
B K \\
B K\end{array}$ & $\begin{array}{l}17 \\
19 \\
20 \\
21 \\
22\end{array}$ & $\begin{array}{l}\text { ONEIDA AVENUE } \\
\text { NICOLLS ROAD } \\
\text { DOOLINGS PATH } \\
\text { FAWN LANE } \\
\text { PENN STREET }\end{array}$ & $\begin{array}{l}\text { GIRARD STREET } \\
\text { FORTUNE LANE } \\
\text { BLUETOP ROAD } \\
\text { TRACHER LANE } \\
\text { NEW YORK AVENUE }\end{array}$ & & & & $\begin{array}{l}5058980 \\
1178080 \\
395868 \\
132368 \\
2898680\end{array}$ & $\begin{array}{l}515408 \\
133000 \\
309900 \\
127000 \\
349008\end{array}$ \\
\hline $\begin{array}{l}B K \\
B K \\
B K \\
B K \\
B K\end{array}$ & $\begin{array}{l}23 \\
24 \\
25 \\
26 \\
27\end{array}$ & $\begin{array}{l}\text { COMERFORD } \\
\text { FAWN LANE } \\
\text { NICOLLS ROAD } \\
\text { HAMILTON ROAD } \\
\text { SETAUKET ROAD }\end{array}$ & $\begin{array}{l}\text { NEW YORK AVENUE } \\
\text { LONGBOW LANE } \\
\text { DANIEL WEB'R DR. } \\
\text { WASHINGTON ROAD } \\
\text { SHEEP PASTURE RD }\end{array}$ & 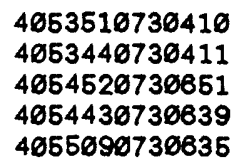 & $\begin{array}{l}6207 \\
6207 \\
640203 \\
640203 \\
640203\end{array}$ & $\begin{array}{l}- \\
- \\
-\end{array}$ & $\begin{array}{l}166406 \\
129708 \\
4608008 \\
7268000 \\
-\end{array}$ & $\begin{array}{l}170000 \\
145000 \\
468000 \\
724000 \\
-\end{array}$ \\
\hline $\begin{array}{l}B K \\
B K \\
B K \\
B K \\
B K\end{array}$ & $\begin{array}{l}28 \\
29 \\
30 \\
31 \\
32\end{array}$ & $\begin{array}{l}\text { FIRST STREET } \\
\text { STORY LAND LANE } \\
\text { CINDERELLA LANE } \\
\text { MERLIN LANE } \\
\text { SETAUKET ROAD }\end{array}$ & $\begin{array}{l}\text { FIRST AVENUE } \\
\text { STORY BOOK LANE } \\
\text { MERLIN LANE } \\
\text { STORY LAND LANE } \\
\text { 25TH. STREET }\end{array}$ & $\begin{array}{l}4 ø 51020730241 \\
4654510736611 \\
4654580730557 \\
4655646736558 \\
4053470736548\end{array}$ & $\begin{array}{l}649128 \\
610630 \\
6396 \\
6386 \\
671221\end{array}$ & $\begin{array}{l}- \\
- \\
- \\
-\end{array}$ & $\begin{array}{l}92208 \\
3923080 \\
1626880 \\
1358080 \\
-\end{array}$ & $\begin{array}{l}144400 \\
4808080 \\
185000 \\
1458000 \\
-\end{array}$ \\
\hline $\begin{array}{l}B K \\
B K \\
B K \\
B K \\
B K\end{array}$ & $\begin{array}{l}33 \\
34 \\
36 \\
36 \\
37\end{array}$ & $\begin{array}{l}\text { LOCUST AVENUE } \\
\text { TREE ROAD } \\
\text { HAYKINS ROAD } \\
\text { STRATTON COURT } \\
\text { SYCAMORE CIRCLE }\end{array}$ & $\begin{array}{l}\text { 1ST. AVENUE } \\
\text { HILBURN ROAD } \\
\text { EASTWOOD BLVD. } \\
\text { STRATTON LANE } \\
\text { SHEPPARD LANE }\end{array}$ & 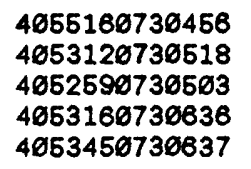 & $\begin{array}{l}671026 \\
640109 \\
646109 \\
631210 \\
631210\end{array}$ & $\begin{array}{l}- \\
- \\
- \\
-\end{array}$ & $\begin{array}{r}107000 \\
110000 \\
1920080 \\
7150800 \\
1168080\end{array}$ & $\begin{array}{r}1080800 \\
136060 \\
2420800 \\
7506000 \\
12370080\end{array}$ \\
\hline $\begin{array}{l}B K \\
B K \\
B K \\
B K \\
B K\end{array}$ & $\begin{array}{l}38 \\
39 \\
46 \\
41 \\
42\end{array}$ & $\begin{array}{l}\text { SILVER SPRUCE LA } \\
\text { SYCAMORE CIRCLE } \\
\text { POND PATH } \\
\text { HASKEL LANE } \\
\text { OXHEAD ROAD }\end{array}$ & $\begin{array}{l}\text { STRATTON LANE } \\
\text { SHAWMONT LANE } \\
\text { MCGAW AVENUE } \\
\text { HASTINGS DRIVE } \\
\text { BARNWELL LANE }\end{array}$ & $\begin{array}{l}4053220730611 \\
4053370730559 \\
4052200730638 \\
4054680739656 \\
4053290730657\end{array}$ & $\begin{array}{l}641217 \\
646206 \\
670209 \\
651061 \\
650419\end{array}$ & $\begin{array}{l}- \\
- \\
-\end{array}$ & $\begin{array}{l}769000 \\
2500080 \\
3490800 \\
199600 \\
675000\end{array}$ & $\begin{array}{l}808080 \\
252200 \\
342080 \\
202580 \\
725980\end{array}$ \\
\hline $\begin{array}{l}B K \\
B K \\
B K \\
B K \\
B K\end{array}$ & $\begin{array}{l}43 \\
44 \\
46 \\
46 \\
47\end{array}$ & $\begin{array}{l}\text { RONNIE LANE } \\
\text { BLACKWELL LANE } \\
\text { PALFREY STREET } \\
\text { HUNTING LANE } \\
\text { MARWOOD PLACE }\end{array}$ & $\begin{array}{l}\text { BEAVER DALE LANE } \\
\text { BLACKWELL COURT } \\
\text { PEMBROOK LANE } \\
\text { BARNWELL LANE } \\
\text { MAYMONT LANE }\end{array}$ & 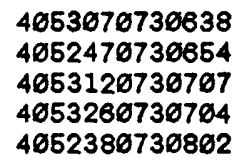 & $\begin{array}{l}650419 \\
650419 \\
651202 \\
651202 \\
650604\end{array}$ & $\begin{array}{l}- \\
\overline{-} \\
-\end{array}$ & $\begin{array}{l}826500 \\
336600 \\
335080 \\
290800 \\
-\end{array}$ & $\begin{array}{l}950000 \\
342000 \\
365000 \\
310000 \\
-\end{array}$ \\
\hline $\begin{array}{l}B K \\
B K \\
B K \\
B K \\
B K\end{array}$ & $\begin{array}{l}49 \\
56 \\
53 \\
54 \\
56\end{array}$ & $\begin{array}{l}\text { STONY BROOK ROAD } \\
\text { MILSTREAM LANE } \\
\text { VALIANT DRIVE } \\
\text { HAWKINS ROAD } \\
\text { HAWKINS ROAD }\end{array}$ & $\begin{array}{l}\text { GROVE STREET } \\
\text { ONYX DRIVE } \\
\text { HOLIDAY PARK DR. } \\
\text { SMITHTOWN BLVD. } \\
\text { COLEMAN ROAD }\end{array}$ & 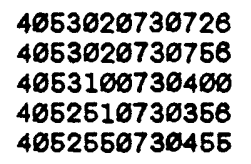 & $\begin{array}{l}661108 \\
661215 \\
680881 \\
646801 \\
680881\end{array}$ & $\begin{array}{l}- \\
- \\
-\end{array}$ & $\begin{array}{r}13338080 \\
485680 \\
1017680 \\
285680 \\
291606\end{array}$ & $\begin{array}{c}\overline{760080} \\
1088080 \\
\overline{2} \\
294800\end{array}$ \\
\hline $\begin{array}{l}B K \\
B K \\
B K \\
B K \\
B K\end{array}$ & $\begin{array}{l}66 \\
57 \\
58 \\
68 \\
61\end{array}$ & $\begin{array}{l}\text { HAWKINS ROAD } \\
\text { OLD HOMESTEAD DR } \\
\text { BEACH AVENUE } \\
\text { JEANNE AVENUE } \\
\text { JEFFERSON BLVD. }\end{array}$ & $\begin{array}{l}\text { COLEMAN ROAD } \\
\text { SANDS LANE } \\
28 T H \text { STREET } \\
\text { MARK STREET } \\
\text { 13TH. STREET }\end{array}$ & 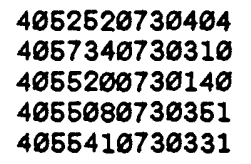 & $\begin{array}{l}681021 \\
630702 \\
650623 \\
641017 \\
620331\end{array}$ & $\begin{array}{l}- \\
- \\
-\end{array}$ & $\begin{array}{l}544080 \\
- \\
1760800 \\
285808 \\
168080\end{array}$ & $\begin{array}{l}562000 \\
- \\
188100 \\
322080 \\
168080\end{array}$ \\
\hline $\begin{array}{l}B K \\
B K \\
B K \\
B K \\
B K\end{array}$ & $\begin{array}{l}62 \\
63 \\
64 \\
65 \\
66\end{array}$ & $\begin{array}{l}\text { JEANNE AVENUE } \\
\text { CAROLYN ROAD } \\
\text { PINE HILL ROAD } \\
\text { ARDMER DRIVE } \\
\text { NORWOOD AVENUE }\end{array}$ & $\begin{array}{l}\text { JOHN STREET } \\
\text { LINDA STREET } \\
\text { CRYSTAL BRK. HOL } \\
\text { GAYMORE ROAD } \\
\text { CHARM CITY DRIVE }\end{array}$ & $\begin{array}{l}4055190739348 \\
4 \varnothing 5510673 \varnothing 424 \\
4056310730227 \\
405527073 ø 318 \\
4055139730320\end{array}$ & $\begin{array}{l}640516 \\
640510 \\
67092.8 \\
650522 \\
650522\end{array}$ & $\begin{array}{l}- \\
- \\
-\end{array}$ & $\begin{array}{l}256600 \\
- \\
203080 \\
303500 \\
126000\end{array}$ & $\begin{array}{l}305000 \\
- \\
2670000 \\
310000 \\
135000\end{array}$ \\
\hline $\begin{array}{l}B K \\
B K \\
B K \\
B K \\
B K\end{array}$ & $\begin{array}{l}67 \\
68 \\
69 \\
70 \\
71\end{array}$ & $\begin{array}{l}\text { CLINTON PLACE } \\
\text { FOREST AVENUE } \\
\text { WILLIAM STREET } \\
\text { CRYSTAL BRK.HOL. } \\
\text { JUNARD STREET }\end{array}$ & $\begin{array}{l}\text { CHEREB LANE } \\
\text { NORWOOD AVENUE } \\
\text { COLUMBIA STREET } \\
\text { SHENANDOAH BLVD. } \\
\text { CANAL ROAD }\end{array}$ & $\begin{array}{l}4655420736317 \\
46550767364 \varnothing 2 \\
465541673624 \varnothing \\
4655410736260 \\
4655260730204\end{array}$ & $\begin{array}{c}591015 \\
- \\
681018 \\
630115 \\
638115\end{array}$ & $\begin{array}{l}- \\
- \\
-\end{array}$ & $\begin{array}{l}1640800 \\
249800 \\
\overline{8} \\
1926800\end{array}$ & $\begin{array}{l}1678000 \\
373180 \\
- \\
91080 \\
183580\end{array}$ \\
\hline $\begin{array}{l}B K \\
B K \\
B K \\
B K \\
B K\end{array}$ & $\begin{array}{l}72 \\
73 \\
74 \\
75 \\
78\end{array}$ & $\begin{array}{l}\text { CANAL ROAD } \\
\text { NORWOOD AVENUE } \\
\text { ANDOVER DRIVE } \\
\text { YALE STREET } \\
\text { CLINTON AVENUE }\end{array}$ & $\begin{array}{l}\text { SHENANDOAH BLVD. } \\
\text { JEFFERSON BLVD. } \\
\text { EATON DRIVE } \\
\text { RHETTA LANE } \\
\text { LONG STREET }\end{array}$ & $\begin{array}{l}4055180730154 \\
4 ø 5511073033 \emptyset \\
4055060730153 \\
4054360730319 \\
4054230730226\end{array}$ & $\begin{array}{l}6504 \\
660510 \\
6584 \\
650213 \\
\quad-\end{array}$ & $\begin{array}{l}- \\
- \\
-\end{array}$ & $\begin{array}{l}138080 \\
113800 \\
175500 \\
396080 \\
-\end{array}$ & $\begin{array}{l}133080 \\
115000 \\
180000 \\
4100000 \\
-\end{array}$ \\
\hline
\end{tabular}


N.Y. [Dash indicates no data. Altitudes are in feet above sea level.] (cont.)

\begin{tabular}{|c|c|c|c|c|c|c|c|c|c|c|c|c|}
\hline \multicolumn{2}{|c|}{$\begin{array}{l}\text { BASIN } \\
\text { NUMBER }\end{array}$} & $\begin{array}{l}\text { MAX. } \\
\text { AREA } \\
\text { (squar }\end{array}$ & $\begin{array}{c}\text { BASIN } \\
\text { AREA } \\
\text { re feet) }\end{array}$ & RIM & $\begin{array}{l}\text {-ALT } \\
\text { OVER } \\
\text { FLOW }\end{array}$ & $\begin{array}{l}\text { ITUDE--- } \\
-\quad \text { BOT- } \\
\text { TOM }\end{array}$ & $\begin{array}{l}\text { WATER } \\
\text { TABLE }\end{array}$ & $\begin{array}{c}\text { DRAIN. } \\
\text { AREA } \\
\text { (acres) }\end{array}$ & \multicolumn{2}{|c|}{$\begin{array}{l}---B A S I N-- \\
\text { STATUS USE }\end{array}$} & $\begin{array}{l}\text { GEO. } \\
\text { UNIT }\end{array}$ & $\begin{array}{l}\text { SOIL } \\
\text { UNIT }\end{array}$ \\
\hline $\begin{array}{l}\text { BK } \\
\text { BK } \\
\text { BK } \\
\text { BK } \\
\text { BK }\end{array}$ & $\begin{array}{l}17 \\
19 \\
20 \\
21 \\
22\end{array}$ & $\begin{array}{l}41200 \\
13300 \\
21300 \\
12700 \\
34000\end{array}$ & $\begin{array}{l}82800 \\
23800 \\
504 \varnothing \emptyset \\
184 \varnothing \emptyset \\
34100\end{array}$ & $\begin{array}{r}133.5 \\
148.0 \\
56.0 \\
107.0 \\
109.0\end{array}$ & $\begin{array}{r}131.3 \\
- \\
50.0 \\
- \\
-\end{array}$ & $\begin{array}{r}119.8 \\
-\overline{5} \\
35.5 \\
96.0 \\
98.0\end{array}$ & $\begin{array}{l}52.0 \\
49.0 \\
33.0 \\
52.0 \\
52.0\end{array}$ & $\begin{array}{l}79.5 \\
18.4 \\
48.0 \\
24.1 \\
33.0\end{array}$ & $\begin{array}{l}1 \\
1 \\
1 \\
1 \\
1\end{array}$ & $\begin{array}{l}1 \\
1 \\
1 \\
1 \\
1\end{array}$ & $\begin{array}{l}1 \\
4 \\
3 \\
1 \\
1\end{array}$ & $\begin{array}{l}1 \\
7 \\
7 \\
5 \\
7\end{array}$ \\
\hline $\begin{array}{l}\text { BK } \\
\text { BK } \\
\text { BK } \\
\text { BK } \\
\text { BK }\end{array}$ & $\begin{array}{l}23 \\
24 \\
25 \\
26 \\
27\end{array}$ & $\begin{array}{c}17000 \\
14500 \\
46800 \\
72000 \\
-\end{array}$ & $\begin{array}{r}21800 \\
22100 \\
46800 \\
105600 \\
41800\end{array}$ & $\begin{array}{r}139.0 \\
109.0 \\
110.0 \\
120.0 \\
97.0\end{array}$ & $\begin{array}{l}\text { - } \\
\text { - } \\
\text { - }\end{array}$ & $\begin{array}{r}124.0 \\
96.0 \\
109.0 \\
95.8 \\
86.0\end{array}$ & $\begin{array}{l}52.0 \\
52.0 \\
43.0 \\
42.0 \\
39.0\end{array}$ & $\begin{array}{r}19.0 \\
22.0 \\
72.4 \\
113.3 \\
-\end{array}$ & $\begin{array}{l}1 \\
1 \\
1 \\
1 \\
1\end{array}$ & $\begin{array}{l}1 \\
1 \\
1 \\
1 \\
1\end{array}$ & $\begin{array}{l}1 \\
1 \\
3 \\
3 \\
3\end{array}$ & $\begin{array}{l}7 \\
7 \\
1 \\
7 \\
5\end{array}$ \\
\hline $\begin{array}{l}\text { BK } \\
\text { BK } \\
\text { BK } \\
\text { BK } \\
\text { BK }\end{array}$ & $\begin{array}{l}28 \\
29 \\
30 \\
31 \\
32\end{array}$ & $\begin{array}{c}144 \varnothing 0 \\
4 \varnothing \varnothing \varnothing \varnothing \\
185 \varnothing \varnothing \\
14500 \\
-\end{array}$ & $\begin{array}{r}16500 \\
105000 \\
67200 \\
36000 \\
75600\end{array}$ & $\begin{array}{r}- \\
157.0 \\
135 . \varnothing \\
118.5\end{array}$ & $\begin{array}{r}- \\
- \\
118 . \overline{5}\end{array}$ & $\begin{array}{r}- \\
- \\
141.8 \\
120.0 \\
102.5\end{array}$ & $\begin{array}{r}58.6 \\
43.6 \\
41.6 \\
57 . \overline{6}\end{array}$ & $\begin{array}{r}14.5 \\
42.0 \\
18.5 \\
15.5 \\
-\end{array}$ & $\begin{array}{l}1 \\
1 \\
1 \\
0 \\
1\end{array}$ & $\begin{array}{l}1 \\
1 \\
1 \\
3 \\
1\end{array}$ & $\begin{array}{l}6 \\
3 \\
3 \\
3 \\
1\end{array}$ & $\begin{array}{l}5 \\
8 \\
3 \\
3 \\
3\end{array}$ \\
\hline $\begin{array}{l}\text { BK } \\
\text { BK } \\
\text { BK } \\
\text { BK } \\
\text { BK }\end{array}$ & $\begin{array}{l}33 \\
34 \\
35 \\
36 \\
37\end{array}$ & $\begin{array}{r}10800 \\
13600 \\
24200 \\
75000 \\
112500\end{array}$ & $\begin{array}{r}28800 \\
28800 \\
57 \varnothing \varnothing \emptyset \\
95 \varnothing \varnothing \varnothing \\
2830 \varnothing \emptyset\end{array}$ & $\begin{array}{l}159.5 \\
115.5 \\
101.5 \\
120.0 \\
129.0\end{array}$ & $\begin{array}{r}- \\
101.5 \\
119.0 \\
129.0\end{array}$ & $\begin{array}{r}146.0 \\
104.0 \\
88.5 \\
109.0 \\
118.0\end{array}$ & $\begin{array}{l}42.0 \\
53.0 \\
53.0 \\
52.0 \\
50.0\end{array}$ & $\begin{array}{r}16.8 \\
17.3 \\
30.2 \\
112.6 \\
134.6\end{array}$ & $\begin{array}{l}1 \\
1 \\
1 \\
1 \\
1\end{array}$ & $\begin{array}{l}1 \\
1 \\
1 \\
1 \\
1\end{array}$ & $\begin{array}{l}4 \\
1 \\
1 \\
1 \\
1\end{array}$ & $\begin{array}{l}6 \\
1 \\
5 \\
7 \\
7\end{array}$ \\
\hline $\begin{array}{l}\text { BK } \\
\text { BK } \\
\text { BK } \\
\text { BK } \\
\text { BK }\end{array}$ & $\begin{array}{l}38 \\
39 \\
40 \\
41 \\
42\end{array}$ & $\begin{array}{l}55200 \\
18000 \\
29700 \\
16900 \\
65900\end{array}$ & $\begin{array}{l}80000 \\
52000 \\
51000 \\
36300 \\
98000\end{array}$ & $\begin{array}{l}128.0 \\
122.0 \\
116.0 \\
153.0 \\
129 . \varnothing\end{array}$ & $\begin{array}{l}116.0 \\
120.0 \\
113.0 \\
153.0 \\
126 . \varnothing\end{array}$ & $\begin{array}{l}101.5 \\
106.0 \\
101.5 \\
141.0 \\
115.0\end{array}$ & $\begin{array}{l}52.0 \\
52.0 \\
55.0 \\
49.0 \\
51.0\end{array}$ & $\begin{array}{r}86.8 \\
28.7 \\
62.6 \\
22.8 \\
123.6\end{array}$ & $\begin{array}{l}1 \\
1 \\
1 \\
1 \\
1\end{array}$ & $\begin{array}{l}1 \\
1 \\
3 \\
1 \\
1\end{array}$ & $\begin{array}{l}1 \\
1 \\
1 \\
4 \\
1\end{array}$ & $\begin{array}{l}7 \\
7 \\
3 \\
7 \\
7\end{array}$ \\
\hline $\begin{array}{l}\text { BK } \\
\text { BK } \\
\text { BK } \\
\text { BK } \\
B K\end{array}$ & $\begin{array}{l}43 \\
44 \\
45 \\
46 \\
47\end{array}$ & $\begin{array}{c}864 \varnothing \varnothing \\
274 \varnothing \varnothing \\
365 \varnothing \emptyset \\
25800 \\
-\end{array}$ & $\begin{array}{r}108800 \\
64800 \\
52500 \\
6500 \varnothing \\
4 \varnothing 000\end{array}$ & $\begin{array}{l}114.0 \\
132.0 \\
136.0 \\
133.0 \\
107.7\end{array}$ & $\begin{array}{l}114.0 \\
129.5 \\
133.5 \\
130.0 \\
105.7\end{array}$ & $\begin{array}{r}103.0 \\
117.0 \\
123.5 \\
118.0 \\
97.0\end{array}$ & $\begin{array}{l}54.0 \\
55.0 \\
51.0 \\
51.0 \\
56.0\end{array}$ & $\begin{array}{r}94.4 \\
38.1 \\
52.7 \\
45.7 \\
-\end{array}$ & $\begin{array}{l}1 \\
0 \\
1 \\
1 \\
1\end{array}$ & $\begin{array}{l}1 \\
3 \\
1 \\
1 \\
1\end{array}$ & $\begin{array}{l}1 \\
1 \\
1 \\
1 \\
1\end{array}$ & $\begin{array}{l}7 \\
7 \\
7 \\
7 \\
7\end{array}$ \\
\hline $\begin{array}{l}\text { BK } \\
\text { BK } \\
\text { BK } \\
\text { BK } \\
\text { BK }\end{array}$ & $\begin{array}{l}49 \\
50 \\
53 \\
54 \\
55\end{array}$ & $\begin{array}{c}\overline{-} \\
65000 \\
106800 \\
- \\
29500\end{array}$ & $\begin{array}{r}150800 \\
7 \varnothing \varnothing \varnothing \varnothing \\
104000 \\
60800 \\
42000\end{array}$ & $\begin{array}{r}135.5 \\
129.0 \\
102.0 \\
93.5 \\
102.0\end{array}$ & $\begin{array}{r}135.5 \\
126.5 \\
\overline{9} \\
91.5 \\
-\end{array}$ & $\begin{array}{r}124.5 \\
114.8 \\
84.0 \\
75.5 \\
83.5\end{array}$ & $\begin{array}{r}52.0 \\
53.0 \\
182.0 \\
54.6 \\
54.6\end{array}$ & $\begin{array}{r}153.0 \\
55.0 \\
160.1 \\
32.6 \\
32.3\end{array}$ & $\begin{array}{l}1 \\
1 \\
1 \\
1 \\
1\end{array}$ & $\begin{array}{l}1 \\
1 \\
1 \\
1 \\
1\end{array}$ & $\begin{array}{l}1 \\
1 \\
1 \\
1 \\
1\end{array}$ & $\begin{array}{l}1 \\
1 \\
5 \\
5 \\
5\end{array}$ \\
\hline $\begin{array}{l}\text { BK } \\
\text { BK } \\
\text { BK } \\
\text { BK } \\
\text { BK }\end{array}$ & $\begin{array}{l}56 \\
57 \\
58 \\
60 \\
61\end{array}$ & $\begin{array}{c}51100 \\
- \\
15700 \\
32200 \\
14100\end{array}$ & $\begin{array}{r}64500 \\
42300 \\
49600 \\
32000\end{array}$ & $\begin{array}{l}113.0 \\
150.0 \\
145.0 \\
171.0 \\
178.0\end{array}$ & $\begin{array}{r}110.0 \\
141.0 \\
176.4\end{array}$ & $\begin{array}{r}99.0 \\
75.0 \\
129.0 \\
160.0 \\
164.5\end{array}$ & $\begin{array}{l}11 . \overline{6} \\
43.0 \\
43.0 \\
42.0\end{array}$ & $\begin{array}{r}85.6 \\
-\overline{1} \\
19.0 \\
44.9 \\
26.4\end{array}$ & $\begin{array}{l}1 \\
1 \\
1 \\
1 \\
1\end{array}$ & $\begin{array}{l}1 \\
1 \\
3 \\
1 \\
1\end{array}$ & $\begin{array}{l}1 \\
3 \\
1 \\
1 \\
1\end{array}$ & $\begin{array}{l}5 \\
1 \\
3 \\
7 \\
7\end{array}$ \\
\hline $\begin{array}{l}\text { BK } \\
\text { BK } \\
\text { BK } \\
\text { BK } \\
\text { BK }\end{array}$ & $\begin{array}{l}62 \\
63 \\
64 \\
65 \\
66\end{array}$ & $\begin{array}{c}30500 \\
- \\
24300 \\
31000 \\
13500\end{array}$ & $\begin{array}{r}504 \varnothing \varnothing \\
- \\
88 \varnothing \varnothing \varnothing \\
504 \varnothing \emptyset \\
224 \varnothing \emptyset\end{array}$ & $\begin{array}{r}174.3 \\
175.0 \\
90.9 \\
173.6 \\
172.0\end{array}$ & $\begin{array}{r}- \\
\overline{-} \\
85.9 \\
-\end{array}$ & $\begin{array}{r}164.5 \\
162.0 \\
74.9 \\
160.5 \\
158.5\end{array}$ & $\begin{array}{l}43.0 \\
44.0 \\
30.6 \\
43.0 \\
43.0\end{array}$ & $\begin{array}{r}40.4 \\
-\overline{5} \\
35.6 \\
13.5\end{array}$ & $\begin{array}{l}1 \\
1 \\
1 \\
1 \\
1\end{array}$ & $\begin{array}{l}1 \\
1 \\
3 \\
1 \\
1\end{array}$ & $\begin{array}{l}1 \\
1 \\
3 \\
1 \\
1\end{array}$ & $\begin{array}{l}7 \\
1 \\
5 \\
7 \\
7\end{array}$ \\
\hline $\begin{array}{l}\text { BK } \\
\text { BK } \\
\text { BK } \\
\text { BK } \\
\text { BK }\end{array}$ & $\begin{array}{l}87 \\
68 \\
69 \\
76 \\
71\end{array}$ & $\begin{array}{c}16700 \\
37300 \\
- \\
7000 \\
18400\end{array}$ & $\begin{array}{r}19500 \\
52000 \\
- \\
8800 \\
33600\end{array}$ & $\begin{array}{l}181.0 \\
170.0 \\
177.7 \\
154.0 \\
145.0\end{array}$ & $\begin{array}{r}- \\
- \\
- \\
150.0 \\
142.5\end{array}$ & $\begin{array}{l}165.0 \\
158.0 \\
163.0 \\
137.0 \\
129.5\end{array}$ & $\begin{array}{l}41.0 \\
44.0 \\
41.0 \\
41.0 \\
43.0\end{array}$ & $\begin{array}{r}28.0 \\
39.3 \\
=\overline{13} \\
30.3\end{array}$ & $\begin{array}{l}1 \\
1 \\
1 \\
1 \\
1\end{array}$ & $\begin{array}{l}1 \\
1 \\
1 \\
1 \\
1\end{array}$ & $\begin{array}{l}1 \\
1 \\
1 \\
1 \\
1\end{array}$ & $\begin{array}{l}7 \\
7 \\
7 \\
1 \\
7\end{array}$ \\
\hline $\begin{array}{l}\text { BK } \\
\text { BK } \\
\text { BK } \\
\text { BK } \\
\text { BK }\end{array}$ & $\begin{array}{l}72 \\
73 \\
74 \\
75 \\
76\end{array}$ & $\begin{array}{c}22200 \\
11500 \\
18000 \\
41000 \\
-\end{array}$ & $\begin{array}{r}44000 \\
12700 \\
36000 \\
56000 \\
-\end{array}$ & $\begin{array}{l}141 . \varnothing \\
171 . \varnothing \\
142 . \varnothing \\
153.8 \\
145.3\end{array}$ & $\begin{array}{r}137.0 \\
140 . \overline{-} \\
- \\
-\end{array}$ & $\begin{array}{r}131 . \varnothing \\
130 . \overline{5} \\
140 . \varnothing \\
132 . \varnothing\end{array}$ & $\begin{array}{l}43.0 \\
43.0 \\
43.0 \\
47.0 \\
50.0\end{array}$ & $\begin{array}{r}14.7 \\
13.0 \\
27.6 \\
62.3 \\
-\end{array}$ & $\begin{array}{l}1 \\
1 \\
1 \\
1 \\
1\end{array}$ & $\begin{array}{l}1 \\
1 \\
1 \\
1 \\
1\end{array}$ & $\begin{array}{l}1 \\
1 \\
1 \\
1 \\
1\end{array}$ & $\begin{array}{l}7 \\
1 \\
1 \\
1 \\
7\end{array}$ \\
\hline
\end{tabular}


Appendix.--Location and basic physical features of recharge basins on Long Island,

\begin{tabular}{|c|c|c|c|c|c|c|c|c|}
\hline \multicolumn{2}{|c|}{$\begin{array}{l}\text { BASIN } \\
\text { NUMBER }\end{array}$} & \multicolumn{2}{|c|}{ NEAREST INTERSECTION } & $\begin{array}{l}\text { LATITUDE } \\
\text { LONGITUDE } \\
0_{n}^{\prime}, n\end{array}$ & $\begin{array}{l}\text { DATE } \\
\text { BUILT } \\
\text { YrMOD }\end{array}$ & COMMNITY & \multicolumn{2}{|c|}{$\begin{array}{l}\text { DESIGN ACTUAL } \\
\text { CAPACITY CAPACITY } \\
\text { (cubic feet) }\end{array}$} \\
\hline $\begin{array}{l}\text { BK } \\
\text { BK } \\
\text { BK } \\
\text { BK } \\
\text { BK }\end{array}$ & $\begin{array}{l}77 \\
78 \\
79 \\
80 \\
81\end{array}$ & $\begin{array}{l}\text { MARLO ROAD } \\
\text { HALSEY STREET } \\
\text { HALF MILE ROAD } \\
\text { JOLINE ROAD } \\
\text { ALPINE STREET }\end{array}$ & $\begin{array}{l}\text { CHARTER ROAD } \\
\text { BOSTON AVENUE } \\
\text { OLD TOWN ROAD } \\
\text { NEWPORT DRIVE } \\
\text { LINCOLN PLACE }\end{array}$ & $\begin{array}{l}4053250730311 \\
4054330730466 \\
4054130730318 \\
4854370730236 \\
405437 \varnothing 730227\end{array}$ & $\begin{array}{l}600206 \\
660207 \\
650522 \\
6207 \\
6297\end{array}$ & $\begin{array}{l}- \\
- \\
- \\
-\end{array}$ & $\begin{array}{c}252200 \\
285000 \\
420500 \\
- \\
141800\end{array}$ & $\begin{array}{c}253000 \\
289400 \\
453000 \\
- \\
142000\end{array}$ \\
\hline $\begin{array}{l}\text { BK } \\
\text { BK } \\
\text { BK } \\
\text { BK } \\
\text { BK }\end{array}$ & $\begin{array}{l}82 \\
83 \\
84 \\
85 \\
86\end{array}$ & $\begin{array}{l}\text { KENT ROAD } \\
\text { SCHOOLDRIVE } \\
\text { WENMORE LANE } \\
\text { CHAMPLAIN STREET } \\
\text { CORNELL STREET }\end{array}$ & $\begin{array}{l}\text { NEWPORT DRIVE } \\
\text { UNIVERSITY DRIVE } \\
\text { GREENHAVEN DRIVE } \\
\text { NORTON LANE } \\
\text { OLD TOMN ROAD }\end{array}$ & $\begin{array}{l}4854390730240 \\
4055080730257 \\
4054380730323 \\
4053490730235 \\
4054200730331\end{array}$ & $\begin{array}{c}6207 \\
8089 \\
- \\
6083 \\
640415\end{array}$ & $\begin{array}{l}- \\
- \\
- \\
-\end{array}$ & $\begin{array}{l}126000 \\
116000 \\
761000 \\
- \\
166000\end{array}$ & $\begin{array}{l}233000 \\
117000 \\
86000 \\
- \\
382000\end{array}$ \\
\hline $\begin{array}{l}\text { BK } \\
\text { BK } \\
\text { BK } \\
\text { BK } \\
\text { BK }\end{array}$ & $\begin{array}{l}87 \\
88 \\
89 \\
9 \varnothing \\
91\end{array}$ & $\begin{array}{l}\text { GREENHAVEN DRIVE } \\
\text { CROWN STREET } \\
\text { THAMES STREET } \\
\text { BROADWAY } \\
\text { THAMES STREET }\end{array}$ & $\begin{array}{l}\text { YALE STREET } \\
\text { PORT JEFFERSON R } \\
\text { BROADWAY } \\
\text { CARROLL STREET } \\
\text { WOODHULL AVE. }\end{array}$ & $\begin{array}{l}4054270730331 \\
4054380730357 \\
4054590730404 \\
4054530730359 \\
4055010730340\end{array}$ & $\begin{array}{l}620410 \\
640415 \\
640401 \\
610401 \\
591128\end{array}$ & $\begin{array}{l}- \\
\overline{-} \\
249\end{array}$ & $\begin{array}{l}170400 \\
384000 \\
- \\
- \\
-\end{array}$ & $\begin{array}{l}1717000 \\
3876000 \\
- \\
- \\
-\end{array}$ \\
\hline $\begin{array}{l}\text { BK } \\
\text { BK } \\
\text { BK } \\
\text { BK } \\
\text { BK }\end{array}$ & $\begin{array}{l}93 \\
94 \\
95 \\
96 \\
98\end{array}$ & $\begin{array}{l}\text { ROSS STREET } \\
\text { HEWES STREET } \\
\text { ROLLING ROAD } \\
\text { MILLER PLACE RD. } \\
\text { PIPE STAVE }\end{array}$ & $\begin{array}{l}\text { NEW YORK AVENUE } \\
\text { NEW YORK AVENUE } \\
\text { TREE ROAD } \\
\text { CEDAR PLACE } \\
\text { HILDALE AVENUE }\end{array}$ & $\begin{array}{l}4 \varnothing 53260730350 \\
4853490730482 \\
4856578725950 \\
4 \varnothing 57179725941 \\
4057110730053\end{array}$ & $\begin{array}{l}650819 \\
650819 \\
678525 \\
670725 \\
608130\end{array}$ & $\begin{array}{l}- \\
\overline{-} \\
\overline{-}\end{array}$ & $\begin{array}{l}410800 \\
868000 \\
575800 \\
662000 \\
130000\end{array}$ & $\begin{array}{l}472000 \\
889000 \\
587700 \\
688000 \\
139900\end{array}$ \\
\hline $\begin{array}{l}\text { BK } \\
\text { BK } \\
\text { BK } \\
\text { BK } \\
\text { BK }\end{array}$ & $\begin{array}{r}99 \\
100 \\
101 \\
103 \\
104\end{array}$ & $\begin{array}{l}\text { WOODY PLACE } \\
\text { VIDONI DRIVE } \\
\text { VIDONI DRIVE } \\
\text { CANAL ROAD } \\
\text { BERRY LANE }\end{array}$ & $\begin{array}{l}\text { VIDONI DRIVE } \\
\text { WINOMILL LANE } \\
\text { BARNABY LANE } \\
\text { MOUNT SINAI ROAD } \\
\text { LOCUST COURT }\end{array}$ & $\begin{array}{l}4856100730157 \\
4056010730155 \\
4056090730139 \\
4055140730123 \\
4856540725935\end{array}$ & $\begin{array}{l}640617 \\
640617 \\
640714 \\
6703 \\
6610\end{array}$ & $\begin{array}{l}- \\
- \\
-\end{array}$ & $\begin{array}{r}213800 \\
228000 \\
581900 \\
342000 \\
68300\end{array}$ & $\begin{array}{r}214000 \\
222700 \\
581400 \\
353000 \\
88500\end{array}$ \\
\hline $\begin{array}{l}\text { BK } \\
\text { BK } \\
\text { BK } \\
\text { BK } \\
\text { BK }\end{array}$ & $\begin{array}{l}106 \\
106 \\
107 \\
109 \\
110\end{array}$ & $\begin{array}{l}\text { PIPE STAVE ROAD } \\
\text { DOGWOOD LANE } \\
\text { HONEY LANE } \\
\text { N.ROCKY POINT RD } \\
\text { N.ROCKY POINT RD }\end{array}$ & $\begin{array}{l}\text { EVANS LANE } \\
\text { MAGNOLIA LANE } \\
\text { PIPE STAVE ROAD } \\
\text { SOUNDWAY DRIVE } \\
\text { CADDY PLACE }\end{array}$ & $\begin{array}{l}4056340730013 \\
4057170730039 \\
4057070730042 \\
4057340725718 \\
4057400725727\end{array}$ & $\begin{array}{l}6410 \\
660511 \\
680412 \\
670613 \\
661065\end{array}$ & $\begin{array}{l}- \\
- \\
- \\
-\end{array}$ & $\begin{array}{r}332000 \\
92000 \\
81500 \\
376000 \\
1050000\end{array}$ & $\begin{array}{r}346300 \\
116000 \\
83000 \\
496000 \\
1090000\end{array}$ \\
\hline $\begin{array}{l}\text { BK } \\
\text { BK } \\
\text { BK } \\
\text { BK } \\
\text { BK }\end{array}$ & $\begin{array}{l}111 \\
112 \\
113 \\
114 \\
115\end{array}$ & $\begin{array}{l}\text { MILLER AVENUE } \\
\text { ORCHARO PLACE } \\
\text { BUNKER STREET } \\
\text { BABYLON DRIVE } \\
\text { KINGS ROAD }\end{array}$ & $\begin{array}{l}\text { JOHN STREET } \\
\text { SOUNDWAY DRIVE } \\
\text { GREENWAY DRIVE } \\
\text { NORTH COUNTRY RD } \\
\text { ROYAL WAY }\end{array}$ & $\begin{array}{l}4056520725321 \\
4 \varnothing 574707257 \varnothing 9 \\
4056500725646 \\
4 \varnothing 56450725748 \\
4 \varnothing 56500725311\end{array}$ & $\begin{array}{l}630518 \\
\quad- \\
6509 \\
668620 \\
650525\end{array}$ & $\begin{array}{l}- \\
- \\
-\end{array}$ & $\begin{array}{r}4360000 \\
70000 \\
878000 \\
120000 \\
736000\end{array}$ & $\begin{array}{r}4906000 \\
108000 \\
89700 \\
121000 \\
808000\end{array}$ \\
\hline $\begin{array}{l}\text { BK } \\
\text { BK } \\
\text { BK } \\
\text { BK } \\
\text { BK }\end{array}$ & $\begin{array}{l}116 \\
117 \\
118 \\
119 \\
120\end{array}$ & $\begin{array}{l}\text { MILLS ROAD } \\
\text { ROCKY POINT ROAD } \\
\text { MAHOGANY ROAD } \\
\text { MAHOGANY ROAD } \\
\text { PATCHOGUE DRIVE }\end{array}$ & $\begin{array}{l}\text { GATEWAY DRIVE } \\
\text { NORTH COUNTRY RO } \\
\text { WHITEWOOD DRIVE } \\
\text { SHORT LANE } \\
\text { LAUREL ROAD }\end{array}$ & $\begin{array}{l}4856430725106 \\
4056180725622 \\
4857060725701 \\
4057150725659 \\
4057109725717\end{array}$ & $\begin{array}{l}6504 \\
680914 \\
648320 \\
640320 \\
680608\end{array}$ & $\begin{array}{l}- \\
- \\
-\end{array}$ & $\begin{array}{l}236000 \\
169000 \\
895000 \\
- \\
282000\end{array}$ & $\begin{array}{l}241000 \\
178080 \\
975000 \\
480800 \\
290000\end{array}$ \\
\hline $\begin{array}{l}\text { BK } \\
\text { BK } \\
\text { BK } \\
\text { BK } \\
\text { BK }\end{array}$ & $\begin{array}{l}121 \\
122 \\
123 \\
124 \\
125\end{array}$ & $\begin{array}{l}\text { NORTH COUNTRY RD } \\
\text { STERLING DRIVE } \\
\text { PARSNIP POND RD. } \\
\text { SARAH DRIVE } \\
\text { HY PLACE }\end{array}$ & $\begin{array}{l}\text { ROCKY POINT ROAD } \\
\text { WADE DRIVE } \\
\text { GLENN WAY } \\
\text { KATHY LANE } \\
\text { BRANDY LANE }\end{array}$ & $\begin{array}{l}4056390725624 \\
4051170730717 \\
4051320730729 \\
4051170730728 \\
4 \varnothing 5126073 \varnothing 7 \varnothing 1\end{array}$ & $\begin{array}{l}680914 \\
6410 \\
6410 \\
6410 \\
650303\end{array}$ & $\begin{array}{l}- \\
- \\
-\end{array}$ & $\begin{array}{l}88000 \\
430500 \\
147000 \\
37500 \\
-\end{array}$ & $\begin{array}{l}90300 \\
430500 \\
147000 \\
46000 \\
-\end{array}$ \\
\hline $\begin{array}{l}\text { BK } \\
\text { BK } \\
\text { BK } \\
\text { BK } \\
\text { BK }\end{array}$ & $\begin{array}{l}126 \\
129 \\
130 \\
131 \\
132\end{array}$ & $\begin{array}{l}\text { WADE DRIVE } \\
\text { LEHIGH AVENUE } \\
\text { KATHRYN LANE } \\
\text { HURON STREET } \\
\text { B AVENUE }\end{array}$ & $\begin{array}{l}\text { HY PLACE } \\
\text { LAURIE BOULEVARD } \\
\text { RICHARD PLACE } \\
\text { MILL ROAD } \\
\text { 6TH STREET }\end{array}$ & $\begin{array}{l}4051240730710 \\
4051240730637 \\
4049110730551 \\
4048540730554 \\
4049120730430\end{array}$ & $\begin{array}{l}650303 \\
6409 \\
651207 \\
651207 \\
6405\end{array}$ & $\begin{array}{l}- \\
- \\
-\end{array}$ & $\begin{array}{l}39800 \\
286000 \\
- \\
- \\
1917008\end{array}$ & $\begin{array}{l}504000 \\
298008 \\
- \\
- \\
192000\end{array}$ \\
\hline $\begin{array}{l}\text { BK } \\
\text { BK } \\
\text { BK } \\
\text { BK } \\
\text { BK }\end{array}$ & $\begin{array}{l}133 \\
134 \\
135 \\
136 \\
137\end{array}$ & $\begin{array}{l}\text { TOPAZ DRIVE } \\
\text { PEAK STREET } \\
\text { DIANE STREET } \\
\text { RADBURN DRIVE } \\
\text { GAYMORE LANE }\end{array}$ & $\begin{array}{l}\text { JOY PLACE } \\
\text { VALLEY STREET } \\
\text { HOLLY AVENUE } \\
\text { ARDEN LANE } \\
\text { RADBURN DRIVE }\end{array}$ & 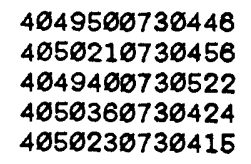 & $\begin{array}{l}610919 \\
641208 \\
610220 \\
680813 \\
650506\end{array}$ & $\begin{array}{l}- \\
- \\
-\end{array}$ & $\begin{array}{r}177700 \\
210000 \\
81500 \\
671000 \\
437000\end{array}$ & $\begin{array}{r}1779000 \\
216000 \\
48000 \\
685000 \\
478000\end{array}$ \\
\hline
\end{tabular}


N.Y. [Dash indicates no data. Altitudes are in feet above sea level.] (cont.)

\begin{tabular}{|c|c|c|c|c|c|c|c|c|c|c|c|c|}
\hline \multicolumn{2}{|c|}{$\begin{array}{l}\text { BASIN } \\
\text { NUMBER }\end{array}$} & $\begin{array}{l}\text { MAX. } \\
\text { AREA } \\
\text { (squar }\end{array}$ & $\begin{array}{c}\text { BASIN } \\
\text { AREA } \\
\text { feet) }\end{array}$ & RIM & $\begin{array}{l}\text { OVER- } \\
\text { FLOW }\end{array}$ & $\begin{array}{l}\text { BOT- } \\
\text { TOM }\end{array}$ & $\begin{array}{l}\text { WATER } \\
\text { TABLE }\end{array}$ & $\begin{array}{c}\text { DRAIN. } \\
\text { AREA } \\
\text { (acres) }\end{array}$ & \multicolumn{2}{|c|}{$\begin{array}{l}---B A S I N-- \\
\text { STATUS USE }\end{array}$} & $\begin{array}{l}\text { GEO. } \\
\text { UNIT }\end{array}$ & $\begin{array}{l}\text { SOIL } \\
\text { UNIT }\end{array}$ \\
\hline $\begin{array}{l}\text { BK } \\
B K \\
B K \\
B K \\
B K\end{array}$ & $\begin{array}{l}77 \\
78 \\
79 \\
80 \\
81\end{array}$ & $\begin{array}{c}211000 \\
20800 \\
45300 \\
- \\
13700\end{array}$ & $\begin{array}{l}34800 \\
51800 \\
54800 \\
33600 \\
27200\end{array}$ & $\begin{array}{l}118.0 \\
149.8 \\
143.7 \\
144.3 \\
150.0\end{array}$ & $\begin{array}{r}116.0 \\
144.9 \\
143 . \overline{0} \\
146.4\end{array}$ & $\begin{array}{l}104.0 \\
131.0 \\
126.0 \\
133.0 \\
136.0\end{array}$ & $\begin{array}{l}53.0 \\
46.0 \\
51.0 \\
48 . \varnothing \\
48 . \varnothing\end{array}$ & $\begin{array}{r}27.0 \\
44.9 \\
66.2 \\
- \\
22.3\end{array}$ & $\begin{array}{l}0 \\
1 \\
1 \\
1 \\
1\end{array}$ & $\begin{array}{l}1 \\
1 \\
1 \\
1 \\
1\end{array}$ & $\begin{array}{l}1 \\
1 \\
1 \\
1 \\
1\end{array}$ & $\begin{array}{l}7 \\
1 \\
7 \\
7 \\
7\end{array}$ \\
\hline $\begin{array}{l}B K \\
B K \\
B K \\
B K \\
B K\end{array}$ & $\begin{array}{l}82 \\
83 \\
84 \\
85 \\
86\end{array}$ & $\begin{array}{c}9900 \\
9800 \\
8600 \\
- \\
38200\end{array}$ & $\begin{array}{l}3660 \emptyset \\
2340 \varnothing \\
1260 \emptyset \\
494 \varnothing \emptyset \\
5980 \varnothing\end{array}$ & $\begin{array}{l}149.3 \\
168.0 \\
160.0 \\
120.5 \\
149.9\end{array}$ & $\begin{array}{r}149.5 \\
166.3 \\
- \\
- \\
-\end{array}$ & $\begin{array}{l}136.0 \\
154.3 \\
145.0 \\
106.7 \\
137.4\end{array}$ & $\begin{array}{l}48 . \varnothing \\
45 . \varnothing \\
47 . \varnothing \\
52 . \varnothing \\
50 . \varnothing\end{array}$ & $\begin{array}{r}16.3 \\
18.3 \\
13.0 \\
- \\
26.1\end{array}$ & $\begin{array}{l}1 \\
1 \\
1 \\
1 \\
1\end{array}$ & $\begin{array}{l}1 \\
1 \\
1 \\
1 \\
1\end{array}$ & $\begin{array}{l}1 \\
1 \\
1 \\
1 \\
1\end{array}$ & $\begin{array}{l}7 \\
7 \\
7 \\
7 \\
7\end{array}$ \\
\hline $\begin{array}{l}\text { BK } \\
B K \\
B K \\
B K \\
B K\end{array}$ & $\begin{array}{l}87 \\
88 \\
89 \\
90 \\
91\end{array}$ & $\begin{array}{c}17200 \\
38600 \\
- \\
- \\
-\end{array}$ & $\begin{array}{r}34800 \\
48000 \\
- \\
- \\
-\end{array}$ & $\begin{array}{l}151.0 \\
160.0 \\
170.0 \\
165.0 \\
170.0\end{array}$ & $\begin{array}{l}- \\
- \\
-\end{array}$ & $\begin{array}{r}141.7 \\
142.0 \\
- \\
- \\
-\end{array}$ & $\begin{array}{l}48 . \varnothing \\
46 . \varnothing \\
45 . \varnothing \\
45 . \varnothing \\
45 . \varnothing\end{array}$ & $\begin{array}{r}26.8 \\
60.4 \\
- \\
- \\
-\end{array}$ & $\begin{array}{l}1 \\
1 \\
1 \\
1 \\
1\end{array}$ & $\begin{array}{l}1 \\
1 \\
1 \\
1 \\
1\end{array}$ & $\begin{array}{l}1 \\
1 \\
1 \\
1 \\
1\end{array}$ & $\begin{array}{l}7 \\
7 \\
7 \\
7 \\
7\end{array}$ \\
\hline $\begin{array}{l}\text { BK } \\
B K \\
B K \\
B K \\
B K\end{array}$ & $\begin{array}{l}93 \\
94 \\
95 \\
96 \\
98\end{array}$ & $\begin{array}{l}33700 \\
59300 \\
58800 \\
68800 \\
14000\end{array}$ & $\begin{array}{r}66600 \\
115000 \\
57500 \\
102400 \\
31500\end{array}$ & $\begin{array}{l}118.0 \\
106.0 \\
160.0 \\
160.0 \\
110.0\end{array}$ & $\begin{array}{r}120.0 \\
109.3 \\
160.1 \\
131.5 \\
87.0\end{array}$ & $\begin{array}{r}184.0 \\
91.0 \\
142.0 \\
115.0 \\
74.0\end{array}$ & $\begin{array}{l}51 . \emptyset \\
52 . \varnothing \\
23 . \emptyset \\
20 . \emptyset \\
18 . \varnothing\end{array}$ & $\begin{array}{l}40.1 \\
85.0 \\
62.0 \\
71.0 \\
20.5\end{array}$ & $\begin{array}{l}1 \\
1 \\
1 \\
1 \\
1\end{array}$ & $\begin{array}{l}1 \\
1 \\
1 \\
3 \\
1\end{array}$ & $\begin{array}{l}1 \\
1 \\
4 \\
4 \\
3\end{array}$ & $\begin{array}{l}3 \\
3 \\
3 \\
3 \\
6\end{array}$ \\
\hline $\begin{array}{l}\text { BK } \\
\text { BK } \\
\text { BK } \\
\text { BK } \\
\text { BK }\end{array}$ & $\begin{array}{r}99 \\
106 \\
101 \\
103 \\
104\end{array}$ & $\begin{array}{r}23300 \\
20200 \\
44700 \\
27200 \\
8900\end{array}$ & $\begin{array}{l}46000 \\
55 i 00 \\
72900 \\
57800 \\
19200\end{array}$ & $\begin{array}{l}145.6 \\
137.0 \\
139.5 \\
134.8 \\
175.0\end{array}$ & $\begin{array}{l}147.3 \\
139.4 \\
144.0 \\
138.0 \\
168.0\end{array}$ & $\begin{array}{l}135.8 \\
126.0 \\
126.5 \\
121.8 \\
156.0\end{array}$ & $\begin{array}{l}39 . \varnothing \\
39 . \varnothing \\
39.0 \\
44.0 \\
24.0\end{array}$ & $\begin{array}{r}22.9 \\
23.5 \\
62.3 \\
35.6 \\
7.8\end{array}$ & $\begin{array}{l}1 \\
1 \\
1 \\
1 \\
1\end{array}$ & $\begin{array}{l}1 \\
1 \\
1 \\
1 \\
3\end{array}$ & $\begin{array}{l}4 \\
4 \\
4 \\
1 \\
4\end{array}$ & $\begin{array}{l}1 \\
1 \\
1 \\
1 \\
3\end{array}$ \\
\hline $\begin{array}{l}\text { BK } \\
B K \\
B K \\
B K \\
B K\end{array}$ & $\begin{array}{l}105 \\
106 \\
107 \\
109 \\
110\end{array}$ & $\begin{array}{r}34600 \\
8900 \\
8300 \\
49600 \\
68100\end{array}$ & $\begin{array}{r}60000 \\
39600 \\
26100 \\
76800 \\
180800\end{array}$ & $\begin{array}{r}150.0 \\
133.5 \\
162.0 \\
70.0 \\
50.0\end{array}$ & $\begin{array}{r}154.0 \\
166 . \overline{0} \\
50.0 \\
59.0\end{array}$ & $\begin{array}{r}138.0 \\
120.5 \\
152.0 \\
34.5 \\
34.0\end{array}$ & $\begin{array}{r}33.0 \\
20.0 \\
20.0 \\
10.0 \\
5.0\end{array}$ & $\begin{array}{l}35.6 \\
14.5 \\
12.8 \\
59.0 \\
64.3\end{array}$ & $\begin{array}{l}1 \\
1 \\
1 \\
0 \\
0\end{array}$ & $\begin{array}{l}1 \\
1 \\
1 \\
1 \\
3\end{array}$ & $\begin{array}{l}4 \\
4 \\
4 \\
4 \\
4\end{array}$ & $\begin{array}{l}1 \\
3 \\
7 \\
6 \\
6\end{array}$ \\
\hline $\begin{array}{l}\text { BK } \\
B K \\
B K \\
B K \\
B K\end{array}$ & $\begin{array}{l}111 \\
112 \\
113 \\
114 \\
115\end{array}$ & $\begin{array}{r}49000 \\
10300 \\
7500 \\
10100 \\
56900\end{array}$ & $\begin{array}{l}57000 \\
28600 \\
16900 \\
33900 \\
63800\end{array}$ & $\begin{array}{r}120.0 \\
50.5 \\
102.5 \\
118.0 \\
122.2\end{array}$ & $\begin{array}{r}123.0 \\
51.5 \\
103.5 \\
121.0 \\
127.0\end{array}$ & $\begin{array}{r}112.0 \\
40.0 \\
90.5 \\
106.0 \\
108.0\end{array}$ & $\begin{array}{r}25.0 \\
4.0 \\
27.0 \\
27.0 \\
25.0\end{array}$ & $\begin{array}{l}28.7 \\
11.0 \\
15.0 \\
13.8 \\
45.1\end{array}$ & $\begin{array}{l}1 \\
1 \\
1 \\
1 \\
1\end{array}$ & $\begin{array}{l}3 \\
1 \\
1 \\
3 \\
3\end{array}$ & $\begin{array}{l}1 \\
4 \\
4 \\
4 \\
1\end{array}$ & $\begin{array}{l}3 \\
7 \\
3 \\
8 \\
3\end{array}$ \\
\hline $\begin{array}{l}\text { BK } \\
\text { BK } \\
\text { BK } \\
\text { BK } \\
\text { BK }\end{array}$ & $\begin{array}{l}116 \\
117 \\
118 \\
119 \\
120\end{array}$ & $\begin{array}{l}17200 \\
11700 \\
88600 \\
48000 \\
20700\end{array}$ & $\begin{array}{r}36000 \\
30000 \\
128000 \\
54000 \\
60000\end{array}$ & $\begin{array}{r}128.0 \\
104.5 \\
79.0 \\
64.0 \\
118.0\end{array}$ & $\begin{array}{r}131.0 \\
106.5 \\
83.0 \\
67.0 \\
119.2\end{array}$ & $\begin{array}{r}114.0 \\
90.0 \\
68.0 \\
54.0 \\
104.0\end{array}$ & $\begin{array}{l}44.0 \\
35.0 \\
25.0 \\
25.0 \\
22.0\end{array}$ & $\begin{array}{r}26.0 \\
26.6 \\
140.9 \\
75.6 \\
44.4\end{array}$ & $\begin{array}{l}1 \\
1 \\
0 \\
1 \\
1\end{array}$ & $\begin{array}{l}1 \\
1 \\
1 \\
1 \\
1\end{array}$ & $\begin{array}{l}1 \\
1 \\
4 \\
4 \\
4\end{array}$ & $\begin{array}{l}1 \\
1 \\
3 \\
7 \\
6\end{array}$ \\
\hline $\begin{array}{l}\text { BK } \\
\text { BK } \\
\text { BK } \\
\text { BK } \\
\text { BK }\end{array}$ & $\begin{array}{l}121 \\
122 \\
123 \\
124 \\
125\end{array}$ & $\begin{array}{c}6500 \\
506 \varnothing \emptyset \\
3340 \emptyset \\
460 \emptyset \\
-\end{array}$ & $\begin{array}{r}19500 \\
115200 \\
48000 \\
16900 \\
27300\end{array}$ & $\begin{array}{r}142.0 \\
75.5 \\
71.0 \\
90.0 \\
125.0\end{array}$ & $\begin{array}{r}147.0 \\
92.3 \\
72.2 \\
77.4 \\
117.1\end{array}$ & $\begin{array}{r}128.0 \\
67.0 \\
66.6 \\
68.4 \\
194.5\end{array}$ & $\begin{array}{l}30.0 \\
52.0 \\
52 . \varnothing \\
52 . \varnothing \\
52.0\end{array}$ & $\begin{array}{r}5.4 \\
47.5 \\
23.1 \\
43.0 \\
-\end{array}$ & $\begin{array}{l}1 \\
1 \\
1 \\
1 \\
1\end{array}$ & $\begin{array}{l}3 \\
1 \\
1 \\
1 \\
1\end{array}$ & $\begin{array}{l}1 \\
1 \\
1 \\
1 \\
1\end{array}$ & $\begin{array}{l}6 \\
6 \\
6 \\
5 \\
6\end{array}$ \\
\hline $\begin{array}{l}\text { BK } \\
\text { BK } \\
\text { BK } \\
\text { BK } \\
\text { BK }\end{array}$ & $\begin{array}{l}126 \\
129 \\
130 \\
131 \\
132\end{array}$ & $\begin{array}{c}5000 \\
20700 \\
- \\
- \\
19200\end{array}$ & $\begin{array}{l}11000 \\
39000 \\
72200 \\
24200 \\
50400\end{array}$ & $\begin{array}{r}115.0 \\
98.0 \\
112.0 \\
112.0 \\
120.0\end{array}$ & $\begin{array}{l}103.4 \\
101.0 \\
115.6 \\
115.0 \\
118.0\end{array}$ & $\begin{array}{r}90.0 \\
84.0 \\
98.0 \\
102.0 \\
105.7\end{array}$ & $\begin{array}{l}52.0 \\
54.0 \\
47.0 \\
47 . \varnothing \\
47.0\end{array}$ & $\begin{array}{r}4.3 \\
28.0 \\
- \\
- \\
20.0\end{array}$ & $\begin{array}{l}1 \\
1 \\
1 \\
1 \\
1\end{array}$ & $\begin{array}{l}1 \\
1 \\
1 \\
1 \\
1\end{array}$ & $\begin{array}{l}1 \\
1 \\
1 \\
1 \\
1\end{array}$ & $\begin{array}{l}3 \\
6 \\
3 \\
3 \\
5\end{array}$ \\
\hline $\begin{array}{l}\text { BK } \\
\text { BK } \\
\text { BK } \\
\text { BK } \\
\text { BK }\end{array}$ & $\begin{array}{l}133 \\
134 \\
135 \\
136 \\
137\end{array}$ & $\begin{array}{r}16200 \\
16000 \\
4000 \\
68500 \\
47000\end{array}$ & $\begin{array}{r}34000 \\
30800 \\
10200 \\
123200 \\
48600\end{array}$ & $\begin{array}{r}131.5 \\
170.0 \\
150.0 \\
95.0 \\
93.3\end{array}$ & $\begin{array}{r}131.5 \\
139.0 \\
151.0 \\
- \\
-\end{array}$ & $\begin{array}{r}120.5 \\
124.0 \\
138.0 \\
68.5 \\
81.5\end{array}$ & $\begin{array}{l}52.0 \\
54.0 \\
51.0 \\
55.0 \\
53.0\end{array}$ & $\begin{array}{r}20.4 \\
33.1 \\
9.0 \\
65.3 \\
47.0\end{array}$ & $\begin{array}{l}1 \\
1 \\
1 \\
1 \\
1\end{array}$ & $\begin{array}{l}1 \\
1 \\
1 \\
1 \\
1\end{array}$ & $\begin{array}{l}1 \\
6 \\
1 \\
1 \\
6\end{array}$ & $\begin{array}{l}6 \\
5 \\
7 \\
5 \\
5\end{array}$ \\
\hline
\end{tabular}


Appendix.--Location and basic physical features of recharge basins on Long Island,

\begin{tabular}{|c|c|c|c|c|c|c|c|c|}
\hline \multicolumn{2}{|c|}{$\begin{array}{l}\text { BASIN } \\
\text { NUMBER }\end{array}$} & \multicolumn{2}{|c|}{ NEAREST INTERSECTION } & $\begin{array}{l}\text { LATITUDE } \\
\text { LONGITUDE } \\
0,0^{*},\end{array}$ & $\begin{array}{l}\text { DATE } \\
\text { BUILT } \\
\text { YrMOD }\end{array}$ & COMMNITY & \multicolumn{2}{|c|}{$\begin{array}{l}\text { DESIGN ACTUAL } \\
\text { CAPACITY CAPACITY } \\
\text { (cubic fot) }\end{array}$} \\
\hline $\begin{array}{l}B K \\
B K \\
B K \\
B K \\
B K\end{array}$ & $\begin{array}{l}138 \\
139 \\
148 \\
141 \\
142\end{array}$ & $\begin{array}{l}\text { MOONEY POND ROAD } \\
\text { LAKESIDE DTREET } \\
\text { LOLLY LANE W. } \\
\text { UNIVERSITY DRIVE } \\
\text { BLUE POINT ROAD }\end{array}$ & $\begin{array}{l}\text { RADBURN DRIVE } \\
\text { PACKARD STREET } \\
\text { LOLLY LANE } \\
\text { WASHINGTON AVE. } \\
\text { N. AVENUE }\end{array}$ & $\begin{array}{l}4850320736483 \\
4852216736652 \\
4850286736544 \\
484951073042 \varnothing \\
4849356736551\end{array}$ & $\begin{array}{c}650506 \\
- \\
621023 \\
670721 \\
670501\end{array}$ & $\begin{array}{l}- \\
- \\
-\end{array}$ & $\begin{array}{l}160600 \\
- \\
364200 \\
65300 \\
-\end{array}$ & $\begin{array}{l}280600 \\
- \\
375800 \\
78060 \\
-\end{array}$ \\
\hline $\begin{array}{l}B K \\
B K \\
B K \\
B K \\
B K\end{array}$ & $\begin{array}{l}143 \\
144 \\
145 \\
146 \\
147\end{array}$ & $\begin{array}{l}\text { FOREST AVENUE } \\
\text { HARDWICK STREET } \\
\text { DEERFIELD DRIVE } \\
\text { HAWKINS AVENUE } \\
\text { HY PLACE }\end{array}$ & $\begin{array}{l}\text { LAURELTON AVENUE } \\
\text { VIRGINIA AVENUE } \\
\text { ALPINE PLACE } \\
\text { SYCAMORE AVENUE } \\
\text { VIE PLACE }\end{array}$ & $\begin{array}{l}4050440730734 \\
4050320730629 \\
4050470730627 \\
4050510730646 \\
4051310730703\end{array}$ & $\begin{array}{l}670103 \\
650720 \\
650720 \\
650720 \\
96800\end{array}$ & $\begin{array}{l}- \\
- \\
- \\
227\end{array}$ & $\begin{array}{r}386000 \\
166000 \\
276000 \\
236000 \\
96800\end{array}$ & $\begin{array}{l}420000 \\
360000 \\
541000 \\
251000 \\
106000\end{array}$ \\
\hline $\begin{array}{l}B K \\
B K \\
B K \\
B K \\
B K\end{array}$ & $\begin{array}{l}148 \\
149 \\
150 \\
152 \\
153\end{array}$ & $\begin{array}{l}\text { MIDDLE COUNTRY R } \\
\text { SWAIN STREET } \\
\text { PATCHOGUE AVENUE } \\
\text { HAMMOND AVENUE } \\
\text { HOLBROOK ROAD }\end{array}$ & $\begin{array}{l}\text { HALLOCK ROAD } \\
\text { VIRGINIA AVENUE } \\
\text { POND ROAD } \\
\text { HAWKINS ROAD } \\
\text { LENORE LANE }\end{array}$ & $\begin{array}{l}4051420730722 \\
4050260730629 \\
4048580730533 \\
4052520730431 \\
4 \varnothing 51190730501\end{array}$ & $\begin{array}{l}661108 \\
670804 \\
680116 \\
680416 \\
620315\end{array}$ & $\begin{array}{l}- \\
- \\
- \\
-\end{array}$ & $\begin{array}{r}\overline{9} \\
934600 \\
184600 \\
94800 \\
158800\end{array}$ & $\begin{array}{l}- \\
108100 \\
208000 \\
102000 \\
174800\end{array}$ \\
\hline $\begin{array}{l}\text { BK } \\
\text { BK } \\
\text { BK } \\
\text { BK } \\
\text { BK }\end{array}$ & $\begin{array}{l}154 \\
155 \\
156 \\
157 \\
158\end{array}$ & $\begin{array}{l}\text { LEHIGH AVENUE } \\
\text { TUCKER LANE } \\
\text { HEIZEN AVENUE } \\
\text { WASHBURN STREET } \\
\text { AVONDALE DRIVE }\end{array}$ & $\begin{array}{l}\text { LAURIE BOULEVARD } \\
\text { DIANE AVENUE } \\
\text { TUCKER LANE } \\
\text { BIRCH STREET } \\
\text { ALDEN LANE }\end{array}$ & $\begin{array}{l}4051240730640 \\
4051160730629 \\
4051230730624 \\
4051120730642 \\
4052440730436\end{array}$ & $\begin{array}{l}600318 \\
660318 \\
600318 \\
641030 \\
600814\end{array}$ & $\begin{array}{l}- \\
- \\
-\end{array}$ & $\begin{array}{l}- \\
- \\
\overline{82008} \\
328008\end{array}$ & $\begin{array}{l}- \\
- \\
- \\
879080 \\
3290008\end{array}$ \\
\hline $\begin{array}{l}\text { BK } \\
B K \\
B K \\
B K \\
B K\end{array}$ & $\begin{array}{l}159 \\
160 \\
161 \\
162 \\
163\end{array}$ & $\begin{array}{l}\text { HAMMOND AVENUE } \\
\text { WARD AVENUE } \\
\text { STONEHURST } \\
\text { SAXON ROAD } \\
\text { NORTHFIELD DR. }\end{array}$ & $\begin{array}{l}\text { ELWOOD DRIVE } \\
\text { HAMMOND DRIVE } \\
\text { CIRCLE STREET } \\
\text { HAMMOND AVENUE } \\
\text { RIDGE ROAD }\end{array}$ & $\begin{array}{l}4052340730429 \\
4052240730437 \\
4052460730419 \\
4052090730425 \\
4050210730516\end{array}$ & $\begin{array}{l}600814 \\
660814 \\
680723 \\
660104 \\
660329\end{array}$ & $\begin{array}{l}- \\
- \\
-\end{array}$ & $\begin{array}{r}202000 \\
253900 \\
251800 \\
99000 \\
183800\end{array}$ & $\begin{array}{l}204000 \\
260000 \\
267000 \\
114000 \\
184000\end{array}$ \\
\hline $\begin{array}{l}\text { BK } \\
\text { BK } \\
\text { BK } \\
\text { BK } \\
\text { BK }\end{array}$ & $\begin{array}{l}164 \\
165 \\
166 \\
187 \\
168\end{array}$ & $\begin{array}{l}\text { HOWELL AVENUE } \\
\text { NORTHVIEW COURT } \\
\text { CHARLES STREET } \\
\text { HAMMOND AVENUE } \\
\text { BISCAYNE DRIVE }\end{array}$ & $\begin{array}{l}\text { MIDOLE COUNTRY R } \\
\text { SAXON ROAD } \\
\text { WASHINGTON AVE. } \\
\text { SMITH STREET } \\
\text { MONTCLAIR DRIVE }\end{array}$ & $\begin{array}{l}4052030730427 \\
4052180730438 \\
4051020730525 \\
4052060730420 \\
4653190730316\end{array}$ & $\begin{array}{l}671120 \\
671127 \\
680605 \\
680523 \\
640724\end{array}$ & $\begin{array}{l}- \\
- \\
-\end{array}$ & $\begin{array}{c}54900 \\
- \\
126500 \\
75800 \\
589000\end{array}$ & $\begin{array}{r}57800 \\
140600 \\
133500 \\
78060 \\
695000\end{array}$ \\
\hline $\begin{array}{l}\text { BK } \\
\text { BK } \\
\text { BK } \\
\text { BK } \\
\text { BK }\end{array}$ & $\begin{array}{l}169 \\
170 \\
171 \\
172 \\
173\end{array}$ & $\begin{array}{l}\text { OAK STREET } \\
\text { WILLOW STREET } \\
\text { RULAND ROAD } \\
\text { QUAKER LANE } \\
\text { JANE BOULEVARD }\end{array}$ & $\begin{array}{l}\text { CEDAR STREET } \\
\text { APPLE STREET } \\
\text { DON LANE } \\
\text { STUYVESANT DRIVE } \\
\text { 14TH STREET }\end{array}$ & $\begin{array}{l}4053010730327 \\
4053100730329 \\
4053010730338 \\
4053210730255 \\
4053310730241\end{array}$ & $\begin{array}{c}651229 \\
631229 \\
- \\
6203 \\
6203\end{array}$ & $\begin{array}{l}- \\
- \\
-\end{array}$ & $\begin{array}{r}327000 \\
57060 \\
464200 \\
250200 \\
221500\end{array}$ & $\begin{array}{r}433000 \\
57500 \\
475000 \\
365000 \\
226000\end{array}$ \\
\hline $\begin{array}{l}\text { BK } \\
\text { BK } \\
\text { BK } \\
\text { BK } \\
\text { BK }\end{array}$ & $\begin{array}{l}174 \\
175 \\
176 \\
177 \\
178\end{array}$ & $\begin{array}{l}\text { POINT AVENUE } \\
\text { ROSS PLACE } \\
\text { WASHINGTON AVE. } \\
\text { FISK ROAD } \\
\text { PIEDMONT DRIVE }\end{array}$ & $\begin{array}{l}\text { JANICE LANE } \\
\text { EMERY AVENUE } \\
\text { CHOATE AVENUE } \\
\text { ALMA AVENUE } \\
\text { CLINTON PLACE }\end{array}$ & $\begin{array}{l}4051530730206 \\
4051460730204 \\
4052370730326 \\
4052210730306 \\
4055510730342\end{array}$ & $\begin{array}{l}650697 \\
650607 \\
641020 \\
661031 \\
621117\end{array}$ & $\begin{array}{l}- \\
- \\
-\end{array}$ & $\begin{array}{l}280600 \\
234060 \\
706000 \\
513000 \\
172908\end{array}$ & $\begin{array}{l}284080 \\
252000 \\
858000 \\
514000 \\
175000\end{array}$ \\
\hline $\begin{array}{l}\text { BK } \\
B K \\
B K \\
B K \\
B K\end{array}$ & $\begin{array}{l}179 \\
180 \\
181 \\
182 \\
183\end{array}$ & $\begin{array}{l}\text { SELDEN BOULEVARD } \\
\text { 10TH STREET } \\
\text { EAGLE STREET } \\
\text { SOUTH BICYCLE PA } \\
\text { GALAXIE LANE }\end{array}$ & $\begin{array}{l}\text { BELLROSE AVENUE } \\
\text { DAY STREET } \\
\text { GLADYS STREET } \\
\text { CLEARVIEW AVENUE } \\
\text { AMBASSADOR LANE }\end{array}$ & $\begin{array}{l}4052160730345 \\
4055280730116 \\
4051330730135 \\
4051030730126 \\
4051220730243\end{array}$ & $\begin{array}{l}650211 \\
660204 \\
630223 \\
630223 \\
660202\end{array}$ & $\begin{array}{l}- \\
- \\
-\end{array}$ & $\begin{array}{c}332360 \\
106500 \\
362080 \\
- \\
458060\end{array}$ & $\begin{array}{c}435700 \\
105060 \\
368000 \\
- \\
4776000\end{array}$ \\
\hline $\begin{array}{l}B K \\
B K \\
B K \\
B K \\
B K\end{array}$ & $\begin{array}{l}185 \\
186 \\
187 \\
188 \\
189\end{array}$ & $\begin{array}{l}\text { FISK ROAD } \\
\text { WASHINGTON HGTS. } \\
\text { SELDEN BOLLEVARD } \\
\text { MAGNOLIA ROAD } \\
\text { COLONY ROAD }\end{array}$ & $\begin{array}{l}\text { CARSTON STREET } \\
\text { FISK ROAD } \\
\text { HENRY AVENUE } \\
\text { CONTINENTAL ROAD } \\
\text { PRISCILLA LANE }\end{array}$ & $\begin{array}{l}4052090730300 \\
4052090730241 \\
4051560730341 \\
4053390730245 \\
40535007303 \varnothing \varnothing\end{array}$ & $\begin{array}{l}641019 \\
641019 \\
650211 \\
631204 \\
631204\end{array}$ & $\begin{array}{l}- \\
- \\
-\end{array}$ & $\begin{array}{l}- \\
\overline{176300} \\
- \\
-\end{array}$ & $\begin{array}{l}- \\
\overline{215200} \\
- \\
-\end{array}$ \\
\hline $\begin{array}{l}\text { BK } \\
\text { BK } \\
\text { BK } \\
\text { BK } \\
\text { BK }\end{array}$ & $\begin{array}{l}190 \\
191 \\
192 \\
193 \\
194\end{array}$ & $\begin{array}{l}\text { HART DRIVE } \\
\text { MARCH COURT } \\
\text { REMINGTON STREET } \\
\text { FRANKLIN AVENUE } \\
\text { STRAUSS STREET }\end{array}$ & $\begin{array}{l}\text { SEMINOLE STREET } \\
\text { WANDA PLACE } \\
\text { FISK ROAD } \\
\text { MAGNOLIA ROAD } \\
\text { FISK ROAD }\end{array}$ & $\begin{array}{l}4052130730328 \\
4052200730224 \\
4052440730301 \\
4 \varnothing 52390730244 \\
405231073030 \varnothing\end{array}$ & $\begin{array}{l}6212 \\
600308 \\
621017 \\
621017 \\
641017\end{array}$ & $\begin{array}{l}- \\
- \\
-\end{array}$ & $\begin{array}{l}124000 \\
85800 \\
236800 \\
322000 \\
-\end{array}$ & $\begin{array}{r}1670000 \\
90000 \\
249000 \\
385000 \\
-\end{array}$ \\
\hline
\end{tabular}


N.Y. [Dash indicates no data. Altitudes are in feet above sea level.] (cont.)

\begin{tabular}{|c|c|c|c|c|c|c|c|c|c|c|c|c|}
\hline \multicolumn{2}{|c|}{$\begin{array}{l}\text { BASIN } \\
\text { NUMBER }\end{array}$} & $\begin{array}{l}\text { MAX. } \\
\text { AREA } \\
\text { (squar }\end{array}$ & $\begin{array}{c}\text { BASIN } \\
\text { AREA } \\
\text { feet) }\end{array}$ & RIM & $\begin{array}{l}\text { OVEF } \\
\text { FLOY }\end{array}$ & $\begin{array}{l}\text { BE--- } \\
\text { TOT- } \\
\text { TOM }\end{array}$ & $\begin{array}{l}\text { WATER } \\
\text { TABLE }\end{array}$ & $\begin{array}{c}\text { DRAIN. } \\
\text { AREA } \\
\text { (acres) }\end{array}$ & \multicolumn{2}{|c|}{$\begin{array}{l}---B A S I N-- \\
\text { STATUS USE }\end{array}$} & $\begin{array}{l}\text { GEO. } \\
\text { UNIT }\end{array}$ & $\begin{array}{l}\text { SOIL } \\
\text { UNIT }\end{array}$ \\
\hline $\begin{array}{l}\text { BK } \\
\text { BK } \\
\text { BK } \\
\text { BK } \\
\text { BK }\end{array}$ & $\begin{array}{l}138 \\
139 \\
140 \\
141 \\
142\end{array}$ & $\begin{array}{c}20000 \\
- \\
37500 \\
7800 \\
-\end{array}$ & $\begin{array}{l}52800 \\
12800 \\
66000 \\
36100 \\
36000\end{array}$ & $\begin{array}{r}140.0 \\
92.7 \\
134.0 \\
110.0 \\
150 . \varnothing\end{array}$ & $\begin{array}{r}\overline{-} \\
\overline{-} \\
121 . \bar{\varnothing}\end{array}$ & $\begin{array}{r}117.0 \\
80.0 \\
122.0 \\
99.3 \\
112.0\end{array}$ & $\begin{array}{l}55.0 \\
60.0 \\
53.0 \\
51.0 \\
51.0\end{array}$ & $\begin{array}{r}17.0 \\
-\overline{0} \\
7.2 \\
-\end{array}$ & $\begin{array}{l}1 \\
1 \\
1 \\
1 \\
1\end{array}$ & $\begin{array}{l}1 \\
1 \\
1 \\
1 \\
1\end{array}$ & $\begin{array}{l}8 \\
1 \\
1 \\
1 \\
1\end{array}$ & $\begin{array}{l}5 \\
3 \\
5 \\
5 \\
1\end{array}$ \\
\hline $\begin{array}{l}\text { BK } \\
\text { BK } \\
\text { BK } \\
\text { BK } \\
\text { BK }\end{array}$ & $\begin{array}{l}143 \\
144 \\
145 \\
146 \\
147\end{array}$ & $\begin{array}{r}2600 \\
2800 \\
54100 \\
20900 \\
10600\end{array}$ & $\begin{array}{l}52700 \\
52000 \\
74800 \\
52000 \\
23400\end{array}$ & $\begin{array}{r}87.0 \\
121.0 \\
129.0 \\
117.0 \\
121.5\end{array}$ & $\begin{array}{r}85 . \varnothing \\
118.5 \\
114.0 \\
-\end{array}$ & $\begin{array}{r}69.0 \\
105.5 \\
110.0 \\
102.0 \\
106.0\end{array}$ & $\begin{array}{l}51.0 \\
53.0 \\
53.0 \\
53.0 \\
53.0\end{array}$ & $\begin{array}{l}60.8 \\
19.0 \\
31.0 \\
27.0 \\
15.1\end{array}$ & $\begin{array}{l}1 \\
1 \\
1 \\
0 \\
1\end{array}$ & $\begin{array}{l}1 \\
1 \\
1 \\
3 \\
1\end{array}$ & $\begin{array}{l}1 \\
1 \\
1 \\
1 \\
1\end{array}$ & $\begin{array}{l}3 \\
6 \\
3 \\
7 \\
3\end{array}$ \\
\hline $\begin{array}{l}\text { BK } \\
\text { BK } \\
\text { BK } \\
\text { BK } \\
\text { BK }\end{array}$ & $\begin{array}{l}148 \\
149 \\
150 \\
152 \\
153\end{array}$ & $\begin{array}{r}- \\
9800 \\
16000 \\
10700 \\
14600\end{array}$ & $\begin{array}{r}160000 \\
28400 \\
38400 \\
27000 \\
27000\end{array}$ & $\begin{array}{r}66.5 \\
108.0 \\
119.0 \\
99.0 \\
99.0\end{array}$ & $\begin{array}{r}- \\
106 . \varnothing \\
115.5 \\
95.0 \\
95 . \varnothing\end{array}$ & $\begin{array}{r}62.0 \\
95.0 \\
103.0 \\
85.5 \\
83.0\end{array}$ & $\begin{array}{l}51.0 \\
53.0 \\
47.0 \\
54.0 \\
54.0\end{array}$ & $\begin{array}{l}10 . \overline{-} \\
19.8 \\
14.9 \\
25.0\end{array}$ & $\begin{array}{l}1 \\
1 \\
1 \\
1 \\
1\end{array}$ & $\begin{array}{l}4 \\
1 \\
1 \\
1 \\
1\end{array}$ & $\begin{array}{l}1 \\
1 \\
1 \\
1 \\
1\end{array}$ & $\begin{array}{l}6 \\
3 \\
3 \\
5 \\
6\end{array}$ \\
\hline $\begin{array}{l}\text { BK } \\
\text { BK } \\
\text { BK } \\
\text { BK } \\
\text { BK }\end{array}$ & $\begin{array}{l}154 \\
155 \\
156 \\
157 \\
158\end{array}$ & $\begin{array}{c}- \\
- \\
- \\
8800 \\
32900\end{array}$ & $\begin{array}{l}3500 \varnothing \\
2940 \varnothing \\
3750 \varnothing \\
19200 \\
4400 \varnothing\end{array}$ & $\begin{array}{r}90.0 \\
110.0 \\
110.0 \\
125.0 \\
100.0\end{array}$ & $\begin{array}{l}- \\
\text { - } \\
\text { - }\end{array}$ & $\begin{array}{r}70.1 \\
76.9 \\
75.0 \\
112.0 \\
83.7\end{array}$ & $\begin{array}{l}53.0 \\
53 . \varnothing \\
53 . \varnothing \\
52 . \varnothing \\
54 . \varnothing\end{array}$ & $\begin{array}{r}\overline{-} \\
\overline{-} \\
12.9 \\
34.2\end{array}$ & $\begin{array}{l}1 \\
1 \\
1 \\
1 \\
1\end{array}$ & $\begin{array}{l}1 \\
1 \\
1 \\
1 \\
1\end{array}$ & $\begin{array}{l}1 \\
1 \\
1 \\
1 \\
1\end{array}$ & $\begin{array}{l}6 \\
3 \\
3 \\
7 \\
5\end{array}$ \\
\hline $\begin{array}{l}\text { BK } \\
\text { BK } \\
\text { BK } \\
\text { BK } \\
\text { BK }\end{array}$ & $\begin{array}{l}159 \\
160 \\
161 \\
162 \\
163\end{array}$ & $\begin{array}{l}20400 \\
26000 \\
23600 \\
11400 \\
15300\end{array}$ & $\begin{array}{l}300 \varnothing \varnothing \\
286 \varnothing 0 \\
5440 \varnothing \\
30 \varnothing \varnothing 0 \\
42500\end{array}$ & $\begin{array}{r}101.0 \\
100.5 \\
96.4 \\
97.0 \\
129.0\end{array}$ & $\begin{array}{r}\overline{-} \\
94 . \overline{4} \\
127 . \bar{\theta}\end{array}$ & $\begin{array}{r}83.3 \\
83.0 \\
83.5 \\
82.0 \\
115 . \varnothing\end{array}$ & $\begin{array}{l}54.0 \\
55.0 \\
55.0 \\
55.0 \\
53.8\end{array}$ & $\begin{array}{l}21.0 \\
30.7 \\
39.6 \\
15.6 \\
19.0\end{array}$ & $\begin{array}{l}1 \\
1 \\
1 \\
1 \\
1\end{array}$ & $\begin{array}{l}1 \\
1 \\
1 \\
1 \\
1\end{array}$ & $\begin{array}{l}1 \\
1 \\
1 \\
1 \\
6\end{array}$ & $\begin{array}{l}5 \\
5 \\
5 \\
5 \\
5\end{array}$ \\
\hline $\begin{array}{l}\text { BK } \\
\text { BK } \\
\text { BK } \\
\text { BK } \\
\text { BK }\end{array}$ & $\begin{array}{l}164 \\
165 \\
166 \\
167 \\
168\end{array}$ & $\begin{array}{r}4200 \\
9400 \\
13400 \\
7100 \\
69500\end{array}$ & $\begin{array}{l}17300 \\
29700 \\
35700 \\
19500 \\
84800\end{array}$ & $\begin{array}{r}95.4 \\
55.0 \\
117.4 \\
95.0 \\
120.0\end{array}$ & $\begin{array}{r}93.5 \\
95.8 \\
115.8 \\
93.0 \\
-\end{array}$ & $\begin{array}{r}80.0 \\
94.0 \\
105.8 \\
82.0 \\
103.0\end{array}$ & $\begin{array}{l}54.0 \\
55.2 \\
55.0 \\
54.0 \\
54.1\end{array}$ & $\begin{array}{r}6.3 \\
22.1 \\
14.8 \\
11.9 \\
92.7\end{array}$ & $\begin{array}{l}1 \\
1 \\
1 \\
1 \\
1\end{array}$ & $\begin{array}{l}1 \\
1 \\
1 \\
1 \\
1\end{array}$ & $\begin{array}{l}1 \\
1 \\
1 \\
1 \\
1\end{array}$ & $\begin{array}{l}5 \\
5 \\
6 \\
5 \\
8\end{array}$ \\
\hline $\begin{array}{l}\text { BK } \\
\text { BK } \\
\text { BK } \\
\text { BK } \\
\text { BK }\end{array}$ & $\begin{array}{l}169 \\
170 \\
171 \\
172 \\
173\end{array}$ & $\begin{array}{r}36100 \\
5800 \\
47500 \\
36500 \\
22600\end{array}$ & $\begin{array}{r}72500 \\
18800 \\
81200 \\
70200 \\
-\end{array}$ & $\begin{array}{l}105.5 \\
125.0 \\
112.9 \\
127.0 \\
118.5\end{array}$ & $\begin{array}{r}103.0 \\
122.0 \\
= \\
=\end{array}$ & $\begin{array}{r}91.0 \\
112.0 \\
92.6 \\
108.5 \\
105.5\end{array}$ & $\begin{array}{l}56.0 \\
56.0 \\
56.0 \\
53.0 \\
53.0\end{array}$ & $\begin{array}{r}51.5 \\
9.0 \\
73.1 \\
58.0 \\
23.0\end{array}$ & $\begin{array}{l}1 \\
1 \\
1 \\
1 \\
1\end{array}$ & $\begin{array}{l}1 \\
1 \\
1 \\
1 \\
1\end{array}$ & $\begin{array}{l}1 \\
1 \\
1 \\
1 \\
1\end{array}$ & $\begin{array}{l}5 \\
5 \\
5 \\
7 \\
7\end{array}$ \\
\hline $\begin{array}{l}\text { BK } \\
\text { BK } \\
\text { BK } \\
\text { BK } \\
\text { BK }\end{array}$ & $\begin{array}{l}174 \\
175 \\
176 \\
177 \\
178\end{array}$ & $\begin{array}{l}28400 \\
25200 \\
71500 \\
51400 \\
17500\end{array}$ & $\begin{array}{r}44100 \\
25200 \\
105600 \\
91000 \\
30000\end{array}$ & $\begin{array}{r}73.1 \\
121.5 \\
96.0 \\
93.2 \\
185.5\end{array}$ & $\begin{array}{r}- \\
93 . \overline{0} \\
88.3 \\
-\end{array}$ & $\begin{array}{r}64.0 \\
107.0 \\
81.0 \\
78.3 \\
170.0\end{array}$ & $\begin{array}{l}54 . \varnothing \\
54.0 \\
56.0 \\
55.0 \\
4 \varnothing . \varnothing\end{array}$ & $\begin{array}{r}29.0 \\
24.0 \\
110.2 \\
31.4 \\
13.7\end{array}$ & $\begin{array}{l}1 \\
1 \\
1 \\
1 \\
1\end{array}$ & $\begin{array}{l}1 \\
1 \\
1 \\
3 \\
3\end{array}$ & $\begin{array}{l}1 \\
6 \\
1 \\
1 \\
1\end{array}$ & $\begin{array}{l}8 \\
8 \\
5 \\
5 \\
1\end{array}$ \\
\hline $\begin{array}{l}\text { BK } \\
\text { BK } \\
\text { BK } \\
\text { BK } \\
\text { BK }\end{array}$ & $\begin{array}{l}179 \\
180 \\
181 \\
182 \\
183\end{array}$ & $\begin{array}{c}31100 \\
10500 \\
33500 \\
- \\
47700\end{array}$ & $\begin{array}{r}58580 \\
43200 \\
65100 \\
50400\end{array}$ & $\begin{array}{r}86.8 \\
150.0 \\
124.0 \\
208.4 \\
108.1\end{array}$ & $\begin{array}{r}85.5 \\
110.0 \\
118.0 \\
- \\
-\end{array}$ & $\begin{array}{r}71.5 \\
99.9 \\
107.8 \\
193.5 \\
81.0\end{array}$ & $\begin{array}{l}55.0 \\
44.8 \\
60.0 \\
54.0 \\
58.0\end{array}$ & $\begin{array}{r}52.3 \\
16.8 \\
57.0 \\
-\overline{0}\end{array}$ & $\begin{array}{l}1 \\
1 \\
0 \\
1 \\
1\end{array}$ & $\begin{array}{l}1 \\
1 \\
1 \\
1 \\
1\end{array}$ & $\begin{array}{l}1 \\
1 \\
6 \\
6 \\
1\end{array}$ & $\begin{array}{l}5 \\
1 \\
5 \\
5 \\
5\end{array}$ \\
\hline $\begin{array}{l}\text { BK } \\
\text { BK } \\
\text { BK } \\
\text { BK } \\
\text { BK }\end{array}$ & $\begin{array}{l}185 \\
186 \\
187 \\
188 \\
189\end{array}$ & $\begin{array}{c}\overline{-} \\
21500 \\
- \\
-\end{array}$ & $\begin{array}{r}50000 \\
37500 \\
30600 \\
18200 \\
-\end{array}$ & $\begin{array}{r}92.1 \\
94.0 \\
99.8 \\
135.8 \\
140.0\end{array}$ & $\begin{array}{l}\text { - } \\
\text { - } \\
-\end{array}$ & $\begin{array}{r}73.5 \\
83.0 \\
87.0 \\
123.4 \\
125.0\end{array}$ & $\begin{array}{l}58 . \varnothing \\
58 . \varnothing \\
54 . \varnothing \\
52 . \varnothing \\
52 . \varnothing\end{array}$ & $\begin{array}{r}- \\
\overline{-} \\
-\end{array}$ & $\begin{array}{l}1 \\
1 \\
1 \\
1 \\
\varnothing\end{array}$ & $\begin{array}{l}1 \\
3 \\
1 \\
1 \\
2\end{array}$ & $\begin{array}{l}1 \\
1 \\
1 \\
1 \\
1\end{array}$ & $\begin{array}{l}5 \\
5 \\
5 \\
7 \\
7\end{array}$ \\
\hline $\begin{array}{l}\text { BK } \\
\text { BK } \\
\text { BK } \\
\text { BK } \\
\text { BK }\end{array}$ & $\begin{array}{l}190 \\
191 \\
192 \\
193 \\
194\end{array}$ & $\begin{array}{r}16700 \\
9000 \\
24900 \\
38500 \\
-\end{array}$ & $\begin{array}{r}27800 \\
12600 \\
20400 \\
35200 \\
-\end{array}$ & $\begin{array}{r}170.0 \\
100.0 \\
94.7 \\
99.0 \\
90.0\end{array}$ & $\begin{array}{l}- \\
- \\
-\end{array}$ & $\begin{array}{r}156.7 \\
75.0 \\
82.5 \\
82.0 \\
-\end{array}$ & $\begin{array}{l}58 . \varnothing \\
57 . \varnothing \\
57 . \varnothing \\
57 . \varnothing \\
54 . \varnothing\end{array}$ & $\begin{array}{r}22.0 \\
13.4 \\
37.2 \\
50.7 \\
-\end{array}$ & $\begin{array}{l}1 \\
1 \\
1 \\
1 \\
1\end{array}$ & $\begin{array}{l}1 \\
1 \\
1 \\
1 \\
1\end{array}$ & $\begin{array}{l}6 \\
1 \\
1 \\
1 \\
1\end{array}$ & $\begin{array}{l}8 \\
5 \\
5 \\
5 \\
8\end{array}$ \\
\hline
\end{tabular}


Appendix.--Location and basic physical features of recharge basins on Long Island,

\begin{tabular}{|c|c|c|c|c|c|c|c|c|}
\hline \multicolumn{2}{|c|}{$\begin{array}{l}\text { BASIN } \\
\text { NUMBER }\end{array}$} & \multicolumn{2}{|c|}{ NEAREST INTERSECTION } & $\begin{array}{l}\text { LATITUDE } \\
\text { LONGITUDE } \\
\text { ०," }, "\end{array}$ & $\begin{array}{l}\text { DATE } \\
\text { BUILT } \\
\text { YrMoD }\end{array}$ & COMMUNITY & \multicolumn{2}{|c|}{$\begin{array}{l}\text { DESIGN ACTUAL } \\
\text { CAPACITY CAPACITY } \\
\text { (cubic feet) }\end{array}$} \\
\hline $\begin{array}{l}\text { BK } \\
B K \\
B K \\
B K \\
B K\end{array}$ & $\begin{array}{l}195 \\
196 \\
198 \\
199 \\
200\end{array}$ & $\begin{array}{l}\text { MALLARD AVENUE } \\
\text { HAWKINS ROAD } \\
\text { LARRY ROAD } \\
\text { WYANET STREET } \\
\text { MOONEY POND ROAD }\end{array}$ & $\begin{array}{l}\text { TILDEN AVENUE } \\
\text { CAMPO AVENUE } \\
\text { RULAND ROAD } \\
\text { HIGHVIEW DRIVE } \\
\text { BEAR STREET }\end{array}$ & 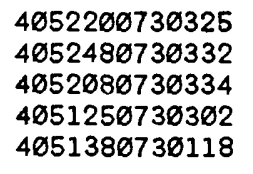 & $\begin{array}{l}6003 \\
6003 \\
6210 \\
660427 \\
680429\end{array}$ & $\begin{array}{l}- \\
- \\
- \\
-\end{array}$ & $\begin{array}{r}248000 \\
502000 \\
252700 \\
54000 \\
288000\end{array}$ & $\begin{array}{r}255000 \\
508000 \\
252300 \\
65000 \\
295000\end{array}$ \\
\hline $\begin{array}{l}B K \\
B K \\
B K \\
B K \\
B K\end{array}$ & $\begin{array}{l}201 \\
202 \\
203 \\
205 \\
207\end{array}$ & $\begin{array}{l}\text { WOODBURY DRIVE } \\
\text { WOODBURY DRIVE } \\
\text { PORTION ROAD } \\
\text { CRESTWOOD COURT } \\
\text { HORSE BLOCK ROAD }\end{array}$ & $\begin{array}{l}\text { STRATFORD ROAD } \\
\text { PINE TREE ROAD } \\
\text { LEEDS BOULEVARD } \\
\text { CRESTWOOD LANE } \\
\text { WAVERLY AVENUE }\end{array}$ & 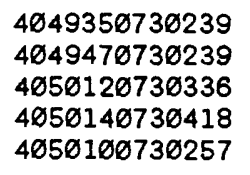 & $\begin{array}{l}6307 \varnothing 2 \\
6307 \varnothing 2 \\
660817 \\
6503 \emptyset 5 \\
670501\end{array}$ & $\begin{array}{l}- \\
- \\
-\end{array}$ & $\begin{array}{l}2980 \varnothing \varnothing \\
1190 \varnothing \emptyset \\
164 \varnothing \varnothing \varnothing \\
183 \varnothing \varnothing \emptyset \\
46 \varnothing \varnothing \varnothing \emptyset\end{array}$ & $\begin{array}{l}30 \varnothing \varnothing \varnothing \varnothing \\
1280 \varnothing \varnothing \\
175 \varnothing \varnothing \varnothing \\
1910 \varnothing \varnothing \\
5 \varnothing \varnothing \varnothing \varnothing \varnothing\end{array}$ \\
\hline $\begin{array}{l}\text { BK } \\
B K \\
B K \\
B K \\
B K\end{array}$ & $\begin{array}{l}208 \\
289 \\
210 \\
211 \\
212\end{array}$ & $\begin{array}{l}\text { LENORE LANE } \\
\text { HOLBROOK ROAD } \\
\text { HOLBROOK ROAD } \\
\text { HILLBERRY LANE } \\
\text { HONEYSUCKLE LA. }\end{array}$ & $\begin{array}{l}\text { HORSE BLOCK RD. } \\
\text { 6TH STREET } \\
\text { 4TH STREET } \\
\text { HORNLEAF LANE } \\
\text { DIVISION STREET }\end{array}$ & 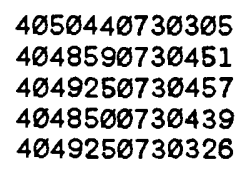 & $\begin{array}{l}678501 \\
660127 \\
668127 \\
66 \varnothing 127 \\
678911\end{array}$ & $\begin{array}{l}- \\
- \\
-\end{array}$ & $\begin{array}{l}145000 \\
945000 \\
117000 \\
231000 \\
122500\end{array}$ & $\begin{array}{l}146000 \\
950000 \\
128000 \\
2327 \varnothing 0 \\
132000\end{array}$ \\
\hline $\begin{array}{l}B K \\
B K \\
B K \\
B K \\
B K\end{array}$ & $\begin{array}{l}213 \\
214 \\
215 \\
216 \\
217\end{array}$ & $\begin{array}{l}\text { GRANNY ROAD } \\
\text { KNICKERBOCKER AV } \\
\text { HORSE BLOCK ROAD } \\
\text { KNICKERBOCKER AV } \\
\text { COMMERCIAL BLVD. }\end{array}$ & $\begin{array}{l}\text { TUNNEL ROAD } \\
\text { SMITH AVENUE } \\
\text { SIPP AVENUE } \\
\text { SIPP AVENUE } \\
\text { ISLAND ROAD }\end{array}$ & 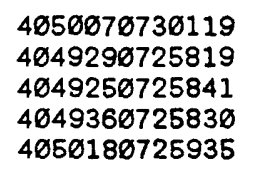 & $\begin{array}{c}680605 \\
661007 \\
- \\
601025 \\
630415\end{array}$ & $\begin{array}{l}- \\
- \\
-\end{array}$ & $\begin{array}{l}566000 \\
143000 \\
- \\
\overline{248000}\end{array}$ & $\begin{array}{l}698000 \\
145000 \\
- \\
\overline{249000}\end{array}$ \\
\hline $\begin{array}{l}B K \\
B K \\
B K \\
B K \\
B K\end{array}$ & $\begin{array}{l}218 \\
219 \\
220 \\
221 \\
222\end{array}$ & $\begin{array}{l}\text { GLENWOOD AVENUE } \\
\text { BEECH AVENUE } \\
\text { EVERGREEN AVENUE } \\
\text { OAKDALE AVENUE } \\
\text { MARURE AVENUE }\end{array}$ & $\begin{array}{l}\text { 6TH STREET } \\
\text { 4TH STREET } \\
\text { 1ST STREET } \\
\text { HORSE BLOCK ROAD } \\
\text { SOUTHAVEN AVENUE }\end{array}$ & 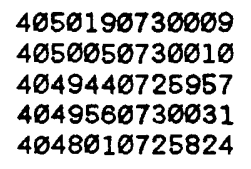 & $\begin{array}{l}650720 \\
660624 \\
66 \emptyset 720 \\
661118 \\
670117\end{array}$ & $\begin{array}{l}- \\
- \\
- \\
-\end{array}$ & $\begin{array}{r}452300 \\
495000 \\
659400 \\
69200 \\
340200\end{array}$ & $\begin{array}{r}453100 \\
50810 \emptyset \\
68030 \emptyset \\
6920 \emptyset \\
363500\end{array}$ \\
\hline $\begin{array}{l}\text { BK } \\
\text { BK } \\
\text { BK } \\
\text { BK } \\
\text { BK }\end{array}$ & $\begin{array}{l}225 \\
226 \\
227 \\
228 \\
229\end{array}$ & $\begin{array}{l}\text { RICHMOND AVENUE } \\
\text { TREMONT AVENUE } \\
\text { L.I.R.R. } \\
\text { PATCHOGUE ROAD } \\
\text { ROBINSON AVENUE }\end{array}$ & $\begin{array}{l}\text { WOODSIDE AVENUE } \\
\text { BARTON AVENUE } \\
\text { SOUTH COUNTRY RD } \\
\text { BROOKHAVEN ROAD } \\
\text { SUNRISE HIGHWAY }\end{array}$ & 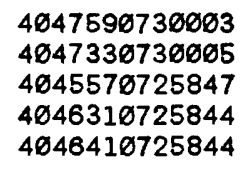 & $\begin{array}{c}- \\
669329 \\
660829 \\
- \\
-\end{array}$ & $\begin{array}{l}- \\
- \\
-\end{array}$ & $\begin{array}{l}- \\
144000 \\
87100 \\
- \\
-\end{array}$ & $\begin{array}{l}- \\
157300 \\
110000 \\
- \\
-\end{array}$ \\
\hline $\begin{array}{l}\text { BK } \\
\text { BK } \\
\text { BK } \\
\text { BK } \\
B K\end{array}$ & $\begin{array}{l}230 \\
231 \\
232 \\
233 \\
234\end{array}$ & $\begin{array}{l}\text { CORKY COURT } \\
\text { DEBBIE LANE } \\
\text { WOODSIDE AVENUE } \\
\text { RICHMOND AVENUE } \\
\text { COLUMBUS AVENUE }\end{array}$ & $\begin{array}{l}\text { DENA DRIVE } \\
\text { SHARRON LANE } \\
\text { MEDFORO AVENUE } \\
\text { BARTON AVENUE } \\
\text { BROOKHAVEN AVE. }\end{array}$ & 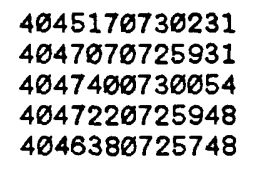 & $\begin{array}{l}- \\
680226 \\
680617 \\
680605 \\
600418\end{array}$ & $\begin{array}{l}- \\
- \\
- \\
-\end{array}$ & $\begin{array}{r}51800 \\
185000 \\
62300 \\
76000 \\
277300\end{array}$ & $\begin{array}{r}52200 \\
178000 \\
627 \varnothing \emptyset \\
76000 \\
277400\end{array}$ \\
\hline $\begin{array}{l}\text { BK } \\
\text { BK } \\
\text { BK } \\
\text { BK } \\
\text { BK }\end{array}$ & $\begin{array}{l}235 \\
236 \\
237 \\
238 \\
239\end{array}$ & $\begin{array}{l}\text { AMSTERDAM AVENUE } \\
\text { HEAD OF THE NECK } \\
\text { HEAD OF THE NECK } \\
\text { N.Y.S. ROUTE } \\
\text { GOLDEN GATE DR. }\end{array}$ & $\begin{array}{l}\text { LOUISE AVENUE } \\
\text { COUNTRY CLUB RD. } \\
\text { MUNSELL ROAD } \\
\text { PAQUATUCK AVENUE } \\
\text { SMITH ROAD }\end{array}$ & 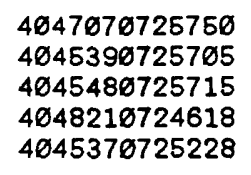 & $\begin{array}{l}601018 \\
678501 \\
6705 \\
660427 \\
620322\end{array}$ & $\begin{array}{l}- \\
- \\
-\end{array}$ & $\begin{array}{r}175700 \\
98000 \\
201000 \\
111000 \\
21500\end{array}$ & $\begin{array}{r}175400 \\
131100 \\
230000 \\
147000 \\
25100\end{array}$ \\
\hline $\begin{array}{l}B K \\
B K \\
B K \\
B K \\
B K\end{array}$ & $\begin{array}{l}240 \\
241 \\
242 \\
243 \\
244\end{array}$ & $\begin{array}{l}\text { EDWARD LAKE } \\
\text { CHANEL DRIVE } \\
\text { ARPEGE DRIVE } \\
\text { ALCOLADE DRIVE } \\
\text { ARPAGE DRIVE }\end{array}$ & $\begin{array}{l}\text { CANAL DRIVE } \\
\text { COLLINGWOOD ROAD } \\
\text { COLLINGWOOD ROAD } \\
\text { COLLINGWOOD ROAD } \\
\text { COLLINGWOOD ROAD }\end{array}$ & $\begin{array}{l}4 \emptyset 475 \emptyset 0724759 \\
4 \emptyset 46 \emptyset 2 \emptyset 725142 \\
4 \emptyset 45550725141 \\
4 \emptyset 46 \emptyset 2 \varnothing 725136 \\
4 \varnothing 4557 \varnothing 725136\end{array}$ & $\begin{array}{l}- \\
600217 \\
600217 \\
600217 \\
600217\end{array}$ & $\begin{array}{l}- \\
- \\
-\end{array}$ & $\begin{array}{l}- \\
98900 \\
61900 \\
98300 \\
63400\end{array}$ & $\begin{array}{l}- \\
\overline{-} \\
\overline{-}\end{array}$ \\
\hline $\begin{array}{l}\text { BK } \\
\text { BK } \\
\text { BK } \\
\text { BK } \\
\text { BK }\end{array}$ & $\begin{array}{l}245 \\
246 \\
247 \\
248 \\
249\end{array}$ & $\begin{array}{l}\text { CHANEL DRIVE } \\
\text { FLOYD ROAD } \\
\text { WINTERS DRIVE } \\
\text { GIRARD DRIVE } \\
\text { CAMPBELL DRIVE }\end{array}$ & $\begin{array}{l}\text { FLOYD ROAD } \\
\text { ARPAGE DRIVE } \\
\text { MARCELLA DRIVE } \\
\text { CARLIN ORIVE } \\
\text { ABERDEAN DRIVE }\end{array}$ & $\begin{array}{l}4046 \emptyset 40725121 \\
4046 \varnothing 00725112 \\
4049530725154 \\
4049450725151 \\
4049590725138\end{array}$ & $\begin{array}{l}600217 \\
600217 \\
600217 \\
600217 \\
600217\end{array}$ & $\begin{array}{l}- \\
- \\
- \\
-\end{array}$ & $\begin{array}{r}95680 \\
98500 \\
138800 \\
166100 \\
56700\end{array}$ & $\begin{array}{l}- \\
- \\
141200 \\
191600 \\
58400\end{array}$ \\
\hline $\begin{array}{l}\text { BK } \\
\text { BK } \\
\text { BK } \\
\text { BK } \\
\text { BK }\end{array}$ & $\begin{array}{l}250 \\
251 \\
252 \\
253 \\
254\end{array}$ & $\begin{array}{l}\text { TITMUS DRIVE } \\
\text { TITMUS DRIVE } \\
\text { SMITH ROAD } \\
\text { APPEL DRIVE } \\
\text { BRESTON DRIVE }\end{array}$ & $\begin{array}{l}\text { MARCELLA DRIVE } \\
\text { MARCELLA DRIVE } \\
\text { RANCH DRIVE } \\
\text { MARGIN DRIVE } \\
\text { HELENE DRIVE }\end{array}$ & $\begin{array}{l}4 \emptyset 4951 \varnothing 725128 \\
4 \varnothing 495 \varnothing \varnothing 725124 \\
4 \varnothing 4634 \varnothing 725228 \\
4 \varnothing 4635 \emptyset 725159 \\
4 \varnothing 4639 \emptyset 725153\end{array}$ & $\begin{array}{l}600217 \\
600217 \\
600519 \\
600519 \\
600519\end{array}$ & $\begin{array}{c}- \\
\overline{23} \\
- \\
-\end{array}$ & $\begin{array}{r}163200 \\
219400 \\
56000 \\
160000 \\
49600\end{array}$ & $\begin{array}{l}- \\
\overline{-} \\
\overline{-}\end{array}$ \\
\hline
\end{tabular}


N.Y. [Dash indicates no data. Altitudes are in feet above sea level.] (cont.)

\begin{tabular}{|c|c|c|c|c|c|c|c|c|c|c|c|c|}
\hline \multicolumn{2}{|c|}{$\begin{array}{l}\text { BASIN } \\
\text { NUMBER }\end{array}$} & $\begin{array}{l}\text { MAX. } \\
\text { AREA } \\
\text { (squar }\end{array}$ & $\begin{array}{c}\text { BASIN } \\
\text { AREA } \\
\text { feet) }\end{array}$ & RIM & $\begin{array}{l}-A L T I \\
\text { OVER- } \\
\text { FLOW }\end{array}$ & $\begin{array}{l}\text { BOT- } \\
\text { TOM }\end{array}$ & $\begin{array}{l}\text { WATER } \\
\text { TABLE }\end{array}$ & $\begin{array}{c}\text { DRAIN. } \\
\text { AREA } \\
\text { (acres) }\end{array}$ & \multicolumn{2}{|c|}{$\begin{array}{l}---B A S I N-- \\
\text { STATUS USE }\end{array}$} & $\begin{array}{l}\text { GEO. } \\
\text { UNIT }\end{array}$ & $\begin{array}{l}\text { SOIL } \\
\text { UNIT }\end{array}$ \\
\hline $\begin{array}{l}B K \\
B K \\
B K \\
B K \\
B K\end{array}$ & $\begin{array}{l}195 \\
196 \\
198 \\
199 \\
200\end{array}$ & $\begin{array}{r}2550 \emptyset \\
41300 \\
25200 \\
8100 \\
29500\end{array}$ & $\begin{array}{l}32400 \\
56600 \\
31600 \\
15600 \\
33000\end{array}$ & $\begin{array}{r}91.7 \\
90.0 \\
93.4 \\
143.0 \\
135.0\end{array}$ & $\begin{array}{r}93 . \overline{3} \\
\overline{-} \\
141.0 \\
-\end{array}$ & $\begin{array}{r}78.0 \\
81.0 \\
77.5 \\
133.0 \\
105.0\end{array}$ & $\begin{array}{l}57.0 \\
56.0 \\
56.0 \\
59.0 \\
60.0\end{array}$ & $\begin{array}{r}39.0 \\
79.0 \\
39.8 \\
8.5 \\
31.0\end{array}$ & $\begin{array}{l}1 \\
1 \\
1 \\
1 \\
1\end{array}$ & $\begin{array}{l}1 \\
1 \\
1 \\
1 \\
3\end{array}$ & $\begin{array}{l}1 \\
1 \\
1 \\
6 \\
6\end{array}$ & $\begin{array}{l}8 \\
5 \\
8 \\
5 \\
5\end{array}$ \\
\hline $\begin{array}{l}\text { BK } \\
B K \\
B K \\
B K \\
B K\end{array}$ & $\begin{array}{l}201 \\
202 \\
203 \\
205 \\
207\end{array}$ & $\begin{array}{l}30000 \\
12800 \\
23800 \\
17400 \\
62500\end{array}$ & $\begin{array}{l}28600 \\
14000 \\
24800 \\
34400 \\
63200\end{array}$ & $\begin{array}{r}113.0 \\
133.0 \\
150.0 \\
- \\
-\end{array}$ & $\begin{array}{r}- \\
\overline{-} \\
145 . \\
-\end{array}$ & $\begin{array}{r}101.0 \\
120.0 \\
132.7 \\
- \\
-\end{array}$ & $\begin{array}{l}48.0 \\
48.0 \\
52.0 \\
53.0 \\
52.0\end{array}$ & $\begin{array}{l}32.0 \\
17.0 \\
10.0 \\
28.8 \\
72.4\end{array}$ & $\begin{array}{l}1 \\
1 \\
1 \\
1 \\
0\end{array}$ & $\begin{array}{l}1 \\
1 \\
3 \\
1 \\
0\end{array}$ & $\begin{array}{l}1 \\
1 \\
1 \\
1 \\
0\end{array}$ & $\begin{array}{l}7 \\
7 \\
5 \\
5 \\
0\end{array}$ \\
\hline $\begin{array}{l}B K \\
B K \\
B K \\
B K \\
B K\end{array}$ & $\begin{array}{l}208 \\
209 \\
210 \\
211 \\
212\end{array}$ & $\begin{array}{l}15600 \\
95000 \\
12800 \\
23300 \\
11500\end{array}$ & $\begin{array}{r}24800 \\
110800 \\
8600 \\
26400 \\
22500\end{array}$ & $\begin{array}{l}155.0 \\
118.0 \\
130.0 \\
110.0 \\
140.0\end{array}$ & $\begin{array}{r}151.3 \\
- \\
- \\
\overline{-}\end{array}$ & $\begin{array}{r}142.0 \\
101.0 \\
117.5 \\
85.5 \\
106.0\end{array}$ & $\begin{array}{l}56.0 \\
47.0 \\
12.6 \\
47.0 \\
50.0\end{array}$ & $\begin{array}{r}22.8 \\
148.8 \\
12.6 \\
24.0 \\
14.1\end{array}$ & $\begin{array}{l}1 \\
1 \\
1 \\
1 \\
1\end{array}$ & $\begin{array}{l}1 \\
1 \\
1 \\
3 \\
1\end{array}$ & $\begin{array}{l}6 \\
1 \\
1 \\
1 \\
1\end{array}$ & $\begin{array}{l}5 \\
3 \\
3 \\
3 \\
1\end{array}$ \\
\hline $\begin{array}{l}\text { BK } \\
B K \\
B K \\
B K \\
B K\end{array}$ & $\begin{array}{l}213 \\
214 \\
215 \\
216 \\
217\end{array}$ & $\begin{array}{c}53100 \\
14500 \\
- \\
\overline{-} \\
24900\end{array}$ & $\begin{array}{r}80900 \\
36000 \\
- \\
-\end{array}$ & $\begin{array}{r}- \\
90.0 \\
80.0 \\
95.0 \\
105.0\end{array}$ & $\begin{array}{r}129.0 \\
87.0 \\
- \\
- \\
-\end{array}$ & $\begin{array}{r}116.0 \\
77.0 \\
69.0 \\
70.0 \\
89.8\end{array}$ & $\begin{array}{l}49.0 \\
41.0 \\
41.0 \\
41.0 \\
47.0\end{array}$ & $\begin{array}{r}34.6 \\
22.5 \\
- \\
39.0\end{array}$ & $\begin{array}{l}1 \\
1 \\
1 \\
1 \\
1\end{array}$ & $\begin{array}{l}3 \\
1 \\
1 \\
1 \\
1\end{array}$ & $\begin{array}{l}1 \\
1 \\
1 \\
1 \\
1\end{array}$ & $\begin{array}{l}3 \\
3 \\
3 \\
3 \\
6\end{array}$ \\
\hline $\begin{array}{l}B K \\
B K \\
B K \\
B K \\
B K\end{array}$ & $\begin{array}{l}218 \\
219 \\
220 \\
221 \\
222\end{array}$ & $\begin{array}{r}56600 \\
38500 \\
85000 \\
8700 \\
36400\end{array}$ & $\begin{array}{l}60400 \\
58100 \\
9980 \emptyset \\
17400 \\
68600\end{array}$ & $\begin{array}{r}126.0 \\
110.0 \\
98.0 \\
97.0 \\
68.0\end{array}$ & $\begin{array}{r}123.5 \\
112.5 \\
95.5 \\
95.0 \\
66.0\end{array}$ & $\begin{array}{r}115.5 \\
99.5 \\
87.5 \\
87.0 \\
56.0\end{array}$ & $\begin{array}{l}53.0 \\
52.0 \\
44.0 \\
50.0 \\
35.0\end{array}$ & $\begin{array}{l}50.0 \\
53.0 \\
70.6 \\
74.1 \\
37.1\end{array}$ & $\begin{array}{l}1 \\
1 \\
1 \\
1 \\
1\end{array}$ & $\begin{array}{l}1 \\
1 \\
1 \\
3 \\
1\end{array}$ & $\begin{array}{l}1 \\
1 \\
1 \\
1 \\
1\end{array}$ & $\begin{array}{l}3 \\
6 \\
6 \\
3 \\
3\end{array}$ \\
\hline $\begin{array}{l}B K \\
B K \\
B K \\
B K \\
B K\end{array}$ & $\begin{array}{l}225 \\
226 \\
227 \\
228 \\
229\end{array}$ & $\begin{array}{c}- \\
17500 \\
18300 \\
- \\
-\end{array}$ & $\begin{array}{r}25600 \\
25700 \\
15700\end{array}$ & $\begin{array}{l}70.0 \\
61.0 \\
15.5 \\
25.0 \\
32.0\end{array}$ & $\begin{array}{r}59 . \overline{0} \\
13.3 \\
- \\
-\end{array}$ & $\begin{array}{r}50 . \overline{0} \\
7.3 \\
20.0 \\
32.5\end{array}$ & $\begin{array}{r}36.0 \\
36.0 \\
7.0 \\
20.0 \\
-\end{array}$ & $\begin{array}{r}- \\
15.4 \\
5.3 \\
- \\
-\end{array}$ & $\begin{array}{l}1 \\
1 \\
1 \\
1 \\
1\end{array}$ & $\begin{array}{l}3 \\
1 \\
3 \\
1 \\
1\end{array}$ & $\begin{array}{l}1 \\
1 \\
1 \\
1 \\
1\end{array}$ & $\begin{array}{l}3 \\
3 \\
3 \\
3 \\
3\end{array}$ \\
\hline $\begin{array}{l}B K \\
B K \\
B K \\
B K \\
B K\end{array}$ & $\begin{array}{l}230 \\
231 \\
232 \\
233 \\
234\end{array}$ & $\begin{array}{r}5200 \\
18700 \\
7400 \\
12700 \\
23100\end{array}$ & $\begin{array}{l}11300 \\
33300 \\
16900 \\
21800 \\
35200\end{array}$ & $\begin{array}{l}31.6 \\
36.0 \\
42.0 \\
40.0 \\
38.0\end{array}$ & $\begin{array}{l}31 . \overline{0} \\
38.5 \\
31.0 \\
37.5\end{array}$ & $\begin{array}{l}20.0 \\
21.5 \\
30.0 \\
25.0 \\
25.5\end{array}$ & $\begin{array}{l}20.0 \\
21.0 \\
30.0 \\
25.0 \\
16.0\end{array}$ & $\begin{array}{r}8.2 \\
33.8 \\
71.5 \\
12.0 \\
43.7\end{array}$ & $\begin{array}{l}1 \\
1 \\
1 \\
1 \\
1\end{array}$ & $\begin{array}{l}1 \\
3 \\
1 \\
1 \\
1\end{array}$ & $\begin{array}{l}1 \\
1 \\
1 \\
1 \\
1\end{array}$ & $\begin{array}{l}6 \\
3 \\
3 \\
7 \\
7\end{array}$ \\
\hline $\begin{array}{l}\text { BK } \\
B K \\
B K \\
B K \\
B K\end{array}$ & $\begin{array}{l}235 \\
236 \\
237 \\
238 \\
239\end{array}$ & $\begin{array}{r}21900 \\
14300 \\
24200 \\
23500 \\
2500\end{array}$ & $\begin{array}{r}34900 \\
22900 \\
33900 \\
40600 \\
7700\end{array}$ & $\begin{array}{l}38.4 \\
28.5 \\
21.0 \\
26.0 \\
19.0\end{array}$ & $\begin{array}{r}36.8 \\
26.2 \\
19.5 \\
17.7 \\
-\end{array}$ & $\begin{array}{r}28.8 \\
17.0 \\
10.0 \\
11.5 \\
6.0\end{array}$ & $\begin{array}{r}28.0 \\
8.0 \\
8.0 \\
12.0 \\
4.0\end{array}$ & $\begin{array}{r}27.7 \\
119.1 \\
31.6 \\
6.8 \\
3.4\end{array}$ & $\begin{array}{l}1 \\
1 \\
1 \\
1 \\
1\end{array}$ & $\begin{array}{l}1 \\
3 \\
1 \\
3 \\
1\end{array}$ & $\begin{array}{l}1 \\
1 \\
1 \\
1 \\
1\end{array}$ & $\begin{array}{l}1 \\
3 \\
3 \\
3 \\
3\end{array}$ \\
\hline $\begin{array}{l}\text { BK } \\
\text { BK } \\
\text { BK } \\
\text { BK } \\
\text { BK }\end{array}$ & $\begin{array}{l}240 \\
241 \\
242 \\
243 \\
244\end{array}$ & $\begin{array}{l}- \\
- \\
-\end{array}$ & $\begin{array}{r}- \\
11500 \\
7100 \\
6900 \\
7000\end{array}$ & $\begin{array}{l}20.0 \\
20.0 \\
16.0 \\
19.0 \\
16.0\end{array}$ & $\begin{array}{l}15.6 \\
16.6 \\
17.4 \\
16.1\end{array}$ & $\begin{array}{r}10.0 \\
7.0 \\
7.0 \\
7.5 \\
7.0\end{array}$ & $\begin{array}{r}10.0 \\
7.0 \\
6.0 \\
7.0 \\
6.0\end{array}$ & $\begin{array}{r}-\overline{8} \\
11.8 \\
7.9 \\
12.1 \\
6.4\end{array}$ & $\begin{array}{l}1 \\
1 \\
1 \\
1 \\
1\end{array}$ & $\begin{array}{l}1 \\
1 \\
1 \\
1 \\
1\end{array}$ & $\begin{array}{l}1 \\
1 \\
1 \\
1 \\
1\end{array}$ & $\begin{array}{l}6 \\
3 \\
3 \\
3 \\
3\end{array}$ \\
\hline $\begin{array}{l}B K \\
B K \\
B K \\
B K \\
B K\end{array}$ & $\begin{array}{l}245 \\
246 \\
247 \\
248 \\
249\end{array}$ & $\begin{array}{c}- \\
- \\
14100 \\
19200 \\
5800\end{array}$ & $\begin{array}{l}17900 \\
23500 \\
25800 \\
28800 \\
11300\end{array}$ & $\begin{array}{l}18.0 \\
15.0 \\
87.0 \\
95.0 \\
86.0\end{array}$ & $\begin{array}{l}12.3 \\
10.2 \\
81.7 \\
75.9 \\
85.0\end{array}$ & $\begin{array}{r}7.0 \\
6.0 \\
71.7 \\
65.9 \\
75.0\end{array}$ & $\begin{array}{r}5.0 \\
4.0 \\
30.0 \\
29.0 \\
31.0\end{array}$ & $\begin{array}{l}15.6 \\
10.2 \\
25.5 \\
30.5 \\
10.4\end{array}$ & $\begin{array}{l}1 \\
1 \\
1 \\
1 \\
1\end{array}$ & $\begin{array}{l}1 \\
1 \\
1 \\
1 \\
1\end{array}$ & $\begin{array}{l}1 \\
1 \\
1 \\
1 \\
1\end{array}$ & $\begin{array}{l}3 \\
3 \\
3 \\
3 \\
3\end{array}$ \\
\hline $\begin{array}{l}\text { BK } \\
\text { BK } \\
\text { BK } \\
\text { BK } \\
\text { BK }\end{array}$ & $\begin{array}{l}250 \\
251 \\
252 \\
253 \\
254\end{array}$ & $\begin{array}{l}- \\
\overline{-} \\
\overline{-}\end{array}$ & $\begin{array}{r}27700 \\
40300 \\
12400 \\
16000 \\
5000\end{array}$ & $\begin{array}{l}65.9 \\
68.0 \\
30.0 \\
32.0 \\
33.0\end{array}$ & $\begin{array}{l}64.5 \\
64.5 \\
27.5 \\
29.7 \\
30.5\end{array}$ & $\begin{array}{l}54.5 \\
54.5 \\
23.0 \\
20.0 \\
21.0\end{array}$ & $\begin{array}{r}31.0 \\
31.0 \\
6.0 \\
7.0 \\
8.0\end{array}$ & $\begin{array}{r}25.7 \\
34.5 \\
66.8 \\
169.6 \\
6.4\end{array}$ & $\begin{array}{l}1 \\
1 \\
1 \\
1 \\
1\end{array}$ & $\begin{array}{l}1 \\
1 \\
1 \\
1 \\
1\end{array}$ & $\begin{array}{l}1 \\
1 \\
1 \\
1 \\
1\end{array}$ & $\begin{array}{l}3 \\
3 \\
3 \\
6 \\
6\end{array}$ \\
\hline
\end{tabular}


Appendix.--Location and basic physical features of recharge basins on Long Island,

\begin{tabular}{|c|c|c|c|c|c|c|c|c|}
\hline \multicolumn{2}{|c|}{$\begin{array}{l}\text { BASIN } \\
\text { NUMBER }\end{array}$} & \multicolumn{2}{|c|}{ NEAREST INTERSECTION } & $\begin{array}{l}\text { LATITUDE } \\
\text { LONGITUDE, } \\
0, " 0, n\end{array}$ & $\begin{array}{l}\text { DATE } \\
\text { BUILT } \\
\text { YrMOD }\end{array}$ & COMMNITY & \multicolumn{2}{|c|}{$\begin{array}{l}\text { DESIGN ACTUAL } \\
\text { CAPACITY CAPACITY } \\
\text { (cubic feet) }\end{array}$} \\
\hline $\begin{array}{l}B K \\
B K \\
B K \\
B K \\
B K\end{array}$ & $\begin{array}{l}255 \\
256 \\
257 \\
258 \\
259\end{array}$ & $\begin{array}{l}\text { APPEL ORIVE } \\
\text { MARGIN DRIVE } \\
\text { BRESTON DRIVE } W . \\
\text { CARLIN DRIVE } \\
\text { MASON DRIVE }\end{array}$ & $\begin{array}{l}\text { HOUNSLOW ROAD } \\
\text { BRESTON ORIVE W. } \\
\text { MARGIN DRIVE } \\
\text { TITMUS DRIVE } \\
\text { MINTI DRIVE }\end{array}$ & $\begin{array}{l}4846330725138 \\
4846350725204 \\
4846480725205 \\
4849430725127 \\
4052510725943\end{array}$ & $\begin{array}{l}800519 \\
860519 \\
600519 \\
680517 \\
680427\end{array}$ & $\begin{array}{l}230 \\
- \\
- \\
-\end{array}$ & $\begin{array}{l}281700 \\
183480 \\
195060 \\
- \\
689800\end{array}$ & $\begin{array}{l}- \\
- \\
- \\
705000\end{array}$ \\
\hline $\begin{array}{l}B K \\
B K \\
B K \\
B K \\
B K\end{array}$ & $\begin{array}{l}260 \\
261 \\
262 \\
263 \\
264\end{array}$ & $\begin{array}{l}\text { BOLIN ROAD } \\
\text { WINFIEID DAVIS } \\
\text { DENIS LANE } \\
\text { COMMUNITY ROAD } \\
\text { PATCHOQUE ROAD }\end{array}$ & $\begin{array}{l}\text { GRADY LANE } \\
\text { BEECHER AVENUE } \\
\text { OAK LANE } \\
\text { FORREST LANE } \\
\text { PINE ROAD }\end{array}$ & $\begin{array}{l}4 \varnothing 52350736014 \\
4652320725932 \\
4652490725909 \\
4652550725901 \\
4653200736050\end{array}$ & $\begin{array}{l}680427 \\
650716 \\
650716 \\
670130 \\
6803\end{array}$ & $\begin{array}{l}- \\
218 \\
- \\
-\end{array}$ & $\begin{array}{l}5808000 \\
254000 \\
- \\
277000 \\
359000\end{array}$ & $\begin{array}{l}6000800 \\
2700000 \\
- \\
299000 \\
375800\end{array}$ \\
\hline $\begin{array}{l}B K \\
B K \\
B K \\
B K \\
B K\end{array}$ & $\begin{array}{l}266 \\
266 \\
267 \\
268 \\
269\end{array}$ & $\begin{array}{l}\text { HANSON STREET } \\
\text { MT.SINAI-CORAM R } \\
\text { OLD TOWN ROAD } \\
\text { PENNAQUID ROAD } \\
\text { LAUREL AVENUE }\end{array}$ & $\begin{array}{l}\text { WESTFIELD ROAD } \\
\text { CORAM-SWEZEYTOWN } \\
\text { CLARK STREET } \\
\text { HAWKINS ROAD } \\
\text { JOHN STREET }\end{array}$ & $\begin{array}{l}4 \varnothing 53180725846 \\
4853290725966 \\
4652440730135 \\
4653030730145 \\
4 \varnothing 53360730159\end{array}$ & $\begin{array}{c}\overline{-} \\
- \\
\overline{6} \\
661031 \\
661031\end{array}$ & $\begin{array}{l}-\overline{218} \\
- \\
-\end{array}$ & $\begin{array}{l}214400 \\
569900 \\
875000 \\
552000 \\
990000\end{array}$ & $\begin{array}{r}225800 \\
576200 \\
876060 \\
638000 \\
1100000\end{array}$ \\
\hline $\begin{array}{l}B K \\
B K \\
B K \\
B K \\
B K\end{array}$ & $\begin{array}{l}270 \\
271 \\
272 \\
273 \\
275\end{array}$ & $\begin{array}{l}\text { PINE STREET } \\
\text { PINE STREET } \\
\text { HOWE ROAD } \\
\text { BAILEY ROAD } \\
\text { FLICKER DRIVE }\end{array}$ & $\begin{array}{l}\text { HOWE ROAD } \\
\text { HOWE ROAD } \\
\text { PORT JEFFERSON R } \\
\text { ROCKY POINT ROAD } \\
\text { MAPLE ROAD }\end{array}$ & $\begin{array}{l}4653246736126 \\
4653366736124 \\
4653470736116 \\
4653246725668 \\
4 \varnothing 53156725622\end{array}$ & $\begin{array}{l}670103 \\
678163 \\
688219 \\
658481 \\
6482\end{array}$ & $\begin{array}{l}- \\
- \\
-\end{array}$ & $\begin{array}{l}197000 \\
189800 \\
450800 \\
236800 \\
136800\end{array}$ & $\begin{array}{l}210000 \\
191000 \\
460000 \\
251000 \\
134300\end{array}$ \\
\hline $\begin{array}{l}B K \\
B K \\
B K \\
B K \\
B K\end{array}$ & $\begin{array}{l}276 \\
277 \\
278 \\
279 \\
280\end{array}$ & $\begin{array}{l}\text { ANTIOCH DRIVE } \\
\text { HIGHWOOD LANE } \\
\text { HIGHWOOD LANE } \\
\text { SOUTHAMPTON PATH } \\
\text { HILLTOP LANE }\end{array}$ & $\begin{array}{l}\text { RIDER LANE } \\
\text { CREST DRIVE } \\
\text { CREST DRIVE } \\
\text { WADING RIVER RD. } \\
\text { APEX LANE }\end{array}$ & $\begin{array}{l}4055470725330 \\
4055070725319 \\
4054546725361 \\
4654438725251 \\
4054216725317\end{array}$ & $\begin{array}{l}680118 \\
6501 \\
6501 \\
6501 \\
6501\end{array}$ & $\begin{array}{c}- \\
\overline{2} \\
-\end{array}$ & $\begin{array}{l}489000 \\
390000 \\
632000 \\
400100 \\
446500\end{array}$ & $\begin{array}{l}493500 \\
407000 \\
671000 \\
489000 \\
500000\end{array}$ \\
\hline $\begin{array}{l}\text { BK } \\
\text { BK } \\
\text { BK } \\
\text { BK } \\
\text { BK }\end{array}$ & $\begin{array}{l}281 \\
282 \\
283 \\
285 \\
286\end{array}$ & $\begin{array}{l}\text { LONG POND DRIVE } \\
\text { GARDEN LANE } \\
\text { HORSE BLOCK ROAD } \\
\text { HOWELL AVENUE } \\
\text { HOWELL AVENUE }\end{array}$ & $\begin{array}{l}\text { STEPHEN DRIVE } \\
\text { TERRACE COURT } \\
\text { BELLPORT ROAD } \\
\text { HORSE BLOCK ROAD } \\
\text { HORSE BLOCK ROAD }\end{array}$ & $\begin{array}{l}4655446725649 \\
465622672564 \varnothing \\
4648456725738 \\
4 \varnothing 56560736418 \\
4650470736413\end{array}$ & $\begin{array}{l}878468 \\
620818 \\
680716 \\
681213 \\
681212\end{array}$ & $\begin{array}{l}250 \\
- \\
- \\
-\end{array}$ & $\begin{array}{r}270000 \\
52800 \\
283500 \\
78800 \\
162000\end{array}$ & $\begin{array}{r}286606 \\
38600 \\
284380 \\
92060 \\
1750600\end{array}$ \\
\hline $\begin{array}{l}\text { BK } \\
\text { BK } \\
\text { BK } \\
\text { BK } \\
\text { BK }\end{array}$ & $\begin{array}{l}288 \\
289 \\
290 \\
291 \\
292\end{array}$ & $\begin{array}{l}\text { PINE HILL ROAD } \\
\text { CRYSTAL BRK.HOL. } \\
\text { WILSON STREET } \\
\text { CLEMATIS STREET } \\
\text { QUINCY STREET }\end{array}$ & $\begin{array}{l}\text { ELLEN DRIVE } \\
\text { PINE HILL ROAD } \\
\text { MARCY AVENUE } \\
\text { NORWOOD AVENUE } \\
\text { CHAMBERLAND PATH }\end{array}$ & $\begin{array}{l}4656280736250 \\
4656370730233 \\
4653260730348 \\
4646230736411 \\
4058660736729\end{array}$ & $\begin{array}{c}680910 \\
680910 \\
651021 \\
- \\
6506\end{array}$ & $\begin{array}{l}249 \\
249 \\
- \\
- \\
-\end{array}$ & $\begin{array}{l}89800 \\
221000 \\
420000 \\
245880 \\
158100\end{array}$ & $\begin{array}{r}85000 \\
225000 \\
430000 \\
373100 \\
173000\end{array}$ \\
\hline $\begin{array}{l}B K \\
B K \\
B K \\
B K \\
B K\end{array}$ & $\begin{array}{l}293 \\
294 \\
295 \\
296 \\
297\end{array}$ & $\begin{array}{l}\text { MOUNT HURON CT. } \\
\text { RIDGEWAY AVENUE } \\
\text { A AVENUE } \\
\text { BEAVERDAM ROAD } \\
\text { WEST BROAOWAY DR }\end{array}$ & $\begin{array}{l}\text { ALLEGHENY DRIVE } \\
\text { BENNETS ROAD } \\
\text { 4TH STREET } \\
\text { ARTHUR AVENUE } \\
\text { FAIRWAY DRIVE }\end{array}$ & $\begin{array}{l}4049566736104 \\
4656056730714 \\
4849276736445 \\
4646260725529 \\
4654120736344\end{array}$ & \begin{tabular}{l}
\multicolumn{1}{c}{-} \\
670622 \\
681024 \\
8868 \\
641117
\end{tabular} & $\begin{array}{l}- \\
- \\
- \\
-\end{array}$ & $\begin{array}{l}446500 \\
- \\
142000 \\
1674800 \\
123000\end{array}$ & $\begin{array}{l}459700 \\
- \\
148200 \\
172006 \\
128000\end{array}$ \\
\hline $\begin{array}{l}B K \\
B K \\
B K \\
B K \\
B K\end{array}$ & $\begin{array}{l}298 \\
299 \\
300 \\
301 \\
302\end{array}$ & $\begin{array}{l}\text { CONCORD STREET } \\
\text { THE BEND } \\
\text { BLACKFOOT TRAIL } \\
\text { BRANCH LANE } \\
\text { SETAUKET ROAD }\end{array}$ & $\begin{array}{l}\text { QUEENS AVENUE } \\
\text { VEE JAY DRIVE } \\
\text { VEE JAY DRIVE } \\
\text { FIRESIDE LANE } \\
\text { 2OTH STREET }\end{array}$ & $\begin{array}{l}4656460725911 \\
4656360725249 \\
4656270725313 \\
4654176736448 \\
4653296736556\end{array}$ & $\begin{array}{l}- \\
\overline{-} \\
-\end{array}$ & $\begin{array}{l}- \\
\overline{-} \\
-\end{array}$ & $\begin{array}{l}183000 \\
- \\
- \\
363400 \\
135100\end{array}$ & $\begin{array}{l}191000 \\
- \\
- \\
385000 \\
135900\end{array}$ \\
\hline $\begin{array}{l}B K \\
B K \\
B K \\
B K \\
B K\end{array}$ & $\begin{array}{l}303 \\
304 \\
305 \\
306 \\
307\end{array}$ & $\begin{array}{l}\text { MISSOURI PLACE } \\
\text { MAIN ROAD } \\
\text { SETALCUTT PLACE } \\
\text { LOWER DEPOT ROAD } \\
\text { UPPER DEPOT ROAD }\end{array}$ & $\begin{array}{l}\text { CORAM ROAD } \\
\text { HUBB ROAD } \\
\text { SETALCUTT PL. N. } \\
\text { MILLS LANE } \\
\text { HIGHLAND AVENUE }\end{array}$ & $\begin{array}{l}4654556725936 \\
4 \varnothing 54346736617 \\
4655466736787 \\
46563187386 \varnothing 2 \\
4656256736615\end{array}$ & $\bar{z}$ & $\bar{z}$ & $\begin{array}{l}381800 \\
188600 \\
43600 \\
- \\
-\end{array}$ & $\begin{array}{l}4148000 \\
203800 \\
44060 \\
- \\
-\end{array}$ \\
\hline $\begin{array}{l}B K \\
B K \\
B K \\
B K \\
B K\end{array}$ & $\begin{array}{l}308 \\
309 \\
310 \\
320 \\
321\end{array}$ & $\begin{array}{l}\text { OLD TOWN ROAD } \\
\text { SHEEP PASTURE RD } \\
\text { SETAUKET ROAD } \\
\text { THOMPSONS PATH } \\
\text { WILLIAM PENN DR. }\end{array}$ & $\begin{array}{l}\text { LYNX LANE } \\
\text { BENNETS ROAD } \\
\text { LIRR } \\
\text { TUDOR DRIVE } \\
\text { YORKSHIRE AVENUE }\end{array}$ & $\begin{array}{l}4655476736514 \\
4655176736655 \\
4655426736653 \\
4655346736736 \\
4655476736742\end{array}$ & $\begin{array}{l}- \\
\overline{-} \\
\overline{-}\end{array}$ & $\begin{array}{l}- \\
\overline{-} \\
\overline{-}\end{array}$ & $\begin{array}{l}- \\
\overline{-} \\
\overline{-}\end{array}$ & $\begin{array}{l}- \\
\overline{-} \\
-\end{array}$ \\
\hline
\end{tabular}


N.Y. [Dash indicates no data. Altitudes are in feet above sea level.] (cont.)

\begin{tabular}{|c|c|c|c|c|c|c|c|c|c|c|c|c|}
\hline \multicolumn{2}{|c|}{$\begin{array}{l}\text { BASIN } \\
\text { NUMBER }\end{array}$} & $\begin{array}{l}\text { MAX. } \\
\text { AREA } \\
\text { (square }\end{array}$ & $\begin{array}{c}\text { BASIN } \\
\text { AREA } \\
\text { feet) }\end{array}$ & RIM & $\begin{array}{l}\text { OVER- } \\
\text { FLOW }\end{array}$ & TOM & $\begin{array}{l}\text { WATER } \\
\text { TABLE }\end{array}$ & $\begin{array}{c}\text { DRAIN. } \\
\text { AREA } \\
\text { (acres) }\end{array}$ & \multicolumn{2}{|c|}{$\begin{array}{l}-- \text { BASIN-- } \\
\text { STATUS USE }\end{array}$} & $\begin{array}{l}\text { GEO. } \\
\text { UNIT }\end{array}$ & $\begin{array}{l}\text { SOIL } \\
\text { UNIT }\end{array}$ \\
\hline $\begin{array}{l}\text { BK } \\
\text { BK } \\
\text { BK } \\
\text { BK } \\
\text { BK }\end{array}$ & $\begin{array}{l}255 \\
256 \\
257 \\
258 \\
259\end{array}$ & $\begin{array}{c}- \\
\overline{-} \\
\overline{70500}\end{array}$ & $\begin{array}{r}20200 \\
10300 \\
19500 \\
47300 \\
-\end{array}$ & $\begin{array}{l}30.0 \\
33.0 \\
35.0 \\
75.0 \\
70.0\end{array}$ & $\begin{array}{r}27.7 \\
29.9 \\
27.7 \\
66.5 \\
-\end{array}$ & $\begin{array}{l}20 . \overline{0} \\
18.0 \\
56.5 \\
60.5\end{array}$ & $\begin{array}{r}7.0 \\
7.0 \\
8.0 \\
30.0 \\
53.0\end{array}$ & $\begin{array}{r}279.4 \\
22.0 \\
30.7 \\
-\overline{105} .3\end{array}$ & $\begin{array}{l}1 \\
1 \\
1 \\
1 \\
1\end{array}$ & $\begin{array}{l}1 \\
1 \\
1 \\
1 \\
1\end{array}$ & $\begin{array}{l}1 \\
1 \\
1 \\
1 \\
1\end{array}$ & $\begin{array}{l}3 \\
3 \\
6 \\
3 \\
3\end{array}$ \\
\hline $\begin{array}{l}\text { BK } \\
\text { BK } \\
\text { BK } \\
\text { BK } \\
\text { BK }\end{array}$ & $\begin{array}{l}260 \\
261 \\
262 \\
263 \\
264\end{array}$ & $\begin{array}{c}60000 \\
17000 \\
- \\
20800 \\
37500\end{array}$ & $\begin{array}{r}- \\
30300 \\
37400 \\
21300\end{array}$ & $\begin{array}{r}70.0 \\
78.5 \\
90.0 \\
85.0 \\
100.0\end{array}$ & $\begin{array}{r}75 . \overline{9} \\
\overline{4} \\
71 . \overline{4}\end{array}$ & $\begin{array}{r}60 . \overline{0} \\
57 . \overline{0} \\
-\end{array}$ & $\begin{array}{l}53.0 \\
53.0 \\
52.0 \\
52.0 \\
52.0\end{array}$ & $\begin{array}{r}55.2 \\
40.0 \\
-\overline{-} \\
43.6 \\
41.0\end{array}$ & $\begin{array}{l}1 \\
1 \\
1 \\
1 \\
1\end{array}$ & $\begin{array}{l}1 \\
1 \\
1 \\
1 \\
1\end{array}$ & $\begin{array}{l}1 \\
1 \\
1 \\
1 \\
1\end{array}$ & $\begin{array}{l}5 \\
7 \\
5 \\
5 \\
5\end{array}$ \\
\hline $\begin{array}{l}\text { BK } \\
\text { BK } \\
B K \\
B K \\
B K\end{array}$ & $\begin{array}{l}265 \\
266 \\
267 \\
268 \\
269\end{array}$ & $\begin{array}{r}19300 \\
57600 \\
87600 \\
63800 \\
110000\end{array}$ & $\begin{array}{r}35680 \\
- \\
- \\
- \\
-\end{array}$ & $\begin{array}{r}80.0 \\
95.0 \\
90.0 \\
90.0 \\
120.0\end{array}$ & $\begin{array}{r}81.2 \\
- \\
- \\
- \\
-\end{array}$ & $\begin{array}{l}69.5 \\
73.0 \\
71.5 \\
86.0 \\
96.6\end{array}$ & $\begin{array}{l}52.0 \\
52.0 \\
54.0 \\
54.0 \\
54.0\end{array}$ & $\begin{array}{l}17.8 \\
52.4 \\
97.2 \\
61.2 \\
10.3\end{array}$ & $\begin{array}{l}1 \\
1 \\
1 \\
1 \\
1\end{array}$ & $\begin{array}{l}1 \\
1 \\
1 \\
3 \\
1\end{array}$ & $\begin{array}{l}1 \\
1 \\
1 \\
1 \\
1\end{array}$ & $\begin{array}{l}6 \\
0 \\
5 \\
5 \\
1\end{array}$ \\
\hline $\begin{array}{l}B K \\
B K \\
B K \\
B K \\
B K\end{array}$ & $\begin{array}{l}270 \\
271 \\
272 \\
273 \\
275\end{array}$ & $\begin{array}{l}2100 \emptyset \\
1910 \emptyset \\
4600 \emptyset \\
17300 \\
11200\end{array}$ & $\begin{array}{r}- \\
- \\
33600 \\
22100\end{array}$ & $\begin{array}{r}120.0 \\
120.0 \\
110.0 \\
90.0 \\
88.5\end{array}$ & $\begin{array}{r}- \\
- \\
87 . \overline{0} \\
86.0\end{array}$ & $\begin{array}{r}100.0 \\
102.0 \\
85.0 \\
72.5 \\
74.0\end{array}$ & $\begin{array}{l}54.0 \\
54.0 \\
51.0 \\
50.0 \\
50.0\end{array}$ & $\begin{array}{r}22.5 \\
21.0 \\
49.8 \\
14.5 \\
152 . \varnothing\end{array}$ & $\begin{array}{l}1 \\
1 \\
1 \\
1 \\
1\end{array}$ & $\begin{array}{l}3 \\
3 \\
3 \\
3 \\
3\end{array}$ & $\begin{array}{l}1 \\
1 \\
1 \\
1 \\
1\end{array}$ & $\begin{array}{l}3 \\
3 \\
5 \\
1 \\
1\end{array}$ \\
\hline $\begin{array}{l}\text { BK } \\
\text { BK } \\
\text { BK } \\
\text { BK } \\
\text { BK }\end{array}$ & $\begin{array}{l}276 \\
277 \\
278 \\
279 \\
28 \varnothing\end{array}$ & $\begin{array}{l}49400 \\
40700 \\
67100 \\
48900 \\
58000\end{array}$ & $\begin{array}{l}\text { 41400 } \\
64800 \\
61000 \\
46600\end{array}$ & $\begin{array}{r}185.0 \\
75.0 \\
77.0 \\
83.0 \\
81.0\end{array}$ & $\begin{array}{l}- \\
- \\
- \\
-\end{array}$ & $\begin{array}{l}-\overline{8} \\
63.8 \\
50.8 \\
69.5 \\
66.5\end{array}$ & $\begin{array}{l}40.0 \\
43.0 \\
45.0 \\
46.0 \\
47.0\end{array}$ & $\begin{array}{l}55.0 \\
43.0 \\
71.0 \\
46.7 \\
51.0\end{array}$ & $\begin{array}{l}1 \\
1 \\
0 \\
1 \\
1\end{array}$ & $\begin{array}{l}1 \\
1 \\
1 \\
3 \\
1\end{array}$ & $\begin{array}{l}1 \\
1 \\
1 \\
1 \\
1\end{array}$ & $\begin{array}{l}1 \\
3 \\
0 \\
3 \\
6\end{array}$ \\
\hline $\begin{array}{l}B K \\
B K \\
B K \\
B K \\
B K\end{array}$ & $\begin{array}{l}281 \\
282 \\
283 \\
285 \\
286\end{array}$ & $\begin{array}{r}31200 \\
34900 \\
26400 \\
9200 \\
17500\end{array}$ & $\begin{array}{r}37500 \\
6600 \\
- \\
12800 \\
22500\end{array}$ & $\begin{array}{r}49.5 \\
50.5 \\
95.0 \\
135.0 \\
130.0\end{array}$ & $\begin{array}{r}49.0 \\
67.5 \\
- \\
- \\
-\end{array}$ & $\begin{array}{r}40.0 \\
46.6 \\
78.5 \\
104 . \overline{0}\end{array}$ & $\begin{array}{r}40.0 \\
38.0 \\
38.0 \\
114.0 \\
56.0\end{array}$ & $\begin{array}{r}42.5 \\
8.3 \\
10.8 \\
9.0 \\
18.5\end{array}$ & $\begin{array}{l}1 \\
1 \\
1 \\
1 \\
1\end{array}$ & $\begin{array}{l}1 \\
1 \\
3 \\
1 \\
1\end{array}$ & $\begin{array}{l}1 \\
1 \\
1 \\
6 \\
6\end{array}$ & $\begin{array}{l}0 \\
3 \\
3 \\
5 \\
5\end{array}$ \\
\hline $\begin{array}{l}\text { BK } \\
B K \\
B K \\
B K \\
B K\end{array}$ & $\begin{array}{l}288 \\
289 \\
290 \\
291 \\
292\end{array}$ & $\begin{array}{r}8600 \\
18000 \\
43800 \\
37300 \\
17300\end{array}$ & $\begin{array}{r}16700 \\
32500 \\
- \\
26900\end{array}$ & $\begin{array}{r}163.0 \\
78.0 \\
115.0 \\
175.0 \\
107.5\end{array}$ & $\begin{array}{r}159.0 \\
75.5 \\
- \\
180.0\end{array}$ & $\begin{array}{r}149.0 \\
63.0 \\
-\overline{158.0} \\
90.0\end{array}$ & $\begin{array}{l}30.0 \\
30.0 \\
52.0 \\
44.0 \\
27.0\end{array}$ & $\begin{array}{r}4.2 \\
13.5 \\
41.1 \\
38.7 \\
27.0\end{array}$ & $\begin{array}{l}1 \\
1 \\
1 \\
1 \\
1\end{array}$ & $\begin{array}{l}3 \\
3 \\
1 \\
1 \\
1\end{array}$ & $\begin{array}{l}3 \\
3 \\
1 \\
1 \\
3\end{array}$ & $\begin{array}{l}5 \\
5 \\
3 \\
7 \\
5\end{array}$ \\
\hline $\begin{array}{l}B K \\
B K \\
8 K \\
B K \\
B K\end{array}$ & $\begin{array}{l}293 \\
294 \\
295 \\
296 \\
297\end{array}$ & $\begin{array}{c}46000 \\
\overline{1} \\
11900 \\
172 \varnothing \varnothing \\
12600\end{array}$ & $\begin{array}{r}- \\
24200 \overline{-} \\
14900\end{array}$ & $\begin{array}{r}120.0 \\
50.0 \\
132.2 \\
47.0 \\
146.3\end{array}$ & $\begin{array}{r}62 . \overline{-} \\
130 . \overline{5} \\
-\end{array}$ & $\begin{array}{r}106.5 \\
49.0 \\
117.0 \\
132 . \overline{0}\end{array}$ & $\begin{array}{l}50.0 \\
27.0 \\
51.0 \\
14.0 \\
52.0\end{array}$ & $\begin{array}{r}70.3 \\
- \\
19.2 \\
30.5 \\
22.3\end{array}$ & $\begin{array}{l}1 \\
1 \\
1 \\
1 \\
1\end{array}$ & $\begin{array}{l}1 \\
3 \\
1 \\
3 \\
1\end{array}$ & $\begin{array}{l}1 \\
3 \\
1 \\
1 \\
1\end{array}$ & $\begin{array}{l}7 \\
5 \\
6 \\
3 \\
7\end{array}$ \\
\hline $\begin{array}{l}\text { BK } \\
8 K \\
\text { BK } \\
8 K \\
B K\end{array}$ & $\begin{array}{l}298 \\
299 \\
300 \\
301 \\
302\end{array}$ & $\begin{array}{c}15900 \\
\overline{-} \\
38 \overline{5} 00 \\
13600\end{array}$ & $\begin{array}{l}26400 \\
18900 \\
29200 \\
57600 \\
28800\end{array}$ & $\begin{array}{l}145.2 \\
120.0 \\
120.0 \\
141.0 \\
116.0\end{array}$ & $\begin{array}{r}142.0 \\
- \\
=\end{array}$ & $\begin{array}{r}130.0 \\
104.0 \\
109.0 \\
127.0 \\
-\end{array}$ & $\begin{array}{l}31.0 \\
28.0 \\
28.0 \\
50.0 \\
52.0\end{array}$ & $\begin{array}{r}18.0 \\
- \\
- \\
41.5 \\
15.5\end{array}$ & $\begin{array}{l}1 \\
1 \\
1 \\
1 \\
1\end{array}$ & $\begin{array}{l}1 \\
1 \\
1 \\
1 \\
1\end{array}$ & $\begin{array}{l}1 \\
1 \\
1 \\
1 \\
1\end{array}$ & $\begin{array}{l}3 \\
3 \\
1 \\
7 \\
3\end{array}$ \\
\hline $\begin{array}{l}\text { BK } \\
8 K \\
B K \\
B K \\
B K\end{array}$ & $\begin{array}{l}303 \\
304 \\
305 \\
306 \\
307\end{array}$ & $\begin{array}{c}41500 \\
204 \varnothing \varnothing \\
448 \varnothing \\
- \\
-\end{array}$ & $\begin{array}{r}72800 \\
51200 \\
25680 \\
- \\
-\end{array}$ & $\begin{array}{r}140.0 \\
132.0 \\
97.0 \\
15.0 \\
15.0\end{array}$ & $\begin{array}{r}134.8 \\
127.0 \\
91.0 \\
- \\
-\end{array}$ & $\begin{array}{r}116 . \overline{0} \\
81.0 \\
- \\
-\end{array}$ & $\begin{array}{r}48.0 \\
44.0 \\
30.0 \\
5.0 \\
5.0\end{array}$ & $\begin{array}{r}34.0 \\
19.6 \\
7.9 \\
- \\
-\end{array}$ & $\begin{array}{l}1 \\
1 \\
1 \\
1 \\
1\end{array}$ & $\begin{array}{l}1 \\
1 \\
1 \\
1 \\
1\end{array}$ & $\begin{array}{l}1 \\
3 \\
3 \\
3 \\
3\end{array}$ & $\begin{array}{l}5 \\
6 \\
6 \\
4 \\
4\end{array}$ \\
\hline $\begin{array}{l}\text { BK } \\
B K \\
B K \\
B K \\
B K\end{array}$ & $\begin{array}{l}308 \\
309 \\
310 \\
320 \\
321\end{array}$ & $\begin{array}{l}- \\
- \\
-\end{array}$ & $\begin{array}{l}\text { - } \\
\overline{-} \\
\overline{-}\end{array}$ & $\begin{array}{r}110.0 \\
90.0 \\
50.0 \\
90.0 \\
150.0\end{array}$ & $\begin{array}{l}- \\
- \\
-\end{array}$ & $\begin{array}{l}- \\
\overline{-} \\
-\end{array}$ & $\begin{array}{l}35.0 \\
38.0 \\
33.0 \\
32.0 \\
31.0\end{array}$ & - & $\begin{array}{l}1 \\
1 \\
1 \\
1 \\
1\end{array}$ & $\begin{array}{l}3 \\
3 \\
1 \\
1 \\
1\end{array}$ & $\begin{array}{l}3 \\
3 \\
3 \\
3 \\
3\end{array}$ & $\begin{array}{l}6 \\
1 \\
3 \\
7 \\
7\end{array}$ \\
\hline
\end{tabular}


Appendix.--Location and basic physical features of recharge basins on Long Island,

\begin{tabular}{|c|c|c|c|c|c|c|c|c|}
\hline \multicolumn{2}{|c|}{$\begin{array}{l}\text { BASIN } \\
\text { NUMBER }\end{array}$} & \multicolumn{2}{|c|}{ NEAREST INTERSECTION } & $\begin{array}{l}\text { LATITUDE } \\
\text { LONGITUDE } \\
0, n 0^{\prime},\end{array}$ & $\begin{array}{l}\text { DATE } \\
\text { BUILT } \\
\text { YrMOD }\end{array}$ & COMMUNITY & \multicolumn{2}{|c|}{$\begin{array}{l}\text { DESIGN ACTUAL } \\
\text { CAPACITY CAPACITY } \\
\text { (cubic feet) }\end{array}$} \\
\hline $\begin{array}{l}B K \\
B K \\
B K \\
B K \\
B K\end{array}$ & $\begin{array}{l}322 \\
323 \\
324 \\
326 \\
327\end{array}$ & $\begin{array}{l}\text { OLD TOWN ROAD } \\
\text { MOUNT SINAI AVE. } \\
\text { POPLAR STREET. } \\
\text { GRAND AVENUE } \\
\text { GRAND AVENUE }\end{array}$ & $\begin{array}{l}\text { LIRR } \\
\text { CANAL ROAD } \\
\text { RED AVENUE } \\
\text { DEWEY STREET } \\
\text { DEWEY STREET }\end{array}$ & $\begin{array}{l}4056390730005 \\
4055260730058 \\
4055380730250 \\
4055480730251 \\
4055470730251\end{array}$ & $\begin{array}{l}- \\
\overline{-} \\
-\end{array}$ & $\begin{array}{c}- \\
249 \\
- \\
-\end{array}$ & $\begin{array}{l}- \\
- \\
-\end{array}$ & $\begin{array}{l}- \\
- \\
-\end{array}$ \\
\hline $\begin{array}{l}\text { BK } \\
\text { BK } \\
\text { BK } \\
\text { BK } \\
\text { BK }\end{array}$ & $\begin{array}{l}328 \\
329 \\
330 \\
331 \\
332\end{array}$ & $\begin{array}{l}\text { GRAND AVENUE } \\
\text { PARDAM KNOLL RD. } \\
\text { HALLOCK LANDING } \\
\text { 2ND AVENUE } \\
\text { SYCAMORE ROAD }\end{array}$ & $\begin{array}{l}\text { DEWEY STREET } \\
\text { EVANS LANE } \\
N \text {. ROCKY POINT R } \\
\text { LANDING ROAD } \\
\text { SOUND VIEW DRIVE }\end{array}$ & $\begin{array}{l}4855460730253 \\
4856370730008 \\
4057850725633 \\
4057050725630 \\
4057430725547\end{array}$ & $\begin{array}{l}- \\
\overline{-} \\
-\end{array}$ & $\begin{array}{c}- \\
- \\
251 \\
251 \\
256\end{array}$ & $\begin{array}{l}- \\
- \\
-\end{array}$ & $\begin{array}{l}- \\
- \\
- \\
-\end{array}$ \\
\hline $\begin{array}{l}\text { BK } \\
\text { BK } \\
\text { BK } \\
\text { BK } \\
\text { BK }\end{array}$ & $\begin{array}{l}333 \\
334 \\
335 \\
336 \\
337\end{array}$ & $\begin{array}{l}\text { PECONIC ROAD } \\
\text { PORT JEFFERSON R } \\
\text { WILDWOOD DRIVE } \\
\text { IROQUOIS TRAIL } \\
\text { IROQUOIS TRAIL }\end{array}$ & $\begin{array}{l}\text { PLANDOME ROAD } \\
\text { YAPHANK ROAD } \\
\text { NEW YORK AVENUE } \\
\text { LONG POND ROAD } \\
\text { LONG POND ROAD }\end{array}$ & $\begin{array}{l}4657000725749 \\
4057510725815 \\
4057130725905 \\
4056460725109 \\
4055480725111\end{array}$ & $\begin{array}{l}- \\
- \\
-\end{array}$ & $\begin{array}{l}257 \\
257 \\
- \\
- \\
-\end{array}$ & $\begin{array}{l}- \\
- \\
- \\
-\end{array}$ & $\begin{array}{l}- \\
- \\
-\end{array}$ \\
\hline $\begin{array}{l}\text { BK } \\
B K \\
B K \\
B K \\
B K\end{array}$ & $\begin{array}{l}338 \\
339 \\
340 \\
341 \\
342\end{array}$ & $\begin{array}{l}\text { IROQUOIS TRAIL } \\
\text { CHEEMAUN TRAIL } \\
\text { CHEEMAUN TRAIL } \\
\text { LAKEVIEW TRAIL } \\
\text { WOODVILLE ROAD }\end{array}$ & $\begin{array}{l}\text { LONG POND ROAD } \\
\text { LONG POND ROAD } \\
\text { LONG POND ROAD } \\
\text { DORIS TRAIL } \\
\text { CIRCLE DRIVE }\end{array}$ & $\begin{array}{l}4855480725113 \\
4055480725116 \\
4055460725116 \\
4055350725659 \\
4057100725425\end{array}$ & $\begin{array}{l}- \\
\overline{-} \\
\overline{-}\end{array}$ & $\begin{array}{l}- \\
- \\
\overline{256}\end{array}$ & $\begin{array}{l}- \\
- \\
- \\
-\end{array}$ & $\begin{array}{l}- \\
- \\
- \\
-\end{array}$ \\
\hline $\begin{array}{l}B K \\
B K \\
B K \\
B K \\
B K\end{array}$ & $\begin{array}{l}343 \\
344 \\
346 \\
346 \\
347\end{array}$ & $\begin{array}{l}\text { NORMAN AVENUE } \\
\text { WOODVILLE ROAD } \\
\text { MARKET STREET } \\
\text { MARK TREE ROAD } \\
\text { DAWN DRIVE }\end{array}$ & $\begin{array}{l}\text { FREDERICK DRIVE } \\
\text { SUFFOLK DOWN } \\
\text { NOEL DRIVE } \\
\text { LINDA DRIVE } \\
\text { STANLEY DRIVE }\end{array}$ & $\begin{array}{l}4057140725346 \\
4057180725422 \\
485134873 \varnothing 521 \\
485158073 \varnothing 511 \\
485157 \varnothing 73 \varnothing 515\end{array}$ & $\begin{array}{l}- \\
- \\
- \\
-\end{array}$ & $\begin{array}{l}- \\
256 \\
- \\
-\end{array}$ & $\begin{array}{l}- \\
- \\
- \\
-\end{array}$ & $\begin{array}{l}- \\
- \\
- \\
-\end{array}$ \\
\hline $\begin{array}{l}\text { BK } \\
B K \\
B K \\
B K \\
B K\end{array}$ & $\begin{array}{l}348 \\
349 \\
356 \\
351 \\
352\end{array}$ & $\begin{array}{l}\text { 53RD. STREET } \\
\text { STANLEY DRIVE } \\
\text { MARK TREE ROAD } \\
\text { OXHEAD ROAD } \\
\text { CRAFT ROAD }\end{array}$ & $\begin{array}{l}\text { NOEL DRIVE } \\
\text { 49TH STREET } \\
\text { 47TH STREET } \\
\text { EDWARD STREET } \\
\text { EDWARD STREET }\end{array}$ & $\begin{array}{l}405157073 \varnothing 524 \\
4052060730525 \\
4052130730525 \\
4851420730536 \\
4051370730544\end{array}$ & $\begin{array}{l}- \\
- \\
- \\
-\end{array}$ & $\begin{array}{l}216 \\
216 \\
216 \\
216 \\
216\end{array}$ & $\begin{array}{l}- \\
- \\
-\end{array}$ & $\begin{array}{l}- \\
- \\
- \\
-\end{array}$ \\
\hline $\begin{array}{l}B K \\
B K \\
B K \\
B K \\
B K\end{array}$ & $\begin{array}{l}353 \\
354 \\
355 \\
356 \\
367\end{array}$ & $\begin{array}{l}\text { VAN BERGEN BLVD. } \\
\text { MIDLAND AVENUE } \\
\text { MCGRAW AVENUE } \\
\text { ORIENTA AVENUE } \\
\text { STONY BROOK RD. }\end{array}$ & $\begin{array}{l}\text { EVELYN LANE } \\
\text { WASHINGTON ST. } \\
\text { PENN STREET } \\
\text { HAMPTON STREET } \\
\text { STEVEN PLACE }\end{array}$ & $\begin{array}{l}4051100730554 \\
4050410730610 \\
4051400730633 \\
4050450730728 \\
4052420730709\end{array}$ & $\begin{array}{l}- \\
- \\
- \\
-\end{array}$ & $\begin{array}{l}\overline{216} \\
- \\
-\end{array}$ & $\begin{array}{l}- \\
- \\
-\end{array}$ & $\begin{array}{l}- \\
- \\
- \\
-\end{array}$ \\
\hline $\begin{array}{l}B K \\
B K \\
B K \\
B K \\
B K\end{array}$ & $\begin{array}{l}358 \\
369 \\
360 \\
361 \\
362\end{array}$ & $\begin{array}{l}\text { STONY BROOK ROAD } \\
\text { FIRESIDE LANE } \\
\text { CABIN LANE } \\
\text { W. OF UNIVERSITY } \\
\text { TUDOR COURT }\end{array}$ & $\begin{array}{l}\text { STEVEN PLACE } \\
\text { MAYFLOWER LANE } \\
\text { STALKER LANE } \\
\text { UNIVERSITY DRIVE }\end{array}$ & $\begin{array}{l}4852420736709 \\
4853800730451 \\
4854410730435 \\
4854280736851 \\
4854280736811\end{array}$ & $\begin{array}{l}- \\
- \\
-\end{array}$ & $\begin{array}{l}- \\
\overline{-} \\
\overline{-}\end{array}$ & $\begin{array}{l}- \\
- \\
- \\
-\end{array}$ & $\begin{array}{l}- \\
- \\
-\end{array}$ \\
\hline $\begin{array}{l}\text { BK } \\
\text { BK } \\
\text { BK } \\
\text { BK } \\
\text { BK }\end{array}$ & $\begin{array}{l}363 \\
364 \\
365 \\
366 \\
367\end{array}$ & $\begin{array}{l}\text { N. OF ACORN LA. } \\
\text { STATE HWY.25A } \\
\text { DRAKE ROAD } \\
\text { WEST COURT DR. } \\
\text { LAURA DRIVE }\end{array}$ & $\begin{array}{l}\text { MOUNT ROAD } \\
\text { BOWDEN ROAD } \\
\text { 34TH STREET } \\
\text { ESTHER }\end{array}$ & $\begin{array}{l}4 \varnothing 54240730828 \\
4 \varnothing 5431073 \emptyset 745 \\
4 \varnothing 52560730516 \\
4 \varnothing 5241073 \emptyset 529 \\
4 \varnothing 51530730411\end{array}$ & $\begin{array}{l}- \\
- \\
-\end{array}$ & $\begin{array}{l}- \\
- \\
-\end{array}$ & $\begin{array}{l}- \\
- \\
-\end{array}$ & $\begin{array}{l}\overline{-} \\
\overline{-} \\
\overline{-}\end{array}$ \\
\hline $\begin{array}{l}\text { BK } \\
\text { BK } \\
\text { BK } \\
\text { BK } \\
\text { BK }\end{array}$ & $\begin{array}{l}368 \\
369 \\
370 \\
371 \\
372\end{array}$ & $\begin{array}{l}\text { POND PATH } \\
\text { POND PATH } \\
\text { N. OF TUDOR ROAD } \\
\text { SUNSET DRIVE } \\
\text { TREE ROAD }\end{array}$ & $\begin{array}{l}\text { PEARLESS DRIVE } \\
\text { PEARLESS DRIVE } \\
\text { MORNING DRIVE } \\
\text { LEONARD LANE }\end{array}$ & $\begin{array}{l}405204073065 \emptyset \\
4 \varnothing 5204 \varnothing 736853 \\
4 \varnothing 51280730447 \\
4 \varnothing 52070730511 \\
4052130730510\end{array}$ & $\begin{array}{l}- \\
- \\
-\end{array}$ & $\begin{array}{l}- \\
\overline{-} \\
\overline{234}\end{array}$ & $\begin{array}{l}- \\
- \\
-\end{array}$ & $\begin{array}{l}- \\
- \\
-\end{array}$ \\
\hline $\begin{array}{l}\text { BK } \\
\text { BK } \\
\text { BK } \\
\text { BK } \\
\text { BK }\end{array}$ & $\begin{array}{l}373 \\
374 \\
375 \\
376 \\
377\end{array}$ & $\begin{array}{l}\text { BARBARA DRIVE } \\
\text { EASTWOOD BLVD. } \\
\text { WAGON LANE SOUTH } \\
\text { WAGON LANE EAST } \\
\text { S. OF FLOWER LA. }\end{array}$ & $\begin{array}{l}\text { BERNARD PLACE } \\
\text { SCHOOL STREET } \\
\text { WAGON LANE EAST } \\
\text { EASTWOOD BLVD }\end{array}$ & $\begin{array}{l}4052110730455 \\
4051540730444 \\
4052190730451 \\
4052340730456 \\
4052350730504\end{array}$ & $\begin{array}{l}- \\
- \\
-\end{array}$ & $\begin{array}{c}216 \\
- \\
216 \\
216 \\
-\end{array}$ & $\begin{array}{l}- \\
- \\
-\end{array}$ & $\begin{array}{l}- \\
- \\
-\end{array}$ \\
\hline
\end{tabular}


N.Y. [Dash indicates no data. Altitudes are in feet above sea level.] (cont.)

\begin{tabular}{|c|c|c|c|c|c|c|c|c|c|c|c|c|}
\hline & $\begin{array}{l}\text { SIN } \\
\text { MBER }\end{array}$ & $\begin{array}{l}\text { MAX. } \\
\text { AREA } \\
\text { (square }\end{array}$ & $\begin{array}{l}\text { BASIN } \\
\text { AREA } \\
\text { fe日t) }\end{array}$ & RIM & $\begin{array}{l}--A L T I T \\
\text { OVER- } \\
\text { FLOW }\end{array}$ & $\begin{array}{l}\text { E--- } \\
\text { BOT- } \\
\text { TOM }\end{array}$ & $\begin{array}{l}\text { WATER } \\
\text { TABLE }\end{array}$ & $\begin{array}{c}\text { DRAIN. } \\
\text { AREA } \\
\text { (acres) }\end{array}$ & $\begin{array}{l}--B \\
\text { STAT }\end{array}$ & $\begin{array}{l}\text { IN-- } \\
\text { USE }\end{array}$ & $\begin{array}{l}\text { GEO } \\
\text { UNIT }\end{array}$ & $\begin{array}{l}\text { SOIL } \\
\text { UNIT }\end{array}$ \\
\hline $\begin{array}{l}\text { BK } \\
\text { BK } \\
\text { BK } \\
\text { BK } \\
\text { BK }\end{array}$ & $\begin{array}{l}322 \\
323 \\
324 \\
326 \\
327\end{array}$ & $\begin{array}{l}- \\
- \\
-\end{array}$ & $\begin{array}{l}- \\
- \\
- \\
-\end{array}$ & $\begin{array}{l}100.0 \\
150.0 \\
180.0 \\
175.0 \\
175.0\end{array}$ & $\begin{array}{l}- \\
- \\
- \\
-\end{array}$ & $\begin{array}{l}- \\
- \\
- \\
-\end{array}$ & $\begin{array}{l}32.0 \\
42.0 \\
43.0 \\
41.0 \\
41.0\end{array}$ & $\begin{array}{l}- \\
- \\
- \\
-\end{array}$ & $\begin{array}{l}1 \\
1 \\
1 \\
1 \\
1\end{array}$ & $\begin{array}{l}1 \\
1 \\
1 \\
1 \\
1\end{array}$ & $\begin{array}{l}3 \\
3 \\
1 \\
1 \\
1\end{array}$ & $\begin{array}{l}5 \\
1 \\
7 \\
7 \\
7\end{array}$ \\
\hline $\begin{array}{l}\text { BK } \\
B K \\
B K \\
B K \\
B K\end{array}$ & $\begin{array}{l}328 \\
329 \\
330 \\
331 \\
332\end{array}$ & $\begin{array}{l}- \\
- \\
-\end{array}$ & $\begin{array}{l}- \\
- \\
-\end{array}$ & $\begin{array}{l}175.0 \\
150.0 \\
115.0 \\
115.0 \\
110.0\end{array}$ & $\begin{array}{l}- \\
- \\
- \\
-\end{array}$ & $\begin{array}{l}- \\
- \\
- \\
-\end{array}$ & $\begin{array}{r}41.0 \\
32.0 \\
24.0 \\
24.0 \\
2.0\end{array}$ & $\begin{array}{l}- \\
- \\
-\end{array}$ & $\begin{array}{l}1 \\
1 \\
1 \\
1 \\
1\end{array}$ & $\begin{array}{l}1 \\
1 \\
1 \\
1 \\
1\end{array}$ & $\begin{array}{l}1 \\
4 \\
4 \\
4 \\
1\end{array}$ & $\begin{array}{l}7 \\
7 \\
7 \\
7 \\
6\end{array}$ \\
\hline $\begin{array}{l}B K \\
B K \\
B K \\
B K \\
B K\end{array}$ & $\begin{array}{l}333 \\
334 \\
335 \\
336 \\
337\end{array}$ & $\begin{array}{l}- \\
- \\
- \\
-\end{array}$ & $\begin{array}{l}- \\
- \\
- \\
-\end{array}$ & $\begin{array}{r}180.0 \\
90.0 \\
110.0 \\
60.0 \\
65.0\end{array}$ & $\begin{array}{l}- \\
- \\
- \\
-\end{array}$ & $\begin{array}{l}- \\
- \\
- \\
-\end{array}$ & $\begin{array}{r}25.0 \\
3.0 \\
22.0 \\
42.0 \\
42.0\end{array}$ & $\begin{array}{l}- \\
- \\
- \\
-\end{array}$ & $\begin{array}{l}1 \\
1 \\
1 \\
1 \\
1\end{array}$ & $\begin{array}{l}1 \\
1 \\
1 \\
1 \\
1\end{array}$ & $\begin{array}{l}4 \\
4 \\
4 \\
1 \\
1\end{array}$ & $\begin{array}{l}5 \\
6 \\
3 \\
3 \\
3\end{array}$ \\
\hline $\begin{array}{l}B K \\
B K \\
B K \\
B K \\
B K\end{array}$ & $\begin{array}{l}338 \\
339 \\
34 \emptyset \\
341 \\
342\end{array}$ & $\begin{array}{l}- \\
- \\
- \\
-\end{array}$ & $\begin{array}{l}- \\
- \\
- \\
-\end{array}$ & $\begin{array}{r}65 . \varnothing \\
65.0 \\
7 \varnothing . \varnothing \\
45 . \varnothing \\
140.0\end{array}$ & $\begin{array}{l}- \\
- \\
- \\
-\end{array}$ & $\begin{array}{l}- \\
- \\
- \\
-\end{array}$ & $\begin{array}{l}44.0 \\
44.0 \\
44.0 \\
44.0 \\
18.0\end{array}$ & $\begin{array}{l}- \\
- \\
- \\
-\end{array}$ & $\begin{array}{l}1 \\
1 \\
1 \\
1 \\
1\end{array}$ & $\begin{array}{l}1 \\
1 \\
1 \\
1 \\
1\end{array}$ & $\begin{array}{l}1 \\
1 \\
1 \\
1 \\
4\end{array}$ & $\begin{array}{l}3 \\
1 \\
1 \\
1 \\
5\end{array}$ \\
\hline $\begin{array}{l}\text { BK } \\
B K \\
B K \\
B K \\
B K\end{array}$ & $\begin{array}{l}343 \\
344 \\
345 \\
346 \\
347\end{array}$ & $\begin{array}{l}- \\
- \\
- \\
-\end{array}$ & $\begin{array}{l}- \\
- \\
-\end{array}$ & $\begin{array}{r}160.0 \\
100.0 \\
100.0 \\
95.0 \\
100.0\end{array}$ & $\begin{array}{l}- \\
- \\
- \\
-\end{array}$ & $\begin{array}{l}- \\
- \\
- \\
-\end{array}$ & $\begin{array}{l}15.0 \\
14.0 \\
54.0 \\
54.0 \\
54.0\end{array}$ & $\begin{array}{l}- \\
- \\
- \\
-\end{array}$ & $\begin{array}{l}1 \\
1 \\
1 \\
1 \\
1\end{array}$ & $\begin{array}{l}1 \\
1 \\
1 \\
1 \\
1\end{array}$ & $\begin{array}{l}4 \\
4 \\
1 \\
1 \\
1\end{array}$ & $\begin{array}{l}5 \\
6 \\
5 \\
8 \\
8\end{array}$ \\
\hline $\begin{array}{l}\text { BK } \\
\text { BK } \\
\text { BK } \\
\text { BK } \\
\text { BK }\end{array}$ & $\begin{array}{l}348 \\
349 \\
350 \\
351 \\
352\end{array}$ & $\begin{array}{l}- \\
- \\
- \\
-\end{array}$ & $\begin{array}{l}- \\
- \\
-\end{array}$ & $\begin{array}{r}105.0 \\
110.0 \\
100.0 \\
100.0 \\
95.0\end{array}$ & $\begin{array}{l}- \\
- \\
- \\
-\end{array}$ & $\begin{array}{l}- \\
- \\
- \\
-\end{array}$ & $\begin{array}{l}54.0 \\
55.0 \\
55 . \varnothing \\
54 . \varnothing \\
54 . \varnothing\end{array}$ & $\begin{array}{l}- \\
- \\
- \\
-\end{array}$ & $\begin{array}{l}1 \\
1 \\
1 \\
1 \\
1\end{array}$ & $\begin{array}{l}1 \\
1 \\
1 \\
1 \\
1\end{array}$ & $\begin{array}{l}1 \\
1 \\
1 \\
1 \\
1\end{array}$ & $\begin{array}{l}5 \\
5 \\
5 \\
5 \\
6\end{array}$ \\
\hline $\begin{array}{l}B K \\
B K \\
B K \\
B K \\
B K\end{array}$ & $\begin{array}{l}353 \\
354 \\
355 \\
356 \\
357\end{array}$ & $\begin{array}{l}- \\
- \\
- \\
-\end{array}$ & $\begin{array}{l}- \\
\overline{-} \\
\overline{-}\end{array}$ & $\begin{array}{r}95 . \varnothing \\
135.0 \\
1 \varnothing \varnothing . \varnothing \\
10 \varnothing . \varnothing \\
140 . \varnothing\end{array}$ & $\begin{array}{l}- \\
- \\
- \\
-\end{array}$ & $\begin{array}{l}- \\
- \\
- \\
-\end{array}$ & $\begin{array}{l}56.0 \\
53.0 \\
57.0 \\
53.0 \\
56.0\end{array}$ & $\begin{array}{l}- \\
- \\
- \\
-\end{array}$ & $\begin{array}{l}1 \\
1 \\
1 \\
1 \\
1\end{array}$ & $\begin{array}{l}1 \\
1 \\
1 \\
1 \\
1\end{array}$ & $\begin{array}{l}1 \\
1 \\
1 \\
1 \\
1\end{array}$ & $\begin{array}{l}7 \\
5 \\
5 \\
3 \\
1\end{array}$ \\
\hline $\begin{array}{l}B K \\
B K \\
B K \\
B K \\
B K\end{array}$ & $\begin{array}{l}358 \\
359 \\
36 \varnothing \\
361 \\
362\end{array}$ & $\begin{array}{l}- \\
- \\
- \\
-\end{array}$ & $\begin{array}{l}- \\
- \\
- \\
-\end{array}$ & $\begin{array}{l}140.0 \\
155 . \varnothing \\
165.0 \\
2 \varnothing 0 . \varnothing \\
170.0\end{array}$ & $\begin{array}{l}- \\
- \\
- \\
-\end{array}$ & $\begin{array}{l}- \\
- \\
- \\
-\end{array}$ & $\begin{array}{l}56.0 \\
46.0 \\
46.0 \\
46.0 \\
45.0\end{array}$ & $\begin{array}{l}- \\
- \\
- \\
-\end{array}$ & $\begin{array}{l}1 \\
1 \\
1 \\
1 \\
1\end{array}$ & $\begin{array}{l}1 \\
1 \\
1 \\
1 \\
1\end{array}$ & $\begin{array}{l}1 \\
1 \\
1 \\
1 \\
4\end{array}$ & $\begin{array}{l}1 \\
7 \\
3 \\
6 \\
3\end{array}$ \\
\hline $\begin{array}{l}B K \\
B K \\
B K \\
B K \\
B K\end{array}$ & $\begin{array}{l}363 \\
364 \\
365 \\
366 \\
367\end{array}$ & $\begin{array}{l}- \\
- \\
- \\
-\end{array}$ & $\begin{array}{l}- \\
- \\
-\end{array}$ & $\begin{array}{r}130.0 \\
175.0 \\
110.0 \\
110 . \varnothing \\
95 . \varnothing\end{array}$ & $\begin{array}{l}- \\
- \\
-\end{array}$ & $\begin{array}{l}- \\
- \\
- \\
-\end{array}$ & $\begin{array}{l}32.0 \\
37.0 \\
54.0 \\
54.0 \\
54.0\end{array}$ & $\begin{array}{l}- \\
- \\
-\end{array}$ & $\begin{array}{l}1 \\
1 \\
1 \\
1 \\
1\end{array}$ & $\begin{array}{l}1 \\
1 \\
1 \\
1 \\
1\end{array}$ & $\begin{array}{l}4 \\
1 \\
1 \\
1 \\
1\end{array}$ & $\begin{array}{l}7 \\
7 \\
5 \\
5 \\
5\end{array}$ \\
\hline $\begin{array}{l}B K \\
B K \\
B K \\
B K \\
B K\end{array}$ & $\begin{array}{l}368 \\
369 \\
37 \emptyset \\
371 \\
372\end{array}$ & $\begin{array}{l}- \\
- \\
- \\
-\end{array}$ & $\begin{array}{l}- \\
- \\
-\end{array}$ & $\begin{array}{l}110.0 \\
110.0 \\
100.0 \\
100.0 \\
100.0\end{array}$ & $\begin{array}{l}- \\
- \\
-\end{array}$ & $\begin{array}{l}- \\
- \\
- \\
-\end{array}$ & $\begin{array}{l}57.0 \\
57.0 \\
54.0 \\
55.0 \\
55.0\end{array}$ & $\begin{array}{l}- \\
- \\
- \\
-\end{array}$ & $\begin{array}{l}1 \\
1 \\
0 \\
1 \\
1\end{array}$ & $\begin{array}{l}1 \\
1 \\
1 \\
1 \\
1\end{array}$ & $\begin{array}{l}1 \\
1 \\
1 \\
1 \\
1\end{array}$ & $\begin{array}{l}7 \\
7 \\
5 \\
8 \\
8\end{array}$ \\
\hline $\begin{array}{l}\text { BK } \\
\text { BK } \\
\text { BK } \\
\text { BK } \\
\text { BK }\end{array}$ & $\begin{array}{l}373 \\
374 \\
375 \\
376 \\
377\end{array}$ & $\begin{array}{l}- \\
- \\
-\end{array}$ & $\begin{array}{l}- \\
- \\
- \\
-\end{array}$ & $\begin{array}{r}100.0 \\
95.0 \\
100.0 \\
105.0 \\
95.0\end{array}$ & $\begin{array}{l}- \\
- \\
- \\
-\end{array}$ & $\begin{array}{l}- \\
- \\
- \\
-\end{array}$ & $\begin{array}{l}55.0 \\
54.0 \\
55.0 \\
56.0 \\
53.0\end{array}$ & $\begin{array}{l}- \\
- \\
-\end{array}$ & $\begin{array}{l}1 \\
1 \\
1 \\
1 \\
1\end{array}$ & $\begin{array}{l}1 \\
1 \\
1 \\
1 \\
1\end{array}$ & $\begin{array}{l}1 \\
1 \\
1 \\
1 \\
1\end{array}$ & $\begin{array}{l}8 \\
8 \\
8 \\
8 \\
5\end{array}$ \\
\hline
\end{tabular}


Appendix.--Location and basic physical features of recharge basins on Long Island,

\begin{tabular}{|c|c|c|c|c|c|c|c|c|}
\hline \multicolumn{2}{|c|}{$\begin{array}{l}\text { BASIN } \\
\text { NUMBER }\end{array}$} & \multicolumn{2}{|c|}{ NEAREST INTERSECTION } & $\begin{array}{l}\text { LATITUDE } \\
\text { LONGITUDE } \\
0_{0}^{\prime}, n\end{array}$ & $\begin{array}{l}\text { DATE } \\
\text { BUILT } \\
\text { YrMOD }\end{array}$ & COMMUNITY & $\begin{array}{l}\text { DESIGN } \\
\text { CAPACITY } \\
\text { (cubic }\end{array}$ & $\begin{array}{l}\text { ACTUAL } \\
\text { CAPACITY } \\
\text { feet) }\end{array}$ \\
\hline $\begin{array}{l}B K \\
B K \\
B K \\
B K \\
B K\end{array}$ & $\begin{array}{l}378 \\
379 \\
380 \\
381 \\
382\end{array}$ & $\begin{array}{l}\text { ARBOR LANE } \\
\text { STIRRUP LANE } \\
\text { HAWKINS ROAD } \\
\text { MARK TREE ROAD } \\
\text { PUBLIC ROAD }\end{array}$ & $\begin{array}{l}\text { WOODS LANE } \\
\text { SADDLE LANE } \\
\text { 29TH STREET } \\
\text { 23RD. STREET } \\
\text { ROSLYN AVENUE }\end{array}$ & $\begin{array}{l}4 \varnothing 52440730580 \\
4 \varnothing 5247073 \varnothing 5 \varnothing 4 \\
4 \varnothing 5251073 \varnothing 53 \varnothing \\
4 \varnothing 53430730549 \\
4 \varnothing 5149073 \varnothing 138\end{array}$ & $\begin{array}{l}- \\
- \\
- \\
-\end{array}$ & $\begin{array}{l}216 \\
216 \\
- \\
- \\
-\end{array}$ & $\begin{array}{l}- \\
- \\
-\end{array}$ & $\begin{array}{l}- \\
- \\
-\end{array}$ \\
\hline $\begin{array}{l}\text { BK } \\
\text { BK } \\
\text { BK } \\
\text { BK } \\
\text { BK }\end{array}$ & $\begin{array}{l}383 \\
384 \\
385 \\
386 \\
387\end{array}$ & $\begin{array}{l}\text { CEDARHURST AVE. } \\
\text { HIGHVIEW DRIVE } \\
\text { PAULA BOULEVARD } \\
\text { W.OF CAMP DRIVE } \\
\text { PAULA DRIVE }\end{array}$ & $\begin{array}{l}\text { EVERGREEN } \\
\text { WYANET STREET } \\
\text { JOY ROAD } \\
\text { MIDDLE COUNTRY R }\end{array}$ & $\begin{array}{l}4051440730236 \\
4 \varnothing 51250730309 \\
4 \varnothing 5119073 \varnothing 324 \\
4 \varnothing 5137073 \varnothing 332 \\
4051370730336\end{array}$ & $\begin{array}{l}- \\
- \\
- \\
-\end{array}$ & $\begin{array}{c}- \\
\overline{253} \\
\overline{253}\end{array}$ & $\begin{array}{l}- \\
- \\
-\end{array}$ & $\begin{array}{l}- \\
- \\
- \\
-\end{array}$ \\
\hline $\begin{array}{l}B K \\
B K \\
B K \\
B K \\
B K\end{array}$ & $\begin{array}{l}388 \\
389 \\
398 \\
391 \\
392\end{array}$ & $\begin{array}{l}\text { ROSEMARY LANE } \\
\text { ROSEMARY LANE } \\
\text { W. OF JOAN AVE. } \\
\text { NW OF FRAN LANE } \\
\text { DURST DRIVE }\end{array}$ & $\begin{array}{l}\text { POWERS AVENUE } \\
\text { POWERS AVENUE } \\
\text { NEWTON AVENUE }\end{array}$ & $\begin{array}{l}4051350730348 \\
4051320730348 \\
4051206730344 \\
4051110730342 \\
4051510730331\end{array}$ & $\begin{array}{l}- \\
- \\
- \\
-\end{array}$ & $\begin{array}{c}253 \\
253 \\
- \\
- \\
244\end{array}$ & $\begin{array}{l}- \\
- \\
-\end{array}$ & $\begin{array}{l}- \\
- \\
-\end{array}$ \\
\hline $\begin{array}{l}\text { BK } \\
\text { BK } \\
\text { BK } \\
\text { BK } \\
\text { BK }\end{array}$ & $\begin{array}{l}393 \\
394 \\
395 \\
396 \\
397\end{array}$ & $\begin{array}{l}\text { LARRY ROAD } \\
\text { ALMA AVENUE } \\
\text { NE OF MARCH CT. } \\
\text { JACKSON AVENUE } \\
\text { HAWKINS ROAD }\end{array}$ & $\begin{array}{l}\text { HENRY AVENUE } \\
\text { MAGNOLIA DRIVE } \\
\text { TAYLOR AVENUE } \\
\text { SMITHTOWN BLVD. }\end{array}$ & $\begin{array}{l}4 \varnothing 52010738334 \\
4 \varnothing 52210730242 \\
4 \varnothing 52200738221 \\
4052390730350 \\
4 \varnothing 52470730359\end{array}$ & $\begin{array}{l}- \\
- \\
-\end{array}$ & $\begin{array}{c}244 \\
244 \\
- \\
244 \\
-\end{array}$ & $\begin{array}{l}- \\
\overline{-} \\
\overline{-}\end{array}$ & $\begin{array}{l}- \\
- \\
-\end{array}$ \\
\hline $\begin{array}{l}\text { BK } \\
B K \\
B K \\
B K \\
B K\end{array}$ & $\begin{array}{l}398 \\
399 \\
400 \\
401 \\
402\end{array}$ & $\begin{array}{l}\text { E. OF ARROWHEAD } \\
\text { NOSTRAND AVENUE } \\
\text { JAYNE BOULEVARD } \\
\text { OLD TOWN ROAD } \\
\text { CAMPSITE LANE }\end{array}$ & $\begin{array}{l}\text { TAYLOR STREET } \\
\text { LESTER STREET } \\
\text { GREENHAVEN DRIVE } \\
\text { ARROWHEAD LANE }\end{array}$ & $\begin{array}{l}4054100730417 \\
4053400730334 \\
4054470730255 \\
4054190730329 \\
4054410730422\end{array}$ & $\begin{array}{l}- \\
- \\
- \\
-\end{array}$ & $\begin{array}{l}- \\
- \\
-\end{array}$ & $\begin{array}{l}- \\
- \\
-\end{array}$ & $\begin{array}{l}- \\
- \\
-\end{array}$ \\
\hline $\begin{array}{l}\text { BK } \\
\text { BK } \\
\text { BK } \\
\text { BK } \\
\text { BK }\end{array}$ & $\begin{array}{l}403 \\
404 \\
405 \\
406 \\
407\end{array}$ & $\begin{array}{l}\text { E. OF NOREN LANE } \\
\text { E. OF CEDAR BRAN } \\
\text { POINSETTA AVENUE } \\
\text { WHISKEY ROAD } \\
\text { S. OF MONTAUK DR }\end{array}$ & $\begin{array}{l}\text { PINECONE STREET } \\
\text { MILLER PLACE }\end{array}$ & $\begin{array}{l}4051560725744 \\
4053330725811 \\
405324 \varnothing 725816 \\
4 \varnothing 54 \varnothing 8 \emptyset 72572 \varnothing \\
4054360725044\end{array}$ & $\begin{array}{l}- \\
- \\
- \\
-\end{array}$ & $\begin{array}{c}- \\
\overline{218} \\
218 \\
-\end{array}$ & $\begin{array}{l}- \\
- \\
- \\
-\end{array}$ & $\begin{array}{l}- \\
- \\
- \\
-\end{array}$ \\
\hline $\begin{array}{l}\text { BK } \\
\text { BK } \\
\text { BK } \\
\text { BK } \\
\text { BK }\end{array}$ & $\begin{array}{l}408 \\
409 \\
410 \\
411 \\
412\end{array}$ & $\begin{array}{l}\text { TARKILL TRAIL } \\
\text { WAUWPEX TRAIL } \\
\text { WAUWPEX TRAIL } \\
\text { SUNRISE TRAIL } \\
\text { SETAUKET TRAIL }\end{array}$ & $\begin{array}{l}\text { TARKILL } \\
\text { TARKILL TRAIL } \\
\text { TARKILL TRAIL } \\
\text { ROCKAWAY } \\
\text { ROCKAWAY }\end{array}$ & $\begin{array}{l}4054520725040 \\
4054540725048 \\
4054520725051 \\
4 \varnothing 55020725049 \\
4055020725049\end{array}$ & $\begin{array}{l}- \\
\overline{-} \\
\overline{-}\end{array}$ & $\begin{array}{l}- \\
- \\
- \\
-\end{array}$ & $\begin{array}{l}- \\
- \\
-\end{array}$ & $\begin{array}{l}- \\
\overline{-} \\
\overline{-}\end{array}$ \\
\hline $\begin{array}{l}\text { BK } \\
\text { BK } \\
\text { BK } \\
\text { BK } \\
\text { BK }\end{array}$ & $\begin{array}{l}413 \\
414 \\
415 \\
416 \\
417\end{array}$ & $\begin{array}{l}\text { ROCKAWAY } \\
\text { ROCKAWAY } \\
\text { FOREST TRAIL } \\
\text { WYANDANCH } \\
\text { UNION AVENUE }\end{array}$ & $\begin{array}{l}\text { SUNRISE TRAIL } \\
\text { WAMPUM TRAIL } \\
\text { MATINECUCK } \\
\text { SUNRISE TRAIL } \\
\text { KNICKERBOCKER }\end{array}$ & $\begin{array}{l}4055110725046 \\
4655110725056 \\
4655060725108 \\
4 \varnothing 55000725042 \\
4 \varnothing 48360730524\end{array}$ & $\begin{array}{l}- \\
- \\
- \\
-\end{array}$ & $\begin{array}{l}- \\
- \\
- \\
-\end{array}$ & $\begin{array}{l}- \\
- \\
- \\
-\end{array}$ & $\begin{array}{l}- \\
- \\
- \\
-\end{array}$ \\
\hline $\begin{array}{l}\text { BK } \\
\text { BK } \\
\text { BK } \\
\text { BK } \\
\text { BK }\end{array}$ & $\begin{array}{l}418 \\
419 \\
420 \\
421 \\
422\end{array}$ & $\begin{array}{l}\text { UNION AVENUE } \\
\text { W. OF DUANE DR. } \\
\text { SW OF BARBARA RD } \\
\text { BERGEN STREET } \\
\text { RONKONKOMA AVE. }\end{array}$ & $\begin{array}{l}\text { CARROL AVENUE } \\
\text { LIBERTY STREET } \\
\text { HILLCREST STREET }\end{array}$ & $\begin{array}{l}4048350730611 \\
4048580730612 \\
4049020730613 \\
4048350730634 \\
4049450730642\end{array}$ & $\begin{array}{l}- \\
- \\
-\end{array}$ & $\begin{array}{c}252 \\
- \\
252 \\
252\end{array}$ & $\begin{array}{l}- \\
\overline{-} \\
\overline{-}\end{array}$ & $\begin{array}{l}- \\
- \\
- \\
-\end{array}$ \\
\hline $\begin{array}{l}\text { BK } \\
\text { BK } \\
\text { BK } \\
\text { BK } \\
\text { BK }\end{array}$ & $\begin{array}{l}423 \\
424 \\
425 \\
426 \\
427\end{array}$ & $\begin{array}{l}\text { RONKONKOMA AVE. } \\
\text { ACKERLY ROAD } \\
\text { SW OF HOLLO DR } \\
\text { SE MAPLECREST DR } \\
\text { PORTION ROAD }\end{array}$ & $\begin{array}{l}\text { HILLCREST STREET } \\
\text { HANS BOULEVARD } \\
\text { MAPLECREST DR. S }\end{array}$ & 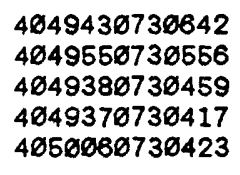 & $\begin{array}{l}- \\
- \\
-\end{array}$ & $\begin{array}{l}252 \\
229 \\
229 \\
- \\
-\end{array}$ & $\begin{array}{l}- \\
- \\
- \\
-\end{array}$ & $\begin{array}{l}- \\
- \\
- \\
-\end{array}$ \\
\hline $\begin{array}{l}\text { BK } \\
\text { BK } \\
\text { BK } \\
\text { BK } \\
\text { BK }\end{array}$ & $\begin{array}{l}428 \\
429 \\
430 \\
431 \\
432\end{array}$ & $\begin{array}{l}\text { N. OF WARNER LA. } \\
\text { N. OF WARNER LA. } \\
\text { COOKE AVENUE } \\
\text { RIDGEDALE AVENUE } \\
\text { OHIO AVENUE }\end{array}$ & $\begin{array}{l}\text { WENDY DRIVE } \\
\text { ABNER DRIVE } \\
\text { PECONIC AVENUE }\end{array}$ & 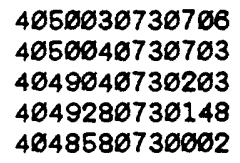 & $\begin{array}{l}- \\
- \\
-\end{array}$ & $\begin{array}{l}253 \\
253 \\
226 \\
226 \\
232\end{array}$ & $\begin{array}{l}- \\
- \\
-\end{array}$ & $\begin{array}{l}- \\
- \\
- \\
-\end{array}$ \\
\hline
\end{tabular}


N.Y. [Dash indicates no data. Altitudes are in feet above sea level.] (cont.)

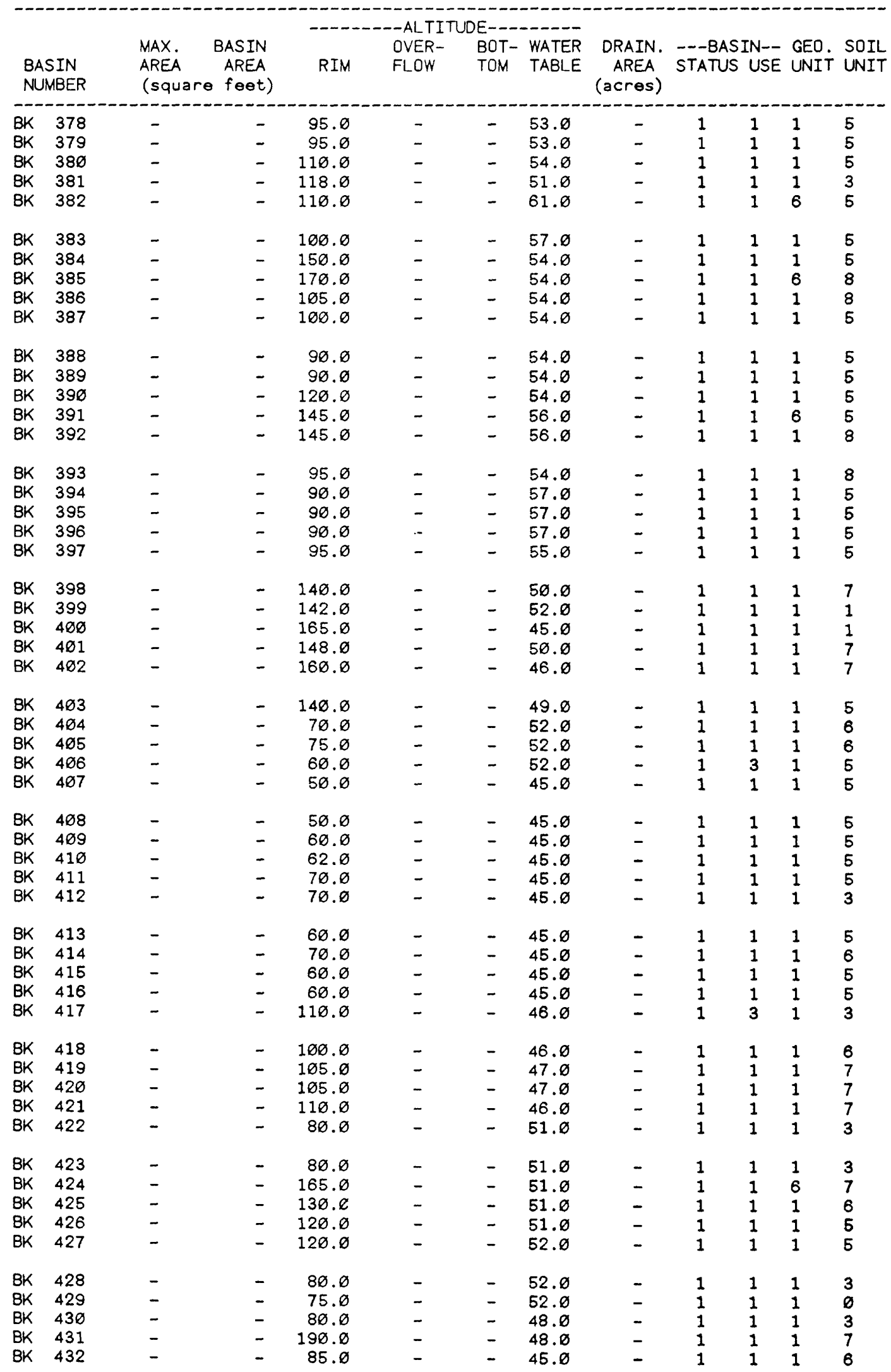


Appondix.--Location and basic physical foatures of rochargo basins on Long Island,

\begin{tabular}{|c|c|c|c|c|c|c|c|c|}
\hline \multicolumn{2}{|c|}{$\begin{array}{l}\text { BASIN } \\
\text { NUMBER }\end{array}$} & \multicolumn{2}{|c|}{ NEAREST INTERSECTION } & $\begin{array}{l}\text { LATITUDE } \\
\text { LONGITUDE, } \\
\circ, " \circ\end{array}$ & $\begin{array}{l}\text { DATE } \\
\text { BUILT } \\
\text { YrMOD }\end{array}$ & COMMNITY & \multicolumn{2}{|c|}{$\begin{array}{l}\text { DESIGN ACTUAL } \\
\text { CAPACITY CAPACITY } \\
\text { (cubic feet) }\end{array}$} \\
\hline $\begin{array}{l}\text { BK } \\
\text { BK } \\
\text { BK } \\
\text { BK } \\
\text { BK }\end{array}$ & $\begin{array}{l}433 \\
434 \\
435 \\
436 \\
437\end{array}$ & $\begin{array}{l}\text { PHILLIPS DRIVE } \\
\text { DEVON AVENUE } \\
\text { POINT ROAD } \\
\text { JOSEPH COURT } \\
\text { TUCK LANE }\end{array}$ & $\begin{array}{l}\text { RHODE ISLAND AVE } \\
\text { RACE AVENUE } \\
\text { CEDAR OAKS AVE } \\
\text { NICOLLS ROAD } \\
\text { LEEDS BLVD }\end{array}$ & 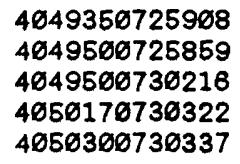 & $\begin{array}{l}- \\
- \\
-\end{array}$ & $\begin{array}{c}232 \\
232 \\
-\overline{24} \\
-\end{array}$ & $\begin{array}{l}- \\
- \\
- \\
-\end{array}$ & $\begin{array}{l}- \\
- \\
- \\
-\end{array}$ \\
\hline $\begin{array}{l}\text { BK } \\
\text { BK } \\
\text { BK } \\
\text { BK } \\
\text { BK }\end{array}$ & $\begin{array}{l}438 \\
439 \\
440 \\
441 \\
442\end{array}$ & $\begin{array}{l}\text { TUCK LANE } \\
\text { LIEBER BOULEVARD } \\
\text { FOXBORA AVENUE } \\
\text { KANE AVENUE } \\
\text { RAIMOND STREET }\end{array}$ & $\begin{array}{l}\text { MORRIS AVENUE } \\
\text { MORRIS AVENUE } \\
\text { RACE AVENUE } \\
\text { SCHOOL STREET }\end{array}$ & 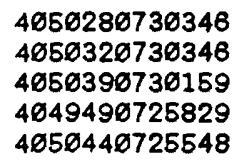 & $\begin{array}{l}- \\
- \\
-\end{array}$ & $\begin{array}{l}- \\
- \\
224 \\
232 \\
281\end{array}$ & $\begin{array}{l}- \\
- \\
-\end{array}$ & $\begin{array}{l}- \\
- \\
-\end{array}$ \\
\hline $\begin{array}{l}\text { BK } \\
B K \\
B K \\
B K \\
B K\end{array}$ & $\begin{array}{l}443 \\
444 \\
445 \\
446 \\
447\end{array}$ & $\begin{array}{l}\text { FRANK AVENUE } \\
\text { DONALD AVENUE } \\
\text { CHARLES STREET } \\
\text { CHARLES STREET } \\
\text { ROBERTA LANE }\end{array}$ & $\begin{array}{l}\text { RAIMOND STREET } \\
\text { SHANNON BLVD. } \\
\text { VALERIE COURT } \\
\text { VALERIE COURT } \\
\text { TILNEY AVENUE }\end{array}$ & 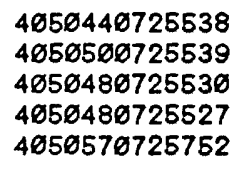 & $\begin{array}{l}\overline{-} \\
\overline{-}\end{array}$ & $\begin{array}{l}261 \\
281 \\
281 \\
281 \\
-\end{array}$ & $\begin{array}{l}- \\
- \\
- \\
-\end{array}$ & $\begin{array}{l}- \\
- \\
-\end{array}$ \\
\hline $\begin{array}{l}\text { BK } \\
B K \\
B K \\
B K \\
B K\end{array}$ & $\begin{array}{l}448 \\
449 \\
450 \\
451 \\
452\end{array}$ & $\begin{array}{l}\text { CANDIDO AVENUE } \\
\text { FLOWER HILL DR. } \\
\text { BURNEY BOULEVARD } \\
\text { BLUE POINT AVE. } \\
\text { SWAN LAKE DRIVE }\end{array}$ & $\begin{array}{l}\text { MONTAUK HIGHWAY } \\
\text { STARLIGHT DRIVE } \\
\text { CLAFLIN STREET } \\
\text { ROGERS STREET } \\
\text { PHYLLIS DRIVE }\end{array}$ & 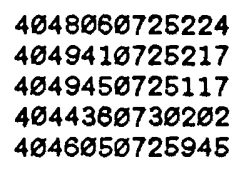 & $\begin{array}{l}- \\
- \\
-\end{array}$ & $\begin{array}{c}- \\
- \\
214 \\
221\end{array}$ & $\begin{array}{l}- \\
- \\
-\end{array}$ & $\begin{array}{l}- \\
- \\
-\end{array}$ \\
\hline $\begin{array}{l}\mathrm{BK} \\
\mathrm{BK} \\
\mathrm{BK} \\
\mathrm{BK} \\
\mathrm{BK}\end{array}$ & $\begin{array}{l}453 \\
454 \\
455 \\
456 \\
457\end{array}$ & $\begin{array}{l}\text { SWAN LAKE DRIVE } \\
\text { SWAN LAKE DRIVE } \\
\text { CLARK STREET } \\
\text { FRANKLIN STREET } \\
\text { HERBERT CIRCLE }\end{array}$ & $\begin{array}{l}\text { CELIA STREET } \\
\text { WASHINGTON PLACE } \\
\text { ANNANIAS AVENUE } \\
\text { DAVIDSON AVENUE }\end{array}$ & $\begin{array}{l}4046280725941 \\
4046350725941 \\
4046570730010 \\
4046410730019 \\
4046590730046\end{array}$ & $\begin{array}{l}- \\
- \\
-\end{array}$ & $\begin{array}{c}221 \\
221 \\
221 \\
- \\
221\end{array}$ & $\begin{array}{l}- \\
- \\
- \\
-\end{array}$ & $\begin{array}{l}- \\
\overline{-} \\
-\end{array}$ \\
\hline $\begin{array}{l}\text { BK } \\
\text { BK } \\
\text { BK } \\
\text { BK } \\
\text { BK }\end{array}$ & $\begin{array}{l}458 \\
459 \\
46 \varnothing \\
461 \\
462\end{array}$ & $\begin{array}{l}\text { S. OF HARRIS ST. } \\
\text { EVERETT STREET } \\
\text { SNABER ROAD } \\
\text { BARTON AVENUE } \\
\text { HEAD OF THE NECK }\end{array}$ & $\begin{array}{l}\text { CLINTON AVENUE } \\
\text { MAPLE AVENUE } \\
\text { MT. VERNON AVE. } \\
\text { MUNSEL ROAD }\end{array}$ & 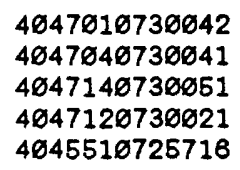 & $\begin{array}{l}- \\
- \\
-\end{array}$ & $\begin{array}{l}- \\
221 \\
- \\
221 \\
213\end{array}$ & $\begin{array}{l}- \\
- \\
-\end{array}$ & $\begin{array}{l}- \\
- \\
-\end{array}$ \\
\hline $\begin{array}{l}\mathrm{BK} \\
\mathrm{BK} \\
\mathrm{BK} \\
\mathrm{BK} \\
\mathrm{BK}\end{array}$ & $\begin{array}{l}463 \\
464 \\
465 \\
466 \\
468\end{array}$ & $\begin{array}{l}\text { E. MASEM SQUARE } \\
\text { LENOX AVENUE } \\
\text { SCHELPLER AVENUE } \\
\text { ASSOCIATION RD. } \\
\text { POST AVENUE }\end{array}$ & $\begin{array}{l}\text { S. MASEM SQUARE } \\
\text { ATLANTIC AVENUE } \\
\text { PATCHOGUE AVENUE } \\
\text { POST AVENUE } \\
\text { PATCHOGUE AVENUE }\end{array}$ & $\begin{array}{l}4 \emptyset 4535 \varnothing 725728 \\
4 \varnothing 4627 \emptyset 725734 \\
4 \varnothing 4633 \emptyset 725727 \\
4 \varnothing 4618 \varnothing 72564 \varnothing \\
4 \varnothing 4638 \emptyset 725642\end{array}$ & $\begin{array}{l}- \\
- \\
-\end{array}$ & $\begin{array}{l}213 \\
236 \\
213 \\
236 \\
236\end{array}$ & $\begin{array}{l}- \\
- \\
-\end{array}$ & $\begin{array}{l}- \\
- \\
-\end{array}$ \\
\hline $\begin{array}{l}\text { BK } \\
B K \\
B K \\
B K \\
B K\end{array}$ & $\begin{array}{l}469 \\
470 \\
471 \\
472 \\
473\end{array}$ & $\begin{array}{l}\text { MACDONALD AVE. } \\
\text { MACDONALD AVE. } \\
\text { FLOYD ROAD } \\
\text { RANCH DRIVE } \\
\text { PROPOSE ROAD }\end{array}$ & $\begin{array}{l}\text { BROOKHAVEN AVE. } \\
\text { BROOKHAVEN AVE. } \\
\text { PRESFORD DRIVE } \\
\text { PROPOSE ROAD } \\
\text { PROBST DRIVE }\end{array}$ & $\begin{array}{l}4046420725715 \\
4046400725715 \\
4 \varnothing 46410725120 \\
4 \varnothing 4646 \varnothing 725221 \\
4046520725229\end{array}$ & $\begin{array}{l}- \\
- \\
-\end{array}$ & $\begin{array}{l}236 \\
236 \\
230 \\
255 \\
255\end{array}$ & $\begin{array}{l}- \\
- \\
-\end{array}$ & $\begin{array}{l}- \\
- \\
- \\
-\end{array}$ \\
\hline $\begin{array}{l}\text { BK } \\
\text { BK } \\
B K \\
B K \\
B K\end{array}$ & $\begin{array}{l}474 \\
475 \\
476 \\
477 \\
478\end{array}$ & $\begin{array}{l}\text { WESTWOOD DRIVE } \\
\text { WESTWOOD DRIVE } \\
\text { MALBA DRIVE } \\
\text { MALBA DRIVE } \\
\text { MALBA DRIVE }\end{array}$ & $\begin{array}{l}\text { PROPOSE ROAD } \\
\text { PROPOSE ROAD } \\
\text { PROPOSE ROAD } \\
\text { PROPOSE DRIVE } \\
\text { PROPOSE ROAD }\end{array}$ & $\begin{array}{l}4 \emptyset 46550725224 \\
4 \varnothing 4654 \varnothing 725228 \\
4 \varnothing 4657 \emptyset 725225 \\
4 \varnothing 4658 \emptyset 725228 \\
4 \varnothing 46590725226\end{array}$ & $\begin{array}{l}- \\
- \\
-\end{array}$ & $\begin{array}{l}255 \\
255 \\
255 \\
255 \\
255\end{array}$ & $\begin{array}{l}- \\
- \\
-\end{array}$ & $\begin{array}{l}- \\
- \\
- \\
-\end{array}$ \\
\hline $\begin{array}{l}\mathrm{BK} \\
\mathrm{BK} \\
\mathrm{BK} \\
\mathrm{BK} \\
\mathrm{BK}\end{array}$ & $\begin{array}{l}479 \\
480 \\
483 \\
484 \\
485\end{array}$ & $\begin{array}{l}\text { E. MORICHES\&MANOR } \\
\text { LANDING LANE } \\
\text { MORICH-RVERHD.RD } \\
\text { MORICH-RVERHD.RD } \\
\text { MORICH-RVERHD.RD }\end{array}$ & $\begin{array}{l}\text { COVELA LANE } \\
\text { TOWN LINE } \\
\text { TOPPINGS PATH } \\
\text { TOPPINGS PATH }\end{array}$ & 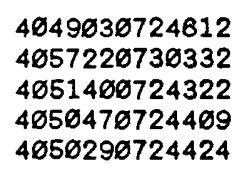 & $\begin{array}{l}- \\
- \\
- \\
-\end{array}$ & $\begin{array}{l}220 \\
- \\
219 \\
219\end{array}$ & $\begin{array}{l}- \\
\overline{-} \\
-\end{array}$ & $\begin{array}{l}- \\
- \\
- \\
-\end{array}$ \\
\hline $\begin{array}{l}\text { BK } \\
\text { BK } \\
\text { BK } \\
\text { BK } \\
\text { BK }\end{array}$ & $\begin{array}{l}486 \\
487 \\
488 \\
489 \\
490\end{array}$ & $\begin{array}{l}\text { LAUREL } \\
\text { HALLOCK ROAD } \\
\text { HALLOCK ROAD } \\
\text { MOUNT ROAD } \\
\text { NICHOLLS ROAD }\end{array}$ & $\begin{array}{l}\text { BIRCH DRIVE } \\
\text { MIDDLE COUNTRY } \\
\text { MIDDLE COUNTRY } \\
\text { N. COUNTRY RD. } \\
\text { SYCAMORE CIRCLE }\end{array}$ & 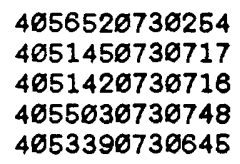 & $\begin{array}{l}- \\
- \\
-\end{array}$ & $\begin{array}{l}248 \\
227 \\
227 \\
28 \emptyset \\
259\end{array}$ & $\begin{array}{l}- \\
- \\
-\end{array}$ & $\begin{array}{l}- \\
- \\
-\end{array}$ \\
\hline
\end{tabular}


N.Y. [Dash indicates no data. Altitudes are in feet above sea level.] (cont.)

\begin{tabular}{|c|c|c|c|c|c|c|c|c|c|c|c|c|}
\hline & $\begin{array}{l}\text { SIN } \\
\text { MBER }\end{array}$ & $\begin{array}{l}\text { MAX. } \\
\text { AREA } \\
\text { (square }\end{array}$ & $\begin{array}{c}\text { BASIN } \\
\text { AREA } \\
\text { feet) }\end{array}$ & RIM & $\begin{array}{l}\text {-ALTIT } \\
\text { OVER- } \\
\text { FLOW }\end{array}$ & $\begin{array}{l}\text { OE---. } \\
\text { BOT- } \\
\text { TOM }\end{array}$ & $\begin{array}{l}\text { WATER } \\
\text { TABLE }\end{array}$ & $\begin{array}{c}\text { DRAIN. } \\
\text { AREA } \\
\text { (acres) }\end{array}$ & $\begin{array}{l}---B A S \\
\text { STATUS }\end{array}$ & $\begin{array}{l}\text { IN-- } \\
\text { USE }\end{array}$ & $\begin{array}{l}\text { GEO. } \\
\text { UNIT }\end{array}$ & $\begin{array}{l}\text { SOIL } \\
\text { UNIT }\end{array}$ \\
\hline $\begin{array}{l}\text { BK } \\
\text { BK } \\
\text { BK } \\
\text { BK } \\
\text { BK }\end{array}$ & $\begin{array}{l}433 \\
434 \\
435 \\
436 \\
437\end{array}$ & $\begin{array}{l}- \\
- \\
- \\
-\end{array}$ & $\begin{array}{l}- \\
\text { - } \\
-\end{array}$ & $\begin{array}{r}100.0 \\
90.0 \\
120.0 \\
160.0 \\
180.0\end{array}$ & $\begin{array}{l}- \\
- \\
- \\
-\end{array}$ & $\begin{array}{l}- \\
- \\
- \\
-\end{array}$ & $\begin{array}{l}42.0 \\
42.0 \\
48.0 \\
52.0 \\
52.0\end{array}$ & $\begin{array}{l}- \\
- \\
-\end{array}$ & $\begin{array}{l}1 \\
1 \\
1 \\
1 \\
1\end{array}$ & $\begin{array}{l}1 \\
1 \\
1 \\
1 \\
1\end{array}$ & $\begin{array}{l}1 \\
1 \\
1 \\
1 \\
6\end{array}$ & $\begin{array}{l}7 \\
7 \\
1 \\
5 \\
5\end{array}$ \\
\hline $\begin{array}{l}\text { BK } \\
\text { BK } \\
\text { BK } \\
\text { BK } \\
\text { BK }\end{array}$ & $\begin{array}{l}438 \\
439 \\
440 \\
441 \\
442\end{array}$ & $\begin{array}{l}- \\
- \\
- \\
-\end{array}$ & $\begin{array}{l}- \\
- \\
-\end{array}$ & $\begin{array}{r}160.0 \\
160.0 \\
290.0 \\
95.0 \\
70.0\end{array}$ & $\begin{array}{l}- \\
- \\
- \\
-\end{array}$ & $\begin{array}{l}- \\
- \\
-\end{array}$ & $\begin{array}{l}54.0 \\
54.0 \\
53.0 \\
42.0 \\
39.0\end{array}$ & $\begin{array}{l}- \\
- \\
-\end{array}$ & $\begin{array}{l}1 \\
1 \\
1 \\
1 \\
1\end{array}$ & $\begin{array}{l}1 \\
1 \\
1 \\
1 \\
1\end{array}$ & $\begin{array}{l}6 \\
6 \\
6 \\
1 \\
1\end{array}$ & $\begin{array}{l}5 \\
5 \\
5 \\
7 \\
3\end{array}$ \\
\hline $\begin{array}{l}\text { BK } \\
\text { BK } \\
\text { BK } \\
\text { BK } \\
\text { BK }\end{array}$ & $\begin{array}{l}443 \\
444 \\
445 \\
446 \\
447\end{array}$ & $\begin{array}{l}- \\
- \\
- \\
-\end{array}$ & $\begin{array}{l}- \\
- \\
- \\
-\end{array}$ & $\begin{array}{r}80.0 \\
105.0 \\
130.0 \\
130.0 \\
150.0\end{array}$ & $\begin{array}{l}- \\
- \\
-\end{array}$ & $\begin{array}{l}- \\
- \\
- \\
-\end{array}$ & $\begin{array}{l}39.0 \\
39.0 \\
39.0 \\
39.0 \\
45.0\end{array}$ & $\begin{array}{l}- \\
- \\
- \\
-\end{array}$ & $\begin{array}{l}1 \\
1 \\
1 \\
1 \\
1\end{array}$ & $\begin{array}{l}1 \\
1 \\
1 \\
1 \\
1\end{array}$ & $\begin{array}{l}1 \\
6 \\
6 \\
6 \\
1\end{array}$ & $\begin{array}{l}7 \\
7 \\
7 \\
7 \\
6\end{array}$ \\
\hline $\begin{array}{l}\text { BK } \\
\text { BK } \\
\text { BK } \\
\text { BK } \\
\text { BK }\end{array}$ & $\begin{array}{l}448 \\
449 \\
450 \\
451 \\
452\end{array}$ & $\begin{array}{l}- \\
- \\
-\end{array}$ & $\begin{array}{l}\text { - } \\
\text { - } \\
\text { - }\end{array}$ & $\begin{array}{r}45.0 \\
75.0 \\
65.0 \\
5.0 \\
20.0\end{array}$ & $\begin{array}{l}- \\
- \\
-\end{array}$ & $\begin{array}{l}- \\
\overline{-} \\
-\end{array}$ & $\begin{array}{l}15.0 \\
27.0 \\
30.0 \\
10 . \overline{0}\end{array}$ & $\begin{array}{l}- \\
\overline{-} \\
-\end{array}$ & $\begin{array}{l}1 \\
1 \\
1 \\
0 \\
\varnothing\end{array}$ & $\begin{array}{l}1 \\
1 \\
1 \\
1 \\
1\end{array}$ & $\begin{array}{l}1 \\
1 \\
1 \\
1 \\
1\end{array}$ & $\begin{array}{l}3 \\
3 \\
3 \\
7 \\
5\end{array}$ \\
\hline $\begin{array}{l}B K \\
B K \\
B K \\
B K \\
B K\end{array}$ & $\begin{array}{l}453 \\
454 \\
455 \\
456 \\
457\end{array}$ & $\begin{array}{l}- \\
\overline{-} \\
-\end{array}$ & - & $\begin{array}{l}18.0 \\
18.0 \\
40.0 \\
30.0 \\
40.0\end{array}$ & $\begin{array}{l}- \\
\overline{-} \\
\overline{-}\end{array}$ & $\begin{array}{l}- \\
- \\
-\end{array}$ & $\begin{array}{l}12.0 \\
12.0 \\
30.0 \\
21.0 \\
30.0\end{array}$ & $\begin{array}{l}- \\
- \\
- \\
-\end{array}$ & $\begin{array}{l}1 \\
1 \\
1 \\
1 \\
1\end{array}$ & $\begin{array}{l}1 \\
1 \\
1 \\
1 \\
1\end{array}$ & $\begin{array}{l}1 \\
1 \\
1 \\
1 \\
1\end{array}$ & $\begin{array}{l}4 \\
4 \\
6 \\
6 \\
6\end{array}$ \\
\hline $\begin{array}{l}\text { BK } \\
\text { BK } \\
\text { BK } \\
\text { BK } \\
\text { BK }\end{array}$ & $\begin{array}{l}458 \\
459 \\
460 \\
461 \\
462\end{array}$ & $\begin{array}{l}- \\
- \\
- \\
-\end{array}$ & $\begin{array}{l}\bar{z} \\
\bar{z}\end{array}$ & $\begin{array}{l}45.0 \\
45.0 \\
40 . \varnothing \\
50.0 \\
20.0\end{array}$ & $\begin{array}{l}- \\
- \\
- \\
-\end{array}$ & $\begin{array}{l}- \\
- \\
- \\
-\end{array}$ & $\begin{array}{r}30.0 \\
30.0 \\
35.0 \\
32.0 \\
8.0\end{array}$ & $\begin{array}{l}- \\
- \\
- \\
-\end{array}$ & $\begin{array}{l}1 \\
1 \\
1 \\
1 \\
1\end{array}$ & $\begin{array}{l}1 \\
1 \\
1 \\
1 \\
1\end{array}$ & $\begin{array}{l}1 \\
1 \\
1 \\
1 \\
1\end{array}$ & $\begin{array}{l}7 \\
7 \\
7 \\
3 \\
7\end{array}$ \\
\hline $\begin{array}{l}\text { BK } \\
\text { BK } \\
\text { BK } \\
\text { BK } \\
\text { BK }\end{array}$ & $\begin{array}{l}463 \\
464 \\
465 \\
466 \\
468\end{array}$ & $\begin{array}{l}- \\
- \\
- \\
-\end{array}$ & $\begin{array}{l}- \\
- \\
-\end{array}$ & $\begin{array}{l}14.0 \\
35.0 \\
40.0 \\
45.0 \\
46.0\end{array}$ & $\begin{array}{l}- \\
- \\
- \\
-\end{array}$ & $\begin{array}{l}- \\
- \\
- \\
-\end{array}$ & $\begin{array}{r}8.0 \\
22.0 \\
23.0 \\
23.0 \\
24.0\end{array}$ & $\begin{array}{l}- \\
- \\
- \\
-\end{array}$ & $\begin{array}{l}1 \\
1 \\
1 \\
1 \\
1\end{array}$ & $\begin{array}{l}1 \\
1 \\
1 \\
1 \\
1\end{array}$ & $\begin{array}{l}1 \\
1 \\
1 \\
1 \\
1\end{array}$ & $\begin{array}{l}7 \\
7 \\
7 \\
3 \\
7\end{array}$ \\
\hline $\begin{array}{l}\text { BK } \\
\text { BK } \\
\text { BK } \\
\text { BK } \\
\text { BK }\end{array}$ & $\begin{array}{l}469 \\
470 \\
471 \\
472 \\
473\end{array}$ & $\begin{array}{l}- \\
- \\
- \\
-\end{array}$ & $\begin{array}{l}- \\
- \\
-\end{array}$ & $\begin{array}{l}45.0 \\
45.0 \\
45.0 \\
35.0 \\
30.0\end{array}$ & $\begin{array}{l}- \\
- \\
- \\
-\end{array}$ & $\begin{array}{l}- \\
- \\
- \\
-\end{array}$ & $\begin{array}{l}24 . \varnothing \\
29 . \varnothing \\
29 . \varnothing \\
10 . \varnothing \\
11 . \varnothing\end{array}$ & $\begin{array}{l}- \\
- \\
- \\
-\end{array}$ & $\begin{array}{l}1 \\
1 \\
1 \\
1 \\
1\end{array}$ & $\begin{array}{l}1 \\
1 \\
1 \\
1 \\
1\end{array}$ & $\begin{array}{l}1 \\
1 \\
1 \\
1 \\
1\end{array}$ & $\begin{array}{l}7 \\
7 \\
7 \\
3 \\
3\end{array}$ \\
\hline $\begin{array}{l}\text { BK } \\
\text { BK } \\
\text { BK } \\
\text { BK } \\
\text { BK }\end{array}$ & $\begin{array}{l}474 \\
475 \\
476 \\
477 \\
478\end{array}$ & $\begin{array}{l}- \\
- \\
- \\
-\end{array}$ & $\begin{array}{l}- \\
\bar{z} \\
\text { - }\end{array}$ & $\begin{array}{l}30.0 \\
30.0 \\
27.0 \\
30.0 \\
30.0\end{array}$ & $\begin{array}{l}- \\
- \\
- \\
-\end{array}$ & $\begin{array}{l}- \\
- \\
- \\
-\end{array}$ & $\begin{array}{l}12.0 \\
12.0 \\
12.0 \\
12.0 \\
12.0\end{array}$ & $\begin{array}{l}- \\
- \\
- \\
-\end{array}$ & $\begin{array}{l}1 \\
1 \\
1 \\
1 \\
1\end{array}$ & $\begin{array}{l}1 \\
1 \\
1 \\
1 \\
1\end{array}$ & $\begin{array}{l}1 \\
1 \\
1 \\
1 \\
1\end{array}$ & $\begin{array}{l}3 \\
3 \\
3 \\
3 \\
3\end{array}$ \\
\hline $\begin{array}{l}\text { BK } \\
B K \\
B K \\
B K \\
B K\end{array}$ & $\begin{array}{l}479 \\
480 \\
483 \\
484 \\
485\end{array}$ & $\begin{array}{l}- \\
- \\
- \\
-\end{array}$ & $\begin{array}{l}- \\
\text { - } \\
\text { - }\end{array}$ & $\begin{array}{r}40.0 \\
185.0 \\
95.0 \\
70.0 \\
50.0\end{array}$ & $\begin{array}{l}- \\
- \\
- \\
-\end{array}$ & $\begin{array}{l}- \\
- \\
- \\
-\end{array}$ & $\begin{array}{l}21.0 \\
20.0 \\
54.0 \\
43.0 \\
33.0\end{array}$ & $\begin{array}{l}- \\
- \\
-\end{array}$ & $\begin{array}{l}1 \\
1 \\
1 \\
1 \\
1\end{array}$ & $\begin{array}{l}1 \\
1 \\
1 \\
1 \\
1\end{array}$ & $\begin{array}{l}1 \\
3 \\
1 \\
1 \\
1\end{array}$ & $\begin{array}{l}3 \\
7 \\
3 \\
1 \\
5\end{array}$ \\
\hline $\begin{array}{l}B K \\
B K \\
B K \\
B K \\
B K\end{array}$ & $\begin{array}{l}486 \\
487 \\
488 \\
489 \\
499\end{array}$ & $\begin{array}{l}\bar{z} \\
\bar{z} \\
\bar{z}\end{array}$ & $\begin{array}{l}- \\
- \\
-\end{array}$ & $\begin{array}{l}180.0 \\
150.0 \\
110.0 \\
100.0 \\
130.0\end{array}$ & $\begin{array}{l}\overline{-} \\
\bar{z} \\
\text { - }\end{array}$ & $\begin{array}{l}- \\
\overline{-} \\
-\end{array}$ & $\begin{array}{l}22 . \varnothing \\
57 . \varnothing \\
57 . \varnothing \\
34 . \varnothing \\
50 . \varnothing\end{array}$ & $\begin{array}{l}\overline{-} \\
\bar{z} \\
\bar{z}\end{array}$ & $\begin{array}{l}1 \\
1 \\
1 \\
1 \\
1\end{array}$ & $\begin{array}{l}1 \\
1 \\
1 \\
1 \\
1\end{array}$ & $\begin{array}{l}3 \\
1 \\
1 \\
3 \\
1\end{array}$ & $\begin{array}{l}6 \\
6 \\
6 \\
7 \\
3\end{array}$ \\
\hline
\end{tabular}


Appendix.--Location and basic physical features of recharge basins on Long Island,

\begin{tabular}{|c|c|c|c|c|c|c|c|c|}
\hline \multicolumn{2}{|c|}{$\begin{array}{l}\text { BASIN } \\
\text { NUMBER }\end{array}$} & \multicolumn{2}{|c|}{ NEAREST INTERSECTION } & $\begin{array}{l}\text { LATITUDE } \\
\text { LONGITUDE } \\
\circ, " \circ\end{array}$ & $\begin{array}{l}\text { DATE } \\
\text { BUILT } \\
\text { YrMOD }\end{array}$ & COMMNITY & \multicolumn{2}{|c|}{$\begin{array}{l}\text { DESIGN ACTUAL } \\
\text { CAPACITY CAPACITY } \\
\text { (cubic feet) }\end{array}$} \\
\hline $\begin{array}{l}B K \\
B K \\
B K \\
B K \\
B K\end{array}$ & $\begin{array}{l}491 \\
492 \\
493 \\
494 \\
495\end{array}$ & $\begin{array}{l}\text { VICEROY PLACE } \\
\text { SUPERIOR STREET } \\
\text { HURON STREET } \\
\text { MIDDLE COUNTRY } \\
\text { ØLD TOWN ROAD }\end{array}$ & $\begin{array}{l}\text { JAYNE BOULEVVARD } \\
\text { DILLON AVENUE } \\
\text { SQUIRE AVENUE } \\
\text { WOODVILLE ROAD } \\
\text { L.I.R.R. }\end{array}$ & 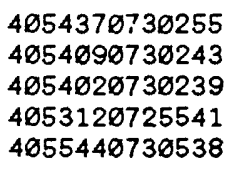 & $\begin{array}{l}- \\
- \\
-\end{array}$ & $\begin{array}{c}- \\
\overline{-} \\
233\end{array}$ & $\begin{array}{l}- \\
\overline{-} \\
-\end{array}$ & $\begin{array}{l}- \\
\overline{-} \\
-\end{array}$ \\
\hline $\begin{array}{l}E \\
E \\
E \\
E M \\
H\end{array}$ & $\begin{array}{l}1 \\
2 \\
4 \\
2 \\
1\end{array}$ & $\begin{array}{l}\text { YEW STREET } \\
\text { L.I.R.R. } \\
\text { SPRINGY BANKS RD } \\
\text { OLD WESTBURY RD. } \\
\text { ROCKLYN COURT }\end{array}$ & $\begin{array}{l}\text { INKBERRY STREET } \\
\text { WINDMILL LANE } \\
\text { OYSTER POND LANE } \\
\text { SALISBURY PK.DR. } \\
\text { PLEASANT COURT }\end{array}$ & $\begin{array}{l}4 \emptyset 58310721117 \\
4 \varnothing 5845 \emptyset 720854 \\
4100180721151 \\
4 \varnothing 44 \oslash 10733252 \\
4 \varnothing 52120732335\end{array}$ & $\begin{array}{c}- \\
- \\
- \\
560127\end{array}$ & $\begin{array}{r}319 \\
317 \\
328 \\
8 \\
-\end{array}$ & $\begin{array}{l}- \\
- \\
- \\
\overline{7}\end{array}$ & $\begin{array}{l}- \\
- \\
\overline{9} \\
95000\end{array}$ \\
\hline $\begin{array}{l}H \\
H \\
H \\
H \\
H\end{array}$ & $\begin{array}{l}2 \\
3 \\
4 \\
5 \\
6\end{array}$ & $\begin{array}{l}\text { WILLOUGHBY PATH } \\
\text { EMERSON STREET } \\
\text { WINDMILL DRIVE } \\
\text { BLUEBIRD LANE } \\
\text { LANCASTER DRIVE }\end{array}$ & $\begin{array}{l}\text { VINCENT STREET } \\
\text { WOODEDGE PLACE } \\
\text { WILLWOOD DRIVE } \\
\text { SPARROW LANE } \\
\text { MELVILLE ROAD }\end{array}$ & $\begin{array}{l}4 \emptyset 49460731944 \\
4 \varnothing 46250732 \emptyset 34 \\
4 \varnothing 52320732311 \\
4 \varnothing 49360732616 \\
4 \varnothing 492007324 \varnothing 8\end{array}$ & $\begin{array}{l}620815 \\
650512 \\
600722 \\
550129 \\
540915\end{array}$ & $\begin{array}{c}\overline{159} \\
\overline{154} \\
-\end{array}$ & $\begin{array}{l}163500 \\
154100 \\
400000 \\
- \\
54900\end{array}$ & $\begin{array}{l}170000 \\
160800 \\
405000 \\
- \\
68100\end{array}$ \\
\hline $\begin{array}{l}H \\
H \\
H \\
H \\
H\end{array}$ & $\begin{array}{r}7 \\
8 \\
9 \\
10 \\
13\end{array}$ & $\begin{array}{l}\text { SELLGER COURT } \\
\text { HARMON DRIVE } \\
\text { ATHENS COURT } \\
\text { LESLIE LANE } \\
\text { LANCASTER DRIVE }\end{array}$ & $\begin{array}{l}\text { BELTANE DRIVE } \\
\text { LITTLE PLAINS RD } \\
\text { 1OTH AVENUE } \\
\text { HARRIET AVENUE } \\
\text { DERBY COURT }\end{array}$ & $\begin{array}{l}4 \varnothing 49200732210 \\
4 \varnothing 5 \varnothing 56 \varnothing 73202 \emptyset \\
4 \varnothing 5244 \varnothing 732012 \\
4952450732432 \\
4 \varnothing 4929073241 \varnothing\end{array}$ & $\begin{array}{l}560824 \\
630225 \\
6382 \\
5506 \\
541206\end{array}$ & $\begin{array}{c}133 \\
139 \\
- \\
146\end{array}$ & $\begin{array}{l}23500 \varnothing \\
25010 \varnothing \\
894 \varnothing \varnothing \\
- \\
-\end{array}$ & $\begin{array}{l}260000 \\
269000 \\
92600 \\
- \\
-\end{array}$ \\
\hline $\begin{array}{l}H \\
H \\
H \\
H \\
H\end{array}$ & $\begin{array}{l}14 \\
17 \\
18 \\
20 \\
21\end{array}$ & $\begin{array}{l}\text { FIFTH AVENUE } \\
\text { CHARTER AVENUE } \\
\text { RAYBOR ROAD } \\
\text { RONDEL LANE } \\
\text { BURTON LANE }\end{array}$ & $\begin{array}{l}\text { ESTHER PLACE } \\
\text { EUCLID AVENUE } \\
\text { DEL LANE } \\
\text { WICKS ROAD } \\
\text { BURFORD DRIVE }\end{array}$ & 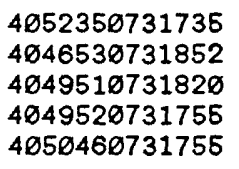 & $\begin{array}{c}560504 \\
- \\
610502 \\
601221 \\
550527\end{array}$ & $\begin{array}{l}- \\
- \\
169 \\
169 \\
169\end{array}$ & $\begin{array}{l}- \\
775000 \\
119500 \\
169500 \\
-\end{array}$ & $\begin{array}{l}89500 \\
899000 \\
136000 \\
170500 \\
-\end{array}$ \\
\hline $\begin{array}{l}H \\
H \\
H \\
H \\
H\end{array}$ & $\begin{array}{l}22 \\
23 \\
26 \\
27 \\
28\end{array}$ & $\begin{array}{l}\text { BURRWOOD COURT } \\
\text { INGOLD DRIVE } \\
\text { E END OF S.LANE } \\
\text { WINTERGREEN DR. } \\
\text { DORSET LANE }\end{array}$ & $\begin{array}{l}\text { BURR ROAD } \\
\text { PLYMOUTH ROAD } \\
\text { OLD COUNTRY ROAD } \\
\text { WINDHAM DRIVE }\end{array}$ & $\begin{array}{l}4051 \varnothing 607318 \emptyset 7 \\
4048490732225 \\
4050590732202 \\
404824 \varnothing 732318 \\
404848 \emptyset 732341\end{array}$ & $\begin{array}{l}610117 \\
560717 \\
641019 \\
620302 \\
600102\end{array}$ & $\begin{array}{l}169 \\
133 \\
- \\
- \\
151\end{array}$ & $\begin{array}{l}- \\
\overline{81200} \\
\overline{65500}\end{array}$ & $\begin{array}{l}- \\
136400 \\
92500 \\
- \\
91000\end{array}$ \\
\hline $\begin{array}{l}H \\
H \\
H \\
H \\
H\end{array}$ & $\begin{array}{l}29 \\
30 \\
31 \\
32 \\
33\end{array}$ & $\begin{array}{l}\text { PIDGEON HILL RD. } \\
\text { VILLANOVA LANE } \\
\text { FRONTIER LANE } \\
\text { CLAY PITS ROAD } \\
\text { HARBOR COURT }\end{array}$ & $\begin{array}{l}\text { WOLF HILL ROAD } \\
\text { DEER PARK ROAD } \\
\text { COMPTON STREET } \\
\text { GRAYSTONE DRIVE } \\
\text { HARBOR RIDGE RD. }\end{array}$ & $\begin{array}{l}4948510732321 \\
404954 \varnothing 732015 \\
4051290732015 \\
4 \oslash 5157 \varnothing 7319 \varnothing \emptyset \\
405416 \varnothing 732223\end{array}$ & $\begin{array}{l}600121 \\
611025 \\
611025 \\
540825 \\
590924\end{array}$ & $\begin{array}{l}161 \\
133 \\
169 \\
137 \\
130\end{array}$ & $\begin{array}{l}351000 \\
- \\
- \\
490000 \\
-\end{array}$ & $\begin{array}{l}580000 \\
- \\
- \\
- \\
90000\end{array}$ \\
\hline $\begin{array}{l}H \\
H \\
H \\
H \\
H\end{array}$ & $\begin{array}{l}34 \\
35 \\
36 \\
37 \\
38\end{array}$ & $\begin{array}{l}\text { OASIS PLACE } \\
\text { COPPERDALE LANE } \\
\text { SOUTH KETAY AVE. } \\
\text { HONEY LANE } \\
\text { CHAUCER DRIVE }\end{array}$ & $\begin{array}{l}\text { CARAVAN COURT } \\
\text { WEST NECK ROAD } \\
\text { KETAY DRIVE } \\
\text { KIM LANE } \\
\text { DRESSLER ROAD }\end{array}$ & $\begin{array}{l}4052140731807 \\
4053010732618 \\
4050580731851 \\
4042410731819 \\
4051070732138\end{array}$ & $\begin{array}{c}630328 \\
551020 \\
- \\
600301 \\
540729\end{array}$ & $\begin{array}{l}137 \\
\overline{169} \\
137 \\
141\end{array}$ & $\begin{array}{l}47400 \varnothing \\
- \\
286000 \\
- \\
-\end{array}$ & $\begin{array}{l}528000 \\
154000 \\
- \\
- \\
140000\end{array}$ \\
\hline $\begin{array}{l}H \\
H \\
H \\
H \\
H\end{array}$ & $\begin{array}{l}39 \\
49 \\
42 \\
43 \\
44\end{array}$ & $\begin{array}{l}\text { MANETTO HILL RD. } \\
\text { CONNELLY ROAD } \\
\text { DUFFY LANE } \\
\text { CROOKED HILL RD. } \\
\text { PLANE TREE LANE }\end{array}$ & $\begin{array}{l}\text { MANETTO COURT } \\
\text { HOWARD ROAD } \\
\text { CARROLL PLACE } \\
\text { VIDONI PLACE } \\
\text { PEAR ROAD }\end{array}$ & $\begin{array}{l}4 \emptyset 4824 \emptyset 73265 \emptyset \\
4 \varnothing 5234 \varnothing 732243 \\
4 \emptyset 5133 \emptyset 732127 \\
4 \varnothing 5144 \varnothing 73244 \emptyset \\
4 \emptyset 4843 \emptyset 732127\end{array}$ & $\begin{array}{l}610516 \\
590722 \\
540921 \\
550209 \\
630815\end{array}$ & $\begin{array}{l}\overline{13 \emptyset} \\
14 \overline{1} \\
146 \\
133\end{array}$ & $\begin{array}{l}130000 \\
377000 \\
- \\
30000 \\
111000\end{array}$ & $\begin{array}{l}132000 \\
389000 \\
- \\
32000 \\
119000\end{array}$ \\
\hline $\begin{array}{l}H \\
H \\
H \\
H \\
H\end{array}$ & $\begin{array}{l}45 \\
48 \\
47 \\
48 \\
49\end{array}$ & $\begin{array}{l}\text { PLEASANT STREET } \\
\text { LOWICK PLACE } \\
\text { ANDOVER COURT } \\
\text { VILLAGE DRIVE } \\
\text { MILLET STREET }\end{array}$ & $\begin{array}{l}\text { BERRYWOOD DRIVE } \\
\text { COLDEN STREET } \\
\text { PARK AVENUE } \\
\text { WEATHERVANE WAY } \\
\text { PINE ACRE BLVD. }\end{array}$ & $\begin{array}{l}4 \varnothing 5 \varnothing 24 \varnothing 732236 \\
4 \varnothing 48530732346 \\
4 \varnothing 5 \emptyset 410732239 \\
4 \varnothing 4717 \emptyset 731946 \\
4046560731935\end{array}$ & $\begin{array}{l}570403 \\
561107 \\
550906 \\
650819 \\
650106\end{array}$ & $\begin{array}{l}141 \\
151 \\
- \\
- \\
-\end{array}$ & $\begin{array}{l}183000 \\
- \\
- \\
277000 \\
321000\end{array}$ & $\begin{array}{l}190000 \\
64000 \\
- \\
292000 \\
347000\end{array}$ \\
\hline $\begin{array}{l}H \\
H \\
H \\
H \\
H\end{array}$ & $\begin{array}{l}50 \\
51 \\
52 \\
53 \\
54\end{array}$ & $\begin{array}{l}\text { SQUIRE AVENUE } \\
\text { DUNLOP ROAD } \\
\text { HAMMOND ROAD } \\
\text { GLENRIDGE LANE } \\
\text { OLD BRIDGE ROAD }\end{array}$ & $\begin{array}{l}\text { JEANNE PLACE } \\
\text { ASHLAND DRIVE } \\
\text { SHARI LANE } \\
\text { STEINBECK } \\
\text { PULASKI ROAD }\end{array}$ & $\begin{array}{l}4052340731908 \\
4051560732332 \\
4051106731929 \\
4652090732009 \\
4652550731804\end{array}$ & $\begin{array}{l}570507 \\
550527 \\
630115 \\
550824 \\
550810\end{array}$ & $\begin{array}{c}137 \\
- \\
139 \\
\overline{137}\end{array}$ & $\begin{array}{l}- \\
- \\
- \\
-\end{array}$ & $\begin{array}{l}- \\
\overline{-} \\
465000 \\
34000 \\
112000\end{array}$ \\
\hline
\end{tabular}


N.Y. [Dash indicates no data. Altitudes are in feet above sea level.] (cont.)

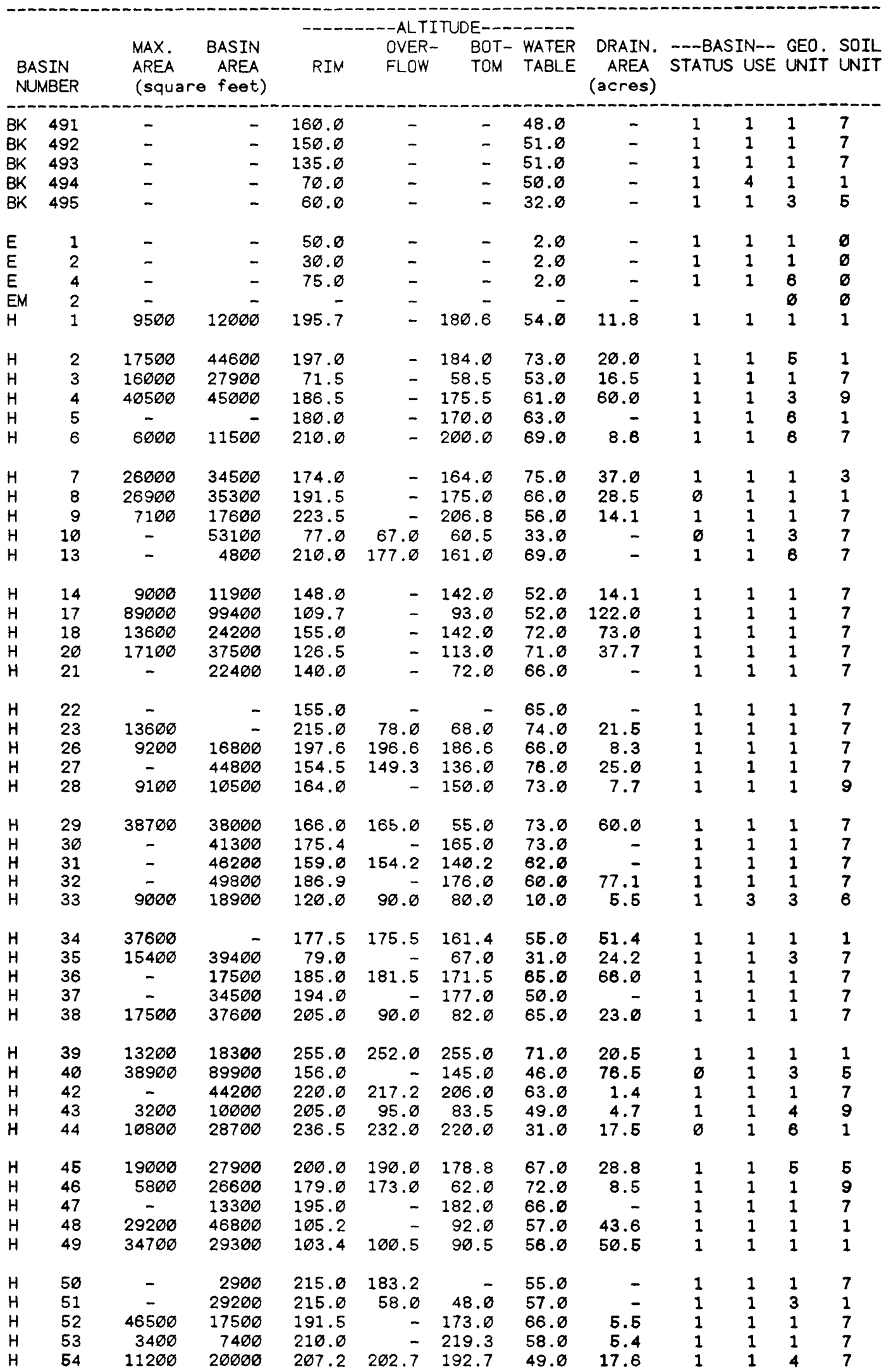


Appendix.--Location and basic physical features of recharge basins on Long Island,

\begin{tabular}{|c|c|c|c|c|c|c|c|c|}
\hline \multicolumn{2}{|c|}{$\begin{array}{l}\text { BASIN } \\
\text { NUMBER }\end{array}$} & \multicolumn{2}{|c|}{ NEAREST INTERSECTION } & $\begin{array}{l}\text { LATITUDE } \\
\text { LONGITUDE, } \\
\circ, " \circ, "\end{array}$ & $\begin{array}{l}\text { DATE } \\
\text { BUILT } \\
\text { YrMoD }\end{array}$ & COMMNITY & \multicolumn{2}{|c|}{$\begin{array}{l}\text { DESIGN ACTUAL } \\
\text { CAPACITY CAPACITY } \\
\text { (cubic foot) }\end{array}$} \\
\hline $\begin{array}{l}H \\
H \\
H \\
H \\
H \\
H\end{array}$ & $\begin{array}{l}56 \\
56 \\
57 \\
58 \\
59\end{array}$ & $\begin{array}{l}\text { MULBERRY DRIVE } \\
\text { ICELAND DRIVE } \\
\text { TIPPIN DRIVE } \\
\text { POND ROAD } \\
\text { BELLAFIORE DRIVE }\end{array}$ & $\begin{array}{l}\text { VINEYARD ROAD } \\
\text { GREENLAND DRIVE } \\
\text { 1ITH AVENUE } \\
\text { CLARMAN COURT } \\
\text { FRANCIS COURT }\end{array}$ & $\begin{array}{l}4 \varnothing 53340732434 \\
4 \varnothing 49350732436 \\
4 \varnothing 5 \varnothing 690732531 \\
4 \varnothing 5602 \varnothing 732349 \\
4 \varnothing 522 \varnothing 6731851\end{array}$ & $\begin{array}{l}580819 \\
681167 \\
5504 \\
648225 \\
591814\end{array}$ & $\begin{array}{c}142 \\
151 \\
151 \\
\overline{137}\end{array}$ & $\begin{array}{l}78080 \\
- \\
- \\
61200 \\
170580\end{array}$ & $\begin{array}{l}85000 \\
- \\
- \\
64000 \\
190000\end{array}$ \\
\hline $\begin{array}{l}\mathrm{H} \\
\mathrm{H} \\
\mathrm{H} \\
\mathrm{H} \\
\mathrm{H}\end{array}$ & $\begin{array}{l}60 \\
62 \\
64 \\
65 \\
68\end{array}$ & $\begin{array}{l}\text { LANDVIEW DRIVE } \\
\text { TIANA PLACE } \\
\text { GAY LORE DRIVE } \\
\text { LUCILLE LANE } \\
\text { JANET LANE }\end{array}$ & $\begin{array}{l}\text { FOLGER LANE } \\
\text { MEMAS COURT } \\
\text { OLD COUNTRY ROAD } \\
\text { IRENE LANE } \\
\text { SHELDON PLACE }\end{array}$ & $\begin{array}{l}40480107321 \varnothing 1 \\
40481 \varnothing 6732 \varnothing 4 \varnothing \\
4 \varnothing 4943 \varnothing 732144 \\
4 \varnothing 5 \varnothing 63 \varnothing 731806 \\
4 \varnothing 5 \varnothing 14 \varnothing 731808\end{array}$ & $\begin{array}{l}601025 \\
580717 \\
550484 \\
570819 \\
570819\end{array}$ & $\begin{array}{l}133 \\
133 \\
133 \\
169 \\
169\end{array}$ & $\begin{array}{l}3870600 \\
- \\
- \\
335000 \\
-\end{array}$ & $\begin{array}{l}396600 \\
- \\
- \\
467600 \\
-\end{array}$ \\
\hline $\begin{array}{l}H \\
H \\
H \\
H \\
H\end{array}$ & $\begin{array}{l}67 \\
68 \\
69 \\
78 \\
71\end{array}$ & $\begin{array}{l}\text { VESTRY COURT } \\
\text { ELEANOR PLACE } \\
\text { TURKEY LANE } \\
\text { BENNETT AVENUE } \\
\text { RENEE PLACE }\end{array}$ & $\begin{array}{l}\text { GRENLEAF DRIVE } \\
\text { MARIE AVENUE } \\
\text { RIDGE ROAD } \\
\text { E. ROGUES PATH } \\
\text { SUGAR WOOD PLACE }\end{array}$ & $\begin{array}{l}4051060732100 \\
4053250732546 \\
4052090732708 \\
4050530732386 \\
4 \varnothing 50280731815\end{array}$ & $\begin{array}{l}631213 \\
560309 \\
630114 \\
650511 \\
6010\end{array}$ & $\begin{array}{l}141 \\
- \\
131 \\
141 \\
169\end{array}$ & $\begin{array}{l}- \\
- \\
- \\
90800 \\
75000\end{array}$ & $\begin{array}{l}328300 \\
80000 \\
- \\
117000 \\
79000\end{array}$ \\
\hline $\begin{array}{l}H \\
H \\
H \\
H \\
H\end{array}$ & $\begin{array}{l}72 \\
73 \\
74 \\
75 \\
76\end{array}$ & $\begin{array}{l}\text { HEDGEROW COURT } \\
\text { DOVECOTE LANE } \\
\text { BRIDLE LANE } \\
\text { DOE LANE } \\
\text { WARRENTON COURT }\end{array}$ & $\begin{array}{l}\text { MANGIN ROAD } \\
\text { KEVIN ROAD } \\
\text { COE STREET } \\
\text { SHELDON PLACE } \\
\text { LAIRE DRIVE }\end{array}$ & $\begin{array}{l}4 \varnothing 5 \varnothing 416731753 \\
4856446731736 \\
4 \varnothing 48446732349 \\
4 \varnothing 5 \varnothing 17 \varnothing 731819 \\
4 \varnothing 52266732444\end{array}$ & $\begin{array}{l}681126 \\
680726 \\
590289 \\
6102 \\
531118\end{array}$ & $\begin{array}{l}169 \\
169 \\
151 \\
169 \\
-\end{array}$ & $\begin{array}{r}268060 \\
269300 \\
283600 \\
345660 \\
52960\end{array}$ & $\begin{array}{r}300080 \\
224600 \\
290000 \\
349000 \\
53300\end{array}$ \\
\hline $\begin{array}{l}H \\
H \\
H \\
H \\
H\end{array}$ & $\begin{array}{l}77 \\
78 \\
79 \\
89 \\
81\end{array}$ & $\begin{array}{l}\text { HARRIET LANE } \\
\text { PARK AVENUE } \\
\text { POLLY DRIVE } \\
\text { COTTONTAIL ROAD } \\
\text { BEECH PLACE }\end{array}$ & $\begin{array}{l}\text { ELVIRA COURT } \\
\text { LAKE ROAD } \\
\text { POLLY COURT } \\
\text { BRAMBLE LANE } \\
\text { BRIARWOOD DRIVE }\end{array}$ & $\begin{array}{l}4852330732435 \\
4 \varnothing 51130732386 \\
4 \varnothing 52206732434 \\
4 \varnothing 47 \varnothing 6 \varnothing 7326 \varnothing \emptyset \\
4 \varnothing 51516732602\end{array}$ & $\begin{array}{l}550505 \\
551024 \\
616417 \\
660465 \\
651210\end{array}$ & $\begin{array}{l}- \\
\overline{148} \\
146\end{array}$ & $\begin{array}{l}93600 \\
- \\
145500 \\
93000 \\
97500\end{array}$ & $\begin{array}{l}93700 \\
- \\
146100 \\
98000 \\
98200\end{array}$ \\
\hline $\begin{array}{l}H \\
H \\
H \\
H \\
H\end{array}$ & $\begin{array}{l}82 \\
83 \\
84 \\
85 \\
86\end{array}$ & $\begin{array}{l}\text { BRAMBLE LANE } \\
\text { BRIARFIELD LANE } \\
\text { GABLES COURT } \\
\text { PHILLIP COURT } \\
\text { WOODMONT ROAD }\end{array}$ & $\begin{array}{l}\text { TREEVIEW DRIVE } \\
\text { RED DEER LANE } \\
\text { E. DEER PARK RD. } \\
\text { DAVID COURT } \\
\text { WILMINGTON DRIVE }\end{array}$ & $\begin{array}{l}4 \varnothing 47 \varnothing 86732541 \\
4 \varnothing 5218673235 \varnothing \\
4 \varnothing 49410732 \varnothing 29 \\
4 \varnothing 51320732423 \\
4 \varnothing 4621 \varnothing 73232 \varnothing\end{array}$ & $\begin{array}{l}840520 \\
600815 \\
638605 \\
551611 \\
6511\end{array}$ & $\begin{array}{l}148 \\
- \\
133 \\
146 \\
143\end{array}$ & $\begin{array}{l}470360 \\
293800 \\
- \\
- \\
1508400\end{array}$ & $\begin{array}{c}470400 \\
302000 \\
- \\
- \\
1577000\end{array}$ \\
\hline $\begin{array}{l}H \\
H \\
H \\
H \\
H\end{array}$ & $\begin{array}{l}87 \\
88 \\
89 \\
90 \\
91\end{array}$ & $\begin{array}{l}\text { L.I.E. } \\
\text { L.I.E. } \\
\text { MONTROSE PLACE } \\
\text { ARLEIGH ROAD } \\
\text { BRIARWOOD DRIVE }\end{array}$ & $\begin{array}{l}\text { BEAUMONT DRIVE } \\
\text { ROUNDTREE DRIVE } \\
\text { ROUNDTREE DRIVE } \\
\text { ARLEIGH COURT } \\
\text { BEECH PLACE }\end{array}$ & $\begin{array}{l}4047120732252 \\
4047100732304 \\
4046420732257 \\
4 \varnothing 52250731722 \\
4051480732553\end{array}$ & $\begin{array}{l}650106 \\
650186 \\
650186 \\
630801 \\
640309\end{array}$ & $\begin{array}{l}- \\
143 \\
143 \\
137 \\
146\end{array}$ & $\begin{array}{r}323200 \\
196000 \\
215500 \\
212600 \\
26500\end{array}$ & $\begin{array}{r}325000 \\
205300 \\
248000 \\
279000 \\
20600\end{array}$ \\
\hline $\begin{array}{l}H \\
H \\
H \\
H \\
H\end{array}$ & $\begin{array}{l}92 \\
93 \\
94 \\
95 \\
96\end{array}$ & $\begin{array}{l}\text { DIANE LANE } \\
\text { DIANE LANE } \\
\text { LAMPLIGHT LANE } \\
\text { CRAVEN STREET } \\
\text { HOLLY DRIVE }\end{array}$ & $\begin{array}{l}\text { MEDFORD LANE } \\
\text { NEWBROOK LANE } \\
\text { CANDLE STICK CT. } \\
\text { STEPAR PLACE } \\
\text { RODERICK COURT }\end{array}$ & $\begin{array}{l}4 \varnothing 5127 \varnothing 731752 \\
4 \varnothing 51180731743 \\
4 \varnothing 46480732604 \\
4 \varnothing 5 \varnothing 2 \varnothing 6732533 \\
4 \varnothing 51316731804\end{array}$ & $\begin{array}{l}620514 \\
620514 \\
601801 \\
560823 \\
641020\end{array}$ & $\begin{array}{c}169 \\
169 \\
- \\
169\end{array}$ & $\begin{array}{c}3613000 \\
335800 \\
157000 \\
- \\
139000\end{array}$ & $\begin{array}{l}36400 \varnothing \\
355000 \\
160000 \\
12600 \emptyset \\
1394 \varnothing \varnothing\end{array}$ \\
\hline $\begin{array}{l}H \\
H \\
H \\
H \\
H\end{array}$ & $\begin{array}{r}97 \\
98 \\
160 \\
101 \\
102\end{array}$ & $\begin{array}{l}\text { THORNEY AVENUE } \\
\text { OLD COUNTRY ROAD } \\
\text { PLAINWOOD ROAD } \\
\text { CAXTON STREET } \\
\text { LANTERN STREET }\end{array}$ & $\begin{array}{l}\text { E. 25TH STREET } \\
\text { LINFORD LANE } \\
\text { ELDERWOOD ROAD } \\
\text { SYCAMORE STREET } \\
\text { BROADWAY }\end{array}$ & $\begin{array}{l}4049520732359 \\
40482 \varnothing 6732339 \\
4 \varnothing 48016732431 \\
4 \varnothing 48296732354 \\
4050470732208\end{array}$ & $\begin{array}{l}- \\
568918 \\
558401 \\
590108 \\
620321\end{array}$ & $\begin{array}{c}151 \\
151 \\
\overline{151} \\
-\end{array}$ & $\begin{array}{l}- \\
562500 \\
\overline{598000} \\
58300\end{array}$ & $\begin{array}{l}\overline{719000} \\
\overline{8} \\
880080 \\
58800\end{array}$ \\
\hline $\begin{array}{l}H \\
H \\
H \\
H \\
H\end{array}$ & $\begin{array}{l}103 \\
104 \\
105 \\
107 \\
108\end{array}$ & $\begin{array}{l}\text { HASTINGS STREET } \\
\text { LONGFELLOW DRIVE } \\
\text { PENFIELD ORIVE } \\
\text { CANDLEWOOD PATH } \\
\text { ARISTA DRIVE }\end{array}$ & $\begin{array}{l}\text { STRAIGHT PATH } \\
\text { JERICHO TURNPIKE } \\
\text { LARKFIELD ROAD } \\
\text { CHELSEA PLACE } \\
\text { BRYCEWOOD DRIVE }\end{array}$ & $\begin{array}{l}4 \varnothing 46386732 \varnothing 32 \\
4 \varnothing 5 \varnothing 67 \varnothing 7323 \varnothing 8 \\
4 \varnothing 5 \varnothing 58 \varnothing 7319 \varnothing 6 \\
4 \varnothing 4809 \varnothing 731938 \\
4 \varnothing 4935073214 \varnothing\end{array}$ & $\begin{array}{c}631121 \\
- \\
600502 \\
630924 \\
620417\end{array}$ & $\begin{array}{c}143 \\
151 \\
139 \\
- \\
133\end{array}$ & $\begin{array}{l}252000 \\
- \\
190800 \\
421200 \\
70000\end{array}$ & $\begin{array}{l}2940 \varnothing \emptyset \\
- \\
2160 \varnothing \varnothing \\
47110 \varnothing \\
1360 \varnothing \emptyset\end{array}$ \\
\hline $\begin{array}{l}H \\
H \\
H \\
H \\
H\end{array}$ & $\begin{array}{l}169 \\
110 \\
112 \\
113 \\
115\end{array}$ & $\begin{array}{l}\text { CANDLEWOOD PATH } \\
\text { OLD COUNRTY ROAD } \\
\text { PUMPKIN STREET } \\
\text { NEW YORK AVENUE } \\
\text { OLD COUNTRY ROAD }\end{array}$ & $\begin{array}{l}\text { CHELSEA PLACE } \\
\text { LONGWOOD DRIVE } \\
\text { SPUR DRIVE } \\
\text { TODD COURT } \\
\text { DARBY DRIVE }\end{array}$ & $\begin{array}{l}4 \varnothing 482 \varnothing \varnothing 73195 \emptyset \\
4 \varnothing 4843 \varnothing 7323 \varnothing 9 \\
4 \varnothing 514 \varnothing \emptyset 7319 \emptyset 1 \\
4 \varnothing 4919 \emptyset 732426 \\
4 \varnothing 49 \varnothing 7 \varnothing 732234\end{array}$ & $\begin{array}{l}620417 \\
608220 \\
570917 \\
631118 \\
610215\end{array}$ & $\begin{array}{l}- \\
- \\
137 \\
151 \\
-\end{array}$ & 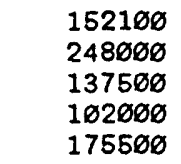 & $\begin{array}{r}2656 \varnothing \\
51508 \varnothing \\
14508 \emptyset \\
18200 \varnothing \\
4506 \varnothing \varnothing\end{array}$ \\
\hline
\end{tabular}


N.Y. [Dash indicates no data. Altitudes are in feet above sea level.] (cont.)

\begin{tabular}{|c|c|c|c|c|c|c|c|c|c|c|c|c|}
\hline \multicolumn{2}{|c|}{$\begin{array}{l}\text { BASIN } \\
\text { NUMBER }\end{array}$} & $\begin{array}{l}\text { MAX. } \\
\text { AREA } \\
\text { (square }\end{array}$ & $\begin{array}{c}\text { BASIN } \\
\text { AREA } \\
\text { feet) }\end{array}$ & RIM & $\begin{array}{l}\text { OVER } \\
\text { FLOW }\end{array}$ & $\begin{array}{l}\text { BOT- } \\
\text { TOM }\end{array}$ & $\begin{array}{l}\text { WATER } \\
\text { TABLE }\end{array}$ & $\begin{array}{c}\text { DRAIN. } \\
\text { AREA } \\
\text { (acres) }\end{array}$ & $\begin{array}{l}--B A S I \\
\text { STATUS }\end{array}$ & $\begin{array}{l}\text { IN-- } \\
\text { USE }\end{array}$ & $\begin{array}{l}\text { GEO. } \\
\text { UNIT }\end{array}$ & $\begin{array}{l}\text { SOIL } \\
\text { UNIT }\end{array}$ \\
\hline $\begin{array}{l}H \\
H \\
H\end{array}$ & $\begin{array}{l}55 \\
56 \\
57 \\
58 \\
59\end{array}$ & $\begin{array}{c}8500 \\
- \\
- \\
6400 \\
19000\end{array}$ & $\begin{array}{l}13000 \\
16300 \\
27200 \\
13800 \\
28600\end{array}$ & $\begin{array}{r}43.0 \\
168.0 \\
182.0 \\
185.0 \\
205.0\end{array}$ & $\begin{array}{r}- \\
- \\
175 . \varnothing \\
-\end{array}$ & $\begin{array}{r}30.0 \\
154.4 \\
171.5 \\
165.0 \\
188.7\end{array}$ & $\begin{array}{l}31.0 \\
67.0 \\
62.0 \\
67.0 \\
57.0\end{array}$ & $\begin{array}{r}9.0 \\
- \\
-\overline{6} \\
9.6\end{array}$ & $\begin{array}{l}1 \\
1 \\
1 \\
1 \\
1\end{array}$ & $\begin{array}{l}1 \\
4 \\
1 \\
1 \\
1\end{array}$ & $\begin{array}{l}3 \\
1 \\
1 \\
1 \\
1\end{array}$ & $\begin{array}{l}1 \\
7 \\
7 \\
9 \\
7\end{array}$ \\
\hline $\mathrm{H}$ & $\begin{array}{l}60 \\
62 \\
64 \\
65 \\
66\end{array}$ & $\begin{array}{c}39600 \\
- \\
\overline{4} \\
46700 \\
-\end{array}$ & $\begin{array}{l}43400 \\
406 \varnothing 0 \\
12000 \\
624 \varnothing 0 \\
17600\end{array}$ & $\begin{array}{l}180.0 \\
186.0 \\
185.0 \\
136.0 \\
150.0\end{array}$ & $\begin{array}{r}- \\
182 . \varnothing \\
- \\
-\end{array}$ & $\begin{array}{l}131.5 \\
170.0 \\
175.0 \\
123.8 \\
127.8\end{array}$ & $\begin{array}{l}63.0 \\
64.0 \\
72.0 \\
71.0 \\
70.0\end{array}$ & $\begin{array}{r}78.6 \\
\overline{-} \\
61.0 \\
-\end{array}$ & $\begin{array}{l}1 \\
1 \\
1 \\
1 \\
1\end{array}$ & $\begin{array}{l}1 \\
1 \\
1 \\
1 \\
1\end{array}$ & $\begin{array}{l}8 \\
1 \\
1 \\
1 \\
1\end{array}$ & $\begin{array}{l}7 \\
1 \\
5 \\
7 \\
7\end{array}$ \\
\hline $\begin{array}{l}H \\
H\end{array}$ & $\begin{array}{l}67 \\
68 \\
69 \\
70 \\
71\end{array}$ & $\begin{array}{r}32800 \\
8000 \\
25800 \\
11700 \\
7900\end{array}$ & $\begin{array}{l}- \\
16800 \\
26400 \\
27200 \\
16300\end{array}$ & $\begin{array}{r}265.0 \\
80.5 \\
75.0 \\
189.7 \\
149.5\end{array}$ & $\begin{array}{l}- \\
- \\
- \\
-\end{array}$ & $\begin{array}{r}240.0 \\
68.0 \\
65.0 \\
175.5 \\
136.0\end{array}$ & $\begin{array}{l}65.0 \\
20.0 \\
41.0 \\
64.0 \\
70.0\end{array}$ & $\begin{array}{r}51.7 \\
12.6 \\
-\bar{\varnothing} \\
11.0 \\
5.2\end{array}$ & $\begin{array}{l}1 \\
1 \\
0 \\
1 \\
1\end{array}$ & $\begin{array}{l}1 \\
1 \\
1 \\
1 \\
1\end{array}$ & $\begin{array}{l}1 \\
1 \\
3 \\
1 \\
1\end{array}$ & $\begin{array}{l}1 \\
7 \\
7 \\
7 \\
7\end{array}$ \\
\hline $\begin{array}{l}\mathrm{H} \\
\mathrm{H} \\
\mathrm{H} \\
\mathrm{H} \\
\mathrm{H}\end{array}$ & $\begin{array}{l}72 \\
73 \\
74 \\
75 \\
76\end{array}$ & $\begin{array}{r}30000 \\
22500 \\
29000 \\
34900 \\
5300\end{array}$ & $\begin{array}{r}39200 \\
34 \varnothing \varnothing \emptyset \\
494 \varnothing \emptyset \\
44 \varnothing \varnothing \varnothing \\
52 \varnothing \varnothing\end{array}$ & $\begin{array}{r}149.5 \\
140.0 \\
161.5 \\
159.0\end{array}$ & $\begin{array}{l}- \\
- \\
- \\
-\end{array}$ & $\begin{array}{r}131.0 \\
111.0 \\
147.0 \\
141.5 \\
-\end{array}$ & $\begin{array}{l}67.0 \\
68.0 \\
73.0 \\
72 . \varnothing \\
34.0\end{array}$ & $\begin{array}{r}28.0 \\
21.3 \\
57.2 \\
36.8 \\
8.2\end{array}$ & $\begin{array}{l}1 \\
1 \\
1 \\
1 \\
1\end{array}$ & $\begin{array}{l}1 \\
1 \\
1 \\
1 \\
1\end{array}$ & $\begin{array}{l}1 \\
1 \\
1 \\
1 \\
1\end{array}$ & $\begin{array}{l}7 \\
1 \\
7 \\
7 \\
7\end{array}$ \\
\hline $\begin{array}{l}H \\
H \\
H \\
H \\
H\end{array}$ & $\begin{array}{l}77 \\
78 \\
79 \\
80 \\
81\end{array}$ & $\begin{array}{l}9400 \\
\overline{9} \\
9100 \\
7100 \\
9800\end{array}$ & $\begin{array}{l}23400 \\
32000 \\
32400 \\
28000 \\
23300\end{array}$ & $\begin{array}{r}105.0 \\
200.0 \\
- \\
175.0 \\
106.9\end{array}$ & $\begin{array}{l}105.0 \\
151.0 \\
172.6 \\
174.0 \\
105 . \varnothing\end{array}$ & $\begin{array}{r}93.0 \\
138.0 \\
155.0 \\
160.2 \\
95 . \varnothing\end{array}$ & $\begin{array}{l}40 . \varnothing \\
63 . \varnothing \\
37 . \varnothing \\
71.0 \\
38 . \varnothing\end{array}$ & $\begin{array}{r}16 . \varnothing \\
-\overline{4} \\
19.4 \\
5.7 \\
15.4\end{array}$ & $\begin{array}{l}1 \\
1 \\
1 \\
1 \\
0\end{array}$ & $\begin{array}{l}1 \\
1 \\
1 \\
3 \\
1\end{array}$ & $\begin{array}{l}3 \\
1 \\
1 \\
8 \\
1\end{array}$ & $\begin{array}{l}7 \\
7 \\
7 \\
5 \\
7\end{array}$ \\
\hline $\begin{array}{l}H \\
H \\
H \\
H \\
H\end{array}$ & $\begin{array}{l}82 \\
83 \\
84 \\
85 \\
86\end{array}$ & $\begin{array}{c}29480 \\
20200 \\
- \\
- \\
157700\end{array}$ & $\begin{array}{r}50000 \\
43500 \\
4 \varnothing 200 \\
18100 \\
197600\end{array}$ & $\begin{array}{l}137.5 \\
193.4 \\
194 . \varnothing \\
215.0 \\
160 . \varnothing\end{array}$ & $\begin{array}{r}136.5 \\
191 . \varnothing \\
- \\
94.5 \\
131.7\end{array}$ & $\begin{array}{r}120.5 \\
176.0 \\
- \\
84.0 \\
115.0\end{array}$ & $\begin{array}{l}71 . \varnothing \\
51 . \varnothing \\
73 . \varnothing \\
55 . \varnothing \\
61 . \varnothing\end{array}$ & $\begin{array}{r}74.0 \\
46.2 \\
- \\
154.0\end{array}$ & $\begin{array}{l}1 \\
0 \\
0 \\
1 \\
1\end{array}$ & $\begin{array}{l}1 \\
1 \\
1 \\
1 \\
2\end{array}$ & $\begin{array}{l}8 \\
1 \\
1 \\
1 \\
8\end{array}$ & $\begin{array}{l}8 \\
9 \\
1 \\
9 \\
5\end{array}$ \\
\hline $\begin{array}{l}H \\
H \\
H \\
H \\
H\end{array}$ & $\begin{array}{l}87 \\
88 \\
89 \\
99 \\
91\end{array}$ & $\begin{array}{r}25000 \\
17100 \\
19100 \\
27900 \\
2100\end{array}$ & $\begin{array}{l}364 \varnothing \emptyset \\
24 \varnothing \varnothing \emptyset \\
5 \varnothing \varnothing \varnothing \emptyset \\
625 \varnothing \emptyset \\
13 \varnothing \varnothing \emptyset\end{array}$ & $\begin{array}{l}178 . \emptyset \\
158 . \varnothing \\
185.5 \\
135 . \varnothing \\
190 . \varnothing\end{array}$ & $\begin{array}{l}156.5 \\
157.0 \\
185 . \varnothing \\
136.5 \\
154.0\end{array}$ & $\begin{array}{l}143.5 \\
145 . \varnothing \\
173 . \varnothing \\
124 . \varnothing \\
144 . \varnothing\end{array}$ & $\begin{array}{l}67 . \varnothing \\
67 . \varnothing \\
62 . \varnothing \\
52 . \varnothing \\
39 . \varnothing\end{array}$ & $\begin{array}{r}33.0 \\
30.9 \\
33.0 \\
26.0 \\
3.2\end{array}$ & $\begin{array}{l}1 \\
1 \\
0 \\
1 \\
1\end{array}$ & $\begin{array}{l}1 \\
1 \\
1 \\
1 \\
1\end{array}$ & $\begin{array}{l}8 \\
8 \\
8 \\
1 \\
1\end{array}$ & $\begin{array}{l}9 \\
9 \\
9 \\
7 \\
7\end{array}$ \\
\hline $\begin{array}{l}H \\
H \\
H \\
H \\
H\end{array}$ & $\begin{array}{l}92 \\
93 \\
94 \\
95 \\
96\end{array}$ & $\begin{array}{r}36400 \\
35500 \\
16000 \\
12000 \\
9300\end{array}$ & $\begin{array}{l}484 \varnothing \emptyset \\
633 \varnothing \emptyset \\
38 \varnothing \emptyset \emptyset \\
198 \varnothing \emptyset \\
231 \varnothing \emptyset\end{array}$ & $\begin{array}{r}151.0 \\
149.5 \\
95.0 \\
180.0 \\
165.0\end{array}$ & $\begin{array}{r}- \\
- \\
- \\
153.5\end{array}$ & $\begin{array}{r}136.0 \\
130 . \varnothing \\
172.0 \\
138.3\end{array}$ & $\begin{array}{l}63 . \varnothing \\
63 . \varnothing \\
55 . \varnothing \\
61 . \varnothing \\
63 . \varnothing\end{array}$ & $\begin{array}{l}42.0 \\
35 . \varnothing \\
24.7 \\
18.9 \\
17.0\end{array}$ & $\begin{array}{l}1 \\
1 \\
1 \\
1 \\
1\end{array}$ & $\begin{array}{l}1 \\
1 \\
1 \\
1 \\
1\end{array}$ & $\begin{array}{l}1 \\
1 \\
1 \\
1 \\
1\end{array}$ & $\begin{array}{l}7 \\
7 \\
7 \\
8 \\
7\end{array}$ \\
\hline $\begin{array}{l}\mathrm{H} \\
\mathrm{H} \\
\mathrm{H} \\
\mathrm{H} \\
\mathrm{H}\end{array}$ & $\begin{array}{r}97 \\
98 \\
100 \\
101 \\
102\end{array}$ & $\begin{array}{c}\overline{71900} \\
\overline{8} \\
80000 \\
5900\end{array}$ & $\begin{array}{r}- \\
93600 \\
49000 \\
88000 \\
1600\end{array}$ & $\begin{array}{r}200.0 \\
146.5 \\
147.8 \\
195.0\end{array}$ & $\begin{array}{l}- \\
- \\
- \\
-\end{array}$ & $\begin{array}{r}- \\
130.1 \\
127.0 \\
175 . \varnothing\end{array}$ & $\begin{array}{l}67.0 \\
76 . \varnothing \\
75.0 \\
72.0 \\
66 . \varnothing\end{array}$ & $\begin{array}{r}50 . \overline{0} \\
-\overline{4} \\
85 . \overline{2} \\
9 . \overline{2}\end{array}$ & $\begin{array}{l}1 \\
1 \\
1 \\
1 \\
1\end{array}$ & $\begin{array}{l}1 \\
1 \\
1 \\
1 \\
1\end{array}$ & $\begin{array}{l}1 \\
1 \\
1 \\
1 \\
1\end{array}$ & $\begin{array}{l}7 \\
7 \\
7 \\
7 \\
7\end{array}$ \\
\hline $\begin{array}{l}H \\
H \\
H \\
H \\
H\end{array}$ & $\begin{array}{l}103 \\
104 \\
105 \\
107 \\
108\end{array}$ & $\begin{array}{c}24500 \\
- \\
21600 \\
42800 \\
13000\end{array}$ & $\begin{array}{r}44200 \\
- \\
49300 \\
79200 \\
34000\end{array}$ & $\begin{array}{r}80 . \varnothing \\
220 . \varnothing \\
183.4 \\
223 . \varnothing\end{array}$ & $\begin{array}{r}76.7 \\
- \\
- \\
- \\
222.0\end{array}$ & $\begin{array}{r}64.7 \\
- \\
170 . \varnothing \\
212 . \overline{-}\end{array}$ & $\begin{array}{l}54.0 \\
67.0 \\
67.0 \\
57 . \varnothing \\
73 . \varnothing\end{array}$ & $\begin{array}{r}39.7 \\
- \\
35.0 \\
72.0 \\
12.0\end{array}$ & $\begin{array}{l}1 \\
1 \\
1 \\
0 \\
1\end{array}$ & $\begin{array}{l}1 \\
1 \\
1 \\
1 \\
1\end{array}$ & $\begin{array}{l}1 \\
1 \\
1 \\
1 \\
6\end{array}$ & $\begin{array}{l}7 \\
7 \\
7 \\
7 \\
9\end{array}$ \\
\hline $\begin{array}{l}H \\
H \\
H \\
H \\
H\end{array}$ & $\begin{array}{l}109 \\
110 \\
112 \\
113 \\
115\end{array}$ & $\begin{array}{r}25200 \\
34300 \\
14500 \\
7800 \\
40900\end{array}$ & $\begin{array}{l}3910 \varnothing \\
344 \varnothing \varnothing \\
101 \varnothing \varnothing \\
258 \varnothing \varnothing \\
429 \varnothing \varnothing\end{array}$ & $\begin{array}{l}170.0 \\
157.0 \\
171.0 \\
159.0 \\
166.0\end{array}$ & $\begin{array}{r}153 . \bar{\varnothing} \\
157 . \overline{3} \\
160.0\end{array}$ & $\begin{array}{l}138 . \bar{\varnothing} \\
159.8 \\
144 . \varnothing \\
149.8\end{array}$ & $\begin{array}{l}58 . \varnothing \\
75 . \varnothing \\
62 . \varnothing \\
68 . \varnothing \\
65 . \varnothing\end{array}$ & $\begin{array}{r}26.0 \\
42.3 \\
25.0 \\
6.2 \\
20.0\end{array}$ & $\begin{array}{l}1 \\
1 \\
1 \\
1 \\
1\end{array}$ & $\begin{array}{l}1 \\
1 \\
1 \\
4 \\
1\end{array}$ & $\begin{array}{l}1 \\
1 \\
1 \\
1 \\
1\end{array}$ & $\begin{array}{l}1 \\
1 \\
7 \\
7 \\
7\end{array}$ \\
\hline
\end{tabular}


Appendix.--Location and basic physical features of recharge basins on Long Island,

\begin{tabular}{|c|c|c|c|c|c|c|c|c|}
\hline \multicolumn{2}{|c|}{$\begin{array}{l}\text { BASIN } \\
\text { NUMBER }\end{array}$} & \multicolumn{2}{|c|}{ NEAREST INTERSECTION } & $\begin{array}{l}\text { LATITUDE } \\
\text { LONGITUDE } \\
\circ, " \circ, "\end{array}$ & $\begin{array}{l}\text { DATE } \\
\text { BUILT } \\
\text { YrMOD }\end{array}$ & COMMUNITY & \multicolumn{2}{|c|}{$\begin{array}{l}\text { DESIGN ACTUAL } \\
\text { CAPACITY CAPACITY } \\
\text { (cubic feet) }\end{array}$} \\
\hline $\begin{array}{l}\mathrm{H} \\
\mathrm{H} \\
\mathrm{H} \\
\mathrm{H} \\
\mathrm{H}\end{array}$ & $\begin{array}{l}116 \\
117 \\
119 \\
120 \\
121\end{array}$ & $\begin{array}{l}\text { CORTLAND COURT } \\
\text { END OF MAXESS RD } \\
\text { SILVER AVENUE } \\
\text { CORDELL PLACE } \\
\text { CORDELL PLACE }\end{array}$ & $\begin{array}{l}\text { ASSY LANE } \\
\text { LODGE AVENUE } \\
\text { HULL PLACE } \\
\text { HULL PLACE }\end{array}$ & $\begin{array}{l}4 \emptyset 4921 \varnothing 732348 \\
4 \emptyset 4628 \emptyset 732447 \\
4 \varnothing 5 \emptyset 15 \emptyset 732259 \\
4 \varnothing 5239 \emptyset 731826 \\
4 \varnothing 5232 \varnothing 731837\end{array}$ & $\begin{array}{l}551026 \\
631018 \\
530908 \\
5503 \\
580821\end{array}$ & $\begin{array}{l}151 \\
148 \\
151 \\
137 \\
137\end{array}$ & $\begin{array}{l}105300 \\
277700 \\
102000 \\
- \\
122000\end{array}$ & $\begin{array}{l}- \\
4124 \varnothing \varnothing \\
104000 \\
173000 \\
155000\end{array}$ \\
\hline $\begin{array}{l}\mathrm{H} \\
\mathrm{H} \\
\mathrm{H} \\
\mathrm{H} \\
\mathrm{H}\end{array}$ & $\begin{array}{l}122 \\
123 \\
124 \\
125 \\
126\end{array}$ & $\begin{array}{l}\text { E. END OF SQUIRE } \\
\text { GILDARE DRIVE } \\
\text { NEW YORK AVENUE } \\
\text { DANVILLE DRIVE } \\
\text { KETAY DRIVE SO. }\end{array}$ & $\begin{array}{l}\text { CLAY PITTS ROAD } \\
\text { NEWTOWN LANE } \\
\text { DARROW LANE } \\
\text { HASKEL LANE }\end{array}$ & 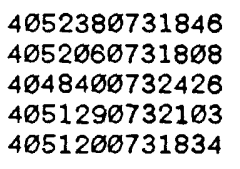 & $\begin{array}{c}- \\
590821 \\
580527 \\
640617 \\
591105\end{array}$ & $\begin{array}{l}137 \\
137 \\
151 \\
141 \\
169\end{array}$ & $\begin{array}{l}- \\
371100 \\
\overline{13} \\
2546000\end{array}$ & $\begin{array}{l}- \\
380000 \\
- \\
190000 \\
274000\end{array}$ \\
\hline $\begin{array}{l}\mathrm{H} \\
\mathrm{H} \\
\mathrm{H} \\
\mathrm{H} \\
\mathrm{H}\end{array}$ & $\begin{array}{l}127 \\
128 \\
129 \\
130 \\
131\end{array}$ & $\begin{array}{l}\text { DANVILLE DRIVE } \\
\text { GREG LANE } \\
\text { HASKIN PLACE } \\
\text { POLO STREET } \\
\text { MCNULTY STREET }\end{array}$ & $\begin{array}{l}\text { DARROW LANE } \\
\text { BURR ROAD } \\
\text { CEDAR CREST DR. } \\
\text { KILMER AVENUE } \\
\text { LAUREN AVENUE }\end{array}$ & $\begin{array}{l}4 \varnothing 5123073205 \emptyset \\
4 \varnothing 51 \varnothing 90731743 \\
4 \emptyset 4746 \varnothing 7318 \emptyset 9 \\
4 \varnothing 47430731745 \\
4 \varnothing 4753 \emptyset 731756\end{array}$ & $\begin{array}{l}640617 \\
6012 \\
591627 \\
591027 \\
591027\end{array}$ & $\begin{array}{l}141 \\
169 \\
- \\
- \\
-\end{array}$ & $\begin{array}{l}1755 \varnothing \varnothing \\
13 \varnothing 8 \varnothing \varnothing \\
475 \varnothing \varnothing \varnothing \\
2 \varnothing 5 \varnothing \varnothing \varnothing \\
545 \varnothing \varnothing \varnothing\end{array}$ & $\begin{array}{l}190000 \\
136800 \\
482000 \\
215000 \\
555000\end{array}$ \\
\hline $\begin{array}{l}\mathrm{H} \\
\mathrm{H} \\
\mathrm{H} \\
\mathrm{H} \\
\mathrm{H}\end{array}$ & $\begin{array}{l}132 \\
133 \\
134 \\
135 \\
136\end{array}$ & $\begin{array}{l}\text { PINE HILL LANE } \\
\text { KINSELLA STREET } \\
\text { NORWOOD ROAD } \\
\text { STARLIT DRIVE } \\
\text { GREENVALE DRIVE }\end{array}$ & $\begin{array}{l}\text { DULCE LANE } \\
\text { PINE HILL LANE } \\
\text { MOONEDGE ROAD } \\
\text { NORWOOD ROAD } \\
\text { ELMBARK LANE }\end{array}$ & $\begin{array}{l}464746 \varnothing 731832 \\
4 \varnothing 4736 \varnothing 73181 \emptyset \\
465434 \varnothing 731921 \\
4 \varnothing 5438 \varnothing 731935 \\
4 \varnothing 514 \varnothing \emptyset 731939\end{array}$ & $\begin{array}{l}591627 \\
591027 \\
600606 \\
620629 \\
630416\end{array}$ & $\begin{array}{l}- \\
\overline{149} \\
149 \\
134\end{array}$ & $\begin{array}{l}329000 \\
250000 \\
343100 \\
170000 \\
186400\end{array}$ & $\begin{array}{l}335000 \\
255000 \\
377000 \\
180000 \\
201000\end{array}$ \\
\hline $\begin{array}{l}H \\
H \\
H \\
H \\
H\end{array}$ & $\begin{array}{l}137 \\
138 \\
139 \\
146 \\
141\end{array}$ & $\begin{array}{l}\text { BURTON LANE } \\
\text { EDCRIS LANE } \\
\text { PULASKI ROAD } \\
\text { ESTHER PLACE } \\
\text { GODFREY LANE }\end{array}$ & $\begin{array}{l}\text { DRYDEN WAY } \\
\text { OAKWOOD ROAD } \\
\text { GUN HILL DRIVE } \\
\text { 5TH AVENUE } \\
\text { WARNER ROAD }\end{array}$ & $\begin{array}{l}4050440731739 \\
4 \varnothing 50580732557 \\
4 \varnothing 5257 \emptyset 731744 \\
4 \varnothing 52410731733 \\
4 \varnothing 5 \emptyset 24 \emptyset 732 \emptyset 42\end{array}$ & $\begin{array}{l}580228 \\
568503 \\
570409 \\
6207 \\
550228\end{array}$ & $\begin{array}{c}169 \\
- \\
137 \\
\overline{139}\end{array}$ & $\begin{array}{l}- \\
- \\
- \\
138300\end{array}$ & $\begin{array}{l}\overline{84500} \\
\overline{6} \\
150000 \\
156000\end{array}$ \\
\hline $\begin{array}{l}H \\
H \\
H \\
H \\
H\end{array}$ & $\begin{array}{l}142 \\
143 \\
144 \\
145 \\
146\end{array}$ & $\begin{array}{l}\text { GODFREY LANE } \\
\text { SHORTHILL LANE } \\
\text { LITTLE PLAINS RD } \\
\text { BUNKERHILL DRIVE } \\
\text { BUNKERHILL ORIVE }\end{array}$ & $\begin{array}{l}\text { BROOKHILL LANE } \\
\text { BROOKHILL LANE } \\
\text { GREENHILL LANE } \\
\text { STONEHILL LANE } \\
\text { HEATHERHILL LA. }\end{array}$ & 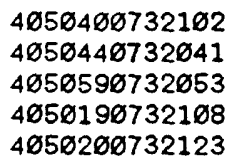 & $\begin{array}{c}- \\
- \\
- \\
578527 \\
578527\end{array}$ & $\begin{array}{l}139 \\
139 \\
139 \\
139 \\
139\end{array}$ & $\begin{array}{l}- \\
- \\
\overline{288000} \\
86500\end{array}$ & $\begin{array}{l}- \\
- \\
- \\
102500\end{array}$ \\
\hline $\begin{array}{l}H \\
H \\
H \\
H \\
H\end{array}$ & $\begin{array}{l}147 \\
148 \\
149 \\
150 \\
151\end{array}$ & $\begin{array}{l}\text { BUNKERHILL DRIVE } \\
\text { WOLF HILL ROAD } \\
\text { WHITE BIRCH DR. } \\
\text { PLANE TREE LANE } \\
\text { BALSAM DRIVE }\end{array}$ & $\begin{array}{l}\text { GODFREY LANE } \\
\text { WHITE BIRCH DR. } \\
\text { RED MAPLE LANE } \\
\text { PEAR ROAD } \\
\text { MAGNOLIA LANE }\end{array}$ & $\begin{array}{l}4 \emptyset 5 \varnothing 27 \varnothing 732055 \\
4 \emptyset 4844 \varnothing 732 \emptyset 44 \\
4 \emptyset 4857 \varnothing 732048 \\
4 \varnothing 4847 \varnothing 732136 \\
4 \varnothing 4852 \varnothing 732118\end{array}$ & $\begin{array}{l}576527 \\
680622 \\
600622 \\
598224 \\
560719\end{array}$ & $\begin{array}{l}139 \\
133 \\
133 \\
133 \\
133\end{array}$ & $\begin{array}{l}- \\
111200 \\
1287 \varnothing \varnothing \\
1810 \varnothing \varnothing \\
403800\end{array}$ & $\begin{array}{l}- \\
175000 \\
160000 \\
230000 \\
455000\end{array}$ \\
\hline $\begin{array}{l}H \\
H \\
H \\
H \\
H\end{array}$ & $\begin{array}{l}152 \\
153 \\
154 \\
155 \\
156\end{array}$ & $\begin{array}{l}\text { CEDRUS AVENUE } \\
\text { MANNING DRIVE } \\
\text { FLOWER HILL ROAD } \\
\text { CEDAR ROAD } \\
\text { CEDAR ROAD }\end{array}$ & $\begin{array}{l}\text { OLMSTEAD LANE } \\
\text { YEOMAN DRIVE } \\
\text { FLOWERFIELD CT. } \\
\text { FORSYTHE DRIVE } \\
\text { RICHLEE DRIVE }\end{array}$ & $\begin{array}{l}4051490731802 \\
4 ø 51000731838 \\
4 \emptyset 53460732341 \\
4 ø 5137 \varnothing 731807 \\
4 \varnothing 51380731759\end{array}$ & $\begin{array}{l}690609 \\
570601 \\
560320 \\
680613 \\
600613\end{array}$ & $\begin{array}{l}169 \\
169 \\
- \\
169 \\
169\end{array}$ & $\begin{array}{l}6 \varnothing \varnothing \varnothing \varnothing \varnothing ~ \\
- \\
\overline{13 \varnothing 8 \varnothing \varnothing} \\
-\end{array}$ & $\begin{array}{l}630000 \\
- \\
220000 \\
142000 \\
-\end{array}$ \\
\hline $\begin{array}{l}H \\
H \\
H \\
H \\
H\end{array}$ & $\begin{array}{l}157 \\
158 \\
159 \\
160 \\
161\end{array}$ & $\begin{array}{l}\text { CLAY PITTS ROAD } \\
\text { LANTERN STREET } \\
\text { SHELBOURNE LANE } \\
\text { OAKWOOD ROAD } \\
\text { CORNFIELD LANE }\end{array}$ & $\begin{array}{l}\text { ELWOOD ROAD } \\
\text { DEKALB PLACE } \\
\text { LOCKSLEY LANE } \\
\text { KNOLL LANE } \\
\text { HEDGEROW LANE }\end{array}$ & $\begin{array}{l}4 \varnothing 5144 \varnothing 731955 \\
4 \varnothing 5048 \varnothing 732223 \\
4 \varnothing 5 \varnothing 310731849 \\
4 \varnothing 51190732546 \\
4 \varnothing 5 \varnothing 37 \varnothing 731816\end{array}$ & $\begin{array}{l}630909 \\
550531 \\
591287 \\
550527 \\
6503\end{array}$ & $\begin{array}{c}- \\
\overline{169} \\
146 \\
169\end{array}$ & $\begin{array}{l}236300 \\
- \\
236000 \\
- \\
273000\end{array}$ & $\begin{array}{l}252500 \\
610000 \\
247000 \\
150000 \\
292200\end{array}$ \\
\hline $\begin{array}{l}H \\
H \\
H \\
H \\
H\end{array}$ & $\begin{array}{l}163 \\
164 \\
165 \\
166 \\
167\end{array}$ & $\begin{array}{l}\text { LARKFIELD ROAD } \\
\text { HEDGEROW LANE } \\
\text { N OF MANSFIELD L } \\
\text { LORIJEAN LANE } \\
\text { VISTA LANE }\end{array}$ & $\begin{array}{l}\text { ALICE LANE } \\
\text { WISTERIA WAY } \\
\text { ROCKROSE LANE } \\
\text { TREEVIEW LANE }\end{array}$ & $\begin{array}{l}4 \varnothing 5 \varnothing 16 \varnothing 731843 \\
4 \varnothing 5 \varnothing 46 \varnothing 731866 \\
4 \varnothing 51396731924 \\
4 \varnothing 5133 \varnothing 73194 \emptyset \\
4 \varnothing 47430732517\end{array}$ & $\begin{array}{l}680829 \\
680829 \\
631121 \\
631121 \\
5788\end{array}$ & $\begin{array}{l}- \\
169 \\
139 \\
139 \\
148\end{array}$ & $\begin{array}{r}900 \varnothing 0 \\
88400 \\
5990 \varnothing 0 \\
226580 \\
296 \varnothing \varnothing \varnothing\end{array}$ & $\begin{array}{r}120000 \\
104800 \\
676300 \\
243000 \\
23500\end{array}$ \\
\hline $\begin{array}{l}H \\
H \\
H \\
H \\
H\end{array}$ & $\begin{array}{l}169 \\
170 \\
171 \\
172 \\
173\end{array}$ & $\begin{array}{l}\text { LOCUST COURT } \\
\text { HORIZON DRIVE } \\
\text { ALLISON COURT } \\
\text { PANORAMA DRIVE } \\
\text { BURR ROAD }\end{array}$ & $\begin{array}{l}\text { TALL OAK DRIVE } \\
\text { PANORAMA DRIVE } \\
\text { TOWER STREET } \\
\text { TERRACE DRIVE } \\
\text { TERESA PLACE }\end{array}$ & $\begin{array}{l}4 \varnothing 512507326 \varnothing 4 \\
4651210732521 \\
4 \varnothing 512207325 \varnothing 4 \\
4 \varnothing 510867325 \varnothing 8 \\
4656510731938\end{array}$ & $\begin{array}{l}600126 \\
590917 \\
590917 \\
596917 \\
610802\end{array}$ & $\begin{array}{l}146 \\
146 \\
146 \\
146 \\
139\end{array}$ & $\begin{array}{r}- \\
4170000 \\
64800 \\
171000 \\
245300\end{array}$ & $\begin{array}{l}- \\
417000 \\
83060 \\
244600 \\
2496 \varnothing 0\end{array}$ \\
\hline
\end{tabular}


N.Y. [Dash indicates no data. Altitudes are in feet above sea level.] (cont.)

\begin{tabular}{|c|c|c|c|c|c|c|c|c|c|c|c|c|}
\hline & $\begin{array}{l}\text { SIN } \\
\text { ABER }\end{array}$ & $\begin{array}{l}\text { MAX. } \\
\text { AREA } \\
\text { (squar }\end{array}$ & $\begin{array}{c}\text { BASIN } \\
\text { AREA } \\
\text { feet) }\end{array}$ & RIM & $\begin{array}{l}\text {-ALT } \\
\text { OVER } \\
\text { FLOW }\end{array}$ & $\begin{array}{c}\text { UDE--- } \\
\text { BOT- } \\
\text { TOM }\end{array}$ & $\begin{array}{l}\text { WATER } \\
\text { TABLE }\end{array}$ & $\begin{array}{c}\text { DRAIN. } \\
\text { AREA } \\
\text { (acres) }\end{array}$ & $\begin{array}{l}-- \text {-BASI } \\
\text { STATUS }\end{array}$ & $\begin{array}{l}\text { IN-- } \\
\text { USE }\end{array}$ & $\begin{array}{l}\text { GEO. } \\
\text { UNIT }\end{array}$ & $\begin{array}{l}\text { SOIL } \\
\text { UNIT }\end{array}$ \\
\hline $\begin{array}{l}H \\
H \\
H\end{array}$ & $\begin{array}{l}116 \\
117 \\
119 \\
120 \\
121\end{array}$ & $\begin{array}{l}\overline{412 \theta 0} \\
104 \theta 0 \\
157 \varnothing 0 \\
15500\end{array}$ & $\begin{array}{l}14300 \\
49500 \\
38500 \\
30000 \\
24700\end{array}$ & $\begin{array}{l}225.0 \\
116.0 \\
250 . \emptyset \\
192.2 \\
196.7\end{array}$ & $\begin{array}{r}- \\
\overline{-} \\
190 . \overline{7} \\
-\end{array}$ & $\begin{array}{r}175.4 \\
100.5 \\
- \\
192.2 \\
184.5\end{array}$ & $\begin{array}{l}70.0 \\
65.0 \\
66.0 \\
53.0 \\
52.0\end{array}$ & $\begin{array}{r}16.6 \\
8.5 \\
14.0 \\
27.2 \\
16.0\end{array}$ & $\begin{array}{l}1 \\
1 \\
1 \\
1 \\
1\end{array}$ & $\begin{array}{l}1 \\
1 \\
1 \\
1 \\
1\end{array}$ & $\begin{array}{l}1 \\
1 \\
5 \\
1 \\
1\end{array}$ & $\begin{array}{l}9 \\
0 \\
7 \\
7 \\
7\end{array}$ \\
\hline $\begin{array}{l}\mathrm{H} \\
\mathrm{H} \\
\mathrm{H} \\
\mathrm{H} \\
\mathrm{H}\end{array}$ & $\begin{array}{l}122 \\
123 \\
124 \\
125 \\
126\end{array}$ & $\begin{array}{c}\overline{-} \\
27100 \\
\overline{-} \\
19000 \\
27400\end{array}$ & $\begin{array}{l}- \\
38000 \\
50000 \\
34000 \\
35700\end{array}$ & $\begin{array}{l}205 . \emptyset \\
176 . \emptyset \\
152.1 \\
226.5 \\
16 \varnothing . \emptyset\end{array}$ & $\begin{array}{r}175 . \overline{5} \\
- \\
- \\
-\end{array}$ & $\begin{array}{l}- \\
161.5 \\
140.0 \\
215.0 \\
147.0\end{array}$ & $\begin{array}{l}52.0 \\
57.0 \\
72.0 \\
63.0 \\
62.0\end{array}$ & $\begin{array}{r}58 . \overline{4} \\
\overline{-} \\
15.0 \\
29.0\end{array}$ & $\begin{array}{l}1 \\
1 \\
1 \\
1 \\
1\end{array}$ & $\begin{array}{l}1 \\
1 \\
1 \\
1 \\
1\end{array}$ & $\begin{array}{l}1 \\
1 \\
1 \\
1 \\
1\end{array}$ & $\begin{array}{l}7 \\
7 \\
7 \\
7 \\
7\end{array}$ \\
\hline $\begin{array}{l}H \\
H \\
H \\
H \\
H \\
H\end{array}$ & $\begin{array}{l}127 \\
128 \\
129 \\
130 \\
131\end{array}$ & $\begin{array}{l}19000 \\
13600 \\
48200 \\
21500 \\
55500\end{array}$ & $\begin{array}{l}34200 \\
32000 \\
70000 \\
30400 \\
61600\end{array}$ & $\begin{array}{l}270.0 \\
145.6 \\
108.1 \\
106.4 \\
113.0\end{array}$ & $\begin{array}{r}- \\
- \\
106.0 \\
105.5 \\
112.5\end{array}$ & $\begin{array}{r}223.0 \\
135.5 \\
96.0 \\
95.5 \\
102.5\end{array}$ & $\begin{array}{l}66.0 \\
66.0 \\
55 . \varnothing \\
53.0 \\
55 . \varnothing\end{array}$ & $\begin{array}{l}20.0 \\
16.0 \\
74.8 \\
32.3 \\
85.8\end{array}$ & $\begin{array}{l}0 \\
1 \\
1 \\
1 \\
1\end{array}$ & $\begin{array}{l}1 \\
1 \\
1 \\
1 \\
1\end{array}$ & $\begin{array}{l}5 \\
1 \\
1 \\
1 \\
1\end{array}$ & $\begin{array}{l}1 \\
7 \\
7 \\
7 \\
7\end{array}$ \\
\hline $\begin{array}{l}\mathrm{H} \\
\mathrm{H} \\
\mathrm{H} \\
\mathrm{H} \\
\mathrm{H}\end{array}$ & $\begin{array}{l}132 \\
133 \\
134 \\
135 \\
136\end{array}$ & $\begin{array}{l}33500 \\
25500 \\
37700 \\
18000 \\
20100\end{array}$ & $\begin{array}{l}64000 \\
46500 \\
36400 \\
34100 \\
33600\end{array}$ & $\begin{array}{l}121.0 \\
104.1 \\
104.0 \\
105.0 \\
185.0\end{array}$ & $\begin{array}{r}119.8 \\
102.5 \\
- \\
- \\
-\end{array}$ & $\begin{array}{r}109.0 \\
92.5 \\
92.5 \\
91.0 \\
64.2\end{array}$ & $\begin{array}{l}56.0 \\
54.8 \\
26.8 \\
25.8 \\
62.8\end{array}$ & $\begin{array}{l}51.8 \\
39.4 \\
39.1 \\
26.0 \\
29.3\end{array}$ & $\begin{array}{l}1 \\
1 \\
1 \\
1 \\
1\end{array}$ & $\begin{array}{l}1 \\
1 \\
1 \\
1 \\
1\end{array}$ & $\begin{array}{l}1 \\
1 \\
3 \\
1 \\
1\end{array}$ & $\begin{array}{l}7 \\
7 \\
7 \\
7 \\
7\end{array}$ \\
\hline $\begin{array}{l}H \\
H \\
H \\
H \\
H\end{array}$ & $\begin{array}{l}137 \\
138 \\
139 \\
148 \\
141\end{array}$ & $\begin{array}{c}- \\
5900 \\
- \\
63000 \\
15600\end{array}$ & $\begin{array}{l}- \\
40000 \\
28000 \\
62400 \\
21600\end{array}$ & $\begin{array}{l}140.0 \\
223.0 \\
185.0 \\
167.5 \\
232.2\end{array}$ & $\begin{array}{r}- \\
222.4 \\
- \\
- \\
-\end{array}$ & $\begin{array}{l}- \\
208.0 \\
181.3 \\
146.0 \\
222.0\end{array}$ & $\begin{array}{l}68.0 \\
55.8 \\
50.0 \\
50.0 \\
66.0\end{array}$ & $\begin{array}{r}13 . \overline{3} \\
-\overline{2} \\
99 . \overline{8} \\
72.0\end{array}$ & $\begin{array}{l}1 \\
1 \\
1 \\
1 \\
1\end{array}$ & $\begin{array}{l}1 \\
1 \\
1 \\
1 \\
1\end{array}$ & $\begin{array}{l}1 \\
1 \\
1 \\
1 \\
5\end{array}$ & $\begin{array}{l}7 \\
7 \\
7 \\
7 \\
1\end{array}$ \\
\hline $\begin{array}{l}\mathrm{H} \\
\mathrm{H} \\
\mathrm{H} \\
\mathrm{H} \\
\mathrm{H}\end{array}$ & $\begin{array}{l}142 \\
143 \\
144 \\
145 \\
146\end{array}$ & $\begin{array}{c}- \\
- \\
- \\
10300\end{array}$ & $\begin{array}{l}28000 \\
12800 \\
27200 \\
32000 \\
26300\end{array}$ & $\begin{array}{l}274.3 \\
245.18 \\
227.5 \\
268.0 \\
228.0\end{array}$ & $\begin{array}{l}- \\
- \\
- \\
-\end{array}$ & $\begin{array}{l}265.0 \\
235.0 \\
217.0 \\
258.5 \\
215.0\end{array}$ & $\begin{array}{l}66.0 \\
66.0 \\
65.0 \\
67.0 \\
67.0\end{array}$ & $\begin{array}{r}25.2 \\
- \\
- \\
25.5 \\
9.0\end{array}$ & $\begin{array}{l}1 \\
1 \\
1 \\
0 \\
1\end{array}$ & $\begin{array}{l}1 \\
1 \\
1 \\
1 \\
1\end{array}$ & $\begin{array}{l}5 \\
5 \\
5 \\
5 \\
5\end{array}$ & $\begin{array}{l}1 \\
1 \\
3 \\
1 \\
5\end{array}$ \\
\hline $\begin{array}{l}\mathrm{H} \\
\mathrm{H} \\
\mathrm{H} \\
\mathrm{H} \\
\mathrm{H}\end{array}$ & $\begin{array}{l}147 \\
148 \\
149 \\
150 \\
151\end{array}$ & $\begin{array}{c}- \\
1750 \varnothing \\
16 \varnothing \emptyset \emptyset \\
230 \varnothing \emptyset \\
4550 \emptyset\end{array}$ & $\begin{array}{l}32000 \\
21800 \\
21600 \\
44000 \\
21500\end{array}$ & $\begin{array}{l}250.6 \\
263.0 \\
261 . \varnothing \\
245.5 \\
258 . \varnothing\end{array}$ & $\begin{array}{l}- \\
- \\
-\end{array}$ & $\begin{array}{l}230.2 \\
251.0 \\
251.0 \\
230 . \varnothing \\
242 . \varnothing\end{array}$ & $\begin{array}{l}66.0 \\
72.0 \\
73.0 \\
73.0 \\
74.0\end{array}$ & $\begin{array}{l}19.0 \\
22.0 \\
31.0 \\
69.0\end{array}$ & $\begin{array}{l}1 \\
1 \\
1 \\
1 \\
1\end{array}$ & $\begin{array}{l}1 \\
1 \\
1 \\
1 \\
1\end{array}$ & $\begin{array}{l}5 \\
1 \\
6 \\
6 \\
3\end{array}$ & $\begin{array}{l}5 \\
1 \\
9 \\
9 \\
9\end{array}$ \\
\hline $\begin{array}{l}\mathrm{H} \\
\mathrm{H} \\
\mathrm{H} \\
\mathrm{H} \\
\mathrm{H}\end{array}$ & $\begin{array}{l}152 \\
153 \\
154 \\
155 \\
156\end{array}$ & $\begin{array}{c}63000 \\
- \\
22000 \\
14200 \\
-\end{array}$ & $\begin{array}{r}66000 \\
125000 \\
38480 \\
248010 \\
2990 \varnothing\end{array}$ & $\begin{array}{l}171 . \varnothing \\
177.4 \\
167.8 \\
170 . \varnothing \\
168 . \varnothing\end{array}$ & $\begin{array}{l}- \\
- \\
- \\
-\end{array}$ & $\begin{array}{l}155.0 \\
166 . \varnothing \\
163.4 \\
153 . \varnothing \\
151.0\end{array}$ & $\begin{array}{l}67 . \overline{0} \\
30.0 \\
62.0 \\
61.0\end{array}$ & $\begin{array}{r}110.0 \\
- \\
34.6 \\
24.0 \\
-\end{array}$ & $\begin{array}{l}1 \\
1 \\
1 \\
1 \\
1\end{array}$ & $\begin{array}{l}1 \\
1 \\
1 \\
1 \\
1\end{array}$ & $\begin{array}{l}1 \\
1 \\
3 \\
1 \\
1\end{array}$ & $\begin{array}{l}7 \\
7 \\
1 \\
7 \\
7\end{array}$ \\
\hline $\begin{array}{l}H \\
H \\
H \\
H \\
H\end{array}$ & $\begin{array}{l}157 \\
158 \\
159 \\
160 \\
161\end{array}$ & $\begin{array}{l}194 \varnothing \emptyset \\
6780 \emptyset \\
2 \varnothing 90 \emptyset \\
1880 \emptyset \\
2920 \emptyset\end{array}$ & $\begin{array}{r}41600 \\
129600 \\
47600 \\
39900 \\
59800\end{array}$ & $\begin{array}{l}183.8 \\
195.0 \\
173.2 \\
255.0 \\
160.0\end{array}$ & $\begin{array}{r}181.1 \\
134.0 \\
165.3 \\
151.8 \\
-\end{array}$ & $\begin{array}{l}168.1 \\
125.8 \\
153.5 \\
142.8 \\
149.8\end{array}$ & $\begin{array}{l}62.0 \\
62.0 \\
70.0 \\
51.0 \\
67.0\end{array}$ & $\begin{array}{l}37.2 \\
96.0 \\
36.0 \\
23.6 \\
27.0\end{array}$ & $\begin{array}{l}1 \\
1 \\
1 \\
1 \\
1\end{array}$ & $\begin{array}{l}1 \\
1 \\
1 \\
1 \\
1\end{array}$ & $\begin{array}{l}1 \\
1 \\
1 \\
4 \\
1\end{array}$ & $\begin{array}{l}7 \\
7 \\
7 \\
8 \\
7\end{array}$ \\
\hline $\begin{array}{l}H \\
H \\
H \\
H \\
H\end{array}$ & $\begin{array}{l}163 \\
164 \\
165 \\
166 \\
167\end{array}$ & $\begin{array}{l}12000 \\
10500 \\
67600 \\
19600 \\
23500\end{array}$ & $\begin{array}{r}24200 \\
25000 \\
106400 \\
33600 \\
39200\end{array}$ & $\begin{array}{l}163.5 \\
145.7 \\
179.6 \\
185.7 \\
200.0\end{array}$ & $\begin{array}{r}- \\
- \\
175 . \emptyset \\
183.4 \\
143.0\end{array}$ & $\begin{array}{l}149.7 \\
133.1 \\
165.0 \\
171 . \emptyset \\
128 . \emptyset\end{array}$ & $\begin{array}{l}71.0 \\
68.0 \\
62.0 \\
63.0 \\
72.0\end{array}$ & $\begin{array}{r}14.2 \\
9.0 \\
47.6 \\
27.7 \\
30.0\end{array}$ & $\begin{array}{l}1 \\
1 \\
1 \\
1 \\
1\end{array}$ & $\begin{array}{l}1 \\
1 \\
1 \\
1 \\
1\end{array}$ & $\begin{array}{l}1 \\
1 \\
1 \\
1 \\
8\end{array}$ & $\begin{array}{l}7 \\
7 \\
7 \\
7 \\
5\end{array}$ \\
\hline $\begin{array}{l}H \\
H \\
H \\
H \\
H\end{array}$ & $\begin{array}{l}169 \\
17 \varnothing \\
171 \\
172 \\
173\end{array}$ & $\begin{array}{r}- \\
417 \varnothing 0 \\
8300 \\
12200 \\
24900\end{array}$ & $\begin{array}{l}19200 \\
42000 \\
32400 \\
28000 \\
50000\end{array}$ & $\begin{array}{l}285.0 \\
230 . \emptyset \\
240 . \emptyset \\
235 . \emptyset \\
185 . \emptyset\end{array}$ & $\begin{array}{r}249 . \varnothing \\
224 . \varnothing \\
235 . \varnothing \\
-\end{array}$ & $\begin{array}{l}237 . \varnothing \\
202 . \varnothing \\
212.0 \\
215.0 \\
171.5\end{array}$ & $\begin{array}{l}48.0 \\
52.0 \\
53.0 \\
55.0 \\
68.0\end{array}$ & $\begin{array}{r}37 . \bar{\sigma} \\
8 . \overline{5} \\
15.2 \\
30 . \varnothing\end{array}$ & $\begin{array}{l}1 \\
0 \\
1 \\
1 \\
1\end{array}$ & $\begin{array}{l}1 \\
4 \\
1 \\
1 \\
1\end{array}$ & $\begin{array}{l}1 \\
4 \\
4 \\
4 \\
1\end{array}$ & $\begin{array}{l}8 \\
7 \\
8 \\
7 \\
7\end{array}$ \\
\hline
\end{tabular}


Appendix.--Location and basic physical features of recharge basins on Long Island,

\begin{tabular}{|c|c|c|c|c|c|c|c|c|}
\hline \multicolumn{2}{|c|}{$\begin{array}{l}\text { BASIN } \\
\text { NUMBER }\end{array}$} & \multicolumn{2}{|c|}{ NEAREST INTERSECTION } & $\begin{array}{l}\text { LATITUDE } \\
\text { LONGITUDE } \\
\circ, ", *\end{array}$ & $\begin{array}{l}\text { DATE } \\
\text { BUILT } \\
\text { YrMOD }\end{array}$ & COMMUNITY & \multicolumn{2}{|c|}{$\begin{array}{l}\text { DESIGN ACTUAL } \\
\text { CAPACITY CAPACITY } \\
\text { (cubic feet) }\end{array}$} \\
\hline $\begin{array}{l}H \\
H \\
H \\
H \\
H\end{array}$ & $\begin{array}{l}174 \\
175 \\
176 \\
178 \\
179\end{array}$ & $\begin{array}{l}\text { PUMPKIN STREET } \\
\text { CYRIL DRIVE } \\
\text { HOFSTRA DRIVE } \\
\text { FORT SALONGA RD. } \\
\text { LANDVIEW DRIVE }\end{array}$ & $\begin{array}{l}\text { GREYSTONE DRIVE } \\
\text { EDCRIS LANE } \\
\text { MONETT PLACE } \\
\text { TOMPKINS STREET } \\
\text { BEATRICE COURT }\end{array}$ & $\begin{array}{l}4 \varnothing 5151 \varnothing 731856 \\
4 \varnothing 5 \varnothing 5607326 \varnothing 8 \\
4 \varnothing 5127 \varnothing 732242 \\
4 \varnothing 53280732 \varnothing 29 \\
4 \varnothing 48090732059\end{array}$ & $\begin{array}{l}5707 \varnothing 8 \\
630813 \\
641106 \\
6107 \\
621016\end{array}$ & $\begin{array}{l}- \\
146 \\
141 \\
149 \\
-\end{array}$ & $\begin{array}{l}270100 \\
141700 \\
- \\
110500 \\
298400\end{array}$ & $\begin{array}{l}320000 \\
224000 \\
- \\
174000 \\
303600\end{array}$ \\
\hline $\begin{array}{l}\mathrm{H} \\
\mathrm{H} \\
\mathrm{H} \\
\mathrm{H} \\
\mathrm{H}\end{array}$ & $\begin{array}{l}180 \\
181 \\
182 \\
183 \\
184\end{array}$ & $\begin{array}{l}\text { CAROLINE DRIVE } \\
\text { LIE SERVICE ROAD } \\
\text { STOOTHOFF ROAD } \\
\text { DANVILLE DRIVE } \\
\text { CARDINAL LANE }\end{array}$ & $\begin{array}{l}\text { REGINA AVENUE } \\
\text { MCCULLOCH DRIVE } \\
\text { LEWIS AVENUE } \\
\text { DANVILLE COURT } \\
\text { JAN PLACE }\end{array}$ & $\begin{array}{l}4 \varnothing 4807 \varnothing 731900 \\
404802 \varnothing 731839 \\
4052260731754 \\
4 \varnothing 51350732108 \\
4 \varnothing 5 \varnothing 510731949\end{array}$ & $\begin{array}{l}650928 \\
650928 \\
580210 \\
640629 \\
630219\end{array}$ & $\begin{array}{c}133 \\
- \\
137 \\
141\end{array}$ & $\begin{array}{c}126700 \\
137400 \\
- \\
80700 \\
214600\end{array}$ & $\begin{array}{r}141200 \\
151200 \\
60000 \\
94200 \\
240000\end{array}$ \\
\hline $\begin{array}{l}\mathrm{H} \\
\mathrm{H} \\
\mathrm{H} \\
\mathrm{H} \\
\mathrm{H}\end{array}$ & $\begin{array}{l}185 \\
187 \\
188 \\
189 \\
190\end{array}$ & $\begin{array}{l}\text { EAST GATE DRIVE } \\
\text { LEEDS STREET } \\
\text { DOROTHEA STREET } \\
\text { WICKS ROAD } \\
\text { HIGHFIELD DRIVE }\end{array}$ & $\begin{array}{l}\text { COLDPORT DRIVE } \\
\text { NOAH PLACE } \\
\text { RACHEL AVENUE } \\
\text { DALY ROAD } \\
\text { ETNA LANE }\end{array}$ & $\begin{array}{l}4049570732711 \\
404917 \varnothing 732352 \\
4050250731741 \\
4049540731904 \\
4047500732200\end{array}$ & $\begin{array}{l}630813 \\
560216 \\
5602 \\
620202 \\
580331\end{array}$ & $\frac{-}{169}$ & $\begin{array}{l}87600 \\
60000 \\
- \\
190000 \\
490000\end{array}$ & $\begin{array}{l}110000 \\
68000 \\
- \\
234000 \\
615000\end{array}$ \\
\hline $\begin{array}{l}H \\
H \\
H \\
H \\
H\end{array}$ & $\begin{array}{l}191 \\
192 \\
193 \\
194 \\
195\end{array}$ & $\begin{array}{l}\text { GRAND HAVEN DR. } \\
\text { MAPLEWOOD ROAD } \\
\text { LIE SERVICE ROAD } \\
\text { TITUS LANE } \\
\text { HALF HOLLOW ROAD }\end{array}$ & $\begin{array}{l}\text { SUN HAVEN LANE } \\
\text { BENNETT ROAD } \\
\text { STRAIGHT PATH RD } \\
\text { VALENTINE COURT } \\
\text { WESTCLIFF DRIVE }\end{array}$ & $\begin{array}{l}4049580731833 \\
4 \varnothing 5037 \emptyset 7323 \emptyset 4 \\
4 \varnothing 4803 \emptyset 731910 \\
4 \varnothing 52330732714 \\
4847470732231\end{array}$ & $\begin{array}{l}548615 \\
618911 \\
648127 \\
55113 \emptyset \\
561824\end{array}$ & $\begin{array}{c}169 \\
- \\
131\end{array}$ & $\begin{array}{l}- \\
98000 \\
127500 \\
- \\
-\end{array}$ & $\begin{array}{l}- \\
102300 \\
154000 \\
150000 \\
148000\end{array}$ \\
\hline $\begin{array}{l}\mathrm{H} \\
\mathrm{H} \\
\mathrm{H} \\
\mathrm{H} \\
\mathrm{H}\end{array}$ & $\begin{array}{l}196 \\
197 \\
198 \\
199 \\
290\end{array}$ & $\begin{array}{l}\text { BAY DRIVE } \\
\text { ALGONQUIN DRIVE } \\
\text { BIRCH PLACE } \\
\text { BUTTERFIELD DR. } \\
\text { WICKS DRIVE }\end{array}$ & $\begin{array}{l}\text { SOUNDVIEW DRIVE } \\
\text { MEROKE COURT } \\
\text { GRANGE STREET } \\
\text { FRAZER COURT } \\
\text { WICKS ROAD }\end{array}$ & $\begin{array}{l}4854020732415 \\
4 \varnothing 5106073234 \emptyset \\
4 \varnothing 51190732214 \\
4 \varnothing 51300732221 \\
4 \varnothing 50020731756\end{array}$ & $\begin{array}{l}541005 \\
600326 \\
640904 \\
640904 \\
620724\end{array}$ & $\begin{array}{c}145 \\
- \\
141 \\
169\end{array}$ & $\begin{array}{l}\overline{6} \\
264500 \\
655200 \\
225000\end{array}$ & $\begin{array}{r}- \\
83000 \\
280500 \\
739800 \\
275000\end{array}$ \\
\hline $\begin{array}{l}H \\
H \\
H \\
H \\
H\end{array}$ & $\begin{array}{l}202 \\
203 \\
204 \\
205 \\
206\end{array}$ & $\begin{array}{l}\text { VANDERBILT PKWY. } \\
\text { MCCULLOCH DRIVE } \\
\text { COLBY DRIVE } \\
\text { VILLAGE HILL DR. } \\
\text { VILLAGE HILL DR. }\end{array}$ & $\begin{array}{l}\text { MCLANE DRIVE } \\
\text { BLAINE DRIVE } \\
\text { VILLAGE HILL DR. } \\
\text { CAMPBELL DRIVE } \\
\text { OLNEY PLACE }\end{array}$ & $\begin{array}{l}4 \emptyset 49060731804 \\
4048380731818 \\
4048300731829 \\
4 \emptyset 48150731843 \\
4048290731844\end{array}$ & $\begin{array}{l}640824 \\
640824 \\
64 \varnothing 824 \\
640824 \\
640824\end{array}$ & $\begin{array}{c}- \\
\overline{133} \\
-\end{array}$ & $\begin{array}{l}435300 \\
915500 \\
440700 \\
270900 \\
192600\end{array}$ & $\begin{array}{r}664600 \\
1110000 \\
629500 \\
279800 \\
224000\end{array}$ \\
\hline $\begin{array}{l}H \\
H \\
H \\
H \\
H\end{array}$ & $\begin{array}{l}207 \\
208 \\
209 \\
210 \\
211\end{array}$ & $\begin{array}{l}\text { COLBY DRIVE } \\
\text { VICTOR DRIVE } \\
\text { ELCHESTER DRIVE } \\
\text { COLONIAL STREET } \\
\text { VANDERBILT PKWY. }\end{array}$ & $\begin{array}{l}\text { RANDOLPH DRIVE } \\
\text { MADISON COURT } \\
\text { ELWOOD ROAD } \\
\text { ELTON DRIVE } \\
\text { VILLAGE HILL DR. }\end{array}$ & $\begin{array}{l}4048480731837 \\
4 \varnothing 5 \varnothing 030731901 \\
4 \varnothing 50260732001 \\
4 \varnothing 50290731932 \\
4048490731907\end{array}$ & $\begin{array}{l}640824 \\
551206 \\
540913 \\
570102 \\
640824\end{array}$ & $\begin{array}{l}- \\
139 \\
139 \\
139 \\
133\end{array}$ & $\begin{array}{l}1577000 \\
- \\
- \\
126000 \\
112000\end{array}$ & $\begin{array}{r}180600 \\
28000 \\
240000 \\
140000 \\
145000\end{array}$ \\
\hline $\begin{array}{l}H \\
H \\
H \\
H \\
H\end{array}$ & $\begin{array}{l}212 \\
213 \\
214 \\
215 \\
216\end{array}$ & $\begin{array}{l}\text { CHERRYWOOD DRIVE } \\
\text { LIE SERVICE ROAD } \\
\text { MCCULLOCH DRIVE } \\
\text { EASTOVER DRIVE } \\
\text { LISA DRIVE }\end{array}$ & $\begin{array}{l}\text { PUMPKIN STREET } \\
\text { MCCULLOCH DRIVE } \\
\text { SEWARD DRIVE } \\
\text { HEMLOCK STREET } \\
\text { NORMA LANE }\end{array}$ & $\begin{array}{l}4 \varnothing 51370731853 \\
4 \varnothing 48020731834 \\
4 \varnothing 48050731807 \\
4 \varnothing 5133 \emptyset 731831 \\
4 \varnothing 4844 \varnothing 732139\end{array}$ & $\begin{array}{l}620207 \\
640824 \\
640824 \\
610289 \\
640225\end{array}$ & $\begin{array}{c}- \\
\overline{-} \\
169 \\
133\end{array}$ & $\begin{array}{l}384200 \\
205500 \\
237400 \\
220700 \\
255000\end{array}$ & $\begin{array}{l}387300 \\
205800 \\
278500 \\
272000 \\
292000\end{array}$ \\
\hline $\begin{array}{l}H \\
H \\
H \\
H \\
H\end{array}$ & $\begin{array}{l}217 \\
218 \\
219 \\
22 \varnothing \\
221\end{array}$ & $\begin{array}{l}\text { WOLF HILL ROAD } \\
\text { ARBOR LANE } \\
\text { WOODBURY ROAD } \\
\text { ORIOLE WAY } \\
\text { DEER PARK AVENUE }\end{array}$ & $\begin{array}{l}\text { CALEDONIA ROAD } \\
\text { LISA DRIVE } \\
\text { WOODCHUCK HOL.RD } \\
\text { LYRIC PLACE } \\
\text { CALEDONIA ROAD }\end{array}$ & $\begin{array}{l}4048360732203 \\
4048510732153 \\
4051210732632 \\
4049190732141 \\
4049180732044\end{array}$ & $\begin{array}{l}6201 \\
6201 \\
570423 \\
550316 \\
530415\end{array}$ & $\begin{array}{c}133 \\
- \\
133 \\
133\end{array}$ & $\begin{array}{l}675700 \\
199000 \\
- \\
- \\
-\end{array}$ & $\begin{array}{l}713100 \\
206000 \\
260000 \\
- \\
-\end{array}$ \\
\hline $\begin{array}{l}\mathrm{H} \\
\mathrm{H} \\
\mathrm{H} \\
\mathrm{H} \\
\mathrm{H}\end{array}$ & $\begin{array}{l}222 \\
223 \\
224 \\
225 \\
226\end{array}$ & $\begin{array}{l}\text { JACKSON AV.S END } \\
\text { OLD BRIDGE ROAD } \\
\text { GUNTHER DRIVE } \\
\text { YEOMAN DRIVE } \\
\text { KETAY DRIVE }\end{array}$ & $\begin{array}{l}\text { GUN CLUB ROAD } \\
\text { STACY STREET } \\
\text { ROMANY WAY } \\
\text { PAULA STREET }\end{array}$ & $\begin{array}{l}4052100732457 \\
4053060731802 \\
4052150731836 \\
4051030731818 \\
4051190731851\end{array}$ & $\begin{array}{l}- \\
660608 \\
590624 \\
600523 \\
621285\end{array}$ & $\begin{array}{l}146 \\
137 \\
137 \\
169 \\
169\end{array}$ & $\begin{array}{l}- \\
191900 \\
196200 \\
245300 \\
-\end{array}$ & $\begin{array}{l}- \\
211000 \\
214000 \\
276000 \\
-\end{array}$ \\
\hline $\begin{array}{l}H \\
H \\
H \\
H \\
H\end{array}$ & $\begin{array}{l}227 \\
228 \\
229 \\
231 \\
232\end{array}$ & $\begin{array}{l}\text { BANBURY LANE } \\
\text { SARINA DRIVE } \\
\text { JULIA COURT } \\
\text { ELWOOD ROAD } \\
\text { PULASKI ROAD }\end{array}$ & $\begin{array}{l}\text { MARIDON LANE } \\
\text { BETTE LANE } \\
\text { ZORANNE DRIVE } \\
\text { 2ND. AVENUE } \\
\text { FRAZER DRIVE }\end{array}$ & $\begin{array}{l}4850560731803 \\
4850410731849 \\
4050380731900 \\
4 \varnothing 51550732066 \\
4851390732235\end{array}$ & $\begin{array}{l}570318 \\
570117 \\
5504 \\
661128 \\
641014\end{array}$ & $\begin{array}{l}169 \\
169 \\
139 \\
137 \\
141\end{array}$ & $\begin{array}{l}185700 \\
285000 \\
- \\
- \\
-\end{array}$ & $\begin{array}{l}183400 \\
298000 \\
- \\
86800 \\
-\end{array}$ \\
\hline
\end{tabular}


N.Y. [Dash indicates no data. Altitudes are in feet above sea level.] (cont.)

\begin{tabular}{|c|c|c|c|c|c|c|c|c|c|c|c|c|}
\hline \multicolumn{2}{|c|}{$\begin{array}{l}\text { BASIN } \\
\text { NUMBER }\end{array}$} & $\begin{array}{l}\text { MAX. } \\
\text { AREA } \\
\text { (squar }\end{array}$ & $\begin{array}{c}\text { BASIN } \\
\text { AREA } \\
\text { feet) }\end{array}$ & RIM & $\begin{array}{l}\text { OVLT } \\
\text { OVER } \\
\text { FLOW }\end{array}$ & $\begin{array}{l}\text { ITUDE--- } \\
-\quad \text { BOT- } \\
\text { TOM }\end{array}$ & $\begin{array}{l}\text { WATER } \\
\text { TABLE }\end{array}$ & $\begin{array}{c}\text { DRAIN. } \\
\text { AREA } \\
\text { (acres) }\end{array}$ & \multicolumn{2}{|c|}{$\begin{array}{l}---B A S I N-- \\
\text { STATUS USE }\end{array}$} & $\begin{array}{l}\text { GEO. } \\
\text { UNIT }\end{array}$ & $\begin{array}{l}\text { SOIL } \\
\text { UNIT }\end{array}$ \\
\hline $\begin{array}{l}H \\
H \\
H \\
H \\
H\end{array}$ & $\begin{array}{l}174 \\
175 \\
176 \\
178 \\
179\end{array}$ & $\begin{array}{c}22900 \\
22400 \\
- \\
13600 \\
20200\end{array}$ & $\begin{array}{l}396 \varnothing \varnothing \\
36900 \\
39 \varnothing \varnothing \varnothing \\
264 \varnothing \varnothing \\
561 \varnothing \varnothing\end{array}$ & $\begin{array}{l}185.0 \\
250.0 \\
215.5 \\
175.0 \\
225.0\end{array}$ & $\begin{array}{l}176.5 \\
181.6 \\
216.1 \\
168.8 \\
198.0\end{array}$ & $\begin{array}{l}162.5 \\
165.5 \\
206.1 \\
175.0 \\
183.0\end{array}$ & $\begin{array}{l}61.0 \\
55.0 \\
63.0 \\
40.0 \\
63.0\end{array}$ & $\begin{array}{r}24.8 \\
26.0 \\
- \\
17.4 \\
47.8\end{array}$ & $\begin{array}{l}1 \\
1 \\
1 \\
1 \\
1\end{array}$ & $\begin{array}{l}1 \\
1 \\
1 \\
1 \\
1\end{array}$ & $\begin{array}{l}1 \\
4 \\
1 \\
3 \\
8\end{array}$ & $\begin{array}{l}7 \\
1 \\
8 \\
7 \\
5\end{array}$ \\
\hline $\begin{array}{l}\mathrm{H} \\
\mathrm{H} \\
\mathrm{H} \\
\mathrm{H} \\
\mathrm{H}\end{array}$ & $\begin{array}{l}180 \\
181 \\
182 \\
183 \\
184\end{array}$ & $\begin{array}{r}8000 \\
13700 \\
6000 \\
9400 \\
24000\end{array}$ & $\begin{array}{l}392 \varnothing 0 \\
306 \varnothing \varnothing \\
17100 \\
24 \varnothing \varnothing \varnothing \\
456 \varnothing \varnothing\end{array}$ & $\begin{array}{l}153.0 \\
148 . \varnothing \\
186.0 \\
224.1 \\
186.5\end{array}$ & $\begin{array}{r}149.7 \\
145 . \varnothing \\
- \\
223 . \varnothing \\
-\end{array}$ & $\begin{array}{l}132 . \varnothing \\
134 . \varnothing \\
172.8 \\
213 . \varnothing \\
171.7\end{array}$ & $\begin{array}{l}58 . \emptyset \\
58 . \emptyset \\
52 . \varnothing \\
62 . \varnothing \\
67 . \varnothing\end{array}$ & $\begin{array}{r}19.9 \\
8.4 \\
9.4 \\
12.7 \\
25.2\end{array}$ & $\begin{array}{l}1 \\
1 \\
0 \\
1 \\
1\end{array}$ & $\begin{array}{l}1 \\
3 \\
1 \\
1 \\
1\end{array}$ & $\begin{array}{l}1 \\
1 \\
1 \\
1 \\
1\end{array}$ & $\begin{array}{l}1 \\
6 \\
1 \\
7 \\
7\end{array}$ \\
\hline $\begin{array}{l}H \\
H \\
H \\
H \\
H\end{array}$ & $\begin{array}{l}185 \\
187 \\
188 \\
189 \\
190\end{array}$ & $\begin{array}{c}102 \varnothing 0 \\
6800 \\
- \\
167 \varnothing 0 \\
526 \varnothing 0\end{array}$ & $\begin{array}{r}38 \varnothing \varnothing \varnothing \\
132 \varnothing \emptyset \\
42 \varnothing \varnothing \varnothing \\
- \\
55 \varnothing \varnothing \varnothing\end{array}$ & $\begin{array}{l}14 \varnothing . \varnothing \\
210 . \varnothing \\
134 . \varnothing \\
139 . \varnothing \\
165 . \varnothing\end{array}$ & $\begin{array}{r}120.3 \\
172.2 \\
-\overline{4} \\
137.4 \\
162.7\end{array}$ & $\begin{array}{l}109.5 \\
162.2 \\
117.0 \\
123.4 \\
151 . \varnothing\end{array}$ & $\begin{array}{l}57.0 \\
71.0 \\
69.0 \\
73.0 \\
63.0\end{array}$ & $\begin{array}{r}12.0 \\
9.4 \\
-\overline{9} \\
29.9 \\
77.1\end{array}$ & $\begin{array}{l}1 \\
1 \\
1 \\
1 \\
1\end{array}$ & $\begin{array}{l}1 \\
1 \\
1 \\
1 \\
1\end{array}$ & $\begin{array}{l}1 \\
6 \\
1 \\
1 \\
1\end{array}$ & $\begin{array}{l}7 \\
7 \\
7 \\
7 \\
1\end{array}$ \\
\hline $\begin{array}{l}H \\
H \\
H \\
H \\
H\end{array}$ & $\begin{array}{l}191 \\
192 \\
193 \\
194 \\
195\end{array}$ & $\begin{array}{c}- \\
128 \varnothing \varnothing \\
2 \varnothing 6 \varnothing \varnothing \\
15 \varnothing \varnothing \varnothing \\
14 \varnothing \varnothing \varnothing\end{array}$ & $\begin{array}{l}304 \varnothing 0 \\
2 \varnothing 8 \varnothing \varnothing \\
399 \varnothing 0 \\
30 \varnothing \varnothing \varnothing \\
32 \varnothing \varnothing \varnothing\end{array}$ & $\begin{array}{l}160.0 \\
187 . \varnothing \\
149 . \varnothing \\
130 . \varnothing \\
170 . \varnothing\end{array}$ & $\begin{array}{r}- \\
180.5 \\
148.4 \\
124.9\end{array}$ & $\begin{array}{l}135.0 \\
172.5 \\
141.0 \\
120.0 \\
114.9\end{array}$ & $\begin{array}{l}72.0 \\
66.0 \\
58 . \varnothing \\
36.0 \\
71.0\end{array}$ & $\begin{array}{r}- \\
15.4 \\
7.8 \\
23.6 \\
16.0\end{array}$ & $\begin{array}{l}1 \\
1 \\
1 \\
1 \\
1\end{array}$ & $\begin{array}{l}1 \\
1 \\
3 \\
1 \\
1\end{array}$ & $\begin{array}{l}1 \\
1 \\
1 \\
3 \\
6\end{array}$ & $\begin{array}{l}7 \\
7 \\
1 \\
1 \\
1\end{array}$ \\
\hline $\begin{array}{l}\mathrm{H} \\
\mathrm{H} \\
\mathrm{H} \\
\mathrm{H} \\
\mathrm{H}\end{array}$ & $\begin{array}{l}196 \\
197 \\
198 \\
199 \\
200\end{array}$ & $\begin{array}{c}\overline{8} \\
800 \\
25500 \\
82200 \\
34400\end{array}$ & $\begin{array}{r}16000 \\
17000 \\
48000 \\
170400 \\
45000\end{array}$ & $\begin{array}{l}100.0 \\
190.0 \\
206.0 \\
214.0 \\
132.0\end{array}$ & $\begin{array}{r}- \\
- \\
202.0 \\
206.0 \\
-\end{array}$ & $\begin{array}{r}82.0 \\
176.9 \\
191.0 \\
197.0 \\
121.0\end{array}$ & $\begin{array}{l}23.0 \\
62.0 \\
67.0 \\
66.0 \\
71.0\end{array}$ & $\begin{array}{r}- \\
9.7 \\
41.7 \\
103.1 \\
35.4\end{array}$ & $\begin{array}{l}0 \\
1 \\
1 \\
1 \\
1\end{array}$ & $\begin{array}{l}1 \\
1 \\
1 \\
1 \\
1\end{array}$ & $\begin{array}{l}3 \\
1 \\
1 \\
1 \\
1\end{array}$ & $\begin{array}{l}7 \\
7 \\
7 \\
7 \\
7\end{array}$ \\
\hline $\begin{array}{l}\mathrm{H} \\
\mathrm{H} \\
\mathrm{H} \\
\mathrm{H} \\
\mathrm{H}\end{array}$ & $\begin{array}{l}202 \\
203 \\
204 \\
205 \\
206\end{array}$ & $\begin{array}{r}475 \varnothing \varnothing \\
25 \varnothing \varnothing \\
685 \varnothing \varnothing \\
187 \varnothing \varnothing \\
224 \varnothing \varnothing\end{array}$ & $\begin{array}{r}95200 \\
122200 \\
62800 \\
39600 \\
41800\end{array}$ & $\begin{array}{l}160.0 \\
164.0 \\
165.0 \\
159.5 \\
167.0\end{array}$ & $\begin{array}{l}157.5 \\
161.3 \\
163.1 \\
158.5 \\
166.2\end{array}$ & $\begin{array}{l}143.5 \\
149.3 \\
152.7 \\
143.5 \\
156.2\end{array}$ & $\begin{array}{l}71.0 \\
59.0 \\
58.0 \\
58.0 \\
58.0\end{array}$ & $\begin{array}{r}68.5 \\
144.1 \\
69.4 \\
38.3 \\
30.3\end{array}$ & $\begin{array}{l}0 \\
1 \\
1 \\
1 \\
1\end{array}$ & $\begin{array}{l}1 \\
1 \\
1 \\
1 \\
1\end{array}$ & $\begin{array}{l}6 \\
6 \\
1 \\
1 \\
1\end{array}$ & $\begin{array}{l}6 \\
7 \\
7 \\
7 \\
7\end{array}$ \\
\hline $\begin{array}{l}\mathrm{H} \\
\mathrm{H} \\
\mathrm{H} \\
\mathrm{H} \\
\mathrm{H}\end{array}$ & $\begin{array}{l}207 \\
208 \\
209 \\
210 \\
211\end{array}$ & $\begin{array}{r}18100 \\
9600 \\
24000 \\
15900 \\
14500\end{array}$ & $\begin{array}{l}384 \varnothing \varnothing \\
247 \varnothing \varnothing \\
384 \varnothing \varnothing \\
3510 \varnothing \\
429 \varnothing \varnothing\end{array}$ & $\begin{array}{l}180.0 \\
140.0 \\
175.0 \\
160.0 \\
231.5\end{array}$ & $\begin{array}{r}178.7 \\
- \\
- \\
82.3 \\
230.5\end{array}$ & $\begin{array}{r}168.7 \\
85.5 \\
92.0 \\
73.5 \\
220.5\end{array}$ & $\begin{array}{l}70.0 \\
72.0 \\
69.0 \\
68.0 \\
71.0\end{array}$ & $\begin{array}{l}24.8 \\
15.4 \\
37.8 \\
23.0 \\
17.6\end{array}$ & $\begin{array}{l}1 \\
1 \\
1 \\
1 \\
0\end{array}$ & $\begin{array}{l}1 \\
1 \\
1 \\
1 \\
1\end{array}$ & $\begin{array}{l}1 \\
1 \\
1 \\
1 \\
6\end{array}$ & $\begin{array}{l}7 \\
7 \\
7 \\
7 \\
3\end{array}$ \\
\hline $\begin{array}{l}H \\
H \\
H \\
H \\
H\end{array}$ & $\begin{array}{l}212 \\
213 \\
214 \\
215 \\
216\end{array}$ & $\begin{array}{l}38700 \\
206 \varnothing 0 \\
27200 \\
27200 \\
29200\end{array}$ & $\begin{array}{l}57600 \\
35700 \\
33000 \\
48400 \\
44000\end{array}$ & $\begin{array}{l}174.5 \\
180.0 \\
153.4 \\
171.5 \\
235.5\end{array}$ & $\begin{array}{r}- \\
149.5 \\
153.4 \\
170.0 \\
-\end{array}$ & $\begin{array}{l}159.5 \\
139.5 \\
143.4 \\
160.0 \\
222.0\end{array}$ & $\begin{array}{l}62.0 \\
57.0 \\
56.0 \\
63 . \varnothing \\
73.0\end{array}$ & $\begin{array}{l}47.0 \\
12.6 \\
14.5 \\
27 . \varnothing \\
29.3\end{array}$ & $\begin{array}{l}1 \\
1 \\
0 \\
1 \\
1\end{array}$ & $\begin{array}{l}1 \\
3 \\
3 \\
1 \\
1\end{array}$ & $\begin{array}{l}1 \\
1 \\
1 \\
1 \\
6\end{array}$ & $\begin{array}{l}7 \\
6 \\
7 \\
7 \\
9\end{array}$ \\
\hline $\begin{array}{l}\mathrm{H} \\
\mathrm{H}\end{array}$ & $\begin{array}{l}217 \\
218 \\
219 \\
220 \\
221\end{array}$ & $\begin{array}{c}71300 \\
20600 \\
2600 \\
- \\
-\end{array}$ & $\begin{array}{r}66700 \\
476 \varnothing 0 \\
5800 \emptyset \\
616 \varnothing \varnothing \\
-\end{array}$ & $\begin{array}{l}211.5 \\
249.5 \\
193.0 \\
226.0 \\
220 . \varnothing\end{array}$ & $\begin{array}{r}- \\
- \\
- \\
211.0\end{array}$ & $\begin{array}{l}192.5 \\
234.0 \\
182.0 \\
210 . \varnothing \\
198 . \emptyset\end{array}$ & $\begin{array}{r}73.0 \\
- \\
50 . \varnothing \\
74.0 \\
73.0\end{array}$ & $\begin{array}{r}77.0 \\
73.0 \\
46.9 \\
- \\
-\end{array}$ & $\begin{array}{l}1 \\
0 \\
1 \\
1 \\
1\end{array}$ & $\begin{array}{l}1 \\
1 \\
1 \\
1 \\
1\end{array}$ & $\begin{array}{l}6 \\
6 \\
6 \\
6 \\
1\end{array}$ & $\begin{array}{l}7 \\
7 \\
0 \\
5 \\
1\end{array}$ \\
\hline $\begin{array}{l}\mathrm{H} \\
\mathrm{H} \\
\mathrm{H} \\
\mathrm{H} \\
\mathrm{H}\end{array}$ & $\begin{array}{l}222 \\
223 \\
224 \\
225 \\
226\end{array}$ & $\begin{array}{c}- \\
19200 \\
21400 \\
27600 \\
-\end{array}$ & $\begin{array}{l}- \\
4480 \varnothing \\
3040 \varnothing \\
312 \varnothing \varnothing \\
7250 \varnothing\end{array}$ & $\begin{array}{l}125 . \varnothing \\
190 . \emptyset \\
193 . \emptyset \\
165 . \varnothing \\
170 . \varnothing\end{array}$ & $\begin{array}{r}- \\
136.0 \\
- \\
-\end{array}$ & $\begin{array}{l}115 . \varnothing \\
125 . \emptyset \\
179 . \varnothing \\
152.5 \\
141.5\end{array}$ & $\begin{array}{l}36.0 \\
47 . \varnothing \\
58 . \emptyset \\
67 . \varnothing \\
63.0\end{array}$ & $\begin{array}{l}30 . \overline{2} \\
24.0 \\
45.0 \\
13.9\end{array}$ & $\begin{array}{l}1 \\
1 \\
1 \\
1 \\
1\end{array}$ & $\begin{array}{l}1 \\
1 \\
1 \\
1 \\
1\end{array}$ & $\begin{array}{l}1 \\
4 \\
1 \\
1 \\
1\end{array}$ & $\begin{array}{l}8 \\
7 \\
7 \\
7 \\
7\end{array}$ \\
\hline $\mathrm{H}$ & $\begin{array}{l}227 \\
228 \\
229 \\
231 \\
232\end{array}$ & $\begin{array}{c}18300 \\
29600 \\
- \\
8700 \\
-\end{array}$ & $\begin{array}{r}30000 \\
38400 \\
38000 \\
9000 \\
40000\end{array}$ & $\begin{array}{l}150 . \emptyset \\
174.2 \\
178 . \varnothing \\
200 . \varnothing \\
220 . \varnothing\end{array}$ & $\begin{array}{l}- \\
- \\
- \\
-\end{array}$ & $\begin{array}{l}134.0 \\
162.0 \\
166.0 \\
181.5 \\
210.0\end{array}$ & $\begin{array}{l}67 . \varnothing \\
67 . \varnothing \\
68 . \varnothing \\
61 . \emptyset \\
62 . \varnothing\end{array}$ & $\begin{array}{r}29.0 \\
38.0 \\
- \\
13.7 \\
-\end{array}$ & $\begin{array}{l}1 \\
1 \\
1 \\
1 \\
1\end{array}$ & $\begin{array}{l}1 \\
1 \\
1 \\
1 \\
1\end{array}$ & $\begin{array}{l}1 \\
1 \\
1 \\
1 \\
1\end{array}$ & $\begin{array}{l}7 \\
7 \\
7 \\
7 \\
7\end{array}$ \\
\hline
\end{tabular}


Appendix.--Location and basic physical features of recharge basins on Long Island,

\begin{tabular}{|c|c|c|c|c|c|c|c|c|}
\hline \multicolumn{2}{|c|}{$\begin{array}{l}\text { BASIN } \\
\text { NUMBER }\end{array}$} & \multicolumn{2}{|c|}{ NEAREST INTERSECTION } & $\begin{array}{l}\text { LATITUDE } \\
\text { LONGITUDE } \\
0, "{ }^{\prime}, "\end{array}$ & $\begin{array}{l}\text { DATE } \\
\text { BUILT } \\
\text { YrMOD }\end{array}$ & COMMUNITY & \multicolumn{2}{|c|}{$\begin{array}{l}\text { DESIGN ACTUAL } \\
\text { CAPACITY CAPACITY } \\
\text { (cubic feet) }\end{array}$} \\
\hline $\begin{array}{l}H \\
H \\
H \\
H \\
H\end{array}$ & $\begin{array}{l}233 \\
234 \\
238 \\
237 \\
238\end{array}$ & $\begin{array}{l}\text { CARMAN ROAD } \\
\text { MARKWOOD LANE } \\
\text { KATHY COURT } \\
\text { E. ROGUES PATH } \\
\text { MARION OPLACE }\end{array}$ & $\begin{array}{l}\text { ESTATES PLACE } \\
\text { FOOTHILL LANE } \\
\text { KATHY LANE } \\
\text { HARTLAND AVENUE } \\
\text { BEVERLY ROAD }\end{array}$ & $\begin{array}{l}4 \emptyset 4755 \emptyset 732246 \\
4 \emptyset 5121 \emptyset 731948 \\
4 \emptyset 5354 \varnothing 731949 \\
4 \emptyset 5 \emptyset 49 \emptyset 732257 \\
4 \emptyset 4925 \emptyset 732232\end{array}$ & $\begin{array}{c}661024 \\
640311 \\
- \\
620924 \\
-\end{array}$ & $\begin{array}{l}133 \\
139 \\
152 \\
141 \\
151\end{array}$ & $\begin{array}{l}\overline{171700} \\
\overline{158100} \\
-\end{array}$ & $\begin{array}{l}216000 \\
177000 \\
- \\
158400 \\
-\end{array}$ \\
\hline $\begin{array}{l}H \\
H \\
H \\
H \\
H\end{array}$ & $\begin{array}{l}239 \\
242 \\
243 \\
244 \\
245\end{array}$ & $\begin{array}{l}\text { BURR ROAD } \\
\text { BROADWAY } \\
\text { OAKLEDGE DRIVE } \\
\text { OLD COUNTRY RD. } \\
\text { PASHEN PLACE }\end{array}$ & $\begin{array}{l}\text { COMMACK ROAD } \\
\text { CUBA HILL ROAD } \\
\text { STACY STREET } \\
\text { DIX HILLS ROAD } \\
\text { LAUREN AVENUE }\end{array}$ & $\begin{array}{l}4051100731733 \\
4051410732153 \\
405222073183 \emptyset \\
404932073215 \emptyset \\
4047550731819\end{array}$ & $\begin{array}{c}610502 \\
640127 \\
571207 \\
620417 \\
-\end{array}$ & $\begin{array}{l}169 \\
141 \\
137 \\
- \\
-\end{array}$ & $\begin{array}{l}\overline{358400} \\
- \\
\overline{101500}\end{array}$ & $\begin{array}{l}- \\
372500 \\
- \\
\overline{185000}\end{array}$ \\
\hline $\begin{array}{l}\mathrm{H} \\
\mathrm{H} \\
\mathrm{H} \\
\mathrm{H} \\
\mathrm{H}\end{array}$ & $\begin{array}{l}246 \\
247 \\
248 \\
249 \\
250\end{array}$ & $\begin{array}{l}\text { UDELL WAY } \\
\text { TOMPKINS STREET } \\
\text { MEADOWRUE LANE } \\
\text { MAYAPPLE LANE } \\
\text { ROUTE } 11 \varnothing\end{array}$ & $\begin{array}{l}\text { OAKLEDGE DRIVE } \\
\text { CAYUGA AVENUE } \\
\text { CORNFLOWER LANE } \\
\text { WINTERCRESS LANE } \\
\text { OVERHILL ROAD }\end{array}$ & $\begin{array}{l}4 \emptyset 52080731837 \\
4 \varnothing 53180731837 \\
4 \varnothing 51540731738 \\
4 \varnothing 51470731737 \\
4048390732437\end{array}$ & $\begin{array}{c}- \\
- \\
650122 \\
660111 \\
-\end{array}$ & $\begin{array}{l}137 \\
134 \\
169 \\
169 \\
151\end{array}$ & $\begin{array}{l}- \\
\overline{-} \\
274580 \\
-\end{array}$ & $\begin{array}{l}- \\
- \\
\overline{285000} \\
-\end{array}$ \\
\hline $\begin{array}{l}H \\
H \\
H \\
H \\
H\end{array}$ & $\begin{array}{l}251 \\
252 \\
253 \\
254 \\
255\end{array}$ & $\begin{array}{l}\text { W. END HAZEL CT. } \\
\text { ROUTE } 110 \\
\text { JERICHO TURNPIKE } \\
\text { SWEET HOLLOW RD. } \\
\text { JERICHO TURNPIKE }\end{array}$ & $\begin{array}{l}\text { WESTON STREET } \\
\text { WEST HILLS ROAD } \\
\text { OAKLEY PLACE } \\
\text { OAKLEY PLACE }\end{array}$ & $\begin{array}{l}4 \emptyset 4815 \emptyset 732447 \\
4 \varnothing 4928 \emptyset 7325 \emptyset \emptyset \\
4 \emptyset 4937 \emptyset 73251 \emptyset \\
4 \emptyset 4945 \emptyset 7325 \emptyset 9 \\
4 \emptyset 49460732452\end{array}$ & $\begin{array}{l}- \\
- \\
- \\
-\end{array}$ & $\begin{array}{l}- \\
151 \\
151 \\
- \\
-\end{array}$ & $\begin{array}{l}- \\
- \\
- \\
-\end{array}$ & $\begin{array}{l}- \\
\overline{-} \\
-\end{array}$ \\
\hline $\begin{array}{l}H \\
H \\
H \\
H \\
H\end{array}$ & $\begin{array}{l}256 \\
257 \\
258 \\
259 \\
260\end{array}$ & $\begin{array}{l}\text { W. 22ND. ST. } \\
\text { W. } 15 T H . \text { ST. } \\
\text { OAKWOOD ROAD } \\
\text { HAMILTON LANE } \\
\text { STEPAR PLACE }\end{array}$ & $\begin{array}{l}\text { LONGLEY PLACE } \\
\text { LOCKWOOD AVENUE } \\
\text { 22ND. STREET } \\
\text { CARREN CIRCLE } \\
\text { NORDEN LANE }\end{array}$ & 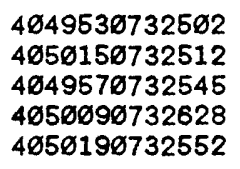 & $\begin{array}{l}- \\
\overline{-} \\
\overline{-}\end{array}$ & $\begin{array}{c}151 \\
- \\
- \\
-\end{array}$ & $\begin{array}{l}- \\
- \\
- \\
-\end{array}$ & $\begin{array}{l}- \\
- \\
-\end{array}$ \\
\hline $\begin{array}{l}H \\
H \\
H \\
H \\
H\end{array}$ & $\begin{array}{l}261 \\
262 \\
263 \\
264 \\
265\end{array}$ & $\begin{array}{l}\text { W. IOTH. ST. } \\
\text { PIDGEON HILL RD. } \\
\text { PIDGEON HILL RD. } \\
\text { ALDRICH STREET } \\
\text { LENOX ROAD }\end{array}$ & $\begin{array}{l}\text { 3RD. AVENUE } \\
\text { JERICHO TURNPIKE } \\
\text { SUNNYWOODS DR. } \\
\text { OAK AVENUE } \\
\text { INGERSOLL STREET }\end{array}$ & $\begin{array}{l}4 \emptyset 50440732449 \\
4 \emptyset 4957 \varnothing 732319 \\
4 \emptyset 49060732317 \\
4 \emptyset 50390732320 \\
4 \emptyset 50290732344\end{array}$ & $\begin{array}{l}- \\
- \\
- \\
-\end{array}$ & $\begin{array}{l}- \\
\overline{151} \\
151 \\
151\end{array}$ & $\begin{array}{l}- \\
- \\
-\end{array}$ & $\begin{array}{l}- \\
- \\
-\end{array}$ \\
\hline $\begin{array}{l}\mathrm{H} \\
\mathrm{H} \\
\mathrm{H} \\
\mathrm{H} \\
\mathrm{H}\end{array}$ & $\begin{array}{l}266 \\
267 \\
268 \\
269 \\
270\end{array}$ & $\begin{array}{l}\text { LENOX ROAD } \\
\text { JERICHO TURNPIKE } \\
\text { OAKWOOD ROAD } \\
\text { WEST NECK ROAD } \\
\text { YOUNGS HILL ROAD }\end{array}$ & $\begin{array}{l}\text { JOSHUA STREET } \\
\text { SAPPHIRE PLACE } \\
\text { TALL OAK DRIVE } \\
\text { AMHERST COURT } \\
\text { GLENVIEW PLACE }\end{array}$ & $\begin{array}{l}4 \varnothing 5 \varnothing 210732341 \\
4 \varnothing 4955073225 \emptyset \\
4 \varnothing 5120 \varnothing 732556 \\
4 \varnothing 52490732629 \\
4 \varnothing 5321073244 \emptyset\end{array}$ & $\begin{array}{l}- \\
- \\
- \\
-\end{array}$ & $\begin{array}{l}151 \\
- \\
146 \\
131 \\
142\end{array}$ & $\begin{array}{l}- \\
- \\
- \\
-\end{array}$ & $\begin{array}{l}- \\
- \\
- \\
-\end{array}$ \\
\hline $\begin{array}{l}H \\
H \\
H \\
H \\
H\end{array}$ & $\begin{array}{l}271 \\
272 \\
273 \\
274 \\
275\end{array}$ & $\begin{array}{l}\text { OLD FIELD ROAD } \\
\text { CUBA HILL ROAD } \\
\text { WESTPARK DRIVE } \\
\text { CALEDONIA ROAD } \\
\text { NORTHERN ST. PKW }\end{array}$ & $\begin{array}{l}\text { TILDEN LANE } \\
\text { BURNS COURT } \\
\text { E. 14TH STREET } \\
\text { KENDRICK LANE } \\
\text { WILLOUGHBY PATH }\end{array}$ & $\begin{array}{l}4052140732244 \\
4651220732139 \\
465027 \varnothing 732422 \\
4048090732152 \\
4049550731944\end{array}$ & $\begin{array}{l}- \\
- \\
- \\
-\end{array}$ & $\begin{array}{l}130 \\
141 \\
151 \\
133 \\
133\end{array}$ & $\begin{array}{l}- \\
- \\
- \\
-\end{array}$ & $\begin{array}{l}- \\
- \\
-\end{array}$ \\
\hline $\begin{array}{l}H \\
H \\
H \\
H \\
H\end{array}$ & $\begin{array}{l}278 \\
277 \\
278 \\
279 \\
280\end{array}$ & $\begin{array}{l}\text { ALISTER CIRCLE } \\
\text { ELWOOD ROAD } \\
\text { CLAY PITTS ROAD } \\
\text { N/O 5TH STREET } \\
\text { PULASKI ROAD }\end{array}$ & $\begin{array}{l}\text { DALY ROAD } \\
\text { WARREN ROAD } \\
\text { TALCOTT DRIVE } \\
\text { E/O WICKS AVENUE } \\
\text { 7TH AVENUE WEST }\end{array}$ & 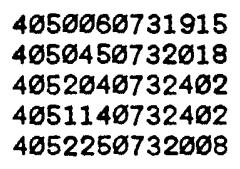 & $\begin{array}{l}- \\
- \\
- \\
-\end{array}$ & $\begin{array}{c}- \\
\overline{134} \\
\overline{134}\end{array}$ & $\begin{array}{l}- \\
- \\
- \\
-\end{array}$ & $\overline{-}$ \\
\hline $\begin{array}{l}H \\
H \\
H \\
H \\
H\end{array}$ & $\begin{array}{l}281 \\
282 \\
283 \\
284 \\
285\end{array}$ & $\begin{array}{l}\text { CLAY PITTS ROAD } \\
\text { LITTLE PLAINS RD } \\
\text { CARRIAGE COURT } \\
\text { OLD COUNTRY ROAD } \\
\text { PULASKI ROAD }\end{array}$ & $\begin{array}{l}\text { IST STREET } \\
\text { SEXTON COURT } \\
\text { LANDVIEW DRIVE } \\
\text { SINNOCK COURT } \\
\text { CULLEN DRIVE }\end{array}$ & $\begin{array}{l}4051530731921 \\
4 \varnothing 51020732145 \\
4047406732050 \\
46481107324 \varnothing 5 \\
4652500731853\end{array}$ & $\begin{array}{l}- \\
- \\
- \\
-\end{array}$ & $\begin{array}{c}134 \\
141 \\
133 \\
- \\
137\end{array}$ & $\begin{array}{l}- \\
- \\
- \\
-\end{array}$ & $\begin{array}{l}- \\
\overline{-} \\
-\end{array}$ \\
\hline $\begin{array}{l}H \\
I \\
I \\
I \\
I\end{array}$ & $\begin{array}{r}286 \\
1 \\
2 \\
3 \\
4\end{array}$ & $\begin{array}{l}\text { LAUREL ROAD } \\
\text { POTTER BOULEVARD } \\
\text { LINCOLN BLVD. } \\
\text { LINCOLN BLVD. } \\
\text { PINE ACRES BLVD. }\end{array}$ & $\begin{array}{l}\text { CEDAR HILL DRIVE } \\
\text { SPUR DRIVE } \\
\text { PEACH PLACE } \\
\text { HEMLOCK DRIVE } \\
\text { LOUISE DRIVE }\end{array}$ & 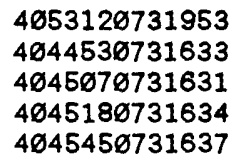 & $\begin{array}{l}\quad- \\
550125 \\
550125 \\
550125 \\
5508\end{array}$ & $\begin{array}{c}134 \\
- \\
- \\
-\end{array}$ & $\begin{array}{l}- \\
- \\
- \\
\overline{970000}\end{array}$ & $\begin{array}{c}- \\
- \\
\overline{-} \\
1035000\end{array}$ \\
\hline
\end{tabular}


N.Y. [Dash indicates no data. Altitudes are in feet above sea level.] (cont.)

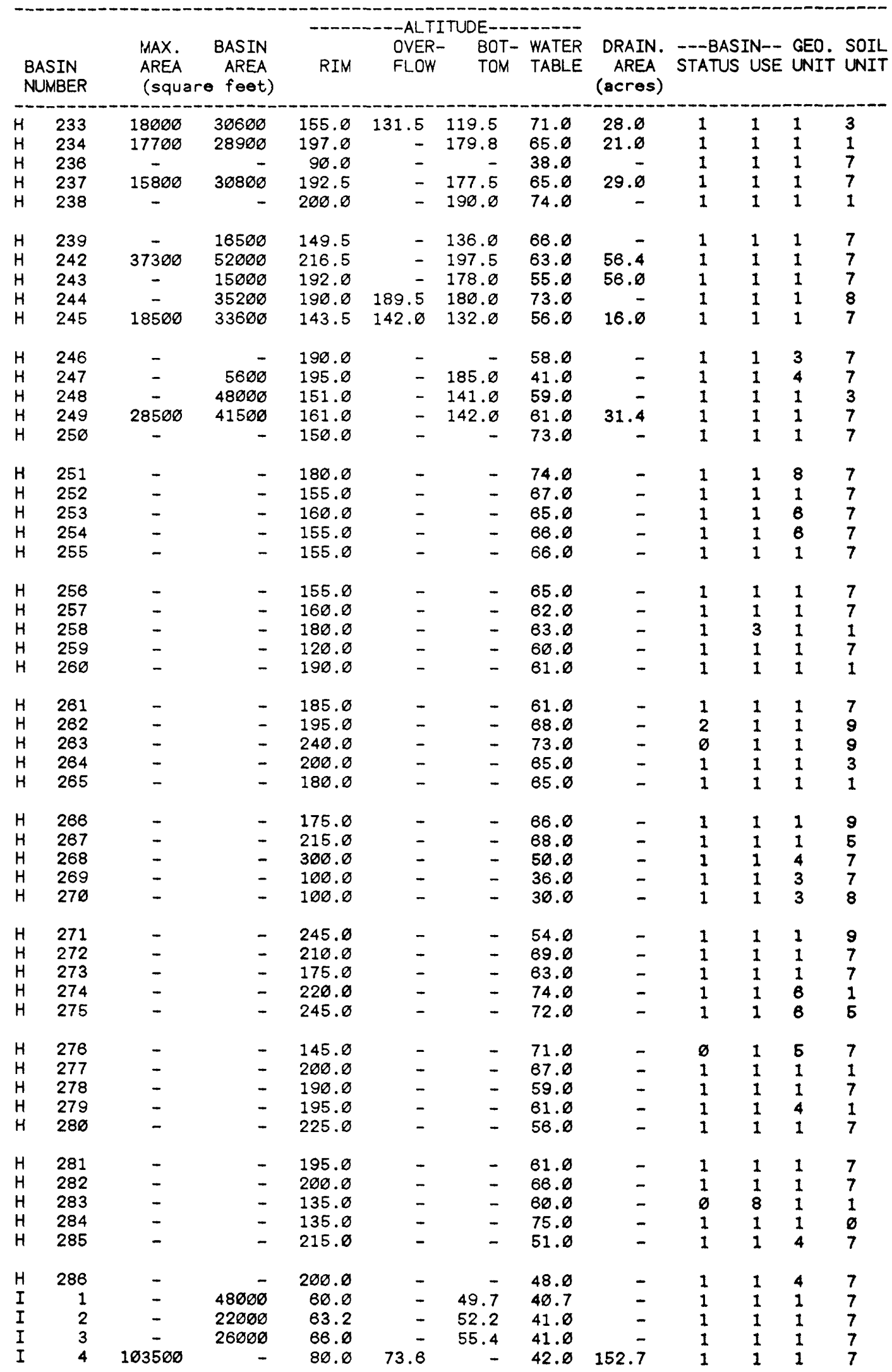


Appendix.--Location and basic physical features of recharge basins on Long Island,

\begin{tabular}{|c|c|c|c|c|c|c|c|c|}
\hline \multicolumn{2}{|c|}{$\begin{array}{l}\text { BASIN } \\
\text { NUMBER }\end{array}$} & \multicolumn{2}{|c|}{ NEAREST INTERSECTION } & $\begin{array}{l}\text { LATITUDE } \\
\text { LONGITUDE } \\
0, n \text {, n }\end{array}$ & $\begin{array}{l}\text { DATE } \\
\text { BUILT } \\
\text { YrMoD }\end{array}$ & COMMNITY & $\begin{array}{l}\text { DESIGN } \\
\text { CAPACITY } \\
\text { (cubic fe }\end{array}$ & $\begin{array}{l}\text { ACTUAL } \\
\text { CAPACITY } \\
\text { t) }\end{array}$ \\
\hline $\begin{array}{l}I \\
I \\
I \\
I \\
I\end{array}$ & $\begin{array}{r}5 \\
9 \\
10 \\
11 \\
12\end{array}$ & $\begin{array}{l}\text { LOCUST DRIVE } \\
\text { 23RD AVENUE } \\
\text { MADISON AVENUE } \\
\text { MADISON AVENUE } \\
\text { MADISON AVENUE }\end{array}$ & $\begin{array}{l}\text { ELSIE LANE } \\
\text { SPUR LANE } \\
\text { BARLEAU STREET } \\
\text { FLOYD STREET } \\
\text { RUTLEDGE STREET }\end{array}$ & $\begin{array}{l}4 \emptyset 4541 \emptyset 73162 \emptyset \\
4 \emptyset 45 \emptyset 5 \emptyset 731452 \\
4 \emptyset 4726 \varnothing 731517 \\
4 \emptyset 4735 \emptyset 731518 \\
4 \emptyset 475 \varnothing \emptyset 731519\end{array}$ & $\begin{array}{l}56 \varnothing 5 \\
531204 \\
558982 \\
550982 \\
570481\end{array}$ & $\begin{array}{l}189 \\
189 \\
189 \\
189 \\
189\end{array}$ & $\begin{array}{l}- \\
- \\
69406 \varnothing \\
7430 \varnothing \emptyset \\
6156 \varnothing \varnothing\end{array}$ & $\begin{array}{r}12500 \varnothing \\
- \\
94606 \varnothing \\
106 \varnothing 600 \\
667300\end{array}$ \\
\hline $\begin{array}{l}I \\
I \\
I \\
I \\
I\end{array}$ & $\begin{array}{l}14 \\
15 \\
16 \\
17 \\
18\end{array}$ & $\begin{array}{l}\text { MADISON AVENUE } \\
\text { ORIENT AVENUE } \\
\text { EVERGREEN AVENUE } \\
\text { NOSTRAND AVENUE } \\
\text { COMMERCIAL BLVD. }\end{array}$ & $\begin{array}{l}\text { WHITE STREET } \\
\text { ELDRIDGE STREET } \\
\text { VITA DRIVE } \\
\text { BIRCHGROVE DRIVE } \\
\text { GATES AVENUE }\end{array}$ & $\begin{array}{l}4 \varnothing 48 \varnothing 8 \varnothing 731517 \\
4 \varnothing 48 \varnothing \varnothing \varnothing 73143 \emptyset \\
4 \varnothing 4724 \varnothing 731252 \\
4 \varnothing 4733 \varnothing 731256 \\
4 \varnothing 4754 \varnothing 731257\end{array}$ & $\begin{array}{c}560229 \\
5506 \\
- \\
- \\
5506\end{array}$ & $\begin{array}{l}189 \\
189 \\
191 \\
191 \\
191\end{array}$ & $\begin{array}{l}1019300 \\
424100 \\
- \\
590900\end{array}$ & $\begin{array}{l}1214800 \\
277300 \\
- \\
- \\
533800\end{array}$ \\
\hline $\begin{array}{l}I \\
I \\
I \\
I \\
I\end{array}$ & $\begin{array}{l}19 \\
20 \\
21 \\
22 \\
24\end{array}$ & $\begin{array}{l}\text { FRONT AVENUE } \\
\text { GRAND BOULEVARD } \\
\text { WILSON BOULEVARD } \\
\text { MCFARLAND AVENUE } \\
\text { PROSPECT AVENUE }\end{array}$ & $\begin{array}{l}\text { DIXON STREET } \\
\text { JEWEL STREET } \\
\text { CEDAR STREET } \\
\text { CEDAR STREET } \\
\text { ROSEWOOD STREET }\end{array}$ & $\begin{array}{l}4 \varnothing 4617 \varnothing 731326 \\
4 \varnothing 46288731319 \\
4 \varnothing 4444 \varnothing 731213 \\
4 \varnothing 4633 \varnothing 7312 \varnothing 6 \\
4 \varnothing 4644 \varnothing 731114\end{array}$ & $\begin{array}{r}\overline{-} \\
\overline{-} \\
-\end{array}$ & $\begin{array}{l}189 \\
189 \\
198 \\
191 \\
191\end{array}$ & $\begin{array}{l}3324408 \\
- \\
-\end{array}$ & $\begin{array}{l}\overline{-} \\
- \\
- \\
-\end{array}$ \\
\hline $\begin{array}{l}I \\
I \\
I \\
I\end{array}$ & $\begin{array}{l}26 \\
27 \\
28 \\
29 \\
30\end{array}$ & $\begin{array}{l}\text { PROSPECT AVENUE } \\
\text { PROSPECT AVENUE } \\
\text { PROSPECT AVENUE } \\
\text { ADAMS STREET E. } \\
\text { SEYMOUR AVENUE }\end{array}$ & $\begin{array}{l}\text { HICKORY STREET } \\
\text { TAMARACK STREET } \\
\text { MAGNOLIA STREET } \\
\text { SHERWOOD DRIVE } \\
\text { MADISON STREET }\end{array}$ & $\begin{array}{l}4 \varnothing 4638 \varnothing 731112 \\
4 \varnothing 4634 \varnothing 731113 \\
4 \varnothing 463 \varnothing \varnothing 731112 \\
4 \varnothing 4428 \varnothing 7311 \varnothing \varnothing \\
4 \varnothing 4433 \varnothing 731 \varnothing 36\end{array}$ & $\begin{array}{c}588424 \\
- \\
- \\
-\end{array}$ & $\begin{array}{l}191 \\
191 \\
191 \\
193 \\
193\end{array}$ & $\begin{array}{l}- \\
- \\
-\end{array}$ & $\begin{array}{l}- \\
- \\
-\end{array}$ \\
\hline $\begin{array}{l}I \\
I \\
I \\
I \\
I\end{array}$ & $\begin{array}{l}31 \\
32 \\
33 \\
34 \\
35\end{array}$ & $\begin{array}{l}\text { CARLETON AVENUE } \\
\text { LOCUST AVENUE } \\
\text { RIVERDALE DRIVE } \\
\text { RIVERDALE AVENUE } \\
\text { WYANDANCH ROAD }\end{array}$ & $\begin{array}{l}\text { ADAMS STREET } \\
\text { IVY HILL ROAD } \\
\text { AMBOY ROAD } \\
\text { PRESIDENT STREET } \\
\text { LAWRENCE STREET }\end{array}$ & $\begin{array}{l}4 \varnothing 4424 \varnothing 73113 \emptyset \\
4 \varnothing 45 \emptyset 4 \varnothing 73 \varnothing 652 \\
4 \varnothing 4454 \varnothing 73 \varnothing 7 \varnothing \emptyset \\
4 \varnothing 4436 \varnothing 73 \varnothing 7 \varnothing 9 \\
4 \varnothing 4513 \varnothing 73 \varnothing 413\end{array}$ & $\begin{array}{l}-\overline{551007} \\
551007 \\
551067 \\
581001\end{array}$ & $\begin{array}{l}193 \\
205 \\
205 \\
205 \\
207\end{array}$ & $\begin{array}{l}- \\
- \\
-\end{array}$ & $\begin{array}{l}- \\
2207 \varnothing \varnothing \\
265 \varnothing \varnothing \varnothing \\
5358 \varnothing \varnothing \\
2382 \varnothing \varnothing\end{array}$ \\
\hline $\begin{array}{l}I \\
I \\
I \\
I \\
I\end{array}$ & $\begin{array}{l}36 \\
37 \\
38 \\
39 \\
40\end{array}$ & $\begin{array}{l}\text { TUCKER DRIVE } \\
\text { CANDLEWOOD ROAD } \\
\text { BRENTWOOD ROAD } \\
\text { WYNDANCH ROAD } \\
\text { KATHERINE PLACE }\end{array}$ & $\begin{array}{l}\text { STEPHEN ROAD } \\
\text { LLOYD DRIVE } \\
\text { NEW JERSEY AVE. } \\
\text { MCNEIL STREET } \\
\text { RACE PLACE }\end{array}$ & $\begin{array}{l}4 \varnothing 45 \varnothing 3 \varnothing 73 \varnothing 328 \\
4 \emptyset 4548 \varnothing 731446 \\
4 \emptyset 4623 \varnothing 73144 \emptyset \\
4 \varnothing 45 \emptyset 1 \varnothing 73 \varnothing 412 \\
4 \emptyset 4425 \varnothing 73 \varnothing 714\end{array}$ & $\begin{array}{l}5605 \\
- \\
- \\
5405 \\
551025\end{array}$ & $\begin{array}{l}186 \\
189 \\
189 \\
267 \\
2 \varnothing 5\end{array}$ & $\begin{array}{l}- \\
- \\
- \\
-\end{array}$ & $\begin{array}{l}- \\
- \\
-\end{array}$ \\
\hline $\begin{array}{l}I \\
I \\
I \\
I\end{array}$ & $\begin{array}{l}41 \\
42 \\
44 \\
45 \\
46\end{array}$ & $\begin{array}{l}\text { CHAPEL HILL DR. } \\
\text { DALE DRIVE } \\
\text { CRESCENT DRIVE } \\
\text { CRANBERRY STREET } \\
\text { TONI PLACE }\end{array}$ & $\begin{array}{l}\text { CHANEL DRIVE } \\
\text { CRANBERRY STREET } \\
\text { CRESCENT DRIVE } \\
\text { LACE LANE }\end{array}$ & $\begin{array}{l}4 \varnothing 4738 \varnothing 731547 \\
4 \varnothing 4354 \varnothing 73 \varnothing 63 \varnothing \\
4 \varnothing 4626 \varnothing 73112 \emptyset \\
4 \varnothing 4627 \varnothing 731114 \\
4 \varnothing 4747 \varnothing 731246\end{array}$ & $\begin{array}{l}58 \\
57 \varnothing 3 \\
580630 \\
581202 \\
571004\end{array}$ & $\begin{array}{l}189 \\
191 \\
191 \\
191 \\
191\end{array}$ & $\begin{array}{l}1454000 \\
- \\
- \\
- \\
-\end{array}$ & $\begin{array}{l}1461500 \\
- \\
16 \varnothing \varnothing \varnothing \varnothing \\
216 \varnothing \varnothing \varnothing \\
36 \varnothing \varnothing \varnothing\end{array}$ \\
\hline $\begin{array}{l}I \\
I \\
I \\
I\end{array}$ & $\begin{array}{l}47 \\
48 \\
49 \\
50 \\
51\end{array}$ & $\begin{array}{l}\text { PINE ACRES BLVD. } \\
\text { SYCAMORE AVENUE } \\
\text { WICHARD DRIVE } \\
\text { MUNSON LANE } \\
\text { CRANBERRY STREET }\end{array}$ & $\begin{array}{l}\text { LAUREL DRIVE } \\
\text { WESTWIND DRIVE } \\
\text { OCEAN AVENUE } \\
\text { MONTAUK HIGHWAY } \\
\text { NICOLL AVENUE }\end{array}$ & $\begin{array}{l}4 \varnothing 4458 \varnothing 73162 \varnothing \\
4 \varnothing 4624 \varnothing 73 \varnothing 71 \varnothing \\
4 \varnothing 4466 \varnothing 73 \varnothing 656 \\
4 \varnothing 4347 \varnothing 73 \varnothing 613 \\
4 \varnothing 463 \varnothing \varnothing 731 \varnothing 51\end{array}$ & $\begin{array}{l}611029 \\
580921 \\
590713 \\
598225 \\
6112\end{array}$ & $\begin{array}{l}189 \\
265 \\
2 ø 5 \\
211 \\
191\end{array}$ & $\begin{array}{l}- \\
102000 \\
- \\
-\end{array}$ & $\begin{array}{l}251100 \\
1200 \varnothing \varnothing \\
- \\
1 \overline{1 \sigma \varnothing \varnothing \varnothing \varnothing ~}\end{array}$ \\
\hline $\begin{array}{l}I \\
I \\
I \\
I\end{array}$ & $\begin{array}{l}52 \\
53 \\
54 \\
55 \\
56\end{array}$ & $\begin{array}{l}\text { E. ELM STREET } \\
\text { E. ELM STREET } \\
\text { QUAIL DRIVE } \\
\text { ARBELL DRIVE } \\
\text { CRAIG PLACE }\end{array}$ & $\begin{array}{l}\text { LOWELL AVENUE } \\
\text { NICOLL AVENUE } \\
\text { WICKS ROAD } \\
\text { CANDLEWOOD ROAD } \\
\text { CRAIG ROAD }\end{array}$ & $\begin{array}{l}4 \emptyset 47 \varnothing 1 \varnothing 731126 \\
4 \varnothing 47 \varnothing 3 \varnothing 731 \varnothing 58 \\
4 \varnothing 4747 \varnothing 7316 \varnothing 2 \\
4 \varnothing 4552 \varnothing 731452 \\
4 \varnothing 45 \varnothing 2 \varnothing 73 \varnothing 954\end{array}$ & $\begin{array}{l}620403 \\
620103 \\
6 \varnothing 10 \\
596389 \\
591210\end{array}$ & $\begin{array}{l}191 \\
191 \\
189 \\
189 \\
199\end{array}$ & $\begin{array}{l}1098 \varnothing \varnothing \\
10980 \varnothing \\
2955 \varnothing \varnothing \\
556780 \\
31960 \varnothing\end{array}$ & $\begin{array}{l}- \\
169800 \\
299200 \\
577700 \\
321000\end{array}$ \\
\hline $\begin{array}{l}I \\
I \\
I \\
I\end{array}$ & $\begin{array}{l}57 \\
58 \\
59 \\
60 \\
61\end{array}$ & $\begin{array}{l}\text { ELSIE LANE } \\
\text { WOODCLIFF ROAD } \\
\text { OTIS ROAD } \\
\text { NASSAU STREET } \\
\text { NASSAU STREET }\end{array}$ & $\begin{array}{l}\text { LOCUST DRIVE } \\
\text { EVERGREEN STREET } \\
\text { SELEY CROSS } \\
\text { SATELITE DRIVE } \\
\text { COMMERCIAL AVE. }\end{array}$ & $\begin{array}{l}4045100731 \varnothing 04 \\
4 \varnothing 45200731 \varnothing 16 \\
4045360731016 \\
404453 \varnothing 731 \varnothing 38 \\
4044580731018\end{array}$ & $\begin{array}{l}59121 \varnothing \\
591210 \\
591210 \\
638410 \\
680422\end{array}$ & $\begin{array}{l}189 \\
- \\
199 \\
199 \\
199\end{array}$ & $\begin{array}{l}16808 \emptyset \\
2073 \varnothing \varnothing \\
1886 \varnothing \emptyset \\
3851 \varnothing \emptyset \\
5236 \varnothing \emptyset\end{array}$ & $\begin{array}{l}173600 \\
213000 \\
193060 \\
467500 \\
528080\end{array}$ \\
\hline $\begin{array}{l}I \\
I \\
I\end{array}$ & $\begin{array}{l}62 \\
63 \\
64 \\
65 \\
66\end{array}$ & $\begin{array}{l}\text { HEAD DRIVE } \\
\text { BROADWAY } \\
\text { CANDLEWOOD ROAD } \\
\text { KELLY AVENUE } \\
\text { URN COURT }\end{array}$ & $\begin{array}{l}\text { OLD BROADWAY } \\
\text { BARBER STREET } \\
\text { OWENS STREET } \\
\text { PETERS BOULEVARD } \\
\text { WURZ STREET }\end{array}$ & $\begin{array}{l}4045200730351 \\
4 \emptyset 46490731344 \\
4 \emptyset 455907315 \emptyset 3 \\
4046500731231 \\
4045260731304\end{array}$ & $\begin{array}{l}590482 \\
680601 \\
608511 \\
681013 \\
608323\end{array}$ & $\begin{array}{l}186 \\
189 \\
189 \\
191 \\
189\end{array}$ & $\overline{-}$ & $\begin{array}{l}- \\
- \\
65300 \\
-\end{array}$ \\
\hline
\end{tabular}


N.Y. [Dash indicates no data. Altitudes are in feet above sea level.] (cont.)

\begin{tabular}{|c|c|c|c|c|c|c|c|c|c|c|c|c|}
\hline & & $\begin{array}{l}\text { MAX. } \\
\text { AREA } \\
\text { (squar }\end{array}$ & $\begin{array}{r}\text { BASIN } \\
\text { AREA } \\
\text { feot) }\end{array}$ & RIM & $\begin{array}{l}\text {-ALTI } \\
\text { OVER- } \\
\text { FLOW }\end{array}$ & $\begin{array}{l}\text { DDE-- } \\
\text { BOT- } \\
\text { TOM }\end{array}$ & $\begin{array}{l}\text { WATER } \\
\text { TABLE }\end{array}$ & $\begin{array}{c}\text { DRAIN. } \\
\text { AREA } \\
\text { (acres) }\end{array}$ & $\begin{array}{l}---B \\
\text { STAT }\end{array}$ & IN-- & $\begin{array}{l}\text { GEO. } \\
\text { UNIT }\end{array}$ & $\begin{array}{l}\text { SOIL } \\
\text { UNIT }\end{array}$ \\
\hline & $\begin{array}{r}5 \\
9 \\
10 \\
11 \\
12\end{array}$ & $\begin{array}{c}12500 \\
- \\
117500 \\
105300 \\
27800\end{array}$ & $\begin{array}{r}20800 \\
36080 \\
104000 \\
104000 \\
-\end{array}$ & $\begin{array}{r}75.0 \\
45.0 \\
184.9 \\
108.9 \\
130.0\end{array}$ & $\begin{array}{r}74.8 \\
- \\
103.0 \\
106.5 \\
107.0\end{array}$ & $\begin{array}{r}- \\
85 . \overline{0} \\
87.0 \\
83.0\end{array}$ & $\begin{array}{l}43.0 \\
33.0 \\
47.0 \\
47.0 \\
47.0\end{array}$ & $\begin{array}{r}19.7 \\
- \\
85.0 \\
91.0 \\
96.9\end{array}$ & $\begin{array}{l}1 \\
1 \\
1 \\
0 \\
0\end{array}$ & $\begin{array}{l}1 \\
1 \\
1 \\
1 \\
1\end{array}$ & $\begin{array}{l}1 \\
1 \\
1 \\
1 \\
1\end{array}$ & $\begin{array}{l}7 \\
7 \\
7 \\
7 \\
7\end{array}$ \\
\hline 1 & $\begin{array}{l}14 \\
15 \\
16 \\
17 \\
18\end{array}$ & $\begin{array}{c}40500 \\
27700 \\
- \\
- \\
53300\end{array}$ & $\begin{array}{r}48000 \\
- \\
- \\
-\end{array}$ & $\begin{array}{r}135.0 \\
115.0 \\
75.0 \\
80.0 \\
90.0\end{array}$ & $\begin{array}{r}126.0 \\
- \\
- \\
- \\
-\end{array}$ & $\begin{array}{r}96.0 \\
- \\
- \\
- \\
-\end{array}$ & $\begin{array}{l}47.0 \\
45.0 \\
38.0 \\
39.0 \\
39.0\end{array}$ & $\begin{array}{r}160.5 \\
46.5 \\
- \\
93 . \overline{0}\end{array}$ & $\begin{array}{l}1 \\
1 \\
1 \\
1 \\
0\end{array}$ & $\begin{array}{l}1 \\
1 \\
1 \\
1 \\
1\end{array}$ & $\begin{array}{l}1 \\
1 \\
1 \\
1 \\
1\end{array}$ & $\begin{array}{l}7 \\
7 \\
3 \\
7 \\
7\end{array}$ \\
\hline I & $\begin{array}{l}19 \\
20 \\
21 \\
22 \\
24\end{array}$ & $\begin{array}{c}- \\
33700 \\
- \\
- \\
-\end{array}$ & $\begin{array}{l}- \\
- \\
-\end{array}$ & $\begin{array}{l}55.0 \\
70 . \varnothing \\
30.0 \\
65 . \varnothing \\
55.0\end{array}$ & $\begin{array}{l}- \\
- \\
- \\
-\end{array}$ & $\begin{array}{r}43 . \overline{5} \\
- \\
- \\
-\end{array}$ & $\begin{array}{l}38.0 \\
38.0 \\
16.0 \\
33.0 \\
32.0\end{array}$ & $\begin{array}{r}51.1 \\
- \\
-\end{array}$ & $\begin{array}{l}1 \\
1 \\
1 \\
1 \\
1\end{array}$ & $\begin{array}{l}1 \\
1 \\
1 \\
1 \\
1\end{array}$ & $\begin{array}{l}1 \\
1 \\
1 \\
1 \\
1\end{array}$ & $\begin{array}{l}7 \\
3 \\
7 \\
3 \\
7\end{array}$ \\
\hline T & $\begin{array}{l}26 \\
27 \\
28 \\
29 \\
30\end{array}$ & $\begin{array}{l}- \\
- \\
- \\
-\end{array}$ & $\begin{array}{l}- \\
- \\
- \\
-\end{array}$ & $\begin{array}{l}55.0 \\
50.0 \\
50.0 \\
25.0 \\
25.0\end{array}$ & $\begin{array}{l}- \\
- \\
- \\
-\end{array}$ & $\begin{array}{l}- \\
- \\
- \\
-\end{array}$ & $\begin{array}{l}32.0 \\
31.0 \\
31.0 \\
16.0 \\
16.0\end{array}$ & $\begin{array}{l}- \\
- \\
-\end{array}$ & $\begin{array}{l}1 \\
1 \\
1 \\
1 \\
1\end{array}$ & $\begin{array}{l}1 \\
1 \\
1 \\
1 \\
1\end{array}$ & $\begin{array}{l}1 \\
1 \\
1 \\
1 \\
1\end{array}$ & $\begin{array}{l}7 \\
6 \\
6 \\
7 \\
7\end{array}$ \\
\hline$I$ & $\begin{array}{l}31 \\
32 \\
33 \\
34 \\
35\end{array}$ & $\begin{array}{l}- \\
22100 \\
26500 \\
53600 \\
23800\end{array}$ & $\begin{array}{r}- \\
28800 \\
22480 \\
73600 \\
33600\end{array}$ & $\begin{array}{l}25.0 \\
42.0 \\
35.0 \\
30.0 \\
30.0\end{array}$ & $\begin{array}{l}- \\
- \\
-\end{array}$ & $\begin{array}{r}20 . \overline{0} \\
18.5 \\
7 . \overline{0}\end{array}$ & $\begin{array}{l}13.0 \\
20.0 \\
19.0 \\
19.0 \\
18.0\end{array}$ & $\begin{array}{l}-\overline{4} \\
30.4 \\
36.5 \\
73.8 \\
37.5\end{array}$ & $\begin{array}{l}1 \\
1 \\
1 \\
1 \\
1\end{array}$ & $\begin{array}{l}1 \\
1 \\
1 \\
1 \\
1\end{array}$ & $\begin{array}{l}1 \\
1 \\
1 \\
1 \\
1\end{array}$ & $\begin{array}{l}7 \\
7 \\
7 \\
7 \\
7\end{array}$ \\
\hline 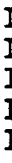 & $\begin{array}{l}36 \\
37 \\
38 \\
39 \\
48\end{array}$ & $\begin{array}{l}- \\
- \\
- \\
-\end{array}$ & $\begin{array}{r}- \\
- \\
9480 \\
-\end{array}$ & $\begin{array}{l}77.0 \\
65.0 \\
50.0 \\
25.0 \\
25.0\end{array}$ & $\begin{array}{r}- \\
- \\
14 . \overline{0} \\
-\end{array}$ & $\begin{array}{r}- \\
- \\
6 . \bar{\sigma} \\
-\end{array}$ & $\begin{array}{l}36.0 \\
40.0 \\
35.0 \\
17.0 \\
11.0\end{array}$ & $\begin{array}{l}- \\
- \\
- \\
-\end{array}$ & $\begin{array}{l}1 \\
1 \\
1 \\
1 \\
1\end{array}$ & $\begin{array}{l}1 \\
1 \\
1 \\
1 \\
1\end{array}$ & $\begin{array}{l}1 \\
1 \\
1 \\
1 \\
1\end{array}$ & $\begin{array}{l}6 \\
7 \\
7 \\
7 \\
7\end{array}$ \\
\hline ] & $\begin{array}{l}41 \\
42 \\
44 \\
45 \\
46\end{array}$ & $\begin{array}{c}146100 \\
- \\
10000 \\
21000 \\
3600\end{array}$ & $\begin{array}{l}- \\
17600 \\
17100 \\
36000 \\
21600\end{array}$ & $\begin{array}{r}101.0 \\
11.0 \\
50.0 \\
48.6 \\
98.5\end{array}$ & $\begin{array}{l}- \\
- \\
-\end{array}$ & $\begin{array}{l}89.0 \\
63.5 \\
36.0 \\
35.0 \\
87.5\end{array}$ & $\begin{array}{r}47.0 \\
4.0 \\
38.0 \\
38.0 \\
38.0\end{array}$ & $\begin{array}{r}228.9 \\
- \\
15.7 \\
33.1 \\
5.7\end{array}$ & $\begin{array}{l}0 \\
1 \\
1 \\
1 \\
1\end{array}$ & $\begin{array}{l}1 \\
1 \\
1 \\
1 \\
1\end{array}$ & $\begin{array}{l}1 \\
1 \\
1 \\
1 \\
1\end{array}$ & $\begin{array}{l}7 \\
7 \\
6 \\
6 \\
7\end{array}$ \\
\hline 1 & $\begin{array}{l}47 \\
48 \\
49 \\
50 \\
51\end{array}$ & $\begin{array}{c}17900 \\
12000 \\
- \\
- \\
10000\end{array}$ & $\begin{array}{r}33800 \\
19500 \\
57000 \\
25800\end{array}$ & $\begin{array}{l}62.1 \\
45.0 \\
40.0 \\
17.0 \\
49.7\end{array}$ & $\begin{array}{r}59.0 \\
- \\
- \\
-\end{array}$ & $\begin{array}{r}45 . \varnothing \\
32.0 \\
- \\
- \\
37 . \varnothing\end{array}$ & $\begin{array}{r}40.0 \\
27.0 \\
19.0 \\
5.0 \\
30.0\end{array}$ & $\begin{array}{r}39.5 \\
15.6 \\
- \\
- \\
12.7\end{array}$ & $\begin{array}{l}1 \\
1 \\
1 \\
1 \\
1\end{array}$ & $\begin{array}{l}1 \\
1 \\
1 \\
1 \\
1\end{array}$ & $\begin{array}{l}1 \\
1 \\
1 \\
1 \\
1\end{array}$ & $\begin{array}{l}7 \\
7 \\
7 \\
7 \\
6\end{array}$ \\
\hline & $\begin{array}{l}52 \\
53 \\
54 \\
55 \\
56\end{array}$ & $\begin{array}{c}- \\
16900 \\
29900 \\
57800 \\
27900\end{array}$ & $\begin{array}{r}22500 \\
23800 \\
- \\
-\end{array}$ & $\begin{array}{r}55.0 \\
56.3 \\
120.0 \\
7 \varnothing .0 \\
18.5\end{array}$ & $\begin{array}{r}- \\
- \\
- \\
18 . \overline{0}\end{array}$ & $\begin{array}{r}42.5 \\
43.0 \\
- \\
6.5\end{array}$ & $\begin{array}{l}34.0 \\
32.0 \\
47.0 \\
40.0 \\
12.0\end{array}$ & $\begin{array}{l}15.0 \\
15.0 \\
40.0 \\
87.6 \\
50.3\end{array}$ & $\begin{array}{l}1 \\
1 \\
0 \\
1 \\
1\end{array}$ & $\begin{array}{l}1 \\
1 \\
1 \\
1 \\
1\end{array}$ & $\begin{array}{l}1 \\
1 \\
1 \\
1 \\
1\end{array}$ & $\begin{array}{l}7 \\
6 \\
7 \\
7 \\
7\end{array}$ \\
\hline & $\begin{array}{l}57 \\
58 \\
59 \\
60 \\
61\end{array}$ & $\begin{array}{l}15700 \\
19400 \\
17500 \\
39200 \\
52400\end{array}$ & $\begin{array}{l}27000 \\
29300 \\
28600 \\
59800 \\
39000\end{array}$ & $\begin{array}{l}23.0 \\
28.5 \\
29.5 \\
32.3 \\
27.8\end{array}$ & $\begin{array}{r}21.0 \\
28.0 \\
29.5 \\
28.4 \\
-\end{array}$ & $\begin{array}{l}10.0 \\
17.0 \\
18.5 \\
18.0 \\
12.0\end{array}$ & $\begin{array}{l}12.0 \\
20.0 \\
21.0 \\
22.0 \\
10.0\end{array}$ & $\begin{array}{r}26.4 \\
32.6 \\
29.6 \\
1.8 \\
68.8\end{array}$ & $\begin{array}{l}1 \\
1 \\
1 \\
1 \\
1\end{array}$ & $\begin{array}{l}1 \\
1 \\
1 \\
3 \\
1\end{array}$ & $\begin{array}{l}1 \\
1 \\
1 \\
1 \\
1\end{array}$ & $\begin{array}{l}7 \\
7 \\
7 \\
7 \\
7\end{array}$ \\
\hline & $\begin{array}{l}62 \\
63 \\
64 \\
65 \\
66\end{array}$ & $\begin{array}{l}- \\
- \\
\overline{5} \\
- \\
-\end{array}$ & $\begin{array}{r}26600 \\
96000 \\
- \\
15400 \\
-\end{array}$ & $\begin{array}{l}35.0 \\
75 . \varnothing \\
75 . \varnothing \\
65 . \varnothing \\
35 . \varnothing\end{array}$ & $\begin{array}{r}-\overline{0} \\
68 . \\
65.0 \\
-\end{array}$ & $\begin{array}{r}56 . \bar{\varnothing} \\
-\bar{\varnothing} \\
55 .\end{array}$ & $\begin{array}{l}18.0 \\
41.0 \\
42.0 \\
36.0 \\
32.0\end{array}$ & $\begin{array}{r}3 . \overline{3} \\
\overline{-} \\
10 . \overline{3} \\
-\end{array}$ & $\begin{array}{l}1 \\
1 \\
1 \\
1 \\
1\end{array}$ & $\begin{array}{l}1 \\
1 \\
1 \\
1 \\
1\end{array}$ & $\begin{array}{l}1 \\
1 \\
1 \\
1 \\
1\end{array}$ & $\begin{array}{l}7 \\
7 \\
7 \\
3 \\
7\end{array}$ \\
\hline
\end{tabular}


Appendix.--Location and basic physical features of recharge basins on Long Island,

\begin{tabular}{|c|c|c|c|c|c|c|c|c|}
\hline \multicolumn{2}{|c|}{$\begin{array}{l}\text { BASIN } \\
\text { NUMBER }\end{array}$} & \multicolumn{2}{|c|}{ NEAREST INTERSECTION } & $\begin{array}{l}\text { LATITUDE } \\
\text { LONGITUDE, } \\
\circ, " \circ, "\end{array}$ & $\begin{array}{l}\text { DATE } \\
\text { BUILT } \\
\text { YrMOD }\end{array}$ & COMMUNITY & \multicolumn{2}{|c|}{$\begin{array}{l}\text { DESIGN ACTUAL } \\
\text { CAPACITY CAPACITY } \\
\text { (cubic feet) }\end{array}$} \\
\hline $\begin{array}{l}I \\
I \\
I \\
I \\
I\end{array}$ & $\begin{array}{l}67 \\
68 \\
69 \\
70 \\
71\end{array}$ & $\begin{array}{l}\text { TOWN LINE ROAD } \\
\text { L.I.E. } \\
\text { WHEELER ROAD } \\
\text { PINE GROVE BLVD. } \\
\text { RIDDLE STREET }\end{array}$ & $\begin{array}{l}\text { VETERANS HIGHWAY } \\
\text { WHEELER ROAD } \\
\text { WOODLAND AVENUE } \\
\text { PROSPECT PLACE } \\
\text { RADCLIFF DRIVE }\end{array}$ & $\begin{array}{l}4 \varnothing 4924 \varnothing 731219 \\
4 \varnothing 4826 \varnothing 731257 \\
4 \varnothing 4837 \varnothing 7313 \varnothing \varnothing \\
4 \varnothing 4557 \varnothing 731627 \\
4 \varnothing 455 \varnothing \varnothing 731322\end{array}$ & $\begin{array}{l}686314 \\
620129 \\
6104 \\
591124 \\
661104\end{array}$ & $\begin{array}{c}196 \\
196 \\
- \\
189 \\
189\end{array}$ & $\begin{array}{l}117000 \\
215060 \\
- \\
235000 \\
331700\end{array}$ & $\begin{array}{c}126 \varnothing \varnothing \varnothing \\
217 \varnothing \varnothing \varnothing \\
- \\
2465 \varnothing \varnothing \\
34290 \varnothing\end{array}$ \\
\hline $\begin{array}{l}I \\
I \\
I \\
I \\
I\end{array}$ & $\begin{array}{l}72 \\
73 \\
74 \\
75 \\
76\end{array}$ & $\begin{array}{l}\text { DELAWARE AVENUE } \\
\text { HENDERSON PLACE } \\
\text { SWALLOW LANE } \\
\text { WILLEN LANE } \\
\text { ELAYNE AVENUE }\end{array}$ & $\begin{array}{l}\text { INDIANA AVENUE } \\
\text { WILLIAMS STREET } \\
\text { THRUSH DRIVE } \\
\text { THRUSH DRIVE } \\
\text { NEVADA AVENUE }\end{array}$ & $\begin{array}{l}40452507314 \varnothing 3 \\
4 \emptyset 47 \varnothing 50731557 \\
4 \varnothing 480207316 \varnothing 2 \\
4 \varnothing 48090731539 \\
4 \varnothing 4416 \varnothing 731436\end{array}$ & $\begin{array}{l}610317 \\
6511 \\
610710 \\
610710 \\
5911\end{array}$ & $\begin{array}{l}189 \\
189 \\
189 \\
189 \\
282\end{array}$ & $\begin{array}{l}- \\
179700 \\
- \\
75200 \\
-\end{array}$ & $\begin{array}{l}390000 \\
184000 \\
- \\
77800 \\
7800\end{array}$ \\
\hline $\begin{array}{l}I \\
I \\
I \\
I \\
I\end{array}$ & $\begin{array}{l}77 \\
78 \\
79 \\
80 \\
81\end{array}$ & $\begin{array}{l}\text { BALDWIN BLVD. } \\
\text { FREEMAN AVENUE } \\
\text { WINSTON DRIVE } \\
\text { DOVECOTE LANE } \\
\text { SUGARWOOD LANE }\end{array}$ & $\begin{array}{l}\text { OSWEGO DRIVE } \\
\text { APPLE STREET } \\
\text { FREEMAN AVENUE } \\
\text { L.I.R.R. } \\
\text { 2ND. PLACE }\end{array}$ & $\begin{array}{l}4 \varnothing 44320731621 \\
4 \varnothing 46100731259 \\
4 \varnothing 45230731259 \\
4 \varnothing 47380731122 \\
4 \varnothing 4744 \varnothing 731113\end{array}$ & $\begin{array}{l}6804 \\
630418 \\
6105 \\
610803 \\
601023\end{array}$ & $\begin{array}{l}282 \\
189 \\
189 \\
191 \\
191\end{array}$ & $\begin{array}{l}- \\
292300 \\
2 \\
229400 \\
340000\end{array}$ & $\begin{array}{l}-\overline{209200} \\
- \\
234060 \\
370000\end{array}$ \\
\hline $\begin{array}{l}I \\
I \\
I \\
I \\
I\end{array}$ & $\begin{array}{l}82 \\
83 \\
84 \\
85 \\
87\end{array}$ & $\begin{array}{l}\text { VILLAGE LANE } \\
\text { EE. 3RD. AVENUE } \\
\text { HAUPPAUGE ROAD } \\
\text { SQUAW LANE } \\
\text { CONNECTICUT AVE. }\end{array}$ & $\begin{array}{l}\text { MARLON LANE } \\
\text { BELDON LANE } \\
\text { HUBBS AVENUE } \\
\text { INDIAN HEAD DR. } \\
\text { OHIO AVENUE }\end{array}$ & $\begin{array}{l}4 \varnothing 4927 \varnothing 731123 \\
4 \varnothing 4444 \varnothing 731528 \\
4 \varnothing 4967 \varnothing 731245 \\
4 \varnothing 4528073 \varnothing 353 \\
4 \varnothing 4534 \varnothing 731419\end{array}$ & $\begin{array}{l}8209 \\
610720 \\
661121 \\
600519 \\
6063\end{array}$ & $\begin{array}{c}196 \\
202 \\
- \\
207 \\
189\end{array}$ & $\begin{array}{l}- \\
\overline{73200} \\
\overline{120080}\end{array}$ & $\begin{array}{l}- \\
77060 \\
78000 \\
\overline{130600}\end{array}$ \\
\hline $\begin{array}{l}I \\
I \\
I \\
I \\
I\end{array}$ & $\begin{array}{l}88 \\
89 \\
90 \\
91 \\
92\end{array}$ & $\begin{array}{l}\text { DALE DRIVE } \\
\text { HEMLOCK DRIVE } \\
\text { BRIGHT SHORE BLV } \\
\text { BIRCH STREET } \\
\text { NOLIN STREET }\end{array}$ & $\begin{array}{l}\text { DALE COURT } \\
\text { BRIGHT SHORE BLV } \\
\text { CEDAR DRIVE } \\
\text { MILLAY LANE } \\
\text { BROADWAY }\end{array}$ & $\begin{array}{l}4 \emptyset 4853 \emptyset 731253 \\
4 \emptyset 4522 \varnothing 731611 \\
4 \emptyset 451 \varnothing \emptyset 7316 \emptyset 7 \\
4 \emptyset 4449 \emptyset 7315 \varnothing 7 \\
4 \varnothing 4623 \varnothing 731343\end{array}$ & $\begin{array}{l}638410 \\
618606 \\
610686 \\
650489 \\
618711\end{array}$ & $\begin{array}{l}196 \\
189 \\
189 \\
282 \\
189\end{array}$ & $\begin{array}{r}1612 \varnothing \varnothing \\
196100 \\
7540 \varnothing \\
9440 \varnothing \\
32900\end{array}$ & $\begin{array}{r}280060 \\
186280 \\
128300 \\
108300 \\
32100\end{array}$ \\
\hline $\begin{array}{l}I \\
I \\
I \\
I \\
I\end{array}$ & $\begin{array}{l}93 \\
94 \\
95 \\
96 \\
97\end{array}$ & $\begin{array}{l}\text { LOEFELER STREET } \\
\text { RANICK ROAD } \\
\text { BRIDGE ROAD } \\
\text { MILFORD DRIVE } \\
\text { OAKLAND AVENUE }\end{array}$ & $\begin{array}{l}\text { BROADWAY } \\
\text { KINGS HIGHWAY } \\
\text { HIGHLAND ROAD } \\
\text { LIL LANE } \\
\text { EVERGREEN AVENUE }\end{array}$ & $\begin{array}{l}4 \varnothing 4649 \emptyset 731345 \\
4 \varnothing 4842 \varnothing 731349 \\
4 \varnothing 4811 \varnothing 731318 \\
4 \varnothing 48 \emptyset 4 \varnothing 7312 \varnothing 3 \\
4 \varnothing 4725 \varnothing 731242\end{array}$ & $\begin{array}{l}610711 \\
6589 \\
671220 \\
\quad- \\
658920\end{array}$ & $\begin{array}{c}189 \\
196 \\
- \\
191 \\
191\end{array}$ & $\begin{array}{l}724 \varnothing 0 \\
- \\
- \\
16 \varnothing \varnothing \varnothing \varnothing\end{array}$ & $\begin{array}{l}6890 \varnothing \\
- \\
- \\
\overline{165600}\end{array}$ \\
\hline $\begin{array}{l}I \\
I \\
I \\
I \\
I\end{array}$ & $\begin{array}{r}98 \\
99 \\
100 \\
101 \\
102\end{array}$ & $\begin{array}{l}\text { NORTHFIELD ROAD } \\
\text { PLYMOUTH ROAD } \\
\text { FAIRBANKS AVENUE } \\
\text { ONTARIO STREET } \\
\text { BARLEAU STREET }\end{array}$ & $\begin{array}{l}\text { TOWN LINE ROAD } \\
\text { HALF MILE ROAD } \\
\text { GARDEN CITY ST. } \\
\text { SHELTER STREET } \\
\text { LINCOLN AVENUE }\end{array}$ & $\begin{array}{l}4 \varnothing 4922 \varnothing 731341 \\
4 \varnothing 4856 \varnothing 731324 \\
4 \varnothing 4513 \emptyset 731729 \\
4 \varnothing 49 \varnothing 5 \varnothing 736739 \\
4 \varnothing 4721 \varnothing 731526\end{array}$ & $\begin{array}{c}610804 \\
638219 \\
- \\
- \\
620801\end{array}$ & $\begin{array}{l}196 \\
196 \\
189 \\
208 \\
189\end{array}$ & $\begin{array}{l}365000 \\
268900 \\
- \\
- \\
-\end{array}$ & $\begin{array}{l}382000 \\
287500 \\
- \\
- \\
-\end{array}$ \\
\hline $\begin{array}{l}I \\
I \\
I \\
I\end{array}$ & $\begin{array}{l}103 \\
104 \\
105 \\
106 \\
107\end{array}$ & $\begin{array}{l}\text { STUB STREET } \\
\text { BECK COURT } \\
\text { GIBBS ROAD } \\
\text { BETSY DRIVE } \\
\text { MATTHEWS ROAD }\end{array}$ & $\begin{array}{l}\text { SHERMAN STREET } \\
\text { SHERMAN STREET } \\
\text { BOW LANE } \\
\text { MONTAUK HIGHWAY } \\
\text { WEAVER ROAD }\end{array}$ & 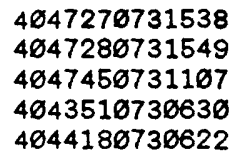 & $\begin{array}{l}610217 \\
610217 \\
620307 \\
520766 \\
620687\end{array}$ & $\begin{array}{l}189 \\
189 \\
191 \\
211 \\
211\end{array}$ & $\begin{array}{l}- \\
\overline{89060} \\
45900 \\
233000\end{array}$ & $\begin{array}{l}- \\
- \\
1148808 \\
48080 \\
244808\end{array}$ \\
\hline $\begin{array}{l}I \\
I \\
I\end{array}$ & $\begin{array}{l}108 \\
189 \\
110 \\
111 \\
112\end{array}$ & $\begin{array}{l}\text { NEW YORK AVENUE } \\
\text { COMMACK ROAD } \\
\text { COMMACK ROAD } \\
\text { CANDLEWOOD ROAD } \\
\text { PELHAM DRIVE }\end{array}$ & $\begin{array}{l}\text { ILLINOIS AVENUE } \\
\text { MONTAUK AVENUE } \\
\text { CONCORD STREET } \\
\text { CHURCHILL DRIVE } \\
\text { HUDSON AVENUE }\end{array}$ & $\begin{array}{l}4 \varnothing 4526 \varnothing 731455 \\
4 \varnothing 45480731418 \\
4 \varnothing 4613 \emptyset 731335 \\
4 \emptyset 45290731359 \\
4 \emptyset 4862 \varnothing 731448\end{array}$ & $\begin{array}{l}658521 \\
62 \varnothing 313 \\
64 \varnothing 323 \\
62 \varnothing 5 \varnothing 2 \\
610126\end{array}$ & $\begin{array}{l}189 \\
189 \\
198 \\
189 \\
189\end{array}$ & $\begin{array}{l}- \\
\overline{121600} \\
18200 \emptyset \\
-\end{array}$ & 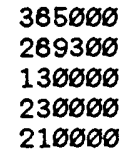 \\
\hline $\begin{array}{l}I \\
I \\
I\end{array}$ & $\begin{array}{l}113 \\
114 \\
116 \\
117 \\
118\end{array}$ & $\begin{array}{l}\text { ORANGE STREET } \\
\text { WINSTON DRIVE } \\
\text { LOCUST AVENUE } \\
\text { LOWELL AVENUE } \\
\text { JUNIPER STREET }\end{array}$ & $\begin{array}{l}\text { MAYFLOWER AVENUE } \\
\text { COMMACK ROAD } \\
\text { OCEAN AVENUE } \\
\text { E. CHERRY STREET } \\
\text { NICOLL AVENUE }\end{array}$ & 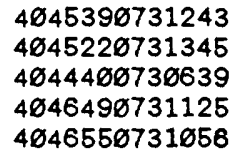 & $\begin{array}{l}620321 \\
630524 \\
5867 \\
610710 \\
606836\end{array}$ & $\begin{array}{l}189 \\
189 \\
285 \\
191 \\
191\end{array}$ & $\begin{array}{l}175200 \\
- \\
- \\
- \\
75000\end{array}$ & $\begin{array}{l}223280 \\
- \\
- \\
98080 \\
109200\end{array}$ \\
\hline I & $\begin{array}{l}121 \\
122 \\
123 \\
124 \\
125\end{array}$ & $\begin{array}{l}\text { KANSAS AVENUE } \\
\text { LAUREL AVENUE } \\
\text { STEPHEN ROAD } \\
\text { BARTON LANE } \\
\text { GRISSOM WAY }\end{array}$ & $\begin{array}{l}\text { OHIO AVENUE } \\
\text { UNION BOULEVARD } \\
\text { STEPHEN COURT } \\
\text { JENNEY ROAD } \\
\text { TELSTAR LANE }\end{array}$ & 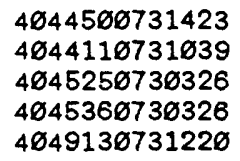 & $\begin{array}{l}610915 \\
\quad- \\
651022 \\
6310 \\
620412\end{array}$ & $\begin{array}{l}202 \\
193 \\
186 \\
186 \\
196\end{array}$ & $\begin{array}{l}173900 \\
- \\
252280 \\
242800 \\
196000\end{array}$ & $\begin{array}{l}37306 \varnothing \\
- \\
256000 \\
250000 \\
2060 \varnothing \varnothing\end{array}$ \\
\hline
\end{tabular}


N.Y. [Dash indicates no data. Altitudes are in feet above sea level.] (cont.)

\begin{tabular}{|c|c|c|c|c|c|c|c|c|c|c|c|c|}
\hline \multicolumn{2}{|c|}{$\begin{array}{l}\text { BASIN } \\
\text { NUMBER }\end{array}$} & $\begin{array}{l}\text { MAX. } \\
\text { AREA } \\
\text { (square }\end{array}$ & $\begin{array}{c}\text { BASIN } \\
\text { AREA } \\
\theta \text { fe日t) }\end{array}$ & RIM & $\begin{array}{l}\text { OVER } \\
\text { FLOW }\end{array}$ & $\begin{array}{l}\text { BOT- } \\
\text { TOM }\end{array}$ & $\begin{array}{l}\text { WATER } \\
\text { TABLE }\end{array}$ & $\begin{array}{c}\text { DRAIN. } \\
\text { AREA } \\
\text { (acres) }\end{array}$ & $\begin{array}{l}---B A S \\
\text { STATUS }\end{array}$ & $\begin{array}{l}\text { IN-- } \\
\text { USE }\end{array}$ & $\begin{array}{l}\text { GEO. } \\
\text { UNIT }\end{array}$ & $\begin{array}{l}\text { SOIL } \\
\text { UNIT }\end{array}$ \\
\hline $\begin{array}{l}I \\
I \\
I \\
I \\
I\end{array}$ & $\begin{array}{l}67 \\
68 \\
69 \\
70 \\
71\end{array}$ & $\begin{array}{c}12600 \\
18100 \\
- \\
24700 \\
25400\end{array}$ & $\begin{array}{r}24000 \\
50400 \\
46800 \\
46800\end{array}$ & $\begin{array}{r}65.0 \\
105 . \varnothing \\
90.0 \\
85.0 \\
49.5\end{array}$ & $\begin{array}{r}95 . \overline{0} \\
\overline{-} \\
49 . \overline{0}\end{array}$ & $\begin{array}{r}83 . \overline{0} \\
- \\
71 . \overline{5} \\
35 . \overline{5}\end{array}$ & $\begin{array}{l}50 . \varnothing \\
41 . \varnothing \\
42 . \varnothing \\
43 . \varnothing \\
36.0\end{array}$ & $\begin{array}{r}16 . \varnothing \\
21 . \varnothing \\
-\overline{27} \\
45.6\end{array}$ & $\begin{array}{l}1 \\
\varnothing \\
0 \\
1 \\
\varnothing\end{array}$ & $\begin{array}{l}1 \\
3 \\
3 \\
1 \\
1\end{array}$ & $\begin{array}{l}1 \\
6 \\
6 \\
1 \\
1\end{array}$ & $\begin{array}{l}6 \\
7 \\
3 \\
7 \\
7\end{array}$ \\
\hline $\begin{array}{l}I \\
I \\
I \\
I \\
I\end{array}$ & $\begin{array}{l}72 \\
73 \\
74 \\
75 \\
76\end{array}$ & $\begin{array}{c}30000 \\
19400 \\
- \\
7800 \\
700\end{array}$ & $\begin{array}{r}44600 \\
37800 \\
67200 \\
22500 \\
5400\end{array}$ & $\begin{array}{r}50.3 \\
135.0 \\
130.0 \\
135.0 \\
25.0\end{array}$ & $\begin{array}{r}- \\
101.2 \\
124.0 \\
133.4 \\
-\end{array}$ & $\begin{array}{r}35.2 \\
91.7 \\
114.0 \\
24 . \overline{0}\end{array}$ & $\begin{array}{l}33.0 \\
47.0 \\
48.0 \\
47.0 \\
23.0\end{array}$ & $\begin{array}{r}47.2 \\
28.3 \\
- \\
11.8 \\
1.1\end{array}$ & $\begin{array}{l}1 \\
1 \\
1 \\
1 \\
1\end{array}$ & $\begin{array}{l}1 \\
1 \\
1 \\
1 \\
1\end{array}$ & $\begin{array}{l}1 \\
1 \\
1 \\
1 \\
1\end{array}$ & $\begin{array}{l}7 \\
7 \\
7 \\
7 \\
7\end{array}$ \\
\hline $\begin{array}{l}I \\
I \\
I\end{array}$ & $\begin{array}{l}77 \\
78 \\
79 \\
80 \\
81\end{array}$ & $\begin{array}{c}\overline{1} \overline{300} \\
\overline{-} \\
23400 \\
37000\end{array}$ & $\begin{array}{r}5900 \\
26100 \\
- \\
34200 \\
30400\end{array}$ & $\begin{array}{l}51.1 \\
50.5 \\
35.0 \\
58.5 \\
59.0\end{array}$ & $\begin{array}{l}- \\
\text { - } \\
\text { - }\end{array}$ & $\begin{array}{r}44.0 \\
42.0 \\
- \\
46 . \varnothing \\
44.0\end{array}$ & $\begin{array}{l}35.0 \\
34.0 \\
30.0 \\
35.0 \\
35.0\end{array}$ & $\begin{array}{r}31 . \overline{9} \\
- \\
26 . \overline{2} \\
39.5\end{array}$ & $\begin{array}{l}1 \\
1 \\
1 \\
1 \\
1\end{array}$ & $\begin{array}{l}1 \\
1 \\
1 \\
1 \\
1\end{array}$ & $\begin{array}{l}1 \\
1 \\
1 \\
1 \\
1\end{array}$ & $\begin{array}{l}7 \\
7 \\
7 \\
6 \\
6\end{array}$ \\
\hline $\begin{array}{l}\text { I } \\
\text { I } \\
\text { I } \\
\text { I } \\
\text { I }\end{array}$ & $\begin{array}{l}82 \\
83 \\
84 \\
85 \\
87\end{array}$ & $\begin{array}{c}- \\
8300 \\
7800 \\
- \\
13000\end{array}$ & $\begin{array}{l}19900 \\
14900 \\
36100 \\
18000\end{array}$ & $\begin{array}{l}84.5 \\
45.3 \\
85.0 \\
38.0 \\
54.0\end{array}$ & $\begin{array}{r}- \\
43.7 \\
- \\
- \\
-\end{array}$ & $\begin{array}{r}65.0 \\
34.0 \\
- \\
- \\
39.0\end{array}$ & $\begin{array}{l}52.0 \\
33.0 \\
44.0 \\
18.0 \\
36.0\end{array}$ & $\begin{array}{r}- \\
12.4 \\
9.5 \\
18.0 \\
18.9\end{array}$ & $\begin{array}{l}1 \\
1 \\
1 \\
1 \\
1\end{array}$ & $\begin{array}{l}1 \\
1 \\
1 \\
1 \\
1\end{array}$ & $\begin{array}{l}1 \\
1 \\
1 \\
1 \\
1\end{array}$ & $\begin{array}{l}7 \\
7 \\
3 \\
6 \\
7\end{array}$ \\
\hline I & $\begin{array}{l}88 \\
89 \\
90 \\
91 \\
92\end{array}$ & $\begin{array}{r}20000 \\
12400 \\
12300 \\
10200 \\
4000\end{array}$ & $\begin{array}{l}34200 \\
28000 \\
28000 \\
23400 \\
11500\end{array}$ & $\begin{array}{l}88.0 \\
64.8 \\
60.5 \\
46.7 \\
66.0\end{array}$ & $\begin{array}{l}64 . \overline{5} \\
58.9 \\
44.4 \\
64.7\end{array}$ & $\begin{array}{l}73.0 \\
49.5 \\
48.5 \\
34.0 \\
56.7\end{array}$ & $\begin{array}{l}42.0 \\
42.0 \\
42.0 \\
34.0 \\
38.0\end{array}$ & $\begin{array}{r}18.5 \\
26.8 \\
11.9 \\
12.1 \\
4.5\end{array}$ & $\begin{array}{l}1 \\
1 \\
0 \\
1 \\
1\end{array}$ & $\begin{array}{l}1 \\
1 \\
1 \\
1 \\
1\end{array}$ & $\begin{array}{l}1 \\
1 \\
1 \\
1 \\
1\end{array}$ & $\begin{array}{l}7 \\
1 \\
3 \\
3 \\
7\end{array}$ \\
\hline $\begin{array}{l}\text { I } \\
\text { I }\end{array}$ & $\begin{array}{l}93 \\
94 \\
95 \\
96 \\
97\end{array}$ & $\begin{array}{c}6200 \\
- \\
- \\
16600\end{array}$ & $\begin{array}{l}12000 \\
13600 \\
12600 \\
72000 \\
36000\end{array}$ & $\begin{array}{r}67.8 \\
115.0 \\
121.5 \\
95.0 \\
82.7\end{array}$ & $\begin{array}{r}67.2 \\
109.4 \\
95 . \overline{0}\end{array}$ & $\begin{array}{r}58.0 \\
104.0 \\
111.9 \\
84.0 \\
70.0\end{array}$ & $\begin{array}{l}38.0 \\
44.0 \\
41.0 \\
37.0 \\
37.0\end{array}$ & $\begin{array}{r}9.9 \\
\overline{-} \\
25 . \overline{2}\end{array}$ & $\begin{array}{l}1 \\
1 \\
1 \\
1 \\
1\end{array}$ & $\begin{array}{l}1 \\
1 \\
1 \\
1 \\
1\end{array}$ & $\begin{array}{l}1 \\
6 \\
1 \\
1 \\
1\end{array}$ & $\begin{array}{l}7 \\
3 \\
3 \\
7 \\
7\end{array}$ \\
\hline $\begin{array}{l}\text { I } \\
\text { I } \\
\text { I }\end{array}$ & $\begin{array}{r}98 \\
99 \\
100 \\
101 \\
102\end{array}$ & $\begin{array}{c}38200 \\
41100 \\
- \\
- \\
-\end{array}$ & $\begin{array}{r}83200 \\
69300 \\
- \\
- \\
-\end{array}$ & $\begin{array}{r}48.2 \\
57.0 \\
65.0 \\
100.0 \\
100.0\end{array}$ & $\begin{array}{r}57.0 \\
- \\
-\end{array}$ & $\begin{array}{r}41.4 \\
50.0 \\
- \\
-\end{array}$ & $\begin{array}{l}39.0 \\
43.0 \\
42.0 \\
51.0 \\
45.0\end{array}$ & $\begin{array}{r}67.5 \\
42.3 \\
- \\
- \\
-\end{array}$ & $\begin{array}{l}1 \\
1 \\
1 \\
1 \\
1\end{array}$ & $\begin{array}{l}1 \\
1 \\
1 \\
1 \\
1\end{array}$ & $\begin{array}{l}1 \\
1 \\
1 \\
6 \\
1\end{array}$ & $\begin{array}{l}6 \\
6 \\
7 \\
3 \\
7\end{array}$ \\
\hline I & $\begin{array}{l}103 \\
104 \\
105 \\
106 \\
107\end{array}$ & $\begin{array}{c}- \\
\overline{1} \\
1400 \\
4800 \\
24400\end{array}$ & $\begin{array}{r}- \\
20000 \\
10800 \\
27900\end{array}$ & $\begin{array}{r}105.0 \\
100.0 \\
58.0 \\
13.0 \\
20.0\end{array}$ & $\begin{array}{l}- \\
- \\
-\end{array}$ & $\begin{array}{r}- \\
\overline{-} \\
45.0 \\
8.0 \\
14.0\end{array}$ & $\begin{array}{r}46.0 \\
46.0 \\
37.0 \\
4.0 \\
7.0\end{array}$ & $\begin{array}{r}- \\
12 . \overline{2} \\
6.2 \\
25.2\end{array}$ & $\begin{array}{l}1 \\
1 \\
1 \\
1 \\
1\end{array}$ & $\begin{array}{l}1 \\
1 \\
1 \\
1 \\
1\end{array}$ & $\begin{array}{l}1 \\
1 \\
1 \\
1 \\
1\end{array}$ & $\begin{array}{l}7 \\
7 \\
6 \\
7 \\
7\end{array}$ \\
\hline I & $\begin{array}{l}108 \\
109 \\
110 \\
111 \\
112\end{array}$ & $\begin{array}{l}36500 \\
29800 \\
24100 \\
21500 \\
26300\end{array}$ & $\begin{array}{l}50480 \\
43700 \\
37800 \\
33600 \\
43400\end{array}$ & $\begin{array}{r}60.0 \\
58.6 \\
35.1 \\
51.1 \\
122.0\end{array}$ & $\begin{array}{r}54 . \overline{3} \\
33.5 \\
50.2 \\
121.0\end{array}$ & $\begin{array}{r}42.0 \\
45.3 \\
28.1 \\
39.5 \\
113.0\end{array}$ & $\begin{array}{l}36.0 \\
38.0 \\
32.0 \\
34.0 \\
46.0\end{array}$ & $\begin{array}{r}57.5 \\
1.2 \\
14.9 \\
28.7 \\
33.1\end{array}$ & $\begin{array}{l}1 \\
1 \\
1 \\
1 \\
1\end{array}$ & $\begin{array}{l}1 \\
1 \\
1 \\
1 \\
1\end{array}$ & $\begin{array}{l}1 \\
1 \\
1 \\
1 \\
1\end{array}$ & $\begin{array}{l}7 \\
7 \\
3 \\
7 \\
7\end{array}$ \\
\hline I & $\begin{array}{l}113 \\
114 \\
116 \\
117 \\
118\end{array}$ & $\begin{array}{c}26600 \\
- \\
- \\
10300 \\
11500\end{array}$ & $\begin{array}{r}37300 \\
- \\
37200 \\
27000 \\
14000\end{array}$ & $\begin{array}{l}45.0 \\
35.0 \\
37.0 \\
55.0 \\
54.8\end{array}$ & $\begin{array}{r}39.9 \\
- \\
54.4 \\
53.0\end{array}$ & $\begin{array}{r}31.5 \\
- \\
21.0 \\
45.7 \\
43.5\end{array}$ & $\begin{array}{l}30.0 \\
32.0 \\
12.0 \\
33.0 \\
31.0\end{array}$ & $\begin{array}{r}26.5 \\
- \\
- \\
14.2 \\
11.8\end{array}$ & $\begin{array}{l}1 \\
1 \\
1 \\
1 \\
1\end{array}$ & $\begin{array}{l}1 \\
1 \\
3 \\
1 \\
1\end{array}$ & $\begin{array}{l}1 \\
1 \\
1 \\
1 \\
1\end{array}$ & $\begin{array}{l}7 \\
7 \\
3 \\
7 \\
6\end{array}$ \\
\hline 1 & $\begin{array}{l}121 \\
122 \\
123 \\
124 \\
125\end{array}$ & $\begin{array}{c}93400 \\
- \\
19700 \\
17900 \\
28000\end{array}$ & $\begin{array}{r}\overline{-} \\
41600 \\
36400 \\
44000\end{array}$ & $\begin{array}{l}34.9 \\
23.0 \\
29.0 \\
32 . \varnothing \\
93.0\end{array}$ & $\begin{array}{r}32.0 \\
27 . \overline{-} \\
30.0 \\
-\end{array}$ & $\begin{array}{l}28.0 \\
14 . \overline{-} \\
16.0 \\
80.0\end{array}$ & $\begin{array}{l}33.0 \\
15.0 \\
20.0 \\
20.0 \\
51.0\end{array}$ & $\begin{array}{r}57.5 \\
27 . \overline{-} \\
26 . \varnothing \\
24 . \varnothing\end{array}$ & $\begin{array}{l}1 \\
1 \\
1 \\
1 \\
1\end{array}$ & $\begin{array}{l}1 \\
1 \\
1 \\
1 \\
1\end{array}$ & $\begin{array}{l}1 \\
1 \\
1 \\
1 \\
6\end{array}$ & $\begin{array}{l}7 \\
7 \\
6 \\
5 \\
6\end{array}$ \\
\hline
\end{tabular}


Appendix.--Location and basic physical features of recharge basins on Long Island,

\begin{tabular}{|c|c|c|c|c|c|c|c|c|}
\hline \multicolumn{2}{|c|}{$\begin{array}{l}\text { 8ASIN } \\
\text { NUMBER }\end{array}$} & \multicolumn{2}{|c|}{ NEAREST INTERSECTION } & $\begin{array}{l}\text { LATITUDE } \\
\text { LONGITUDE, } \\
\circ, " \circ\end{array}$ & $\begin{array}{l}\text { DATE } \\
\text { BUILT } \\
\text { YrMOD }\end{array}$ & COMMUNITY & \multicolumn{2}{|c|}{$\begin{array}{l}\text { DESIGN ACTUAL } \\
\text { CAPACITY CAPACITY } \\
\text { (cubic foot) }\end{array}$} \\
\hline $\begin{array}{l}I \\
I \\
I \\
I \\
I\end{array}$ & $\begin{array}{l}126 \\
127 \\
128 \\
129 \\
130\end{array}$ & $\begin{array}{l}\text { CARPENTER LANE } \\
\text { LOWELL ROAD } \\
\text { HILL DRIVE } \\
\text { E.FORKS ROAD } \\
\text { ALISA LANE }\end{array}$ & $\begin{array}{l}\text { ATLANTIC PLACE } \\
\text { WYANDANCH ROAD } \\
\text { NATHAN DRIVE } \\
\text { OAKRIDGE DRIVE } \\
\text { OAKRIDGE DRIVE }\end{array}$ & $\begin{array}{l}4 \emptyset 4855 \emptyset 731234 \\
4 \varnothing 4534 \varnothing 73 \emptyset 418 \\
4 \emptyset 47 \varnothing 5 \varnothing 73 \varnothing 849 \\
4 \varnothing 45 \varnothing 2 \varnothing 7315 \varnothing 6 \\
4 \varnothing 4457 \varnothing 731516\end{array}$ & $\begin{array}{l}640311 \\
620928 \\
640225 \\
621026 \\
621026\end{array}$ & $\begin{array}{l}196 \\
2 ø 7 \\
188 \\
202 \\
2 \varnothing 2\end{array}$ & $\begin{array}{l}180500 \\
- \\
\overline{108600} \\
91500\end{array}$ & $\begin{array}{l}183000 \\
- \\
- \\
114300 \\
97400\end{array}$ \\
\hline $\begin{array}{l}I \\
I \\
I \\
I \\
I\end{array}$ & $\begin{array}{l}131 \\
132 \\
133 \\
135 \\
136\end{array}$ & $\begin{array}{l}\text { JULBET DRIVE } \\
\text { ALBA COURT } \\
\text { ARLINE LANE } \\
\text { AERO WAY } \\
\text { WYNDHAM ROAD }\end{array}$ & $\begin{array}{l}\text { RICHMAR DRIVE } \\
\text { LUCILLE DRIVE } \\
\text { TIMBER POINT RD. } \\
\text { JOHNSON AVENUE } \\
\text { FORE COURT }\end{array}$ & $\begin{array}{l}4 \emptyset 4541073 \emptyset 44 \emptyset \\
4 \emptyset 45480730429 \\
4 \emptyset 43280731047 \\
4 \emptyset 4648073 \varnothing 543 \\
4047330731444\end{array}$ & $\begin{array}{l}6209 \\
6209 \\
631003 \\
620730 \\
621227\end{array}$ & $\begin{array}{l}287 \\
207 \\
195 \\
188 \\
189\end{array}$ & $\begin{array}{l}250100 \\
- \\
96600 \\
172900 \\
120000\end{array}$ & $\begin{array}{l}264300 \\
\overline{101000} \\
- \\
160000\end{array}$ \\
\hline $\begin{array}{l}I \\
I \\
I \\
I \\
I\end{array}$ & $\begin{array}{l}139 \\
140 \\
141 \\
142 \\
143\end{array}$ & $\begin{array}{l}\text { FAIRFAX AVENUE } \\
\text { SILVERLEAF LANE } \\
\text { ST. MARKS CIRCLE } \\
\text { BOHEMIA PARKWAY } \\
\text { ARGOSY STREET }\end{array}$ & $\begin{array}{l}\text { CLAY AVENUE } \\
\text { SCOTCH PINE DR. } \\
\text { PARK PLACE } \\
\text { ISLAND BOULEVARD } \\
\text { ROLAN COURT }\end{array}$ & 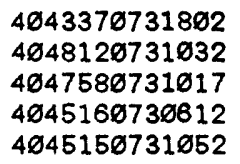 & $\begin{array}{l}5902 \\
63 \varnothing 3 \\
640427 \\
630209 \\
631203\end{array}$ & $\begin{array}{l}204 \\
191 \\
191 \\
205 \\
199\end{array}$ & $\begin{array}{l}77700 \\
448000 \\
534000 \\
25200 \\
-\end{array}$ & $\begin{array}{l}90900 \\
507000 \\
650000 \\
54800 \\
-\end{array}$ \\
\hline $\begin{array}{l}I \\
I \\
I \\
I \\
I\end{array}$ & $\begin{array}{l}144 \\
145 \\
146 \\
147 \\
149\end{array}$ & $\begin{array}{l}\text { BENING LANE } \\
\text { ARCH DRIVE } \\
\text { NORMANDY DRIVE } \\
\text { MARK DRIVE } \\
\text { ARLINE LANE }\end{array}$ & $\begin{array}{l}\text { GRUNDY AVENUE } \\
\text { LOU COURT } \\
\text { CHURCH STREET } \\
\text { COATES AVENUE } \\
\text { TIMBER POINT RD. }\end{array}$ & $\begin{array}{l}4 \varnothing 47290730432 \\
4 \varnothing 46 \varnothing 5 \varnothing 73044 \varnothing \\
4 \varnothing 4615 \varnothing 73 \varnothing 437 \\
4 \varnothing 4627 \varnothing 730441 \\
4 \varnothing 43140731042\end{array}$ & $\begin{array}{c}640429 \\
- \\
- \\
631003\end{array}$ & $\begin{array}{l}225 \\
225 \\
225 \\
225 \\
193\end{array}$ & $\begin{array}{r}63900 \\
139400 \\
389000 \\
299500 \\
63200\end{array}$ & $\begin{array}{r}66800 \\
145700 \\
549000 \\
443700 \\
86400\end{array}$ \\
\hline $\begin{array}{l}I \\
I \\
I \\
I \\
I\end{array}$ & $\begin{array}{l}150 \\
151 \\
152 \\
153 \\
155\end{array}$ & $\begin{array}{l}\text { EISENHOWER AVE. } \\
\text { BELVEDERE DRIVE } \\
\text { KINGS HIGHWAY } \\
\text { GILPIN AVENUE } \\
\text { BALLAD CIRCLE }\end{array}$ & $\begin{array}{l}\text { SUFFOLK AVENUE } \\
\text { SEVENTH STREET } \\
\text { RIDGEFIELD ROAD } \\
\text { TETART STREET } \\
\text { FLUTE LANE }\end{array}$ & 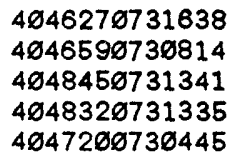 & $\begin{array}{l}630710 \\
648228 \\
\quad- \\
6211 \\
680423\end{array}$ & $\begin{array}{l}189 \\
188 \\
196 \\
196 \\
225\end{array}$ & $\begin{array}{l}92900 \\
- \\
232000 \\
168000 \\
279200\end{array}$ & $\begin{array}{l}142000 \\
- \\
315000 \\
250000 \\
345000\end{array}$ \\
\hline $\begin{array}{l}I \\
I \\
I \\
I\end{array}$ & $\begin{array}{l}157 \\
158 \\
159 \\
16 \varnothing \\
161\end{array}$ & $\begin{array}{l}\text { CRESTWOOD ROAD } \\
\text { KOPF STREET } \\
\text { MADISON AVENUE } \\
\text { SCAMP COURT } \\
\text { MADISON AVENUE }\end{array}$ & $\begin{array}{l}\text { WINDWOOO ROAD } \\
\text { GRAND BOULEVARD } \\
\text { PINE STREET } \\
\text { STUDLEY STREET } \\
\text { CLARKE STREET }\end{array}$ & $\begin{array}{l}4 \varnothing 453207307 \varnothing 1 \\
4 \varnothing 46250731313 \\
4 \varnothing 47050731511 \\
4 \varnothing 45460731352 \\
404757 \varnothing 731519\end{array}$ & $\begin{array}{l}649515 \\
640526 \\
640904 \\
649614 \\
640618\end{array}$ & $\begin{array}{l}188 \\
189 \\
189 \\
189 \\
189\end{array}$ & $\begin{array}{l}112000 \\
- \\
90400 \\
- \\
-\end{array}$ & $\begin{array}{l}1160000 \\
- \\
89800 \\
- \\
-\end{array}$ \\
\hline $\begin{array}{l}I \\
I \\
I \\
I\end{array}$ & $\begin{array}{l}162 \\
163 \\
164 \\
165 \\
166\end{array}$ & $\begin{array}{l}\text { CLARINET LANE } \\
\text { WILSHIRE LANE } \\
\text { OAKDALE ROAD } \\
\text { LURCOTT LANE } \\
\text { WHEELER ROAD }\end{array}$ & $\begin{array}{l}\text { FORD STREET } \\
\text { OAKDALE ROAD } \\
\text { WILSHIRE AVENUE } \\
\text { FRANCISCO AVE. } \\
\text { GRISSOM WAY }\end{array}$ & $\begin{array}{l}4047 \varnothing 5073 \varnothing 50 \emptyset \\
4 \varnothing 4437 \varnothing 730724 \\
4044420730734 \\
4 \varnothing 4736 \varnothing 731137 \\
404917 \varnothing 731233\end{array}$ & $\begin{array}{l}641106 \\
658317 \\
650317 \\
641218 \\
650622\end{array}$ & $\begin{array}{l}225 \\
205 \\
205 \\
191 \\
196\end{array}$ & $\begin{array}{l}286900 \\
- \\
261800 \\
66600 \\
96300\end{array}$ & $\begin{array}{r}284900 \\
55800 \\
295400 \\
88000 \\
100000\end{array}$ \\
\hline $\begin{array}{l}I \\
I \\
I \\
I\end{array}$ & $\begin{array}{l}167 \\
168 \\
169 \\
17 \varnothing \\
171\end{array}$ & $\begin{array}{l}\text { BEL VEDERE DRIVE } \\
\text { CONNETQUOT AVE. } \\
\text { WOOD AVENUE } \\
\text { KATHERINE PLACE } \\
\text { NEWHAM AVENUE }\end{array}$ & $\begin{array}{l}\text { ARON DRIVE } \\
\text { ALLWOOD AVENUE } \\
\text { HAWTHORNE AVENUE } \\
\text { RACE PLACE } \\
\text { COMMACK ROAD }\end{array}$ & 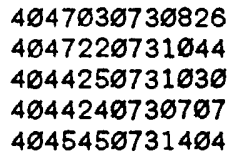 & $\begin{array}{l}648225 \\
651108 \\
650823 \\
650803 \\
651105\end{array}$ & $\begin{array}{l}188 \\
293 \\
193 \\
295 \\
189\end{array}$ & $\begin{array}{l}\overline{42200} \\
98500 \\
- \\
222500\end{array}$ & $\begin{array}{l}\overline{45900} \\
96100 \\
\overline{396100}\end{array}$ \\
\hline $\begin{array}{l}I \\
I \\
I \\
I\end{array}$ & $\begin{array}{l}172 \\
173 \\
174 \\
175 \\
176\end{array}$ & $\begin{array}{l}\text { SPENCE AVENUE } \\
\text { JEFFERSON AVENUE } \\
\text { PEARL STREET } \\
\text { POND ROAD } \\
\text { TIMBERLINE DRIVE }\end{array}$ & $\begin{array}{l}\text { GREENBELT PKWY. } \\
\text { THOMAS AVENUE } \\
\text { S. ROBERTS ST. } \\
\text { KARSHICK STREET } \\
\text { PLUNKET STREET }\end{array}$ & 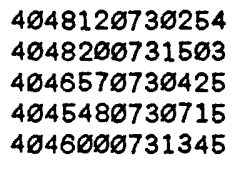 & $\begin{array}{l}651201 \\
64 \varnothing 724 \\
651106 \\
6386 \\
6407\end{array}$ & $\begin{array}{l}228 \\
189 \\
225 \\
188 \\
189\end{array}$ & $\begin{array}{r}225700 \\
77200 \\
278300 \\
87800 \\
-\end{array}$ & $\begin{array}{r}228000 \\
78000 \\
280200 \\
92700 \\
-\end{array}$ \\
\hline$\frac{I}{I}$ & $\begin{array}{l}177 \\
178 \\
179 \\
180 \\
182\end{array}$ & $\begin{array}{l}\text { ROMA AVENUE } \\
\text { ROMA AVENUE } \\
\text { S. BEDFORD DRIVE } \\
\text { CHERIL DRIVE } \\
\text { LORI WAY }\end{array}$ & $\begin{array}{l}\text { BABYLON STREET } \\
\text { ROCKAWAY STREET } \\
\text { HANCOCK STREET } \\
\text { CAMILLE LANE } \\
\text { HUBBS AVENUE }\end{array}$ & 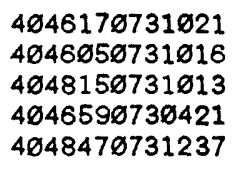 & $\begin{array}{l}650504 \\
649218 \\
640427 \\
6503 \\
620827\end{array}$ & $\begin{array}{l}293 \\
293 \\
293 \\
225 \\
196\end{array}$ & $\begin{array}{l}111000 \\
229800 \\
681300 \\
378800 \\
256000\end{array}$ & $\begin{array}{l}107009 \\
350000 \\
690009 \\
380909 \\
336409\end{array}$ \\
\hline $\begin{array}{l}I \\
I \\
I\end{array}$ & $\begin{array}{l}183 \\
184 \\
185 \\
186 \\
187\end{array}$ & $\begin{array}{l}\text { HELLER PLACE } \\
\text { TERN COURT } \\
\text { MATINECOCK AVE. } \\
\text { WINFIELD AVENUE } \\
\text { GARDINER DRIVE }\end{array}$ & $\begin{array}{l}\text { DIONE LANE } \\
\text { QUAIL RUN } \\
\text { SHAMOKIN LANE } \\
\text { STUYVESANT ST. } \\
\text { SOUTH COUNTRY RD }\end{array}$ & $\begin{array}{l}4 \varnothing 48370731250 \\
4 \varnothing 4322 \varnothing 731115 \\
4 \varnothing 43410731 \varnothing 51 \\
4 \varnothing 4811 \varnothing 731437 \\
4 \varnothing 4212073163 \varnothing\end{array}$ & $\begin{array}{l}630116 \\
670117 \\
631093 \\
66 \varnothing 811 \\
650827\end{array}$ & $\begin{array}{l}196 \\
193 \\
193 \\
189 \\
209\end{array}$ & $\begin{array}{l}- \\
110000 \\
206300 \\
6 \varnothing 700 \\
-\end{array}$ & $\begin{array}{l}- \\
- \\
220000 \\
63000 \\
-\end{array}$ \\
\hline
\end{tabular}


N.Y. [Dash indicates no data. Altitudes are in foet above sea level.] (cont.)

\begin{tabular}{|c|c|c|c|c|c|c|c|c|c|c|c|c|}
\hline & $\begin{array}{l}\text { SIN } \\
\text { MBER }\end{array}$ & $\begin{array}{l}\text { MAX. } \\
\text { AREA } \\
\text { (square }\end{array}$ & $\begin{array}{c}\text { BASIN } \\
\text { AREA } \\
\text { feet) }\end{array}$ & RIM & $\begin{array}{l}- \text {-ALTI } \\
\text { OVER- } \\
\text { FLOW }\end{array}$ & $\begin{array}{l}\text { [TUDE--- } \\
\text { BOT- } \\
\text { TOM }\end{array}$ & $\begin{array}{l}\text { WATER } \\
\text { TABLE }\end{array}$ & $\begin{array}{c}\text { DRAIN. } \\
\text { AREA } \\
\text { (acres) }\end{array}$ & $\begin{array}{l}-- \text { BAS } \\
\text { STATUS }\end{array}$ & $\begin{array}{l}\text { IN-- } \\
\text { USE }\end{array}$ & $\begin{array}{l}\text { GEO. } \\
\text { UNIT }\end{array}$ & $\begin{array}{l}\text { SOIL } \\
\text { UNIT }\end{array}$ \\
\hline I & $\begin{array}{l}126 \\
127 \\
128 \\
129 \\
130\end{array}$ & $\begin{array}{c}18300 \\
- \\
- \\
17900 \\
13900\end{array}$ & $\begin{array}{r}19800 \\
- \\
- \\
13600 \\
19600\end{array}$ & $\begin{array}{r}108.0 \\
35.0 \\
60.0 \\
41.4 \\
45.0\end{array}$ & $\begin{array}{r}- \\
- \\
- \\
41.4 \\
48.0\end{array}$ & $\begin{array}{r}94.0 \\
- \\
35.0 \\
41.0\end{array}$ & $\begin{array}{l}45 . \varnothing \\
25 . \varnothing \\
36 . \varnothing \\
38 . \varnothing \\
34 . \varnothing\end{array}$ & $\begin{array}{r}22.0 \\
- \\
13.9 \\
11.1\end{array}$ & $\begin{array}{l}1 \\
1 \\
1 \\
1 \\
0\end{array}$ & $\begin{array}{l}1 \\
1 \\
1 \\
1 \\
1\end{array}$ & $\begin{array}{l}6 \\
1 \\
1 \\
1 \\
1\end{array}$ & $\begin{array}{l}7 \\
7 \\
7 \\
7 \\
7\end{array}$ \\
\hline$\frac{I}{I}$ & $\begin{array}{l}131 \\
132 \\
133 \\
135 \\
136\end{array}$ & $\begin{array}{c}47200 \\
\overline{10100} \\
- \\
16000\end{array}$ & $\begin{array}{r}88800 \\
- \\
29900 \\
25500 \\
36400\end{array}$ & $\begin{array}{r}35.0 \\
42.0 \\
14.5 \\
60.0 \\
106.5\end{array}$ & $\begin{array}{r}31.6 \\
35.0 \\
- \\
- \\
-\end{array}$ & $\begin{array}{r}26.0 \\
7.0 \\
92.3\end{array}$ & $\begin{array}{r}25.0 \\
25.0 \\
8.0 \\
35.0 \\
45.0\end{array}$ & $\begin{array}{r}29.7 \\
- \\
17.6 \\
7.0 \\
19.0\end{array}$ & $\begin{array}{l}1 \\
1 \\
1 \\
1 \\
1\end{array}$ & $\begin{array}{l}1 \\
1 \\
1 \\
3 \\
1\end{array}$ & $\begin{array}{l}1 \\
1 \\
1 \\
1 \\
1\end{array}$ & $\begin{array}{l}6 \\
3 \\
3 \\
5 \\
7\end{array}$ \\
\hline $\begin{array}{l}I \\
I \\
I \\
I \\
I\end{array}$ & $\begin{array}{l}139 \\
140 \\
141 \\
142 \\
143\end{array}$ & $\begin{array}{c}10100 \\
50700 \\
65000 \\
5500 \\
-\end{array}$ & $\begin{array}{r}21000 \\
69000 \\
84000 \\
7500 \\
37800\end{array}$ & $\begin{array}{l}38.5 \\
76.0 \\
60.0 \\
30.0 \\
35.2\end{array}$ & $\begin{array}{r}38.0 \\
- \\
- \\
-\end{array}$ & $\begin{array}{r}29.0 \\
- \\
- \\
19.0\end{array}$ & $\begin{array}{l}27.0 \\
37.0 \\
35.0 \\
21.0 \\
23.0\end{array}$ & $\begin{array}{r}12.2 \\
48.0 \\
61.2 \\
4.0 \\
-\end{array}$ & $\begin{array}{l}1 \\
1 \\
1 \\
1 \\
1\end{array}$ & $\begin{array}{l}1 \\
1 \\
1 \\
1 \\
1\end{array}$ & $\begin{array}{l}1 \\
1 \\
1 \\
1 \\
1\end{array}$ & $\begin{array}{l}3 \\
7 \\
7 \\
7 \\
7\end{array}$ \\
\hline $\begin{array}{l}I \\
I \\
I\end{array}$ & $\begin{array}{l}144 \\
145 \\
146 \\
147 \\
149\end{array}$ & $\begin{array}{r}5500 \\
17100 \\
54000 \\
44400 \\
8600\end{array}$ & $\begin{array}{r}9800 \\
25500 \\
64400 \\
64400 \\
24200\end{array}$ & $\begin{array}{l}85.1 \\
41.5 \\
50.3 \\
43.9 \\
13.0\end{array}$ & $\begin{array}{r}84.2 \\
40.5 \\
48.0 \\
43.0 \\
-\end{array}$ & $\begin{array}{r}72.0 \\
32.0 \\
38.0 \\
7.0\end{array}$ & $\begin{array}{r}39.0 \\
30.0 \\
34.0 \\
34.0 \\
7.0\end{array}$ & $\begin{array}{r}8.1 \\
17.1 \\
48.2 \\
37.0 \\
11.5\end{array}$ & $\begin{array}{l}1 \\
1 \\
1 \\
1 \\
1\end{array}$ & $\begin{array}{l}1 \\
1 \\
1 \\
1 \\
1\end{array}$ & $\begin{array}{l}1 \\
1 \\
1 \\
1 \\
1\end{array}$ & $\begin{array}{l}3 \\
3 \\
0 \\
6 \\
3\end{array}$ \\
\hline $\begin{array}{l}I \\
I \\
I \\
I \\
I\end{array}$ & $\begin{array}{l}150 \\
151 \\
152 \\
153 \\
155\end{array}$ & $\begin{array}{c}14200 \\
- \\
31500 \\
25000 \\
20100\end{array}$ & $\begin{array}{r}26500 \\
52000 \\
28800 \\
42000\end{array}$ & $\begin{array}{r}95.0 \\
75.0 \\
115.0 \\
150.0 \\
79.3\end{array}$ & $\begin{array}{r}94.0 \\
- \\
- \\
78 .\end{array}$ & $\begin{array}{r}84.0 \\
- \\
34.0 \\
110.0 \\
61.0\end{array}$ & $\begin{array}{l}45 . \varnothing \\
34 . \varnothing \\
44 . \varnothing \\
43 . \varnothing \\
39 . \varnothing\end{array}$ & $\begin{array}{r}4.3 \\
-\overline{36} \\
17.0 \\
35.4\end{array}$ & $\begin{array}{l}1 \\
1 \\
1 \\
0 \\
1\end{array}$ & $\begin{array}{l}1 \\
1 \\
1 \\
1 \\
1\end{array}$ & $\begin{array}{l}1 \\
1 \\
6 \\
6 \\
1\end{array}$ & $\begin{array}{l}7 \\
7 \\
3 \\
5 \\
3\end{array}$ \\
\hline $\begin{array}{l}I \\
I \\
I \\
I \\
I\end{array}$ & $\begin{array}{l}157 \\
158 \\
159 \\
160 \\
161\end{array}$ & $\begin{array}{c}11600 \\
- \\
7800 \\
- \\
-\end{array}$ & $\begin{array}{r}23000 \\
29200 \\
20000 \\
27000 \\
-\end{array}$ & $\begin{array}{r}45.4 \\
65.0 \\
84.2 \\
40.0 \\
125.0\end{array}$ & $\begin{array}{r}- \\
82 . \overline{5} \\
- \\
-\end{array}$ & $\begin{array}{r}31.4 \\
42.0 \\
71.0 \\
- \\
-\end{array}$ & $\begin{array}{l}27.0 \\
38.0 \\
45.0 \\
36.0 \\
47.0\end{array}$ & $\begin{array}{r}11.0 \\
9 . \overline{-} \\
-\end{array}$ & $\begin{array}{l}1 \\
1 \\
1 \\
0 \\
0\end{array}$ & $\begin{array}{l}1 \\
1 \\
1 \\
1 \\
1\end{array}$ & $\begin{array}{l}1 \\
1 \\
1 \\
1 \\
1\end{array}$ & $\begin{array}{l}7 \\
6 \\
7 \\
7 \\
7\end{array}$ \\
\hline I & $\begin{array}{l}162 \\
163 \\
164 \\
165 \\
166\end{array}$ & $\begin{array}{r}28700 \\
5600 \\
29500 \\
8000 \\
10000\end{array}$ & $\begin{array}{r}21500 \\
16800 \\
15000 \\
13200\end{array}$ & $\begin{array}{l}70.0 \\
24.5 \\
27.7 \\
81.5 \\
67.0\end{array}$ & $\begin{array}{r}- \\
23.0 \\
25.0 \\
81.0 \\
-\end{array}$ & $\begin{array}{l}55.3 \\
13.0 \\
15.0 \\
70.0 \\
55.0\end{array}$ & $\begin{array}{l}36.0 \\
19.0 \\
10.0 \\
35.0 \\
45.0\end{array}$ & $\begin{array}{r}22.0 \\
8.9 \\
28.2 \\
49.0 \\
11.0\end{array}$ & $\begin{array}{l}1 \\
1 \\
1 \\
1 \\
1\end{array}$ & $\begin{array}{l}1 \\
1 \\
1 \\
1 \\
1\end{array}$ & $\begin{array}{l}1 \\
1 \\
1 \\
1 \\
1\end{array}$ & $\begin{array}{l}6 \\
1 \\
7 \\
3 \\
3\end{array}$ \\
\hline I & $\begin{array}{l}167 \\
168 \\
169 \\
170 \\
171\end{array}$ & $\begin{array}{c}\overline{-} \\
7100 \\
32000 \\
- \\
39600\end{array}$ & $\begin{array}{r}17600 \\
25000 \\
66000\end{array}$ & $\begin{array}{l}70.0 \\
45.0 \\
25.0 \\
25.0 \\
56.2\end{array}$ & $\begin{array}{r}38 . \overline{5} \\
17.0 \\
-\overline{3}\end{array}$ & $\begin{array}{r}32 . \overline{0} \\
14.0 \\
44 . \overline{3}\end{array}$ & $\begin{array}{l}34.0 \\
32.0 \\
16.0 \\
11.0 \\
38.0\end{array}$ & $\begin{array}{r}6 . \overline{6} \\
15.5 \\
-\overline{1} \\
13 .\end{array}$ & $\begin{array}{l}1 \\
1 \\
1 \\
1 \\
1\end{array}$ & $\begin{array}{l}1 \\
1 \\
1 \\
1 \\
1\end{array}$ & $\begin{array}{l}1 \\
1 \\
1 \\
1 \\
1\end{array}$ & $\begin{array}{l}7 \\
6 \\
7 \\
7 \\
7\end{array}$ \\
\hline I & $\begin{array}{l}172 \\
173 \\
174 \\
175 \\
176\end{array}$ & $\begin{array}{c}15200 \\
10000 \\
20000 \\
9300 \\
-\end{array}$ & $\begin{array}{r}29700 \\
19000 \\
26900 \\
14400 \\
-\end{array}$ & $\begin{array}{r}91.0 \\
143.8 \\
70.3 \\
59.0 \\
55.0\end{array}$ & $\begin{array}{r}88.0 \\
141.8 \\
68.5 \\
- \\
-\end{array}$ & $\begin{array}{r}73.0 \\
134.0 \\
54.5 \\
45.8 \\
-\end{array}$ & $\begin{array}{l}48.0 \\
47.0 \\
37.0 \\
28.0 \\
38.0\end{array}$ & $\begin{array}{r}21.4 \\
9.3 \\
43.8 \\
16.0 \\
-\end{array}$ & $\begin{array}{l}0 \\
1 \\
1 \\
1 \\
1\end{array}$ & $\begin{array}{l}1 \\
1 \\
1 \\
1 \\
1\end{array}$ & $\begin{array}{l}1 \\
1 \\
1 \\
1 \\
1\end{array}$ & $\begin{array}{l}6 \\
7 \\
6 \\
7 \\
7\end{array}$ \\
\hline$I$ & $\begin{array}{l}177 \\
178 \\
179 \\
180 \\
182\end{array}$ & $\begin{array}{r}9700 \\
35000 \\
69000 \\
29300 \\
24900\end{array}$ & $\begin{array}{l}11900 \\
45000 \\
78000 \\
46800 \\
62400\end{array}$ & $\begin{array}{l}35.4 \\
43.5 \\
66.5 \\
73.2 \\
92.0\end{array}$ & $\begin{array}{r}34.0 \\
- \\
- \\
69.2 \\
91.5\end{array}$ & $\begin{array}{l}23.0 \\
24.0 \\
50.0 \\
56.2 \\
78.0\end{array}$ & $\begin{array}{l}23.0 \\
23.0 \\
37.0 \\
37.0 \\
44.0\end{array}$ & $\begin{array}{l}10.8 \\
31.4 \\
72.5 \\
59.6 \\
35.0\end{array}$ & $\begin{array}{l}1 \\
0 \\
1 \\
1 \\
1\end{array}$ & $\begin{array}{l}1 \\
1 \\
1 \\
1 \\
1\end{array}$ & $\begin{array}{l}1 \\
1 \\
1 \\
1 \\
1\end{array}$ & $\begin{array}{l}3 \\
7 \\
7 \\
6 \\
6\end{array}$ \\
\hline I & $\begin{array}{l}183 \\
184 \\
185 \\
186 \\
187\end{array}$ & $\begin{array}{c}- \\
- \\
22000 \\
6600 \\
-\end{array}$ & $\begin{array}{r}37400 \\
62400 \\
33600 \\
15400 \\
-\end{array}$ & $\begin{array}{r}112.0 \\
10.0 \\
15.5 \\
120.2 \\
10.0\end{array}$ & $\begin{array}{r}107.0 \\
9.3 \\
-\overline{5} \\
119.5\end{array}$ & $\begin{array}{r}95.0 \\
6.5 \\
8.5 \\
110.0 \\
-\end{array}$ & $\begin{array}{r}42.0 \\
5.0 \\
11.0 \\
4.0 \\
5.0\end{array}$ & $\begin{array}{r}1 . \overline{4} \\
10.0 \\
7.6 \\
-\end{array}$ & $\begin{array}{l}1 \\
\emptyset \\
0 \\
1 \\
0\end{array}$ & $\begin{array}{l}1 \\
1 \\
1 \\
1 \\
1\end{array}$ & $\begin{array}{l}6 \\
1 \\
1 \\
1 \\
1\end{array}$ & $\begin{array}{l}7 \\
3 \\
3 \\
1 \\
4\end{array}$ \\
\hline
\end{tabular}


Appendix.--Location and basic physical features of recharge basins on Long Island,

\begin{tabular}{|c|c|c|c|c|c|c|c|c|}
\hline \multicolumn{2}{|c|}{$\begin{array}{l}\text { BASIN } \\
\text { NUMBER }\end{array}$} & \multicolumn{2}{|c|}{ NEAREST INTERSECTION } & $\begin{array}{l}\text { LATITUDE } \\
\text { LONGITUDE } \\
\circ, " \text { " " }\end{array}$ & $\begin{array}{l}\text { DATE } \\
\text { BUILT } \\
\text { YrMOD }\end{array}$ & COMMUNITY & \multicolumn{2}{|c|}{$\begin{array}{l}\text { DESIGN ACTUAL } \\
\text { CAPACITY CAPACITY } \\
\text { (cubic feet) }\end{array}$} \\
\hline $\begin{array}{l}I \\
I \\
I \\
I\end{array}$ & $\begin{array}{l}188 \\
189 \\
191 \\
192 \\
193\end{array}$ & $\begin{array}{l}\text { LAKEVIEW AVENUE } \\
\text { BRIDGE ROAD } \\
\text { EVY COURT } \\
\text { GRAND BOULEVARD } \\
\text { OCEAN AVENUE }\end{array}$ & $\begin{array}{l}\text { SOUTH COUNTRY RD } \\
\text { HIGHLAND ROAD } \\
\text { LOOP DRIVE } \\
\text { ORAY STREET } \\
\text { WOODY LANE }\end{array}$ & $\begin{array}{l}4 \varnothing 42010731654 \\
4 \varnothing 48080731317 \\
4 \varnothing 4544 \varnothing 73 \varnothing 421 \\
4 \varnothing 4614 \varnothing 73132 \varnothing \\
4 \varnothing 4438 \varnothing 73 \varnothing 653\end{array}$ & $\begin{array}{l}650827 \\
671220 \\
620920 \\
578718 \\
551807\end{array}$ & $\begin{array}{l}289 \\
191 \\
207 \\
189 \\
285\end{array}$ & $\begin{array}{l}- \\
56000 \\
242280 \\
456200 \\
-\end{array}$ & $\begin{array}{l}- \\
70000 \\
283500 \\
596700 \\
261700\end{array}$ \\
\hline $\begin{array}{l}I \\
I \\
I \\
I \\
\text { MB }\end{array}$ & $\begin{array}{r}194 \\
195 \\
196 \\
197 \\
2\end{array}$ & $\begin{array}{l}\text { PECK AVENUE } \\
\text { CARDINAL CT. S. } \\
\text { INDIANA AVENUE } \\
\text { RADIGAN AVENUE } \\
\text { MELVILLE ROAD }\end{array}$ & $\begin{array}{l}\text { PROSPECT PLACE } \\
\text { PROSPECT PLACE } \\
\text { AMFER COURT } \\
\text { L.I.E. } \\
\text { BROAD HOLLOW RD. }\end{array}$ & $\begin{array}{l}404601073161 \emptyset \\
404554 \varnothing 731621 \\
40451907314 \varnothing 4 \\
404823 \varnothing 731338 \\
404517 \varnothing 732523\end{array}$ & $\begin{array}{c}640122 \\
640327 \\
611027 \\
611027 \\
-\end{array}$ & $\begin{array}{l}189 \\
189 \\
189 \\
196 \\
-\end{array}$ & $\begin{array}{l}- \\
80200 \\
- \\
- \\
-\end{array}$ & $\begin{array}{l}- \\
81600 \\
-\end{array}$ \\
\hline $\begin{array}{l}M B \\
M B \\
M B \\
M B \\
M B\end{array}$ & $\begin{array}{l}3 \\
4 \\
5 \\
6 \\
7\end{array}$ & $\begin{array}{l}\text { MELVILLE ROAD } \\
\text { LINCOLN AVENUE } \\
\text { BETHPAGE ROAD } \\
\text { STRAIGHT PATH } \\
\text { BETHPAGE ROAD }\end{array}$ & $\begin{array}{l}\text { BROAD HOLLOW RD. } \\
\text { GRANT STREET } \\
\text { STRAIGHT PATH } \\
\text { SUNRISE HIGHWAY } \\
\text { IMOLA PLACE }\end{array}$ & $\begin{array}{l}4045130732523 \\
4042410732454 \\
404118073235 \emptyset \\
4041410732336 \\
4041110732352\end{array}$ & $\begin{array}{l}- \\
- \\
- \\
-\end{array}$ & $\begin{array}{l}- \\
- \\
-\end{array}$ & $\begin{array}{l}- \\
- \\
- \\
-\end{array}$ & $\begin{array}{l}- \\
- \\
-\end{array}$ \\
\hline $\begin{array}{l}\text { MBK } \\
\text { MBK } \\
\text { MBK } \\
\text { MBK } \\
\text { MBK }\end{array}$ & $\begin{array}{l}1 \\
2 \\
3 \\
4 \\
5\end{array}$ & $\begin{array}{l}\text { NICOLLS ROAD } \\
\text { ROUTE } 347 \\
\text { MIDDLE COUNTRY } \\
\text { WOODS ROAD } \\
\text { MIDDLE COUNTRY }\end{array}$ & $\begin{array}{l}\text { ST. GEORGE CLUB } \\
\text { OXHEAD ROAD } \\
\text { E. OF SELDEN SCH } \\
\text { MAGNOLIA STREET } \\
\text { COLLEGE ROAD }\end{array}$ & $\begin{array}{l}405507 \varnothing 730702 \\
405302073 \varnothing 627 \\
405210073014 \emptyset \\
4051500730616 \\
4851450730251\end{array}$ & $\begin{array}{l}- \\
- \\
-\end{array}$ & $\begin{array}{c}- \\
- \\
216 \\
253\end{array}$ & $\begin{array}{l}- \\
- \\
-\end{array}$ & $\begin{array}{l}- \\
- \\
- \\
-\end{array}$ \\
\hline $\begin{array}{l}\text { MBK } \\
\text { MBK } \\
\text { MBK } \\
\text { MBK } \\
\text { MBK }\end{array}$ & $\begin{array}{r}6 \\
7 \\
8 \\
9 \\
10\end{array}$ & $\begin{array}{l}\text { B AVENUE } \\
\text { S/O MORICHES-YAP } \\
\text { S/O MORICHES-YAP } \\
\text { WINSTON DRIVE } \\
\text { MIDDLE COUNTRY }\end{array}$ & $\begin{array}{l}\text { 5TH STREET } \\
\text { E/O CRANFORD } \\
\text { E/O CRANFORD } \\
\text { UPTON BOULEVARD } \\
\text { CORAM-YAPHANK RD }\end{array}$ & $\begin{array}{l}4 \varnothing 4919 \emptyset 73 \emptyset 43 \emptyset \\
4 \emptyset 493207251 \varnothing 8 \\
4 \emptyset 49250725198 \\
4 \varnothing 4826 \varnothing 725221 \\
4 \varnothing 52050725958\end{array}$ & $\begin{array}{l}- \\
- \\
-\end{array}$ & $\begin{array}{l}- \\
- \\
- \\
-\end{array}$ & $\begin{array}{l}- \\
- \\
-\end{array}$ & $\begin{array}{l}- \\
- \\
- \\
-\end{array}$ \\
\hline $\begin{array}{l}\text { MBK } \\
\text { MBK } \\
\text { MBK } \\
\text { MBK } \\
\text { MBK }\end{array}$ & $\begin{array}{l}11 \\
12 \\
13 \\
14 \\
15\end{array}$ & $\begin{array}{l}\text { STATION ROAD } \\
\text { BROOKHAVEN LABS. } \\
\text { BROOKHAVEN LABS. } \\
\text { BROOKHAVEN LABS. } \\
\text { BROOKHAVEN LABS. }\end{array}$ & AGAMEMNON AVENUE & 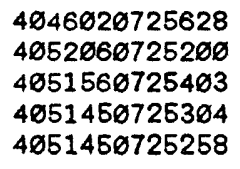 & $\begin{array}{l}- \\
- \\
-\end{array}$ & $\begin{array}{c}213 \\
- \\
- \\
-\end{array}$ & $\begin{array}{l}- \\
- \\
-\end{array}$ & $\begin{array}{l}- \\
- \\
-\end{array}$ \\
\hline $\begin{array}{l}\text { MBK } \\
\text { MH } \\
M H \\
M H \\
M H\end{array}$ & $\begin{array}{r}16 \\
1 \\
2 \\
3 \\
4\end{array}$ & $\begin{array}{l}\text { BROOKHAVEN LABS. } \\
\text { MCKAY ROAD } \\
\text { STH AVENUE } \\
\text { W. 23RD. STREET } \\
\text { W. 22ND STREET }\end{array}$ & $\begin{array}{l}\text { BISCAYNE DRIVE } \\
\text { 1ITH STREET } \\
\text { JONES LANE } \\
\text { GRAYLEY PLACE }\end{array}$ & 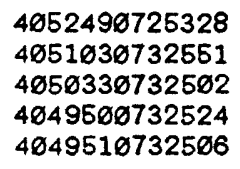 & $\begin{array}{l}- \\
- \\
-\end{array}$ & $\begin{array}{c}\overline{146} \\
\overline{151}\end{array}$ & $\begin{array}{l}- \\
- \\
-\end{array}$ & $\begin{array}{l}- \\
- \\
-\end{array}$ \\
\hline $\begin{array}{l}\text { MH } \\
M H \\
M H \\
M H \\
M H\end{array}$ & $\begin{array}{l}5 \\
6 \\
7 \\
8 \\
9\end{array}$ & $\begin{array}{l}\text { ELMTREE LANE } \\
\text { SPRUCETREE LANE } \\
\text { WALT WHITMAN S.C } \\
\text { WALT WHITMAN S.C } \\
\text { LODGE AVENUE }\end{array}$ & $\begin{array}{l}\text { PEARTREE LANE } \\
\text { OAKTREE LANE } \\
\text { NICHOLS PLACE }\end{array}$ & $\begin{array}{l}4 \emptyset 4926 \emptyset 732505 \\
4 \emptyset 4922 \emptyset 732451 \\
4 \emptyset 4934 \varnothing 73244 \emptyset \\
4 \emptyset 4935 \emptyset 732437 \\
4 \emptyset 5 \emptyset 23 \emptyset 732258\end{array}$ & $\begin{array}{l}- \\
- \\
-\end{array}$ & $\begin{array}{l}151 \\
151 \\
151 \\
151 \\
151\end{array}$ & $\begin{array}{l}- \\
- \\
-\end{array}$ & $\begin{array}{l}- \\
- \\
-\end{array}$ \\
\hline $\begin{array}{l}\mathrm{MH} \\
\mathrm{MH} \\
\mathrm{MH} \\
\mathrm{MH} \\
\mathrm{MH}\end{array}$ & $\begin{array}{l}10 \\
11 \\
14 \\
15 \\
16\end{array}$ & $\begin{array}{l}\text { WALT WHITMAN S.C } \\
\text { WALT WHITMAN S.C } \\
\text { ALTAMORE STREET } \\
\text { GOLDSMITH AVENUE } \\
\text { SWEET HOLLOW RD. }\end{array}$ & $\begin{array}{l}\text { CARY COURT } \\
\text { EUGENE STREET } \\
\text { WALT WHITMAN RD. }\end{array}$ & 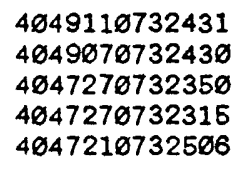 & $\begin{array}{l}- \\
- \\
- \\
-\end{array}$ & $\begin{array}{c}151 \\
151 \\
- \\
148 \\
148\end{array}$ & $\begin{array}{l}- \\
- \\
-\end{array}$ & $\begin{array}{l}- \\
- \\
-\end{array}$ \\
\hline $\begin{array}{l}\text { MH } \\
\text { MH } \\
\text { MH } \\
\text { MH } \\
\text { MH }\end{array}$ & $\begin{array}{l}17 \\
18 \\
19 \\
20 \\
21\end{array}$ & $\begin{array}{l}\text { SWEET HOLLOW RD. } \\
\text { NORTH SIDE LIE } \\
\text { NORTHERN STATE } \\
\text { OLD EAST NECK RD } \\
\text { BAYLIS ROAD }\end{array}$ & $\begin{array}{l}\text { WALT WHITMAN RD. } \\
\text { WALT WHITMAN RD. } \\
\text { BROAD HOLLOW RD. } \\
\text { PINE LAWN ROAD } \\
\text { PINE LAWN ROAD }\end{array}$ & 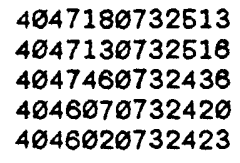 & $\begin{array}{l}- \\
- \\
-\end{array}$ & $\begin{array}{l}148 \\
148 \\
148 \\
148 \\
148\end{array}$ & $\begin{array}{l}- \\
- \\
-\end{array}$ & $\begin{array}{l}- \\
- \\
-\end{array}$ \\
\hline $\begin{array}{l}\text { MH } \\
\text { MH } \\
\text { MH } \\
\text { MH } \\
\text { MH }\end{array}$ & $\begin{array}{l}22 \\
23 \\
24 \\
25 \\
26\end{array}$ & $\begin{array}{l}\text { COLONIAL SPRINGS } \\
\text { ABBOTT DRIVE } \\
\text { POLLY COURT } \\
\text { LINDA PLACE } \\
\text { OAKWOOD ROAD }\end{array}$ & $\begin{array}{l}\text { WELLWOOD ROAD } \\
\text { GLADES WAY } \\
\text { POLLY DRIVE } \\
\text { HARRIET LANE } \\
\text { WOODCHUCK HOL.RD }\end{array}$ & $\begin{array}{l}4 \oslash 4557 ø 732414 \\
4 \varnothing 53810732469 \\
4052220732355 \\
4 \varnothing 5216 \varnothing 732417 \\
4051158732557\end{array}$ & $\begin{array}{l}- \\
- \\
-\end{array}$ & $\begin{array}{l}- \\
142 \\
142 \\
142 \\
146\end{array}$ & $\begin{array}{l}- \\
- \\
-\end{array}$ & $\begin{array}{l}- \\
- \\
-\end{array}$ \\
\hline
\end{tabular}


N.Y. [Dash indicates no data. Altitudes are in feet above sea level.] (cont.)

\begin{tabular}{|c|c|c|c|c|c|c|c|c|c|c|c|c|}
\hline \multicolumn{2}{|c|}{$\begin{array}{l}\text { BASIN } \\
\text { NUMBER }\end{array}$} & $\begin{array}{l}\text { MAX. } \\
\text { AREA } \\
\text { (square }\end{array}$ & $\begin{array}{l}\text { BASIN } \\
\text { AREA } \\
\text { feet) }\end{array}$ & RIM & $\begin{array}{l}-- \text { ALTI } \\
\text { OVER- } \\
\text { FLOW }\end{array}$ & $\begin{array}{l}\text { TUDE- } \\
\text { BOT- } \\
\text { TOM }\end{array}$ & $\begin{array}{l}\text { WATER } \\
\text { TABLE }\end{array}$ & $\begin{array}{c}\text { DRAIN. } \\
\text { AREA } \\
\text { (acres) }\end{array}$ & $\begin{array}{l}---B \\
\text { STAT }\end{array}$ & $\begin{array}{l}\text { IN-- } \\
\text { USE }\end{array}$ & $\begin{array}{l}\text { GEO. } \\
\text { UNIT }\end{array}$ & $\begin{array}{l}\text { SOIL } \\
\text { UNIT }\end{array}$ \\
\hline $\begin{array}{l}I \\
I \\
I \\
I \\
I\end{array}$ & $\begin{array}{l}188 \\
189 \\
191 \\
192 \\
193\end{array}$ & $\begin{array}{c}- \\
7000 \\
29200 \\
33100 \\
26200\end{array}$ & $\begin{array}{l}13200 \\
45000 \\
48000 \\
48600\end{array}$ & $\begin{array}{r}10.0 \\
121.5 \\
43.0 \\
63.0 \\
31.4\end{array}$ & $\begin{array}{r}- \\
- \\
40.7 \\
- \\
-\end{array}$ & $\begin{array}{r}109 . \overline{5} \\
31 . \varnothing \\
44 . \varnothing \\
20.7\end{array}$ & $\begin{array}{r}5.0 \\
41.0 \\
25.0 \\
37.0 \\
12.0\end{array}$ & $\begin{array}{r}8 . \overline{8} \\
30.2 \\
71.5 \\
38.8\end{array}$ & $\begin{array}{l}0 \\
1 \\
1 \\
1 \\
1\end{array}$ & $\begin{array}{l}1 \\
1 \\
1 \\
1 \\
1\end{array}$ & $\begin{array}{l}1 \\
6 \\
1 \\
1 \\
1\end{array}$ & $\begin{array}{l}4 \\
3 \\
7 \\
3 \\
7\end{array}$ \\
\hline $\begin{array}{l}I \\
I \\
I \\
I \\
M B\end{array}$ & $\begin{array}{r}194 \\
195 \\
196 \\
197 \\
2\end{array}$ & $\begin{array}{c}26100 \\
8600 \\
- \\
- \\
-\end{array}$ & $\begin{array}{r}29400 \\
21000 \\
20000 \\
- \\
-\end{array}$ & $\begin{array}{r}85.0 \\
85 . \emptyset \\
45 . \emptyset \\
150 . \emptyset \\
85 . \varnothing\end{array}$ & $\begin{array}{r}80 . \overline{5} \\
- \\
- \\
-\end{array}$ & $\begin{array}{r}-\overline{0} \\
- \\
-\end{array}$ & $\begin{array}{l}44.0 \\
44.0 \\
32.0 \\
43.0 \\
59.0\end{array}$ & $\begin{array}{r}5 . \overline{4} \\
- \\
- \\
-\end{array}$ & $\begin{array}{l}1 \\
1 \\
1 \\
1 \\
1\end{array}$ & $\begin{array}{l}1 \\
1 \\
1 \\
1 \\
1\end{array}$ & $\begin{array}{l}1 \\
1 \\
1 \\
1 \\
1\end{array}$ & $\begin{array}{l}7 \\
7 \\
7 \\
5 \\
6\end{array}$ \\
\hline $\begin{array}{l}M B \\
M B \\
M B \\
M B \\
M B\end{array}$ & $\begin{array}{l}3 \\
4 \\
5 \\
6 \\
7\end{array}$ & $\begin{array}{l}- \\
- \\
- \\
-\end{array}$ & $\begin{array}{l}- \\
- \\
-\end{array}$ & $\begin{array}{l}85.0 \\
50.0 \\
25.0 \\
35.0 \\
20.0\end{array}$ & $\begin{array}{l}- \\
- \\
- \\
-\end{array}$ & $\begin{array}{l}- \\
- \\
- \\
-\end{array}$ & $\begin{array}{l}59.0 \\
41 . \emptyset \\
22.0 \\
25.0 \\
17.0\end{array}$ & $\begin{array}{l}- \\
- \\
-\end{array}$ & $\begin{array}{l}1 \\
1 \\
1 \\
1 \\
1\end{array}$ & $\begin{array}{l}1 \\
1 \\
1 \\
1 \\
1\end{array}$ & $\begin{array}{l}1 \\
1 \\
1 \\
1 \\
1\end{array}$ & $\begin{array}{l}6 \\
1 \\
7 \\
7 \\
7\end{array}$ \\
\hline $\begin{array}{l}\text { MBK } \\
\text { MBK } \\
\text { MBK } \\
\text { MBK } \\
\text { MBK }\end{array}$ & $\begin{array}{l}1 \\
2 \\
3 \\
4 \\
5\end{array}$ & $\begin{array}{l}- \\
- \\
- \\
-\end{array}$ & $\begin{array}{l}- \\
- \\
-\end{array}$ & $\begin{array}{r}80.0 \\
115 . \varnothing \\
85 . \varnothing \\
185 . \varnothing \\
110.0\end{array}$ & $\begin{array}{l}- \\
- \\
- \\
-\end{array}$ & $\begin{array}{l}- \\
- \\
- \\
-\end{array}$ & $\begin{array}{l}39.0 \\
53.0 \\
58.0 \\
57.0 \\
57.0\end{array}$ & $\begin{array}{l}- \\
\overline{-} \\
\overline{-}\end{array}$ & $\begin{array}{l}1 \\
1 \\
1 \\
1 \\
1\end{array}$ & $\begin{array}{l}1 \\
1 \\
1 \\
1 \\
1\end{array}$ & $\begin{array}{l}3 \\
1 \\
1 \\
1 \\
1\end{array}$ & $\begin{array}{l}1 \\
5 \\
6 \\
5 \\
5\end{array}$ \\
\hline $\begin{array}{l}\text { MBK } \\
\text { MBK } \\
\text { MBK } \\
\text { MBK } \\
\text { MBK }\end{array}$ & $\begin{array}{r}6 \\
7 \\
8 \\
9 \\
10\end{array}$ & $\begin{array}{l}- \\
\overline{-} \\
\overline{-}\end{array}$ & $\begin{array}{l}- \\
- \\
-\end{array}$ & $\begin{array}{r}125.0 \\
60.0 \\
60.0 \\
58 . \varnothing \\
140.0\end{array}$ & $\begin{array}{l}- \\
- \\
- \\
-\end{array}$ & $\begin{array}{l}- \\
- \\
- \\
-\end{array}$ & $\begin{array}{l}48.0 \\
25.0 \\
24.0 \\
21.0 \\
56.0\end{array}$ & $\begin{array}{l}- \\
- \\
-\end{array}$ & $\begin{array}{l}1 \\
1 \\
1 \\
1 \\
0\end{array}$ & $\begin{array}{l}1 \\
1 \\
1 \\
1 \\
4\end{array}$ & $\begin{array}{l}1 \\
1 \\
1 \\
1 \\
1\end{array}$ & $\begin{array}{l}5 \\
3 \\
3 \\
3 \\
5\end{array}$ \\
\hline $\begin{array}{l}\text { MBK } \\
\text { MBK } \\
\text { MBK } \\
\text { MBK } \\
\text { MBK }\end{array}$ & $\begin{array}{l}11 \\
12 \\
13 \\
14 \\
15\end{array}$ & $\begin{array}{l}- \\
- \\
-\end{array}$ & $\begin{array}{l}- \\
\overline{-} \\
\overline{-}\end{array}$ & $\begin{array}{l}40.0 \\
75.0 \\
90.0 \\
88.0 \\
84.0\end{array}$ & $\begin{array}{l}- \\
\overline{-} \\
\overline{-}\end{array}$ & $\begin{array}{l}- \\
- \\
- \\
-\end{array}$ & $\begin{array}{l}20.0 \\
38.0 \\
42.0 \\
40.0 \\
40.0\end{array}$ & $\begin{array}{l}- \\
- \\
- \\
-\end{array}$ & $\begin{array}{l}0 \\
1 \\
1 \\
1 \\
1\end{array}$ & $\begin{array}{l}4 \\
2 \\
2 \\
2 \\
2\end{array}$ & $\begin{array}{l}1 \\
1 \\
1 \\
1 \\
1\end{array}$ & $\begin{array}{l}7 \\
3 \\
3 \\
3 \\
3\end{array}$ \\
\hline $\begin{array}{l}\text { MBK } \\
M H \\
M H \\
M H \\
M H\end{array}$ & $\begin{array}{r}16 \\
1 \\
2 \\
3 \\
4\end{array}$ & $\begin{array}{l}- \\
- \\
-\end{array}$ & $\begin{array}{l}- \\
- \\
- \\
-\end{array}$ & $\begin{array}{r}60.0 \\
270.0 \\
180.0 \\
170.0 \\
155.0\end{array}$ & $\begin{array}{l}- \\
- \\
- \\
-\end{array}$ & $\begin{array}{l}- \\
- \\
- \\
-\end{array}$ & $\begin{array}{l}40.0 \\
55.0 \\
61.0 \\
64.0 \\
65.0\end{array}$ & $\begin{array}{l}- \\
- \\
- \\
-\end{array}$ & $\begin{array}{l}1 \\
1 \\
1 \\
1 \\
1\end{array}$ & $\begin{array}{l}2 \\
1 \\
1 \\
1 \\
1\end{array}$ & $\begin{array}{l}1 \\
4 \\
1 \\
1 \\
1\end{array}$ & $\begin{array}{l}3 \\
7 \\
7 \\
7 \\
7\end{array}$ \\
\hline $\begin{array}{l}M H \\
M H \\
M H \\
M H \\
M H\end{array}$ & $\begin{array}{l}5 \\
6 \\
7 \\
8 \\
9\end{array}$ & $\begin{array}{l}- \\
\overline{-} \\
-\end{array}$ & $\begin{array}{l}- \\
- \\
- \\
-\end{array}$ & $\begin{array}{l}155.0 \\
155 . \varnothing \\
155 . \varnothing \\
155.0 \\
220 . \varnothing\end{array}$ & $\begin{array}{l}- \\
- \\
-\end{array}$ & $\begin{array}{l}- \\
- \\
- \\
-\end{array}$ & $\begin{array}{l}67.0 \\
65.0 \\
67.0 \\
67.0 \\
67.0\end{array}$ & $\begin{array}{l}- \\
- \\
- \\
-\end{array}$ & $\begin{array}{l}1 \\
1 \\
0 \\
0 \\
1\end{array}$ & $\begin{array}{l}1 \\
1 \\
4 \\
4 \\
1\end{array}$ & $\begin{array}{l}1 \\
1 \\
1 \\
1 \\
5\end{array}$ & $\begin{array}{l}7 \\
7 \\
0 \\
0 \\
9\end{array}$ \\
\hline $\begin{array}{l}\text { MH } \\
M H \\
M H \\
M H \\
M H\end{array}$ & $\begin{array}{l}10 \\
11 \\
14 \\
15 \\
16\end{array}$ & $\begin{array}{l}- \\
\overline{-} \\
\overline{-}\end{array}$ & $\begin{array}{l}- \\
- \\
- \\
-\end{array}$ & $\begin{array}{l}155.0 \\
155.0 \\
135.0 \\
135.0 \\
125.0\end{array}$ & $\begin{array}{l}- \\
- \\
- \\
-\end{array}$ & $\begin{array}{l}- \\
\overline{-} \\
-\end{array}$ & $\begin{array}{l}69.0 \\
69.0 \\
71.0 \\
69.0 \\
72.0\end{array}$ & $\begin{array}{l}- \\
\overline{-} \\
\overline{-}\end{array}$ & $\begin{array}{l}0 \\
0 \\
1 \\
1 \\
1\end{array}$ & $\begin{array}{l}4 \\
1 \\
1 \\
1 \\
1\end{array}$ & $\begin{array}{l}1 \\
4 \\
1 \\
1 \\
1\end{array}$ & $\begin{array}{l}0 \\
1 \\
3 \\
7 \\
9\end{array}$ \\
\hline $\begin{array}{l}M H \\
M H \\
M H \\
M H \\
M H\end{array}$ & $\begin{array}{l}17 \\
18 \\
19 \\
20 \\
21\end{array}$ & $\begin{array}{l}- \\
\overline{-} \\
\overline{-}\end{array}$ & $\begin{array}{l}- \\
\overline{-} \\
-\end{array}$ & $\begin{array}{l}130.0 \\
125 . \varnothing \\
155.0 \\
100.0 \\
105 . \varnothing\end{array}$ & $\begin{array}{l}- \\
- \\
- \\
-\end{array}$ & $\begin{array}{l}- \\
- \\
- \\
-\end{array}$ & $\begin{array}{l}72.0 \\
71.0 \\
73.0 \\
62.0 \\
62.0\end{array}$ & $\begin{array}{l}- \\
- \\
- \\
-\end{array}$ & $\begin{array}{l}1 \\
1 \\
1 \\
1 \\
1\end{array}$ & $\begin{array}{l}1 \\
3 \\
3 \\
1 \\
1\end{array}$ & $\begin{array}{l}1 \\
1 \\
1 \\
1 \\
1\end{array}$ & $\begin{array}{l}9 \\
1 \\
1 \\
6 \\
6\end{array}$ \\
\hline $\begin{array}{l}M H \\
M H \\
M H \\
M H \\
M H\end{array}$ & $\begin{array}{l}22 \\
23 \\
24 \\
25 \\
26\end{array}$ & $\begin{array}{l}- \\
- \\
-\end{array}$ & $\begin{array}{l}- \\
- \\
- \\
-\end{array}$ & $\begin{array}{r}100.0 \\
28.0 \\
200.0 \\
180.0 \\
245.0\end{array}$ & $\begin{array}{l}- \\
- \\
- \\
-\end{array}$ & $\begin{array}{l}- \\
- \\
- \\
-\end{array}$ & $\begin{array}{l}61.0 \\
22 . \emptyset \\
48 . \emptyset \\
45 . \emptyset \\
62 . \emptyset\end{array}$ & $\begin{array}{l}- \\
- \\
- \\
-\end{array}$ & $\begin{array}{l}1 \\
1 \\
1 \\
1 \\
1\end{array}$ & $\begin{array}{l}1 \\
1 \\
1 \\
1 \\
1\end{array}$ & $\begin{array}{l}1 \\
1 \\
1 \\
1 \\
4\end{array}$ & $\begin{array}{l}6 \\
8 \\
9 \\
9 \\
9\end{array}$ \\
\hline
\end{tabular}


Appendix.--Location and basic physical features of recharge basins on Long Island,

\begin{tabular}{|c|c|c|c|c|c|c|c|c|}
\hline \multicolumn{2}{|c|}{$\begin{array}{l}\text { BASIN } \\
\text { NUMBER }\end{array}$} & \multicolumn{2}{|c|}{ NEAREST INTERSECTION } & $\begin{array}{l}\text { LATITTUDE } \\
\text { LONGITUDE, } \\
0, " 0, "\end{array}$ & $\begin{array}{l}\text { DATE } \\
\text { BUILT } \\
\text { YrMOD }\end{array}$ & COMMUNITY & $\begin{array}{l}\text { DESIGN } \\
\text { CAPACITY } \\
\text { (cubic }\end{array}$ & $\begin{array}{c}\text { ACTUAL } \\
\text { CAPACITY } \\
\text { foet) }\end{array}$ \\
\hline $\begin{array}{l}\text { MH } \\
\text { MH } \\
\text { MH } \\
\text { MH } \\
\text { MH }\end{array}$ & $\begin{array}{l}27 \\
28 \\
29 \\
30 \\
31\end{array}$ & $\begin{array}{l}\text { BROAD HOLLOW RD. } \\
\text { ESTATES PLACE } \\
\text { HEMINGWAY DRIVE } \\
\text { ROWENA LANE } \\
\text { L.I.R.R. }\end{array}$ & $\begin{array}{l}\text { RULANO ROAD } \\
\text { CARMAN ROAD } \\
\text { BAGATELLE ROAD } \\
\text { AUTUMN DRIVE } \\
\text { CATHERINE STREET }\end{array}$ & $\begin{array}{l}4045420732515 \\
4 \varnothing 47530732251 \\
4047020732239 \\
4 \oslash 52370731811 \\
4 \varnothing 52480732015\end{array}$ & $\begin{array}{l}\overline{-} \\
\overline{-} \\
\overline{-}\end{array}$ & $\begin{array}{l}148 \\
133 \\
133 \\
137 \\
134\end{array}$ & $\begin{array}{l}\overline{-} \\
= \\
-\end{array}$ & $\begin{array}{l}- \\
\overline{-} \\
-\end{array}$ \\
\hline $\begin{array}{l}\text { MH } \\
\text { MH } \\
\text { MH } \\
\text { MH } \\
\text { MH }\end{array}$ & $\begin{array}{l}32 \\
33 \\
34 \\
35 \\
36\end{array}$ & $\begin{array}{l}\text { CEDAR ROAD } \\
\text { CEDAR ROAD } \\
\text { HOLLY DRIVE } \\
\text { E. END ARISTA CT } \\
\text { WINTHROP DRIVE }\end{array}$ & $\begin{array}{l}\text { NEWMARK STREET } \\
\text { CATON STREET } \\
\text { BEACON LANE } \\
\text { SUNCREST DRIVE }\end{array}$ & $\begin{array}{l}4 \oslash 51190731924 \\
4 \varnothing 51210731917 \\
4 \varnothing 51140731812 \\
4 \varnothing 49460732054 \\
4 \varnothing 48210732153\end{array}$ & $\begin{array}{l}- \\
- \\
- \\
-\end{array}$ & $\begin{array}{l}139 \\
139 \\
169 \\
133 \\
133\end{array}$ & $\begin{array}{l}- \\
- \\
- \\
-\end{array}$ & $\begin{array}{l}- \\
- \\
-\end{array}$ \\
\hline $\begin{array}{l}\text { MH } \\
\text { MH } \\
\text { MH } \\
\text { MH } \\
\text { MH }\end{array}$ & $\begin{array}{l}37 \\
38 \\
39 \\
40 \\
41\end{array}$ & $\begin{array}{l}\text { DEER PARK AVENUE } \\
\text { VANDERBILT PKWY. } \\
\text { CARLLS STRAIGHT } \\
\text { CARLLS STRAIGHT } \\
\text { CARLLS STRAIGHT }\end{array}$ & $\begin{array}{l}\text { PARSONS DRIVE } \\
\text { CANDLEWOOD PATH } \\
\text { CAROLINE DRIVE } \\
\text { GRAYON DRIVE } \\
\text { CORWIN COURT }\end{array}$ & 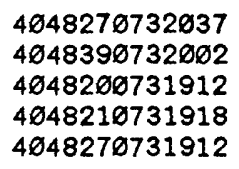 & $\begin{array}{l}- \\
- \\
- \\
-\end{array}$ & $\begin{array}{l}133 \\
133 \\
133 \\
133 \\
133\end{array}$ & $\begin{array}{l}- \\
- \\
- \\
-\end{array}$ & $\begin{array}{l}- \\
- \\
-\end{array}$ \\
\hline $\begin{array}{l}\text { MH } \\
\text { MH } \\
\text { MH } \\
\text { MH } \\
\text { MI }\end{array}$ & $\begin{array}{r}42 \\
44 \\
46 \\
46 \\
1\end{array}$ & $\begin{array}{l}\text { HALF HOLLOW RD. } \\
\text { TRUXTON ROAD } \\
\text { HARMON DRIVE } \\
\text { THADFORD STREET } \\
\text { BRADLEY STREET }\end{array}$ & $\begin{array}{l}\text { SOUTH HOLLOW RD. } \\
\text { EBBTIDE LANE } \\
\text { CIRO STREET } \\
\text { HARLEY AVENUE } \\
\text { WICKS ROAD }\end{array}$ & 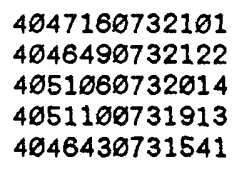 & $\begin{array}{l}- \\
- \\
- \\
-\end{array}$ & $\begin{array}{l}- \\
139 \\
139 \\
189\end{array}$ & $\begin{array}{l}- \\
- \\
- \\
-\end{array}$ & $\begin{array}{l}- \\
- \\
- \\
-\end{array}$ \\
\hline $\begin{array}{l}M I \\
M I \\
M I \\
M I \\
M I\end{array}$ & $\begin{array}{l}2 \\
3 \\
4 \\
5 \\
6\end{array}$ & $\begin{array}{l}\text { FIFTH AVENUE } \\
\text { ALKIER STREET } \\
\text { JEFFERSON AVENUE } \\
\text { WILSON BOULEVARD } \\
\text { MONTAUK AVENUE }\end{array}$ & $\begin{array}{l}\text { RUMPELT AVENUE } \\
\text { GRAND BOULEVARD } \\
\text { 17TH AVENUE } \\
\text { BEECH STREET } \\
\text { OCEANSIDE STREET }\end{array}$ & $\begin{array}{l}4 \emptyset 4443 \varnothing 731549 \\
4 \varnothing 4659 \varnothing 731322 \\
4 \varnothing 4648 \varnothing 736842 \\
4 \varnothing 463 \varnothing \varnothing 731224 \\
4 \varnothing 46 \varnothing 1 \varnothing 731003\end{array}$ & $\begin{array}{l}- \\
- \\
-\end{array}$ & $\begin{array}{l}202 \\
191 \\
188 \\
191 \\
203\end{array}$ & $\begin{array}{l}- \\
- \\
- \\
-\end{array}$ & $\begin{array}{l}- \\
- \\
- \\
-\end{array}$ \\
\hline $\begin{array}{l}M I \\
M I \\
M I \\
M I \\
M I\end{array}$ & $\begin{array}{r}7 \\
8 \\
9 \\
10 \\
11\end{array}$ & $\begin{array}{l}\text { FREEMAN AVENUE } \\
\text { EAST FORKS ROAD } \\
\text { WHEELERS STREET } \\
\text { WHEELERS STREET } \\
\text { POND ROAD }\end{array}$ & $\begin{array}{l}\text { WILEY AVENUE } \\
\text { BROOK AVENUE } \\
\text { SUNRISE HIGHWAY } \\
\text { SOUTH COUNTRY RD } \\
\text { L.I.R.R. }\end{array}$ & 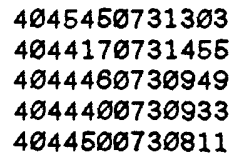 & $\begin{array}{l}- \\
- \\
- \\
-\end{array}$ & $\begin{array}{l}189 \\
202 \\
203 \\
203 \\
205\end{array}$ & $\begin{array}{l}- \\
- \\
- \\
-\end{array}$ & $\begin{array}{l}\overline{-} \\
\overline{-}\end{array}$ \\
\hline $\begin{array}{l}M I \\
M I \\
M I \\
M I \\
M I\end{array}$ & $\begin{array}{l}12 \\
13 \\
14 \\
15 \\
16\end{array}$ & $\begin{array}{l}\text { POND ROAD } \\
\text { VETERANS HIGHWAY } \\
\text { BOHEMIA SPORT CL. } \\
\text { CENTRAL AVENUE } \\
\text { CALEB'S PATH }\end{array}$ & $\begin{array}{l}\text { L.I.R.R. } \\
\text { JOHNSON AVENUE } \\
\text { PURICK STREET } \\
\text { L.I.E. }\end{array}$ & 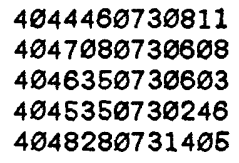 & $\begin{array}{l}- \\
- \\
- \\
-\end{array}$ & $\begin{array}{l}205 \\
188 \\
188 \\
186 \\
191\end{array}$ & $\begin{array}{l}- \\
- \\
- \\
-\end{array}$ & $\begin{array}{l}- \\
- \\
- \\
-\end{array}$ \\
\hline $\begin{array}{l}\text { MN } \\
\text { MN } \\
M N \\
M N \\
M N\end{array}$ & $\begin{array}{r}1 \\
7 \\
8 \\
9 \\
11\end{array}$ & $\begin{array}{l}\text { GRUMMAN AIRPORT } \\
\text { GRUMMAN AIRPORT } \\
\text { GRUMMAN AIRPORT } \\
\text { GRUMMAN AIRPORT } \\
\text { ELMMOOD STREET }\end{array}$ & NASSAU AVENUE & $\begin{array}{l}4 \emptyset 45250732932 \\
4 \emptyset 45180732934 \\
4 \varnothing 4518 \emptyset 732929 \\
4 \varnothing 452 \varnothing \emptyset 732917 \\
4 \varnothing 4630073295 \emptyset\end{array}$ & $\begin{array}{l}- \\
- \\
- \\
-\end{array}$ & $\begin{array}{r}92 \\
92 \\
92 \\
92 \\
122\end{array}$ & $\begin{array}{l}- \\
- \\
-\end{array}$ & $\begin{array}{l}- \\
- \\
-\end{array}$ \\
\hline $\begin{array}{l}\text { MN } \\
\text { MN } \\
M N \\
M N \\
M N\end{array}$ & $\begin{array}{l}12 \\
13 \\
14 \\
18 \\
26\end{array}$ & $\begin{array}{l}\text { BARNUM AVENUE } \\
\text { WINDING ROAD } \\
\text { WINDING ROAD } \\
\text { MITCHELL FIELD } \\
\text { MICHAEL COURT }\end{array}$ & $\begin{array}{l}\text { QUEENS COURT } \\
\text { BETHPAGE SWEET.R } \\
\text { BETHPAGE SWEET.R } \\
\text { FAIRCHILD AVENUE }\end{array}$ & 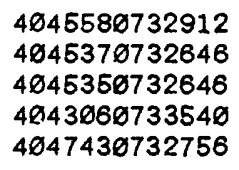 & $\begin{array}{l}- \\
- \\
- \\
-\end{array}$ & $\begin{array}{r}92 \\
116 \\
116 \\
14 \\
122\end{array}$ & $\begin{array}{l}- \\
\overline{-} \\
\overline{-}\end{array}$ & $\begin{array}{l}- \\
- \\
-\end{array}$ \\
\hline $\begin{array}{l}\mathrm{MN} \\
\mathrm{MN} \\
\mathrm{MN} \\
\mathrm{MN} \\
\mathrm{MN}\end{array}$ & $\begin{array}{l}21 \\
22 \\
23 \\
24 \\
25\end{array}$ & $\begin{array}{l}\text { SQUIRREL LANE } \\
\text { GRUMMAN AIRPORT } \\
\text { PENNSYLVANIA AVE } \\
\text { GLEN COVE ROAD } \\
\text { SANDS POINT ROAD }\end{array}$ & $\begin{array}{l}\text { HEMPSTEAD TPKE. } \\
\text { MAPLE DRIVE } \\
\text { VOICE ROAD } \\
\text { OAKTREE LANE }\end{array}$ & $\begin{array}{l}4 \oslash 43320733126 \\
4 \oslash 4446 \oslash 733 \oslash \oslash 5 \\
4 \oslash 4 \oslash 24 \oslash 733556 \\
4 \oslash 4444 \oslash 733715 \\
4 \oslash 51 \oslash 2 \oslash 734259\end{array}$ & $\begin{array}{l}- \\
- \\
-\end{array}$ & $\begin{array}{l}25 \\
92 \\
37 \\
52 \\
86\end{array}$ & $\begin{array}{l}- \\
- \\
- \\
-\end{array}$ & $\begin{array}{l}- \\
- \\
-\end{array}$ \\
\hline $\begin{array}{l}M N \\
M N \\
M N \\
M N \\
M N\end{array}$ & $\begin{array}{l}26 \\
27 \\
28 \\
29 \\
30\end{array}$ & $\begin{array}{l}\text { GLEN COVE DRIVE } \\
\text { FRUITLEDGE ROAD } \\
\text { LINDA COURT } \\
\text { WOODLEA ROAD } \\
\text { CIRCLE ROAD }\end{array}$ & $\begin{array}{l}\text { MAIDEN LANE } \\
\text { TAPPENTOWN LANE } \\
\text { DONNA DRIVE } \\
\text { HUNTERS DRIVE } \\
\text { MUTTONTOWN ROAD }\end{array}$ & 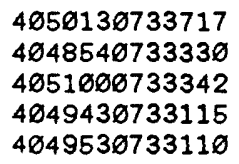 & $\begin{array}{l}- \\
- \\
-\end{array}$ & $\begin{array}{r}101 \\
93 \\
127 \\
115 \\
115\end{array}$ & $\begin{array}{l}- \\
- \\
- \\
-\end{array}$ & $\begin{array}{l}- \\
- \\
-\end{array}$ \\
\hline
\end{tabular}


N.Y. [Dash indicates no data. Altitudes are in feet above sea level.] (cont.)

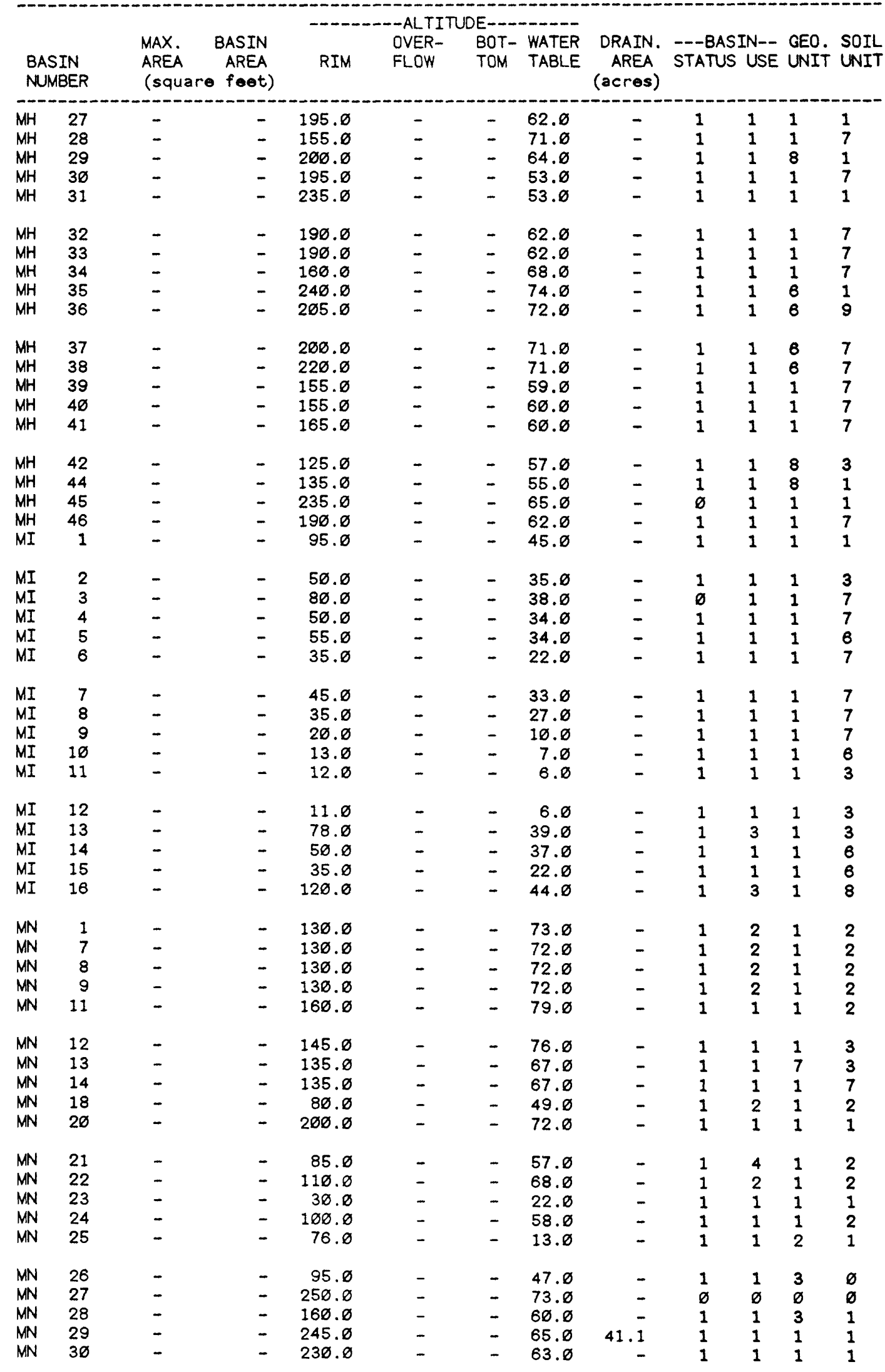


Appendix.--Location and basic physical features of recharge basins on Long Island,

\begin{tabular}{|c|c|c|c|c|c|c|c|c|}
\hline \multicolumn{2}{|c|}{$\begin{array}{l}\text { BASIN } \\
\text { NUMBER }\end{array}$} & \multicolumn{2}{|c|}{ NEAREST INTERSECTION } & $\begin{array}{l}\text { LATITUDE } \\
\text { LONGITUDE } \\
\circ, " 0^{\prime}, "\end{array}$ & $\begin{array}{l}\text { DATE } \\
\text { BUILT } \\
\text { YrMOD }\end{array}$ & COMMUNITY & \multicolumn{2}{|c|}{$\begin{array}{l}\text { DESIGN ACTUAL } \\
\text { CAPACITY CAPACITY } \\
\text { (cubic foot) }\end{array}$} \\
\hline $\begin{array}{l}M N \\
M N \\
M N \\
M N \\
M N\end{array}$ & $\begin{array}{l}31 \\
32 \\
33 \\
34 \\
35\end{array}$ & $\begin{array}{l}\text { NEW SOUTH ROAD } \\
\text { EAST CARMANS RD. } \\
\text { NEWBRIDGE ROAD } \\
\text { SAGAMORE STREET } \\
\text { POPLAR PLACE }\end{array}$ & $\begin{array}{l}\text { KARIN LANE } \\
\text { FARMINGDALE ROAD } \\
\text { HEMPSTEAD TPKE. } \\
\text { OAKWOOD LANE } \\
\text { WOOLSEY AVENUE }\end{array}$ & $\begin{array}{l}4 \emptyset 45310733 \emptyset \emptyset 8 \\
4 \emptyset 4247 \emptyset 7326 \emptyset 1 \\
4 \emptyset 433 \emptyset \emptyset 73323 \emptyset \\
4 \emptyset 4729 \emptyset 732829 \\
4 \emptyset 5225 \emptyset 7338 \varnothing 6\end{array}$ & $\begin{array}{l}- \\
- \\
-\end{array}$ & $\begin{array}{r}104 \\
99 \\
8 \\
122 \\
100\end{array}$ & $\begin{array}{l}- \\
- \\
- \\
200700\end{array}$ & $\begin{array}{l}- \\
- \\
- \\
39400\end{array}$ \\
\hline $\begin{array}{l}\text { MN } \\
\text { MS } \\
\text { MS } \\
\text { MS } \\
\text { MS }\end{array}$ & $\begin{array}{r}36 \\
1 \\
2 \\
3 \\
4\end{array}$ & $\begin{array}{l}\text { MID ISLAND MALL } \\
\text { GATEWOOD DRIVE } \\
\text { EAST LANE } \\
\text { CAMBON AVENUE } \\
\text { HARNED ROAD }\end{array}$ & $\begin{array}{l}\text { GLENWOOD DRIVE } \\
\text { OAKSIDE ROAD } \\
\text { CAMELOT LANE } \\
\text { NEW HIGHWAY }\end{array}$ & $\begin{array}{l}4 \emptyset 461807332 \emptyset 8 \\
4 \emptyset 4944 \varnothing 7316 \varnothing 7 \\
4 \emptyset 5219 \emptyset 731259 \\
4 \emptyset 5156 \emptyset 73 \varnothing 84 \emptyset \\
4 \emptyset 49380731657\end{array}$ & $\begin{array}{l}- \\
- \\
- \\
-\end{array}$ & $\begin{array}{l}104 \\
196 \\
182 \\
180 \\
169\end{array}$ & $\begin{array}{l}- \\
- \\
-\end{array}$ & $\begin{array}{l}- \\
- \\
- \\
-\end{array}$ \\
\hline $\begin{array}{l}\text { MS } \\
\text { MST } \\
\text { MST } \\
\text { MST } \\
\text { MST }\end{array}$ & $\begin{array}{r}5 \\
10 \\
15 \\
16 \\
17\end{array}$ & $\begin{array}{l}\text { MARIE CRESCENT } \\
\text { EAST NORWICH RD. } \\
\text { WADLEIGH AVENUE } \\
\text { MEADOWBROOK PKY. } \\
\text { SUNKEN MEADOW PK }\end{array}$ & $\begin{array}{l}\text { DEWEY ROAD } \\
\text { CEDAR SWAMP ROAD } \\
\text { SOUTHERN ST. PKY } \\
\text { NORTHERN ST. PKY } \\
\text { JERICHO TURNPIKE }\end{array}$ & $\begin{array}{l}4049310731651 \\
4047550733222 \\
4041100733837 \\
4 \varnothing 451607337 \varnothing 4 \\
4050390731558\end{array}$ & $\begin{array}{l}- \\
- \\
- \\
-\end{array}$ & $\begin{array}{r}169 \\
105 \\
23 \\
52 \\
169\end{array}$ & $\begin{array}{l}- \\
- \\
- \\
-\end{array}$ & $\begin{array}{l}- \\
- \\
- \\
-\end{array}$ \\
\hline $\begin{array}{l}\text { MST } \\
\text { MST } \\
N \\
N \\
N\end{array}$ & $\begin{array}{r}18 \\
19 \\
1 \\
2 \\
3\end{array}$ & $\begin{array}{l}\text { TOWN LINE ROAD } \\
\text { COMMACK ROAD } \\
\text { DUTCH BROADWAY } \\
\text { DUTCH BROADWAY } \\
\text { DUTCH BROADWAY }\end{array}$ & $\begin{array}{l}\text { HAVEMEYER LANE } \\
\text { GENESEE DRIVE } \\
\text { RUSSELL AVENUE } \\
\text { NASSAU STREET } \\
\text { EMILY AVENUE }\end{array}$ & 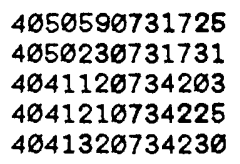 & $\begin{array}{l}- \\
5104 \\
5104 \\
5104\end{array}$ & $\begin{array}{r}169 \\
169 \\
10 \\
10 \\
10\end{array}$ & $\begin{array}{l}- \\
- \\
- \\
-\end{array}$ & $\begin{array}{l}- \\
- \\
184000 \\
130000 \\
110300\end{array}$ \\
\hline $\begin{array}{l}N \\
N \\
N \\
N \\
N\end{array}$ & $\begin{array}{l}4 \\
5 \\
6 \\
7 \\
8\end{array}$ & $\begin{array}{l}\text { KENSINGTON RD } \\
\text { ARRANDALE RD } \\
\text { HICKSVILLE RD } \\
\text { ANTHONY DR } \\
\text { NIGHTINGALE ROAD }\end{array}$ & $\begin{array}{l}\text { ANDREWS AVE } \\
\text { MARTHA BLVD } \\
\text { MARTIN RD } \\
\text { OAK ST } \\
\text { ROBIN COURT }\end{array}$ & $\begin{array}{l}4043040733231 \\
4044230733001 \\
4043450732919 \\
4043230732747 \\
4041000733846\end{array}$ & $\begin{array}{l}5188 \\
5212 \\
5112 \\
5510 \\
5111\end{array}$ & $\begin{array}{r}8 \\
92 \\
92 \\
99 \\
23\end{array}$ & $\begin{array}{l}- \\
- \\
-\end{array}$ & $\begin{array}{r}79000 \\
136200 \\
121000 \\
120600 \\
44000\end{array}$ \\
\hline $\begin{array}{l}N \\
N \\
N \\
N \\
N\end{array}$ & $\begin{array}{r}9 \\
18 \\
11 \\
12 \\
13\end{array}$ & $\begin{array}{l}\text { OVERLOOK TERRACE } \\
\text { HERRICKS ROAD } \\
\text { FALCON ST } \\
\text { LINDEN BLVD } \\
\text { AVOCA AVE }\end{array}$ & $\begin{array}{l}\text { THORNE PL } \\
\text { WILSON BOULEVARD } \\
\text { NEWBRIDGE RD } \\
\text { JERUSALUM AVE } \\
\text { HICKSVILLE RD }\end{array}$ & $\begin{array}{l}4047100733818 \\
4044510733938 \\
4 \varnothing 42410733225 \\
4044510733041 \\
4043560732915\end{array}$ & $\begin{array}{l}5211 \\
5106 \\
5104 \\
5311 \\
5202\end{array}$ & $\begin{array}{r}53 \\
62 \\
8 \\
104 \\
92\end{array}$ & $\begin{array}{l}- \\
- \\
- \\
-\end{array}$ & $\begin{array}{r}446000 \\
2600700 \\
319000 \\
325000 \\
443060\end{array}$ \\
\hline $\begin{array}{l}N \\
N \\
N \\
N \\
N\end{array}$ & $\begin{array}{l}14 \\
15 \\
16 \\
17 \\
18\end{array}$ & $\begin{array}{l}\text { THIRD AVE } \\
\text { MILFORD LA } \\
\text { ASH PLACE } \\
\text { CLAUDY LA } \\
\text { ALBERTSON AVE. }\end{array}$ & $\begin{array}{l}\text { 4TH STREET } \\
\text { CENTRAL AVE } \\
\text { CLIFF ROAD } \\
\text { LAKEVILLE RD } \\
\text { I.U. WILLETS RD. }\end{array}$ & $\begin{array}{l}4043010733249 \\
404 \varnothing 390734232 \\
4041160733401 \\
4045080734156 \\
4046190733835\end{array}$ & $\begin{array}{l}5104 \\
5104 \\
5206 \\
5110 \\
5108\end{array}$ & $\begin{array}{r}8 \\
34 \\
33 \\
71 \\
50\end{array}$ & $\begin{array}{l}- \\
- \\
- \\
-\end{array}$ & $\begin{array}{r}177000 \\
835000 \\
72600 \\
173000 \\
550000\end{array}$ \\
\hline $\begin{array}{l}N \\
N \\
N \\
N \\
N\end{array}$ & $\begin{array}{l}19 \\
20 \\
21 \\
22 \\
23\end{array}$ & $\begin{array}{l}\text { MEADOW LA } \\
\text { HILLSIDE AVE } \\
\text { MARCUS AVE } \\
\text { JERUSALEM AVE } \\
\text { PERIWINKLE RD }\end{array}$ & $\begin{array}{l}\text { JERICHO TPKE } \\
\text { STEWART AVE } \\
\text { NEW HYDE PARK RD } \\
\text { CORNFLOWER RD } \\
\text { CORNFLOWER RD }\end{array}$ & $\begin{array}{l}4 \varnothing 48490732959 \\
4044250734201 \\
4052440733806 \\
4043590733055 \\
4044010733135\end{array}$ & $\begin{array}{l}5108 \\
540601 \\
651011 \\
5106 \\
5105\end{array}$ & $\begin{array}{r}126 \\
71 \\
65 \\
25 \\
25\end{array}$ & $\begin{array}{l}-\overline{452000} \\
- \\
-\end{array}$ & $\begin{array}{c}770200 \\
385000 \\
- \\
550000 \\
1090000\end{array}$ \\
\hline $\begin{array}{l}N \\
N \\
N \\
N \\
N\end{array}$ & $\begin{array}{l}24 \\
25 \\
26 \\
27 \\
28\end{array}$ & $\begin{array}{l}\text { HYACINTH RD } \\
\text { MERIDIAN RD. } \\
\text { HEMPSTEAD TPKE. } \\
\text { GARDINERS AVE } \\
\text { GARDINERS AVE }\end{array}$ & $\begin{array}{l}\text { AZALEA RD } \\
\text { SOLAR LN. } \\
\text { HAMLET RD } \\
\text { REED LA } \\
\text { RANCH LA }\end{array}$ & $\begin{array}{l}4044010733204 \\
4043580733028 \\
4043340733034 \\
4043090733058 \\
4043090733040\end{array}$ & $\begin{array}{l}5105 \\
510924 \\
5104 \\
5104 \\
5105\end{array}$ & $\begin{array}{l}25 \\
25 \\
25 \\
25 \\
25\end{array}$ & $\begin{array}{l}- \\
- \\
-\end{array}$ & $\begin{array}{r}342000 \\
315000 \\
1130000 \\
293000 \\
236000\end{array}$ \\
\hline $\begin{array}{l}N \\
N \\
N \\
N \\
N\end{array}$ & $\begin{array}{l}29 \\
30 \\
31 \\
32 \\
33\end{array}$ & $\begin{array}{l}\text { GRASSY LANE } \\
\text { JERUSALEM AVE. } \\
\text { GARDINERS AVE. } \\
\text { CANTER LA. } \\
\text { WANTAGH AVE. }\end{array}$ & $\begin{array}{l}\text { BUCKET LANE } \\
\text { HEMPSTEAD TPKE. } \\
\text { WATER LA. } \\
\text { HEMPSTEAD TPKE. } \\
\text { MILLER PL. }\end{array}$ & $\begin{array}{l}4043360733120 \\
4043370733053 \\
4042370733039 \\
404257 \varnothing 733136 \\
4043010733005\end{array}$ & $\begin{array}{l}5704 \\
5107 \\
5105 \\
5107 \\
5104\end{array}$ & $\begin{array}{l}25 \\
25 \\
25 \\
25 \\
25\end{array}$ & $\begin{array}{l}- \\
- \\
-\end{array}$ & $\begin{array}{r}212000 \\
389000 \\
1630000 \\
5940600 \\
686000\end{array}$ \\
\hline $\begin{array}{l}N \\
N \\
N \\
N \\
N\end{array}$ & $\begin{array}{l}34 \\
35 \\
36 \\
37 \\
38\end{array}$ & $\begin{array}{l}\text { POLARIS DRIVE } \\
\text { BLOOMINGDALE RD. } \\
\text { POTTER LANE } \\
\text { SCOOTER LANE } \\
\text { CHERRY ST. }\end{array}$ & $\begin{array}{l}\text { JERUSALEM AVE. } \\
\text { SOLAR LA. } \\
\text { SPINDLE ROAD } \\
\text { POET LANE } \\
\text { WINTER LA. }\end{array}$ & 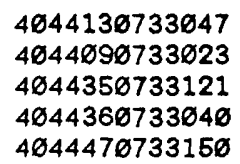 & $\begin{array}{l}5105 \\
5110 \\
5104 \\
5107 \\
5104\end{array}$ & $\begin{array}{l}25 \\
25 \\
25 \\
25 \\
25\end{array}$ & $\begin{array}{l}- \\
- \\
- \\
-\end{array}$ & $\begin{array}{r}1720600 \\
520000 \\
318000 \\
530000 \\
152000\end{array}$ \\
\hline
\end{tabular}


N.Y. [Dash indicates no data. Altitudes are in feet above sea level.] (cont.)

\begin{tabular}{|c|c|c|c|c|c|c|c|c|c|c|c|c|}
\hline \multicolumn{2}{|c|}{$\begin{array}{l}\text { BASIN } \\
\text { NUMBER }\end{array}$} & $\begin{array}{l}\text { MAX. } \\
\text { AREA } \\
\text { (square }\end{array}$ & $\begin{array}{c}\text { BASIN } \\
\text { AREA } \\
\text { feet) }\end{array}$ & RIM & $\begin{array}{l}-- \text { ALT } \\
\text { OVER } \\
\text { FLOW }\end{array}$ & $\begin{array}{l}\text { TUDE--- } \\
\text { BOT- } \\
\text { TOM }\end{array}$ & $\begin{array}{l}\text { WATER } \\
\text { TABLE }\end{array}$ & $\begin{array}{c}\text { DRAIN. } \\
\text { AREA } \\
\text { (acres) }\end{array}$ & \multicolumn{2}{|c|}{$\begin{array}{l}---B A S I N-- \\
\text { STATUS USE }\end{array}$} & $\begin{array}{l}\text { GEO. } \\
\text { UNIT }\end{array}$ & $\begin{array}{l}\text { SOIL } \\
\text { UNIT }\end{array}$ \\
\hline $\begin{array}{l}\text { MN } \\
\text { MN } \\
\text { MN } \\
\text { MN } \\
\text { MN }\end{array}$ & $\begin{array}{l}31 \\
32 \\
33 \\
34 \\
35\end{array}$ & $\begin{array}{l}- \\
- \\
- \\
\overline{5300}\end{array}$ & $\begin{array}{l}- \\
- \\
-\end{array}$ & $\begin{array}{r}130.0 \\
50.0 \\
90.0 \\
167.4 \\
116.0\end{array}$ & $\begin{array}{r}- \\
- \\
- \\
114 . \overline{4}\end{array}$ & $\begin{array}{r}- \\
- \\
- \\
187.0\end{array}$ & $\begin{array}{l}73.0 \\
40.0 \\
58.0 \\
77.0 \\
54.0\end{array}$ & $\begin{array}{r}- \\
- \\
20.7 \\
64.5 \\
39.8\end{array}$ & $\begin{array}{l}1 \\
1 \\
1 \\
1 \\
1\end{array}$ & $\begin{array}{l}1 \\
1 \\
4 \\
1 \\
1\end{array}$ & $\begin{array}{l}1 \\
1 \\
1 \\
1 \\
3\end{array}$ & $\begin{array}{l}2 \\
2 \\
2 \\
1 \\
3\end{array}$ \\
\hline $\begin{array}{l}\text { MN } \\
\text { MS } \\
\text { MS } \\
\text { MS } \\
\text { MS }\end{array}$ & $\begin{array}{r}36 \\
1 \\
2 \\
3 \\
4\end{array}$ & $\begin{array}{l}\bar{z} \\
\bar{z} \\
\overline{-}\end{array}$ & $\begin{array}{l}- \\
- \\
- \\
-\end{array}$ & $\begin{array}{l}145.0 \\
140.0 \\
110.0 \\
120.0 \\
165.0\end{array}$ & $\begin{array}{l}\text { - } \\
\text { - } \\
\text { - }\end{array}$ & $\begin{array}{l}- \\
- \\
-\end{array}$ & $\begin{array}{l}77 . \varnothing \\
56 . \varnothing \\
35.0 \\
63 . \varnothing \\
61 . \varnothing\end{array}$ & $\begin{array}{l}- \\
- \\
-\end{array}$ & $\begin{array}{l}0 \\
1 \\
1 \\
1 \\
0\end{array}$ & $\begin{array}{l}4 \\
1 \\
1 \\
1 \\
3\end{array}$ & $\begin{array}{l}1 \\
1 \\
1 \\
1 \\
5\end{array}$ & $\begin{array}{l}2 \\
5 \\
7 \\
7 \\
3\end{array}$ \\
\hline $\begin{array}{l}\text { MS } \\
\text { MST } \\
\text { MST } \\
\text { MST } \\
\text { MST }\end{array}$ & $\begin{array}{r}5 \\
10 \\
15 \\
16 \\
17\end{array}$ & $\begin{array}{l}- \\
- \\
- \\
-\end{array}$ & $\begin{array}{l}- \\
- \\
- \\
-\end{array}$ & $\begin{array}{r}175.8 \\
220.0 \\
41.0 \\
95.8 \\
140.0\end{array}$ & $\begin{array}{l}- \\
- \\
\text { - }\end{array}$ & $\begin{array}{l}- \\
- \\
-\end{array}$ & $\begin{array}{l}58.0 \\
77.0 \\
22.0 \\
62.0 \\
56.0\end{array}$ & $\begin{array}{l}- \\
- \\
- \\
-\end{array}$ & $\begin{array}{l}0 \\
1 \\
1 \\
1 \\
1\end{array}$ & $\begin{array}{l}1 \\
3 \\
3 \\
3 \\
3\end{array}$ & $\begin{array}{l}5 \\
6 \\
1 \\
1 \\
1\end{array}$ & $\begin{array}{l}8 \\
1 \\
1 \\
2 \\
7\end{array}$ \\
\hline $\begin{array}{l}\text { MST } \\
\text { MST } \\
N \\
N \\
N\end{array}$ & $\begin{array}{r}18 \\
19 \\
1 \\
2 \\
3\end{array}$ & $\begin{array}{c}- \\
- \\
47900 \\
113300 \\
31800\end{array}$ & $\begin{array}{l}- \\
- \\
- \\
-\end{array}$ & $\begin{array}{r}140.0 \\
120.0 \\
35.5 \\
47.0 \\
55.6\end{array}$ & $\begin{array}{l}- \\
- \\
- \\
-\end{array}$ & $\begin{array}{r}- \\
- \\
28 . \emptyset \\
38.0 \\
44.0\end{array}$ & $\begin{array}{l}65.0 \\
67.0 \\
17.0 \\
15.0 \\
14.0\end{array}$ & $\begin{array}{r}- \\
- \\
29.0 \\
20.5 \\
17.4\end{array}$ & $\begin{array}{l}1 \\
1 \\
1 \\
1 \\
1\end{array}$ & $\begin{array}{l}3 \\
3 \\
1 \\
1 \\
1\end{array}$ & $\begin{array}{l}1 \\
1 \\
1 \\
1 \\
1\end{array}$ & $\begin{array}{l}7 \\
7 \\
6 \\
6 \\
6\end{array}$ \\
\hline $\begin{array}{l}N \\
N \\
N \\
N \\
N\end{array}$ & $\begin{array}{l}4 \\
5 \\
6 \\
7 \\
8\end{array}$ & $\begin{array}{l}21200 \\
23800 \\
19700 \\
21700 \\
176 \varnothing 0\end{array}$ & $\begin{array}{l}- \\
- \\
- \\
-\end{array}$ & $\begin{array}{r}76.5 \\
102.0 \\
88.7 \\
67.8 \\
37.0\end{array}$ & $\begin{array}{l}- \\
- \\
- \\
-\end{array}$ & $\begin{array}{l}66.5 \\
91.0 \\
77.0 \\
54.0 \\
27.0\end{array}$ & $\begin{array}{l}50.0 \\
66.0 \\
59.0 \\
50.0 \\
20.0\end{array}$ & $\begin{array}{r}12.4 \\
21.4 \\
19.0 \\
19.0 \\
2.7\end{array}$ & $\begin{array}{l}1 \\
1 \\
0 \\
1 \\
1\end{array}$ & $\begin{array}{l}1 \\
1 \\
1 \\
1 \\
3\end{array}$ & $\begin{array}{l}1 \\
1 \\
1 \\
1 \\
1\end{array}$ & $\begin{array}{l}2 \\
2 \\
1 \\
1 \\
1\end{array}$ \\
\hline $\begin{array}{l}N \\
N \\
N \\
N \\
N\end{array}$ & $\begin{array}{r}9 \\
10 \\
11 \\
12 \\
13\end{array}$ & $\begin{array}{r}57200 \\
265300 \\
65200 \\
61700 \\
55500\end{array}$ & $\begin{array}{l}- \\
- \\
- \\
-\end{array}$ & $\begin{array}{r}158.0 \\
92.0 \\
66.7 \\
121.6 \\
92.4\end{array}$ & $\begin{array}{l}- \\
- \\
- \\
-\end{array}$ & $\begin{array}{r}148.0 \\
82.0 \\
54.0 \\
110.5 \\
80.0\end{array}$ & $\begin{array}{l}51.0 \\
46.0 \\
49.0 \\
69.0 \\
59.0\end{array}$ & $\begin{array}{r}70.2 \\
409.4 \\
50.2 \\
51.2 \\
69.0\end{array}$ & $\begin{array}{l}1 \\
0 \\
1 \\
1 \\
0\end{array}$ & $\begin{array}{l}1 \\
1 \\
1 \\
1 \\
1\end{array}$ & $\begin{array}{l}1 \\
1 \\
1 \\
1 \\
1\end{array}$ & $\begin{array}{l}1 \\
1 \\
1 \\
2 \\
1\end{array}$ \\
\hline $\begin{array}{l}N \\
N \\
N \\
N \\
N\end{array}$ & $\begin{array}{l}14 \\
15 \\
16 \\
17 \\
18\end{array}$ & $\begin{array}{l}35200 \\
37800 \\
18300 \\
39500 \\
69900\end{array}$ & $\begin{array}{l}- \\
- \\
- \\
-\end{array}$ & $\begin{array}{r}75.5 \\
27.0 \\
48.0 \\
124.0 \\
129.0\end{array}$ & $\begin{array}{l}- \\
- \\
- \\
-\end{array}$ & $\begin{array}{r}62 . \varnothing \\
19 . \varnothing \\
114 . \overline{-} \\
116 . \varnothing\end{array}$ & $\begin{array}{l}52.0 \\
11.0 \\
30.0 \\
33.0 \\
54.0\end{array}$ & $\begin{array}{r}27.9 \\
131.4 \\
11.4 \\
27.2 \\
86.6\end{array}$ & $\begin{array}{l}1 \\
0 \\
1 \\
1 \\
0\end{array}$ & $\begin{array}{l}1 \\
1 \\
1 \\
1 \\
1\end{array}$ & $\begin{array}{l}1 \\
1 \\
1 \\
1 \\
1\end{array}$ & $\begin{array}{l}2 \\
6 \\
3 \\
1 \\
1\end{array}$ \\
\hline $\begin{array}{l}N \\
N \\
N \\
N \\
N\end{array}$ & $\begin{array}{l}19 \\
20 \\
21 \\
22 \\
23\end{array}$ & $\begin{array}{r}91600 \\
38400 \\
38000 \\
59200 \\
105000\end{array}$ & $\begin{array}{r}- \\
67800 \\
58000 \\
- \\
-\end{array}$ & $\begin{array}{r}194.0 \\
61.3 \\
115.0 \\
94.2 \\
91.5\end{array}$ & $\begin{array}{r}60 . \overline{0} \\
- \\
- \\
-\end{array}$ & $\begin{array}{r}175.0 \\
50.0 \\
101.5 \\
77.3 \\
72.3\end{array}$ & $\begin{array}{l}72.0 \\
32.0 \\
34.0 \\
62.0 \\
64.0\end{array}$ & $\begin{array}{r}121.2 \\
14.1 \\
74.9 \\
86.6 \\
171.6\end{array}$ & $\begin{array}{l}1 \\
1 \\
1 \\
0 \\
0\end{array}$ & $\begin{array}{l}1 \\
1 \\
1 \\
1 \\
1\end{array}$ & $\begin{array}{l}6 \\
1 \\
3 \\
1 \\
1\end{array}$ & $\begin{array}{l}1 \\
2 \\
1 \\
2 \\
2\end{array}$ \\
\hline $\begin{array}{l}N \\
N \\
N \\
N \\
N\end{array}$ & $\begin{array}{l}24 \\
25 \\
26 \\
27 \\
28\end{array}$ & $\begin{array}{r}4490 \varnothing \\
5680 \varnothing \\
21920 \varnothing \\
4 \varnothing 0 \varnothing \varnothing \\
496 \varnothing \varnothing\end{array}$ & $\begin{array}{l}- \\
- \\
-\end{array}$ & $\begin{array}{l}95 . \varnothing \\
85.0 \\
81.6 \\
82.0 \\
72 . \varnothing\end{array}$ & $\begin{array}{l}- \\
- \\
-\end{array}$ & $\begin{array}{l}75.2 \\
68.0 \\
62.8 \\
57.8 \\
56.8\end{array}$ & $\begin{array}{l}64.0 \\
62.0 \\
57.0 \\
52.0 \\
52.0\end{array}$ & $\begin{array}{r}53.8 \\
49.6 \\
177.9 \\
46.1 \\
37.2\end{array}$ & $\begin{array}{l}1 \\
1 \\
1 \\
0 \\
1\end{array}$ & $\begin{array}{l}1 \\
1 \\
1 \\
1 \\
1\end{array}$ & $\begin{array}{l}1 \\
1 \\
1 \\
1 \\
1\end{array}$ & $\begin{array}{l}2 \\
2 \\
2 \\
2 \\
2\end{array}$ \\
\hline $\begin{array}{l}N \\
N \\
N \\
N \\
N\end{array}$ & $\begin{array}{l}29 \\
30 \\
31 \\
32 \\
33\end{array}$ & $\begin{array}{r}40900 \\
54600 \\
435900 \\
708900 \\
104600\end{array}$ & $\begin{array}{l}- \\
- \\
- \\
-\end{array}$ & $\begin{array}{l}87.5 \\
86.0 \\
57.8 \\
72.0 \\
64.5\end{array}$ & $\begin{array}{l}- \\
- \\
- \\
-\end{array}$ & $\begin{array}{l}69.5 \\
67.0 \\
49.8 \\
55.0 \\
52.8\end{array}$ & $\begin{array}{l}58 . \varnothing \\
58 . \varnothing \\
44.0 \\
51.0 \\
50.0\end{array}$ & $\begin{array}{r}33.4 \\
58.1 \\
256.6 \\
363.6 \\
42 . \varnothing\end{array}$ & $\begin{array}{l}1 \\
0 \\
1 \\
1 \\
0\end{array}$ & $\begin{array}{l}1 \\
1 \\
1 \\
3 \\
3\end{array}$ & $\begin{array}{l}1 \\
1 \\
1 \\
1 \\
1\end{array}$ & $\begin{array}{l}2 \\
2 \\
2 \\
1 \\
2\end{array}$ \\
\hline $\begin{array}{l}N \\
N \\
N \\
N \\
N\end{array}$ & $\begin{array}{l}34 \\
35 \\
36 \\
37 \\
38\end{array}$ & $\begin{array}{r}178700 \\
64900 \\
51400 \\
67700 \\
39500\end{array}$ & $\begin{array}{l}- \\
- \\
-\end{array}$ & $\begin{array}{r}97.0 \\
90.4 \\
106.1 \\
110.1 \\
118.5\end{array}$ & $\begin{array}{l}- \\
- \\
- \\
-\end{array}$ & $\begin{array}{r}85.5 \\
73.2 \\
91.8 \\
95.5 \\
102.5\end{array}$ & $\begin{array}{l}65.0 \\
63.0 \\
68.0 \\
66.0 \\
70.0\end{array}$ & $\begin{array}{r}270.8 \\
81.9 \\
19.5 \\
83.4 \\
23.9\end{array}$ & $\begin{array}{l}0 \\
1 \\
1 \\
1 \\
1\end{array}$ & $\begin{array}{l}1 \\
1 \\
3 \\
1 \\
1\end{array}$ & $\begin{array}{l}1 \\
1 \\
1 \\
1 \\
1\end{array}$ & $\begin{array}{l}2 \\
2 \\
2 \\
2 \\
2 \\
2\end{array}$ \\
\hline
\end{tabular}


Appendix.--Location and basic physical features of recharge basins on Long Island,

\begin{tabular}{|c|c|c|c|c|c|c|c|c|}
\hline \multicolumn{2}{|c|}{$\begin{array}{l}\text { BASIN } \\
\text { NUMBER }\end{array}$} & \multicolumn{2}{|c|}{ NEAREST INTERSECTION } & $\begin{array}{l}\text { LATITUDE } \\
\text { LONGITUDE } \\
0, \text { " }\end{array}$ & $\begin{array}{l}\text { DATE } \\
\text { BUILT } \\
\text { YrMoD }\end{array}$ & COMMUNITY & $\begin{array}{l}\text { DESIGN } \\
\text { CAPACITY } \\
\text { (cubic fe }\end{array}$ & $\begin{array}{l}\text { ACTUAL } \\
\text { CAPACITY } \\
\text { oet) }\end{array}$ \\
\hline $\begin{array}{l}N \\
N \\
N \\
N \\
N\end{array}$ & $\begin{array}{l}39 \\
40 \\
41 \\
42 \\
43\end{array}$ & $\begin{array}{l}\text { DIVISION AVE. } \\
\text { MANSFIELD AVE. } \\
\text { N. STEWART AVE. } \\
\text { PINTAIL LANE } \\
\text { NEWBRIDGE DR. }\end{array}$ & $\begin{array}{l}\text { AUTUMN LA. } \\
\text { PARKER AVE. } \\
\text { LEVITTOWN PKWY. } \\
\text { ORCHID ROAD } \\
\text { SKIMMER LA. }\end{array}$ & $\begin{array}{l}4044460733124 \\
4042470732935 \\
4 \varnothing 4438 \emptyset 733232 \\
4 \varnothing 44160733206 \\
4044140733131\end{array}$ & $\begin{array}{l}5104 \\
521106 \\
5105 \\
5108 \\
5104\end{array}$ & $\begin{array}{r}25 \\
121 \\
25 \\
25 \\
25\end{array}$ & $\begin{array}{l}- \\
378000 \\
- \\
- \\
-\end{array}$ & $\begin{array}{l}1510 \varnothing \varnothing \\
288 \varnothing \varnothing \varnothing \\
571 \varnothing \varnothing \varnothing \\
1785 \varnothing \varnothing \varnothing \\
1254 \varnothing \varnothing \varnothing\end{array}$ \\
\hline $\begin{array}{l}N \\
N \\
N \\
N \\
N\end{array}$ & $\begin{array}{l}44 \\
45 \\
46 \\
47 \\
48\end{array}$ & $\begin{array}{l}\text { WILLIS AVE. } \\
\text { N. STEWART AVE. } \\
\text { ROUND HILL RD. } \\
\text { HEMPSTEAD TPK. } \\
\text { NEWBRIDGE RD. }\end{array}$ & $\begin{array}{l}\text { MEADOW LA. } \\
\text { ACRE LA. } \\
\text { GLENWOOD LA. } \\
\text { DIVISION AVE. } \\
\text { NICHOLAI ST. }\end{array}$ & $\begin{array}{l}4 \varnothing 46350733911 \\
4 \varnothing 4455 \varnothing 733235 \\
4 \varnothing 4714 \varnothing 733753 \\
4 \varnothing 4332 \varnothing 733147 \\
4 \varnothing 4542 \varnothing 733211\end{array}$ & $\begin{array}{l}5104 \\
5104 \\
5308 \\
5104 \\
5111\end{array}$ & $\begin{array}{r}50 \\
104 \\
53 \\
25 \\
104\end{array}$ & $\begin{array}{l}- \\
- \\
- \\
-\end{array}$ & $\begin{array}{l}143000 \\
502000 \\
288000 \\
780000 \\
66200\end{array}$ \\
\hline $\begin{array}{l}N \\
N \\
N \\
N \\
N\end{array}$ & $\begin{array}{l}49 \\
58 \\
51 \\
52 \\
53\end{array}$ & $\begin{array}{l}\text { HAWTHORN ST. } \\
\text { S. CABOT LANE } \\
\text { CARMAN AVENUE } \\
\text { CARMAN AVE } \\
\text { GRACE LA }\end{array}$ & $\begin{array}{l}\text { JOHN ST. } \\
\text { ALICE COURT } \\
\text { OLD COUNTRY ROAD } \\
\text { N. STEWART AVE. } \\
\text { NEWBRIDGE RD }\end{array}$ & 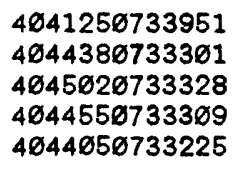 & $\begin{array}{l}531023 \\
5408 \\
530330 \\
5104 \\
5104\end{array}$ & $\begin{array}{r}47 \\
8 \\
8 \\
8 \\
104\end{array}$ & $\begin{array}{l}98000 \\
\overline{133600} \\
- \\
-\end{array}$ & $\begin{array}{l}118600 \\
531000 \\
222400 \\
222000 \\
221000\end{array}$ \\
\hline $\begin{array}{l}N \\
N \\
N \\
N \\
N\end{array}$ & $\begin{array}{l}54 \\
55 \\
56 \\
57 \\
58\end{array}$ & $\begin{array}{l}\text { BALL PARK LANE } \\
\text { CAROL PLACE } \\
\text { BELLMORE RD. } \\
\text { PORT LANE } \\
\text { NEWBRIDGE RD }\end{array}$ & $\begin{array}{l}\text { NEWBRIDGE ROAD } \\
\text { S. ZORRANE DRIVE } \\
\text { MAGNOLIA RD. } \\
\text { SALISBURY PK.DR. } \\
\text { SECOND ST }\end{array}$ & $\begin{array}{l}4 \varnothing 4457 \varnothing 733222 \\
4 \varnothing 43 \varnothing 90732819 \\
4 \varnothing 4132 \varnothing 7332 \varnothing 9 \\
4 \varnothing 4419 \emptyset 73335 \emptyset \\
404313 \varnothing 733255\end{array}$ & $\begin{array}{l}5104 \\
5104 \\
561210 \\
5110 \\
5107\end{array}$ & $\begin{array}{r}104 \\
92 \\
32 \\
8 \\
8\end{array}$ & $\begin{array}{l}- \\
\overline{185000} \\
- \\
-\end{array}$ & $\begin{array}{r}1020000 \\
24700 \\
123000 \\
711000 \\
114000\end{array}$ \\
\hline $\begin{array}{l}N \\
N \\
N \\
N \\
N\end{array}$ & $\begin{array}{l}59 \\
69 \\
61 \\
62 \\
63\end{array}$ & $\begin{array}{l}\text { NEWBRIDGE RD } \\
\text { DWYER PL } \\
\text { E. WILLISTON AVE } \\
\text { WANTAGH ST.PKWY } \\
\text { NORTHERN ST.PKY }\end{array}$ & $\begin{array}{l}\text { SECOND ST } \\
\text { BALDWIN RD } \\
\text { L.I.R.R. } \\
\text { OLD WESTBURY RD } \\
\text { WILLIS AVE }\end{array}$ & 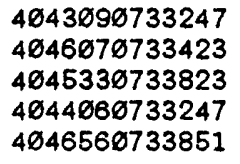 & $\begin{array}{l}5186 \\
540409 \\
5608 \\
5204 \\
5104\end{array}$ & $\begin{array}{r}8 \\
89 \\
54 \\
8 \\
83\end{array}$ & $\begin{array}{l}- \\
350000 \\
- \\
- \\
-\end{array}$ & $\begin{array}{r}- \\
334080 \\
379000 \\
9020000 \\
102200\end{array}$ \\
\hline $\begin{array}{l}N \\
N \\
N \\
N \\
N\end{array}$ & $\begin{array}{l}64 \\
65 \\
66 \\
67 \\
68\end{array}$ & $\begin{array}{l}\text { OLD FARM RD } \\
\text { WESTBURY RD } \\
\text { BUXTON AVE } \\
\text { JERUSALEM AVE } \\
\text { FORDHAM AVE }\end{array}$ & $\begin{array}{l}\text { ROUND HILL RD } \\
\text { OLD BRICK RD } \\
\text { LAWRENCE RD } \\
\text { VALLEY LA } \\
\text { DIVISION AVE }\end{array}$ & $\begin{array}{l}4 \varnothing 47210733741 \\
4 \varnothing 47 \varnothing 7 \varnothing 733746 \\
4 \varnothing 4137 \varnothing 733958 \\
4 \varnothing 44590733121 \\
4 \varnothing 45880733129\end{array}$ & $\begin{array}{l}5308 \\
5203 \\
5211 \\
530608 \\
5404\end{array}$ & $\begin{array}{r}53 \\
53 \\
69 \\
104 \\
104\end{array}$ & $\begin{array}{l}- \\
- \\
- \\
240000 \\
104\end{array}$ & $\begin{array}{r}89200 \\
76600 \\
233000 \\
328000 \\
318000\end{array}$ \\
\hline $\begin{array}{l}N \\
N \\
N \\
N \\
N\end{array}$ & $\begin{array}{l}69 \\
78 \\
71 \\
72 \\
73\end{array}$ & $\begin{array}{l}\text { WOODBURY RD } \\
\text { HAYLOFT LA } \\
\text { SCHOOL HOUSE LA. } \\
\text { ROUND HILL RD } \\
\text { UNDERHILL BLVD. }\end{array}$ & $\begin{array}{l}\text { IRENE LA. SOUTH } \\
\text { NORTHERN ST. PKY } \\
\text { ARBOR LA. } \\
\text { WAGON RD } \\
\text { WILLIS LANE }\end{array}$ & $\begin{array}{l}4 \varnothing 4723 \varnothing 732927 \\
4 \varnothing 464 \varnothing \varnothing 7338 \varnothing 3 \\
4 \varnothing 46 \varnothing 4 \varnothing 7338 \varnothing 2 \\
4 \varnothing 4723 \varnothing 733756 \\
4 \varnothing 4855 \varnothing 733 \varnothing 47\end{array}$ & $\begin{array}{l}530303 \\
5104 \\
5111 \\
5110 \\
581022\end{array}$ & $\begin{array}{r}184 \\
83 \\
83 \\
53 \\
115\end{array}$ & $\begin{array}{l}1510000 \\
- \\
- \\
-\end{array}$ & $\begin{array}{l}156100 \\
530000 \\
1245000 \\
273000 \\
-\end{array}$ \\
\hline $\begin{array}{l}N \\
N \\
N \\
N \\
N\end{array}$ & $\begin{array}{l}74 \\
75 \\
76 \\
77 \\
78\end{array}$ & $\begin{array}{l}\text { NIAGARA ST } \\
\text { PROSPECT AVE } \\
\text { HARRIET ROAD } \\
\text { DEAN ST } \\
\text { HEMPSTEAD TPKE }\end{array}$ & $\begin{array}{l}\text { GREENWAY BLVD } \\
\text { BELLMORE RD } \\
\text { GLORIA ROAD } \\
\text { JERUSALEM AVE } \\
\text { BENSON AVE }\end{array}$ & $\begin{array}{l}4 \oslash 4108 \emptyset 734221 \\
4 \varnothing 4251 \varnothing 733217 \\
4 \varnothing 4247 \varnothing 7329 \varnothing 3 \\
4 \varnothing 4452 \varnothing 733 \varnothing 57 \\
4 \varnothing 4228 \varnothing 734155\end{array}$ & $\begin{array}{l}5205 \\
5302 \\
5109 \\
5309 \\
5909\end{array}$ & $\begin{array}{r}10 \\
8 \\
121 \\
104 \\
10\end{array}$ & $\begin{array}{l}- \\
- \\
- \\
-\end{array}$ & $\begin{array}{r}313000 \\
385000 \\
264 \varnothing 00 \\
456000 \\
94000\end{array}$ \\
\hline $\begin{array}{l}N \\
N \\
N \\
N \\
N\end{array}$ & $\begin{array}{l}79 \\
80 \\
81 \\
82 \\
83\end{array}$ & $\begin{array}{l}\text { SHEPHERD LANE } \\
\text { YALE ST } \\
\text { EVERGREEN AVE } \\
\text { ROSELLE STREET } \\
\text { MIRIAM PARKWAY }\end{array}$ & $\begin{array}{l}\text { SNAPDRAGON LANE } \\
\text { WILLIS AVE } \\
\text { STEWART AVE } \\
\text { SHORTRIDGE DRIVE } \\
\text { MIRIAM COURT }\end{array}$ & $\begin{array}{l}4 \varnothing 4643 \varnothing 733823 \\
4 \varnothing 4633 \varnothing 733838 \\
4 \varnothing 4545 \varnothing 732938 \\
4 \varnothing 4523 \varnothing 733735 \\
4 \varnothing 4132 \varnothing 734204\end{array}$ & $\begin{array}{l}5109 \\
51 \\
5212 \\
6203 \\
5508\end{array}$ & $\begin{array}{l}83 \\
50 \\
92 \\
68 \\
10\end{array}$ & $\begin{array}{l}- \\
- \\
- \\
-\end{array}$ & $\begin{array}{l}193000 \\
124200 \\
187000 \\
127400 \\
122000\end{array}$ \\
\hline $\begin{array}{l}N \\
N \\
N \\
N \\
N\end{array}$ & $\begin{array}{l}84 \\
85 \\
86 \\
87 \\
88\end{array}$ & $\begin{array}{l}\text { STEWART AVE } \\
\text { N. JERUSALEM RD } \\
\text { JEFFERSON ST } \\
\text { CLEARMEADOW RD. } \\
\text { JACKSON AVE. }\end{array}$ & $\begin{array}{l}\text { DENNIS LA } \\
\text { NEWBRIDGE AVE } \\
\text { MERITTS RD } \\
\text { NEWBRIDGE RD. } \\
\text { CONVENT RD. }\end{array}$ & $\begin{array}{l}4 \varnothing 4312 \varnothing 732847 \\
4 \varnothing 4211 \varnothing 73331 \varnothing \\
4 \varnothing 4359 \varnothing 73272 \varnothing \\
4 \varnothing 4333 \varnothing 7333 \varnothing \emptyset \\
4 \varnothing 4924 \varnothing 732955\end{array}$ & $\begin{array}{l}5309 \\
52 \\
5404 \\
5205 \\
571104\end{array}$ & $\begin{array}{r}121 \\
8 \\
99 \\
8 \\
126\end{array}$ & $\begin{array}{l}- \\
- \\
\overline{3} 2200\end{array}$ & $\begin{array}{l}270000 \\
155400 \\
119800 \\
278000 \\
53000\end{array}$ \\
\hline $\begin{array}{l}N \\
N \\
N \\
N \\
N\end{array}$ & $\begin{array}{l}89 \\
90 \\
91 \\
92 \\
93\end{array}$ & $\begin{array}{l}\text { SEARINGTOWN RD. } \\
\text { SEARINGTOWN RD. } \\
\text { OLIVE STREET } \\
\text { OLD WESTBURY RD. } \\
\text { WILLIS AVE. }\end{array}$ & $\begin{array}{l}\text { NORTHERN BLVD. } \\
\text { THE SPUR } \\
\text { TANNERS ROAD } \\
\text { GUINEA WOODS RD. } \\
\text { HOLLOW CT. }\end{array}$ & 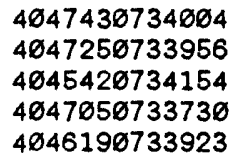 & $\begin{array}{l}5803 \\
5511 \\
5710 \\
5110\end{array}$ & $\begin{array}{l}81 \\
81 \\
65 \\
53 \\
50\end{array}$ & $\begin{array}{l}- \\
- \\
- \\
-\end{array}$ & $\begin{array}{r}18300 \\
64600 \\
129200 \\
234000 \\
86400\end{array}$ \\
\hline
\end{tabular}


N.Y. [Dash indicates no data. Altitudes are in feot above sea level.] (cont.)

\begin{tabular}{|c|c|c|c|c|c|c|c|c|c|c|c|c|}
\hline & & $\begin{array}{l}\text { MAX. } \\
\text { AREA } \\
\text { (square }\end{array}$ & $\begin{array}{c}\text { BASIN } \\
\text { AREA } \\
\text { fe日t) }\end{array}$ & RIM & $\begin{array}{l}--A L T I \\
\text { OVER- } \\
\text { FLOW }\end{array}$ & $\begin{array}{l}\text { ITUDE- } \\
\text { BOT- } \\
\text { TOM }\end{array}$ & $\begin{array}{l}\text { WATER } \\
\text { TABLE }\end{array}$ & $\begin{array}{c}\text { DRAIN. } \\
\text { AREA } \\
\text { (acres) }\end{array}$ & $\begin{array}{l}\text {--BAS } \\
\text { STATUS }\end{array}$ & $\begin{array}{l}\text { IN-- } \\
\text { USE }\end{array}$ & $\begin{array}{l}\text { GEO. } \\
\text { UNIT }\end{array}$ & $\begin{array}{l}\text { SOIL } \\
\text { UNIT }\end{array}$ \\
\hline $\begin{array}{l}N \\
N \\
N \\
N \\
N\end{array}$ & $\begin{array}{l}39 \\
40 \\
41 \\
42 \\
43\end{array}$ & $\begin{array}{r}57800 \\
28700 \\
69100 \\
81100 \\
136600\end{array}$ & $\begin{array}{r}- \\
54560 \\
- \\
-\end{array}$ & $\begin{array}{r}112.9 \\
67.0 \\
111.9 \\
42.0 \\
99.0\end{array}$ & $\begin{array}{r}- \\
62.6 \\
- \\
-\end{array}$ & $\begin{array}{r}101.8 \\
52.0 \\
92.5 \\
33.0 \\
80.5\end{array}$ & $\begin{array}{l}69.0 \\
45.0 \\
68.0 \\
66.0 \\
65.0\end{array}$ & $\begin{array}{r}23.8 \\
59.5 \\
89.9 \\
281.0 \\
197.4\end{array}$ & $\begin{array}{l}1 \\
1 \\
1 \\
0 \\
1\end{array}$ & $\begin{array}{l}1 \\
1 \\
1 \\
1 \\
1\end{array}$ & $\begin{array}{l}1 \\
1 \\
1 \\
1 \\
1\end{array}$ & $\begin{array}{l}2 \\
1 \\
2 \\
2 \\
2\end{array}$ \\
\hline $\begin{array}{l}N \\
N \\
N \\
N \\
N\end{array}$ & $\begin{array}{l}44 \\
45 \\
46 \\
47 \\
48\end{array}$ & $\begin{array}{r}23000 \\
58000 \\
29500 \\
135200 \\
18000\end{array}$ & $\begin{array}{l}- \\
- \\
-\end{array}$ & $\begin{array}{r}126.5 \\
116.7 \\
166.0 \\
79.5 \\
129 . \varnothing\end{array}$ & $\begin{array}{l}\overline{-} \\
\bar{z}\end{array}$ & $\begin{array}{r}113.0 \\
93.6 \\
154.0 \\
58.5 \\
119.5\end{array}$ & $\begin{array}{l}48.0 \\
70.0 \\
55.0 \\
57.0 \\
73.0\end{array}$ & $\begin{array}{r}22.5 \\
79.0 \\
45.3 \\
122.8 \\
10.4\end{array}$ & $\begin{array}{l}1 \\
1 \\
1 \\
0 \\
1\end{array}$ & $\begin{array}{l}1 \\
1 \\
1 \\
1 \\
1\end{array}$ & $\begin{array}{l}2 \\
1 \\
1 \\
1 \\
1\end{array}$ & $\begin{array}{l}1 \\
2 \\
1 \\
2 \\
2\end{array}$ \\
\hline $\begin{array}{l}N \\
N \\
N \\
N \\
N\end{array}$ & $\begin{array}{l}49 \\
50 \\
51 \\
52 \\
53\end{array}$ & $\begin{array}{l}26900 \\
62500 \\
22200 \\
37500 \\
40900\end{array}$ & $\begin{array}{r}30300 \\
- \\
- \\
- \\
-\end{array}$ & $\begin{array}{r}219.5 \\
111.0 \\
113.0 \\
116.3 \\
97.0\end{array}$ & $\begin{array}{r}219.3 \\
- \\
- \\
- \\
-\end{array}$ & $\begin{array}{r}212.0 \\
95.7 \\
97.0 \\
98.3 \\
80.0\end{array}$ & $\begin{array}{l}21.0 \\
68.0 \\
71.0 \\
70.0 \\
64.0\end{array}$ & $\begin{array}{l}18.0 \\
83.6 \\
21.0 \\
34.9 \\
34.8\end{array}$ & $\begin{array}{l}1 \\
1 \\
1 \\
1 \\
1\end{array}$ & $\begin{array}{l}1 \\
1 \\
1 \\
1 \\
1\end{array}$ & $\begin{array}{l}1 \\
1 \\
1 \\
1 \\
1\end{array}$ & $\begin{array}{l}1 \\
2 \\
2 \\
2 \\
2\end{array}$ \\
\hline $\begin{array}{l}N \\
N \\
N \\
N \\
N\end{array}$ & $\begin{array}{l}54 \\
55 \\
56 \\
57 \\
58\end{array}$ & $\begin{array}{r}796000 \\
37500 \\
18900 \\
87500 \\
22100\end{array}$ & $\begin{array}{r}- \\
33500 \\
-\end{array}$ & $\begin{array}{r}117.0 \\
74.6 \\
45.0 \\
110.0 \\
83.0\end{array}$ & $43 . \overline{-}$ & $\begin{array}{r}107.0 \\
62.0 \\
34.0 \\
100.0 \\
100 . \varnothing\end{array}$ & $\begin{array}{l}70.0 \\
51.0 \\
30.0 \\
66.0 \\
54.0\end{array}$ & $\begin{array}{r}160.6 \\
15.1 \\
25.4 \\
111.9 . \\
17.9\end{array}$ & $\begin{array}{l}0 \\
1 \\
1 \\
1 \\
1\end{array}$ & $\begin{array}{l}1 \\
3 \\
1 \\
1 \\
0\end{array}$ & $\begin{array}{l}1 \\
1 \\
1 \\
1 \\
1\end{array}$ & $\begin{array}{l}2 \\
3 \\
1 \\
2 \\
2\end{array}$ \\
\hline $\begin{array}{l}N \\
N \\
N \\
N \\
N\end{array}$ & $\begin{array}{l}59 \\
60 \\
61 \\
62 \\
63\end{array}$ & $\begin{array}{r}26100 \\
38700 \\
73400 \\
592300 \\
18400\end{array}$ & $\begin{array}{r}79200 \\
- \\
-\end{array}$ & $\begin{array}{r}78.9 \\
135.8 \\
109.1 \\
102.0 \\
122.3\end{array}$ & $\begin{array}{r}123.3 \\
- \\
- \\
-\end{array}$ & $\begin{array}{r}66.0 \\
123.3 \\
99.4 \\
79.0 \\
112.8\end{array}$ & $\begin{array}{l}53.0 \\
73.0 \\
51.0 \\
52.0 \\
50.0\end{array}$ & $\begin{array}{r}17.6 \\
48.2 \\
59.7 \\
419.9 \\
16.1\end{array}$ & $\begin{array}{l}1 \\
1 \\
1 \\
1 \\
1\end{array}$ & $\begin{array}{l}1 \\
1 \\
1 \\
1 \\
1\end{array}$ & $\begin{array}{l}1 \\
1 \\
1 \\
1 \\
1\end{array}$ & $\begin{array}{l}2 \\
1 \\
1 \\
1 \\
1\end{array}$ \\
\hline $\begin{array}{l}N \\
N \\
N \\
N \\
N\end{array}$ & $\begin{array}{l}64 \\
65 \\
66 \\
67 \\
68\end{array}$ & $\begin{array}{l}18200 \\
15900 \\
11900 \\
39300 \\
61000\end{array}$ & $\begin{array}{r}- \\
- \\
63480 \\
-\end{array}$ & $\begin{array}{r}163.0 \\
158.0 \\
52.0 \\
119.0 \\
124.8\end{array}$ & $\begin{array}{l}- \\
- \\
- \\
-\end{array}$ & $\begin{array}{r}153.0 \\
143.0 \\
38.0 \\
189.0 \\
110.0\end{array}$ & $\begin{array}{l}58.0 \\
57.0 \\
20.0 \\
71.0 \\
71.0\end{array}$ & $\begin{array}{l}14.0 \\
12.1 \\
14.3 \\
32.7 \\
50.1\end{array}$ & $\begin{array}{l}1 \\
1 \\
1 \\
1 \\
1\end{array}$ & $\begin{array}{l}1 \\
1 \\
4 \\
1 \\
1\end{array}$ & $\begin{array}{l}1 \\
1 \\
1 \\
1 \\
1\end{array}$ & $\begin{array}{l}1 \\
1 \\
1 \\
2 \\
2\end{array}$ \\
\hline $\begin{array}{l}N \\
N \\
N \\
N \\
N\end{array}$ & $\begin{array}{l}69 \\
70 \\
71 \\
72 \\
73\end{array}$ & $\begin{array}{r}17300 \\
89500 \\
201200 \\
51900 \\
23400\end{array}$ & $\begin{array}{r}28080 \\
- \\
- \\
41000\end{array}$ & $\begin{array}{l}176.0 \\
115.8 \\
105.3 \\
171.0 \\
216.3\end{array}$ & $\begin{array}{r}174.5 \\
- \\
- \\
- \\
-\end{array}$ & $\begin{array}{r}164.5 \\
101.4 \\
87.5 \\
157.8 \\
205.0\end{array}$ & $\begin{array}{l}81.0 \\
56.0 \\
56.0 \\
55.0 \\
71.0\end{array}$ & $\begin{array}{r}22.0 \\
83.4 \\
196.0 \\
43.0 \\
42.2\end{array}$ & $\begin{array}{l}1 \\
1 \\
1 \\
1 \\
0\end{array}$ & $\begin{array}{l}1 \\
1 \\
1 \\
1 \\
1\end{array}$ & $\begin{array}{l}1 \\
1 \\
1 \\
6 \\
6\end{array}$ & $\begin{array}{l}2 \\
1 \\
1 \\
1 \\
1\end{array}$ \\
\hline $\begin{array}{l}N \\
N \\
N \\
N \\
N\end{array}$ & $\begin{array}{l}74 \\
75 \\
76 \\
77 \\
78\end{array}$ & $\begin{array}{l}56900 \\
65000 \\
58100 \\
62600 \\
16000\end{array}$ & $\begin{array}{l}- \\
- \\
-\end{array}$ & $\begin{array}{r}39.0 \\
7 \varnothing .7 \\
66.0 \\
120.4 \\
69.0\end{array}$ & $\begin{array}{l}- \\
- \\
- \\
-\end{array}$ & $\begin{array}{r}28.0 \\
59.5 \\
- \\
187.4 \\
56.0\end{array}$ & $\begin{array}{l}15.0 \\
49.0 \\
43.0 \\
69.0 \\
21.0\end{array}$ & $\begin{array}{l}49.3 \\
60.6 \\
41.6 \\
71.8 \\
14.8\end{array}$ & $\begin{array}{l}1 \\
1 \\
0 \\
1 \\
1\end{array}$ & $\begin{array}{l}1 \\
1 \\
1 \\
1 \\
1\end{array}$ & $\begin{array}{l}1 \\
1 \\
1 \\
1 \\
1\end{array}$ & $\begin{array}{l}6 \\
1 \\
3 \\
2 \\
1\end{array}$ \\
\hline $\begin{array}{l}N \\
N \\
N \\
N \\
N\end{array}$ & $\begin{array}{l}79 \\
80 \\
81 \\
82 \\
83\end{array}$ & $\begin{array}{l}47260 \\
77300 \\
39980 \\
28100 \\
24380\end{array}$ & $\begin{array}{l}- \\
- \\
-\end{array}$ & $\begin{array}{r}116.5 \\
122.0 \\
134.2 \\
185.3 \\
42.8\end{array}$ & $\begin{array}{l}- \\
- \\
-\end{array}$ & $\begin{array}{r}99.5 \\
106.0 \\
124.0 \\
90.9 \\
31.0\end{array}$ & $\begin{array}{l}55.0 \\
53.0 \\
78.0 \\
59.0 \\
15.0\end{array}$ & $\begin{array}{l}30.4 \\
19.6 \\
29.4 \\
20.1 \\
19.2\end{array}$ & $\begin{array}{l}1 \\
1 \\
1 \\
1 \\
1\end{array}$ & $\begin{array}{l}1 \\
1 \\
1 \\
1 \\
1\end{array}$ & $\begin{array}{l}1 \\
1 \\
1 \\
1 \\
1\end{array}$ & $\begin{array}{l}1 \\
1 \\
2 \\
2 \\
6\end{array}$ \\
\hline $\begin{array}{l}N \\
N \\
N \\
N \\
N\end{array}$ & $\begin{array}{l}84 \\
85 \\
86 \\
87 \\
88\end{array}$ & $\begin{array}{r}49600 \\
33600 \\
36300 \\
42900 \\
6000\end{array}$ & $\begin{array}{r}- \\
- \\
14400\end{array}$ & $\begin{array}{r}74.2 \\
41.8 \\
73.4 \\
91.5 \\
216.2\end{array}$ & $\begin{array}{r}51 . \overline{5} \\
\overline{-} \\
216.0\end{array}$ & $\begin{array}{r}63.0 \\
44.0 \\
61.0 \\
76.5 \\
288.0\end{array}$ & $\begin{array}{l}49.0 \\
24.5 \\
58.0 \\
59.0 \\
67.0\end{array}$ & $\begin{array}{r}42.5 \\
1.0 \\
18.9 \\
43.4 \\
5.1\end{array}$ & $\begin{array}{l}1 \\
1 \\
1 \\
1 \\
1\end{array}$ & $\begin{array}{l}1 \\
1 \\
1 \\
1 \\
1\end{array}$ & $\begin{array}{l}1 \\
1 \\
1 \\
1 \\
6\end{array}$ & $\begin{array}{l}1 \\
1 \\
1 \\
2 \\
1\end{array}$ \\
\hline $\begin{array}{l}N \\
N \\
N \\
N \\
N\end{array}$ & $\begin{array}{l}89 \\
90 \\
91 \\
92 \\
93\end{array}$ & $\begin{array}{r}6000 \\
21100 \\
45000 \\
50800 \\
18000\end{array}$ & $\begin{array}{l}- \\
- \\
- \\
-\end{array}$ & $\begin{array}{l}186.3 \\
233.4 \\
133 . \varnothing \\
158 . \varnothing \\
124.1\end{array}$ & $\begin{array}{l}- \\
- \\
-\end{array}$ & $\begin{array}{l}176.4 \\
225.4 \\
119 . \varnothing \\
142 . \varnothing \\
110 . \varnothing\end{array}$ & $\begin{array}{l}42.0 \\
43.0 \\
33.0 \\
61.0 \\
40.0\end{array}$ & $\begin{array}{r}2.9 \\
10.2 \\
20.3 \\
36.8 \\
13.6\end{array}$ & $\begin{array}{l}1 \\
1 \\
0 \\
1 \\
0\end{array}$ & $\begin{array}{l}1 \\
1 \\
1 \\
1 \\
1\end{array}$ & $\begin{array}{l}4 \\
4 \\
1 \\
1 \\
6\end{array}$ & $\begin{array}{l}1 \\
1 \\
1 \\
1 \\
1\end{array}$ \\
\hline
\end{tabular}


Appendix.--Location and basic physical features of recharge basins on Long Island,

\begin{tabular}{|c|c|c|c|c|c|c|c|c|}
\hline \multicolumn{2}{|c|}{$\begin{array}{l}\text { BASIN } \\
\text { NUMBER }\end{array}$} & \multicolumn{2}{|c|}{ NEAREST INTERSECTION } & $\begin{array}{l}\text { LATITUDE } \\
\text { LONGITUDE } \\
\circ, " \text { " }\end{array}$ & $\begin{array}{l}\text { DATE } \\
\text { BUILT } \\
\text { YrMOD }\end{array}$ & COMMUNITY & $\begin{array}{l}\text { DESIGN } \\
\text { CAPACITY } \\
\text { (cubic fe }\end{array}$ & $\begin{array}{l}\text { ACTUAL } \\
\text { CAPACITY } \\
\text { (t) }\end{array}$ \\
\hline $\begin{array}{l}N \\
N \\
N \\
N \\
N\end{array}$ & $\begin{array}{l}94 \\
95 \\
96 \\
97 \\
98\end{array}$ & $\begin{array}{l}\text { APPLETREE LA. } \\
\text { COLUMBUS AVE. } \\
\text { PARKSIDE RD. } \\
\text { WANTAGH AVE. } \\
\text { KNOLLWOOD DR. }\end{array}$ & $\begin{array}{l}\text { JAMAICA BLVD. } \\
\text { NEWBRIDGE RD. } \\
\text { ROSLYN RD. } \\
\text { HEMPSTEAD TPKE. } \\
\text { ASBURY AVE. }\end{array}$ & $\begin{array}{l}4045050733650 \\
4 \emptyset 42 \emptyset 4 \varnothing 733231 \\
4046590733829 \\
4 \emptyset 43410732954 \\
4 \varnothing 45100733629\end{array}$ & $\begin{array}{l}520915 \\
670508 \\
51 \\
5104 \\
5111\end{array}$ & $\begin{array}{l}52 \\
32 \\
80 \\
25 \\
52\end{array}$ & $\begin{array}{l}- \\
\overline{-} \\
- \\
-\end{array}$ & $\begin{array}{r}203400 \\
875500 \\
273200 \\
201000 \\
72800\end{array}$ \\
\hline $\begin{array}{l}N \\
N \\
N \\
N \\
N\end{array}$ & $\begin{array}{r}99 \\
100 \\
101 \\
102 \\
103\end{array}$ & $\begin{array}{l}\text { BEECHWOOD DR. } \\
\text { CENTRAL DR. } \\
\text { PARKWAY DRIVE } \\
\text { LAWRENCE LA. } \\
\text { SOUTHERN ST. PKY }\end{array}$ & $\begin{array}{l}\text { GLEN HEAD RD. } \\
\text { GLEN COVE RD. } \\
\text { I.U. WILLETS RD. } \\
\text { FROST POND RD. } \\
\text { GREENWAY BLVD. }\end{array}$ & $\begin{array}{l}4050430733742 \\
4 \emptyset 50370733747 \\
4 \emptyset 46290733736 \\
4 \emptyset 51250733605 \\
4040580734222\end{array}$ & $\begin{array}{l}51 \\
51 \\
51 \\
5209 \\
5210\end{array}$ & $\begin{array}{r}101 \\
101 \\
50 \\
100 \\
10\end{array}$ & $\begin{array}{l}- \\
- \\
-\end{array}$ & $\begin{array}{r}129200 \\
48100 \\
131100 \\
269000 \\
82700\end{array}$ \\
\hline $\begin{array}{l}N \\
N \\
N \\
N \\
N\end{array}$ & $\begin{array}{l}104 \\
105 \\
106 \\
107 \\
108\end{array}$ & $\begin{array}{l}\text { PLYMOUTH DR.N. } \\
\text { OLD WESTBURY RD. } \\
\text { SEAMANS NECK RD. } \\
\text { SEAMANS NECK RD. } \\
\text { WILLETS DR. }\end{array}$ & $\begin{array}{l}\text { GLEN COVE RD. } \\
\text { KINGS DR. } \\
\text { MAXWELL RD. } \\
\text { TOLLGATE LANE } \\
\text { JACKSON AVE. }\end{array}$ & $\begin{array}{l}405033073374 \emptyset \\
4047180733637 \\
4041250732955 \\
404157073294 \varnothing \\
4048510733023\end{array}$ & $\begin{array}{l}5211 \\
5208 \\
5303 \\
5204 \\
5501\end{array}$ & $\begin{array}{r}101 \\
118 \\
46 \\
46 \\
108\end{array}$ & $\begin{array}{l}- \\
- \\
- \\
-\end{array}$ & $\begin{array}{c}133000 \\
112000 \\
248000 \\
- \\
134000\end{array}$ \\
\hline $\begin{array}{l}N \\
N \\
N \\
N \\
N\end{array}$ & $\begin{array}{l}169 \\
110 \\
111 \\
112 \\
113\end{array}$ & $\begin{array}{l}\text { WANTAGH AVE. } \\
\text { WANTAGH AVE. } \\
\text { CARMAN AVENUE } \\
\text { L.I.R.R. } \\
\text { TEMPLE DR. }\end{array}$ & $\begin{array}{l}\text { FARM RANCH RD. } \\
\text { MILLER PL. } \\
\text { GASSER AVENUE } \\
\text { ARIZONA AVE. } \\
\text { HENRY RD. }\end{array}$ & $\begin{array}{l}4044130733008 \\
4042510733007 \\
4 \varnothing 44240733316 \\
4 \emptyset 49310732959 \\
4 \emptyset 4 \varnothing 530732958\end{array}$ & $\begin{array}{l}5112 \\
5201 \\
5204 \\
520317 \\
5202\end{array}$ & $\begin{array}{r}92 \\
25 \\
8 \\
92 \\
46\end{array}$ & $\begin{array}{l}- \\
- \\
89800 \\
-\end{array}$ & $\begin{array}{r}157000 \\
399000 \\
410000 \\
59500 \\
281000\end{array}$ \\
\hline $\begin{array}{l}N \\
N \\
N \\
N \\
N\end{array}$ & $\begin{array}{l}114 \\
115 \\
116 \\
117 \\
118\end{array}$ & $\begin{array}{l}\text { ROXBURY LA. } \\
\text { HEMPSTEAD TPKE. } \\
\text { HEMPSTEAD TPKE. } \\
\text { LUDLAM AVE. } \\
\text { HILLSIDE AVE. }\end{array}$ & $\begin{array}{l}\text { BARBARA LA. } \\
\text { MILLER RD. } \\
\text { COLONIAL DR. } \\
\text { ELMONT RD. } \\
\text { GUINEA WOODS RD. }\end{array}$ & $\begin{array}{l}4043110733109 \\
4043390732812 \\
4043310732748 \\
4042060734325 \\
4045380733718\end{array}$ & $\begin{array}{l}5110 \\
5302 \\
5209 \\
590901 \\
540210\end{array}$ & $\begin{array}{r}25 \\
92 \\
121 \\
10 \\
118\end{array}$ & $\begin{array}{l}- \\
- \\
\overline{84000}\end{array}$ & $\begin{array}{r}108700 \\
69200 \\
106000 \\
- \\
96500\end{array}$ \\
\hline $\begin{array}{l}N \\
N \\
N \\
N \\
N\end{array}$ & $\begin{array}{l}119 \\
120 \\
121 \\
122 \\
123\end{array}$ & $\begin{array}{l}\text { HEMLOCK ST. } \\
\text { RAFF AVENUE } \\
\text { STEWART STREET } \\
\text { ELMONT ROAD } \\
\text { OLD COUNTRY ROAD }\end{array}$ & $\begin{array}{l}\text { PINE TREE DR. } \\
\text { FLORAL PKWY. } \\
\text { COVERT AVE. } \\
\text { DUTCH BROADWAY } \\
\text { ROCKAWAY AVENUE }\end{array}$ & $\begin{array}{l}404308 \emptyset 732751 \\
4042580734145 \\
4043280734142 \\
4 \varnothing 41410734318 \\
4044120733908\end{array}$ & $\begin{array}{l}530224 \\
61 \\
621015 \\
4303 \\
4105\end{array}$ & $\begin{array}{r}121 \\
55 \\
55 \\
10 \\
14\end{array}$ & $\begin{array}{l}153600 \\
- \\
- \\
6286000\end{array}$ & $\begin{array}{l}121000 \\
- \\
- \\
3770000 \\
5720000\end{array}$ \\
\hline $\begin{array}{l}N \\
N \\
N \\
N \\
N\end{array}$ & $\begin{array}{l}124 \\
125 \\
126 \\
127 \\
128\end{array}$ & $\begin{array}{l}\text { CLINTON RD } \\
\text { GARFIELD AVE. } \\
\text { L.I.R.R. } \\
\text { WILLIS AVE. } \\
\text { DUFFY LA. }\end{array}$ & $\begin{array}{l}\text { STEWART AVE. } \\
\text { MARCELLUS RD. } \\
\text { BROWN ST. } \\
\text { HAWTHORNE ST. } \\
\text { OLD COUNTRY RD. }\end{array}$ & $\begin{array}{l}4 \emptyset 4356 \emptyset 7337 \varnothing 6 \\
4 \emptyset 4436 \emptyset 73385 \emptyset \\
4 \emptyset 4533 \emptyset 733827 \\
4 \emptyset 4534 \emptyset 733848 \\
4 \emptyset 4534 \varnothing 733256\end{array}$ & $\begin{array}{l}4604 \\
4104 \\
41 \\
3001 \\
43\end{array}$ & $\begin{array}{r}14 \\
68 \\
90 \\
90 \\
104\end{array}$ & $\begin{array}{l}- \\
- \\
- \\
-\end{array}$ & $\begin{array}{l}- \\
- \\
- \\
156500\end{array}$ \\
\hline $\begin{array}{l}N \\
N \\
N \\
N \\
N\end{array}$ & $\begin{array}{l}129 \\
130 \\
131 \\
132 \\
133\end{array}$ & $\begin{array}{l}\text { DENTON AVE. } \\
\text { HERRICKS RD. } \\
\text { L.I.R.R. } \\
\text { DENTON AVE. } \\
\text { FROST STREET }\end{array}$ & $\begin{array}{l}\text { HILLSIDE AVE. } \\
\text { HILLSIDE AVE. } \\
\text { EDGEMERE ROAD } \\
\text { JERICHO TPKE. } \\
\text { UNQUA ROAD }\end{array}$ & $\begin{array}{l}4044510734031 \\
4045170733929 \\
4043050734016 \\
4044410734018 \\
4040460732619\end{array}$ & $\begin{array}{l}62 \\
38 \\
59 \\
43 \\
540407\end{array}$ & $\begin{array}{r}57 \\
62 \\
14 \\
57 \\
110\end{array}$ & $\begin{array}{l}- \\
- \\
- \\
217008\end{array}$ & $\begin{array}{l}19 \overline{10000} \\
- \\
\overline{218000}\end{array}$ \\
\hline $\begin{array}{l}N \\
N \\
N \\
N \\
N\end{array}$ & $\begin{array}{l}134 \\
135 \\
136 \\
137 \\
138\end{array}$ & $\begin{array}{l}\text { WILLIS AVENUE } \\
\text { STEWART AVE } \\
\text { PINE HOLLOW RO } \\
\text { JEROME AVENUE } \\
\text { JERICHO TPKE }\end{array}$ & $\begin{array}{l}\text { OLD MOTOR PKWY. } \\
\text { ROMSCHO ST } \\
\text { SPLIT ROCK RD } \\
\text { GLEN COVE ROAD } \\
\text { HALSEY AVE }\end{array}$ & $\begin{array}{l}4045620733903 \\
4 \varnothing 44 \varnothing 0 \varnothing 732857 \\
4 \varnothing 51200733204 \\
4 \varnothing 4504 \emptyset 733712 \\
4047260733225\end{array}$ & $\begin{array}{l}660802 \\
541220 \\
630228 \\
37 \\
5407\end{array}$ & $\begin{array}{r}50 \\
92 \\
98 \\
52 \\
105\end{array}$ & $\begin{array}{l}120000 \\
1186000 \\
- \\
-\end{array}$ & $\begin{array}{l}137300 \\
1634300 \\
- \\
-\end{array}$ \\
\hline $\begin{array}{l}N \\
N \\
N \\
N \\
N\end{array}$ & $\begin{array}{l}139 \\
140 \\
141 \\
142 \\
143\end{array}$ & $\begin{array}{l}\text { CHERRY LANE } \\
\text { MASON ST } \\
\text { TULIP AVE } \\
\text { WHITE OAK TREE R } \\
\text { GEORGE STREET }\end{array}$ & $\begin{array}{l}\text { ATLANTIC AVENUE } \\
\text { TENNYSON AVE } \\
\text { JERICHO TPKE } \\
\text { N.HEMPSTEAD TPK. } \\
\text { GUINEA WOODS RD }\end{array}$ & $\begin{array}{l}4 \varnothing 44508733637 \\
4 \varnothing 4504 \varnothing 733427 \\
4 \varnothing 4337 \varnothing 734236 \\
4 \varnothing 5050 \varnothing 732908 \\
4 \varnothing 472107337 \varnothing 3\end{array}$ & $\begin{array}{l}441128 \\
670320 \\
460830 \\
640423 \\
5708\end{array}$ & $\begin{array}{r}52 \\
89 \\
89 \\
107 \\
118\end{array}$ & $\begin{array}{l}\overline{100000} \\
\overline{430000} \\
-\end{array}$ & $\begin{array}{l}\overline{138000} \\
\overline{438000} \\
-\end{array}$ \\
\hline $\begin{array}{l}N \\
N \\
N \\
N \\
N\end{array}$ & $\begin{array}{l}144 \\
145 \\
146 \\
147 \\
148\end{array}$ & $\begin{array}{l}\text { BRUSH HOLLOW RD } \\
\text { OLD COUNTRY RD } \\
\text { JERUSALEM AVE } \\
\text { UNION AVENUE } \\
\text { LINDA RD }\end{array}$ & $\begin{array}{l}\text { NORTHERN ST. PKY } \\
\text { CENTRAL PARK RD } \\
\text { WASHINGTON AVE } \\
\text { WANTAGH ST. PKWY } \\
\text { CARMANS RD }\end{array}$ & $\begin{array}{l}404623 \emptyset 733345 \\
4 \emptyset 4633 \emptyset 73284 \emptyset \\
404124 \emptyset 732916 \\
4 \emptyset 4606 \emptyset 733351 \\
404155 \emptyset 732607\end{array}$ & $\begin{array}{l}531230 \\
5501 \\
6508 \\
471103 \\
560528\end{array}$ & $\begin{array}{r}89 \\
122 \\
38 \\
70 \\
110\end{array}$ & $\begin{array}{l}87300 \\
- \\
- \\
\overline{690000}\end{array}$ & $\begin{array}{l}88700 \\
282000 \\
- \\
- \\
72500\end{array}$ \\
\hline
\end{tabular}


N.Y. [Dash indicates no data. Altitudes are in feet above sea level.] (cont.)

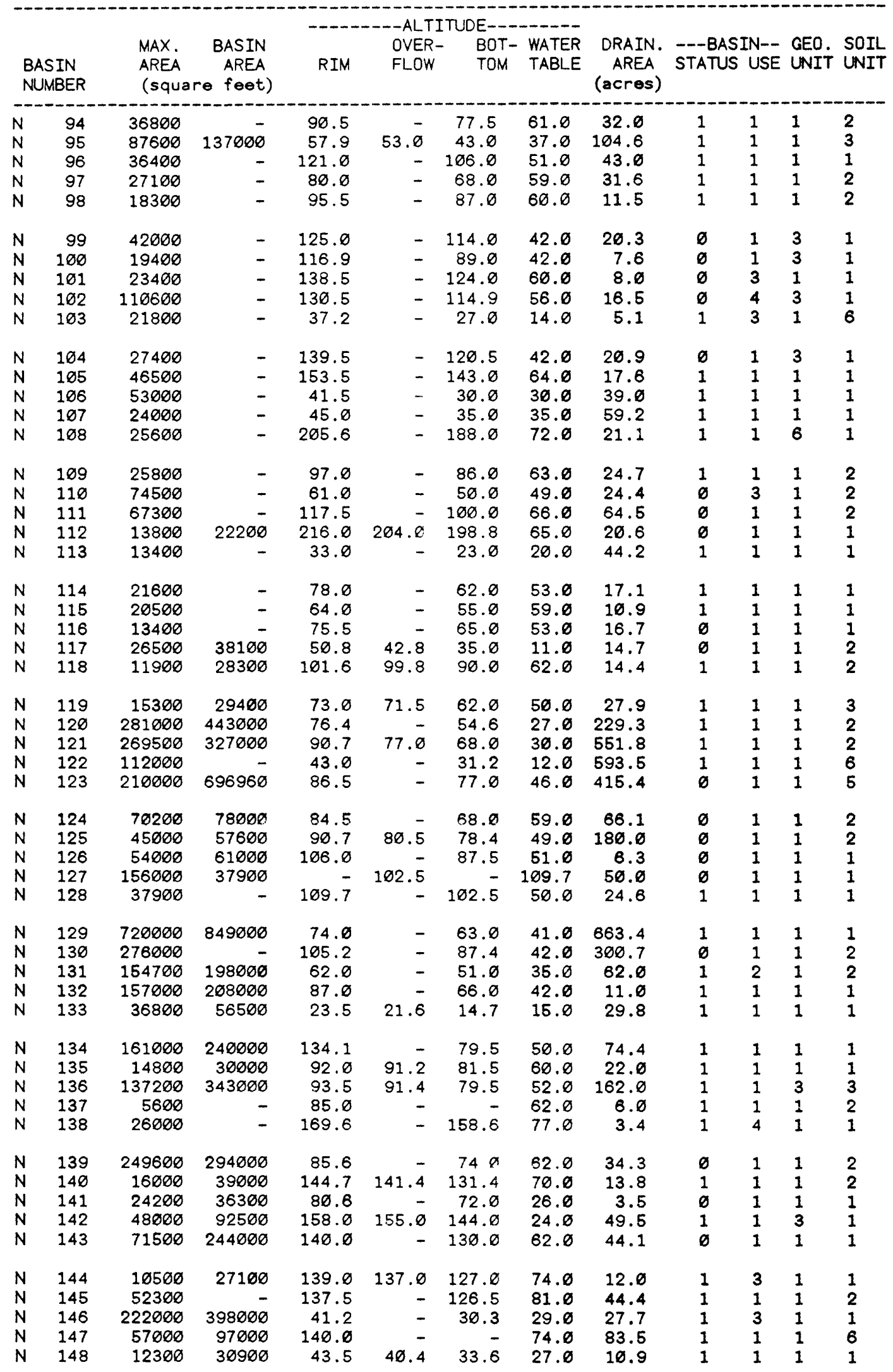


Appendix.--Location and basic physical features of recharge basins on Long Island,

\begin{tabular}{|c|c|c|c|c|c|c|c|c|}
\hline \multicolumn{2}{|c|}{$\begin{array}{l}\text { BASIN } \\
\text { NUMBER }\end{array}$} & \multicolumn{2}{|c|}{ NEAREST INTERSECTION } & $\begin{array}{l}\text { LATITUDE } \\
\text { LONGITUDE } \\
\circ, " 0^{\prime}, "\end{array}$ & $\begin{array}{l}\text { DATE } \\
\text { BUILT } \\
\text { YrMOD }\end{array}$ & COMMUNITY & \multicolumn{2}{|c|}{$\begin{array}{l}\text { DESIGN ACTUAL } \\
\text { CAPACITY CAPACITY } \\
\text { (cubic feet) }\end{array}$} \\
\hline $\begin{array}{l}N \\
N \\
N \\
N \\
N\end{array}$ & $\begin{array}{l}149 \\
150 \\
151 \\
152 \\
153\end{array}$ & $\begin{array}{l}\text { CYPRESS RD } \\
\text { POWELLS LANE } \\
\text { ROSLYN RD } \\
\text { VERNON STREET } \\
\text { PLUM TREE ROAD }\end{array}$ & $\begin{array}{l}\text { ELM ST } \\
\text { JERICHO TURNPIKE } \\
\text { HILLDALE RD } \\
\text { ISLAND STREET } \\
\text { STEWART AVENUE }\end{array}$ & $\begin{array}{l}4 \emptyset 4916 \varnothing 732744 \\
4 \emptyset 4619 \emptyset 733428 \\
4 \emptyset 4614 \varnothing 733816 \\
4 \varnothing 4616 \varnothing 732952 \\
4 \varnothing 4434 \varnothing 7334 \varnothing 5\end{array}$ & $\begin{array}{l}560711 \\
521103 \\
5110 \\
511231 \\
54 \varnothing 52 \varnothing\end{array}$ & $\begin{array}{r}128 \\
89 \\
50 \\
122 \\
42\end{array}$ & $\begin{array}{l}292000 \\
113000 \\
- \\
- \\
2000000\end{array}$ & $\begin{array}{r}127500 \\
166000 \\
29380 \\
10800 \varnothing \\
1897 \varnothing \varnothing \varnothing\end{array}$ \\
\hline $\begin{array}{l}N \\
N \\
N \\
N \\
N\end{array}$ & $\begin{array}{l}154 \\
155 \\
156 \\
157 \\
158\end{array}$ & $\begin{array}{l}\text { CEDAR LANE } \\
\text { PETERS AVE. } \\
\text { NORTH DRIVE } \\
\text { CORNELL AVE. } \\
\text { HILLSIDE AVE. }\end{array}$ & $\begin{array}{l}\text { APPLE LANE } \\
\text { RUGBY RD. } \\
\text { PARKWAY DRIVE } \\
\text { WILOWOOD AVE. } \\
\text { NORTHERN ST. PKY }\end{array}$ & $\begin{array}{l}4 \varnothing 4237 \varnothing 733414 \\
4 \varnothing 4306 \varnothing 733431 \\
4 \varnothing 4532 \varnothing 733628 \\
4 \varnothing 4104 \varnothing 733958 \\
4 \varnothing 4534 \varnothing 733727\end{array}$ & $\begin{array}{l}560810 \\
551017 \\
5505 \\
5408 \\
6109\end{array}$ & $\begin{array}{r}8 \\
8 \\
89 \\
29 \\
54\end{array}$ & $\begin{array}{l}848080 \\
- \\
- \\
- \\
-\end{array}$ & $\begin{array}{l}2528 \varnothing \varnothing \\
- \\
1828 \varnothing \varnothing \\
2228 \varnothing \varnothing \\
1678 \varnothing \varnothing\end{array}$ \\
\hline $\begin{array}{l}N \\
N \\
N \\
N \\
N\end{array}$ & $\begin{array}{l}169 \\
160 \\
161 \\
162 \\
163\end{array}$ & $\begin{array}{l}\text { BLOOMINGDALE AVE } \\
\text { JERICHO TPKE. } \\
\text { REDWOOD LA. } \\
\text { TWISTING LA. } \\
\text { CENTRAL DR. S. }\end{array}$ & $\begin{array}{l}\text { BROADWAY } \\
\text { DEWEY ST. } \\
\text { SEAMANS NECK RD. } \\
\text { SEAMANS NECK RD. } \\
\text { NEWBRIDGE AVE. }\end{array}$ & $\begin{array}{l}4 \varnothing 4503 \emptyset 733 \varnothing 21 \\
4 \varnothing 4713 \varnothing 733238 \\
4 \varnothing 4225 \varnothing 732939 \\
4 \varnothing 4146 \emptyset 732945 \\
4 \varnothing 4216 \varnothing 733256\end{array}$ & $\begin{array}{l}540927 \\
521118 \\
5205 \\
5205 \\
5210\end{array}$ & $\begin{array}{r}104 \\
105 \\
25 \\
46 \\
8\end{array}$ & $\begin{array}{l}\overline{172000} \\
- \\
- \\
-\end{array}$ & $\begin{array}{l}133000 \\
156000 \\
479000 \\
20200 \varnothing \\
66600\end{array}$ \\
\hline $\begin{array}{l}\mathbf{N} \\
\mathbf{N} \\
\mathbf{N} \\
\mathbf{N} \\
\mathbf{N}\end{array}$ & $\begin{array}{l}164 \\
165 \\
166 \\
167 \\
168\end{array}$ & $\begin{array}{l}\text { N.HEMPSTEAD TPK. } \\
\text { SOUTHERN ST. PKY } \\
\text { LINCOLN RD. } \\
\text { ZECKENDORF BLVD. } \\
\text { MINEOLA AVENUE }\end{array}$ & $\begin{array}{l}\text { DAVID CT. } \\
\text { WANTAGH ST. PKWY } \\
\text { OLD COUNTRY RD. } \\
\text { MEADOWBROOK PKWY } \\
\text { CHERRY LANE }\end{array}$ & $\begin{array}{l}4 \emptyset 4333 \emptyset 733247 \\
4 \emptyset 4138 \emptyset 7331 \varnothing 9 \\
4 \varnothing 46 \varnothing 6 \varnothing 7329 \varnothing 9 \\
4 \emptyset 44 \varnothing 8 \varnothing 73363 \emptyset \\
4 \varnothing 4452 \varnothing 733642\end{array}$ & $\begin{array}{l}5211 \\
5207 \\
540914 \\
550413 \\
560904\end{array}$ & $\begin{array}{r}8 \\
46 \\
122 \\
14 \\
52\end{array}$ & $\begin{array}{l}- \\
\overline{246800} \\
- \\
-\end{array}$ & $\begin{array}{l}107600 \\
186000 \\
156000 \\
- \\
-\end{array}$ \\
\hline $\begin{array}{l}N \\
N \\
N \\
N \\
N\end{array}$ & $\begin{array}{l}169 \\
170 \\
171 \\
172 \\
173\end{array}$ & $\begin{array}{l}\text { EILEEN AVE. } \\
\text { WILLETS RD. } \\
\text { HICKSVILLE RD. } \\
\text { JERUSALEM AVE. } \\
\text { JERUSALEM AVE. }\end{array}$ & $\begin{array}{l}\text { JANET AVE. } \\
\text { DUCK POND RD. } \\
\text { MICHIGAN DR. } \\
\text { RAY PL. } \\
\text { WANTAGH AVE. }\end{array}$ & $\begin{array}{l}4 \varnothing 45590732949 \\
4 \emptyset 5137 \varnothing 73361 \emptyset \\
4 \varnothing 4455 \emptyset 733025 \\
4 \emptyset 4144 \varnothing 733155 \\
4 \varnothing 4132 \varnothing 732949\end{array}$ & $\begin{array}{l}5211 \\
5311 \\
5310 \\
6303 \varnothing 5 \\
5211\end{array}$ & $\begin{array}{r}92 \\
100 \\
92 \\
32 \\
46\end{array}$ & $\begin{array}{l}- \\
- \\
\overline{176000} \\
-\end{array}$ & $\begin{array}{r}71600 \\
410000 \\
128800 \\
40800 \\
202000\end{array}$ \\
\hline $\begin{array}{l}N \\
N \\
N \\
N \\
N\end{array}$ & $\begin{array}{l}174 \\
175 \\
178 \\
177 \\
178\end{array}$ & $\begin{array}{l}\text { ELWOOD AVE. } \\
\text { SYLVIA DR. } \\
\text { WEST ST. } \\
\text { S. OYSTER BAY RD } \\
\text { NEW SOUTH RD. }\end{array}$ & $\begin{array}{l}\text { CHERRY ST. } \\
\text { NORMAN DR. } \\
\text { EMMETT ST. } \\
\text { JERICHO TPKE. } \\
\text { OLD COUNTRY RD. }\end{array}$ & $\begin{array}{l}4044590733129 \\
4042100733205 \\
4045170734011 \\
4048190733007 \\
4045570733031\end{array}$ & $\begin{array}{l}540621 \\
530713 \\
541011 \\
540219 \\
490711\end{array}$ & $\begin{array}{r}104 \\
32 \\
62 \\
108 \\
104\end{array}$ & $\begin{array}{l}45600 \varnothing \\
98500 \\
313000 \\
33500 \varnothing \\
-\end{array}$ & $\begin{array}{l}494000 \\
- \\
239800 \\
339500 \\
-\end{array}$ \\
\hline $\begin{array}{l}N \\
N \\
N \\
N \\
N\end{array}$ & $\begin{array}{l}179 \\
180 \\
181 \\
182 \\
183\end{array}$ & $\begin{array}{l}\text { CYNTHIA DR. } \\
\text { MEADOW COURT } \\
\text { COLD SPRING ROAD } \\
\text { HICKSVILLE ROAD } \\
\text { GUINEA WOODS RD. }\end{array}$ & $\begin{array}{l}\text { CHALADAY LA. } \\
\text { SOUTH MEADOW RD. } \\
\text { WOODVALE DRIVE } \\
\text { N. RIDGE GATE } \\
\text { WOODBINE RD. }\end{array}$ & $\begin{array}{l}4 \emptyset 4206 \emptyset 733329 \\
4 \varnothing 4126 \emptyset 73336 \emptyset \\
4 \varnothing 5 \varnothing 33 \emptyset 732849 \\
4 \varnothing 4149 \emptyset 732852 \\
4 \varnothing 4731 \varnothing 733719\end{array}$ & $\begin{array}{l}5305 \\
5307 \\
638619 \\
5310 \\
5308\end{array}$ & $\begin{array}{r}8 \\
33 \\
107 \\
110 \\
53\end{array}$ & $\begin{array}{l}- \\
\overline{432080} \\
- \\
-\end{array}$ & $\begin{array}{r}129500 \\
57200 \\
430000 \\
112000 \\
36300\end{array}$ \\
\hline $\begin{array}{l}N \\
N \\
N \\
N \\
N\end{array}$ & $\begin{array}{l}184 \\
185 \\
186 \\
187 \\
188\end{array}$ & $\begin{array}{l}\text { GUINEA WOODS RD. } \\
\text { HICKSVILLE RD. } \\
\text { FENIMORE PLACE } \\
\text { SOUTHERN ST. PKY } \\
\text { SOUTHERN ST. PKY }\end{array}$ & $\begin{array}{l}\text { HARBOR HILL RD. } \\
\text { WICKS AVENUE } \\
\text { SCHOOL DR. } \\
\text { OAKFIELD AVE. } \\
\text { SEAMANS NECK RD. }\end{array}$ & $\begin{array}{l}4 \varnothing 4738073372 \varnothing \\
4 \varnothing 4151 \varnothing 732858 \\
4 \varnothing 4 \varnothing 53 \varnothing 733632 \\
4 \varnothing 41590733157 \\
4 \varnothing 42 \varnothing 1 \varnothing 732937\end{array}$ & $\begin{array}{l}5308 \\
5506 \\
5305 \\
5306 \\
5210\end{array}$ & $\begin{array}{r}53 \\
110 \\
2 \\
32 \\
46\end{array}$ & $\begin{array}{l}\overline{-} \\
\overline{-}\end{array}$ & $\begin{array}{l}37300 \\
23200 \\
412000 \\
283000 \\
-\end{array}$ \\
\hline $\begin{array}{l}N \\
N \\
N \\
N \\
N\end{array}$ & $\begin{array}{l}189 \\
190 \\
191 \\
192 \\
193\end{array}$ & $\begin{array}{l}\text { HEMPSTEAD TPKE. } \\
\text { WILLIS AVE. } \\
\text { POLARIS DRIVE } \\
\text { N.HEMPSTEAD TPK. } \\
\text { ELLISON AVE. }\end{array}$ & $\begin{array}{l}\text { GARDINERS AVE. } \\
\text { YALE ST. } \\
\text { BLOOMINGDALE RD. } \\
\text { WHITNEY LANE } \\
\text { NORTHERN ST. PKY }\end{array}$ & $\begin{array}{l}4043230733107 \\
4 \varnothing 4634 \varnothing 733858 \\
4044110733035 \\
404907 \varnothing 7336 \varnothing 5 \\
404537 \varnothing 7336 \varnothing 3\end{array}$ & $\begin{array}{l}5501 \\
510512 \\
490321 \\
531014 \\
650111\end{array}$ & $\begin{array}{l}25 \\
50 \\
25 \\
93 \\
89\end{array}$ & $\begin{array}{l}- \\
- \\
\overline{152000}\end{array}$ & $\begin{array}{l}1488000 \\
- \\
- \\
187000 \\
151000\end{array}$ \\
\hline $\begin{array}{l}N \\
N \\
N \\
N \\
N\end{array}$ & $\begin{array}{l}194 \\
195 \\
196 \\
197 \\
198\end{array}$ & $\begin{array}{l}\text { HICKSVILLE RD. } \\
\text { N.HEMPSTEAD TPK. } \\
\text { MASS. -HICKSVILLE } \\
\text { N.WISCONSIN AVE. } \\
\text { SOUTHERN ST. PKY }\end{array}$ & $\begin{array}{l}\text { BRIARWOOD RD. } \\
\text { PINE HOLLOW RD. } \\
\text { SOUTHERN ST. PKY } \\
\text { AMHERST DRIVE } \\
\text { CARMANS RD. }\end{array}$ & 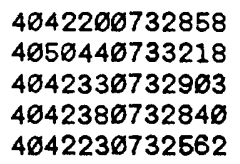 & $\begin{array}{l}521087 \\
5310 \\
530821 \\
5402 \\
5410\end{array}$ & $\begin{array}{r}110 \\
98 \\
121 \\
110 \\
125\end{array}$ & $\begin{array}{l}151800 \\
- \\
231080 \\
- \\
-\end{array}$ & $\begin{array}{r}145500 \\
298880 \\
144980 \\
198000 \\
96680\end{array}$ \\
\hline $\begin{array}{l}N \\
N \\
N \\
N \\
N\end{array}$ & $\begin{array}{l}199 \\
2 ø \varnothing \\
2 ø 1 \\
2 ø 2 \\
2 ø 3\end{array}$ & $\begin{array}{l}\text { CENTRAL AVE. } \\
\text { POLO ROAD } \\
\text { ALOEN DR. } \\
\text { CHERRYWOOD PLACE } \\
\text { BRIARWOOD RD. }\end{array}$ & $\begin{array}{l}\text { WELLS RD. } \\
\text { ARRANDALE AVENUE } \\
\text { CIRCLE DR. } \\
\text { BEECHWOOD PLACE } \\
\text { HICKSVILLE RD. }\end{array}$ & $\begin{array}{l}4 \emptyset 4 \varnothing 5 \varnothing \emptyset 734231 \\
4 \varnothing 482 \varnothing \emptyset 734441 \\
4 \varnothing 4717 \varnothing 733 \varnothing 36 \\
4 \varnothing 4 \varnothing 58 \varnothing 73293 \emptyset \\
4 \varnothing 4222 \varnothing 73283 \emptyset\end{array}$ & $\begin{array}{l}541108 \\
520421 \\
530730 \\
5305 \\
54 \varnothing 8\end{array}$ & $\begin{array}{r}34 \\
140 \\
104 \\
38 \\
110\end{array}$ & $\begin{array}{l}- \\
\overline{-} \\
-\end{array}$ & $\begin{array}{l}- \\
- \\
57000 \\
122000 \\
188000\end{array}$ \\
\hline
\end{tabular}


N.Y. [Dash indicates no data. Altitudes are in foet above sea level.] (cont.)

\begin{tabular}{|c|c|c|c|c|c|c|c|c|c|c|c|c|}
\hline \multicolumn{2}{|c|}{$\begin{array}{l}\text { BASIN } \\
\text { NUMBER }\end{array}$} & $\begin{array}{l}\text { MAX. } \\
\text { AREA } \\
\text { (square }\end{array}$ & $\begin{array}{c}\text { BASIN } \\
\text { AREA } \\
\text { feet) }\end{array}$ & RIM & $\begin{array}{l}\text { OVER- } \\
\text { FLOW }\end{array}$ & $\begin{array}{l}\text { DE-- } \\
\text { BOT- } \\
\text { TOM }\end{array}$ & $\begin{array}{l}\text { WATER } \\
\text { TABLE }\end{array}$ & $\begin{array}{c}\text { DRAIN. } \\
\text { AREA } \\
\text { (acres) }\end{array}$ & \multicolumn{2}{|c|}{$\begin{array}{l}-- \text { BASIN-- } \\
\text { STATUS USE }\end{array}$} & $\begin{array}{l}\text { GEO. } \\
\text { UNIT }\end{array}$ & $\begin{array}{l}\text { SOIL } \\
\text { UNIT }\end{array}$ \\
\hline $\begin{array}{l}N \\
N \\
N \\
N \\
N\end{array}$ & $\begin{array}{l}149 \\
150 \\
151 \\
152 \\
153\end{array}$ & $\begin{array}{r}16000 \\
22200 \\
17500 \\
21000 \\
189700\end{array}$ & $\begin{array}{r}81800 \\
32100 \\
- \\
336700\end{array}$ & $\begin{array}{r}199.0 \\
135.5 \\
116.2 \\
151.1 \\
87.5\end{array}$ & $\begin{array}{r}188.0 \\
134.5 \\
- \\
86 . \varnothing\end{array}$ & $\begin{array}{r}180.0 \\
126.0 \\
104.6 \\
144.0 \\
76.0\end{array}$ & $\begin{array}{l}33.0 \\
73.0 \\
54.0 \\
79.0 \\
66.0\end{array}$ & $\begin{array}{r}33.2 \\
20.5 \\
4.6 \\
17.8 \\
250.0\end{array}$ & $\begin{array}{l}0 \\
1 \\
0 \\
1 \\
1\end{array}$ & $\begin{array}{l}1 \\
3 \\
1 \\
1 \\
1\end{array}$ & $\begin{array}{l}6 \\
1 \\
1 \\
1 \\
1\end{array}$ & $\begin{array}{l}1 \\
1 \\
1 \\
2 \\
2\end{array}$ \\
\hline $\begin{array}{l}N \\
N \\
N \\
N \\
N\end{array}$ & $\begin{array}{l}154 \\
155 \\
156 \\
157 \\
158\end{array}$ & $\begin{array}{c}49300 \\
- \\
36600 \\
47400 \\
29600\end{array}$ & $\begin{array}{r}76900 \\
21600 \\
- \\
-\end{array}$ & $\begin{array}{r}63.0 \\
75.0 \\
115.0 \\
44.8 \\
94.0\end{array}$ & $\begin{array}{r}55.0 \\
- \\
- \\
-\end{array}$ & $\begin{array}{r}51.0 \\
9 . \overline{0} \\
34.0 \\
80.0\end{array}$ & $\begin{array}{l}45.0 \\
50.0 \\
66.0 \\
20.0 \\
60.0\end{array}$ & $\begin{array}{r}114.0 \\
53.5 \\
28.7 \\
34.9 \\
26.3\end{array}$ & $\begin{array}{l}1 \\
1 \\
1 \\
1 \\
1\end{array}$ & $\begin{array}{l}1 \\
1 \\
1 \\
1 \\
1\end{array}$ & $\begin{array}{l}1 \\
1 \\
1 \\
1 \\
1\end{array}$ & $\begin{array}{l}1 \\
2 \\
2 \\
1 \\
2\end{array}$ \\
\hline $\begin{array}{l}N \\
N \\
N \\
N \\
N\end{array}$ & $\begin{array}{l}159 \\
160 \\
161 \\
162 \\
163\end{array}$ & $\begin{array}{r}13400 \\
19100 \\
123800 \\
41400 \\
24100\end{array}$ & $\begin{array}{r}21600 \\
38000 \\
- \\
-\end{array}$ & $\begin{array}{r}116.0 \\
170.6 \\
55.0 \\
45.0 \\
58.0\end{array}$ & $\begin{array}{r}116.0 \\
167.5 \\
- \\
- \\
-\end{array}$ & $\begin{array}{r}185.0 \\
158.6 \\
44.0 \\
35.0 \\
46.8\end{array}$ & $\begin{array}{l}70.0 \\
77.0 \\
41.0 \\
33.0 \\
48.0\end{array}$ & $\begin{array}{l}20.9 \\
41.8 \\
75.4 \\
31.8 \\
10.5\end{array}$ & $\begin{array}{l}1 \\
0 \\
1 \\
1 \\
1\end{array}$ & $\begin{array}{l}1 \\
1 \\
1 \\
1 \\
1\end{array}$ & $\begin{array}{l}1 \\
1 \\
1 \\
1 \\
1\end{array}$ & $\begin{array}{l}2 \\
1 \\
2 \\
1 \\
6\end{array}$ \\
\hline $\begin{array}{l}N \\
N \\
N \\
N \\
N\end{array}$ & $\begin{array}{l}164 \\
165 \\
166 \\
167 \\
168\end{array}$ & $\begin{array}{c}22700 \\
46400 \\
15100 \\
26 \varnothing 0 \varnothing \varnothing \\
-\end{array}$ & $\begin{array}{r}- \\
- \\
38500 \\
338080 \\
38000\end{array}$ & $\begin{array}{r}90.4 \\
4 \varnothing .8 \\
145.7 \\
82.0 \\
95 . \varnothing\end{array}$ & $\begin{array}{r}- \\
- \\
144.1 \\
- \\
-\end{array}$ & $\begin{array}{r}78.0 \\
33.5 \\
132.0 \\
68.5 \\
85.0\end{array}$ & $\begin{array}{l}59.0 \\
37.0 \\
79.0 \\
58.0 \\
62.0\end{array}$ & $\begin{array}{l}16.9 \\
11.4 \\
34.0 \\
57.8 \\
43.7\end{array}$ & $\begin{array}{l}1 \\
1 \\
1 \\
0 \\
1\end{array}$ & $\begin{array}{l}1 \\
3 \\
1 \\
2 \\
1\end{array}$ & $\begin{array}{l}1 \\
1 \\
1 \\
1 \\
1\end{array}$ & $\begin{array}{l}2 \\
1 \\
2 \\
2 \\
2\end{array}$ \\
\hline $\begin{array}{l}N \\
N \\
N \\
N \\
N\end{array}$ & $\begin{array}{l}169 \\
170 \\
171 \\
172 \\
173\end{array}$ & $\begin{array}{r}30400 \\
51500 \\
28800 \\
7800 \\
43700\end{array}$ & $\begin{array}{r}- \\
- \\
- \\
16800 \\
-\end{array}$ & $\begin{array}{r}146.2 \\
140.8 \\
117.8 \\
47.3 \\
42.5\end{array}$ & $\begin{array}{r}- \\
- \\
45.4 \\
-\end{array}$ & $\begin{array}{r}132.0 \\
125.5 \\
186.0 \\
39.0 \\
34.0\end{array}$ & $\begin{array}{l}79.0 \\
56.0 \\
70.0 \\
34.0 \\
31.0\end{array}$ & $\begin{array}{l}11.3 \\
25.1 \\
28.3 \\
27.2 \\
31.8\end{array}$ & $\begin{array}{l}1 \\
0 \\
1 \\
1 \\
1\end{array}$ & $\begin{array}{l}1 \\
4 \\
1 \\
1 \\
1\end{array}$ & $\begin{array}{l}1 \\
3 \\
1 \\
1 \\
1\end{array}$ & $\begin{array}{l}2 \\
1 \\
2 \\
3 \\
1\end{array}$ \\
\hline $\begin{array}{l}N \\
N \\
N \\
N \\
N\end{array}$ & $\begin{array}{l}174 \\
175 \\
176 \\
177 \\
178\end{array}$ & $\begin{array}{c}70200 \\
- \\
29200 \\
36000 \\
248000\end{array}$ & $\begin{array}{r}83000 \\
19700 \\
51000 \\
56 \varnothing 0 \varnothing \\
27400 \varnothing\end{array}$ & $\begin{array}{r}118.2 \\
55.0 \\
107.5 \\
193.0 \\
145.0\end{array}$ & $\begin{array}{r}117.8 \\
- \\
105.0 \\
192.9 \\
-\end{array}$ & $\begin{array}{r}189.5 \\
- \\
95.5 \\
181.5 \\
120.0\end{array}$ & $\begin{array}{l}71.0 \\
40.0 \\
40.0 \\
75.0 \\
77.0\end{array}$ & $\begin{array}{r}21.4 \\
22.4 \\
107.6 \\
61.2 \\
29.3\end{array}$ & $\begin{array}{l}1 \\
1 \\
0 \\
1 \\
0\end{array}$ & $\begin{array}{l}1 \\
1 \\
1 \\
1 \\
1\end{array}$ & $\begin{array}{l}1 \\
1 \\
1 \\
1 \\
1\end{array}$ & $\begin{array}{l}2 \\
3 \\
1 \\
2 \\
2\end{array}$ \\
\hline $\begin{array}{l}N \\
N \\
N \\
N \\
N\end{array}$ & $\begin{array}{l}179 \\
180 \\
181 \\
182 \\
183\end{array}$ & $\begin{array}{l}28800 \\
15500 \\
48100 \\
27000 \\
15900\end{array}$ & $\begin{array}{r}- \\
- \\
80800 \\
-\end{array}$ & $\begin{array}{r}59.7 \\
40.0 \\
231.4 \\
45.0 \\
147.2\end{array}$ & $\begin{array}{r}- \\
- \\
229.6 \\
- \\
-\end{array}$ & $\begin{array}{r}48.0 \\
37.0 \\
219.6 \\
36.0 \\
125.0\end{array}$ & $\begin{array}{l}38.0 \\
29 . \varnothing \\
56.0 \\
33 . \varnothing \\
61.0\end{array}$ & $\begin{array}{r}28.4 \\
3.5 \\
52.8 \\
17.6 \\
5.7\end{array}$ & $\begin{array}{l}0 \\
1 \\
1 \\
1 \\
1\end{array}$ & $\begin{array}{l}1 \\
3 \\
1 \\
1 \\
1\end{array}$ & $\begin{array}{l}1 \\
1 \\
1 \\
1 \\
1\end{array}$ & $\begin{array}{l}6 \\
3 \\
1 \\
3 \\
1\end{array}$ \\
\hline $\begin{array}{l}N \\
N \\
N \\
N \\
N\end{array}$ & $\begin{array}{l}184 \\
185 \\
186 \\
187 \\
188\end{array}$ & $\begin{array}{l}12800 \\
23700 \\
78000 \\
12100 \\
38100\end{array}$ & $\begin{array}{l}- \\
- \\
- \\
-\end{array}$ & $\begin{array}{r}169.0 \\
42.0 \\
45.0 \\
52.8 \\
47.0\end{array}$ & $\begin{array}{l}- \\
- \\
- \\
-\end{array}$ & $\begin{array}{r}157.0 \\
33.0 \\
32.0 \\
43.0 \\
35 . \varnothing\end{array}$ & $\begin{array}{l}61.0 \\
33.0 \\
25.0 \\
38.0 \\
38.0\end{array}$ & $\begin{array}{r}5.9 \\
3.7 \\
64.9 \\
44.5 \\
8.8\end{array}$ & $\begin{array}{l}1 \\
1 \\
1 \\
\varnothing \\
1\end{array}$ & $\begin{array}{l}1 \\
1 \\
1 \\
1 \\
3\end{array}$ & $\begin{array}{l}1 \\
1 \\
1 \\
1 \\
1\end{array}$ & $\begin{array}{l}1 \\
3 \\
1 \\
3 \\
1\end{array}$ \\
\hline $\begin{array}{l}N \\
N \\
N \\
N \\
N\end{array}$ & $\begin{array}{l}189 \\
190 \\
191 \\
192 \\
193\end{array}$ & $\begin{array}{r}2698 \varnothing \emptyset \\
216 \varnothing \varnothing \varnothing \\
975 \varnothing \emptyset \\
375 \varnothing \emptyset \\
171 \varnothing \emptyset\end{array}$ & $\begin{array}{r}- \\
266000 \\
166500 \\
3480 \varnothing\end{array}$ & $\begin{array}{r}82.5 \\
96.0 \\
96.7 \\
158.0 \\
123.0\end{array}$ & $\begin{array}{r}- \\
- \\
122.0\end{array}$ & $\begin{array}{r}67.5 \\
80.0 \\
79.7 \\
146.0 \\
112.0\end{array}$ & $\begin{array}{l}55.8 \\
49.0 \\
50.0 \\
65.0 \\
58.0\end{array}$ & $\begin{array}{r}234.2 \\
4.6 \\
11.8 \\
29.4 \\
21.8\end{array}$ & $\begin{array}{l}1 \\
0 \\
1 \\
1 \\
1\end{array}$ & $\begin{array}{l}1 \\
1 \\
1 \\
1 \\
1\end{array}$ & $\begin{array}{l}1 \\
1 \\
1 \\
3 \\
1\end{array}$ & $\begin{array}{l}1 \\
1 \\
1 \\
1 \\
2\end{array}$ \\
\hline $\begin{array}{l}N \\
N \\
N \\
N \\
N\end{array}$ & $\begin{array}{l}194 \\
195 \\
196 \\
197 \\
198\end{array}$ & $\begin{array}{r}17200 \\
472 \varnothing \emptyset \\
1850 \emptyset \\
424 \varnothing \emptyset \\
94 \varnothing \emptyset\end{array}$ & $\begin{array}{r}28600 \\
- \\
36700 \\
- \\
-\end{array}$ & $\begin{array}{r}59.0 \\
189.0 \\
57.3 \\
63.0 \\
49.0\end{array}$ & $\begin{array}{r}57.4 \\
- \\
55.9 \\
- \\
-\end{array}$ & $\begin{array}{r}47.5 \\
170.0 \\
47.0 \\
51.5 \\
38.0\end{array}$ & $\begin{array}{l}39 . \varnothing \\
56 . \varnothing \\
35.0 \\
40 . \varnothing \\
34 . \varnothing\end{array}$ & $\begin{array}{l}20.6 \\
45.7 \\
42.0 \\
31.2 \\
15.2\end{array}$ & $\begin{array}{l}1 \\
1 \\
1 \\
1 \\
1\end{array}$ & $\begin{array}{l}1 \\
1 \\
1 \\
1 \\
1\end{array}$ & $\begin{array}{l}1 \\
3 \\
1 \\
1 \\
1\end{array}$ & $\begin{array}{l}3 \\
1 \\
1 \\
1 \\
1\end{array}$ \\
\hline $\begin{array}{l}N \\
N \\
N \\
N \\
N\end{array}$ & $\begin{array}{l}199 \\
280 \\
281 \\
282 \\
203\end{array}$ & $\begin{array}{c}\overline{-} \\
229 \varnothing 0 \\
421 \varnothing 0 \\
383 \varnothing 0\end{array}$ & $\begin{array}{r}22500 \\
57600 \\
38100 \\
- \\
-\end{array}$ & $\begin{array}{r}37.0 \\
55.0 \\
182.1 \\
31.2 \\
59.2\end{array}$ & $\begin{array}{r}\overline{-} \\
172 . \overline{0} \\
-\end{array}$ & $\begin{array}{r}\overline{-} \\
170 . \overline{0} \\
23.0 \\
47.4\end{array}$ & $\begin{array}{l}13.0 \\
13.0 \\
81.0 \\
23.0 \\
39.0\end{array}$ & $\begin{array}{r}6.9 \\
14.7 \\
9.8 \\
19.2 \\
29.6\end{array}$ & $\begin{array}{l}1 \\
1 \\
1 \\
1 \\
1\end{array}$ & $\begin{array}{l}1 \\
1 \\
1 \\
1 \\
1\end{array}$ & $\begin{array}{l}1 \\
3 \\
1 \\
1 \\
1\end{array}$ & $\begin{array}{l}3 \\
1 \\
2 \\
1 \\
1\end{array}$ \\
\hline
\end{tabular}


Appendix.--Location and basic physical features of recharge basins on Long Island,

\begin{tabular}{|c|c|c|c|c|c|c|c|c|}
\hline \multicolumn{2}{|c|}{$\begin{array}{l}\text { BASIN } \\
\text { NUMBER }\end{array}$} & \multicolumn{2}{|c|}{ NEAREST INTERSECTION } & $\begin{array}{l}\text { LATITUDE } \\
\text { LONGITUDE } \\
\circ, " \circ, "\end{array}$ & $\begin{array}{l}\text { DATE } \\
\text { BUILT } \\
\text { YrMOD }\end{array}$ & COMMUNITY & $\begin{array}{l}\text { DESIGN } \\
\text { CAPACITY } \\
\text { (cubic } f\end{array}$ & $\begin{array}{l}\text { ACTUAL } \\
\text { CAPACITY } \\
\text { feөt) }\end{array}$ \\
\hline $\begin{array}{l}N \\
N \\
N \\
N \\
N\end{array}$ & $\begin{array}{l}204 \\
205 \\
206 \\
207 \\
2 ø 8\end{array}$ & $\begin{array}{l}\text { DOLPHIN DR, } \\
\text { GLEN COVE RO. } \\
\text { JAMES AVENUE } \\
\text { ROSEMARY DR. } \\
\text { WOOD AVENUE }\end{array}$ & $\begin{array}{l}\text { PLAINVIEW RD. } \\
\text { RAFF AVE. } \\
\text { ROSS LANE } \\
\text { BOSTON AVENUE } \\
\text { PINE STREET }\end{array}$ & $\begin{array}{l}4043460732756 \\
4 \varnothing 45130733711 \\
4 \varnothing 5 \varnothing 350733215 \\
4 \varnothing 4245 \varnothing 732834 \\
4 \varnothing 4126 \varnothing 732823\end{array}$ & $\begin{array}{l}531228 \\
5401 \\
5403 \\
540413 \\
540712\end{array}$ & $\begin{array}{r}99 \\
52 \\
98 \\
121 \\
110\end{array}$ & $\begin{array}{l}296800 \\
- \\
- \\
325000 \\
-\end{array}$ & $\begin{array}{r}40300 \\
124000 \\
138000 \\
350000 \\
-\end{array}$ \\
\hline $\begin{array}{l}N \\
N \\
N \\
N \\
N\end{array}$ & $\begin{array}{l}269 \\
210 \\
211 \\
212 \\
213\end{array}$ & $\begin{array}{l}\text { KNICKERBOCKER RD } \\
\text { SEAMANS NECK RD. } \\
\text { SEAMANS NECK RD. } \\
\text { HICKSVILLE RD. } \\
\text { EMERSON AVENUE }\end{array}$ & $\begin{array}{l}\text { BENTLEY RD. } \\
\text { KINGSBERRY RD. } \\
\text { CORDWOOD ST. } \\
\text { ARLINGTON DR. } \\
\text { MILTON AVENUE }\end{array}$ & $\begin{array}{l}4046500732951 \\
4 \varnothing 42480732928 \\
4 \varnothing 42310732918 \\
4 \varnothing 42160732966 \\
4 \varnothing 43160732929\end{array}$ & $\begin{array}{l}531102 \\
521211 \\
5411 \\
5411 \\
5310\end{array}$ & $\begin{array}{l}122 \\
121 \\
121 \\
110 \\
121\end{array}$ & $\begin{array}{l}481000 \\
226000 \\
- \\
- \\
-\end{array}$ & $\begin{array}{l}452500 \\
29400 \varnothing \\
2577 \varnothing 0 \\
29400 \varnothing \\
182800\end{array}$ \\
\hline $\begin{array}{l}N \\
N \\
N \\
N \\
N\end{array}$ & $\begin{array}{l}214 \\
215 \\
216 \\
217 \\
218\end{array}$ & $\begin{array}{l}\text { N. JERUSALEM RD. } \\
\text { TODD DRIVE EAST } \\
\text { JACKSON AVE. } \\
\text { JACKSON AVE. } \\
\text { ARBOR ROAD }\end{array}$ & $\begin{array}{l}\text { BRUCE DR. } \\
\text { TODD DRIVE NORTH } \\
\text { DEVINE AVE. } \\
\text { TEIBROOK AVENUE } \\
\text { BELMONT CIRCLE }\end{array}$ & $\begin{array}{l}4042090733322 \\
4 \varnothing 5 \varnothing 17 \varnothing 73374 \varnothing \\
4049130733015 \\
4049120733067 \\
4048110733024\end{array}$ & $\begin{array}{l}5402 \\
530109 \\
5203 \\
521187 \\
541105\end{array}$ & $\begin{array}{r}8 \\
181 \\
126 \\
126 \\
168\end{array}$ & $\begin{array}{l}- \\
- \\
- \\
97500 \\
76000\end{array}$ & $\begin{array}{l}92300 \\
147000 \\
- \\
67000 \\
91500\end{array}$ \\
\hline $\begin{array}{l}N \\
N \\
N \\
N \\
N\end{array}$ & $\begin{array}{l}219 \\
220 \\
221 \\
222 \\
223\end{array}$ & $\begin{array}{l}\text { GARY STREET } \\
\text { WHEATLEY RD. } \\
\text { VERNON STREET } \\
\text { SEAMANS NECK RD. } \\
\text { OLD COUNTRY RD. }\end{array}$ & $\begin{array}{l}\text { LINDA DRIVE } \\
\text { MORGAN DR. } \\
\text { ISLAND STREET } \\
\text { PETER STREET } \\
\text { FLORAL AVE. }\end{array}$ & $\begin{array}{l}4 \varnothing 4227 \varnothing 733354 \\
4 \varnothing 47390733532 \\
4 \varnothing 4619073295 \varnothing \\
4 \varnothing 4226 \varnothing 732924 \\
4 \varnothing 455 \varnothing \varnothing 732931\end{array}$ & $\begin{array}{l}521112 \\
650616 \\
5403 \\
5310 \\
5402\end{array}$ & $\begin{array}{r}8 \\
118 \\
122 \\
46 \\
122\end{array}$ & $\begin{array}{l}898080 \\
- \\
- \\
- \\
-\end{array}$ & $\begin{array}{l}117000 \\
352000 \\
262000 \\
101000 \\
621000\end{array}$ \\
\hline $\begin{array}{l}N \\
N \\
N \\
N \\
N\end{array}$ & $\begin{array}{l}224 \\
225 \\
226 \\
227 \\
228\end{array}$ & $\begin{array}{l}\text { SYRACUSE AVE. } \\
\text { WANTAGH AVE. } \\
\text { BLOOMINGDALE RD. } \\
\text { MELISSA PL. } \\
\text { GLENBROOK RD. }\end{array}$ & $\begin{array}{l}\text { WALTER AVE. } \\
\text { REGENT LA. } \\
\text { SCOOTER LA. } \\
\text { NEWBRIDGE RD. } \\
\text { NEWBRIDGE RD. }\end{array}$ & $\begin{array}{l}4042290732833 \\
4042390733009 \\
4044380733026 \\
4 \varnothing 41480733223 \\
4 \varnothing 4515 \varnothing 733138\end{array}$ & $\begin{array}{l}5481 \\
5483 \\
561127 \\
5483 \\
521124\end{array}$ & $\begin{array}{r}110 \\
46 \\
104 \\
32 \\
104\end{array}$ & $\begin{array}{l}- \\
\overline{44060} \\
\overline{209000}\end{array}$ & $\begin{array}{r}132500 \\
195200 \\
52000 \\
66000 \\
243000\end{array}$ \\
\hline $\begin{array}{l}N \\
N \\
N \\
N \\
N\end{array}$ & $\begin{array}{l}229 \\
230 \\
231 \\
232 \\
233\end{array}$ & $\begin{array}{l}\text { SOUTHERN ST. PKY } \\
\text { WALNUT STREET } \\
\text { BERRY HILL RD. } \\
\text { L.I.R.R. } \\
\text { MAGNOLIA DR. }\end{array}$ & $\begin{array}{l}\text { OLD BRITON LANE } \\
\text { CORT PLACE } \\
\text { RENEE RD. } \\
\text { TANNERS POND RD. } \\
\text { HAWTHORNE ST. }\end{array}$ & $\begin{array}{l}4642 \varnothing 2 \varnothing 733219 \\
4 \varnothing 4318 \varnothing 732747 \\
4 \varnothing 5 \varnothing 62 \varnothing 732949 \\
4 \varnothing 435 \varnothing 6734 \varnothing 24 \\
4 \varnothing 4147 \varnothing 73283 \emptyset\end{array}$ & $\begin{array}{l}5403 \\
5404 \\
5407 \\
5386 \\
521822\end{array}$ & $\begin{array}{r}32 \\
125 \\
126 \\
14 \\
110\end{array}$ & $\begin{array}{l}- \\
- \\
- \\
37000\end{array}$ & $\begin{array}{l}194000 \\
197000 \\
125200 \\
- \\
39000\end{array}$ \\
\hline $\begin{array}{l}N \\
N \\
N \\
N \\
N\end{array}$ & $\begin{array}{l}234 \\
235 \\
236 \\
237 \\
238\end{array}$ & $\begin{array}{l}\text { DIVISION AVE. } \\
\text { BROADWAY } \\
\text { W. CHESTTNT ST. } \\
\text { KETCHUM RD. } \\
\text { MILLER PL. }\end{array}$ & $\begin{array}{l}\text { JERUSALEM AVE. } \\
\text { I7TH STREET } \\
\text { CEDAR STREET } \\
\text { CIRCLE DR. } \\
\text { BIRCHWOOD PK. DR }\end{array}$ & $\begin{array}{l}4 \varnothing 45150733124 \\
4 \varnothing 46420733211 \\
4 \varnothing 4324 \varnothing 732715 \\
4 \varnothing 4715 \varnothing 733 \varnothing 35 \\
404726 \varnothing 733 \varnothing 55\end{array}$ & $\begin{array}{l}540729 \\
5311 \\
5682 \\
538721 \\
538488\end{array}$ & $\begin{array}{l}104 \\
105 \\
125 \\
108 \\
108\end{array}$ & $\begin{array}{l}- \\
- \\
362000 \\
97000\end{array}$ & $\begin{array}{l}- \\
80300 \\
159400 \\
322000 \\
199000\end{array}$ \\
\hline $\begin{array}{l}N \\
N \\
N \\
N \\
N\end{array}$ & $\begin{array}{l}239 \\
24 \emptyset \\
241 \\
242 \\
243\end{array}$ & $\begin{array}{l}\text { BLOOMINGDALE RD. } \\
\text { CARMANS RD. } \\
\text { COLD SPRING RD. } \\
\text { GUINEA WOODS RD. } \\
\text { ROBERT STREET }\end{array}$ & $\begin{array}{l}\text { ALAN CREST DRIVE } \\
\text { SOUTHERN ST. PKY } \\
\text { CROCUS DR. } \\
\text { NORTHERN ST. PKY } \\
\text { HAY PATH ROAD }\end{array}$ & $\begin{array}{l}4 \varnothing 4451 \varnothing 733 \varnothing 25 \\
4 \varnothing 4159 \varnothing 732558 \\
4 \varnothing 4938 \varnothing 73295 \emptyset \\
4 \varnothing 4513 \varnothing 7337 \varnothing 3 \\
4 \varnothing 4552 \varnothing 732732\end{array}$ & $\begin{array}{l}530416 \\
530423 \\
521124 \\
601118 \\
541008\end{array}$ & $\begin{array}{l}184 \\
163 \\
126 \\
118 \\
122\end{array}$ & $\begin{array}{l}83000 \\
59000 \\
280000 \\
- \\
513000\end{array}$ & $\begin{array}{r}136800 \\
60 \\
360000 \\
- \\
519000\end{array}$ \\
\hline $\begin{array}{l}N \\
N \\
N \\
N \\
N\end{array}$ & $\begin{array}{l}244 \\
245 \\
246 \\
247 \\
248\end{array}$ & $\begin{array}{l}\text { S.OYSTER BAY RD. } \\
\text { BRIARCLIFF RD. } \\
\text { WANTAGH AVE. } \\
\text { BARKERS POINT RO } \\
\text { MERRITT RD. }\end{array}$ & $\begin{array}{l}\text { KEEN GATE } \\
\text { JERICHO TPKE. } \\
\text { DIANNE ST. } \\
\text { SOUNDVIEW LANE } \\
\text { CLIFFORD DR. }\end{array}$ & $\begin{array}{l}4847390732952 \\
4046290733354 \\
404336073295 \varnothing \\
4851030734325 \\
4043428732723\end{array}$ & $\begin{array}{l}521205 \\
520119 \\
530420 \\
5788 \\
549625\end{array}$ & $\begin{array}{r}126 \\
89 \\
25 \\
67 \\
99\end{array}$ & $\begin{array}{c}506000 \\
233000 \\
136000 \\
- \\
32500\end{array}$ & $\begin{array}{r}416000 \\
236000 \\
8700 \varnothing \\
24200 \varnothing \\
36000\end{array}$ \\
\hline $\begin{array}{l}N \\
N \\
N \\
N \\
N\end{array}$ & $\begin{array}{l}249 \\
250 \\
251 \\
252 \\
253\end{array}$ & $\begin{array}{l}\text { WANTAGH ST.PKY. } \\
\text { PROSPECT AVE. } \\
\text { BOUNDARY AVE. } \\
\text { HICKSVILLE RD. } \\
\text { HICKSVILLE RD. }\end{array}$ & $\begin{array}{l}\text { ALWOOD DR. } \\
\text { BELLMORE AVE. } \\
\text { MOHAWK DR. } \\
\text { GREENWOOD DR. } \\
\text { QUEENS AVE. }\end{array}$ & $\begin{array}{l}4 \varnothing 4333 \varnothing 733227 \\
4 \varnothing 4211 \varnothing 73334 \emptyset \\
4 \varnothing 4244 \varnothing 732847 \\
4 \varnothing 4216 \varnothing 732858 \\
4 \varnothing 4145 \varnothing 732847\end{array}$ & $\begin{array}{l}5587 \\
521125 \\
5410 \\
536506 \\
5402\end{array}$ & $\begin{array}{r}8 \\
8 \\
121 \\
11 \varnothing \\
110\end{array}$ & $\begin{array}{l}\overline{132000} \\
\overline{5} \\
-\end{array}$ & $\begin{array}{r}60400 \\
170000 \\
76900 \\
60000 \\
69500\end{array}$ \\
\hline $\begin{array}{l}N \\
N \\
N \\
N \\
N\end{array}$ & $\begin{array}{l}254 \\
255 \\
256 \\
257 \\
258\end{array}$ & $\begin{array}{l}\text { SOUTHERN ST. PKY } \\
\text { BETHPAGE ROAD } \\
\text { FALCON ST. } \\
\text { CARMANS ROAD } \\
\text { COUNTY LINE RD. }\end{array}$ & $\begin{array}{l}\text { AIKEN AVE. } \\
\text { WILLET AVENUE } \\
\text { MARION DR. } \\
\text { FORD DR. S. } \\
\text { CARMANS RD. }\end{array}$ & $\begin{array}{l}4 \varnothing 4206 \varnothing 732909 \\
4 \varnothing 4644 \varnothing 733143 \\
4 \varnothing 4235 \varnothing 733145 \\
4 \varnothing 4131 \varnothing 732549 \\
4 \varnothing 4234 \varnothing 732555\end{array}$ & $\begin{array}{l}521031 \\
54 \varnothing 7 \\
531022 \\
5602 \\
520112\end{array}$ & $\begin{array}{r}46 \\
104 \\
32 \\
156 \\
125\end{array}$ & $\begin{array}{l}820000 \\
\overline{179000} \\
\overline{5} \\
\overline{5}\end{array}$ & $\begin{array}{r}8200 \varnothing 0 \\
41 \varnothing \varnothing \varnothing \varnothing \varnothing \\
14 \varnothing \varnothing \varnothing \varnothing \varnothing \\
173 \varnothing \varnothing \varnothing \varnothing \\
526 \varnothing \varnothing 6\end{array}$ \\
\hline
\end{tabular}


N.Y. [Dash indicates no data. Altitudes are in feet above sea level.] (cont.)

\begin{tabular}{|c|c|c|c|c|c|c|c|c|c|c|c|c|}
\hline \multicolumn{2}{|c|}{$\begin{array}{l}\text { BASIN } \\
\text { NUMBER }\end{array}$} & $\begin{array}{l}\text { MAX. } \\
\text { AREA } \\
\text { (square }\end{array}$ & $\begin{array}{c}\text { BASIN } \\
\text { AREA } \\
\text { fert) }\end{array}$ & RIM & $\begin{array}{l}\text {-ALT } \\
\text { OVER } \\
\text { FLOW }\end{array}$ & $\begin{array}{l}\text { BDE--- } \\
\text { BOT- } \\
\text { TOM }\end{array}$ & $\begin{array}{l}\text { WATER } \\
\text { TABLE }\end{array}$ & $\begin{array}{c}\text { DRAIN. } \\
\text { AREA } \\
\text { (acres) }\end{array}$ & \multicolumn{2}{|c|}{$\begin{array}{l}-- \text { BASIN-- } \\
\text { STATUS USE }\end{array}$} & $\begin{array}{l}\text { GEO. } \\
\text { UNIT }\end{array}$ & $\begin{array}{l}\text { SOIL } \\
\text { UNIT }\end{array}$ \\
\hline$N$ & 204 & 4000 & 82000 & 84.0 & 80.5 & 70.5 & 57.0 & 4.1 & 1 & 3 & 1 & 1 \\
\hline$N$ & 205 & 30600 & - & 81.4 & - & 85.0 & 60.0 & 7.6 & 1 & 3 & 1 & 2 \\
\hline$N$ & 206 & 40200 & - & 171.0 & - & 158.0 & 58.0 & 21.7 & 1 & 1 & 3 & 1 \\
\hline$N$ & 207 & 34100 & 67400 & 67.5 & 65.5 & 56.5 & 42.0 & 34.4 & 1 & 1 & 1 & 1 \\
\hline$N$ & 208 & - & 40320 & $49 . \varnothing$ & - & - & - & 28.4 & & & $\emptyset$ & $\varnothing$ \\
\hline$N$ & 209 & 47900 & 60000 & 168.9 & 166.6 & 156.0 & 80.0 & 49.4 & 1 & 1 & 1 & 2 \\
\hline$N$ & 210 & 31900 & 53000 & 57.0 & 54.0 & 44.0 & 41.0 & 41.5 & 1 & 1 & 1 & 1 \\
\hline$N$ & 211 & 50800 & - & 64.0 & - & 47.2 & 42.0 & 40.6 & 1 & 1 & 1 & 1 \\
\hline$N$ & 212 & 60500 & - & 56.5 & - & 42.0 & 40.0 & 46.3 & 1 & 1 & 1 & 1 \\
\hline$N$ & 213 & 70000 & - & 79.0 & - & 66.0 & 52.0 & 28.7 & 1 & 1 & 1 & 2 \\
\hline$N$ & 214 & 29100 & - & 56.8 & - & 46.0 & 40.0 & 14.5 & 1 & 1 & 1 & 6 \\
\hline $\mathrm{N}$ & 215 & 17000 & 53700 & 151.0 & 150.0 & 140.0 & 42.0 & 23.1 & 1 & 1 & 3 & 1 \\
\hline$N$ & 216 & 37400 & 52600 & 201.0 & - & 188.0 & 68.0 & 28.8 & 1 & 1 & 1 & 1 \\
\hline$N$ & 217 & 8500 & 16400 & 200.5 & 197.6 & 188.0 & 86.0 & 17.8 & 1 & 1 & 6 & 1 \\
\hline$N$ & 218 & 15300 & 34300 & 190.0 & 188.0 & $182 . \emptyset$ & 77.0 & 10.3 & 1 & 1 & 1 & 2 \\
\hline$N$ & 219 & 13600 & 25900 & 66.7 & 65.5 & 56.5 & 43.0 & 16.3 & 1 & 1 & 1 & 3 \\
\hline$N$ & 220 & 200000 & - & 62.5 & - & 52.0 & 41.0 & 55.4 & 1 & 1 & 1 & 3 \\
\hline$N$ & 221 & 56300 & - & 155.0 & - & 143.0 & 80.0 & 41.2 & 1 & 1 & 1 & 2 \\
\hline$N$ & 222 & 237000 & - & 60.5 & - & $52 . \varnothing$ & 41.0 & 15.9 & 1 & 1 & 1 & 2 \\
\hline$N$ & 223 & 113300 & - & 137.0 & - & 129.0 & 78.0 & 97.8 & 1 & 1 & 1 & 2 \\
\hline$N$ & 224 & 59800 & - & 61.2 & - & 50.0 & 40.0 & 20.9 & 1 & 1 & 1 & 1 \\
\hline$N$ & 225 & 43200 & - & 58.0 & - & 48.0 & 450.0 & 11.9 & 1 & 3 & 1 & 2 \\
\hline$N$ & 228 & 5200 & 12400 & 113.0 & 108.2 & 98.0 & 67.0 & 6.9 & 1 & 1 & 1 & 3 \\
\hline$N$ & 227 & 27700 & - & 52.5 & - & 42.0 & 34.0 & 4.0 & 1 & 3 & 1 & 3 \\
\hline$N$ & 228 & 30400 & 39400 & 129.5 & 127.9 & 118.3 & 72.0 & 32.9 & 1 & 1 & 1 & 2 \\
\hline$N$ & 229 & 42000 & - & 56.9 & - & $45 . \emptyset$ & 40.0 & 30.5 & 1 & 1 & 1 & 3 \\
\hline$N$ & 230 & 37300 & - & 57.5 & - & 47.0 & 50.0 & 31.0 & 1 & 1 & 1 & 1 \\
\hline$N$ & 231 & 31500 & - & 288.0 & - & 198.0 & 61.0 & 19.7 & 1 & 1 & 1 & 1 \\
\hline$N$ & 232 & 318000 & 474000 & 77.5 & - & 67.0 & 38.0 & 77.4 & 1 & 1 & 1 & 2 \\
\hline$N$ & 233 & 5500 & 20200 & 47.8 & 46.1 & 39.0 & 32.0 & 6.8 & 1 & 1 & 1 & 1 \\
\hline$N$ & 234 & 207000 & 275000 & 128.0 & - & 114.0 & 73.0 & 239.0 & 1 & 1 & 1 & 2 \\
\hline$N$ & 235 & 42800 & - & 151.0 & - & 136.5 & 77.0 & 12.6 & 1 & 1 & 1 & 2 \\
\hline$N$ & 238 & 28400 & - & 83.2 & - & 53.0 & 51.0 & 25.1 & 1 & 1 & 1 & 1 \\
\hline$N$ & 237 & 36600 & 60200 & 180.7 & 177.3 & 167.3 & 81.6 & 18.7 & 1 & 1 & 1 & 2 \\
\hline$N$ & 238 & 22700 & 35500 & 175.5 & 173.7 & 163.7 & 82.0 & 30.0 & 1 & 3 & 1 & 2 \\
\hline$N$ & 239 & 20100 & 32100 & 112.6 & 110.0 & 103.0 & 69.0 & 9.8 & 1 & 1 & 1 & 2 \\
\hline$N$ & 240 & 7200 & 17800 & 41.2 & 39.6 & 30.5 & 30.0 & 10.8 & 1 & 1 & 1 & 1 \\
\hline$N$ & 241 & 33600 & 40100 & 213.2 & 212.8 & 201.5 & 84.0 & 51.3 & 1 & 1 & 1 & 1 \\
\hline$N$ & 242 & 252300 & $3540 \varnothing \varnothing$ & 91.5 & - & 79.5 & 61.0 & 45.0 & 1 & 3 & 1 & 2 \\
\hline$N$ & 243 & 51900 & 108000 & 137.0 & 136.0 & 126.0 & 71.0 & 61.6 & 1 & 1 & 8 & 1 \\
\hline$N$ & 244 & 60900 & 81000 & 188.8 & 187.1 & 177.5 & 82.0 & 57.2 & 1 & 1 & 1 & 2 \\
\hline$N$ & 245 & 29900 & 39200 & 151.1 & 147.9 & 138.3 & 74.0 & 42.5 & 1 & 1 & 1 & 1 \\
\hline$N$ & 246 & 14100 & 22400 & 77.5 & 0.1 & 68.0 & 57.0 & 21.9 & 1 & 1 & 1 & 2 \\
\hline$N$ & 247 & 47300 & - & 29.0 & - & 18.0 & 10.0 & 38.1 & 1 & 1 & 3 & 3 \\
\hline$N$ & 248 & 4200 & 10100 & 68.0 & 56.5 & 58.0 & 51.0 & 15.0 & 1 & 1 & 1 & 1 \\
\hline$N$ & 249 & 21600 & - & 90.0 & - & 79.0 & $59 . \varnothing$ & 9.5 & 1 & 1 & 1 & 2 \\
\hline$N$ & 250 & 21400 & 32000 & 63.2 & 57.6 & 48.0 & 41.0 & 23.3 & 1 & 1 & 1 & 3 \\
\hline$N$ & 251 & $4100 \varnothing$ & - & 56.5 & - & 55.5 & 41.0 & 12.1 & 1 & 1 & 1 & 3 \\
\hline$N$ & 252 & 10900 & 16800 & 51.8 & 50.2 & 44.4 & 40.0 & 10.0 & 1 & 1 & 1 & 3 \\
\hline$N$ & 253 & 24000 & - & 45.0 & - & 38.0 & 32.0 & 10.9 & 1 & 1 & 1 & 3 \\
\hline$N$ & 254 & 14100 & 22300 & 51.5 & 48.6 & 42.0 & 36.0 & 11.2 & 1 & 3 & 1 & 1 \\
\hline$N$ & 255 & 61800 & - & 158.8 & - & 146.0 & 78.0 & 64.5 & 1 & 1 & 1 & 2 \\
\hline$N$ & 256 & 19200 & 41600 & 56.3 & 56.3 & 49.0 & 46.0 & 28.2 & 1 & 1 & 1 & 1 \\
\hline$N$ & 257 & 41000 & - & 35.3 & - & 27.0 & 21.0 & 27.2 & 1 & 1 & 1 & 3 \\
\hline$N$ & 258 & 8000 & 16700 & 51.2 & 49.4 & 40.0 & 35.0 & 9.7 & 1 & 1 & 1 & 1 \\
\hline
\end{tabular}


Appendix,--Location and basic physical features of recharge basins on Long Island,

\begin{tabular}{|c|c|c|c|c|c|c|c|c|}
\hline \multicolumn{2}{|c|}{$\begin{array}{l}\text { BASIN } \\
\text { NUMBER }\end{array}$} & \multicolumn{2}{|c|}{ NEAREST INTERSECTION } & $\begin{array}{l}\text { LATITUDE } \\
\text { LONGITUDE } \\
\circ, " \circ "\end{array}$ & $\begin{array}{l}\text { DATE } \\
\text { BUILT } \\
\text { YrMOD }\end{array}$ & COMMUNITY & $\begin{array}{l}\text { DESIGN } \\
\text { CAPACITY } \\
\text { (cubic }\end{array}$ & $\begin{array}{l}\text { ACTUAL } \\
\text { CAPACITY } \\
\text { feet) }\end{array}$ \\
\hline $\begin{array}{l}N \\
N \\
N \\
N \\
N\end{array}$ & $\begin{array}{l}259 \\
260 \\
261 \\
262 \\
263\end{array}$ & $\begin{array}{l}\text { GUINEA WOODS RD. } \\
\text { MITCHELL AVENUE } \\
\text { PLAINVIEW RD. } \\
\text { OLD COUNTRY RD. } \\
\text { NORTHERN ST. PKY }\end{array}$ & $\begin{array}{l}\text { MIDWOOD CR. } \\
\text { SYONEY STREET } \\
\text { HAY PATH RD. } \\
\text { GERHARD RD. } \\
\text { JERICHO TPKE. }\end{array}$ & $\begin{array}{l}4 \varnothing 4815 \varnothing 733747 \\
4 \varnothing 47 \varnothing 2 \varnothing 732843 \\
4 \varnothing 461 \varnothing \varnothing 732823 \\
4 \varnothing 4611 \varnothing 732844 \\
4 \varnothing 4529 \varnothing 733644\end{array}$ & $\begin{array}{l}5612 \\
538216 \\
538217 \\
5710 \\
5585\end{array}$ & $\begin{array}{r}53 \\
122 \\
122 \\
122 \\
89\end{array}$ & $\begin{array}{l}- \\
475060 \\
- \\
- \\
-\end{array}$ & $\begin{array}{r}- \\
168686 \varnothing \varnothing \\
415746 \varnothing \\
828680 \\
96180\end{array}$ \\
\hline $\begin{array}{l}N \\
N \\
N \\
N \\
N\end{array}$ & $\begin{array}{l}264 \\
265 \\
266 \\
267 \\
268\end{array}$ & $\begin{array}{l}\text { POWELLS LA, } \\
\text { ROSE AVE. } \\
\text { SEAMANS NECK RD. } \\
\text { WANTAGH ST. PKWY } \\
\text { WANTAGH ST. PKWY }\end{array}$ & $\begin{array}{l}\text { BALDWIN DR. } \\
\text { HILLSIDE AVE. } \\
\text { JERUSALEM AVE. } \\
\text { GATE LA. } \\
\text { GATE LA. }\end{array}$ & $\begin{array}{l}4 \varnothing 46 \varnothing 2 \varnothing 733434 \\
4 \varnothing 4524 \varnothing 733656 \\
4 \varnothing 4136 \varnothing 732935 \\
4 \varnothing 4418 \varnothing 733236 \\
4 \varnothing 4418 \varnothing 733236\end{array}$ & $\begin{array}{l}536417 \\
538627 \\
538525 \\
521216 \\
521216\end{array}$ & $\begin{array}{l}89 \\
89 \\
46 \\
46 \\
25\end{array}$ & $\begin{array}{r}143000 \\
59000 \\
51200 \\
298000 \\
290600\end{array}$ & $\begin{array}{r}145380 \\
11586 \varnothing \\
546 \varnothing \varnothing \\
388000 \\
386000\end{array}$ \\
\hline $\begin{array}{l}N \\
N \\
N \\
N \\
N\end{array}$ & $\begin{array}{l}269 \\
270 \\
271 \\
272 \\
273\end{array}$ & $\begin{array}{l}\text { JERUSALEM AVE. } \\
\text { JACKSON AVE. } \\
\text { EDGEWOOD DR. } \\
\text { OLD COUNTRY ROAD } \\
\text { KELL PLACE }\end{array}$ & $\begin{array}{l}\text { JAY ST. } \\
\text { IRA ROAD } \\
\text { JERICHO TPKE. } \\
\text { HYACINTH STREET } \\
\text { CORDWOOD LANE }\end{array}$ & $\begin{array}{l}4 \varnothing 45 \varnothing 2 \varnothing 7331 \varnothing 3 \\
4 \varnothing 4929 \varnothing 732959 \\
4 \varnothing 4444 \varnothing 733934 \\
4 \varnothing 45 \varnothing 9 \varnothing 733324 \\
4 \varnothing 424 \varnothing \varnothing 73292 \varnothing\end{array}$ & $\begin{array}{l}5512 \\
521167 \\
53 \varnothing 804 \\
6385 \\
521606\end{array}$ & $\begin{array}{r}104 \\
126 \\
62 \\
8 \\
110\end{array}$ & $\begin{array}{l}\overline{96800} \\
162500 \\
- \\
68000\end{array}$ & $\begin{array}{l}180500 \\
106000 \\
183000 \\
- \\
62000\end{array}$ \\
\hline $\begin{array}{l}N \\
N \\
N \\
N \\
N\end{array}$ & $\begin{array}{l}274 \\
275 \\
276 \\
277 \\
278\end{array}$ & $\begin{array}{l}\text { GREENBRIAR LA. } \\
\text { CONLON RD. } \\
\text { HICKSVILLE ROAD } \\
\text { PITTSBURG AVE. } \\
\text { BEATRICE LA. }\end{array}$ & $\begin{array}{l}\text { SAWMILL RD. } \\
\text { HAUSCH BLVD. } \\
\text { SOUTHERN ST. PKY } \\
\text { PARK LANE } \\
\text { CEDAR SWAMP RD. }\end{array}$ & $\begin{array}{l}40412 \varnothing \varnothing 733211 \\
4 \varnothing 411107335 \varnothing 3 \\
4 \varnothing 4157 \varnothing 732852 \\
4 \varnothing 4131 \varnothing 732614 \\
4 \varnothing 462 \varnothing 0732719\end{array}$ & $\begin{array}{l}538910 \\
54122 \varnothing \\
53891 \varnothing \\
531215 \\
680729\end{array}$ & $\begin{array}{r}32 \\
37 \\
110 \\
111 \\
122\end{array}$ & $\begin{array}{r}65500 \\
191000 \\
182000 \\
424080 \\
85500\end{array}$ & $\begin{array}{r}62080 \\
182808 \\
197080 \\
513700 \\
93000\end{array}$ \\
\hline $\begin{array}{l}N \\
N \\
N \\
N \\
N\end{array}$ & $\begin{array}{l}279 \\
280 \\
281 \\
282 \\
283\end{array}$ & $\begin{array}{l}\text { BETHPAGE ST.PKWY } \\
\text { MAYFLOWER DR. } \\
\text { TWANE ST. } \\
\text { RUTH CT. } \\
\text { SPLIT ROCK RD. }\end{array}$ & $\begin{array}{l}\text { CRESCENT DR. } \\
\text { FORDHAM RD. } \\
\text { HILL RD. } \\
\text { SEAMANS NECK RD. } \\
\text { N.HEMPSTEAD TPK. }\end{array}$ & $\begin{array}{l}4 \varnothing 4341 \varnothing 7328 \varnothing 5 \\
4 \varnothing 4514 \varnothing 733 \varnothing 34 \\
4 \varnothing 441 \varnothing \varnothing 732722 \\
4 \varnothing 4154 \varnothing 732938 \\
4 \varnothing 5 \varnothing 57 \varnothing 733154\end{array}$ & $\begin{array}{l}541019 \\
530914 \\
540908 \\
530914 \\
530904\end{array}$ & $\begin{array}{r}99 \\
184 \\
99 \\
46 \\
119\end{array}$ & $\begin{array}{r}182080 \\
59000 \\
67280 \\
72300 \\
600000\end{array}$ & $\begin{array}{r}101580 \\
73500 \\
61000 \\
98000 \\
501000\end{array}$ \\
\hline $\begin{array}{l}N \\
N \\
N \\
N \\
N\end{array}$ & $\begin{array}{l}284 \\
285 \\
286 \\
287 \\
288\end{array}$ & $\begin{array}{l}\text { COLONY LANE } \\
\text { BRIARWOOD LA. } \\
\text { BOUNDARY AVENUE } \\
\text { ALICIA DR. } \\
\text { OLD MOTOR PKWY. }\end{array}$ & $\begin{array}{l}\text { S. OYSTER BAY RD. } \\
\text { PLAINVIEW RD. } \\
\text { MERRITTS ROAD } \\
\text { CAMBRIDGE AVE. } \\
\text { ROBBY LANE }\end{array}$ & $\begin{array}{l}4 \varnothing 4756 \varnothing 733 \varnothing 28 \\
4 \varnothing 4623 \varnothing 732757 \\
4 \varnothing 43 \varnothing 3 \varnothing 732742 \\
4 \varnothing 4559 \varnothing 733451 \\
4 \varnothing 4547 \varnothing 734 \varnothing 49\end{array}$ & $\begin{array}{l}551229 \\
530914 \\
538226 \\
531116 \\
530919\end{array}$ & $\begin{array}{r}126 \\
122 \\
125 \\
89 \\
62\end{array}$ & $\begin{array}{r}124 \varnothing \varnothing \varnothing \\
2243 \varnothing \varnothing \\
96 \varnothing \varnothing \varnothing \\
3186 \varnothing \varnothing \\
13 \varnothing \varnothing \varnothing \varnothing\end{array}$ & $\begin{array}{r}124000 \\
84500 \\
94000 \\
331000 \\
380000\end{array}$ \\
\hline $\begin{array}{l}N \\
N \\
N \\
N \\
N\end{array}$ & $\begin{array}{l}289 \\
290 \\
291 \\
292 \\
293\end{array}$ & $\begin{array}{l}\text { VISTA RD. } \\
\text { BROADWAY } \\
\text { RUTH PL. } \\
\text { CEDAR DRIVE SO. } \\
\text { WOODLAND DR. }\end{array}$ & $\begin{array}{l}\text { WILLIS AVE. } \\
\text { 17TH ST. } \\
\text { JANET AVE. } \\
\text { OLD COURTHOUSE R } \\
\text { MIDOLE NECK RD. }\end{array}$ & $\begin{array}{l}4 \varnothing 4653 \varnothing 733929 \\
4 \varnothing 4633 \varnothing 73323 \varnothing \\
4 \varnothing 414 \varnothing \varnothing 733145 \\
4 \varnothing 4532 \varnothing 734 \varnothing \varnothing \varnothing \\
4 \varnothing 5123 \varnothing 734248\end{array}$ & $\begin{array}{l}531828 \\
526924 \\
538119 \\
558627 \\
531829\end{array}$ & $\begin{array}{r}83 \\
194 \\
32 \\
62 \\
86\end{array}$ & $\begin{array}{l}67000 \\
79000 \\
52500 \\
- \\
328000\end{array}$ & $\begin{array}{r}59800 \\
95800 \\
82800 \\
38000 \\
387 \varnothing 60\end{array}$ \\
\hline $\begin{array}{l}N \\
N \\
N \\
N \\
N\end{array}$ & $\begin{array}{l}294 \\
295 \\
296 \\
297 \\
298\end{array}$ & $\begin{array}{l}\text { WOOLSEY AVE. } \\
\text { MILLER PLACE } \\
\text { S.OYSTER BAY RD. } \\
\text { PLAINVIEW RD. } \\
\text { FLORGATE RD. }\end{array}$ & $\begin{array}{l}\text { HARWOOD DR. } \\
\text { NORTHERN ST. PKY } \\
\text { WOODBURY RD. } \\
\text { CLEARWATER DR. } \\
\text { HEMPSTEAD TPKE. }\end{array}$ & $\begin{array}{l}4 \varnothing 52198733847 \\
4 \varnothing 4714 \varnothing 733854 \\
4 \varnothing 47 \varnothing 9 \varnothing 732959 \\
4 \varnothing 46 \varnothing 4 \varnothing 732811 \\
4 \varnothing 43398732818\end{array}$ & $\begin{array}{l}531184 \\
530911 \\
5789 \\
538612 \\
5386 \varnothing 8\end{array}$ & $\begin{array}{r}100 \\
108 \\
104 \\
122 \\
99\end{array}$ & $\begin{array}{l}152800 \\
118080 \\
- \\
115680 \\
228080\end{array}$ & $\begin{array}{r}154 \varnothing \varnothing \varnothing \\
145 \varnothing \varnothing \varnothing \\
136 \varnothing \varnothing \varnothing \varnothing \\
1365 \varnothing \varnothing \\
248 \varnothing \varnothing \varnothing\end{array}$ \\
\hline $\begin{array}{l}N \\
N \\
N \\
N \\
N\end{array}$ & $\begin{array}{l}299 \\
380 \\
301 \\
382 \\
303\end{array}$ & $\begin{array}{l}\text { IRIS LANE } \\
\text { CARMAN ROAD } \\
\text { OLD COUNTRY ROAD } \\
\text { OLD COUNTRY RD. } \\
\text { STAUBER DR. }\end{array}$ & $\begin{array}{l}\text { BISMARK AVENUE } \\
\text { WESTWOOD ROAD S. } \\
\text { MAPLE COURT } \\
\text { HOCHMAN BLVD. } \\
\text { CENTRAL PK. RD. }\end{array}$ & $\begin{array}{l}48414 \varnothing 0733214 \\
4 \varnothing 41110732603 \\
4 \varnothing 46220732911 \\
4 \varnothing 464 \varnothing 0732904 \\
4 \varnothing 4626 \varnothing 732903\end{array}$ & $\begin{array}{l}558421 \\
530828 \\
5312 \\
560925 \\
530113\end{array}$ & $\begin{array}{r}32 \\
110 \\
122 \\
122 \\
122\end{array}$ & $\begin{array}{l}79500 \\
498000 \\
- \\
184000 \\
61000\end{array}$ & $\begin{array}{r}165500 \\
350600 \\
105080 \\
129800 \\
52000\end{array}$ \\
\hline $\begin{array}{l}N \\
N \\
N \\
N \\
N\end{array}$ & $\begin{array}{l}304 \\
305 \\
306 \\
307 \\
308\end{array}$ & $\begin{array}{l}\text { WANTAGH AVENUE } \\
\text { HEMPSTEAD TPKE. } \\
\text { AVERY ROAD } \\
\text { INTERVALE } \\
\text { DIANAS TRAIL }\end{array}$ & $\begin{array}{l}\text { HEMPSTEAD TPKE. } \\
\text { HICKSVILLE ROAD } \\
\text { JERICHO TURNPIKE } \\
\text { WARNER AVENUE } \\
\text { THE TULIPS }\end{array}$ & $\begin{array}{l}4 \varnothing 4346 \varnothing 732948 \\
4 \varnothing 4336 \varnothing 732925 \\
4 \varnothing 4911 \varnothing 732711 \\
4 \varnothing 4724 \varnothing 733925 \\
4 \varnothing 4716 \varnothing 733935\end{array}$ & $\begin{array}{l}590817 \\
5512 \\
548310 \\
548511 \\
548517\end{array}$ & $\begin{array}{r}25 \\
25 \\
128 \\
81 \\
81\end{array}$ & $\begin{array}{l}123000 \\
- \\
254000 \\
98000 \\
117000\end{array}$ & $\begin{array}{r}121000 \\
257000 \\
389800 \\
80000 \\
136000\end{array}$ \\
\hline $\begin{array}{l}N \\
N \\
N \\
N \\
N\end{array}$ & $\begin{array}{l}309 \\
310 \\
311 \\
312 \\
313\end{array}$ & $\begin{array}{l}\text { SYOSSET-WOODBURY } \\
\text { WHITMAN AVENUE } \\
\text { GLEN COVE ROAD } \\
\text { FARMERS AVE } \\
\text { VINCENT ROAD }\end{array}$ & $\begin{array}{l}\text { L.I.R.R. } \\
\text { HICKS AVENUE } \\
\text { NORTHERN BLVD. } \\
\text { STEWART AVE } \\
\text { KETCHAM AVENUE }\end{array}$ & $\begin{array}{l}4 \varnothing 4933 \varnothing 732924 \\
4 \varnothing 4814 \varnothing 732952 \\
4 \varnothing 4836 \varnothing 733731 \\
4 \varnothing 4537 \varnothing 7329 \varnothing 2 \\
4 \varnothing 4658 \varnothing 733 \varnothing 5 \varnothing\end{array}$ & $\begin{array}{l}54 \not 0428 \\
5586 \\
54 \varnothing 621 \\
540419 \\
540427\end{array}$ & $\begin{array}{r}126 \\
108 \\
103 \\
92 \\
104\end{array}$ & $\begin{array}{l}4 \varnothing 0000 \\
- \\
296000 \\
532 \varnothing \varnothing \varnothing \\
26 \varnothing \varnothing \varnothing \varnothing\end{array}$ & $\begin{array}{l}430000 \\
310000 \\
325000 \\
714 \varnothing 0 \% \\
275 \varnothing \varnothing \varnothing\end{array}$ \\
\hline
\end{tabular}


N.Y. [Dash indicates no data. Altitudes are in feet above sea level.] (cont.)

\begin{tabular}{|c|c|c|c|c|c|c|c|c|c|c|c|c|}
\hline & $\begin{array}{l}\text { SIN } \\
\text { MBER }\end{array}$ & $\begin{array}{l}\text { MAX. } \\
\text { AREA } \\
\text { (squar }\end{array}$ & $\begin{array}{r}\text { BASIN } \\
\text { AREA } \\
\text { feet) }\end{array}$ & RIM & $\begin{array}{l}-A L T I \\
\text { OVER- } \\
\text { FLOW }\end{array}$ & $\begin{array}{l}\text { JDE-- } \\
\text { BOT- } \\
\text { TOM }\end{array}$ & $\begin{array}{l}\text { WATER } \\
\text { TABLE }\end{array}$ & $\begin{array}{c}\text { DRAIN. } \\
\text { AREA } \\
\text { (acres) }\end{array}$ & $\begin{array}{l}--B A S I \\
\text { STATUS }\end{array}$ & $\begin{array}{l}\text { IN-- } \\
\text { USE }\end{array}$ & $\begin{array}{l}\text { GEO. } \\
\text { UNIT }\end{array}$ & $\begin{array}{l}\text { SOIL } \\
\text { UNIT }\end{array}$ \\
\hline v & $\begin{array}{l}259 \\
260 \\
261 \\
262 \\
263\end{array}$ & $\begin{array}{c}37600 \\
- \\
495000 \\
11070 \varnothing \\
21100\end{array}$ & $\begin{array}{r}40000 \\
780000 \\
- \\
-\end{array}$ & $\begin{array}{l}193.0 \\
124.0 \\
128.7 \\
128.0 \\
109.0\end{array}$ & $\begin{array}{r}- \\
122 . \overline{6} \\
- \\
-\end{array}$ & $\begin{array}{r}185.4 \\
116.0 \\
107.0 \\
109.0 \\
97.0\end{array}$ & $\begin{array}{l}48.0 \\
81.0 \\
76.0 \\
71.0 \\
64.0\end{array}$ & $\begin{array}{r}6.5 \\
65.0 \\
654.5 \\
130.3 \\
15.1\end{array}$ & $\begin{array}{l}1 \\
1 \\
0 \\
0 \\
1\end{array}$ & $\begin{array}{l}1 \\
1 \\
1 \\
1 \\
1\end{array}$ & $\begin{array}{l}3 \\
1 \\
8 \\
0 \\
1\end{array}$ & $\begin{array}{l}1 \\
2 \\
1 \\
0 \\
2\end{array}$ \\
\hline $\begin{array}{l}N \\
N\end{array}$ & $\begin{array}{l}264 \\
265 \\
266 \\
267 \\
268\end{array}$ & $\begin{array}{r}13300 \\
15900 \\
7000 \\
47700 \\
47700\end{array}$ & $\begin{array}{l}29300 \\
27300 \\
16000 \\
68000 \\
68000\end{array}$ & $\begin{array}{r}130.0 \\
97.8 \\
43.0 \\
165.5 \\
105.5\end{array}$ & $\begin{array}{r}225.8 \\
96.8 \\
41.8 \\
100.4 \\
100.4\end{array}$ & $\begin{array}{r}118.8 \\
88.0 \\
91.0 \\
91.0\end{array}$ & $\begin{array}{l}72.0 \\
63.0 \\
31.0 \\
66.0 \\
66.0\end{array}$ & $\begin{array}{r}19.6 \\
8.1 \\
7.8 \\
54.5 \\
54.5\end{array}$ & $\begin{array}{l}1 \\
1 \\
1 \\
1 \\
1\end{array}$ & $\begin{array}{l}1 \\
1 \\
0 \\
1 \\
1\end{array}$ & $\begin{array}{l}1 \\
1 \\
1 \\
1 \\
1\end{array}$ & $\begin{array}{l}1 \\
2 \\
1 \\
2 \\
2\end{array}$ \\
\hline $\begin{array}{l}N \\
N \\
N \\
N \\
N\end{array}$ & $\begin{array}{l}269 \\
270 \\
271 \\
272 \\
273\end{array}$ & $\begin{array}{c}22700 \\
13900 \\
22300 \\
- \\
8600\end{array}$ & $\begin{array}{r}- \\
21400 \\
42700 \\
854600 \\
19100\end{array}$ & $\begin{array}{r}125.0 \\
213.0 \\
103.0 \\
117.0 \\
65.6\end{array}$ & $\begin{array}{r}211 . \overline{8} \\
102.6 \\
63 . \overline{3}\end{array}$ & $\begin{array}{r}112.6 \\
203.0 \\
93.0 \\
53.7\end{array}$ & $\begin{array}{l}71.0 \\
65.0 \\
47.0 \\
72.0 \\
44.0\end{array}$ & $\begin{array}{r}15.8 \\
17.6 \\
29.1 \\
472.2 \\
9.5\end{array}$ & $\begin{array}{l}0 \\
1 \\
1 \\
1 \\
1\end{array}$ & $\begin{array}{l}1 \\
1 \\
1 \\
1 \\
1\end{array}$ & $\begin{array}{l}1 \\
6 \\
1 \\
1 \\
1\end{array}$ & $\begin{array}{l}2 \\
1 \\
1 \\
2 \\
1\end{array}$ \\
\hline $\begin{array}{l}N \\
N \\
N\end{array}$ & $\begin{array}{l}274 \\
275 \\
276 \\
277 \\
278\end{array}$ & $\begin{array}{l}10110 \\
20500 \\
40200 \\
78600 \\
11700\end{array}$ & $\begin{array}{r}20400 \\
30000 \\
64000 \\
104200 \\
25200\end{array}$ & $\begin{array}{r}40.7 \\
46.5 \\
46.3 \\
35 . \emptyset \\
184.2\end{array}$ & $\begin{array}{r}39.5 \\
44.7 \\
45.6 \\
33.4 \\
181.5\end{array}$ & $\begin{array}{r}33.0 \\
34.5 \\
40.0 \\
26.0 \\
171.5\end{array}$ & $\begin{array}{l}27.0 \\
26.0 \\
35.0 \\
21.0 \\
75.0\end{array}$ & $\begin{array}{r}9 . \varnothing \\
26.2 \\
24.8 \\
58.2 \\
11.7\end{array}$ & $\begin{array}{l}1 \\
0 \\
1 \\
1 \\
1\end{array}$ & $\begin{array}{l}1 \\
1 \\
1 \\
1 \\
1\end{array}$ & $\begin{array}{l}1 \\
1 \\
1 \\
1 \\
8\end{array}$ & $\begin{array}{l}1 \\
1 \\
1 \\
1 \\
1\end{array}$ \\
\hline $\begin{array}{l}N \\
N \\
N\end{array}$ & $\begin{array}{l}279 \\
280 \\
281 \\
282 \\
283\end{array}$ & $\begin{array}{r}12500 \\
8700 \\
9500 \\
19000 \\
71900\end{array}$ & $\begin{array}{r}20200 \\
14400 \\
33600 \\
36100 \\
157000\end{array}$ & $\begin{array}{r}68.5 \\
129.5 \\
81.3 \\
45.6 \\
130.0\end{array}$ & $\begin{array}{r}67.5 \\
126.6 \\
79.4 \\
43.0 \\
128.2\end{array}$ & $\begin{array}{r}58.5 \\
117.5 \\
73.0 \\
38.0 \\
120.0\end{array}$ & $\begin{array}{l}60.0 \\
73.0 \\
50.0 \\
34.0 \\
55.0\end{array}$ & $\begin{array}{r}13.9 \\
7.8 \\
13.1 \\
9.8 \\
69.5\end{array}$ & $\begin{array}{l}1 \\
1 \\
1 \\
1 \\
1\end{array}$ & $\begin{array}{l}3 \\
1 \\
1 \\
1 \\
1\end{array}$ & $\begin{array}{l}1 \\
1 \\
1 \\
1 \\
3\end{array}$ & $\begin{array}{l}1 \\
2 \\
1 \\
1 \\
3\end{array}$ \\
\hline $\begin{array}{l}N \\
N \\
N \\
N \\
N\end{array}$ & $\begin{array}{l}284 \\
285 \\
286 \\
287 \\
288\end{array}$ & $\begin{array}{l}15200 \\
50200 \\
14800 \\
33100 \\
22700\end{array}$ & $\begin{array}{l}27500 \\
71500 \\
29500 \\
67200 \\
49000\end{array}$ & $\begin{array}{r}186.5 \\
143.0 \\
65.0 \\
123.0 \\
170.0\end{array}$ & $\begin{array}{r}184.0 \\
141.5 \\
63.5 \\
119.3 \\
163.7\end{array}$ & $\begin{array}{r}175.0 \\
134.5 \\
55.8 \\
109.3 \\
150.0\end{array}$ & $\begin{array}{l}81.0 \\
78.0 \\
48.0 \\
72.0 \\
38.0\end{array}$ & $\begin{array}{l}34.5 \\
30.5 \\
13.2 \\
19.5 \\
17.7\end{array}$ & $\begin{array}{l}1 \\
1 \\
1 \\
1 \\
0\end{array}$ & $\begin{array}{l}1 \\
1 \\
1 \\
3 \\
1\end{array}$ & $\begin{array}{l}1 \\
8 \\
1 \\
1 \\
6\end{array}$ & $\begin{array}{l}2 \\
1 \\
3 \\
1 \\
1\end{array}$ \\
\hline $\begin{array}{l}N \\
N \\
N \\
N \\
N\end{array}$ & $\begin{array}{l}289 \\
290 \\
291 \\
292 \\
293\end{array}$ & $\begin{array}{r}8500 \\
9500 \\
10000 \\
3800 \\
44800\end{array}$ & $\begin{array}{l}25500 \\
1900 \varnothing \\
26500 \\
16500 \\
76 \varnothing 00\end{array}$ & $\begin{array}{r}151.0 \\
147.4 \\
45.5 \\
114.0 \\
59.5\end{array}$ & $\begin{array}{r}150.8 \\
145.6 \\
45.9 \\
117.0 \\
57.0\end{array}$ & $\begin{array}{r}143.0 \\
136.5 \\
36.0 \\
187.0 \\
47.0\end{array}$ & $\begin{array}{r}44.0 \\
7.6 \\
30.0 \\
79.0 \\
17.0\end{array}$ & $\begin{array}{r}12.2 \\
14.5 \\
7.2 \\
6.0 \\
51.6\end{array}$ & $\begin{array}{l}0 \\
1 \\
1 \\
0 \\
1\end{array}$ & $\begin{array}{l}1 \\
3 \\
1 \\
1 \\
1\end{array}$ & $\begin{array}{l}1 \\
1 \\
1 \\
1 \\
2\end{array}$ & $\begin{array}{l}1 \\
2 \\
3 \\
2 \\
1\end{array}$ \\
\hline $\begin{array}{l}N \\
N \\
N \\
N \\
N\end{array}$ & $\begin{array}{l}294 \\
295 \\
296 \\
297 \\
298\end{array}$ & $\begin{array}{r}16500 \\
15600 \\
167900 \\
13700 \\
29000\end{array}$ & $\begin{array}{r}37800 \\
30400 \\
36000 \\
38800\end{array}$ & $\begin{array}{r}95.0 \\
179.5 \\
178.0 \\
144.0 \\
78.4\end{array}$ & $\begin{array}{r}94.0 \\
177.7 \\
- \\
144.0 \\
77.7\end{array}$ & $\begin{array}{r}83.0 \\
167.7 \\
163.5 \\
67.0\end{array}$ & $\begin{array}{l}12.0 \\
80.0 \\
82.0 \\
76.0 \\
57.0\end{array}$ & $\begin{array}{r}20.9 \\
16.2 \\
214.1 \\
18.5 \\
30.3\end{array}$ & $\begin{array}{l}1 \\
1 \\
1 \\
1 \\
1\end{array}$ & $\begin{array}{l}1 \\
1 \\
1 \\
0 \\
3\end{array}$ & $\begin{array}{l}3 \\
1 \\
1 \\
8 \\
1\end{array}$ & $\begin{array}{l}1 \\
2 \\
2 \\
1 \\
1\end{array}$ \\
\hline $\begin{array}{l}N \\
N \\
N \\
N \\
N\end{array}$ & $\begin{array}{l}299 \\
300 \\
301 \\
302 \\
303\end{array}$ & $\begin{array}{l}12600 \\
36100 \\
27600 \\
13400 \\
57700\end{array}$ & $\begin{array}{r}25200 \\
76200 \\
25100 \\
148000\end{array}$ & $\begin{array}{r}48.5 \\
32 . \emptyset \\
152.2 \\
148 . \emptyset \\
145 . \emptyset\end{array}$ & $\begin{array}{r}46.4 \\
27.7 \\
- \\
146.6 \\
141.0\end{array}$ & $\begin{array}{r}38.4 \\
20.5 \\
141.0 \\
137.0 \\
132.0\end{array}$ & $\begin{array}{l}30.0 \\
17.0 \\
80.0 \\
80.0 \\
80.0\end{array}$ & $\begin{array}{r}10.9 \\
67.3 \\
6.4 \\
25.0 \\
8.4\end{array}$ & $\begin{array}{l}1 \\
0 \\
1 \\
1 \\
1\end{array}$ & $\begin{array}{l}1 \\
1 \\
3 \\
1 \\
1\end{array}$ & $\begin{array}{l}1 \\
1 \\
1 \\
1 \\
1\end{array}$ & $\begin{array}{l}1 \\
3 \\
2 \\
2 \\
2\end{array}$ \\
\hline $\begin{array}{l}N \\
N \\
N \\
N \\
N\end{array}$ & $\begin{array}{l}304 \\
305 \\
306 \\
307 \\
308\end{array}$ & $\begin{array}{l}165 \varnothing 0 \\
369 \varnothing \varnothing \\
46 \varnothing \varnothing \varnothing \\
115 \varnothing \varnothing \\
146 \varnothing \varnothing\end{array}$ & $\begin{array}{r}30300 \\
- \\
62000 \\
41400 \\
65500\end{array}$ & $\begin{array}{r}81 . \varnothing \\
87 . \varnothing \\
245.7 \\
236 . \varnothing \\
197 . \varnothing\end{array}$ & $\begin{array}{r}78.9 \\
- \\
247.9 \\
229.5 \\
195.0\end{array}$ & $\begin{array}{r}70 . \emptyset \\
76.0 \\
240.5 \\
222.0 \\
185 . \varnothing\end{array}$ & $\begin{array}{l}59.0 \\
57.0 \\
65.0 \\
45.0 \\
45.0\end{array}$ & $\begin{array}{l}19.4 \\
4 \varnothing .5 \\
35.0 \\
12.2 \\
16.0\end{array}$ & $\begin{array}{l}1 \\
1 \\
1 \\
0 \\
0\end{array}$ & $\begin{array}{l}1 \\
1 \\
3 \\
1 \\
1\end{array}$ & $\begin{array}{l}1 \\
1 \\
6 \\
4 \\
4\end{array}$ & $\begin{array}{l}2 \\
2 \\
1 \\
1 \\
1\end{array}$ \\
\hline $\begin{array}{l}N \\
N \\
N\end{array}$ & $\begin{array}{l}309 \\
310 \\
311 \\
312 \\
313\end{array}$ & $\begin{array}{l}577 \varnothing 0 \\
392 \varnothing 0 \\
379 \varnothing 0 \\
763 \varnothing 0 \\
364 \varnothing 0\end{array}$ & $\begin{array}{r}77200 \\
- \\
69700 \\
107000 \\
61000\end{array}$ & $\begin{array}{l}219.0 \\
204.4 \\
117.4 \\
132.7 \\
173.5\end{array}$ & $\begin{array}{r}216.7 \\
- \\
114.1 \\
30.7 \\
170.8\end{array}$ & $\begin{array}{l}209 . \varnothing \\
190.5 \\
104.5 \\
120.5 \\
162 . \varnothing\end{array}$ & $\begin{array}{l}65.0 \\
77.0 \\
47.0 \\
78.0 \\
81.0\end{array}$ & $\begin{array}{l}54.0 \\
48.8 \\
46.6 \\
73.0 \\
35.7\end{array}$ & $\begin{array}{l}1 \\
1 \\
1 \\
1 \\
1\end{array}$ & $\begin{array}{l}3 \\
1 \\
1 \\
1 \\
1\end{array}$ & $\begin{array}{l}1 \\
1 \\
3 \\
1 \\
1\end{array}$ & $\begin{array}{l}1 \\
2 \\
1 \\
2 \\
2\end{array}$ \\
\hline
\end{tabular}


Appendix.-Location and basic physical features of recharge basins on Long Island,

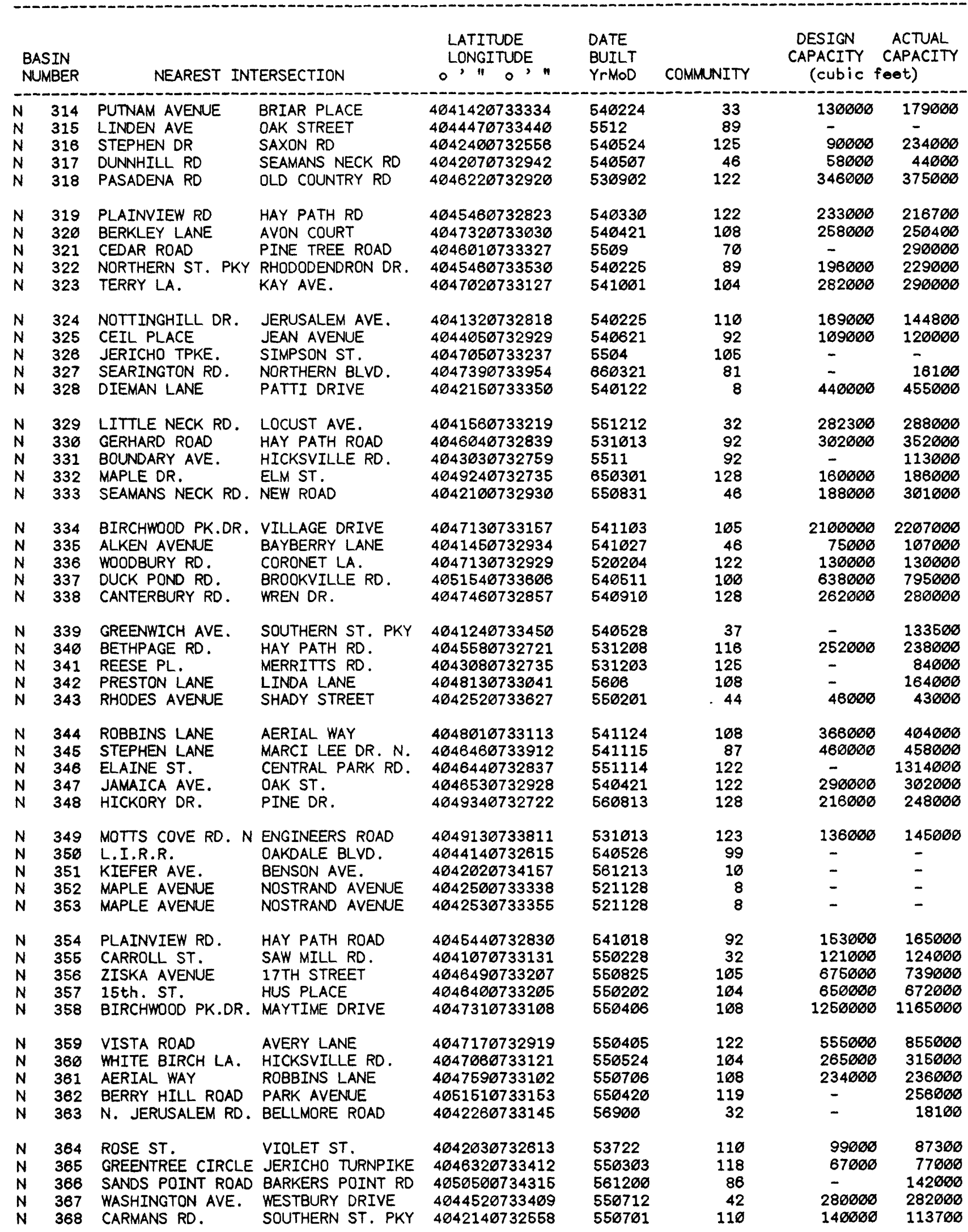


N.Y. [Dash indicates no data. Altitudes are in feet above sea level.] (cont.)

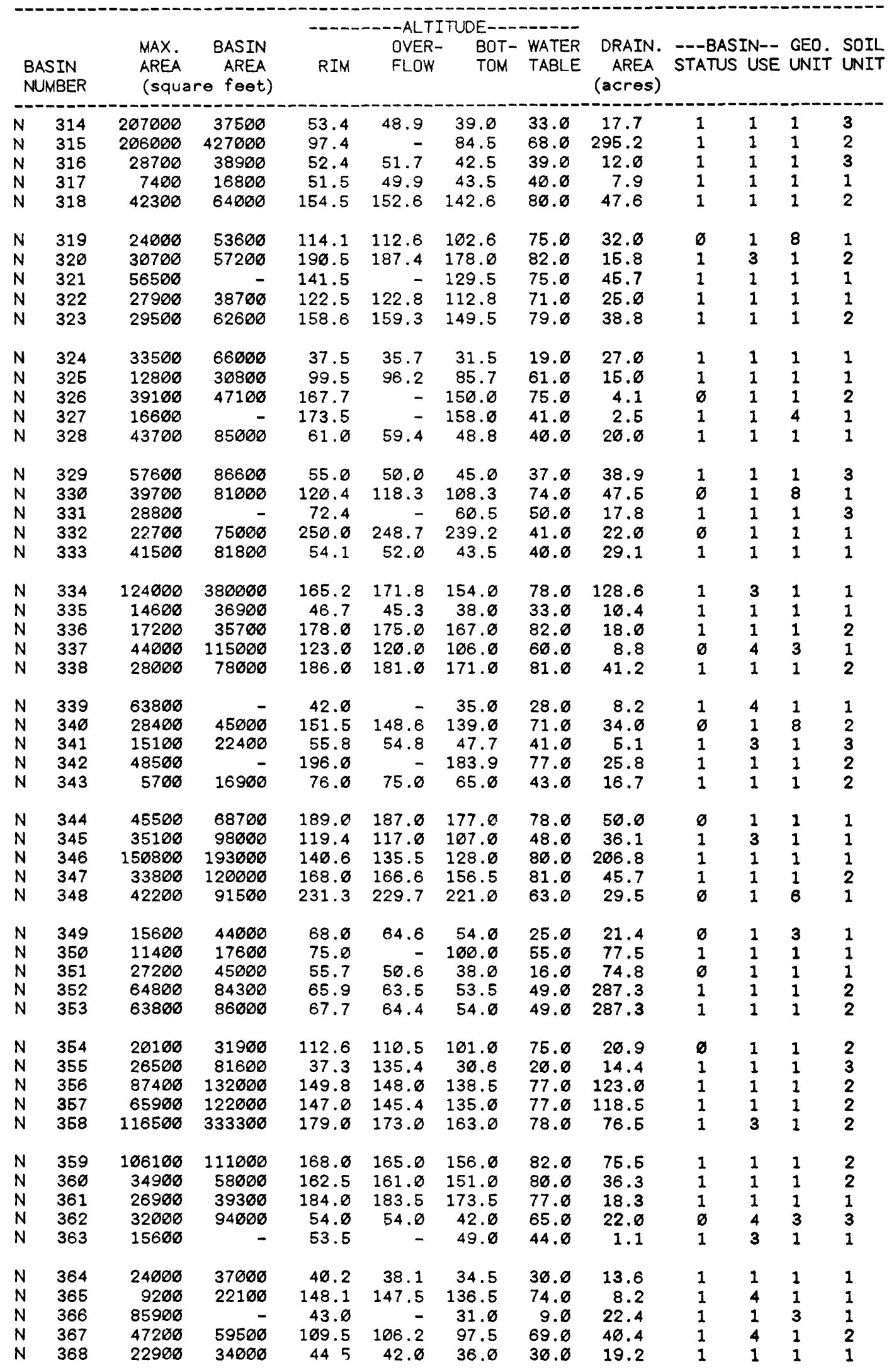


Appendix.--Location and basic physical features of recharge basins on Long Island,

\begin{tabular}{|c|c|c|c|c|c|c|c|c|}
\hline \multicolumn{2}{|c|}{$\begin{array}{l}\text { BASIN } \\
\text { NUMBER }\end{array}$} & \multicolumn{2}{|c|}{ NEAREST INTERSECTION } & $\begin{array}{l}\text { LATITUDE } \\
\text { LONGITUDE } \\
0, ",\end{array}$ & $\begin{array}{l}\text { DATE } \\
\text { BUILT } \\
\text { YrMOD }\end{array}$ & COMMUNITY & $\begin{array}{l}\text { DESIGN } \\
\text { CAPACITY } \\
\text { (cubic }\end{array}$ & $\begin{array}{l}\text { ACTUAL } \\
\text { CAPACITY } \\
\text { feet) }\end{array}$ \\
\hline $\begin{array}{l}N \\
N \\
N \\
N \\
N\end{array}$ & $\begin{array}{l}369 \\
370 \\
371 \\
372 \\
373\end{array}$ & $\begin{array}{l}\text { PLAINVIEW RD. } \\
\text { WASHINGTON AVE. } \\
\text { MILLER PLACE } \\
\text { OLD COUNTRY RD. } \\
\text { SCHULTZ ST. }\end{array}$ & $\begin{array}{l}\text { JERICHO TPKE. } \\
\text { MANETTO HILL RD. } \\
\text { NORTHERN ST. PKY } \\
\text { ROUND SWAMP RD. } \\
\text { JOHN ST. }\end{array}$ & $\begin{array}{l}4 \emptyset 483567327 \varnothing 5 \\
4 \emptyset 46520732756 \\
4047320733 \varnothing 51 \\
494658673265 \emptyset \\
404615073333 \emptyset\end{array}$ & $\begin{array}{l}5610 \\
546819 \\
551627 \\
569420 \\
560425\end{array}$ & $\begin{array}{l}128 \\
122 \\
168 \\
122 \\
104\end{array}$ & $\begin{array}{c}\overline{6} \\
840000 \\
5398060 \\
-\end{array}$ & $\begin{array}{r}187000 \\
172000 \\
85100 \\
5226000 \\
77000\end{array}$ \\
\hline $\begin{array}{l}N \\
N \\
N \\
N \\
N\end{array}$ & $\begin{array}{l}374 \\
375 \\
376 \\
377 \\
378\end{array}$ & $\begin{array}{l}\text { HAMPSHIRE DR. } \\
\text { JERICHO TPKE. } \\
\text { S.OYSTER BAY RD. } \\
\text { MELANIE LANE } \\
\text { CAROL DR. }\end{array}$ & $\begin{array}{l}\text { L.I.R.R. } \\
\text { ORCHARD DR. } \\
\text { L.I.R.R. } \\
\text { CARY STREET } \\
\text { PLAINVIEW RD. }\end{array}$ & $\begin{array}{l}404356073274 \emptyset \\
4 \varnothing 46350733653 \\
4 \varnothing 45206733006 \\
4648166732946 \\
4 \varnothing 48306732822\end{array}$ & $\begin{array}{l}566423 \\
6561 \\
570626 \\
556767 \\
566361\end{array}$ & $\begin{array}{r}99 \\
128 \\
164 \\
168 \\
92\end{array}$ & $\begin{array}{l}137000 \\
- \\
- \\
189600 \\
86500\end{array}$ & $\begin{array}{c}139060 \\
235600 \\
- \\
187600 \\
83760\end{array}$ \\
\hline $\begin{array}{l}N \\
N \\
N \\
N \\
N\end{array}$ & $\begin{array}{l}379 \\
380 \\
381 \\
382 \\
383\end{array}$ & $\begin{array}{l}\text { ROSLYN ROAD } \\
\text { RICHFIELD ST. } \\
\text { DOSORIS LA. } \\
\text { WEST PARK DRIVE } \\
\text { HAY PATH ROAD }\end{array}$ & $\begin{array}{l}\text { CHARLES STREET } \\
\text { PLAINVIEW RD. } \\
\text { NEW WOODS RD. } \\
\text { HAY PATH ROAD } \\
\text { PLAINVIEW RD. }\end{array}$ & $\begin{array}{l}4845176733809 \\
4 \emptyset 47416732844 \\
4 \varnothing 52456733747 \\
4 \varnothing 45376732754 \\
4645476732744\end{array}$ & $\begin{array}{l}6169 \\
551219 \\
556926 \\
556524 \\
5716\end{array}$ & $\begin{array}{r}68 \\
122 \\
160 \\
116 \\
92\end{array}$ & $\begin{array}{l}- \\
155000 \\
78000 \\
392500 \\
-\end{array}$ & $\begin{array}{r}\overline{1} \\
161060 \\
71600 \\
468060 \\
224060\end{array}$ \\
\hline $\begin{array}{l}N \\
N \\
N \\
N \\
N\end{array}$ & $\begin{array}{l}384 \\
385 \\
386 \\
387 \\
388\end{array}$ & $\begin{array}{l}\text { GREENVILLE LA. } \\
\text { OAKFIELD AVE. } \\
\text { TULIP AVE. } \\
\text { FRANKLIN AVE. } \\
\text { PLAINVIEW RD. }\end{array}$ & $\begin{array}{l}\text { BEECHWOOD DR. } \\
\text { JERUSALEM RD. } \\
\text { ANTHONY LA. } \\
\text { FOREST AVE. } \\
\text { SHADOW LANE }\end{array}$ & 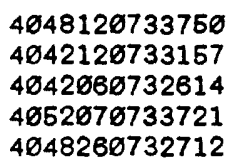 & $\begin{array}{l}580686 \\
561213 \\
560610 \\
576781 \\
586823\end{array}$ & $\begin{array}{r}53 \\
32 \\
110 \\
100 \\
128\end{array}$ & $\begin{array}{l}850006 \\
- \\
397000 \\
- \\
406000\end{array}$ & $\begin{array}{c}1041000 \\
846000 \\
330000 \\
- \\
378600\end{array}$ \\
\hline $\begin{array}{l}N \\
N \\
N \\
N \\
N\end{array}$ & $\begin{array}{l}389 \\
390 \\
391 \\
392 \\
393\end{array}$ & $\begin{array}{l}\text { WOODBURY ROAD } \\
\text { WALNUT ST. } \\
\text { N. UTICA AVENUE } \\
\text { THOMAS AVE. } \\
\text { CENTRAL AVE. }\end{array}$ & $\begin{array}{l}\text { ASHFORD DRIVE } \\
\text { BROADWAY } \\
\text { N. BAY AVENUE } \\
\text { 13TH ST. } \\
\text { JERUSALEM AVE. }\end{array}$ & $\begin{array}{l}4 \varnothing 47456732928 \\
4 \varnothing 42 \varnothing 6 \emptyset 732832 \\
4 \varnothing 4236 \varnothing 73286 \emptyset \\
4 \varnothing 4561 \varnothing 73294 \emptyset \\
4 \varnothing 41350732811\end{array}$ & $\begin{array}{l}568424 \\
548819 \\
579315 \\
558163 \\
570315\end{array}$ & $\begin{array}{r}122 \\
110 \\
110 \\
92 \\
110\end{array}$ & $\begin{array}{l}109000 \\
- \\
- \\
- \\
-\end{array}$ & $\begin{array}{l}189060 \\
- \\
- \\
- \\
-\end{array}$ \\
\hline $\begin{array}{l}N \\
N \\
N \\
N \\
N\end{array}$ & $\begin{array}{l}394 \\
395 \\
396 \\
397 \\
398\end{array}$ & $\begin{array}{l}\text { RUTHERFORD AVE. } \\
\text { EDGEMERE DR. } \\
\text { SYOS-COLD SPR.RD } \\
\text { NEW YORK DRIVE } \\
\text { WOODWARD DR. }\end{array}$ & $\begin{array}{l}\text { N. CENTRAL DRIVE } \\
\text { SEARINGTOWN RD. } \\
\text { S.WOODS ROAD } \\
\text { E.UTICA AVE. } \\
\text { WISCONSIN AVE. }\end{array}$ & $\begin{array}{l}4842246732813 \\
4 \varnothing 46326733959 \\
4 \varnothing 58666732912 \\
4 \varnothing 42296732728 \\
4042438732817\end{array}$ & $\begin{array}{l}576315 \\
541217 \\
566528 \\
5912 \\
591236\end{array}$ & $\begin{array}{r}116 \\
87 \\
128 \\
116 \\
110\end{array}$ & $\begin{array}{l}\overline{1} 24800 \\
- \\
- \\
-\end{array}$ & $\begin{array}{l}- \\
135000 \\
685060 \\
- \\
-\end{array}$ \\
\hline $\begin{array}{l}N \\
N \\
N \\
N \\
N\end{array}$ & $\begin{array}{l}399 \\
460 \\
401 \\
462 \\
403\end{array}$ & $\begin{array}{l}\text { MICHIGAN AVE. } \\
\text { NORTHERN PARKWAY } \\
\text { GLENWOOD ROAD } \\
\text { MEAD AVE. } \\
\text { MEADOWFIELD LA. }\end{array}$ & $\begin{array}{l}\text { BROADWAY } \\
\text { OAK DRIVE } \\
\text { SCUDDERS LANE } \\
\text { STEWART AVE. } \\
\text { FOREST AVE. }\end{array}$ & $\begin{array}{l}4042410732751 \\
4 \varnothing 47 \varnothing 5 \varnothing 732933 \\
4949266733836 \\
4044546732904 \\
405236 \varnothing 733652\end{array}$ & $\begin{array}{l}5705 \\
550921 \\
5769 \\
\quad- \\
590125\end{array}$ & $\begin{array}{r}110 \\
122 \\
123 \\
92 \\
100\end{array}$ & $\begin{array}{l}- \\
152000 \\
- \\
- \\
-\end{array}$ & $\begin{array}{l}- \\
167006 \\
- \\
- \\
-\end{array}$ \\
\hline $\begin{array}{l}N \\
N \\
N \\
N \\
N\end{array}$ & $\begin{array}{l}404 \\
4 \varnothing 5 \\
4 \varnothing 6 \\
4 \varnothing 7 \\
4 \varnothing 8\end{array}$ & $\begin{array}{l}\text { LAUREL HILL DR. } \\
\text { CENTRAL PK. RD. } \\
\text { COLD SP. HARB.RD. } \\
\text { ROUND SWAMP ROAD } \\
\text { WEBSTER AVENUE }\end{array}$ & $\begin{array}{l}\text { HIGHWOOD DR. } \\
\text { PLAINVIEW RD. } \\
\text { WOODS RD. } \\
\text { HAY PATH ROAD } \\
\text { BROOKWOLD DRIVE }\end{array}$ & $\begin{array}{l}4 \varnothing 51138733139 \\
4 \varnothing 47246732856 \\
4 \varnothing 58106732914 \\
4 \varnothing 46 \varnothing 5 \varnothing 732712 \\
4948 \varnothing 86734148\end{array}$ & $\begin{array}{l}566826 \\
561610 \\
576562 \\
570731 \\
58\end{array}$ & $\begin{array}{r}98 \\
122 \\
126 \\
116 \\
76\end{array}$ & $\begin{array}{l}236000 \\
4650600 \\
1602000 \\
108000 \\
-\end{array}$ & $\begin{array}{l}395000 \\
568000 \\
850000 \\
184280 \\
-\end{array}$ \\
\hline $\begin{array}{l}N \\
N \\
N \\
N \\
N\end{array}$ & $\begin{array}{l}469 \\
416 \\
411 \\
412 \\
413\end{array}$ & $\begin{array}{l}\text { ROUND SWAMP RD. } \\
\text { HELEN RD. } \\
\text { CHESTNUT DRIVE } \\
\text { W.DELORES LA. } \\
\text { JOHN STREET }\end{array}$ & $\begin{array}{l}\text { FARRAGUT RD. } \\
\text { VIOLA DR. } \\
\text { LAWN DRIVE } \\
\text { IVY CT. } \\
\text { KUHL AVENUE }\end{array}$ & $\begin{array}{l}49461107327 \varnothing 7 \\
4051546733613 \\
4948166733813 \\
4 \varnothing 44346733906 \\
494559673324 \emptyset\end{array}$ & $\begin{array}{l}576429 \\
576619 \\
566463 \\
566462 \\
596713\end{array}$ & $\begin{array}{r}116 \\
106 \\
53 \\
92 \\
104\end{array}$ & $\begin{array}{l}164700 \\
85000 \\
466000 \\
168000 \\
-\end{array}$ & $\begin{array}{l}109506 \\
78060 \\
428060 \\
188060 \\
-\end{array}$ \\
\hline $\begin{array}{l}N \\
N \\
N \\
N \\
N\end{array}$ & $\begin{array}{l}414 \\
415 \\
416 \\
417 \\
418\end{array}$ & $\begin{array}{l}\text { GERARD AVE. } \\
\text { SHELTER ROCK RD. } \\
\text { LINDEN LA. } \\
\text { SUNNYSIDE LANE } \\
\text { PLAINVIEW RD. }\end{array}$ & $\begin{array}{l}\text { NEW HYDE PK. RD. } \\
\text { EAST STREET } \\
\text { CHAPEL GATE } \\
\text { ROBBINS LANE } \\
\text { SARAH DR. }\end{array}$ & $\begin{array}{l}4045106734129 \\
404537 \varnothing 733958 \\
4850048733453 \\
4046 \varnothing 96733315 \\
4045360732802\end{array}$ & $\begin{array}{l}586314 \\
560813 \\
530529 \\
586811 \\
570702\end{array}$ & $\begin{array}{r}71 \\
62 \\
127 \\
104 \\
116\end{array}$ & $\begin{array}{l}- \\
384000 \\
- \\
415000 \\
130000\end{array}$ & $\begin{array}{l}- \\
427000 \\
147000 \\
343600 \\
141900\end{array}$ \\
\hline $\begin{array}{l}N \\
N \\
N \\
N \\
N\end{array}$ & $\begin{array}{l}419 \\
420 \\
421 \\
422 \\
423\end{array}$ & $\begin{array}{l}\text { FULTON PLACE } \\
\text { SINGWORTH ST. } \\
\text { ORCHARD DRIVE } \\
\text { MILL RIVER ROAD } \\
\text { PARKWAY DR. }\end{array}$ & $\begin{array}{l}\text { ROCKLAND DRIVE } \\
\text { SOUNDVIEW AVE } \\
\text { MAPLE WAY } \\
\text { LAUN LANE } \\
\text { PLAINVIEW RD. }\end{array}$ & $\begin{array}{l}4 \varnothing 46330733238 \\
4 \varnothing 51280733142 \\
4048410732728 \\
4 \varnothing 51036733234 \\
4046050732754\end{array}$ & $\begin{array}{l}571126 \\
581229 \\
591221 \\
591116 \\
551011\end{array}$ & $\begin{array}{l}165 \\
119 \\
128 \\
127 \\
122\end{array}$ & $\begin{array}{r}1180000 \\
277000 \\
181000 \\
580000 \\
77600\end{array}$ & $\begin{array}{r}1352060 \\
269060 \\
178600 \\
538600 \\
75060\end{array}$ \\
\hline
\end{tabular}


N.Y. [Dash indicates no data. Altitudes are in feet above sea lovel.] (cont.)

\begin{tabular}{|c|c|c|c|c|c|c|c|c|c|c|c|c|}
\hline & & $\begin{array}{l}\text { MAX. } \\
\text { AREA } \\
\text { (squar }\end{array}$ & $\begin{array}{c}\text { BASIN } \\
\text { AREA } \\
\text { feet) }\end{array}$ & RIM & $\begin{array}{l}\text { - -ALT } \\
\text { OVER } \\
\text { FLOW }\end{array}$ & $\begin{array}{l}\text { UDE-- } \\
\text { BOT- } \\
\text { TOM }\end{array}$ & $\begin{array}{l}\text { WATER } \\
\text { TABLE }\end{array}$ & $\begin{array}{c}\text { DRAIN. } \\
\text { AREA } \\
\text { (acres) }\end{array}$ & $\begin{array}{l}---B A S I \\
\text { STATUS }\end{array}$ & IN-- & $\begin{array}{l}\text { GEO. } \\
\text { UNIT }\end{array}$ & $\begin{array}{l}\text { SOIL } \\
\text { UNIT }\end{array}$ \\
\hline$N$ & $\begin{array}{l}369 \\
370 \\
371 \\
372 \\
373\end{array}$ & $\begin{array}{r}49300 \\
18000 \\
7200 \\
140000 \\
9200\end{array}$ & $\begin{array}{r}- \\
39500 \\
19800 \\
450000 \\
23400\end{array}$ & $\begin{array}{l}277.0 \\
157.0 \\
190.0 \\
174.0 \\
149.1\end{array}$ & $\begin{array}{l}- \\
156.0 \\
176.5 \\
174.0 \\
147.0\end{array}$ & $\begin{array}{l}265.5 \\
146.5 \\
166.5 \\
159.0 \\
137.0\end{array}$ & $\begin{array}{l}71.0 \\
77.0 \\
82.0 \\
74.0 \\
74.0\end{array}$ & $\begin{array}{r}29.4 \\
8.6 \\
11.5 \\
849.7 \\
12.1\end{array}$ & $\begin{array}{l}1 \\
0 \\
1 \\
1 \\
1\end{array}$ & $\begin{array}{l}1 \\
1 \\
1 \\
1 \\
1\end{array}$ & $\begin{array}{l}6 \\
1 \\
1 \\
1 \\
1\end{array}$ & $\begin{array}{l}1 \\
1 \\
2 \\
1 \\
1\end{array}$ \\
\hline $\begin{array}{l}N \\
N \\
N\end{array}$ & $\begin{array}{l}374 \\
375 \\
376 \\
377 \\
378\end{array}$ & $\begin{array}{l}15700 \\
59500 \\
78800 \\
21000 \\
10400\end{array}$ & $\begin{array}{r}37800 \\
61000 \\
124200 \\
358000 \\
17500\end{array}$ & $\begin{array}{r}85.0 \\
271.0 \\
127.1 \\
205.2 \\
80.0\end{array}$ & $\begin{array}{r}84.0 \\
- \\
- \\
201 . \overline{5} \\
79.0\end{array}$ & $\begin{array}{r}73.0 \\
258.5 \\
116.0 \\
191.5 \\
70.0\end{array}$ & $\begin{array}{l}50.0 \\
81.0 \\
73.0 \\
75.0 \\
58.0\end{array}$ & $\begin{array}{r}25.0 \\
37.0 \\
169.7 \\
90.0 \\
11.8\end{array}$ & $\begin{array}{l}1 \\
0 \\
1 \\
1 \\
1\end{array}$ & $\begin{array}{l}1 \\
1 \\
1 \\
1 \\
1\end{array}$ & $\begin{array}{l}1 \\
1 \\
1 \\
1 \\
1\end{array}$ & $\begin{array}{l}1 \\
2 \\
2 \\
2 \\
6\end{array}$ \\
\hline $\begin{array}{l}N \\
N \\
N\end{array}$ & $\begin{array}{l}379 \\
380 \\
381 \\
382 \\
383\end{array}$ & $\begin{array}{r}26300 \\
19000 \\
8000 \\
47000 \\
65500\end{array}$ & $\begin{array}{r}34600 \\
4500 \emptyset \\
2400 \emptyset \\
75000 \\
-\end{array}$ & $\begin{array}{l}102.6 \\
185.2 \\
122.5 \\
126.0 \\
133.0\end{array}$ & $\begin{array}{r}- \\
179.5 \\
121.0 \\
126.0 \\
-\end{array}$ & $\begin{array}{r}9.0 \\
171.0 \\
111.0 \\
116.0 \\
121.2\end{array}$ & $\begin{array}{l}54.0 \\
80.0 \\
41.0 \\
71.0 \\
70.0\end{array}$ & $\begin{array}{l}24 . \overline{4} \\
10.7 \\
54.0 \\
35.3\end{array}$ & $\begin{array}{l}1 \\
1 \\
1 \\
1 \\
1\end{array}$ & $\begin{array}{l}1 \\
1 \\
1 \\
1 \\
1\end{array}$ & $\begin{array}{l}1 \\
1 \\
1 \\
8 \\
8\end{array}$ & $\begin{array}{l}2 \\
2 \\
1 \\
3 \\
2\end{array}$ \\
\hline $\begin{array}{l}N \\
N \\
N\end{array}$ & $\begin{array}{l}384 \\
385 \\
386 \\
387 \\
388\end{array}$ & $\begin{array}{r}104100 \\
250100 \\
71500 \\
9000 \\
38800\end{array}$ & $\begin{array}{r}175000 \\
350500 \\
106000 \\
27800 \\
79700\end{array}$ & $\begin{array}{r}200.0 \\
52.6 \\
41.0 \\
108.0 \\
275.0\end{array}$ & $\begin{array}{r}198.0 \\
52.0 \\
39.7 \\
270 . \overline{0}\end{array}$ & $\begin{array}{r}188.0 \\
44.8 \\
35.0 \\
90.0 \\
260.0\end{array}$ & $\begin{array}{l}48.0 \\
41.0 \\
29.0 \\
56.0 \\
70.0\end{array}$ & $\begin{array}{r}80.0 \\
132.2 \\
29.8 \\
34.8\end{array}$ & $\begin{array}{l}1 \\
1 \\
1 \\
1 \\
1\end{array}$ & $\begin{array}{l}1 \\
1 \\
1 \\
4 \\
1\end{array}$ & $\begin{array}{l}3 \\
1 \\
1 \\
3 \\
8\end{array}$ & $\begin{array}{l}1 \\
1 \\
1 \\
1 \\
1\end{array}$ \\
\hline $\begin{array}{l}N \\
N \\
N\end{array}$ & $\begin{array}{l}389 \\
390 \\
391 \\
392 \\
393\end{array}$ & $\begin{array}{r}20100 \\
51400 \\
37900 \\
104000 \\
150000\end{array}$ & $\begin{array}{r}38900 \\
85000 \\
62200 \\
137400 \\
186000\end{array}$ & $\begin{array}{r}188.9 \\
46.0 \\
56.2 \\
115.0 \\
39.6\end{array}$ & $\begin{array}{r}186.2 \\
- \\
- \\
- \\
-\end{array}$ & $\begin{array}{r}176.6 \\
34.0 \\
103.0 \\
32.0\end{array}$ & $\begin{array}{l}82.0 \\
31.0 \\
39.0 \\
70.0 \\
27.0\end{array}$ & $\begin{array}{r}15.5 \\
72.3 \\
151.5\end{array}$ & $\begin{array}{l}1 \\
1 \\
1 \\
1 \\
1\end{array}$ & $\begin{array}{l}1 \\
1 \\
1 \\
1 \\
1\end{array}$ & $\begin{array}{l}1 \\
1 \\
1 \\
1 \\
1\end{array}$ & $\begin{array}{l}2 \\
3 \\
3 \\
2 \\
1\end{array}$ \\
\hline $\begin{array}{l}N \\
N \\
N\end{array}$ & $\begin{array}{l}394 \\
395 \\
396 \\
397 \\
398\end{array}$ & $\begin{array}{r}57200 \\
20900 \\
57000 \\
106500 \\
40800\end{array}$ & $\begin{array}{r}83400 \\
44200 \\
121000 \\
170000 \\
68000\end{array}$ & $\begin{array}{r}57.7 \\
153.5 \\
209.8 \\
53.0 \\
39.6\end{array}$ & $\begin{array}{r}- \\
143.0 \\
209.8 \\
- \\
-\end{array}$ & $\begin{array}{r}46.5 \\
135.0 \\
197.8 \\
41.0 \\
32.0\end{array}$ & $\begin{array}{l}38.0 \\
44.0 \\
60.0 \\
38.0 \\
36.0\end{array}$ & $\begin{array}{r}82.0 \\
17.0 \\
41.9 \\
- \\
-\end{array}$ & $\begin{array}{l}1 \\
1 \\
1 \\
1 \\
1\end{array}$ & $\begin{array}{l}1 \\
1 \\
3 \\
1 \\
1\end{array}$ & $\begin{array}{l}1 \\
1 \\
1 \\
1 \\
1\end{array}$ & $\begin{array}{l}1 \\
1 \\
1 \\
3 \\
1\end{array}$ \\
\hline $\begin{array}{l}N \\
N \\
N\end{array}$ & $\begin{array}{l}399 \\
400 \\
401 \\
4 \varnothing 2 \\
403\end{array}$ & $\begin{array}{c}40800 \\
20500 \\
- \\
40800 \\
-\end{array}$ & $\begin{array}{r}68000 \\
39500 \\
31000 \\
60400 \\
-\end{array}$ & $\begin{array}{r}62.0 \\
175.0 \\
23.0 \\
113.2 \\
90.0\end{array}$ & $\begin{array}{r}- \\
173.1 \\
- \\
- \\
-\end{array}$ & $\begin{array}{r}50.0 \\
163.5 \\
13.0 \\
101.0 \\
-\end{array}$ & $\begin{array}{r}41.0 \\
82.0 \\
2.0 \\
58.0 \\
45.0\end{array}$ & $\begin{array}{r}-\overline{9} \\
27.9 \\
20.5 \\
33 . \overline{0}\end{array}$ & $\begin{array}{l}1 \\
1 \\
0 \\
1 \\
0\end{array}$ & $\begin{array}{l}1 \\
1 \\
1 \\
1 \\
1\end{array}$ & $\begin{array}{l}1 \\
1 \\
3 \\
1 \\
3\end{array}$ & $\begin{array}{l}1 \\
3 \\
3 \\
1 \\
1\end{array}$ \\
\hline $\begin{array}{l}N \\
N \\
N \\
N \\
N\end{array}$ & $\begin{array}{l}404 \\
405 \\
406 \\
407 \\
408\end{array}$ & $\begin{array}{l}40400 \\
54600 \\
82500 \\
22900 \\
68100\end{array}$ & $\begin{array}{r}58100 \\
101000 \\
126000 \\
36000 \\
204000\end{array}$ & $\begin{array}{r}197.7 \\
169.1 \\
213.5 \\
155.8 \\
58.5\end{array}$ & $\begin{array}{r}196.0 \\
167.0 \\
209.6 \\
154.2 \\
53.5\end{array}$ & $\begin{array}{r}186.0 \\
157.5 \\
198.0 \\
144.5 \\
46.0\end{array}$ & $\begin{array}{l}52.0 \\
80.0 \\
59.0 \\
70.0 \\
30.0\end{array}$ & $\begin{array}{r}30.0 \\
57.1 \\
95.0 \\
14.9 \\
-\end{array}$ & $\begin{array}{l}0 \\
1 \\
1 \\
0 \\
1\end{array}$ & $\begin{array}{l}1 \\
1 \\
1 \\
1 \\
1\end{array}$ & $\begin{array}{l}3 \\
1 \\
1 \\
8 \\
3\end{array}$ & $\begin{array}{l}1 \\
2 \\
1 \\
3 \\
1\end{array}$ \\
\hline $\begin{array}{l}N \\
N \\
N \\
N \\
N\end{array}$ & $\begin{array}{l}409 \\
410 \\
411 \\
412 \\
413\end{array}$ & $\begin{array}{r}14000 \\
9700 \\
49500 \\
21900 \\
182000\end{array}$ & $\begin{array}{r}34900 \\
22100 \\
94400 \\
57000 \\
540000\end{array}$ & $\begin{array}{l}161.4 \\
136.1 \\
198.5 \\
107.1 \\
131.0\end{array}$ & $\begin{array}{r}160.2 \\
137.6 \\
197.5 \\
105.7 \\
-\end{array}$ & $\begin{array}{r}151.0 \\
128.0 \\
188.5 \\
96.1 \\
111.0\end{array}$ & $\begin{array}{l}70.0 \\
59.0 \\
58.0 \\
66.0 \\
75.0\end{array}$ & $\begin{array}{l}28.4 \\
11.3 \\
40.0 \\
23.2 \\
44.1\end{array}$ & $\begin{array}{l}1 \\
0 \\
0 \\
1 \\
1\end{array}$ & $\begin{array}{l}3 \\
4 \\
1 \\
1 \\
1\end{array}$ & $\begin{array}{l}8 \\
3 \\
4 \\
1 \\
1\end{array}$ & $\begin{array}{l}3 \\
1 \\
1 \\
2 \\
2\end{array}$ \\
\hline $\begin{array}{l}N \\
N \\
N \\
N \\
N\end{array}$ & $\begin{array}{l}414 \\
415 \\
416 \\
417 \\
418\end{array}$ & $\begin{array}{l}49800 \\
76200 \\
15000 \\
55400 \\
17200\end{array}$ & $\begin{array}{l}81000 \\
92000 \\
41600 \\
81000 \\
33700\end{array}$ & $\begin{array}{l}120.0 \\
123.5 \\
168.0 \\
147.0 \\
120.5\end{array}$ & $\begin{array}{l}118.0 \\
162.8 \\
139.9 \\
198.4\end{array}$ & $\begin{array}{r}94.0 \\
112.4 \\
153.0 \\
133.0 \\
108.9\end{array}$ & $\begin{array}{l}36.0 \\
41.0 \\
65.0 \\
75.0 \\
71.0\end{array}$ & $\begin{array}{l}66.1 \\
53.0 \\
23.1 \\
46.0 \\
17.8\end{array}$ & $\begin{array}{l}0 \\
1 \\
1 \\
1 \\
1\end{array}$ & $\begin{array}{l}1 \\
1 \\
1 \\
1 \\
1\end{array}$ & $\begin{array}{l}1 \\
1 \\
3 \\
1 \\
8\end{array}$ & $\begin{array}{l}1 \\
1 \\
3 \\
1 \\
3\end{array}$ \\
\hline $\begin{array}{l}N \\
N \\
N \\
N \\
N\end{array}$ & $\begin{array}{l}419 \\
428 \\
421 \\
422 \\
423\end{array}$ & $\begin{array}{r}135100 \\
79600 \\
20000 \\
61000 \\
71000\end{array}$ & $\begin{array}{r}188000 \\
69600 \\
42000 \\
102000 \\
29700\end{array}$ & $\begin{array}{l}150.4 \\
169.0 \\
295.3 \\
150.0 \\
161.0\end{array}$ & $\begin{array}{l}148.0 \\
167.0 \\
293.5 \\
147.8 \\
160.0\end{array}$ & $\begin{array}{l}138.0 \\
157.0 \\
283.5 \\
137.8 \\
150 . \varnothing\end{array}$ & $\begin{array}{l}75.0 \\
49.0 \\
70.0 \\
55.0 \\
70.0\end{array}$ & $\begin{array}{r}162.0 \\
38.0 \\
27.7 \\
80.0 \\
10.5\end{array}$ & $\begin{array}{l}1 \\
0 \\
1 \\
1 \\
1\end{array}$ & $\begin{array}{l}3 \\
1 \\
1 \\
1 \\
1\end{array}$ & $\begin{array}{l}1 \\
3 \\
6 \\
3 \\
8\end{array}$ & $\begin{array}{l}2 \\
3 \\
1 \\
1 \\
3\end{array}$ \\
\hline
\end{tabular}


Appendix.-Location and basic physical features of recharge basins on Long Island,

\begin{tabular}{|c|c|c|c|c|c|c|c|c|}
\hline \multicolumn{2}{|c|}{$\begin{array}{l}\text { BASIN } \\
\text { NUMBER }\end{array}$} & \multicolumn{2}{|c|}{ NEAREST INTERSECTION } & $\begin{array}{l}\text { LATITUDE } \\
\text { LONGITUDE, } \\
0^{\prime \prime}, 0^{\prime},\end{array}$ & $\begin{array}{l}\text { DATE } \\
\text { BUILT } \\
\text { YrMoD }\end{array}$ & COMMUNITY & $\begin{array}{l}\text { DESIGN } \\
\text { CAPACITY } \\
\text { (cubic }\end{array}$ & $\begin{array}{l}\text { ACTUAL } \\
\text { CAPACITY } \\
\text { feet) }\end{array}$ \\
\hline $\begin{array}{l}N \\
N \\
N \\
N \\
N \\
N\end{array}$ & $\begin{array}{l}424 \\
425 \\
426 \\
427 \\
428\end{array}$ & $\begin{array}{l}\text { MARGINAL ROAD } \\
\text { SOUTHWOODS ROAD } \\
\text { L.I.E. } \\
\text { JERICHO TPKE } \\
\text { KALDA LANE }\end{array}$ & $\begin{array}{l}\text { KEY PLACE } \\
\text { SEARINGTON DRIVE } \\
\text { RITA ST. } \\
\text { CEDAR ST. } \\
\text { OLD COUNTRY ROAD }\end{array}$ & $\begin{array}{l}4 \oslash 4795 \varnothing 733216 \\
4949360732857 \\
4947599732937 \\
4948189732931 \\
494631 \oslash 732852\end{array}$ & $\begin{array}{l}560606 \\
570809 \\
690229 \\
590430 \\
581015\end{array}$ & $\begin{array}{l}105 \\
126 \\
126 \\
126 \\
122\end{array}$ & $\begin{array}{r}374000 \\
187 \varnothing 00 \\
248800 \\
202000 \\
88000\end{array}$ & $\begin{array}{l}441000 \\
192000 \\
367 \varnothing 0 \varnothing \\
20300 \varnothing \\
12000 \varnothing\end{array}$ \\
\hline $\begin{array}{l}N \\
N \\
N \\
N \\
N \\
N\end{array}$ & $\begin{array}{l}429 \\
430 \\
431 \\
432 \\
433\end{array}$ & $\begin{array}{l}\text { MICHAEL DRIVE } \\
\text { WENWOOD DR. } \\
\text { HARBOR HILL RD. } \\
\text { MIMOSA DR. } \\
\text { WHEATLEY ROAD }\end{array}$ & $\begin{array}{l}\text { EILEEN WAY } \\
\text { CEDAR SWAMP RD. } \\
\text { GUINEA WOODS RD. } \\
\text { HARBOR HILL RD. } \\
\text { POST ROAD }\end{array}$ & $\begin{array}{l}4 \varnothing 4806 \varnothing 733101 \\
4 \varnothing 4805 \varnothing 733346 \\
4 \varnothing 47430733754 \\
4 \varnothing 47430733837 \\
4 \varnothing 4747 \varnothing 733513\end{array}$ & $\begin{array}{l}550711 \\
579513 \\
560786 \\
590383 \\
541013\end{array}$ & $\begin{array}{r}108 \\
93 \\
53 \\
53 \\
118\end{array}$ & $\begin{array}{r}1069000 \\
607 \varnothing 00 \\
1090000 \\
275000 \\
67 \varnothing 000\end{array}$ & $\begin{array}{r}1275000 \\
604000 \\
1060000 \\
311000 \\
71600\end{array}$ \\
\hline $\begin{array}{l}N \\
N \\
N \\
N \\
N\end{array}$ & $\begin{array}{l}434 \\
435 \\
436 \\
437 \\
438\end{array}$ & $\begin{array}{l}\text { HOLLY LA. } \\
\text { TERMINAL DRIVE } \\
\text { PRESCOTT PL. } \\
\text { BLOOMINGDALE RD. } \\
\text { WOODBURY RD. }\end{array}$ & $\begin{array}{l}\text { JERICHO TPKE. } \\
\text { SUNNYSIDE BLVD. } \\
\text { ROUND SWAMP RD. } \\
\text { BROADWAY } \\
\text { GLORIA DR. }\end{array}$ & 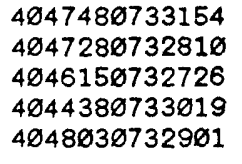 & $\begin{array}{l}580520 \\
590313 \\
590414 \\
560510 \\
590810\end{array}$ & $\begin{array}{l}105 \\
122 \\
116 \\
104 \\
128\end{array}$ & $\begin{array}{l}305000 \\
552000 \\
291000 \\
- \\
1450000\end{array}$ & $\begin{array}{l}294000 \\
606000 \\
307000 \\
- \\
151000\end{array}$ \\
\hline $\begin{array}{l}N \\
N \\
N \\
N \\
N\end{array}$ & $\begin{array}{l}439 \\
44 \varnothing \\
441 \\
442 \\
443\end{array}$ & $\begin{array}{l}\text { COOLIDGE DR. } \\
\text { LEANORE DRIVE } \\
\text { BARRY LANE } \\
\text { JUNEAU BOULEVARD } \\
\text { KODIAK DRIVE }\end{array}$ & $\begin{array}{l}\text { ALBERMARLE AVE. } \\
\text { DANIEL DRIVE } \\
\text { ROUND SWAMP ROAD } \\
\text { BEAUFORT LANE } \\
\text { HEADLEY WAY }\end{array}$ & $\begin{array}{l}4 \varnothing 43 \varnothing 6 \varnothing 7334 \varnothing 7 \\
494254 \varnothing 732558 \\
4 \varnothing 4517 \varnothing 732718 \\
4 \varnothing 4835 \varnothing 732746 \\
494817 \varnothing 732735\end{array}$ & $\begin{array}{l}590407 \\
680627 \\
591823 \\
590219 \\
590219\end{array}$ & $\begin{array}{r}8 \\
125 \\
116 \\
128 \\
128\end{array}$ & $\begin{array}{r}336000 \\
81500 \\
268000 \\
382000 \\
277000\end{array}$ & $\begin{array}{r}28800 \varnothing \\
8 \varnothing 8 \varnothing \varnothing \\
2620 \varnothing \varnothing \\
44 \varnothing \varnothing \varnothing \varnothing \\
2710 \varnothing \varnothing\end{array}$ \\
\hline $\begin{array}{l}N \\
N \\
N \\
N \\
N\end{array}$ & $\begin{array}{l}444 \\
445 \\
446 \\
447 \\
448\end{array}$ & $\begin{array}{l}\text { LINDSAY ST. } \\
\text { CLAREMONT ROAD } \\
\text { EVELYN DR. } \\
\text { ROUND SWAMP ROAD } \\
\text { FOX LANE }\end{array}$ & $\begin{array}{l}\text { PLEASANT AVE. } \\
\text { ROUND SWAMP ROAD } \\
\text { PLAINVIEW RD. } \\
\text { OLD COUNTRY ROAD } \\
\text { JERICHO TPKE. }\end{array}$ & $\begin{array}{l}4047430732832 \\
4045290732737 \\
404535073282 \varnothing \\
4046400732644 \\
4 \varnothing 47550733136\end{array}$ & $\begin{array}{l}690329 \\
591214 \\
590903 \\
611218 \\
591214\end{array}$ & $\begin{array}{l}122 \\
116 \\
116 \\
116 \\
105\end{array}$ & $\begin{array}{r}125000 \\
65000 \\
123000 \\
71600 \\
266000\end{array}$ & $\begin{array}{r}108000 \\
63600 \\
124000 \\
68000 \\
254000\end{array}$ \\
\hline $\begin{array}{l}N \\
N \\
N \\
N \\
N\end{array}$ & $\begin{array}{l}449 \\
450 \\
451 \\
452 \\
453\end{array}$ & $\begin{array}{l}\text { PRIVATE LA. } \\
\text { NEWTOWN ROAD } \\
\text { WINDHORST AVE. } \\
\text { MEADOWBROOK PKWY } \\
\text { WASHINGTON AVE. }\end{array}$ & $\begin{array}{l}\text { SPLIT ROCK RD. } \\
\text { NEWTOWN PLAZA } \\
\text { BROADWAY } \\
\text { IST, ST. } \\
\text { L.I.E. }\end{array}$ & $\begin{array}{l}4 \varnothing 49490733 \varnothing 27 \\
4 \varnothing 46529732796 \\
4 \varnothing 4339 \varnothing 732837 \\
4 \varnothing 449 \varnothing \varnothing 733533 \\
4 \varnothing 47 \varnothing 9 \varnothing 732738\end{array}$ & $\begin{array}{l}590319 \\
640217 \\
600621 \\
600621 \\
620423\end{array}$ & $\begin{array}{r}126 \\
122 \\
92 \\
14 \\
122\end{array}$ & $\begin{array}{r}1249000 \\
51000 \\
88600 \\
252000\end{array}$ & $\begin{array}{r}322000 \\
1311000 \\
77400 \\
90700 \\
251000\end{array}$ \\
\hline $\begin{array}{l}N \\
N \\
N \\
N \\
N\end{array}$ & $\begin{array}{l}454 \\
455 \\
456 \\
457 \\
458\end{array}$ & $\begin{array}{l}\text { ROXTON RD. } \\
\text { BETHPAGE RD. } \\
\text { JUNEAU BLVD. } \\
\text { FAMS COURT } \\
\text { CORNELL PL. }\end{array}$ & $\begin{array}{l}\text { WOODBURY RD. } \\
\text { CLINTON LA. } \\
\text { BERING COURT } \\
\text { CANTIAGUE ROCK R } \\
\text { BETHPAGE ST. PKY }\end{array}$ & $\begin{array}{l}4 \emptyset 47320732932 \\
4 \varnothing 46500733111 \\
4 \emptyset 48110732748 \\
4 \varnothing 46280733310 \\
4 \varnothing 43260732805\end{array}$ & $\begin{array}{l}690203 \\
621231 \\
6062 ø 1 \\
600322 \\
590311\end{array}$ & $\begin{array}{l}128 \\
104 \\
128 \\
105 \\
125\end{array}$ & $\begin{array}{l}70000 \\
115900 \\
280000 \\
126000 \\
373000\end{array}$ & $\begin{array}{r}90000 \\
115600 \\
423000 \\
79000 \\
150000\end{array}$ \\
\hline $\begin{array}{l}N \\
N \\
N \\
N \\
N\end{array}$ & $\begin{array}{l}459 \\
460 \\
461 \\
462 \\
463\end{array}$ & $\begin{array}{l}\text { DOGWOOD ROAD } \\
\text { BASIN ST. } \\
\text { FAIRWAY ORIVE } \\
\text { MARCUS AVE. } \\
\text { OLD COURT HOUSE }\end{array}$ & $\begin{array}{l}\text { PARKVIEW DRIVE } \\
\text { S. TERMINAL DR. } \\
\text { ROUND SWAMP ROAD } \\
\text { MEADOW FARM ROAD } \\
\text { MONTEREY DRIVE }\end{array}$ & $\begin{array}{l}4 \emptyset 46210733938 \\
4 \emptyset 4715 \varnothing 7328 \varnothing 6 \\
4 \emptyset 45390732726 \\
4 \varnothing 4518 \varnothing 734 \varnothing 51 \\
4 \varnothing 4619 \varnothing 734 \varnothing 15\end{array}$ & $\begin{array}{l}580224 \\
600920 \\
650412 \\
600322 \\
6307\end{array}$ & $\begin{array}{r}87 \\
122 \\
116 \\
71 \\
71\end{array}$ & $\begin{array}{l}1040000 \\
112500 \\
634 \varnothing 00 \\
-\end{array}$ & $\begin{array}{r}650000 \\
1270000 \\
116000 \\
627000 \\
240000\end{array}$ \\
\hline $\begin{array}{l}N \\
N \\
N \\
N \\
N\end{array}$ & $\begin{array}{l}464 \\
465 \\
466 \\
467 \\
468\end{array}$ & $\begin{array}{l}\text { WASHINGTON AVE. } \\
\text { HORSE HOLLOW RD. } \\
\text { RUSSELL. PARK RD. } \\
\text { L.I.R.R. } \\
\text { I.U. WILLETS RD. }\end{array}$ & $\begin{array}{l}\text { SORGI COURT } \\
\text { BIRCH HILL RD. } \\
\text { ROBBINS LANE } \\
\text { MERRITTS ROAD } \\
\text { SEARINGTOWN ROAD }\end{array}$ & $\begin{array}{l}4047480732718 \\
4 \varnothing 53190733546 \\
4 \varnothing 48140733131 \\
4 \varnothing 43560732719 \\
4 \varnothing 46110733932\end{array}$ & $\begin{array}{l}686526 \\
610512 \\
610124 \\
6387 \\
650213\end{array}$ & $\begin{array}{r}122 \\
106 \\
108 \\
99 \\
87\end{array}$ & $\begin{array}{l}284000 \\
- \\
46500 \\
- \\
-\end{array}$ & $\begin{array}{l}338600 \\
- \\
63000 \\
- \\
-\end{array}$ \\
\hline $\begin{array}{l}N \\
N \\
N \\
N \\
N\end{array}$ & $\begin{array}{l}469 \\
470 \\
471 \\
472 \\
473\end{array}$ & $\begin{array}{l}\text { ALEX LANE } \\
\text { APPLEGREEN ORIVE } \\
\text { CONLON RD. } \\
\text { MADISON AVE. } \\
\text { WASHINGTON AVE. }\end{array}$ & $\begin{array}{l}\text { VALENTINE AVENUE } \\
\text { WHEATLEY ROAD } \\
\text { CLINTON AVE. } \\
\text { ROCKLAND DR. } \\
\text { L.I.E. }\end{array}$ & $\begin{array}{l}4 \varnothing 5054 \varnothing 733741 \\
4 \varnothing 4727 \varnothing 733617 \\
4 \varnothing 4106 \varnothing 73351 \varnothing \\
4 \varnothing 4632 \varnothing 7333 \varnothing 5 \\
4 \varnothing 47310732725\end{array}$ & $\begin{array}{l}401108 \\
611101 \\
620420 \\
590819 \\
620509\end{array}$ & $\begin{array}{r}100 \\
118 \\
37 \\
105 \\
122\end{array}$ & $\begin{array}{l}\overline{164000} \\
\overline{-} \\
650000 \\
275000\end{array}$ & $\begin{array}{l}38500 \\
15600 \emptyset \\
- \\
53000 \emptyset \\
27380 \varnothing\end{array}$ \\
\hline $\begin{array}{l}N \\
N \\
N \\
N \\
N\end{array}$ & $\begin{array}{l}474 \\
475 \\
476 \\
477 \\
478\end{array}$ & $\begin{array}{l}\text { WOODBURY ROAD } \\
\text { WASHINGTON AVE. } \\
\text { DOGWODD HILL RD. } \\
\text { BOUNDARY AVE. } \\
\text { COLGATE DRIVE }\end{array}$ & $\begin{array}{l}\text { JERICHO TPKE } \\
\text { NORTHERN ST. PKY } \\
\text { WHEATLEY RD. } \\
\text { STEWART AVE. } \\
\text { HARVARD DRIVE }\end{array}$ & $\begin{array}{l}4 \varnothing 4934 \varnothing 732759 \\
4 \varnothing 4816 \varnothing 7327 \emptyset 7 \\
4 \varnothing 49 \varnothing 2 \varnothing 733356 \\
4 \varnothing 42590732855 \\
4 \varnothing 48 \varnothing \varnothing \varnothing 732731\end{array}$ & $\begin{array}{l}631125 \\
620511 \\
620608 \\
541014 \\
620412\end{array}$ & $\begin{array}{r}128 \\
122 \\
93 \\
121 \\
122\end{array}$ & $\begin{array}{r}1416000 \\
112500 \\
22000 \varnothing \\
61500 \emptyset \\
54500 \varnothing\end{array}$ & $\begin{array}{r}1716000 \\
127800 \\
281000 \\
431000 \\
585500\end{array}$ \\
\hline
\end{tabular}


N.Y. [Dash indicates no data. Altitudes are in feet above sea level.] (cont.)

\begin{tabular}{|c|c|c|c|c|c|c|c|c|c|c|c|c|}
\hline \multicolumn{2}{|c|}{$\begin{array}{l}\text { BASIN } \\
\text { NUMBER }\end{array}$} & $\begin{array}{l}\text { MAX. } \\
\text { AREA } \\
\text { (squar }\end{array}$ & $\begin{array}{c}\text { BASIN } \\
\text { AREA } \\
\text { feet) }\end{array}$ & RIM & $\begin{array}{l}\text { OVER } \\
\text { FLOW }\end{array}$ & $\begin{array}{l}\text { ITUDE--- } \\
-\quad \text { BOT- } \\
\text { TOM }\end{array}$ & $\begin{array}{l}\text { WATER } \\
\text { TABLE }\end{array}$ & $\begin{array}{c}\text { DRAIN. } \\
\text { AREA } \\
\text { (acres) }\end{array}$ & \multicolumn{2}{|c|}{$\begin{array}{l}---B A S I N-- \\
\text { STATUS USE }\end{array}$} & $\begin{array}{l}\text { GEO. } \\
\text { UNIT }\end{array}$ & $\begin{array}{l}\text { SOIL } \\
\text { UNIT }\end{array}$ \\
\hline $\begin{array}{l}N \\
N \\
N \\
N \\
N\end{array}$ & $\begin{array}{l}424 \\
425 \\
426 \\
427 \\
428\end{array}$ & $\begin{array}{l}4840 \emptyset \\
2280 \emptyset \\
4360 \emptyset \\
2210 \emptyset \\
14000\end{array}$ & $\begin{array}{l}75000 \\
56000 \\
77500 \\
48300 \\
32400\end{array}$ & $\begin{array}{l}164 . \varnothing \\
2 ø 2 . \varnothing \\
197 . \varnothing \\
208.6 \\
149.3\end{array}$ & $\begin{array}{l}161 . \varnothing \\
201 . \varnothing \\
195 . \varnothing \\
297 . \varnothing \\
151 . \varnothing\end{array}$ & $\begin{array}{l}151.5 \\
191 . \varnothing \\
185 . \varnothing \\
197 . \varnothing \\
139 . \varnothing\end{array}$ & $\begin{array}{l}75.0 \\
64 . \varnothing \\
79 . \varnothing \\
75 . \varnothing \\
81 . \varnothing\end{array}$ & $\begin{array}{l}58.9 \\
26.0 \\
38.6 \\
27.7 \\
12.0\end{array}$ & $\begin{array}{l}1 \\
1 \\
1 \\
1 \\
1\end{array}$ & $\begin{array}{l}1 \\
1 \\
1 \\
1 \\
1\end{array}$ & $\begin{array}{l}1 \\
1 \\
1 \\
1 \\
1\end{array}$ & $\begin{array}{l}1 \\
2 \\
2 \\
2 \\
2\end{array}$ \\
\hline $\begin{array}{l}N \\
N \\
N \\
N \\
N\end{array}$ & $\begin{array}{l}429 \\
430 \\
431 \\
432 \\
433\end{array}$ & $\begin{array}{r}127200 \\
67700 \\
101800 \\
29700 \\
66300\end{array}$ & $\begin{array}{r}190600 \\
130000 \\
28200 \\
78200 \\
110000\end{array}$ & $\begin{array}{l}192 . \varnothing \\
219 . \varnothing \\
219 . \varnothing \\
153 . \varnothing \\
236 . \varnothing\end{array}$ & $\begin{array}{l}191 . \varnothing \\
219 . \varnothing \\
217.6 \\
152 . \varnothing \\
236 . \varnothing\end{array}$ & $\begin{array}{l}172.0 \\
209.0 \\
207.0 \\
140.0 \\
224.0\end{array}$ & $\begin{array}{l}79.0 \\
74.0 \\
54.0 \\
10.0 \\
71.0\end{array}$ & $\begin{array}{r}112.9 \\
83.4 \\
98 . \varnothing \\
25.3 \\
92.0\end{array}$ & $\begin{array}{l}0 \\
1 \\
0 \\
1 \\
1\end{array}$ & $\begin{array}{l}2 \\
1 \\
1 \\
1 \\
1\end{array}$ & $\begin{array}{l}1 \\
8 \\
1 \\
4 \\
8\end{array}$ & $\begin{array}{l}1 \\
1 \\
1 \\
3 \\
1\end{array}$ \\
\hline $\begin{array}{l}N \\
N \\
N \\
N \\
N\end{array}$ & $\begin{array}{l}434 \\
435 \\
436 \\
437 \\
438\end{array}$ & $\begin{array}{r}33000 \\
50500 \\
33900 \\
197000 \\
17800\end{array}$ & $\begin{array}{r}60000 \\
98000 \\
68100 \\
256700 \\
43500\end{array}$ & $\begin{array}{l}203.1 \\
177.5 \\
176.0 \\
106.9 \\
192.0\end{array}$ & $\begin{array}{r}189.0 \\
166.0 \\
174.7 \\
190.5\end{array}$ & $\begin{array}{r}189.0 \\
164.0 \\
164.7 \\
93.5 \\
180.5\end{array}$ & $\begin{array}{l}77.0 \\
80.0 \\
73.0 \\
69.0 \\
77.0\end{array}$ & $\begin{array}{r}42.0 \\
48.3 \\
35.5 \\
19 . \overline{9}\end{array}$ & $\begin{array}{l}0 \\
1 \\
1 \\
1 \\
1\end{array}$ & $\begin{array}{l}1 \\
1 \\
1 \\
1 \\
3\end{array}$ & $\begin{array}{l}1 \\
1 \\
8 \\
1 \\
1\end{array}$ & $\begin{array}{l}1 \\
1 \\
1 \\
2 \\
2\end{array}$ \\
\hline $\begin{array}{l}N \\
N \\
N \\
N \\
N\end{array}$ & $\begin{array}{l}439 \\
440 \\
441 \\
442 \\
443\end{array}$ & $\begin{array}{l}27700 \\
11100 \\
29800 \\
31100 \\
27300\end{array}$ & $\begin{array}{l}554 \varnothing \varnothing \\
2600 \varnothing \\
77 \varnothing \varnothing \varnothing \\
67500 \\
6250 \varnothing\end{array}$ & $\begin{array}{r}79.0 \\
56.6 \\
122.5 \\
257.0 \\
274.8\end{array}$ & $\begin{array}{r}76.5 \\
56.6 \\
120.5 \\
254.0 \\
261.0\end{array}$ & $\begin{array}{r}65 . \varnothing \\
48 . \varnothing \\
110.5 \\
239.0 \\
250 . \varnothing\end{array}$ & $\begin{array}{l}50.0 \\
41.0 \\
67.0 \\
72.0 \\
72.0\end{array}$ & $\begin{array}{r}4.5 \\
11.2 \\
36.9 \\
52.4 \\
38.8\end{array}$ & $\begin{array}{l}1 \\
1 \\
1 \\
0 \\
1\end{array}$ & $\begin{array}{l}1 \\
1 \\
1 \\
0 \\
1\end{array}$ & $\begin{array}{l}1 \\
1 \\
8 \\
1 \\
8\end{array}$ & $\begin{array}{l}2 \\
3 \\
1 \\
1 \\
1\end{array}$ \\
\hline $\begin{array}{l}N \\
N \\
N \\
N \\
N\end{array}$ & $\begin{array}{l}444 \\
445 \\
446 \\
447 \\
448\end{array}$ & $\begin{array}{r}12600 \\
8200 \\
14900 \\
9200 \\
28400\end{array}$ & $\begin{array}{l}34300 \\
22800 \\
33600 \\
25700 \\
55300\end{array}$ & $\begin{array}{l}184.4 \\
149.8 \\
127.0 \\
192.0 \\
201.7\end{array}$ & $\begin{array}{l}183.0 \\
148.0 \\
125.6 \\
188.4 \\
199.2\end{array}$ & $\begin{array}{l}173.0 \\
138.0 \\
115.6 \\
180.4 \\
189.2\end{array}$ & $\begin{array}{l}80.0 \\
71.0 \\
73.0 \\
73.0 \\
78.0\end{array}$ & $\begin{array}{r}17.3 \\
8.9 \\
16.8 \\
9.9 \\
60.8\end{array}$ & $\begin{array}{l}1 \\
1 \\
1 \\
1 \\
0\end{array}$ & $\begin{array}{l}1 \\
1 \\
1 \\
3 \\
1\end{array}$ & $\begin{array}{l}1 \\
8 \\
8 \\
8 \\
1\end{array}$ & $\begin{array}{l}1 \\
1 \\
1 \\
1 \\
1\end{array}$ \\
\hline $\begin{array}{l}N \\
N \\
N \\
N \\
N\end{array}$ & $\begin{array}{l}449 \\
450 \\
451 \\
452 \\
453\end{array}$ & $\begin{array}{r}24300 \\
142300 \\
8700 \\
12400 \\
23200\end{array}$ & $\begin{array}{r}49500 \\
- \\
202000 \\
29000 \\
51200\end{array}$ & $\begin{array}{r}299.0 \\
180.0 \\
86.3 \\
91.3 \\
171.2\end{array}$ & $\begin{array}{r}207.0 \\
160.7 \\
86.3 \\
89.7 \\
168.0\end{array}$ & $\begin{array}{r}193.0 \\
148.7 \\
76.3 \\
79.7 \\
158.0\end{array}$ & $\begin{array}{l}63.0 \\
77.0 \\
55.8 \\
57.0 \\
77.0\end{array}$ & $\begin{array}{r}141.0 \\
98.3 \\
7.0 \\
7.0 \\
27.7\end{array}$ & $\begin{array}{l}1 \\
1 \\
0 \\
1 \\
1\end{array}$ & $\begin{array}{l}1 \\
1 \\
1 \\
4 \\
3\end{array}$ & $\begin{array}{l}1 \\
1 \\
1 \\
1 \\
1\end{array}$ & $\begin{array}{l}1 \\
1 \\
1 \\
2 \\
1\end{array}$ \\
\hline $\begin{array}{l}N \\
N \\
N \\
N \\
N\end{array}$ & $\begin{array}{l}454 \\
455 \\
456 \\
457 \\
458\end{array}$ & $\begin{array}{r}11900 \\
14200 \\
46700 \\
9600 \\
27900\end{array}$ & $\begin{array}{r}290000 \\
- \\
128000 \\
37900 \\
50800\end{array}$ & $\begin{array}{r}181.0 \\
168.8 \\
253.3 \\
158.2 \\
59.0\end{array}$ & $\begin{array}{r}179.7 \\
165.6 \\
253.8 \\
155.7 \\
56.5\end{array}$ & $\begin{array}{r}169.7 \\
156 . \varnothing \\
243 . \varnothing \\
145.7 \\
51 . \varnothing\end{array}$ & $\begin{array}{l}81.0 \\
80.0 \\
73.0 \\
75.0 \\
44.0\end{array}$ & $\begin{array}{r}9.4 \\
15.9 \\
38.4 \\
19.8 \\
50.5\end{array}$ & $\begin{array}{l}1 \\
1 \\
1 \\
1 \\
1\end{array}$ & $\begin{array}{l}1 \\
1 \\
1 \\
1 \\
1\end{array}$ & $\begin{array}{l}1 \\
1 \\
8 \\
1 \\
1\end{array}$ & $\begin{array}{l}2 \\
2 \\
1 \\
2 \\
1\end{array}$ \\
\hline $\begin{array}{l}N \\
N \\
N \\
N \\
N\end{array}$ & $\begin{array}{l}459 \\
460 \\
461 \\
462 \\
463\end{array}$ & $\begin{array}{r}92900 \\
108300 \\
14700 \\
79000 \\
58200\end{array}$ & $\begin{array}{r}104800 \\
230800 \\
344 \varnothing 0 \\
- \\
-\end{array}$ & $\begin{array}{l}124.0 \\
189.0 \\
164.8 \\
107.5 \\
115.7\end{array}$ & $\begin{array}{r}124.0 \\
189.0 \\
160.2 \\
102.0 \\
-\end{array}$ & $\begin{array}{r}118.0 \\
177.0 \\
150.2 \\
92.0 \\
102.0\end{array}$ & $\begin{array}{l}44.0 \\
81.0 \\
78.0 \\
38.0 \\
43 . \varnothing\end{array}$ & $\begin{array}{r}122.8 \\
81.9 \\
15.5 \\
99.8 \\
37.8\end{array}$ & $\begin{array}{l}0 \\
1 \\
1 \\
1 \\
0\end{array}$ & $\begin{array}{l}1 \\
1 \\
1 \\
1 \\
1\end{array}$ & $\begin{array}{l}1 \\
8 \\
8 \\
1 \\
1\end{array}$ & $\begin{array}{l}1 \\
1 \\
1 \\
1 \\
2\end{array}$ \\
\hline $\begin{array}{l}N \\
N \\
N \\
N \\
N\end{array}$ & $\begin{array}{l}464 \\
465 \\
466 \\
467 \\
468\end{array}$ & $\begin{array}{r}37800 \\
55600 \\
7600 \\
157000 \\
226000\end{array}$ & $\begin{array}{r}56300 \\
236000 \\
25500 \\
213000 \\
297000\end{array}$ & $\begin{array}{r}221.5 \\
33.0 \\
219.5 \\
70.0 \\
124.1\end{array}$ & $\begin{array}{r}220 . \varnothing \\
215 . \overline{6} \\
117 . \bar{\varnothing}\end{array}$ & $\begin{array}{r}210.0 \\
23.5 \\
206.0 \\
57.9 \\
108.8\end{array}$ & $\begin{array}{r}75 . \varnothing \\
78.0 \\
56.0 \\
45 . \varnothing\end{array}$ & $\begin{array}{r}39.0 \\
62.0 \\
6.4 \\
151.2 \\
14.7\end{array}$ & $\begin{array}{l}1 \\
1 \\
0 \\
1 \\
0\end{array}$ & $\begin{array}{l}1 \\
1 \\
3 \\
2 \\
1\end{array}$ & $\begin{array}{l}8 \\
3 \\
6 \\
1 \\
1\end{array}$ & $\begin{array}{l}1 \\
1 \\
1 \\
1 \\
1\end{array}$ \\
\hline $\begin{array}{l}N \\
N \\
N \\
N \\
N\end{array}$ & $\begin{array}{l}469 \\
470 \\
471 \\
472 \\
473\end{array}$ & $\begin{array}{r}147700 \\
5600 \\
49700 \\
53000 \\
28400\end{array}$ & $\begin{array}{l}34100 \\
71800 \\
99000 \\
78500\end{array}$ & $\begin{array}{r}115.0 \\
176.0 \\
47.4 \\
157.5 \\
299.3\end{array}$ & $\begin{array}{r}- \\
178 . \varnothing \\
\bar{\varnothing} \\
152 . \varnothing \\
203 . \varnothing\end{array}$ & $\begin{array}{r}104.0 \\
168.0 \\
34.0 \\
142.6 \\
192.0\end{array}$ & $\begin{array}{l}46.0 \\
63.0 \\
47.4 \\
75.0 \\
77.0\end{array}$ & $\begin{array}{r}2.4 \\
25.3 \\
182.8 \\
88.6 \\
38.2\end{array}$ & $\begin{array}{l}1 \\
1 \\
1 \\
1 \\
0\end{array}$ & $\begin{array}{l}4 \\
1 \\
1 \\
1 \\
1\end{array}$ & $\begin{array}{l}3 \\
1 \\
1 \\
1 \\
8\end{array}$ & $\begin{array}{l}1 \\
1 \\
1 \\
2 \\
1\end{array}$ \\
\hline $\begin{array}{l}N \\
N \\
N \\
N \\
N\end{array}$ & $\begin{array}{l}474 \\
475 \\
476 \\
477 \\
478\end{array}$ & $\begin{array}{r}122300 \\
1480 \varnothing \\
287 \varnothing \varnothing \\
4 \varnothing 00 \varnothing \\
63200\end{array}$ & $\begin{array}{r}229000 \\
45800 \\
58000 \\
73000 \\
104000\end{array}$ & $\begin{array}{r}143.3 \\
263.6 \\
228.0 \\
63.0 \\
245.0\end{array}$ & $\begin{array}{r}140.0 \\
261.0 \\
214.0 \\
61.8 \\
236.0\end{array}$ & $\begin{array}{r}126 . \varnothing \\
251 . \varnothing \\
203 . \varnothing \\
50 . \varnothing \\
228 . \varnothing\end{array}$ & $\begin{array}{l}62.0 \\
72.0 \\
73.0 \\
46.0 \\
75.0\end{array}$ & $\begin{array}{r}193.4 \\
12.5 \\
88.5 \\
84.2 \\
80 . \varnothing\end{array}$ & $\begin{array}{l}0 \\
0 \\
1 \\
1 \\
0\end{array}$ & $\begin{array}{l}1 \\
1 \\
1 \\
3 \\
1\end{array}$ & $\begin{array}{l}1 \\
8 \\
4 \\
1 \\
8\end{array}$ & $\begin{array}{l}1 \\
1 \\
1 \\
3 \\
1\end{array}$ \\
\hline
\end{tabular}


Appendix.--Location and basic physical features of recharge basins on Long Island,

\begin{tabular}{|c|c|c|c|c|c|c|c|c|}
\hline \multicolumn{2}{|c|}{$\begin{array}{l}\text { BASIN } \\
\text { NUMBER }\end{array}$} & \multicolumn{2}{|c|}{ NEAREST INTERSECTION } & $\begin{array}{l}\text { LATITUDE } \\
\text { LONGITUDE } \\
\circ, n \circ, n\end{array}$ & $\begin{array}{l}\text { DATE } \\
\text { BUILT } \\
\text { YrMOD }\end{array}$ & COMMUNITY & $\begin{array}{l}\text { DESIGN } \\
\text { CAPACITY } \\
\text { (cubic }\end{array}$ & $\begin{array}{l}\text { ACTUAL } \\
\text { CAPACITY } \\
\text { feet) }\end{array}$ \\
\hline $\begin{array}{l}N \\
N \\
N \\
N \\
N\end{array}$ & $\begin{array}{l}479 \\
488 \\
481 \\
482 \\
483\end{array}$ & $\begin{array}{l}\text { GLEN LANE } \\
\text { FAIRBANKS COURT } \\
\text { ACORN LANE } \\
\text { WYNN COURT } \\
\text { GOULD STREET }\end{array}$ & $\begin{array}{l}\text { OVERLOOK DRIVE } \\
\text { FAIRBANKS BLVD. } \\
\text { CHESTNUT DR. } \\
\text { MUTTONTOWN ROAD } \\
\text { EVERGREEN AVENUE }\end{array}$ & $\begin{array}{l}4 \varnothing 5 \emptyset 44 \varnothing 732804 \\
4 \varnothing 4832 \varnothing 7328 \varnothing 3 \\
4 \varnothing 471 \varnothing \varnothing 732941 \\
4 \varnothing 4934 \varnothing 733 \varnothing 56 \\
4 \varnothing 4433 \varnothing 734 \varnothing 31\end{array}$ & $\begin{array}{l}591023 \\
6204 \\
620411 \\
680714 \\
6208\end{array}$ & $\begin{array}{r}167 \\
128 \\
122 \\
115 \\
57\end{array}$ & $\begin{array}{l}640000 \\
510000 \\
95500 \\
203000 \\
-\end{array}$ & $\begin{array}{l}744000 \\
555000 \\
161000 \\
200000 \\
-\end{array}$ \\
\hline $\begin{array}{l}N \\
N \\
N \\
N \\
N\end{array}$ & $\begin{array}{l}484 \\
485 \\
486 \\
487 \\
488\end{array}$ & $\begin{array}{l}\text { COLGATE LANE } \\
\text { HARVARD DRIVE } \\
\text { JEFFERSON AVE } \\
\text { NEW LATTINGTOWN } \\
\text { PENNSYLVANIA AVE }\end{array}$ & $\begin{array}{l}\text { WOODBURY ROAD } \\
\text { BUCKNELL DRIVE } \\
\text { NEWBRIDGE RD. } \\
\text { DOSORIS LA. } \\
\text { PHILADELPHIA AVE }\end{array}$ & $\begin{array}{l}4 \varnothing 482 \varnothing \varnothing 732859 \\
4 \varnothing 48 \emptyset 7 \varnothing 732728 \\
4 \varnothing 4511 \varnothing 733156 \\
4 \varnothing 53 \varnothing 2 \varnothing 7337 \varnothing 6 \\
4 \varnothing 4 \varnothing 47 \varnothing 732634\end{array}$ & $\begin{array}{l}630211 \\
640914 \\
590729 \\
650202 \\
530612\end{array}$ & $\begin{array}{l}128 \\
122 \\
104 \\
1 \varnothing \varnothing \\
111\end{array}$ & $\begin{array}{c}2033000 \\
149600 \\
- \\
162000 \\
-\end{array}$ & $\begin{array}{c}2021000 \\
149600 \\
- \\
194300 \\
-\end{array}$ \\
\hline $\begin{array}{l}N \\
N \\
N \\
N \\
N\end{array}$ & $\begin{array}{l}489 \\
490 \\
491 \\
492 \\
493\end{array}$ & $\begin{array}{l}\text { MASSACHUSSETS AV } \\
\text { CYPRESS ST. } \\
\text { ILLINOIS AVE } \\
\text { SABINE ROAD } \\
\text { MORRIS ROAD }\end{array}$ & $\begin{array}{l}\text { OCEAN AVE. } \\
\text { PACIFIC ST. } \\
\text { OCEAN AVE. } \\
\text { PALATINE COURT } \\
\text { HUNT PLACE }\end{array}$ & $\begin{array}{l}4 \emptyset 4117 \varnothing 732627 \\
4 \emptyset 41410732637 \\
4 \varnothing 41480732622 \\
4 \varnothing 5 \varnothing 24 \varnothing 732914 \\
4 \varnothing 4309 \emptyset 732833\end{array}$ & $\begin{array}{l}54 \\
540510 \\
541013 \\
630731 \\
630315\end{array}$ & $\begin{array}{l}111 \\
111 \\
111 \\
126 \\
121\end{array}$ & $\begin{array}{l}- \\
- \\
\overline{168080} \\
47200\end{array}$ & $\begin{array}{l}- \\
- \\
\overline{129000} \\
71000\end{array}$ \\
\hline $\begin{array}{l}N \\
N \\
N \\
N \\
N\end{array}$ & $\begin{array}{l}494 \\
495 \\
496 \\
497 \\
498\end{array}$ & $\begin{array}{l}\text { STEWART AVENUE } \\
\text { ARTHUR AVENUE } \\
\text { BETHPAGE RD. } \\
\text { HEMPSTEAD TPKE. } \\
\text { FLOWER LA. }\end{array}$ & $\begin{array}{l}\text { HEMPSTEAD TPKE } \\
\text { STEWART AVENUE } \\
\text { BLUEBIRD LA. } \\
\text { MAKOFSKE AVE. } \\
\text { EAST VIEW AVE. }\end{array}$ & $\begin{array}{l}4 \varnothing 4337 \varnothing 732855 \\
4 \varnothing 4354 \varnothing 73285 \emptyset \\
4 \varnothing 4613 \emptyset 732751 \\
4 \varnothing 42310734216 \\
4 \varnothing 4134 \varnothing 732941\end{array}$ & $\begin{array}{l}678601 \\
65 \\
640903 \\
671217 \\
648601\end{array}$ & $\begin{array}{r}92 \\
92 \\
122 \\
10 \\
46\end{array}$ & $\begin{array}{l}- \\
\overline{6} 4000 \\
\overline{44000}\end{array}$ & $\begin{array}{l}- \\
412000 \\
63000 \\
\overline{54000}\end{array}$ \\
\hline $\begin{array}{l}N \\
N \\
N \\
N \\
N\end{array}$ & $\begin{array}{l}499 \\
500 \\
501 \\
502 \\
503\end{array}$ & $\begin{array}{l}\text { JERICHO-O.B.RD. } \\
\text { MAPLE STREET } \\
\text { EDGE ROAD } \\
\text { TARA DRIVE } \\
\text { N. HEMPSTEAD TPK }\end{array}$ & $\begin{array}{l}\text { MUTTONTOWN ROAD } \\
\text { BROOKLYN AVENUE } \\
\text { E.NORWICH RD. } \\
\text { GLEN COVE ROAD } \\
\text { BERRY HILL ROAD }\end{array}$ & $\begin{array}{l}4 \varnothing 49290733139 \\
4 \varnothing 3928 \varnothing 733711 \\
4 \varnothing 490 \varnothing \varnothing 733144 \\
4 \varnothing 4824 \varnothing 733651 \\
4 \varnothing 5 \varnothing 180733017\end{array}$ & $\begin{array}{l}64 \varnothing 519 \\
65 \varnothing 514 \\
54 \varnothing 619 \\
621203 \\
76 \varnothing 322\end{array}$ & $\begin{array}{r}115 \\
2 \\
115 \\
53 \\
126\end{array}$ & $\begin{array}{l}415000 \\
- \\
963000 \\
435000 \\
-\end{array}$ & $\begin{array}{l}416000 \\
- \\
973000 \\
380000 \\
-\end{array}$ \\
\hline $\begin{array}{l}N \\
N \\
N \\
N \\
N\end{array}$ & $\begin{array}{l}504 \\
505 \\
506 \\
507 \\
508\end{array}$ & $\begin{array}{l}\text { KRISTI LANE } \\
\text { POST ROAD } \\
\text { BROAOWOOD PL. } \\
\text { CEDAR SWAMP ROAD } \\
\text { LINDEN LA. }\end{array}$ & $\begin{array}{l}\text { COLGATE LANE } \\
\text { L.I.E. } \\
\text { KIRKWOOD DR. } \\
\text { N. HEMPSTEAD TPK } \\
\text { JERICHO TPKE. }\end{array}$ & $\begin{array}{l}4 \varnothing 4831073285 \emptyset \\
4 \varnothing 4712 \varnothing 733621 \\
4 \varnothing 523 \varnothing \emptyset 7337 \varnothing 8 \\
4 \varnothing 4923 \emptyset 733454 \\
4 \varnothing 46 \varnothing 10733535\end{array}$ & $\begin{array}{l}671002 \\
5808 \\
650801 \\
650202 \\
730402\end{array}$ & $\begin{array}{r}128 \\
89 \\
100 \\
117 \\
118\end{array}$ & $\begin{array}{l}- \\
\overline{43600} \\
720400 \\
457000\end{array}$ & $\begin{array}{l}250000 \\
- \\
84000 \\
850000 \\
425000\end{array}$ \\
\hline $\begin{array}{l}N \\
N \\
N \\
N \\
N\end{array}$ & $\begin{array}{l}509 \\
512 \\
513 \\
514 \\
515\end{array}$ & $\begin{array}{l}\text { OLD HOUSE LA. } \\
\text { ROLLING DRIVE } \\
\text { OLD WESTBURY RD. } \\
\text { FLAMINGO RD. } \\
\text { BERNICE DRIVE }\end{array}$ & $\begin{array}{l}\text { MIDDLE NECK RD. } \\
\text { WHEATLEY ROAD } \\
\text { TERRACE CT. } \\
\text { NORTHERN BLVD. } \\
\text { BELLMORE ROAD }\end{array}$ & 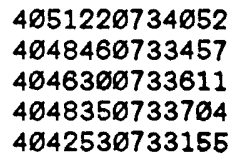 & $\begin{array}{l}640717 \\
641009 \\
660120 \\
650719 \\
640812\end{array}$ & $\begin{array}{r}86 \\
93 \\
118 \\
53 \\
8\end{array}$ & $\begin{array}{r}668000 \\
107000 \\
78900 \\
47800 \\
94000\end{array}$ & $\begin{array}{r}642000 \\
1210000 \\
87000 \\
46000 \\
88000\end{array}$ \\
\hline $\begin{array}{l}N \\
N \\
N \\
N \\
N\end{array}$ & $\begin{array}{l}516 \\
517 \\
518 \\
519 \\
520\end{array}$ & $\begin{array}{l}\text { LINDEN LA. } \\
\text { WOODLEA ROAD } \\
\text { POST ROAD } \\
\text { LAUREL LANE } \\
\text { CECELIA DR. }\end{array}$ & $\begin{array}{l}\text { DOGWOOD COVE } \\
\text { CIRCLE ROAD } \\
\text { L.I.E. } \\
\text { COLD SP. HARB.RD. } \\
\text { MUTTONTOWN RD. }\end{array}$ & $\begin{array}{l}4 \varnothing 5 \varnothing 13 \emptyset 733457 \\
4 \varnothing 4934 \varnothing 733144 \\
4 \varnothing 47 \varnothing 2 \varnothing 73353 \emptyset \\
4 \varnothing 5 \varnothing 31 \varnothing 732813 \\
4 \varnothing 4913 \varnothing 733118\end{array}$ & $\begin{array}{l}690103 \\
600219 \\
560815 \\
581118 \\
670922\end{array}$ & $\begin{array}{r}127 \\
126 \\
89 \\
107 \\
115\end{array}$ & $\begin{array}{l}414000 \\
24800 \emptyset \\
38900 \emptyset \\
7180 \varnothing \emptyset \\
363000\end{array}$ & $\begin{array}{l}42500 \emptyset \\
3060 \varnothing \emptyset \\
2247 \varnothing \emptyset \\
7476 \varnothing \emptyset \\
4150 \varnothing \emptyset\end{array}$ \\
\hline $\begin{array}{l}N \\
N \\
N \\
N \\
N\end{array}$ & $\begin{array}{l}521 \\
522 \\
523 \\
526 \\
527\end{array}$ & $\begin{array}{l}\text { BERRY HILL RD. } \\
\text { FOXHURST CRESENT } \\
\text { CORY CT. } \\
\text { JERICHO TPKE. } \\
\text { WOODHOLLOW COURT }\end{array}$ & $\begin{array}{l}\text { WOODLAND DR. } \\
\text { BERRY HILL RD. } \\
\text { SPLIT ROCK RD. } \\
\text { JUNEAU BLVD. } \\
\text { MUTTONTOWN ROAD }\end{array}$ & $\begin{array}{l}4 \varnothing 51260733106 \\
4 \varnothing 5 \varnothing 270732939 \\
4 \varnothing 5014 \varnothing 73311 \varnothing \\
4 \varnothing 4859073274 \varnothing \\
4 \varnothing 49150733225\end{array}$ & $\begin{array}{l}711129 \\
676620 \\
670718 \\
670405 \\
680515\end{array}$ & $\begin{array}{l}120 \\
115 \\
126 \\
128 \\
115\end{array}$ & $\begin{array}{l}\overline{-} \\
322000 \\
560000 \\
257000 \\
176000\end{array}$ & $\begin{array}{l}- \\
300000 \\
525000 \\
266 \varnothing 0 \varnothing \\
162000\end{array}$ \\
\hline $\begin{array}{l}N \\
N \\
N \\
N \\
N\end{array}$ & $\begin{array}{l}528 \\
529 \\
530 \\
531 \\
532\end{array}$ & $\begin{array}{l}\text { ROUND SWAMP RD. } \\
\text { LEONARD ST. } \\
\text { SCUDDERS LA. } \\
\text { FOXHUNT CRESCENT } \\
\text { DORCHESTER DR. }\end{array}$ & $\begin{array}{l}\text { WINDING RD. } \\
\text { JEROME DR. } \\
\text { SHAUN RIDGE } \\
\text { BERRY HILL RD. } \\
\text { MUTTONTOWN RD. }\end{array}$ & $\begin{array}{l}4 \emptyset 4545 \varnothing 732649 \\
4 \varnothing 4416 \varnothing 732728 \\
4 \varnothing 4932 \varnothing 733824 \\
4 \varnothing 5 \emptyset 12 \emptyset 732931 \\
4 \varnothing 4937 \varnothing 733332\end{array}$ & $\begin{array}{l}630624 \\
650527 \\
680129 \\
680813 \\
670324\end{array}$ & $\begin{array}{r}116 \\
99 \\
102 \\
120 \\
115\end{array}$ & $\begin{array}{r}196000 \\
72400 \\
108000 \\
865000 \\
618600\end{array}$ & $\begin{array}{r}199000 \\
71500 \\
108000 \\
865000 \\
587 \varnothing 00\end{array}$ \\
\hline $\begin{array}{l}N \\
N \\
N \\
N \\
N\end{array}$ & $\begin{array}{l}533 \\
534 \\
535 \\
536 \\
537\end{array}$ & $\begin{array}{l}\text { FOREST DR. } \\
\text { YELLOW COTE RD. } \\
\text { WOODBURY RD. } \\
\text { OLD JERICHO TPKE } \\
\text { HEMPSTEAD TPKE. }\end{array}$ & $\begin{array}{l}\text { MIDDLE NECK RD. } \\
\text { N.HEMPSTEAD TPK. } \\
\text { YUKON RD. } \\
\text { JERICHO TURNPIKE } \\
\text { GLENN CURTISS BL }\end{array}$ & $\begin{array}{l}4 \varnothing 5124 \varnothing 734 \varnothing 44 \\
4 \varnothing 512 \varnothing \varnothing 732955 \\
4 \varnothing 48150732833 \\
4 \varnothing 475 \varnothing \varnothing 7332 \varnothing 7 \\
4 \varnothing 43 \varnothing 5 \varnothing 733515\end{array}$ & $\begin{array}{l}640717 \\
660311 \\
660319 \\
678302 \\
6989\end{array}$ & $\begin{array}{r}86 \\
12 \varnothing \\
128 \\
105 \\
44\end{array}$ & $\begin{array}{l}191000 \\
363000 \\
621000 \\
333000 \\
-\end{array}$ & $\begin{array}{l}18600 \emptyset \\
54000 \emptyset \\
63000 \emptyset \\
3490 \varnothing \emptyset \\
-\end{array}$ \\
\hline
\end{tabular}


N.Y. [Dash indicates no data. Altitudes are in feet above sea level.] (cont.)

\begin{tabular}{|c|c|c|c|c|c|c|c|c|c|c|c|c|}
\hline \multicolumn{2}{|c|}{$\begin{array}{l}\text { BASIN } \\
\text { NUMBER }\end{array}$} & $\begin{array}{l}\text { MAX. } \\
\text { AREA } \\
\text { (squar }\end{array}$ & $\begin{array}{c}\text { BASIN } \\
\text { AREA } \\
\text { feet) }\end{array}$ & RIM & $\begin{array}{l}\text { OVER- } \\
\text { FLOW }\end{array}$ & $\begin{array}{l}\text { BOT- } \\
\text { TOM }\end{array}$ & $\begin{array}{l}\text { WATER } \\
\text { TABLE }\end{array}$ & $\begin{array}{c}\text { DRAIN. } \\
\text { AREA } \\
\text { (acres) }\end{array}$ & \multicolumn{2}{|c|}{$\begin{array}{l}---B A S I N-- \\
\text { STATUS USE }\end{array}$} & $\begin{array}{l}\text { GEO. } \\
\text { UNIT }\end{array}$ & $\begin{array}{l}\text { SOIL } \\
\text { UNIT }\end{array}$ \\
\hline $\begin{array}{l}N \\
N \\
N \\
N \\
N\end{array}$ & $\begin{array}{l}479 \\
480 \\
481 \\
482 \\
483\end{array}$ & $\begin{array}{l}80700 \\
62500 \\
18400 \\
21800 \\
66500\end{array}$ & $\begin{array}{r}170000 \\
88100 \\
37000 \\
42700 \\
83000\end{array}$ & $\begin{array}{r}192.0 \\
234.5 \\
178.4 \\
220.5 \\
77.2\end{array}$ & $\begin{array}{r}190.0 \\
231.0 \\
176.5 \\
220.5 \\
-\end{array}$ & $\begin{array}{r}180.0 \\
221.0 \\
166.1 \\
209.0 \\
64.0\end{array}$ & $\begin{array}{l}45 . \emptyset \\
72 . \varnothing \\
82.0 \\
66 . \varnothing \\
41 . \emptyset\end{array}$ & $\begin{array}{r}70.1 \\
28.5 \\
13.0 \\
28.0 \\
-\end{array}$ & $\begin{array}{l}1 \\
1 \\
1 \\
1 \\
1\end{array}$ & $\begin{array}{l}1 \\
1 \\
1 \\
1 \\
1\end{array}$ & $\begin{array}{l}4 \\
1 \\
1 \\
1 \\
1\end{array}$ & $\begin{array}{l}1 \\
1 \\
2 \\
1 \\
1\end{array}$ \\
\hline $\begin{array}{l}N \\
N \\
N \\
N \\
N\end{array}$ & $\begin{array}{l}484 \\
485 \\
486 \\
487 \\
488\end{array}$ & $\begin{array}{r}138000 \\
15000 \\
78500 \\
41100 \\
115200\end{array}$ & $\begin{array}{r}224000 \\
115000 \\
98000 \\
150000\end{array}$ & $\begin{array}{r}198.0 \\
262.0 \\
128.4 \\
42.0 \\
17.4\end{array}$ & $\begin{array}{r}195.5 \\
261.0 \\
40 . \overline{-} \\
-\end{array}$ & $\begin{array}{r}183.5 \\
251.0 \\
110.4 \\
35.0 \\
12.0\end{array}$ & $\begin{array}{l}75.5 \\
73.0 \\
72.0 \\
30.0 \\
15.0\end{array}$ & $\begin{array}{r}187.0 \\
11.0 \\
25.5 \\
-\end{array}$ & $\begin{array}{l}1 \\
1 \\
1 \\
1 \\
1\end{array}$ & $\begin{array}{l}1 \\
1 \\
1 \\
1 \\
1\end{array}$ & $\begin{array}{l}1 \\
8 \\
1 \\
3 \\
1\end{array}$ & $\begin{array}{l}1 \\
1 \\
2 \\
1 \\
3\end{array}$ \\
\hline $\begin{array}{l}N \\
N \\
N \\
N \\
N\end{array}$ & $\begin{array}{l}489 \\
490 \\
491 \\
492 \\
493\end{array}$ & $\begin{array}{r}39100 \\
13300 \\
39800 \\
14700 \\
9400\end{array}$ & $\begin{array}{l}63100 \\
43600 \\
57300 \\
34800 \\
25600\end{array}$ & $\begin{array}{r}26.4 \\
34.0 \\
34.5 \\
239.0 \\
78.0\end{array}$ & $\begin{array}{r}26.0 \\
30.0 \\
34.5 \\
237.6 \\
75.4\end{array}$ & $\begin{array}{r}20.0 \\
26.0 \\
29.5 \\
227.6 \\
66.0\end{array}$ & $\begin{array}{l}20.0 \\
24.0 \\
25.0 \\
56.0 \\
50.0\end{array}$ & $\begin{array}{r}- \\
- \\
- \\
18 . \overline{6} \\
6.5\end{array}$ & $\begin{array}{l}0 \\
1 \\
1 \\
1 \\
1\end{array}$ & $\begin{array}{l}1 \\
1 \\
1 \\
1 \\
1\end{array}$ & $\begin{array}{l}1 \\
1 \\
1 \\
1 \\
1\end{array}$ & $\begin{array}{l}1 \\
1 \\
1 \\
1 \\
3\end{array}$ \\
\hline $\begin{array}{l}N \\
N \\
N \\
N \\
N\end{array}$ & $\begin{array}{l}494 \\
495 \\
496 \\
497 \\
498\end{array}$ & $\begin{array}{r}110000 \\
141800 \\
6500 \\
98000 \\
5400\end{array}$ & $\begin{array}{r}145000 \\
21100 \\
140000 \\
37500\end{array}$ & $\begin{array}{r}79.0 \\
79.0 \\
168.1 \\
65.0 \\
39.0\end{array}$ & $\begin{array}{r}77.5 \\
\overline{-} \\
166 . \overline{5} \\
38 . \bar{\varnothing}\end{array}$ & $\begin{array}{r}68.0 \\
68.0 \\
156.5 \\
39.0 \\
28.0\end{array}$ & $\begin{array}{l}55.0 \\
60.0 \\
75.0 \\
20.0 \\
31.0\end{array}$ & $\begin{array}{r}64 . \overline{9} \\
8.8 \\
155.6 \\
6.0\end{array}$ & $\begin{array}{l}1 \\
1 \\
1 \\
0 \\
1\end{array}$ & $\begin{array}{l}1 \\
1 \\
1 \\
1 \\
3\end{array}$ & $\begin{array}{l}1 \\
1 \\
8 \\
1 \\
1\end{array}$ & $\begin{array}{l}6 \\
6 \\
3 \\
1 \\
0\end{array}$ \\
\hline $\begin{array}{l}N \\
N \\
N \\
N \\
N\end{array}$ & $\begin{array}{l}499 \\
500 \\
501 \\
502 \\
503\end{array}$ & $\begin{array}{r}44460 \\
83600 \\
116700 \\
55560 \\
72600\end{array}$ & $\begin{array}{r}78000 \\
107000 \\
174000 \\
119000 \\
-\end{array}$ & $\begin{array}{r}217.0 \\
19.0 \\
217.0 \\
179.5 \\
225.4\end{array}$ & $\begin{array}{r}214.5 \\
13.0 \\
214.5 \\
178.0 \\
-\end{array}$ & $\begin{array}{r}203.5 \\
12.0 \\
203.5 \\
170.0 \\
206.3\end{array}$ & $\begin{array}{r}69.0 \\
4.0 \\
70.0 \\
63.0 \\
57.0\end{array}$ & $\begin{array}{r}34.0 \\
32.8 \\
141.0 \\
48.0 \\
141.5\end{array}$ & $\begin{array}{l}1 \\
1 \\
1 \\
1 \\
1\end{array}$ & $\begin{array}{l}1 \\
1 \\
1 \\
1 \\
1\end{array}$ & $\begin{array}{l}1 \\
1 \\
1 \\
3 \\
1\end{array}$ & $\begin{array}{l}3 \\
1 \\
3 \\
3 \\
1\end{array}$ \\
\hline $\begin{array}{l}N \\
N \\
N \\
N \\
N\end{array}$ & $\begin{array}{l}504 \\
505 \\
506 \\
507 \\
508\end{array}$ & $\begin{array}{r}53600 \\
206000 \\
12900 \\
90000 \\
36900\end{array}$ & $\begin{array}{r}- \\
530000 \\
28900 \\
163000 \\
96000\end{array}$ & $\begin{array}{l}216.0 \\
150.0 \\
137.8 \\
136.0 \\
129.2\end{array}$ & $\begin{array}{r}- \\
- \\
136 . \overline{5} \\
136.0 \\
123 . \emptyset\end{array}$ & $\begin{array}{l}203.5 \\
112.0 \\
130.0 \\
126.0 \\
111.5\end{array}$ & $\begin{array}{l}75.0 \\
66.0 \\
45.0 \\
30.0 \\
71.0\end{array}$ & $\begin{array}{r}39.4 \\
- \\
6.0 \\
113.4 \\
63.2\end{array}$ & $\begin{array}{l}0 \\
1 \\
1 \\
1 \\
1\end{array}$ & $\begin{array}{l}1 \\
1 \\
1 \\
1 \\
1\end{array}$ & $\begin{array}{l}1 \\
1 \\
3 \\
3 \\
1\end{array}$ & $\begin{array}{l}1 \\
1 \\
1 \\
1 \\
1\end{array}$ \\
\hline $\begin{array}{l}N \\
N \\
N \\
N \\
N\end{array}$ & $\begin{array}{l}509 \\
512 \\
513 \\
514 \\
515\end{array}$ & $\begin{array}{r}65100 \\
109400 \\
9600 \\
8300 \\
11400\end{array}$ & $\begin{array}{r}149000 \\
16 \varnothing 000 \\
31400 \\
1900 \varnothing \\
23700\end{array}$ & $\begin{array}{r}75.3 \\
181.5 \\
124.0 \\
132.0 \\
61.0\end{array}$ & $\begin{array}{r}72.0 \\
180.5 \\
122.0 \\
130.5 \\
60.0\end{array}$ & $\begin{array}{r}61.0 \\
168.5 \\
112.0 \\
124.0 \\
53 . \varnothing\end{array}$ & $\begin{array}{l}9.0 \\
72.0 \\
67.0 \\
61.0 \\
50.0\end{array}$ & $\begin{array}{r}73.5 \\
146.0 \\
8.0 \\
2.9 \\
13.0\end{array}$ & $\begin{array}{l}1 \\
1 \\
1 \\
1 \\
1\end{array}$ & $\begin{array}{l}4 \\
1 \\
1 \\
4 \\
1\end{array}$ & $\begin{array}{l}3 \\
3 \\
1 \\
3 \\
1\end{array}$ & $\begin{array}{l}1 \\
3 \\
1 \\
1 \\
2\end{array}$ \\
\hline $\begin{array}{l}N \\
N \\
N \\
N \\
N\end{array}$ & $\begin{array}{l}516 \\
517 \\
518 \\
519 \\
520\end{array}$ & $\begin{array}{r}33500 \\
34800 \\
22500 \\
110000 \\
43500\end{array}$ & $\begin{array}{r}70000 \\
70200 \\
41800 \\
166500 \\
95000\end{array}$ & $\begin{array}{l}159.0 \\
217.0 \\
167.6 \\
197.0 \\
216.5\end{array}$ & $\begin{array}{l}157.0 \\
216.0 \\
164.0 \\
193.5 \\
214.5\end{array}$ & $\begin{array}{l}144.0 \\
206.0 \\
154.0 \\
172.0 \\
204.0\end{array}$ & $\begin{array}{l}67.0 \\
68.0 \\
70.0 \\
51.0 \\
68.0\end{array}$ & $\begin{array}{l}28.5 \\
34.0 \\
14.4 \\
78.0 \\
40.0\end{array}$ & $\begin{array}{l}1 \\
1 \\
1 \\
1 \\
1\end{array}$ & $\begin{array}{l}1 \\
1 \\
1 \\
1 \\
1\end{array}$ & $\begin{array}{l}3 \\
4 \\
1 \\
4 \\
6\end{array}$ & $\begin{array}{l}1 \\
3 \\
1 \\
1 \\
1\end{array}$ \\
\hline $\begin{array}{l}N \\
N \\
N \\
N \\
N\end{array}$ & $\begin{array}{l}521 \\
522 \\
523 \\
526 \\
527\end{array}$ & $\begin{array}{l}27100 \\
36900 \\
73400 \\
74600 \\
19200\end{array}$ & $\begin{array}{r}64100 \\
114000 \\
184500 \\
36500\end{array}$ & $\begin{array}{l}160.0 \\
250.6 \\
217.0 \\
258.7 \\
240.0\end{array}$ & $\begin{array}{l}-\overline{3} \\
245.3 \\
215.0 \\
258.0 \\
240 . \emptyset\end{array}$ & $\begin{array}{l}147.4 \\
235.3 \\
206.5 \\
254.8 \\
230.0\end{array}$ & $\begin{array}{l}51.0 \\
57.0 \\
60.0 \\
71.0 \\
71.0\end{array}$ & $\begin{array}{l}17.7 \\
36.5 \\
82.0 \\
69.0 \\
13.5\end{array}$ & $\begin{array}{l}1 \\
1 \\
1 \\
1 \\
1\end{array}$ & $\begin{array}{l}1 \\
1 \\
1 \\
3 \\
1\end{array}$ & $\begin{array}{l}3 \\
1 \\
4 \\
6 \\
1\end{array}$ & $\begin{array}{l}1 \\
1 \\
2 \\
1 \\
1\end{array}$ \\
\hline $\begin{array}{l}N \\
N \\
N \\
N \\
N\end{array}$ & $\begin{array}{l}528 \\
529 \\
530 \\
531 \\
532\end{array}$ & $\begin{array}{r}16500 \\
7200 \\
13000 \\
75000 \\
58700\end{array}$ & $\begin{array}{r}44000 \\
35600 \\
44500 \\
150600 \\
102000\end{array}$ & $\begin{array}{r}132.0 \\
101.0 \\
74.5 \\
208.0 \\
162.7\end{array}$ & $\begin{array}{r}121.0 \\
99.0 \\
54.0 \\
208.0 \\
162.0\end{array}$ & $\begin{array}{r}109.0 \\
89.0 \\
45.0 \\
196.5 \\
152.0\end{array}$ & $\begin{array}{l}66.0 \\
51.0 \\
18.0 \\
58.0 \\
71.0\end{array}$ & $\begin{array}{r}18.0 \\
10.0 \\
20.0 \\
60.0 \\
6.6\end{array}$ & $\begin{array}{l}0 \\
1 \\
0 \\
1 \\
1\end{array}$ & $\begin{array}{l}1 \\
1 \\
1 \\
1 \\
1\end{array}$ & $\begin{array}{l}7 \\
1 \\
3 \\
1 \\
3\end{array}$ & $\begin{array}{l}3 \\
1 \\
1 \\
1 \\
3\end{array}$ \\
\hline $\begin{array}{l}N \\
N \\
N \\
N \\
N\end{array}$ & $\begin{array}{l}533 \\
534 \\
535 \\
536 \\
537\end{array}$ & $\begin{array}{r}21700 \\
46300 \\
87900 \\
50500 \\
201000\end{array}$ & $\begin{array}{r}480 \varnothing \varnothing \\
1410 \varnothing \varnothing \varnothing \\
124 \varnothing \varnothing \varnothing \emptyset \\
777 \varnothing \varnothing \\
-\end{array}$ & $\begin{array}{r}60.0 \\
87.4 \\
207.2 \\
217.1 \\
74.4\end{array}$ & $\begin{array}{r}59.0 \\
87.0 \\
206.0 \\
208.5 \\
-\end{array}$ & $\begin{array}{r}49.0 \\
75.0 \\
196.0 \\
198.5 \\
57.0\end{array}$ & $\begin{array}{r}8.0 \\
50.0 \\
76.0 \\
77.0 \\
50.0\end{array}$ & $\begin{array}{r}21.0 \\
40.0 \\
81.0 \\
23.0 \\
225.2\end{array}$ & $\begin{array}{l}1 \\
1 \\
1 \\
1 \\
1\end{array}$ & $\begin{array}{l}4 \\
4 \\
1 \\
3 \\
1\end{array}$ & $\begin{array}{l}3 \\
1 \\
1 \\
6 \\
1\end{array}$ & $\begin{array}{l}1 \\
3 \\
1 \\
1 \\
2\end{array}$ \\
\hline
\end{tabular}


Appendix.--Location and basic physical features of recharge basins on Long Island,

\begin{tabular}{|c|c|c|c|c|c|c|c|c|}
\hline \multicolumn{2}{|c|}{$\begin{array}{l}\text { BASIN } \\
\text { NUMBER }\end{array}$} & \multicolumn{2}{|c|}{ NEAREST INTERSECTION } & $\begin{array}{l}\text { LATITUDE } \\
\text { LONGITUDE } \\
0^{\prime},{ }^{\prime},\end{array}$ & $\begin{array}{l}\text { DATE } \\
\text { BUILT } \\
\text { YrMoD }\end{array}$ & COMMUNITY & $\begin{array}{l}\text { DESIGN } \\
\text { CAPACITY } \\
\text { (cubic } f\end{array}$ & $\begin{array}{l}\text { ACTUAL } \\
\text { CAPACITY } \\
\text { feet) }\end{array}$ \\
\hline $\begin{array}{l}N \\
N \\
N \\
N \\
N\end{array}$ & $\begin{array}{l}538 \\
539 \\
540 \\
543 \\
544\end{array}$ & $\begin{array}{l}\text { NEW LATTINGTOWN } \\
\text { GLENDALE DR. } \\
\text { OAK STREET } \\
\text { JERICHO O.B. RD. } \\
\text { CROSSWAY }\end{array}$ & $\begin{array}{l}\text { HORSESHOE LA. } \\
\text { N. HEMPSTEAD TPK. } \\
\text { WESTBURY BLVD. } \\
\text { N. HEMPSTEAD TPK. } \\
\text { WEDGEWOOD CT. E. }\end{array}$ & 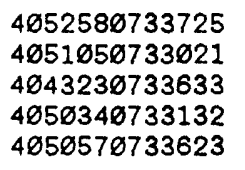 & $\begin{array}{l}671220 \\
749624 \\
721017 \\
729517 \\
758224\end{array}$ & $\begin{array}{r}100 \\
120 \\
44 \\
98 \\
117\end{array}$ & $\begin{array}{l}- \\
2 \overline{3} 2000 \\
- \\
472000 \\
383000\end{array}$ & $\begin{array}{l}264000 \\
318100 \\
- \\
618000 \\
547000\end{array}$ \\
\hline $\begin{array}{l}N \\
N \\
N \\
N \\
N\end{array}$ & $\begin{array}{l}546 \\
546 \\
547 \\
548 \\
549\end{array}$ & $\begin{array}{l}\text { YUKON DRIVE } \\
\text { BERRY HILL RD. } \\
\text { RIDGE COURT } \\
\text { TAMMYS LANE } \\
\text { OLD WESTBURY RD. }\end{array}$ & $\begin{array}{l}\text { ANCHORAGE DRIVE } \\
\text { EAST WOODS RD. } \\
\text { LATTINGTOWN RD. } \\
\text { EAST WOODS ROAD } \\
\text { JERICHO TURNPIKE }\end{array}$ & 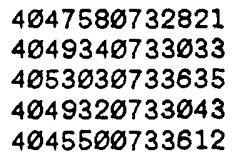 & $\begin{array}{l}698218 \\
740923 \\
780223 \\
750120 \\
780809\end{array}$ & $\begin{array}{l}128 \\
115 \\
106 \\
115 \\
118\end{array}$ & $\begin{array}{l}741200 \\
1450 \varnothing \emptyset \\
155183 \\
102 \varnothing \varnothing \varnothing \\
465 \varnothing \varnothing \varnothing\end{array}$ & $\begin{array}{l}704000 \\
163000 \\
171158 \\
119000 \\
421000\end{array}$ \\
\hline $\begin{array}{l}N \\
N \\
N \\
N \\
N\end{array}$ & $\begin{array}{l}558 \\
551 \\
552 \\
553 \\
554\end{array}$ & $\begin{array}{l}\text { KIRBYS LANE } \\
\text { APPLEGREEN DRIVE } \\
\text { STONE ARCH RD. } \\
\text { TIBER ROAD } \\
\text { BARRY LANE }\end{array}$ & $\begin{array}{l}\text { JERICHO TURNPIKE } \\
\text { WHEATLEY ROAD } \\
\text { HASTINGS RD. } \\
\text { SABINE ROAD } \\
\text { CONVENT ROAD }\end{array}$ & 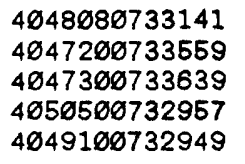 & $\begin{array}{l}780828 \\
750714 \\
740123 \\
770207 \\
770523\end{array}$ & $\begin{array}{l}188 \\
118 \\
118 \\
126 \\
126\end{array}$ & $\begin{array}{l}26900 \varnothing \\
233785 \\
572000 \\
508000 \\
241000\end{array}$ & $\begin{array}{r}299000 \\
222150 \\
616000 \\
5245220 \\
247000\end{array}$ \\
\hline $\begin{array}{l}N \\
N \\
N \\
N \\
N\end{array}$ & $\begin{array}{l}555 \\
556 \\
558 \\
559 \\
560\end{array}$ & $\begin{array}{l}\text { SPLIT ROCK RD. } \\
\text { BERNICE DRIVE } \\
\text { SAMUEL COURT } \\
\text { ARON DR. } \\
\text { SHELTER ROCK RD. }\end{array}$ & $\begin{array}{l}\text { BALSAR CT. } \\
\text { CAMEO COURT } \\
\text { CONVENT ROAD } \\
\text { VICTOR LA. } \\
\text { CARRIAGE RD }\end{array}$ & $\begin{array}{l}4049539733044 \\
4 \varnothing 42598733157 \\
4 \varnothing 49150732935 \\
4 \varnothing 49350732837 \\
4045580734 \varnothing 15\end{array}$ & $\begin{array}{l}748326 \\
758120 \\
750988 \\
790110 \\
798110\end{array}$ & $\begin{array}{r}115 \\
8 \\
126 \\
126 \\
87\end{array}$ & $\begin{array}{l}461000 \\
29000 \\
- \\
213000 \\
129900\end{array}$ & $\begin{array}{l}476000 \\
36000 \\
- \\
234768 \\
133600\end{array}$ \\
\hline $\begin{array}{l}N \\
N \\
N \\
N \\
N\end{array}$ & $\begin{array}{l}561 \\
562 \\
563 \\
567 \\
568\end{array}$ & $\begin{array}{l}\text { POND VIEW DR. } \\
\text { SPRUCE CT. } \\
\text { MOORES HILL RD. } \\
\text { EAST NORWICH RD. } \\
\text { CAT HOLLOW RD. }\end{array}$ & $\begin{array}{l}\text { JERICHO TPKE. } \\
\text { BAYVILLE AVE. } \\
\text { OYS.BAY COVE RD. } \\
\text { BROOKVILLE RD. } \\
\text { GODFREY AVE. }\end{array}$ & 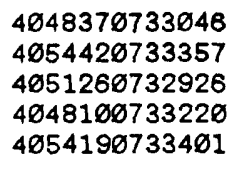 & $\begin{array}{l}860121 \\
746628 \\
7702 \varnothing 9 \\
74 \varnothing 813 \\
761210\end{array}$ & $\begin{array}{r}115 \\
91 \\
107 \\
115 \\
91\end{array}$ & $\begin{array}{r}92500 \\
139000 \\
343060 \\
457 \varnothing 0 \varnothing \\
236000\end{array}$ & $\begin{array}{r}86000 \\
14500 \emptyset \\
34900 \emptyset \\
4580 \varnothing \emptyset \\
25830 \varnothing\end{array}$ \\
\hline $\begin{array}{l}N \\
N \\
N \\
N \\
N\end{array}$ & $\begin{array}{l}569 \\
570 \\
571 \\
572 \\
573\end{array}$ & $\begin{array}{l}\text { UNDERHILL BLVD. } \\
\text { WHEATLEY-BKVL HY } \\
\text { GORDON DRIVE } \\
\text { GILBERT RD. } \\
\text { VON ELM AVE. }\end{array}$ & $\begin{array}{l}\text { JERICHO TURNPIKE } \\
\text { N.HEMPSTEAD TPK. } \\
\text { JERICHO TURNPIKE } \\
\text { STEAM BOAT RD. } \\
\text { PROSPECT AVE. }\end{array}$ & $\begin{array}{l}4 \varnothing 4825 \varnothing 73311 \varnothing \\
4 \varnothing 494 \varnothing \varnothing 733419 \\
4 \varnothing 4813 \varnothing 733 \varnothing 49 \\
4 \varnothing 4834 \varnothing 73443 \emptyset \\
4 \varnothing 4237 \varnothing 733343\end{array}$ & $\begin{array}{l}770131 \\
750325 \\
7803 \varnothing 6 \\
771014 \\
790129\end{array}$ & $\begin{array}{l}115 \\
127 \\
126 \\
140 \\
8\end{array}$ & $\begin{array}{r}27380 \emptyset \\
153680 \\
35458 \emptyset \\
6 \varnothing 571 \\
668 \varnothing \emptyset\end{array}$ & $\begin{array}{r}282652 \\
1780 \varnothing \\
4965 \varnothing \varnothing \\
633 \varnothing \varnothing \\
9209 \emptyset\end{array}$ \\
\hline $\begin{array}{l}N \\
N \\
N \\
N \\
N\end{array}$ & $\begin{array}{l}574 \\
575 \\
576 \\
577 \\
578\end{array}$ & $\begin{array}{l}\text { OLD WESTBURY RD. } \\
\text { SYO.COLD SPR.RD. } \\
\text { SYO.COLD SPR.RD. } \\
\text { BACON ROAD } \\
\text { WHITNEY LANE }\end{array}$ & $\begin{array}{l}\text { DEBRA COURT } \\
\text { SYO. WOODBURY RD. } \\
\text { CEDAR FIELD RD. } \\
\text { I.U. WILLETS RD. } \\
\text { WHEATLEY ROAD }\end{array}$ & $\begin{array}{l}4 \varnothing 46460733622 \\
4 \varnothing 494 \varnothing 0732937 \\
4 \varnothing 5 \varnothing 56 \varnothing 7328 \varnothing 2 \\
4 \varnothing 46 \varnothing 6 \varnothing 7337 \varnothing 3 \\
4 \varnothing 4823 \varnothing 733515\end{array}$ & $\begin{array}{l}780502 \\
791092 \\
740425 \\
791024 \\
7907 \emptyset 2\end{array}$ & $\begin{array}{l}118 \\
126 \\
107 \\
118 \\
118\end{array}$ & $\begin{array}{l}- \\
105000 \\
279149 \\
436000 \\
668000\end{array}$ & $\begin{array}{l}\overline{106000} \\
440000 \\
488000 \\
628000\end{array}$ \\
\hline $\begin{array}{l}N \\
N \\
N \\
N \\
N\end{array}$ & $\begin{array}{l}579 \\
580 \\
581 \\
582 \\
583\end{array}$ & $\begin{array}{l}\text { NORTHERN WOODS R } \\
\text { WOODBURY ROAD } \\
\text { MCCOUNS LANE } \\
\text { CEDAR SWAMP ROAD } \\
\text { MILL RIVER RD. }\end{array}$ & $\begin{array}{l}\text { N. HEMPSTEAD TPK. } \\
\text { L.I.E. } \\
\text { BERRY HILL RD. } \\
\text { NORDEN DRIVE } \\
\text { REMSEN'S LANE }\end{array}$ & $\begin{array}{l}4 \varnothing 48280733715 \\
4 \varnothing 4804 \varnothing 732854 \\
4 \varnothing 5144 \varnothing 733132 \\
4 \varnothing 4814 \varnothing 733314 \\
4 \varnothing 504907333 \varnothing 2\end{array}$ & $\begin{array}{l}790815 \\
800530 \\
790709 \\
800221 \\
791105\end{array}$ & $\begin{array}{l}53 \\
128 \\
119 \\
93 \\
115\end{array}$ & $\begin{array}{l}152000 \\
72600 \\
805000 \\
417000 \\
-\end{array}$ & $\begin{array}{r}14450 \emptyset \\
7800 \emptyset \\
8140 \varnothing \emptyset \\
416 \varnothing \varnothing \emptyset \\
-\end{array}$ \\
\hline $\begin{array}{l}N \\
N \\
N \\
N \\
N\end{array}$ & $\begin{array}{l}584 \\
585 \\
586 \\
587 \\
588\end{array}$ & $\begin{array}{l}\text { AMBER LANE } \\
\text { COACHMAN CT. } \\
\text { SERENITE } \\
\text { BERRY HILL. ROAD } \\
\text { MAGNOLIA BLVD. }\end{array}$ & $\begin{array}{l}\text { N.HEMPSTEAD TPK. } \\
\text { I.U.WILLETS RD. } \\
\text { MUTTONTOWN ROAD } \\
\text { RODEO DRIVE } \\
\text { N.HAWTHORNE ST. }\end{array}$ & 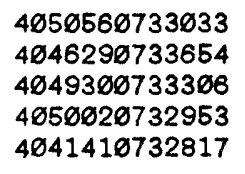 & $\begin{array}{l}800715 \\
800922 \\
80043 \varnothing \\
800319 \\
780717\end{array}$ & $\begin{array}{l}120 \\
118 \\
115 \\
12 \varnothing \\
110\end{array}$ & $\begin{array}{l}657000 \\
552900 \\
374400 \\
- \\
65340\end{array}$ & $\begin{array}{l}661000 \\
577000 \\
432000 \\
- \\
94000\end{array}$ \\
\hline $\begin{array}{l}N \\
N \\
N \\
N \\
N\end{array}$ & $\begin{array}{l}589 \\
590 \\
591 \\
592 \\
593\end{array}$ & $\begin{array}{l}\text { OYSTER BAY COVE } \\
\text { WAGAMON DRIVE } \\
\text { LAKEVIEW ROAD } \\
\text { WAVERLY ROAD } \\
\text { BERRY HILL ROAD }\end{array}$ & $\begin{array}{l}\text { CRAFTON COURT } \\
\text { VICTOR LANE } \\
\text { SUNRISE HIGHWAY } \\
\text { L.I.E. } \\
\text { HICKMAN STREET }\end{array}$ & $\begin{array}{l}4851410732947 \\
4 \varnothing 4936073283 \emptyset \\
4 \varnothing 4 \varnothing 130733112 \\
4 \varnothing 4650 \varnothing 733632 \\
4 \varnothing 5022 \varnothing 733 \varnothing 66\end{array}$ & $\begin{array}{l}831206 \\
798924 \\
81 \\
818219 \\
811281\end{array}$ & $\begin{array}{r}120 \\
128 \\
46 \\
118 \\
12 \varnothing\end{array}$ & $\begin{array}{l}375000 \\
204700 \\
- \\
361600 \\
79000\end{array}$ & $\begin{array}{l}420000 \\
207000 \\
- \\
480000 \\
137000\end{array}$ \\
\hline $\begin{array}{l}N \\
N \\
N \\
N \\
N P\end{array}$ & $\begin{array}{r}594 \\
595 \\
597 \\
598 \\
1\end{array}$ & $\begin{array}{l}\text { GRACE DR. } \\
\text { COURT LANE } \\
\text { MUSEUM ROAD } \\
\text { SEARINGTOWN ROAD } \\
\text { UNDERHILL BLVD. }\end{array}$ & $\begin{array}{l}\text { OLD WESTBURY RD. } \\
\text { COVES RUN } \\
\text { NORTHERN BLVD. } \\
\text { I.U. WILLETS RD. } \\
\text { JERICHO TURNPIKE }\end{array}$ & 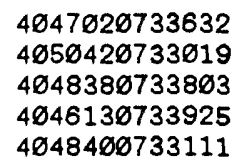 & $\begin{array}{c}820511 \\
811119 \\
801027 \\
811105 \\
-\end{array}$ & $\begin{array}{r}118 \\
120 \\
123 \\
87 \\
115\end{array}$ & $\begin{array}{l}424800 \\
642655 \\
- \\
- \\
-\end{array}$ & $\begin{array}{l}449090 \\
643787 \\
- \\
- \\
-\end{array}$ \\
\hline
\end{tabular}


N.Y. [Dash indicates no data. Altitudes are in feet above sea level.] (cont.)

\begin{tabular}{|c|c|c|c|c|c|c|c|c|c|c|c|c|}
\hline & $\begin{array}{l}\text { SIN } \\
\text { MBER }\end{array}$ & $\begin{array}{l}\text { MAX. } \\
\text { AREA } \\
\text { (squar }\end{array}$ & $\begin{array}{c}\text { BASIN } \\
\text { AREA } \\
\text { feet) }\end{array}$ & RIM & $\begin{array}{l}--A L T \\
\text { OVER } \\
\text { FLOW }\end{array}$ & $\begin{array}{l}\text { DE-- } \\
\text { BOT- } \\
\text { TOM }\end{array}$ & WATER & $\begin{array}{c}\text { DRAIN. } \\
\text { AREA } \\
\text { (acres) }\end{array}$ & $\begin{array}{l}--B A S I \\
\text { STATUS }\end{array}$ & $\begin{array}{l}\text { IN-- } \\
\text { USE }\end{array}$ & $\begin{array}{l}\text { GEO. } \\
\text { UNIT }\end{array}$ & $\begin{array}{l}\text { SOIL } \\
\text { UNIT }\end{array}$ \\
\hline $\begin{array}{l}N \\
N \\
N \\
N \\
N\end{array}$ & $\begin{array}{l}538 \\
539 \\
540 \\
543 \\
544\end{array}$ & $\begin{array}{c}30300 \\
37900 \\
- \\
730 \varnothing 0 \\
666 \varnothing 0\end{array}$ & $\begin{array}{r}59400 \\
85000 \\
- \\
113000 \\
100000\end{array}$ & $\begin{array}{r}136.6 \\
213.0 \\
215.0 \\
141.0\end{array}$ & $\begin{array}{r}135.0 \\
= \\
- \\
-\end{array}$ & $\begin{array}{r}125.0 \\
- \\
204.0 \\
125.0\end{array}$ & $\begin{array}{r}30.0 \\
= \\
= \\
=\end{array}$ & $\begin{array}{r}41.6 \\
18.5 \\
313.0 \\
52.0 \\
26.4\end{array}$ & $\begin{array}{l}1 \\
1 \\
1 \\
1 \\
1\end{array}$ & $\begin{array}{l}1 \\
1 \\
2 \\
1 \\
1\end{array}$ & $\begin{array}{l}3 \\
\emptyset \\
\emptyset \\
\emptyset \\
\varnothing\end{array}$ & $\begin{array}{l}1 \\
0 \\
0 \\
0 \\
0\end{array}$ \\
\hline $\begin{array}{l}N \\
N \\
N \\
N \\
N\end{array}$ & $\begin{array}{l}545 \\
546 \\
547 \\
548 \\
549\end{array}$ & $\begin{array}{l}74200 \\
17600 \\
31916 \\
18200 \\
23810\end{array}$ & $\begin{array}{r}160000 \\
87000 \\
- \\
32400 \\
90200\end{array}$ & $\begin{array}{r}220.5 \\
225.0 \\
49.0 \\
229.4 \\
113.0\end{array}$ & $\begin{array}{r}218.0 \\
217.0 \\
49.0 \\
113.0\end{array}$ & $\begin{array}{l}209.0 \\
206.0 \\
414.0 \\
225.0 \\
102.0\end{array}$ & $\begin{array}{l}- \\
- \\
- \\
-\end{array}$ & $\begin{array}{l}60.0 \\
20 . \varnothing \\
43.5 \\
14.0 \\
21.3\end{array}$ & $\begin{array}{l}1 \\
1 \\
1 \\
1 \\
1\end{array}$ & $\begin{array}{l}1 \\
1 \\
1 \\
1 \\
1\end{array}$ & $\begin{array}{l}\emptyset \\
\emptyset \\
\emptyset \\
\emptyset \\
\varnothing\end{array}$ & $\begin{array}{l}\emptyset \\
\emptyset \\
\varnothing \\
\emptyset \\
\varnothing\end{array}$ \\
\hline $\begin{array}{l}N \\
N \\
N \\
N \\
N\end{array}$ & $\begin{array}{l}550 \\
551 \\
552 \\
553 \\
554\end{array}$ & $\begin{array}{l}15900 \\
31550 \\
72000 \\
48300 \\
31320\end{array}$ & $\begin{array}{r}64500 \\
47900 \\
121800 \\
143000 \\
68400\end{array}$ & $\begin{array}{l}230 . \varnothing \\
181 . \varnothing \\
179.0 \\
213 . \varnothing \\
216 . \varnothing\end{array}$ & $\begin{array}{r}230.0 \\
- \\
172.5 \\
213.0 \\
216.0\end{array}$ & $\begin{array}{l}215.0 \\
165.5 \\
161.5 \\
198.5 \\
201.0\end{array}$ & $\begin{array}{l}- \\
- \\
- \\
-\end{array}$ & $\begin{array}{l}42.5 \\
20.0 \\
77.8 \\
35.0 \\
47.0\end{array}$ & $\begin{array}{l}0 \\
1 \\
1 \\
1 \\
1\end{array}$ & $\begin{array}{l}1 \\
1 \\
1 \\
1 \\
1\end{array}$ & $\begin{array}{l}\emptyset \\
\emptyset \\
\emptyset \\
\emptyset \\
\emptyset\end{array}$ & $\begin{array}{l}\emptyset \\
\emptyset \\
\emptyset \\
\emptyset \\
\emptyset\end{array}$ \\
\hline $\begin{array}{l}N \\
N \\
N \\
N \\
N\end{array}$ & $\begin{array}{l}555 \\
556 \\
558 \\
559 \\
560\end{array}$ & $\begin{array}{r}62730 \\
8000 \\
- \\
33100 \\
21200\end{array}$ & $\begin{array}{r}103000 \\
20500 \\
- \\
52700 \\
39000\end{array}$ & $\begin{array}{r}199.5 \\
78.0 \\
- \\
216.0 \\
152.0\end{array}$ & $\begin{array}{r}199.5 \\
- \\
- \\
-\end{array}$ & $\begin{array}{r}188.5 \\
66.0 \\
- \\
204.0 \\
142.0\end{array}$ & $\begin{array}{l}- \\
- \\
- \\
-\end{array}$ & $\begin{array}{r}64.0 \\
4.0 \\
25.9 \\
29.3 \\
19.7\end{array}$ & $\begin{array}{l}1 \\
1 \\
0 \\
0 \\
1\end{array}$ & $\begin{array}{l}1 \\
1\end{array}$ & $\begin{array}{l}\emptyset \\
\emptyset \\
\emptyset \\
\varnothing \\
\varnothing\end{array}$ & $\begin{array}{l}\emptyset \\
\emptyset \\
\emptyset \\
\emptyset \\
\emptyset\end{array}$ \\
\hline $\begin{array}{l}N \\
N \\
N \\
N \\
N\end{array}$ & $\begin{array}{l}561 \\
562 \\
563 \\
567 \\
568\end{array}$ & $\begin{array}{l}14100 \\
16625 \\
484 \varnothing 0 \\
522 \varnothing 0 \\
30775\end{array}$ & $\begin{array}{r}30500 \\
32600 \\
118000 \\
87100 \\
82000\end{array}$ & $\begin{array}{r}163.0 \\
32 . \varnothing \\
62 . \varnothing \\
131 . \varnothing \\
34.5\end{array}$ & $\begin{array}{r}32 . \bar{\varnothing} \\
62 . \varnothing \\
34 . \overline{5}\end{array}$ & $\begin{array}{r}151.0 \\
2 \varnothing . \varnothing \\
50 . \varnothing \\
112 . \varnothing \\
26 . \varnothing\end{array}$ & $\begin{array}{l}- \\
- \\
- \\
-\end{array}$ & $\begin{array}{l}12.7 \\
30.0 \\
37.8 \\
63.2 \\
29.0\end{array}$ & $\begin{array}{l}1 \\
1 \\
1\end{array}$ & $\begin{array}{l}1 \\
1 \\
1 \\
1 \\
1\end{array}$ & $\begin{array}{l}\emptyset \\
\emptyset \\
\emptyset \\
\varnothing \\
\varnothing\end{array}$ & $\begin{array}{l}\emptyset \\
\emptyset \\
\emptyset \\
\emptyset \\
\emptyset\end{array}$ \\
\hline $\begin{array}{l}N \\
N \\
N \\
N \\
N\end{array}$ & $\begin{array}{l}569 \\
570 \\
571 \\
572 \\
573\end{array}$ & $\begin{array}{l}32100 \\
42200 \\
57400 \\
11000 \\
12045\end{array}$ & $\begin{array}{l}56700 \\
46800 \\
85500 \\
27675 \\
25025\end{array}$ & $\begin{array}{r}160.0 \\
138.7 \\
194.2 \\
26.0 \\
68.0\end{array}$ & $\begin{array}{r}160.0 \\
138.7 \\
190.0 \\
25.0 \\
66.0\end{array}$ & $\begin{array}{r}147.0 \\
129.6 \\
178.0 \\
18.3 \\
57.0\end{array}$ & $\begin{array}{l}- \\
- \\
- \\
-\end{array}$ & $\begin{array}{r}53.0 \\
21.0 \\
27.9 \\
6.0 \\
9.2\end{array}$ & $\begin{array}{l}1 \\
1 \\
1 \\
1 \\
1\end{array}$ & $\begin{array}{l}1 \\
1 \\
2 \\
1 \\
1\end{array}$ & $\begin{array}{l}\emptyset \\
\emptyset \\
\emptyset \\
\emptyset \\
\emptyset\end{array}$ & $\begin{array}{l}\emptyset \\
\emptyset \\
\emptyset \\
\emptyset \\
\emptyset\end{array}$ \\
\hline $\begin{array}{l}N \\
N \\
N \\
N \\
N\end{array}$ & $\begin{array}{l}574 \\
575 \\
576 \\
577 \\
578\end{array}$ & $\begin{array}{c}- \\
16300 \\
52360 \\
59300 \\
79400\end{array}$ & $\begin{array}{r}31300 \\
92400 \\
87000 \\
108000\end{array}$ & $\begin{array}{l}202 . \overline{5} \\
233.0 \\
137.0 \\
179.5\end{array}$ & $\begin{array}{l}199 . \overline{5} \\
233.0 \\
137.0 \\
175.0\end{array}$ & $\begin{array}{l}188 . \overline{5} \\
220.7 \\
126.0 \\
166.0\end{array}$ & $\begin{array}{l}- \\
- \\
- \\
-\end{array}$ & $\begin{array}{r}21.5 \\
9.0 \\
19.9 \\
30.0 \\
74.0\end{array}$ & $\begin{array}{l}1 \\
1 \\
1 \\
1\end{array}$ & $\begin{array}{l}1 \\
1 \\
1 \\
1 \\
1\end{array}$ & $\begin{array}{l}\emptyset \\
\emptyset \\
\emptyset \\
\emptyset \\
\varnothing\end{array}$ & $\begin{array}{l}\emptyset \\
\emptyset \\
\emptyset \\
\theta \\
\varnothing\end{array}$ \\
\hline $\begin{array}{l}N \\
N \\
N \\
N \\
N\end{array}$ & $\begin{array}{l}579 \\
580 \\
581 \\
582 \\
583\end{array}$ & $\begin{array}{r}20600 \\
13000 \\
103400 \\
41200 \\
-\end{array}$ & $\begin{array}{r}33500 \\
24600 \\
140666 \\
75900 \\
-\end{array}$ & $\begin{array}{r}141.7 \\
192.0 \\
108.0 \\
213.0 \\
-\end{array}$ & $\begin{array}{r}141.0 \\
191.5 \\
106.0 \\
208.5 \\
-\end{array}$ & $\begin{array}{r}131.0 \\
181.5 \\
95.0 \\
196.5 \\
-\end{array}$ & $\begin{array}{l}- \\
- \\
-\end{array}$ & $\begin{array}{l}21.0 \\
10.0 \\
88.7 \\
53.0 \\
24.0\end{array}$ & $\begin{array}{l}1 \\
1 \\
1 \\
1\end{array}$ & $\begin{array}{l}1 \\
1 \\
1 \\
1\end{array}$ & $\begin{array}{l}\emptyset \\
\theta \\
\theta \\
\theta \\
\varnothing\end{array}$ & $\begin{array}{l}\emptyset \\
\varnothing \\
\emptyset \\
\emptyset \\
\varnothing\end{array}$ \\
\hline $\begin{array}{l}N \\
N \\
N \\
N \\
N\end{array}$ & $\begin{array}{l}584 \\
585 \\
586 \\
587 \\
588\end{array}$ & $\begin{array}{c}62800 \\
50500 \\
45600 \\
- \\
20223\end{array}$ & $\begin{array}{r}103350 \\
93220 \\
93150 \\
- \\
33936\end{array}$ & $\begin{array}{r}228.5 \\
138.0 \\
202.0 \\
43.3\end{array}$ & $\begin{array}{r}228.0 \\
138.0 \\
201.0 \\
42 . \overline{0}\end{array}$ & $\begin{array}{r}215.0 \\
124.0 \\
188.5 \\
35 . \overline{0}\end{array}$ & $\begin{array}{l}- \\
- \\
-\end{array}$ & $\begin{array}{r}49.1 \\
53.1 \\
43.0 \\
79.2 \\
9.0\end{array}$ & $\begin{array}{l}1 \\
1 \\
0 \\
1\end{array}$ & $\begin{array}{l}1 \\
1 \\
1 \\
1 \\
1\end{array}$ & $\begin{array}{l}0 \\
\theta \\
0 \\
\theta \\
\theta\end{array}$ & $\begin{array}{l}\emptyset \\
\emptyset \\
\emptyset \\
\emptyset \\
\emptyset\end{array}$ \\
\hline $\begin{array}{l}N \\
N \\
N \\
N \\
N\end{array}$ & $\begin{array}{l}589 \\
590 \\
591 \\
592 \\
593\end{array}$ & $\begin{array}{c}36150 \\
21400 \\
- \\
54050 \\
1937 \varnothing\end{array}$ & $\begin{array}{r}73775 \\
43290 \\
8750 \\
35696\end{array}$ & $\begin{array}{r}62.0 \\
215 . \varnothing \\
147 . \overline{5} \\
250 . \varnothing\end{array}$ & $\begin{array}{r}60.6 \\
213.0 \\
147 . \overline{5} \\
249.0\end{array}$ & $\begin{array}{r}48.0 \\
203.0 \\
138.5 \\
239.0\end{array}$ & $\begin{array}{l}- \\
- \\
- \\
-\end{array}$ & $\begin{array}{r}17.2 \\
28.2 \\
126.9 \\
28.8 \\
9.1\end{array}$ & $\begin{array}{l}1 \\
1\end{array}$ & $\begin{array}{l}1 \\
1\end{array}$ & $\begin{array}{l}\emptyset \\
\emptyset \\
\emptyset \\
\emptyset \\
\emptyset\end{array}$ & $\begin{array}{l}\emptyset \\
\emptyset \\
\emptyset \\
\emptyset \\
\emptyset\end{array}$ \\
\hline $\begin{array}{l}N \\
N \\
N \\
N \\
N P\end{array}$ & $\begin{array}{r}594 \\
595 \\
597 \\
598 \\
1\end{array}$ & $\begin{array}{c}63500 \\
72560 \\
- \\
18000 \\
-\end{array}$ & $\begin{array}{r}80000 \\
132000 \\
23750 \\
-\end{array}$ & $\begin{array}{r}141.0 \\
242.2 \\
160.0 \\
124.0 \\
-\end{array}$ & $\begin{array}{r}138.0 \\
238.8 \\
- \\
122.0 \\
-\end{array}$ & $\begin{array}{r}128.0 \\
227.8 \\
113.3 \\
-\end{array}$ & $\begin{array}{r}\overline{-} \\
30 . \bar{\varnothing} \\
80 . \overline{0}\end{array}$ & $\begin{array}{r}76.6 \\
45.9 \\
16.2 \\
15.8 \\
-\end{array}$ & $\begin{array}{l}1 \\
1 \\
0 \\
1 \\
0\end{array}$ & $\begin{array}{l}1 \\
1 \\
1 \\
1 \\
4\end{array}$ & $\begin{array}{l}\emptyset \\
\emptyset \\
\emptyset \\
\emptyset \\
6\end{array}$ & $\begin{array}{l}0 \\
0 \\
0 \\
0 \\
1\end{array}$ \\
\hline
\end{tabular}


Appendix.--Location and basic physical features of recharge basins on Long Island,

\begin{tabular}{|c|c|c|c|c|c|c|c|c|}
\hline \multicolumn{2}{|c|}{$\begin{array}{l}\text { BASIN } \\
\text { NUMBER }\end{array}$} & \multicolumn{2}{|c|}{ NEAREST INTERSECTION } & $\begin{array}{l}\text { LATITUDE } \\
\text { LONGITUDE } \\
\circ, n \text { " }\end{array}$ & $\begin{array}{l}\text { DATE } \\
\text { BUILT } \\
\text { YrMOD }\end{array}$ & COMMUNITY & $\begin{array}{l}\text { DESIGN } \\
\text { CAPACITY } \\
\text { (cubic }\end{array}$ & $\begin{array}{l}\text { ACTUAL } \\
\text { CAPACITY } \\
\text { feot) }\end{array}$ \\
\hline $\begin{array}{l}R \\
R \\
R \\
R \\
R\end{array}$ & $\begin{array}{l}1 \\
2 \\
3 \\
4 \\
5\end{array}$ & $\begin{array}{l}\text { DOGWOOD DRIVE } \\
\text { GULLY ROAD } \\
\text { BEEMER AVENUE } \\
\text { LONG POND ROAD } \\
\text { OAKLEIGH AVENUE }\end{array}$ & $\begin{array}{l}\text { FRANCIS STREET } \\
\text { PARKER ROAD } \\
\text { SOUND AVENUE } \\
\text { STEPHEN DRIVE } \\
\text { NORTH ROAD }\end{array}$ & 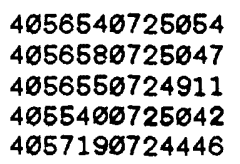 & $\begin{array}{l}- \\
- \\
- \\
-\end{array}$ & $\begin{array}{l}271 \\
271 \\
271 \\
271 \\
263\end{array}$ & $\begin{array}{l}- \\
- \\
- \\
-\end{array}$ & $\begin{array}{l}- \\
- \\
- \\
-\end{array}$ \\
\hline $\begin{array}{l}R \\
R \\
R \\
R \\
R\end{array}$ & $\begin{array}{r}6 \\
7 \\
8 \\
9 \\
10\end{array}$ & $\begin{array}{l}\text { DOLPHIN WAY } \\
\text { ROANOKE AVENUE } \\
\text { NADEL DRIVE } \\
\text { ANDREA COURT } \\
\text { NORTHVILLE TPKE. }\end{array}$ & $\begin{array}{l}\text { NORTH ROAD } \\
\text { JOYCE DRIVE } \\
\text { MIDDLE ROAD } \\
\text { OAKLAND DRIVE SO } \\
\text { DOCTOR'S PATH }\end{array}$ & $\begin{array}{l}4058100724155 \\
4056220724 \emptyset 44 \\
4056150724 \varnothing 3 \emptyset \\
4056420723933 \\
4 \varnothing 56520723926\end{array}$ & $\begin{array}{l}- \\
- \\
- \\
-\end{array}$ & $\begin{array}{l}270 \\
269 \\
269 \\
269 \\
269\end{array}$ & $\begin{array}{l}- \\
- \\
- \\
-\end{array}$ & $\begin{array}{l}- \\
- \\
- \\
-\end{array}$ \\
\hline $\begin{array}{l}R \\
R \\
S \\
S \\
S\end{array}$ & $\begin{array}{r}15 \\
16 \\
2 \\
3 \\
4\end{array}$ & $\begin{array}{l}\text { TUTHILLS LANE } \\
\text { MANOR LANE } \\
\text { ALEXANDER AVENUE } \\
\text { CACTUS LANE } \\
\text { HONEYSUCKLE LANE }\end{array}$ & $\begin{array}{l}\text { APOLLO DRIVE } \\
\text { MAIN ROAD } \\
\text { MILDRED COURT } \\
\text { PRIMROSE LANE } \\
\text { RUMFORD ROAD }\end{array}$ & $\begin{array}{l}4 \varnothing 57140723646 \\
4 \varnothing 57050723501 \\
4051060730759 \\
4 \varnothing 52480731416 \\
4 \varnothing 52580731410\end{array}$ & $\begin{array}{c}\overline{-} \\
\overline{-} \\
880514 \\
680514\end{array}$ & $\begin{array}{l}268 \\
267 \\
177 \\
174 \\
174\end{array}$ & $\begin{array}{l}- \\
- \\
\overline{747200} \\
135500\end{array}$ & $\begin{array}{l}- \\
- \\
\overline{800000} \\
180000\end{array}$ \\
\hline $\begin{array}{l}S \\
S \\
S \\
S \\
S\end{array}$ & $\begin{array}{r}5 \\
6 \\
8 \\
9 \\
10\end{array}$ & $\begin{array}{l}\text { AMHERST LANE } \\
\text { HARVARD LANE } \\
\text { BREAD AND CHEESE } \\
\text { GLENVIEW AVENUE } \\
\text { COTTONWOOD DRIVE }\end{array}$ & $\begin{array}{l}\text { CORNELL DRIVE } \\
\text { CORNELL DRIVE } \\
\text { FIELDVIEW DRIVE } \\
\text { TIMBER LANE } \\
\text { CRABAPPLE LANE }\end{array}$ & $\begin{array}{l}4051030731516 \\
4051010731535 \\
4054090731747 \\
4054260731728 \\
4050520731612\end{array}$ & $\begin{array}{l}630603 \\
650824 \\
651109 \\
651119 \\
5507\end{array}$ & $\begin{array}{l}169 \\
169 \\
171 \\
171 \\
169\end{array}$ & $\begin{array}{r}702300 \\
280200 \\
398000 \\
77000 \\
712800\end{array}$ & $\begin{array}{l}858000 \\
308100 \\
400000 \\
120000 \\
726000\end{array}$ \\
\hline $\begin{array}{l}\text { S } \\
\text { S } \\
\text { S } \\
\text { S } \\
\text { S }\end{array}$ & $\begin{array}{l}11 \\
12 \\
13 \\
14 \\
15\end{array}$ & $\begin{array}{l}\text { CARLDON ROAD } \\
\text { SHIRLEY COURT } \\
\text { LAUREL DRIVE } \\
\text { RADBURN DRIVE } \\
\text { INDIAN HEAD ROAD }\end{array}$ & $\begin{array}{l}\text { ASHLON LANE } \\
\text { VIOLET LANE } \\
\text { SHIRLEY COURT } \\
\text { ROXBURY DRIVE } \\
\text { BYRON ROAD }\end{array}$ & $\begin{array}{l}4 \varnothing 51150731616 \\
4 \varnothing 50280731621 \\
4050240731542 \\
4 \varnothing 5133073162 \varnothing \\
4051330731610\end{array}$ & $\begin{array}{r}5612 \\
5410 \\
- \\
5609 \\
5609\end{array}$ & $\begin{array}{l}169 \\
169 \\
169 \\
169 \\
169\end{array}$ & $\begin{array}{l}117000 \\
- \\
- \\
449300 \\
157500\end{array}$ & $\begin{array}{l}148000 \\
- \\
- \\
246000 \\
73400\end{array}$ \\
\hline $\begin{array}{l}\text { S } \\
\text { S } \\
S \\
S \\
S\end{array}$ & $\begin{array}{l}16 \\
17 \\
18 \\
19 \\
20\end{array}$ & $\begin{array}{l}\text { INDIAN HEAD ROAD } \\
\text { INDIAN HEAD ROAD } \\
\text { FIRST AVENUE } \\
\text { FIRST AVENUE } \\
\text { ST. JOHNLAND RD. }\end{array}$ & $\begin{array}{l}\text { TYRAM STREET } \\
\text { ROXBURY DRIVE } \\
\text { HUDSON DRIVE } \\
\text { BALBOA DRIVE } \\
\text { DAPHNE PLACE }\end{array}$ & $\begin{array}{l}4 \varnothing 51420731601 \\
4 \varnothing 51548731547 \\
4 \varnothing 5250673153 \emptyset \\
4 \varnothing 52420731527 \\
4 \varnothing 52120731310\end{array}$ & $\begin{array}{l}5609 \\
5609 \\
560307 \\
560307 \\
590518\end{array}$ & $\begin{array}{l}169 \\
169 \\
174 \\
174 \\
182\end{array}$ & $\begin{array}{r}186600 \\
292700 \\
32800 \\
111000 \\
-\end{array}$ & $\begin{array}{r}100100 \\
302500 \\
35000 \\
130800 \\
445000\end{array}$ \\
\hline $\begin{array}{l}S \\
S \\
S \\
S \\
S\end{array}$ & $\begin{array}{l}21 \\
22 \\
23 \\
24 \\
25\end{array}$ & $\begin{array}{l}\text { OLD INDIAN HEAD } \\
\text { FOX LANE } \\
\text { 9TH AVENUE } \\
\text { GLEN ROAD } \\
\text { S. PLAISTED AVE. }\end{array}$ & $\begin{array}{l}\text { GEHRIG STREET } \\
\text { HUBBELL STREET } \\
\text { EMILY PLACE } \\
\text { GLEN LANE } \\
\text { SATURN BOULEVARD }\end{array}$ & $\begin{array}{l}405047073171 \varnothing \\
4050490731728 \\
40524 \varnothing 0731610 \\
4052490731638 \\
40494 \varnothing 0731124\end{array}$ & $\begin{array}{c}- \\
5711 \varnothing 4 \\
54 \varnothing 903 \\
631 \varnothing 29 \\
-\end{array}$ & $\begin{array}{l}169 \\
169 \\
174 \\
174 \\
196\end{array}$ & $\begin{array}{l}- \\
313400 \\
87000 \\
108 \varnothing 00 \\
-\end{array}$ & $\begin{array}{l}- \\
5 \varnothing 75 \varnothing \varnothing \\
9000 \varnothing \\
1186 \varnothing \varnothing \\
-\end{array}$ \\
\hline $\begin{array}{l}S \\
S \\
S \\
S \\
S\end{array}$ & $\begin{array}{l}26 \\
27 \\
28 \\
29 \\
30\end{array}$ & $\begin{array}{l}\text { SHEILA DRIVE } \\
\text { SANDRA DRIVE } \\
\text { ORIOLE COURT } \\
\text { OWL LANE } \\
\text { GATEWOOD DRIVE }\end{array}$ & $\begin{array}{l}\text { GARVEY DRIVE } \\
\text { MAUREEN DRIVE } \\
\text { NEW HIGHWAY } \\
\text { CARDINAL LANE } \\
\text { GLENWOOD DRIVE }\end{array}$ & $\begin{array}{l}4049440731059 \\
4 \varnothing 49560731107 \\
4049260731418 \\
4 \varnothing 49170731410 \\
4049390731459\end{array}$ & $\begin{array}{l}641013 \\
6605 \\
570116 \\
610829 \\
601122\end{array}$ & $\begin{array}{l}196 \\
196 \\
169 \\
169 \\
169\end{array}$ & $\begin{array}{r}522200 \\
317800 \\
88000 \\
542000 \\
498000\end{array}$ & $\begin{array}{l}533900 \\
356100 \\
140000 \\
545000 \\
498000\end{array}$ \\
\hline $\begin{array}{l}\text { S } \\
S \\
S \\
S \\
S\end{array}$ & $\begin{array}{l}31 \\
32 \\
33 \\
34 \\
35\end{array}$ & $\begin{array}{l}\text { NEW HIGHWAY } \\
\text { DEWBERRY PLACE } \\
\text { NEW HIGHWAY } \\
\text { VETERANS HIGHWAY } \\
\text { VETERANS HIGHWAY }\end{array}$ & $\begin{array}{l}\text { PINE HOLLOW ROAD } \\
\text { BUTTERFLY DRIVE } \\
\text { ROBIN DRIVE } \\
\text { ROBIN DRIVE } \\
\text { AUTUMN DRIVE }\end{array}$ & $\begin{array}{l}4049190731447 \\
4 \varnothing 49290731512 \\
4049240731425 \\
4049320731427 \\
4049330731434\end{array}$ & $\begin{array}{l}620611 \\
620611 \\
590817 \\
590817 \\
600512\end{array}$ & $\begin{array}{l}169 \\
169 \\
169 \\
169 \\
169\end{array}$ & $\begin{array}{l}195100 \\
156800 \\
105000 \\
147000 \\
210000\end{array}$ & $\begin{array}{l}210000 \\
170800 \\
119000 \\
179000 \\
231000\end{array}$ \\
\hline $\begin{array}{l}\text { S } \\
\text { S } \\
S \\
S \\
S\end{array}$ & $\begin{array}{l}36 \\
37 \\
38 \\
39 \\
40\end{array}$ & $\begin{array}{l}\text { MARQUETTE DRIVE } \\
\text { FIFTY ACRE RD.SO } \\
\text { KNOLL ROAD } \\
\text { APPLE LANE } \\
\text { HOLLY LANE }\end{array}$ & $\begin{array}{l}\text { FIFTY ACRE RD. SO } \\
\text { SAMMIS STREET } \\
\text { WASHINGTON BLVD. } \\
\text { CHESTNUT COURT } \\
\text { LARCH LANE }\end{array}$ & $\begin{array}{l}4052060731010 \\
4 \varnothing 51500731009 \\
4 \varnothing 51310731440 \\
4050100731615 \\
4050110731237\end{array}$ & $\begin{array}{c}- \\
670801 \\
640508 \\
- \\
-\end{array}$ & $\begin{array}{l}180 \\
180 \\
169 \\
169 \\
196\end{array}$ & $\begin{array}{l}- \\
330000 \\
328000 \\
- \\
-\end{array}$ & $\begin{array}{l}- \\
3330000 \\
335000 \\
- \\
-\end{array}$ \\
\hline $\begin{array}{l}\mathrm{S} \\
\mathrm{S} \\
\mathrm{S} \\
\mathrm{S}\end{array}$ & $\begin{array}{l}41 \\
42 \\
43 \\
44 \\
45\end{array}$ & $\begin{array}{l}\text { MERRIVALE DRIVE } \\
\text { MERRIVALE DRIVE } \\
\text { APPLEWOOD ROAD } \\
\text { MORICHES ROAD } \\
\text { ORANGE AVENUE }\end{array}$ & $\begin{array}{l}\text { FOREST LANE } \\
\text { APONE COURT } \\
\text { FLORAL LANE } \\
\text { PLAIN TREE LANE } \\
\text { DELAWARE AVENUE }\end{array}$ & $\begin{array}{l}4049570731233 \\
4049380731236 \\
4053100730901 \\
4 \varnothing 52580730846 \\
4049310731724\end{array}$ & $\begin{array}{c}- \\
\overline{560861} \\
560801 \\
560418\end{array}$ & $\begin{array}{l}198 \\
198 \\
180 \\
180 \\
169\end{array}$ & $\begin{array}{l}- \\
- \\
219500 \\
219500 \\
370000\end{array}$ & $\begin{array}{l}- \\
- \\
2590000 \\
270000 \\
430000\end{array}$ \\
\hline
\end{tabular}


N.Y. [Dash indicates no data. Altitudes are in feet above sea level.] (cont.)

\begin{tabular}{|c|c|c|c|c|c|c|c|c|c|c|c|c|}
\hline & & $\begin{array}{l}\text { MAX. } \\
\text { AREA } \\
\text { (squar }\end{array}$ & $\begin{array}{c}\text { BASIN } \\
\text { AREA } \\
\text { feet) }\end{array}$ & RIM & $\begin{array}{l}--A L T \\
\text { OVER } \\
\text { FLOW }\end{array}$ & $\begin{array}{l}\text { BOTDE-- } \\
\text { TOM }\end{array}$ & $\begin{array}{l}\text { WATER } \\
\text { TABLE }\end{array}$ & $\begin{array}{c}\text { DRAIN. } \\
\text { AREA } \\
\text { (acres) }\end{array}$ & $\begin{array}{l}\text {--BAS } \\
\text { STATUS }\end{array}$ & USE & $\begin{array}{l}\text { GEO. } \\
\text { UNIT }\end{array}$ & $\begin{array}{l}\text { SOIL } \\
\text { UNIT }\end{array}$ \\
\hline $\begin{array}{l}R \\
R \\
R\end{array}$ & $\begin{array}{l}1 \\
2 \\
3 \\
4 \\
5\end{array}$ & $\begin{array}{l}- \\
\overline{-} \\
\overline{-}\end{array}$ & $\begin{array}{l}- \\
- \\
- \\
-\end{array}$ & $\begin{array}{r}150.0 \\
140.0 \\
100.0 \\
60.0 \\
105.0\end{array}$ & $\begin{array}{l}- \\
- \\
-\end{array}$ & $\begin{array}{l}- \\
- \\
- \\
-\end{array}$ & $\begin{array}{l}25.0 \\
24.0 \\
22.0 \\
40.0 \\
14.0\end{array}$ & $\begin{array}{r}39.8 \\
- \\
- \\
-\end{array}$ & $\begin{array}{l}1 \\
1 \\
1 \\
1 \\
1\end{array}$ & $\begin{array}{l}1 \\
1 \\
1 \\
1 \\
1\end{array}$ & $\begin{array}{l}4 \\
4 \\
1 \\
1 \\
1\end{array}$ & $\begin{array}{l}5 \\
5 \\
6 \\
3 \\
1\end{array}$ \\
\hline $\begin{array}{l}R \\
R \\
R\end{array}$ & $\begin{array}{r}6 \\
7 \\
8 \\
9 \\
10\end{array}$ & $\begin{array}{l}- \\
- \\
- \\
-\end{array}$ & $\begin{array}{l}- \\
- \\
-\end{array}$ & $\begin{array}{r}105.0 \\
40.0 \\
30.0 \\
20.0 \\
30 . \varnothing\end{array}$ & $\begin{array}{l}- \\
- \\
- \\
-\end{array}$ & $\begin{array}{l}\bar{z} \\
\bar{z}\end{array}$ & $\begin{array}{r}7.0 \\
18.0 \\
19.0 \\
16.0 \\
15.0\end{array}$ & $\begin{array}{l}- \\
- \\
- \\
-\end{array}$ & $\begin{array}{l}0 \\
0 \\
0 \\
1 \\
1\end{array}$ & $\begin{array}{l}1 \\
1 \\
1 \\
1 \\
1\end{array}$ & $\begin{array}{l}1 \\
1 \\
1 \\
1 \\
1\end{array}$ & $\begin{array}{l}3 \\
3 \\
6 \\
6 \\
3\end{array}$ \\
\hline $\begin{array}{l}R \\
R \\
S \\
S \\
S\end{array}$ & $\begin{array}{r}15 \\
16 \\
2 \\
3 \\
4\end{array}$ & $\begin{array}{c}\overline{-} \\
\overline{-} \\
8 \sigma \sigma \sigma \sigma \\
180 \varnothing \emptyset\end{array}$ & $\begin{array}{r}- \\
- \\
124200 \\
36500\end{array}$ & $\begin{array}{r}30.0 \\
15.0 \\
80.0 \\
150.0 \\
158.0\end{array}$ & $\begin{array}{l}- \\
\overline{-} \\
\text { - }\end{array}$ & $\begin{array}{r}- \\
\overline{-} \\
136 . \bar{\varnothing} \\
149 . \varnothing\end{array}$ & $\begin{array}{r}4.0 \\
3.0 \\
55.0 \\
42.0 \\
40.0\end{array}$ & $\begin{array}{r}\overline{-} \\
117 . \overline{6} \\
21.3\end{array}$ & $\begin{array}{l}1 \\
1 \\
1 \\
1 \\
0\end{array}$ & $\begin{array}{l}1 \\
1 \\
1 \\
1 \\
1\end{array}$ & $\begin{array}{l}1 \\
1 \\
1 \\
4 \\
4\end{array}$ & $\begin{array}{l}0 \\
0 \\
6 \\
3 \\
7\end{array}$ \\
\hline $\begin{array}{l}S \\
S \\
S\end{array}$ & $\begin{array}{r}5 \\
6 \\
8 \\
9 \\
10\end{array}$ & $\begin{array}{l}66500 \\
30800 \\
50000 \\
10000 \\
72600\end{array}$ & $\begin{array}{r}161380 \\
8280 \varnothing \\
96360 \\
35300 \\
1044 \varnothing \varnothing\end{array}$ & $\begin{array}{r}63.0 \\
94.0 \\
48 . \varnothing \\
125 . \varnothing \\
149 . \varnothing\end{array}$ & $\begin{array}{r}62.9 \\
48 . \overline{-} \\
97.0 \\
-\end{array}$ & $\begin{array}{r}- \\
75.5 \\
40.0 \\
89.0 \\
125.0\end{array}$ & $\begin{array}{l}52.0 \\
53.0 \\
18.0 \\
18.0 \\
57.0\end{array}$ & $\begin{array}{r}92.2 \\
44.1 \\
45.0 \\
7.0 \\
112.2\end{array}$ & $\begin{array}{l}0 \\
1 \\
1 \\
1 \\
1\end{array}$ & $\begin{array}{l}1 \\
1 \\
1 \\
1 \\
1\end{array}$ & $\begin{array}{l}1 \\
1 \\
3 \\
3 \\
1\end{array}$ & $\begin{array}{l}5 \\
5 \\
3 \\
3 \\
7\end{array}$ \\
\hline $\begin{array}{l}S \\
S \\
S \\
S \\
S\end{array}$ & $\begin{array}{l}11 \\
12 \\
13 \\
14 \\
15\end{array}$ & $\begin{array}{c}14800 \\
- \\
\overline{18200} \\
7300\end{array}$ & $\begin{array}{l}17100 \\
34000 \\
71500 \\
32000 \\
13800\end{array}$ & $\begin{array}{l}148.0 \\
141 . \varnothing \\
140.7 \\
140 . \varnothing \\
139.2\end{array}$ & $\begin{array}{r}- \\
- \\
136.5 \\
-\end{array}$ & $\begin{array}{l}141 . \varnothing \\
127 . \varnothing \\
112 . \varnothing \\
123 . \varnothing \\
125 . \varnothing\end{array}$ & $\begin{array}{l}58 . \varnothing \\
62 . \varnothing \\
52.0 \\
59 . \varnothing \\
57 . \varnothing\end{array}$ & $\begin{array}{r}16.0 \\
- \\
168 . \overline{0} \\
21.5\end{array}$ & $\begin{array}{l}1 \\
1 \\
1 \\
1 \\
1\end{array}$ & $\begin{array}{l}1 \\
1 \\
1 \\
1 \\
1\end{array}$ & $\begin{array}{l}1 \\
1 \\
1 \\
1 \\
1\end{array}$ & $\begin{array}{l}7 \\
7 \\
7 \\
7 \\
7\end{array}$ \\
\hline $\begin{array}{l}S \\
S \\
S \\
S \\
S\end{array}$ & $\begin{array}{l}16 \\
17 \\
18 \\
19 \\
20\end{array}$ & $\begin{array}{r}8000 \\
30300 \\
4400 \\
9600 \\
44500\end{array}$ & $\begin{array}{l}15800 \\
28900 \\
10300 \\
24000 \\
27500\end{array}$ & $\begin{array}{r}150.5 \\
146.5 \\
150.0 \\
153.2 \\
70.0\end{array}$ & $\begin{array}{r}148.5 \\
- \\
153.0 \\
148.0 \\
-\end{array}$ & $\begin{array}{r}136.0 \\
124.0 \\
145.0 \\
134.5 \\
60.0\end{array}$ & $\begin{array}{l}56 . \emptyset \\
54.0 \\
48 . \varnothing \\
48 . \varnothing \\
37 . \varnothing\end{array}$ & $\begin{array}{r}28.4 \\
40.0 \\
5.5 \\
19.0 \\
70.3\end{array}$ & $\begin{array}{l}1 \\
0 \\
1 \\
1 \\
1\end{array}$ & $\begin{array}{l}1 \\
3 \\
1 \\
1 \\
1\end{array}$ & $\begin{array}{l}1 \\
1 \\
1 \\
3 \\
1\end{array}$ & $\begin{array}{l}7 \\
7 \\
3 \\
7 \\
6\end{array}$ \\
\hline $\begin{array}{l}S \\
S \\
S \\
S \\
S\end{array}$ & $\begin{array}{l}21 \\
22 \\
23 \\
24 \\
25\end{array}$ & $\begin{array}{c}- \\
50800 \\
9800 \\
135580 \\
-\end{array}$ & $\begin{array}{r}- \\
60100 \\
9900 \\
13200 \\
-\end{array}$ & $\begin{array}{r}150.0 \\
148.0 \\
170.0 \\
171.3 \\
70.0\end{array}$ & $\begin{array}{r}- \\
146.2 \\
- \\
169.8 \\
-\end{array}$ & $\begin{array}{r}136 . \overline{2} \\
\overline{-} \\
161 . \varnothing \\
-\end{array}$ & $\begin{array}{l}65.0 \\
66.0 \\
52 . \varnothing \\
48 . \varnothing \\
48 . \varnothing\end{array}$ & $\begin{array}{r}49 . \overline{3} \\
15.6 \\
11.6 \\
-\end{array}$ & $\begin{array}{l}1 \\
1 \\
1 \\
1 \\
1\end{array}$ & $\begin{array}{l}1 \\
1 \\
1 \\
1 \\
1\end{array}$ & $\begin{array}{l}1 \\
1 \\
1 \\
1 \\
1\end{array}$ & $\begin{array}{l}7 \\
7 \\
7 \\
7 \\
7\end{array}$ \\
\hline $\begin{array}{l}S \\
S \\
S \\
S \\
S\end{array}$ & $\begin{array}{l}26 \\
27 \\
28 \\
29 \\
30\end{array}$ & $\begin{array}{r}82100 \\
64700 \\
7800 \\
43100 \\
49800\end{array}$ & $\begin{array}{r}104000 \\
92800 \\
4900 \\
50200 \\
62500\end{array}$ & $\begin{array}{l}53.0 \\
56.4 \\
60.0 \\
61.0 \\
67.5\end{array}$ & $\begin{array}{r}50.5 \\
52.0 \\
69.5 \\
59.3 \\
-\end{array}$ & $\begin{array}{l}44.0 \\
46.5 \\
51.5 \\
47.8 \\
53.0\end{array}$ & $\begin{array}{l}48.0 \\
46.0 \\
44.0 \\
44.0 \\
45.0\end{array}$ & $\begin{array}{l}82.2 \\
50.0 \\
18.0 \\
58.3 \\
78.4\end{array}$ & $\begin{array}{l}0 \\
1 \\
1 \\
1 \\
0\end{array}$ & $\begin{array}{l}1 \\
1 \\
1 \\
1 \\
1\end{array}$ & $\begin{array}{l}1 \\
1 \\
1 \\
1 \\
1\end{array}$ & $\begin{array}{l}3 \\
7 \\
7 \\
6 \\
5\end{array}$ \\
\hline $\begin{array}{l}S \\
S \\
S \\
S \\
S\end{array}$ & $\begin{array}{l}31 \\
32 \\
33 \\
34 \\
35\end{array}$ & $\begin{array}{r}18300 \\
19000 \\
8500 \\
26200 \\
25700\end{array}$ & $\begin{array}{l}42100 \\
44300 \\
13500 \\
33500 \\
37100\end{array}$ & $\begin{array}{r}120.0 \\
121.0 \\
92.6 \\
54.0 \\
58.5\end{array}$ & $\begin{array}{r}114.0 \\
117.0 \\
86.5 \\
53.0 \\
56.0\end{array}$ & $\begin{array}{r}102.5 \\
108.0 \\
72.5 \\
46.2 \\
47.0\end{array}$ & $\begin{array}{l}48.0 \\
48.0 \\
44.0 \\
42.0 \\
42 . \varnothing\end{array}$ & $\begin{array}{r}38.7 \\
24.7 \\
16.5 \\
9.0 \\
33.1\end{array}$ & $\begin{array}{l}1 \\
1 \\
1 \\
1 \\
1\end{array}$ & $\begin{array}{l}1 \\
1 \\
1 \\
3 \\
1\end{array}$ & $\begin{array}{l}1 \\
1 \\
1 \\
1 \\
1\end{array}$ & $\begin{array}{l}3 \\
1 \\
7 \\
6 \\
6\end{array}$ \\
\hline $\begin{array}{l}S \\
S \\
S \\
S \\
S\end{array}$ & $\begin{array}{l}36 \\
37 \\
38 \\
39 \\
40\end{array}$ & $\begin{array}{c}- \\
29000 \\
33500 \\
- \\
-\end{array}$ & $\begin{array}{r}- \\
56000 \\
39000 \\
- \\
-\end{array}$ & $\begin{array}{r}140.0 \\
130.0 \\
60.0 \\
144.0 \\
60.0\end{array}$ & $\begin{array}{l}- \\
- \\
- \\
-\end{array}$ & $\begin{array}{r}- \\
43 . \overline{5} \\
- \\
-\end{array}$ & $\begin{array}{l}41.0 \\
34.0 \\
44.0 \\
58.0 \\
40.0\end{array}$ & $\begin{array}{r}- \\
35.5 \\
38.8 \\
- \\
-\end{array}$ & $\begin{array}{l}1 \\
1 \\
1 \\
1 \\
1\end{array}$ & $\begin{array}{l}1 \\
1 \\
1 \\
1 \\
1\end{array}$ & $\begin{array}{l}1 \\
1 \\
1 \\
1 \\
1\end{array}$ & $\begin{array}{l}7 \\
7 \\
\mathbf{5} \\
7 \\
7\end{array}$ \\
\hline $\begin{array}{l}S \\
S \\
S \\
c\end{array}$ & $\begin{array}{l}41 \\
42 \\
43 \\
44 \\
45\end{array}$ & $\begin{array}{c}\overline{-} \\
24 \overline{7} 00 \\
25700 \\
43000\end{array}$ & $\begin{array}{r}- \\
3380 \overline{-} \\
34500 \\
73500\end{array}$ & $\begin{array}{r}55.0 \\
50.0 \\
135.0 \\
126.0 \\
115.0\end{array}$ & $\begin{array}{r}- \\
131 . \overline{5} \\
123.5 \\
114.0\end{array}$ & $\begin{array}{r}- \\
121 . \bar{\emptyset} \\
113 . \varnothing \\
104 . \varnothing\end{array}$ & $\begin{array}{l}42 . \varnothing \\
44 . \varnothing \\
46 . \varnothing \\
60 . \varnothing \\
65 . \varnothing\end{array}$ & $\begin{array}{r}- \\
30 . \overline{0} \\
34.6 \\
42.5\end{array}$ & $\begin{array}{l}1 \\
1 \\
1 \\
1 \\
1\end{array}$ & $\begin{array}{l}1 \\
1 \\
1 \\
1 \\
1\end{array}$ & $\begin{array}{l}1 \\
1 \\
1 \\
1 \\
1\end{array}$ & $\begin{array}{l}7 \\
6 \\
7 \\
7 \\
7\end{array}$ \\
\hline
\end{tabular}


Appendix.--Location and basic physical features of recharge basins on Long Island,

\begin{tabular}{|c|c|c|c|c|c|c|c|c|}
\hline \multicolumn{2}{|c|}{$\begin{array}{l}\text { BASIN } \\
\text { NUMBER }\end{array}$} & \multicolumn{2}{|c|}{ NEAREST INTERSECTION } & $\begin{array}{l}\text { LATITUDE } \\
\text { LONGITUDE } \\
0, " 1 \text { " } \\
4052496731454\end{array}$ & $\begin{array}{l}\text { DATE } \\
\text { BUILT } \\
\text { YrMOD }\end{array}$ & COMMUNITY & $\begin{array}{l}\text { DESIGN } \\
\text { CAPACITY } \\
\text { (cubic }\end{array}$ & $\begin{array}{l}\text { ACTUAL } \\
\text { CAPACITY } \\
\text { fe日t) }\end{array}$ \\
\hline $\begin{array}{l}\text { S } \\
\text { S } \\
\text { S } \\
\text { S } \\
\text { S }\end{array}$ & $\begin{array}{l}48 \\
49 \\
53 \\
54 \\
55\end{array}$ & $\begin{array}{l}\text { MAGELLAN AVENUE } \\
\text { OLD WILLETS PATH } \\
\text { LAKE AVENUE SO. } \\
\text { TOWN LINE ROAD } \\
\text { CARAMEL ROAD }\end{array}$ & $\begin{array}{l}\text { MARILYN ORIVE } \\
\text { MACKAY DRIVE } \\
\text { BEAU JOL COURT } \\
\text { MARSHMALLOW OR. } \\
\text { MARSHMALLOW DR. }\end{array}$ & $\begin{array}{l}4852496731454 \\
4049156731462 \\
4656416736965 \\
4651576731724 \\
46520167317 \varnothing 5\end{array}$ & $\begin{array}{l}650601 \\
698616 \\
6581 \\
658715 \\
650715\end{array}$ & $\begin{array}{l}174 \\
196 \\
177 \\
169 \\
169\end{array}$ & $\begin{array}{r}14 \varnothing \varnothing \varnothing \varnothing \\
148 \varnothing \varnothing \varnothing \\
493 \varnothing \varnothing \\
2145 \varnothing \varnothing \\
2452 \varnothing \varnothing\end{array}$ & $\begin{array}{r}141400 \\
195000 \\
42300 \\
222000 \\
252000\end{array}$ \\
\hline s & $\begin{array}{l}56 \\
57 \\
58 \\
59 \\
6 \varnothing\end{array}$ & $\begin{array}{l}\text { CRESCENT PLACE } \\
\text { KINGS PARK ROAD } \\
\text { TOWN LINE ROAD } \\
\text { TOWN LINE ROAD } \\
\text { HAYRICK LANE }\end{array}$ & $\begin{array}{l}\text { SEAVER LANE } \\
\text { CHATHAM ROAD } \\
\text { HAVEMEYER LANE } \\
\text { SCHOLAR LANE } \\
\text { SCHOLAR LANE }\end{array}$ & $\begin{array}{l}465067 \varnothing 731265 \\
4651116731559 \\
4651690731726 \\
4651280731727 \\
4651310731649\end{array}$ & $\begin{array}{l}611006 \\
- \\
626422 \\
628422 \\
628422\end{array}$ & $\begin{array}{l}196 \\
169 \\
169 \\
169 \\
169\end{array}$ & $\begin{array}{r}369000 \\
- \\
1452 \varnothing 0 \\
82200 \\
469800\end{array}$ & $\begin{array}{l}374000 \\
\overline{174000} \\
295000 \\
546000\end{array}$ \\
\hline $\begin{array}{l}S \\
S\end{array}$ & $\begin{array}{l}61 \\
62 \\
63 \\
64 \\
65\end{array}$ & $\begin{array}{l}\text { HARVEST LANE } \\
\text { HERON LANE } \\
\text { FINCH COURT } \\
\text { GANNET DRIVE } \\
\text { LAURINDA ORIVE }\end{array}$ & $\begin{array}{l}\text { HAYRICK LANE } \\
\text { PHEASANT DRIVE } \\
\text { PEACOCK LANE } \\
\text { EDSCHO LANE } \\
\text { NEW HIGHWAY }\end{array}$ & $\begin{array}{l}4651160731651 \\
4649460731616 \\
4649416731684 \\
4849366731551 \\
4649446731734\end{array}$ & $\begin{array}{l}- \\
640124 \\
64 \varnothing 124 \\
640124 \\
58 \varnothing 5 \varnothing 8\end{array}$ & $\begin{array}{l}169 \\
169 \\
169 \\
169 \\
169\end{array}$ & $\begin{array}{l}419800 \\
3213 \varnothing 0 \\
23 \varnothing 8 \varnothing 0 \\
136300 \\
-\end{array}$ & $\begin{array}{l}4545 \varnothing \varnothing \\
197 \varnothing \varnothing \varnothing \\
3294 \varnothing \varnothing \\
1795 \varnothing \varnothing \\
-\end{array}$ \\
\hline $\begin{array}{l}\text { S } \\
\text { S } \\
S \\
S \\
S\end{array}$ & $\begin{array}{l}66 \\
67 \\
68 \\
69 \\
70\end{array}$ & $\begin{array}{l}\text { RUSSET COURT } \\
\text { DEEPDALE ORIVE } \\
\text { DEEPDALE DRIVE } \\
\text { BROCTON LANE } \\
\text { AUSTIN BOULEVARD }\end{array}$ & $\begin{array}{l}\text { COUNTRY LANE } \\
\text { SHINBONE LANE } \\
\text { REDLEAF LANE } \\
\text { ASHLAND DRIVE } \\
\text { MOTOR PARKWAY }\end{array}$ & $\begin{array}{l}4653310731532 \\
4 \varnothing 48300731656 \\
4648516731795 \\
4652320731328 \\
4648230731624\end{array}$ & $\begin{array}{l}606624 \\
620426 \\
62042 \varnothing \\
620815 \\
6807 \varnothing 9\end{array}$ & $\begin{array}{l}174 \\
169 \\
169 \\
182 \\
169\end{array}$ & $\begin{array}{r}16200 \varnothing \\
18210 \varnothing \\
15880 \varnothing \\
16106 \varnothing \varnothing \\
44 \varnothing 7 \varnothing \varnothing\end{array}$ & $\begin{array}{rr}x & 1679 \varnothing 0 \\
x & 19600 \varnothing \\
169500 \\
x \\
219600 \varnothing \\
46150 \varnothing\end{array}$ \\
\hline $\begin{array}{l}S \\
S \\
S \\
S \\
S\end{array}$ & $\begin{array}{l}71 \\
72 \\
73 \\
74 \\
75\end{array}$ & $\begin{array}{l}\text { GLENRICH DRIVE } \\
\text { DAMIN CIRCLE } \\
\text { BURHAM DRIVE } \\
\text { NICHOLAS AVENUE } \\
\text { RIVIERA DRIVE }\end{array}$ & $\begin{array}{l}\text { REGAL COURT } \\
\text { GLENRICH ORIVE } \\
\text { ROSE STREET } \\
\text { MONROE STREET } \\
\text { SYBIL PLACE }\end{array}$ & $\begin{array}{l}4652138731165 \\
4652316731113 \\
4652538731254 \\
4652380731361 \\
4652216731360\end{array}$ & $\begin{array}{l}671204 \\
671264 \\
57 \varnothing 715 \\
579715 \\
630523\end{array}$ & $\begin{array}{l}179 \\
179 \\
179 \\
174 \\
179\end{array}$ & $\begin{array}{l}- \\
\overline{11900 \varnothing} \\
30500 \varnothing \\
14800 \varnothing\end{array}$ & $\begin{array}{l}144 \varnothing 0 \varnothing 0 \\
2 \varnothing 9 \varnothing \varnothing \varnothing \\
121 \varnothing \varnothing \varnothing \\
38 \varnothing \varnothing \varnothing \varnothing \\
154 \varnothing \varnothing \varnothing\end{array}$ \\
\hline $\begin{array}{l}\text { S } \\
\text { S } \\
\text { S } \\
\text { S }\end{array}$ & $\begin{array}{l}76 \\
77 \\
78 \\
79 \\
80\end{array}$ & $\begin{array}{l}\text { MEADOW GLEN ROAD } \\
\text { CYGNET DRIVE } \\
\text { BROOKSITE ORIVE } \\
\text { PINEWOOD DRIVE } \\
\text { SEQUOIA DRIVE }\end{array}$ & $\begin{array}{l}\text { JOSEPHINE LANE } \\
\text { FLAMINGO DRIVE } \\
\text { CYGNET DRIVE } \\
\text { CEDARWOOD LANE } \\
\text { NESCONSET HWY. }\end{array}$ & $\begin{array}{l}4653238731634 \\
4856518731362 \\
4656468731233 \\
4848338731647 \\
4649496731148\end{array}$ & $\begin{array}{l}676263 \\
5811 \\
5811 \\
66 \varnothing 218 \\
59 ø 33 \varnothing\end{array}$ & $\begin{array}{l}174 \\
182 \\
182 \\
169 \\
196\end{array}$ & $\begin{array}{l}1450 \varnothing \varnothing \\
9257 \varnothing \varnothing \\
1539 \varnothing \varnothing \\
394 \varnothing \varnothing \varnothing \\
252 \varnothing \varnothing \varnothing\end{array}$ & $\begin{array}{l}1446 \varnothing \varnothing \\
971 \varnothing \varnothing \varnothing \\
1627 \varnothing \varnothing \\
47 \varnothing \varnothing \varnothing \varnothing \\
256 \varnothing \varnothing \varnothing\end{array}$ \\
\hline $\begin{array}{l}\text { S } \\
\text { S } \\
\text { S } \\
\text { S } \\
\text { S }\end{array}$ & $\begin{array}{l}81 \\
82 \\
83 \\
84 \\
85\end{array}$ & $\begin{array}{l}\text { APPLE TREE DRIVE } \\
\text { BOXWOOD DRIVE } \\
\text { PLYMOUTH BLVD. } \\
\text { CONCORD LANE } \\
\text { AMSTERDAM ROAD }\end{array}$ & $\begin{array}{l}\text { SATURN BOULEVARD } \\
\text { ANN COURT } \\
\text { WESTON LANE } \\
\text { QUAKER LANE } \\
\text { JERICHO TURNPIKE }\end{array}$ & $\begin{array}{l}4049386731152 \\
4653278731347 \\
4651246731416 \\
4651130731412 \\
465652 \varnothing 731427\end{array}$ & $\begin{array}{l}598330 \\
676828 \\
61 \varnothing 418 \\
616418 \\
61 \varnothing 418\end{array}$ & $\begin{array}{l}196 \\
181 \\
182 \\
182 \\
182\end{array}$ & $\begin{array}{l}13130 \varnothing \\
4482 \varnothing \varnothing \\
28390 \varnothing \\
747 \varnothing 0 \varnothing \\
243 \varnothing \varnothing \varnothing\end{array}$ & $\begin{array}{l}157000 \\
471406 \\
- \\
760300 \\
256000\end{array}$ \\
\hline $\begin{array}{l}\text { S } \\
\text { S } \\
\text { S } \\
\text { S }\end{array}$ & $\begin{array}{l}86 \\
87 \\
88 \\
89 \\
96\end{array}$ & $\begin{array}{l}\text { HERITAGE PLACE } \\
\text { HARNED ROAD } \\
\text { SHAWNEE PLACE } \\
\text { TRUXTON LANE } \\
\text { TRESCOTT PATH }\end{array}$ & $\begin{array}{l}\text { SOUTH COURT } \\
\text { CUTCHOGUE LANE } \\
\text { SEMINOLE DRIVE } \\
\text { ST. JOHNLAND RD. } \\
\text { TARLETON LANE }\end{array}$ & $\begin{array}{l}4 \varnothing 56320736857 \\
4 \varnothing 495 \varnothing 6731649 \\
464953 \varnothing 731626 \\
465417 \varnothing 731547 \\
465412 \varnothing 73161 \varnothing\end{array}$ & $\begin{array}{l}656716 \\
646968 \\
646968 \\
67 \varnothing 822 \\
676822\end{array}$ & $\begin{array}{l}177 \\
169 \\
169 \\
174 \\
174\end{array}$ & $\begin{array}{r}884 \varnothing 0 \\
579600 \\
3395 \varnothing \varnothing \\
534100 \\
928 \varnothing \varnothing\end{array}$ & $\begin{array}{l}-\overline{60900 \varnothing} \\
346500 \\
560600 \\
157600\end{array}$ \\
\hline $\begin{array}{l}\text { S } \\
\text { S } \\
\text { S } \\
\text { S }\end{array}$ & $\begin{array}{l}91 \\
92 \\
93 \\
94 \\
95\end{array}$ & $\begin{array}{l}\text { OLD WILLETS PATH } \\
\text { COACH DRIVE } \\
\text { BRUCE LANE } \\
\text { PLYMOUTH BLVD. } \\
\text { BAY STREET }\end{array}$ & $\begin{array}{l}\text { VETERANS HIGHWAY } \\
\text { CARRIAGE DRIVE } \\
\text { CORTEZ LANE } \\
\text { MOREWOOD DRIVE } \\
\text { COLUMBUS AVENUE }\end{array}$ & $\begin{array}{l}464927 \varnothing 731469 \\
4653130731483 \\
4652496731569 \\
4651296731419 \\
46523 \varnothing 6731434\end{array}$ & $\begin{array}{l}576116 \\
668269 \\
646508 \\
616563\end{array}$ & $\begin{array}{l}196 \\
174 \\
174 \\
182 \\
174\end{array}$ & $\begin{array}{l}4980000 \\
596 \varnothing \varnothing 0 \\
- \\
2927 \varnothing 0 \\
1620 \varnothing 0\end{array}$ & $\begin{array}{l}619060 \\
607 \varnothing 00 \\
- \\
342000 \\
162500\end{array}$ \\
\hline $\begin{array}{l}S \\
S \\
S \\
S \\
S\end{array}$ & $\begin{array}{r}96 \\
97 \\
98 \\
99 \\
190\end{array}$ & $\begin{array}{l}\text { KRISTI LANE } \\
\text { CORNELIA LANE } \\
\text { LAWRENCE ROAD } \\
\text { URSULAR COURT } \\
\text { URSULAR COURT }\end{array}$ & $\begin{array}{l}\text { SUSSEX LANE } \\
\text { CEDAR AVENUE } \\
\text { LOROLY COURT } \\
\text { STENGEL PLACE } \\
\text { ECKERKAMP DRIVE }\end{array}$ & $\begin{array}{l}4656580730747 \\
4656550730748 \\
4652530731427 \\
4652606731251 \\
465263 \varnothing 73122 \varnothing\end{array}$ & $\begin{array}{r}661 \varnothing 27 \\
661 \varnothing 27 \\
- \\
66 \varnothing 72 \varnothing \\
66672 \varnothing\end{array}$ & $\begin{array}{l}177 \\
177 \\
174 \\
182 \\
182\end{array}$ & $\begin{array}{l}418890 \\
139500 \\
- \\
262780 \\
153300\end{array}$ & $\begin{array}{l}480300 \\
142 \varnothing 00 \\
- \\
3 \varnothing \varnothing 000 \\
22000 \varnothing\end{array}$ \\
\hline $\begin{array}{l}\text { S } \\
S \\
S \\
S \\
S\end{array}$ & $\begin{array}{l}101 \\
102 \\
103 \\
104 \\
105\end{array}$ & $\begin{array}{l}\text { ARLENE PLACE } \\
\text { 1ST AVENUE } \\
\text { MARCUS BOULEVARD } \\
\text { MARCUS BOULEVARD } \\
\text { ALFRED LANE }\end{array}$ & $\begin{array}{l}\text { MARVIN DRIVE } \\
\text { ECHO LANE } \\
\text { OSER AVENUE } \\
\text { MOTOR PARKWAY } \\
\text { SMITHTOWN ROAD }\end{array}$ & $\begin{array}{l}4652280731623 \\
4 \varnothing 52196731541 \\
4 \varnothing 48560731457 \\
4 \varnothing 4834 \varnothing 731454 \\
4652 \varnothing 38731424\end{array}$ & $\begin{array}{l}589968 \\
586968 \\
671616 \\
671916 \\
660718\end{array}$ & $\begin{array}{l}174 \\
174 \\
196 \\
191 \\
182\end{array}$ & $\begin{array}{l}\overline{-} \\
\overline{-} \\
\overline{96000}\end{array}$ & $\begin{array}{l}106006 \\
88460 \\
\overline{-} \\
106300\end{array}$ \\
\hline
\end{tabular}


N.Y. [Dash indicates no data. Altitudes are in feet above sea level.] (cont.)

\begin{tabular}{|c|c|c|c|c|c|c|c|c|c|c|c|c|}
\hline \multicolumn{2}{|c|}{$\begin{array}{l}\text { BASIN } \\
\text { NUMBER }\end{array}$} & $\begin{array}{l}\text { MAX. } \\
\text { AREA } \\
\text { (squar }\end{array}$ & $\begin{array}{c}\text { BASIN } \\
\text { AREA } \\
\text { feet) }\end{array}$ & \multicolumn{2}{|c|}{$\begin{array}{ll} & \text { ALTIT } \\
\text { RIM } & \text { FLER- }\end{array}$} & $\begin{array}{l}\text { BOT- } \\
\text { TOM }\end{array}$ & $\begin{array}{l}\text { WATER } \\
\text { TABLE }\end{array}$ & $\begin{array}{c}\text { DRAIN. } \\
\text { AREA } \\
\text { (acres) }\end{array}$ & \multicolumn{2}{|c|}{$\begin{array}{l}---B A S I N-- \\
\text { STATUS USE }\end{array}$} & $\begin{array}{l}\text { GEO. } \\
\text { UNIT }\end{array}$ & $\begin{array}{l}\text { SOIL } \\
\text { UNIT }\end{array}$ \\
\hline $\begin{array}{l}S \\
S \\
S \\
S \\
S\end{array}$ & $\begin{array}{l}48 \\
49 \\
53 \\
54 \\
55\end{array}$ & $\begin{array}{r}10900 \\
17700 \\
3900 \\
22200 \\
25200\end{array}$ & $\begin{array}{l}247 \varnothing 0 \\
19800 \\
10500 \\
17600 \\
26000\end{array}$ & $\begin{array}{r}140.0 \\
56.0 \\
120.0 \\
155.0 \\
154.0\end{array}$ & $\begin{array}{r}130.0 \\
55.0 \\
113.7 \\
- \\
-\end{array}$ & $\begin{array}{r}117.0 \\
44.0 \\
103.0 \\
141.5 \\
134.0\end{array}$ & $\begin{array}{l}48 . \varnothing \\
41 . \varnothing \\
50.0 \\
56.0 \\
57 . \varnothing\end{array}$ & $\begin{array}{r}22.8 \\
25.5 \\
7.8 \\
33.8 \\
38.6\end{array}$ & $\begin{array}{l}0 \\
1 \\
1 \\
1 \\
1\end{array}$ & $\begin{array}{l}1 \\
1 \\
1 \\
1 \\
1\end{array}$ & $\begin{array}{l}1 \\
1 \\
1 \\
1 \\
1\end{array}$ & $\begin{array}{l}7 \\
6 \\
1 \\
7 \\
7\end{array}$ \\
\hline $\begin{array}{l}S \\
S \\
S \\
S \\
S\end{array}$ & $\begin{array}{l}56 \\
57 \\
58 \\
59 \\
60\end{array}$ & $\begin{array}{c}28800 \\
- \\
1740 \emptyset \\
1860 \emptyset \\
546 \varnothing \emptyset\end{array}$ & $\begin{array}{r}42900 \\
- \\
41600 \\
30000 \\
57500\end{array}$ & $\begin{array}{r}40.0 \\
145.0 \\
150 . \varnothing \\
165.0 \\
149.0\end{array}$ & $\begin{array}{r}- \\
- \\
130 . \overline{7} \\
158.5 \\
143.5\end{array}$ & $\begin{array}{r}- \\
- \\
120 . \overline{7} \\
147.5 \\
133.5\end{array}$ & $\begin{array}{l}32.0 \\
58.0 \\
64.0 \\
62.0 \\
61.0\end{array}$ & $\begin{array}{r}58.1 \\
- \\
22.9 \\
12.9 \\
73.9\end{array}$ & $\begin{array}{l}0 \\
1 \\
1 \\
1 \\
1\end{array}$ & $\begin{array}{l}1 \\
1 \\
1 \\
1 \\
1\end{array}$ & $\begin{array}{l}1 \\
1 \\
1 \\
1 \\
1\end{array}$ & $\begin{array}{l}7 \\
7 \\
7 \\
7 \\
7\end{array}$ \\
\hline$S$ & $\begin{array}{l}61 \\
62 \\
63 \\
64 \\
65\end{array}$ & $\begin{array}{c}37900 \\
197 \varnothing 0 \\
329 \varnothing 0 \\
180 \varnothing \varnothing \\
-\end{array}$ & $\begin{array}{r}41100 \\
15000 \\
17700 \\
17600 \\
-\end{array}$ & $\begin{array}{l}161.0 \\
140.0 \\
140.0 \\
130.0 \\
120.0\end{array}$ & $\begin{array}{r}157.5 \\
- \\
- \\
-\end{array}$ & $\begin{array}{r}145.5 \\
105.5 \\
97.0 \\
112.0 \\
-\end{array}$ & $\begin{array}{l}62.0 \\
56.0 \\
56.0 \\
52.0 \\
68.0\end{array}$ & $\begin{array}{r}66.1 \\
34.4 \\
11.4 \\
14.6 \\
-\end{array}$ & $\begin{array}{l}1 \\
1 \\
1 \\
1 \\
1\end{array}$ & $\begin{array}{l}1 \\
1 \\
1 \\
1 \\
1\end{array}$ & $\begin{array}{l}1 \\
1 \\
1 \\
1 \\
1\end{array}$ & $\begin{array}{l}7 \\
7 \\
5 \\
5 \\
7\end{array}$ \\
\hline $\begin{array}{l}S \\
S \\
S\end{array}$ & $\begin{array}{l}66 \\
67 \\
68 \\
69 \\
70\end{array}$ & $\begin{array}{r}15300 \\
19600 \\
17000 \\
168500 \\
46200\end{array}$ & $\begin{array}{r}24200 \\
21800 \\
30100 \\
161700 \\
59400\end{array}$ & $\begin{array}{l}220.0 \\
147.5 \\
160.0 \\
110.0 \\
150.0\end{array}$ & $\begin{array}{r}163.0 \\
- \\
- \\
148.0\end{array}$ & $\begin{array}{r}152.0 \\
135.5 \\
147.0 \\
- \\
-\end{array}$ & $\begin{array}{l}38.0 \\
52.0 \\
58.0 \\
40.0 \\
50.0\end{array}$ & $\begin{array}{r}25.5 \\
20.8 \\
17.0 \\
159.0 \\
69.4\end{array}$ & $\begin{array}{l}0 \\
1 \\
1 \\
1 \\
1\end{array}$ & $\begin{array}{l}1 \\
1 \\
1 \\
1 \\
1\end{array}$ & $\begin{array}{l}4 \\
1 \\
6 \\
1 \\
1\end{array}$ & $\begin{array}{l}3 \\
7 \\
7 \\
7 \\
7\end{array}$ \\
\hline $\begin{array}{l}S \\
S\end{array}$ & $\begin{array}{l}71 \\
72 \\
73 \\
74 \\
75\end{array}$ & $\begin{array}{l}144 \varnothing \varnothing \\
174 \varnothing \varnothing \\
121 \varnothing \varnothing \\
326 \varnothing \emptyset \\
128 \varnothing \varnothing\end{array}$ & $\begin{array}{r}246 \varnothing \varnothing \\
156 \varnothing \emptyset \\
- \\
299 \varnothing \varnothing \\
144 \varnothing \emptyset\end{array}$ & $\begin{array}{l}101.0 \\
128.0 \\
160.0 \\
141.2 \\
120.0\end{array}$ & $\begin{array}{r}98 . \varnothing \\
126.0 \\
157.6 \\
134.5 \\
110.4\end{array}$ & $\begin{array}{r}88.0 \\
114.0 \\
147.6 \\
123.2 \\
98.4\end{array}$ & $\begin{array}{l}35.0 \\
45.0 \\
30.0 \\
35.0 \\
35.0\end{array}$ & $\begin{array}{l}22.7 \\
32.9 \\
18.7 \\
48.8 \\
16.8\end{array}$ & $\begin{array}{l}1 \\
\varnothing \\
1 \\
1 \\
1\end{array}$ & $\begin{array}{l}1 \\
1 \\
1 \\
1 \\
1\end{array}$ & $\begin{array}{l}1 \\
1 \\
4 \\
1 \\
1\end{array}$ & $\begin{array}{l}5 \\
5 \\
3 \\
7 \\
7\end{array}$ \\
\hline$S$ & $\begin{array}{l}76 \\
77 \\
78 \\
79 \\
80\end{array}$ & $\begin{array}{l}11100 \\
97100 \\
16300 \\
47000 \\
25600\end{array}$ & $\begin{array}{r}23100 \\
107900 \\
12100 \\
47000 \\
23500\end{array}$ & $\begin{array}{r}181.0 \\
50.0 \\
40.0 \\
150.0 \\
61.0\end{array}$ & $\begin{array}{r}179 . \varnothing \\
46.0 \\
39.5 \\
- \\
-\end{array}$ & $\begin{array}{r}166.0 \\
46.5 \\
29.5 \\
135.5 \\
-\end{array}$ & $\begin{array}{l}42 . \varnothing \\
25 . \varnothing \\
32.0 \\
58.0 \\
46 . \varnothing\end{array}$ & $\begin{array}{r}22.8 \\
145.7 \\
24.2 \\
62.8 \\
27.8\end{array}$ & $\begin{array}{l}1 \\
1 \\
0 \\
1 \\
1\end{array}$ & $\begin{array}{l}1 \\
1 \\
1 \\
1 \\
1\end{array}$ & $\begin{array}{l}1 \\
1 \\
1 \\
1 \\
1\end{array}$ & $\begin{array}{l}6 \\
6 \\
7 \\
7 \\
5\end{array}$ \\
\hline$s$ & $\begin{array}{l}81 \\
82 \\
83 \\
84 \\
85\end{array}$ & $\begin{array}{l}157 \varnothing \varnothing \\
377 \varnothing 0 \\
284 \varnothing \varnothing \\
76 \varnothing \varnothing 0 \\
25000\end{array}$ & $\begin{array}{l}16500 \\
62000 \\
28900 \\
81300 \\
33500\end{array}$ & $\begin{array}{l}72.0 \\
31.0 \\
50.6 \\
90.0 \\
60.0\end{array}$ & $\begin{array}{r}30 . \overline{5} \\
-\overline{5} \\
66 .\end{array}$ & $\begin{array}{l}52.5 \\
18.0 \\
40.5 \\
63.5 \\
43.0\end{array}$ & $\begin{array}{l}46.0 \\
15 . \varnothing \\
40.0 \\
34.0 \\
31 . \varnothing\end{array}$ & $\begin{array}{l}15.0 \\
77.1 \\
13.0 \\
80.0 \\
38.3\end{array}$ & $\begin{array}{l}1 \\
1 \\
\emptyset \\
1 \\
1\end{array}$ & $\begin{array}{l}1 \\
1 \\
1 \\
1 \\
1\end{array}$ & $\begin{array}{l}1 \\
3 \\
1 \\
1 \\
1\end{array}$ & $\begin{array}{l}6 \\
3 \\
5 \\
5 \\
5\end{array}$ \\
\hline $\begin{array}{l}S \\
S \\
S \\
S \\
S\end{array}$ & $\begin{array}{l}86 \\
87 \\
88 \\
89 \\
90\end{array}$ & $\begin{array}{l}60900 \\
34600 \\
43100 \\
11300\end{array}$ & $\begin{array}{l}207 \varnothing 0 \\
4680 \varnothing \\
31900 \\
62200 \\
366 \varnothing 0\end{array}$ & $\begin{array}{r}114.0 \\
141.8 \\
140.0 \\
30.0 \\
66.0\end{array}$ & $\begin{array}{r}110.5 \\
- \\
- \\
26.9 \\
56.0\end{array}$ & $\begin{array}{r}100.5 \\
126.0 \\
116.0 \\
13.9 \\
42.0\end{array}$ & $\begin{array}{r}51.0 \\
63 . \varnothing \\
60.0 \\
8.0 \\
10.0\end{array}$ & $\begin{array}{r}9.5 \\
62.0 \\
58.0 \\
84.1 \\
14.6\end{array}$ & $\begin{array}{l}1 \\
\varnothing \\
1 \\
1 \\
1\end{array}$ & $\begin{array}{l}1 \\
1 \\
1 \\
1 \\
1\end{array}$ & $\begin{array}{l}1 \\
5 \\
5 \\
3 \\
3\end{array}$ & $\begin{array}{l}1 \\
7 \\
7 \\
6 \\
6\end{array}$ \\
\hline S & $\begin{array}{l}91 \\
92 \\
93 \\
94 \\
95\end{array}$ & $\begin{array}{c}72800 \\
65300 \\
- \\
34200 \\
12500\end{array}$ & $\begin{array}{r}7160 \varnothing \\
5730 \varnothing \\
51 \varnothing \varnothing \\
294 \varnothing \varnothing \\
236 \varnothing \varnothing\end{array}$ & $\begin{array}{r}48.5 \\
90.0 \\
160.0 \\
49.5 \\
140.0\end{array}$ & $\begin{array}{r}48.5 \\
86 . \varnothing \\
- \\
- \\
142 . \varnothing\end{array}$ & $\begin{array}{r}4 \varnothing . \varnothing \\
76.7 \\
-\overline{8} \\
40 . \varnothing \\
129 . \varnothing\end{array}$ & $\begin{array}{l}41.0 \\
20.0 \\
50.0 \\
40 . \varnothing \\
48.0\end{array}$ & $\begin{array}{r}78.4 \\
68.5 \\
-\overline{8} \\
2.4\end{array}$ & $\begin{array}{l}0 \\
1 \\
1 \\
0 \\
1\end{array}$ & $\begin{array}{l}1 \\
1 \\
1 \\
1 \\
1\end{array}$ & $\begin{array}{l}1 \\
3 \\
1 \\
1 \\
1\end{array}$ & $\begin{array}{l}6 \\
7 \\
7 \\
5 \\
7\end{array}$ \\
\hline$S$ & $\begin{array}{r}96 \\
97 \\
98 \\
99 \\
100\end{array}$ & $\begin{array}{c}366 \varnothing 0 \\
2730 \varnothing \\
- \\
3 \varnothing \varnothing \varnothing \varnothing \\
22 \varnothing 0 \varnothing\end{array}$ & $\begin{array}{r}375 \varnothing \varnothing \\
214 \varnothing \varnothing \\
- \\
225 \varnothing \varnothing \\
284 \varnothing \varnothing\end{array}$ & $\begin{array}{r}80.0 \\
80.0 \\
150.0 \\
72.0 \\
75.0\end{array}$ & $\begin{array}{r}76.5 \\
71.2 \\
- \\
- \\
-\end{array}$ & $\begin{array}{r}65.5 \\
66.0 \\
61 . \overline{0} \\
63.0\end{array}$ & $\begin{array}{l}57.0 \\
57.8 \\
44.8 \\
33.0 \\
29.8\end{array}$ & $\begin{array}{r}51.6 \\
15.0 \\
30.0 \\
17.5\end{array}$ & $\begin{array}{l}1 \\
1 \\
1 \\
1 \\
1\end{array}$ & $\begin{array}{l}1 \\
1 \\
1 \\
1 \\
1\end{array}$ & $\begin{array}{l}1 \\
1 \\
3 \\
1 \\
1\end{array}$ & $\begin{array}{l}3 \\
3 \\
7 \\
7 \\
7\end{array}$ \\
\hline S & $\begin{array}{l}101 \\
102 \\
103 \\
104 \\
105\end{array}$ & $\begin{array}{c}10600 \\
8800 \\
- \\
- \\
8700\end{array}$ & $\begin{array}{r}13200 \\
14800 \\
- \\
11300\end{array}$ & $\begin{array}{l}130.0 \\
110.0 \\
150.0 \\
145.0 \\
102.5\end{array}$ & $\begin{array}{r}90 . \overline{-} \\
\overline{-} \\
-\end{array}$ & $\begin{array}{r}99.0 \\
80.0 \\
- \\
91.0\end{array}$ & $\begin{array}{l}52.0 \\
52.0 \\
48.0 \\
48.0 \\
48.0\end{array}$ & $\begin{array}{r}16.7 \\
13.9 \\
- \\
15.1\end{array}$ & $\begin{array}{l}1 \\
1 \\
1 \\
0 \\
1\end{array}$ & $\begin{array}{l}1 \\
1 \\
1 \\
2 \\
1\end{array}$ & $\begin{array}{l}1 \\
1 \\
1 \\
1 \\
1\end{array}$ & $\begin{array}{l}7 \\
5 \\
6 \\
1 \\
7\end{array}$ \\
\hline
\end{tabular}


Appendix.--Location and basic physical features of recharge basins on Long Island,

\begin{tabular}{|c|c|c|c|c|c|c|c|c|}
\hline \multicolumn{2}{|c|}{$\begin{array}{l}\text { BASIN } \\
\text { NUMBER }\end{array}$} & \multicolumn{2}{|c|}{ NEAREST INTERSECTION } & $\begin{array}{l}\text { LATITUDE } \\
\text { LONGITUDE } \\
0_{0}^{\prime},\end{array}$ & $\begin{array}{l}\text { DATE } \\
\text { BUILT } \\
\text { YrMOD }\end{array}$ & COMMUNITY & $\begin{array}{l}\text { DESIGN } \\
\text { CAPACITY } \\
\text { (cubic } f\end{array}$ & $\begin{array}{l}\text { ACTUAL } \\
\text { CAPACITY } \\
\text { fe日t) }\end{array}$ \\
\hline$S$ & $\begin{array}{l}108 \\
107 \\
108 \\
110 \\
111\end{array}$ & $\begin{array}{l}\text { CURLIN LANE } \\
\text { OLD NORTHPORT RD } \\
\text { ROXBURY DRIVE } \\
\text { BALSAM LANE } \\
\text { MIDDLEVILLE ROAD }\end{array}$ & $\begin{array}{l}\text { COPPER BEECH RD. } \\
\text { PIMLICO DRIVE } \\
\text { BELMAR LANE } \\
\text { SOMERSET DRIVE } \\
\text { DON WAY }\end{array}$ & 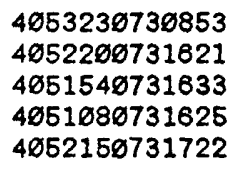 & $\begin{array}{l}881129 \\
67 \varnothing 622 \\
67 \emptyset 622 \\
66 \emptyset 6 \varnothing 2 \\
640423\end{array}$ & $\begin{array}{l}180 \\
169 \\
169 \\
169 \\
169\end{array}$ & $\begin{array}{r}780 \varnothing \emptyset \\
1 \varnothing 83 \varnothing \emptyset \\
1246 \varnothing \emptyset \\
1560 \varnothing \emptyset \\
36438 \emptyset\end{array}$ & $\begin{array}{r}94000 \\
132500 \\
165000 \\
198000 \\
380900\end{array}$ \\
\hline $\mathbf{s}$ & $\begin{array}{l}112 \\
113 \\
114 \\
115 \\
116\end{array}$ & $\begin{array}{l}\text { CARAMEL COURT } \\
\text { NEW HIGHWAY } \\
\text { GREENWICH ROAD } \\
\text { MOOREWOOD ORIVE } \\
\text { CHASSYL ROAD }\end{array}$ & $\begin{array}{l}\text { PEPPERMINT ROAD } \\
\text { NORTHERN ST. PKY } \\
\text { MOREWOOD DRIVE } \\
\text { WOODROW LANE } \\
\text { EDSCHO LANE }\end{array}$ & $\begin{array}{l}4 \varnothing 5214 \varnothing 7317 \varnothing 4 \\
4 \varnothing 4923 \emptyset 731545 \\
4 \varnothing 5122 \varnothing 731438 \\
4 \varnothing 5104 \varnothing 731504 \\
4 \varnothing 4944 \varnothing 731552\end{array}$ & $\begin{array}{l}648423 \\
678807 \\
668497 \\
620985 \\
638610\end{array}$ & $\begin{array}{l}169 \\
169 \\
182 \\
182 \\
169\end{array}$ & $\begin{array}{l}471609 \\
282000 \\
177200 \\
196200 \\
583000\end{array}$ & $\begin{array}{l}476500 \\
210000 \\
197000 \\
203000 \\
515000\end{array}$ \\
\hline S & $\begin{array}{l}117 \\
118 \\
119 \\
120 \\
121\end{array}$ & $\begin{array}{l}\text { PICKWICK DRIVE } \\
\text { CRESCENT PLACE } \\
\text { BASSWOOD LANE } \\
\text { NEW HIGHWAY } \\
\text { TULIPWDOD DRIVE }\end{array}$ & $\begin{array}{l}\text { PALMER LANE } \\
\text { SEAVER LANE } \\
\text { BROOK LANE } \\
\text { EXECUTIVE DRIVE } \\
\text { GLENMERE LANE }\end{array}$ & $\begin{array}{l}4050260731623 \\
405 \oslash 140731203 \\
40502507312 ø 3 \\
4049190731432 \\
4048490731642\end{array}$ & $\begin{array}{l}598611 \\
570183 \\
570103 \\
671218 \\
671218\end{array}$ & $\begin{array}{l}169 \\
182 \\
182 \\
196 \\
169\end{array}$ & $\begin{array}{c}270000 \\
142000 \\
182000 \\
- \\
96300\end{array}$ & $\begin{array}{l}379080 \\
539090 \\
395000 \\
292800 \\
169060\end{array}$ \\
\hline S & $\begin{array}{l}122 \\
123 \\
124 \\
125 \\
126\end{array}$ & $\begin{array}{l}\text { MELWOOD DRIVE } \\
\text { HUNTER PLACE } \\
\text { GANNET DRIVE } \\
\text { MARIE CRESCENT } \\
\text { DANA LANE }\end{array}$ & $\begin{array}{l}\text { MOTOR PARKWAY } \\
\text { HOLLOW DRIVE } \\
\text { HERON LANE } \\
\text { PITTONI DRIVE } \\
\text { STACEY LANE }\end{array}$ & $\begin{array}{l}4048200731635 \\
405055073152 \emptyset \\
404947 \varnothing 731612 \\
40492197317 \varnothing \emptyset \\
4 \varnothing 5104 \varnothing 731513\end{array}$ & $\begin{array}{l}660218 \\
630809 \\
616817 \\
660381 \\
640915\end{array}$ & $\begin{array}{l}169 \\
169 \\
169 \\
169 \\
182\end{array}$ & $\begin{array}{r}5680 \varnothing \\
88090 \\
94980 \\
176900 \\
298800\end{array}$ & $\begin{array}{r}68008 \\
68000 \\
109000 \\
117000 \\
290000\end{array}$ \\
\hline 5 & $\begin{array}{l}127 \\
128 \\
129 \\
139 \\
131\end{array}$ & $\begin{array}{l}\text { MAPLE AVENUE } \\
\text { TISHNER LANE } \\
\text { CHESTNUT COURT } \\
\text { PUMPKIN ROAD } \\
\text { MARS COURT }\end{array}$ & $\begin{array}{l}\text { HAUPPAUGE ROAD } \\
\text { DALE LANE } \\
\text { APPLE LANE } \\
\text { STORYBOOK LANE } \\
\text { WOODLAND LANE }\end{array}$ & $\begin{array}{l}4850110731137 \\
4050250731051 \\
4050140731614 \\
4052010730811 \\
4 \varnothing 5037 \varnothing 731416\end{array}$ & $\begin{array}{l}580909 \\
580929 \\
570606 \\
630523 \\
661006\end{array}$ & $\begin{array}{l}182 \\
182 \\
169 \\
180 \\
169\end{array}$ & $\begin{array}{l}488000 \\
187800 \\
14500 \varnothing \\
17750 \varnothing \\
118200\end{array}$ & $\begin{array}{l}488080 \\
119000 \\
161000 \\
220000 \\
132000\end{array}$ \\
\hline$S$ & $\begin{array}{l}132 \\
133 \\
134 \\
135 \\
136\end{array}$ & $\begin{array}{l}\text { NISSEQUOGUE ROAD } \\
\text { PARKWAY DRIVE N. } \\
\text { FISHER ROAD } \\
\text { PARKWAY ORIVE N. } \\
\text { OLD WILLETTS PTH }\end{array}$ & $\begin{array}{l}\text { TWIXT HILLS ROAD } \\
\text { MARIE CRESCENT } \\
\text { PITTONI DRIVE } \\
\text { MARIE CRESCENT } \\
\text { ENGINEERS ROAD }\end{array}$ & $\begin{array}{l}4 \varnothing 5215073113 \varnothing \\
40491007317 \varnothing 6 \\
4 \varnothing 49180731638 \\
4 \varnothing 4904 \varnothing 73164 \varnothing \\
4 \varnothing 48530731356\end{array}$ & $\begin{array}{l}669517 \\
639493 \\
639493 \\
639493 \\
669818\end{array}$ & $\begin{array}{l}185 \\
169 \\
169 \\
169 \\
196\end{array}$ & $\begin{array}{r}179500 \\
228090 \\
322500 \\
211000 \\
1960000\end{array}$ & $\begin{array}{r}18049 \varnothing \\
24909 \emptyset \\
32650 \varnothing \\
222390 \\
203900 \varnothing\end{array}$ \\
\hline $\begin{array}{l}S \\
S\end{array}$ & $\begin{array}{l}137 \\
138 \\
139 \\
149 \\
141\end{array}$ & $\begin{array}{l}\text { OSAGE LANE } \\
\text { PLANT AVENUE } \\
\text { OLD WILLETTS PTH } \\
\text { PLANT AVENUE } \\
\text { OLD WILLETTS PTH }\end{array}$ & $\begin{array}{l}\text { PONDEROSA LANE } \\
\text { OSER AVENUE } \\
\text { OSER AVENUE } \\
\text { ENGINEERS ROAD } \\
\text { KENNEDY DRIVE }\end{array}$ & 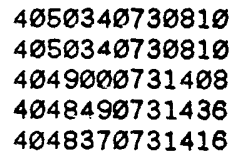 & $\begin{array}{c}669892 \\
- \\
669819 \\
660817 \\
660819\end{array}$ & $\begin{array}{l}177 \\
196 \\
196 \\
196 \\
196\end{array}$ & $\begin{array}{l}113800 \\
433800 \\
625180 \\
585000 \\
198090\end{array}$ & $\begin{array}{l}114000 \\
460000 \\
633100 \\
673000 \\
202080\end{array}$ \\
\hline $\begin{array}{l}\tilde{S} \\
\hat{S} \\
\mathrm{~S}\end{array}$ & $\begin{array}{l}142 \\
143 \\
146 \\
147 \\
148\end{array}$ & $\begin{array}{l}\text { FLORIDA AVENUE } \\
\text { ST. JAMES AVENUE } \\
\text { JILL COURT } \\
\text { PHILSON COURT } \\
\text { SOUTHERN BLVD. }\end{array}$ & $\begin{array}{l}\text { STONY WOOD ROAD } \\
\text { RUTHERFORD ST. } \\
\text { WICHARD BLVD. } \\
\text { WASHINGTON BLVD. } \\
\text { DIANE COURT SO. }\end{array}$ & $\begin{array}{l}40490607317 \varnothing 5 \\
4 \varnothing 51550730949 \\
4 \varnothing 51290731520 \\
4 \varnothing 51350731454 \\
4 \varnothing 5049073092 \varnothing\end{array}$ & $\begin{array}{l}658216 \\
611206 \\
668510 \\
668510 \\
681008\end{array}$ & $\begin{array}{l}169 \\
189 \\
169 \\
169 \\
177\end{array}$ & $\begin{array}{r}249000 \\
255000 \\
338000 \\
76200 \\
96390\end{array}$ & $\begin{array}{l}250000 \\
261000 \\
384600 \\
230080 \\
105000\end{array}$ \\
\hline $\begin{array}{l}S \\
S \\
S \\
S\end{array}$ & $\begin{array}{l}149 \\
150 \\
153 \\
154 \\
155\end{array}$ & $\begin{array}{l}\text { ACORN ROAD } \\
\text { MORELAND ROAD } \\
\text { ALMA LIND LANE } \\
\text { LAKE AVENUE SO. } \\
\text { BREEZY HILL OR. }\end{array}$ & $\begin{array}{l}\text { OAKFIELD ROAD } \\
\text { MOTOR PARKWAY } \\
\text { EMPRESS PINES DR } \\
\text { NESCONSET HWY. } \\
\text { SUNKEN MEADOW RD }\end{array}$ & $\begin{array}{l}4 \varnothing 5328073 \varnothing 980 \\
4 \varnothing 48210731616 \\
4 \varnothing 502307308 \emptyset 4 \\
4 \varnothing 50590730994 \\
4 \varnothing 53560731653\end{array}$ & $\begin{array}{l}660519 \\
660504 \\
641006 \\
680119 \\
648826\end{array}$ & $\begin{array}{l}173 \\
169 \\
229 \\
177 \\
174\end{array}$ & $\begin{array}{l}141000 \\
310080 \\
289508 \\
96390 \\
-\end{array}$ & $\begin{array}{l}155090 \\
358080 \\
388090 \\
128080 \\
-\end{array}$ \\
\hline $\begin{array}{l}S \\
S \\
S \\
S\end{array}$ & $\begin{array}{l}156 \\
157 \\
158 \\
160 \\
161\end{array}$ & $\begin{array}{l}\text { GIBBS POND ROAD } \\
\text { TOWN LINE ROAD } \\
\text { COCONUT DRIVE } \\
\text { MORICHES ROAD } \\
\text { ONEIDA LANE }\end{array}$ & $\begin{array}{l}\text { KNOLLTOP DRIVE } \\
\text { LONGBOW LANE } \\
\text { CROSS BOW LANE } \\
\text { ALEXANDER AVENUE } \\
\text { RENSSELAER DRIVE }\end{array}$ & $\begin{array}{l}4051630730833 \\
4051460731726 \\
4051500731657 \\
4051320730755 \\
4050010731728\end{array}$ & $\begin{array}{c}658485 \\
- \\
608282 \\
680488 \\
581824\end{array}$ & $\begin{array}{l}177 \\
169 \\
169 \\
227 \\
169\end{array}$ & $\begin{array}{l}14809 \emptyset \\
241390 \\
37509 \emptyset \\
- \\
788190\end{array}$ & $\begin{array}{l}152000 \\
251800 \\
400000 \\
- \\
882000\end{array}$ \\
\hline S & $\begin{array}{l}162 \\
163 \\
164 \\
165 \\
166\end{array}$ & $\begin{array}{l}\text { ROCKLAND COURT } \\
\text { VALMONT LANE } \\
\text { STH AVENUE } \\
\text { BRUCE LANE } \\
\text { GAIL COURT }\end{array}$ & $\begin{array}{l}\text { GENESEE DRIVE } \\
\text { NEW HIGHWAY } \\
\text { MARVIN DRIVE } \\
\text { NORMA LANE } \\
\text { ELLEN PLACE }\end{array}$ & $\begin{array}{l}4050160731729 \\
4049330731631 \\
4 \varnothing 52280731551 \\
40524107313 ø 9 \\
4052330731536\end{array}$ & $\begin{array}{c}581024 \\
590715 \\
- \\
591102\end{array}$ & $\begin{array}{l}169 \\
169 \\
174 \\
174 \\
174\end{array}$ & $\begin{array}{l}628200 \\
414500 \\
- \\
- \\
165080\end{array}$ & $\begin{array}{l}815200 \\
425500 \\
- \\
- \\
245000\end{array}$ \\
\hline
\end{tabular}


N.Y. [Dash indicates no data. Altitudes are in feet above sea level.] (cont.)

\begin{tabular}{|c|c|c|c|c|c|c|c|c|c|c|c|c|}
\hline & $\begin{array}{l}\text { IN } \\
\text { BER }\end{array}$ & $\begin{array}{l}\text { MAX. } \\
\text { AREA } \\
\text { (squar }\end{array}$ & $\begin{array}{c}\text { BASIN } \\
\text { AREA } \\
\text { feet) }\end{array}$ & RIM & $\begin{array}{l}- \text {-ALT } \\
\text { OVER } \\
\text { FLOW }\end{array}$ & $\begin{array}{c}\text { ITUDE-- } \\
-\quad \text { BOT- } \\
\text { TOM }\end{array}$ & $\begin{array}{l}\text { WATER } \\
\text { TABLE }\end{array}$ & $\begin{array}{c}\text { DRAIN. } \\
\text { AREA } \\
\text { (acres) }\end{array}$ & $\begin{array}{l}- \text {-BASI } \\
\text { STATUS }\end{array}$ & $\begin{array}{l}\text { IN-- } \\
\text { USE }\end{array}$ & $\begin{array}{l}\text { GEO. } \\
\text { UNIT }\end{array}$ & $\begin{array}{l}\text { SOIL } \\
\text { UNIT }\end{array}$ \\
\hline 5 & $\begin{array}{l}106 \\
107 \\
108 \\
110 \\
111\end{array}$ & $\begin{array}{r}9400 \\
16600 \\
18500 \\
19000 \\
38000\end{array}$ & $\begin{array}{l}12500 \\
237 \varnothing \varnothing \\
246 \varnothing \varnothing \\
158 \varnothing \emptyset \\
4320 \varnothing\end{array}$ & $\begin{array}{l}137 . \emptyset \\
159.5 \\
124.8 \\
120 . \varnothing \\
131 . \varnothing\end{array}$ & $\begin{array}{r}- \\
153 . \varnothing \\
124.0 \\
- \\
-\end{array}$ & $\begin{array}{r}126 . \emptyset \\
145 . \emptyset \\
114 . \varnothing \\
117 . \varnothing\end{array}$ & $\begin{array}{l}44.0 \\
53.0 \\
57.0 \\
57.0 \\
55.0\end{array}$ & $\begin{array}{l}70.0 \\
11.3 \\
13.0 \\
16.7 \\
39.0\end{array}$ & $\begin{array}{l}1 \\
1 \\
1 \\
1 \\
0\end{array}$ & $\begin{array}{l}1 \\
1 \\
1 \\
1 \\
1\end{array}$ & $\begin{array}{l}1 \\
1 \\
1 \\
1 \\
1\end{array}$ & $\begin{array}{l}1 \\
1 \\
3 \\
7 \\
7\end{array}$ \\
\hline $\begin{array}{l}\text { S } \\
\text { S } \\
\text { S }\end{array}$ & $\begin{array}{l}112 \\
113 \\
114 \\
115 \\
116\end{array}$ & $\begin{array}{l}39800 \\
21000 \\
17100 \\
26300 \\
51500\end{array}$ & $\begin{array}{l}42900 \\
28900 \\
15500 \\
17200 \\
44200\end{array}$ & $\begin{array}{r}155 . \varnothing \\
150 . \varnothing \\
107 . \varnothing \\
54 . \varnothing \\
115 . \varnothing\end{array}$ & $\begin{array}{r}149.0 \\
143.0 \\
104.5 \\
103.0\end{array}$ & $\begin{array}{r}- \\
- \\
93.0 \\
42.0 \\
91.0\end{array}$ & $\begin{array}{l}58.0 \\
50.0 \\
44.0 \\
39.0 \\
54.0\end{array}$ & $\begin{array}{l}54.0 \\
31.8 \\
33.0 \\
30.9 \\
53.3\end{array}$ & $\begin{array}{l}1 \\
1 \\
1 \\
0 \\
1\end{array}$ & $\begin{array}{l}1 \\
1 \\
1 \\
1 \\
1\end{array}$ & $\begin{array}{l}1 \\
6 \\
1 \\
1 \\
1\end{array}$ & $\begin{array}{l}7 \\
1 \\
5 \\
6 \\
1\end{array}$ \\
\hline$S$ & $\begin{array}{l}117 \\
118 \\
119 \\
120 \\
121\end{array}$ & $\begin{array}{l}37000 \\
53900 \\
30400 \\
27700 \\
76900\end{array}$ & $\begin{array}{l}27100 \\
14900 \\
27200 \\
28800 \\
25700\end{array}$ & $\begin{array}{r}141.0 \\
50.0 \\
55.0 \\
89 . \varnothing \\
170 . \varnothing\end{array}$ & $\begin{array}{r}52 . \bar{\varnothing} \\
49 . \varnothing \\
89.3 \\
171.5\end{array}$ & $\begin{array}{r}125.0 \\
42.0 \\
36.0 \\
82.0 \\
160.0\end{array}$ & $\begin{array}{l}62.0 \\
40.0 \\
40.0 \\
48.0 \\
58.0\end{array}$ & $\begin{array}{l}46.0 \\
24.5 \\
31.4 \\
31.8 \\
11.0\end{array}$ & $\begin{array}{l}1 \\
0 \\
0 \\
0 \\
1\end{array}$ & $\begin{array}{l}1 \\
1 \\
1 \\
1 \\
1\end{array}$ & $\begin{array}{l}1 \\
1 \\
1 \\
1 \\
6\end{array}$ & $\begin{array}{l}7 \\
7 \\
7 \\
7 \\
7\end{array}$ \\
\hline $\begin{array}{l}\text { S } \\
S\end{array}$ & $\begin{array}{l}122 \\
123 \\
124 \\
125 \\
126\end{array}$ & $\begin{array}{r}8000 \\
6000 \\
10900 \\
11700 \\
22300\end{array}$ & $\begin{array}{r}12200 \\
9300 \\
9100 \\
28000 \\
22800\end{array}$ & $\begin{array}{r}145 . \varnothing \\
14 \varnothing . \varnothing \\
130 . \varnothing \\
85 . \overline{-}\end{array}$ & $\begin{array}{r}\overline{-} \\
\overline{-} \\
63 . \overline{0}\end{array}$ & $\begin{array}{r}134.0 \\
100 . \overline{-} \\
48 . \overline{0}\end{array}$ & $\begin{array}{l}50.0 \\
52.0 \\
58.0 \\
66.0 \\
51.0\end{array}$ & $\begin{array}{r}6.5 \\
9.4 \\
12.5 \\
27.8 \\
21.5\end{array}$ & $\begin{array}{l}1 \\
1 \\
1 \\
0 \\
1\end{array}$ & $\begin{array}{l}1 \\
1 \\
1 \\
1 \\
1\end{array}$ & $\begin{array}{l}1 \\
1 \\
1 \\
6 \\
1\end{array}$ & $\begin{array}{l}7 \\
5 \\
7 \\
7 \\
6\end{array}$ \\
\hline $\begin{array}{l}S \\
S \\
S \\
S \\
S\end{array}$ & $\begin{array}{l}127 \\
128 \\
129 \\
130 \\
131\end{array}$ & $\begin{array}{l}42900 \\
11900 \\
14800 \\
22000 \\
13200\end{array}$ & $\begin{array}{l}38700 \\
13200 \\
35100 \\
18100 \\
15600\end{array}$ & $\begin{array}{r}62 . \varnothing \\
52 . \varnothing \\
137.5 \\
1 \varnothing 7.6 \\
61 . \varnothing\end{array}$ & $\begin{array}{r}58.0 \\
- \\
128.5 \\
- \\
-\end{array}$ & $\begin{array}{r}48.5 \\
45.0 \\
117.5 \\
87.0 \\
43.0\end{array}$ & $\begin{array}{l}43.0 \\
42.0 \\
58.0 \\
64.0 \\
38.0\end{array}$ & $\begin{array}{l}64.2 \\
23.3 \\
22.9 \\
27.9 \\
13.5\end{array}$ & $\begin{array}{l}1 \\
0 \\
1 \\
1 \\
1\end{array}$ & $\begin{array}{l}1 \\
1 \\
1 \\
1 \\
1\end{array}$ & $\begin{array}{l}1 \\
1 \\
1 \\
1 \\
1\end{array}$ & $\begin{array}{l}7 \\
7 \\
7 \\
7 \\
5\end{array}$ \\
\hline $\begin{array}{l}S \\
S \\
S \\
S \\
S\end{array}$ & $\begin{array}{l}132 \\
133 \\
134 \\
135 \\
136\end{array}$ & $\begin{array}{r}15000 \\
34300 \\
39300 \\
24700 \\
203000\end{array}$ & $\begin{array}{r}21600 \\
39200 \\
23800 \\
1500 \emptyset \\
226000\end{array}$ & $\begin{array}{r}42 . \varnothing \\
195 . \emptyset \\
186 . \varnothing \\
183 . \emptyset \\
89 . \varnothing\end{array}$ & $\begin{array}{r}37.0 \\
177.0 \\
179.8 \\
182.0 \\
88.0\end{array}$ & $\begin{array}{r}25.0 \\
170.0 \\
171.5 \\
173.0 \\
78.0\end{array}$ & $\begin{array}{l}15 . \emptyset \\
62 . \emptyset \\
58 . \emptyset \\
58 . \emptyset \\
43 . \emptyset\end{array}$ & $\begin{array}{l}28.3 \\
24.0 \\
34.7 \\
22.7 \\
95.4\end{array}$ & $\begin{array}{l}1 \\
1 \\
1 \\
1 \\
1\end{array}$ & $\begin{array}{l}1 \\
1 \\
2 \\
1 \\
1\end{array}$ & $\begin{array}{l}1 \\
6 \\
6 \\
6 \\
1\end{array}$ & $\begin{array}{l}5 \\
7 \\
7 \\
3 \\
6\end{array}$ \\
\hline $\begin{array}{l}\text { S } \\
\text { S } \\
\text { S } \\
\text { S } \\
\text { S }\end{array}$ & $\begin{array}{l}137 \\
138 \\
139 \\
140 \\
141\end{array}$ & $\begin{array}{l}11400 \\
46000 \\
63300 \\
46400 \\
20200\end{array}$ & $\begin{array}{l}16800 \\
59400 \\
88500 \\
73000 \\
27600\end{array}$ & $\begin{array}{r}79.5 \\
130.0 \\
97.0 \\
123.0 \\
124.0\end{array}$ & $\begin{array}{r}- \\
100.0 \\
92.0 \\
116.5 \\
-\end{array}$ & $\begin{array}{r}63.0 \\
- \\
102.0 \\
110.0\end{array}$ & $\begin{array}{l}48.0 \\
47.0 \\
46.0 \\
47.0 \\
45.0\end{array}$ & $\begin{array}{l}17.9 \\
68.2 \\
3 \varnothing .6 \\
38.3 \\
97.0\end{array}$ & $\begin{array}{l}1 \\
1 \\
1 \\
1 \\
0\end{array}$ & $\begin{array}{l}1 \\
1 \\
1 \\
1 \\
1\end{array}$ & $\begin{array}{l}1 \\
6 \\
1 \\
6 \\
6\end{array}$ & $\begin{array}{l}3 \\
6 \\
6 \\
6 \\
3\end{array}$ \\
\hline $\begin{array}{l}S \\
S \\
S \\
S \\
S\end{array}$ & $\begin{array}{l}142 \\
143 \\
148 \\
147 \\
148\end{array}$ & $\begin{array}{l}25000 \\
26100 \\
34900 \\
23000 \\
10500\end{array}$ & $\begin{array}{l}17100 \\
21200 \\
27000 \\
20000 \\
10500\end{array}$ & $\begin{array}{r}171.0 \\
120.0 \\
89.0 \\
124.0 \\
120.0\end{array}$ & $\begin{array}{r}- \\
- \\
88.0 \\
123.0 \\
-\end{array}$ & $\begin{array}{r}156.0 \\
101.0 \\
77.0 \\
113.0 \\
102.0\end{array}$ & $\begin{array}{l}60 . \emptyset \\
37.0 \\
53.0 \\
48 . \emptyset \\
53 . \varnothing\end{array}$ & $\begin{array}{l}27 . \varnothing \\
39 . \varnothing \\
53.2 \\
12 . \varnothing \\
11 . \varnothing\end{array}$ & $\begin{array}{l}1 \\
1 \\
1 \\
1 \\
1\end{array}$ & $\begin{array}{l}1 \\
1 \\
1 \\
1 \\
1\end{array}$ & $\begin{array}{l}6 \\
1 \\
1 \\
1 \\
1\end{array}$ & $\begin{array}{l}7 \\
7 \\
6 \\
6 \\
1\end{array}$ \\
\hline $\begin{array}{l}S \\
\text { S } \\
S \\
S \\
S\end{array}$ & $\begin{array}{l}149 \\
150 \\
153 \\
154 \\
155\end{array}$ & $\begin{array}{c}15500 \\
35000 \\
30800 \\
12000 \\
-\end{array}$ & $\begin{array}{r}2390 \emptyset \\
35200 \\
37800 \\
18300 \\
-\end{array}$ & $\begin{array}{r}131.0 \\
145.0 \\
77.0 \\
120.0 \\
100.0\end{array}$ & $\begin{array}{l}- \\
- \\
- \\
-\end{array}$ & $\begin{array}{r}116.0 \\
131.5 \\
63.0 \\
107.3 \\
-\end{array}$ & $\begin{array}{l}42 . \emptyset \\
50 . \emptyset \\
48 . \varnothing \\
69 . \varnothing \\
25 . \emptyset\end{array}$ & $\begin{array}{r}22.2 \\
21.8 \\
31.0 \\
11.0 \\
-\end{array}$ & $\begin{array}{l}1 \\
1 \\
1 \\
1 \\
1\end{array}$ & $\begin{array}{l}1 \\
1 \\
1 \\
1 \\
1\end{array}$ & $\begin{array}{l}1 \\
1 \\
1 \\
1 \\
3\end{array}$ & $\begin{array}{l}1 \\
3 \\
3 \\
1 \\
3\end{array}$ \\
\hline $\begin{array}{l}S \\
S \\
S \\
S\end{array}$ & $\begin{array}{l}156 \\
157 \\
158 \\
160 \\
161\end{array}$ & $\begin{array}{c}- \\
25200 \\
33300 \\
- \\
88200\end{array}$ & $\begin{array}{r}22200 \\
34600 \\
576000 \\
- \\
144000\end{array}$ & $\begin{array}{r}93.0 \\
160.0 \\
155.0 \\
85.0 \\
121.0\end{array}$ & $\begin{array}{r}- \\
131 . \overline{0} \\
113 . \overline{5}\end{array}$ & $\begin{array}{r}74.0 \\
145.0 \\
119.0 \\
103.5\end{array}$ & $\begin{array}{l}55.0 \\
58 . \varnothing \\
59 . \varnothing \\
63 . \varnothing \\
69.0\end{array}$ & $\begin{array}{r}23.3 \\
16.9 \\
27.7 \\
- \\
84.8\end{array}$ & $\begin{array}{l}1 \\
1 \\
1 \\
1 \\
1\end{array}$ & $\begin{array}{l}1 \\
1 \\
1 \\
1 \\
1\end{array}$ & $\begin{array}{l}1 \\
1 \\
1 \\
1 \\
1\end{array}$ & $\begin{array}{l}7 \\
7 \\
7 \\
6 \\
7\end{array}$ \\
\hline $\begin{array}{l}\text { S } \\
\text { S } \\
\text { S }\end{array}$ & $\begin{array}{l}162 \\
163 \\
164 \\
165 \\
166\end{array}$ & $\begin{array}{c}81500 \\
37 \varnothing 00 \\
- \\
\overline{2} \\
2400\end{array}$ & $\begin{array}{r}118800 \\
74800 \\
- \\
57600\end{array}$ & $\begin{array}{l}117.5 \\
150 . \varnothing \\
140.0 \\
155.0 \\
110 . \varnothing\end{array}$ & $\begin{array}{r}116.5 \\
147.5 \\
- \\
105.0\end{array}$ & $\begin{array}{r}106.5 \\
136.0 \\
- \\
95.0\end{array}$ & $\begin{array}{l}67 . \varnothing \\
58 . \emptyset \\
52 . \varnothing \\
51 . \varnothing \\
53 . \varnothing\end{array}$ & $\begin{array}{r}98.9 \\
65.2 \\
- \\
32.8\end{array}$ & $\begin{array}{l}1 \\
1 \\
1 \\
1 \\
1\end{array}$ & $\begin{array}{l}1 \\
1 \\
1 \\
1 \\
1\end{array}$ & $\begin{array}{l}1 \\
1 \\
1 \\
1 \\
1\end{array}$ & $\begin{array}{l}7 \\
7 \\
6 \\
7 \\
5\end{array}$ \\
\hline
\end{tabular}


Appendix.--Location and basic physical features of recharge basins on Long Island,

\begin{tabular}{|c|c|c|c|c|c|c|c|c|}
\hline \multicolumn{2}{|c|}{$\begin{array}{l}\text { BASIN } \\
\text { NUMBER }\end{array}$} & \multicolumn{2}{|c|}{ NEAREST INTERSECTION } & $\begin{array}{l}\text { LATITUDE } \\
\text { LONGITUDE } \\
\circ " \text { " " }\end{array}$ & $\begin{array}{l}\text { DATE } \\
\text { BUILT } \\
\text { YrMOD }\end{array}$ & COMMUNITY & $\begin{array}{l}\text { DESIGN } \\
\text { CAPACITY } \\
\text { (cubic f }\end{array}$ & $\begin{array}{l}\text { ACTUAL } \\
\text { CAPACITY } \\
\text { feet) }\end{array}$ \\
\hline $\begin{array}{l}S \\
S \\
S \\
S \\
S\end{array}$ & $\begin{array}{l}167 \\
172 \\
173 \\
174 \\
175\end{array}$ & $\begin{array}{l}\text { L.I. ARENA } \\
\text { DAWN DRIVE } \\
\text { NESCONSET HWY. } \\
\text { LEWIS LANE } \\
\text { ST. JOHNLAND RD. }\end{array}$ & $\begin{array}{l}\text { ECGEWOOD AVENUE } \\
\text { BROOKSITE DRIVE } \\
\text { HUDSON DRIVE } \\
\text { RIVER HEIGHTS DR }\end{array}$ & $\begin{array}{l}4 \varnothing 5 \varnothing 1107317 \varnothing 4 \\
4 \varnothing 51300731218 \\
4 \varnothing 4943 \varnothing 731214 \\
4 \varnothing 5251 \varnothing 731523 \\
4 \varnothing 52010731317\end{array}$ & $\begin{array}{c}- \\
608518 \\
620717 \\
620216 \\
64 \varnothing 3 \varnothing 4\end{array}$ & $\begin{array}{l}169 \\
182 \\
182 \\
174 \\
182\end{array}$ & $\begin{array}{l}- \\
174000 \\
289000 \\
262700 \\
-\end{array}$ & $\begin{array}{l}\overline{185000} \\
290000 \\
280600 \\
-\end{array}$ \\
\hline $\begin{array}{l}S \\
S \\
S \\
S \\
S\end{array}$ & $\begin{array}{l}176 \\
177 \\
178 \\
179 \\
180\end{array}$ & $\begin{array}{l}\text { VETERANS HWY. } \\
\text { MARCHANT DRIVE } \\
\text { OPAL COURT } \\
\text { CARDINAL LANE } \\
\text { SUNKEN MEADOW PK }\end{array}$ & $\begin{array}{l}\text { SUNKEN MEADOW PK } \\
\text { MIDDLE COUNTRY } \\
\text { SMITHTOWN LINE R } \\
\text { NEW HIGHWAY } \\
\text { DALY ROAD }\end{array}$ & $\begin{array}{l}4 \varnothing 501507317 \varnothing 8 \\
4 \varnothing 5127 \varnothing 7310 \varnothing 8 \\
4 \varnothing 4933 ø 731159 \\
4 \varnothing 49220731436 \\
4 \varnothing 49180731734\end{array}$ & $\begin{array}{c}620518 \\
- \\
620116 \\
- \\
590810\end{array}$ & $\begin{array}{l}169 \\
180 \\
196 \\
169 \\
169\end{array}$ & $\begin{array}{l}341700 \\
- \\
- \\
- \\
-\end{array}$ & $\begin{array}{l}389000 \\
- \\
- \\
- \\
330000\end{array}$ \\
\hline $\begin{array}{l}S \\
S \\
S \\
S \\
S\end{array}$ & $\begin{array}{l}181 \\
182 \\
183 \\
184 \\
185\end{array}$ & $\begin{array}{l}\text { HENRY STREET } \\
\text { ROUNDABOUT ROAD } \\
\text { ALEXANDER AVENUE } \\
\text { SHENANDOAH BLVD. } \\
\text { BURHAM DRIVE }\end{array}$ & $\begin{array}{l}\text { E. NORTHPORT RD. } \\
\text { BRIDLEPATH ROAD } \\
\text { MIDDLE COUNTRY R } \\
\text { GLENFIELD LANE } \\
\text { OAKSIDE ROAD }\end{array}$ & $\begin{array}{l}4 \varnothing 5314 \varnothing 731522 \\
4 \varnothing 51280731137 \\
4 \varnothing 5132 \varnothing 73 \varnothing 8 \varnothing 7 \\
4 \varnothing 512 \varnothing 673 \varnothing 815 \\
4 \varnothing 52318731241\end{array}$ & $\begin{array}{l}6210 \\
6382 \\
616615 \\
618615 \\
646121\end{array}$ & $\begin{array}{l}169 \\
185 \\
227 \\
177 \\
182\end{array}$ & $\begin{array}{l}100400 \\
- \\
68000 \\
310060 \\
480600\end{array}$ & $\begin{array}{l}105000 \\
- \\
70000 \\
315000 \\
482000\end{array}$ \\
\hline $\begin{array}{l}S \\
S \\
S \\
S \\
S\end{array}$ & $\begin{array}{l}186 \\
187 \\
188 \\
189 \\
192\end{array}$ & $\begin{array}{l}\text { SHERYL CRESCENT } \\
\text { WALNUT ROAD } \\
\text { NISSEQUOGUE ROAD } \\
\text { BOX PLACE } \\
\text { ST. JOHNLAND RD. }\end{array}$ & $\begin{array}{l}\text { SANDY DRIVE } \\
\text { LANDING AVENUE } \\
\text { LONG HILL ROAD } \\
\text { BOW DRIVE } \\
\text { CRESCENT PLACE }\end{array}$ & $\begin{array}{l}4 \varnothing 52140731350 \\
4 \varnothing 53140731300 \\
4 \varnothing 51460731112 \\
4 \varnothing 49410731049 \\
4 \varnothing 52120731315\end{array}$ & $\begin{array}{l}670117 \\
640214 \\
640313 \\
5906 \\
650908\end{array}$ & $\begin{array}{l}182 \\
181 \\
185 \\
196 \\
182\end{array}$ & $\begin{array}{l}- \\
273700 \\
- \\
149000 \\
-\end{array}$ & $\begin{array}{l}\overline{270000} \\
\overline{150000} \\
-\end{array}$ \\
\hline $\begin{array}{l}S \\
S \\
S \\
S \\
S\end{array}$ & $\begin{array}{l}193 \\
194 \\
195 \\
196 \\
197\end{array}$ & $\begin{array}{l}\text { GIBBS POND ROAD } \\
\text { ATLAS PLACE } \\
\text { FRANKLIN DRIVE } \\
\text { CALLAHAN'S BCH. } \\
\text { OLD MILL ROAD }\end{array}$ & $\begin{array}{l}\text { ROBIN HOOD COURT } \\
\text { GEMINI LANE } \\
\text { HOWELL DRIVE } \\
\text { FORT SALONGA RD. } \\
\text { HIGH WOODS ROAD }\end{array}$ & $\begin{array}{l}4 \varnothing 56430730833 \\
4 \varnothing 5025073 \varnothing 933 \\
4 \varnothing 5124 \varnothing 731027 \\
4 \varnothing 54230731648 \\
4 \varnothing 5234 \varnothing 731059\end{array}$ & $\begin{array}{l}670510 \\
660606 \\
57 \varnothing 989 \\
681120 \\
670106\end{array}$ & $\begin{array}{l}177 \\
177 \\
185 \\
171 \\
180\end{array}$ & $\begin{array}{r}12750 \emptyset \\
21890 \emptyset \\
\overline{186800} \\
81900\end{array}$ & $\begin{array}{r}143400 \\
228800 \\
- \\
186900 \\
84100\end{array}$ \\
\hline $\begin{array}{l}S \\
S \\
S \\
S \\
S\end{array}$ & $\begin{array}{l}198 \\
199 \\
200 \\
201 \\
202\end{array}$ & $\begin{array}{l}\text { HILLTOP DRIVE } \\
\text { TRUVAL LANE } \\
\text { WYANDANCH BLVD. } \\
\text { BAYBERRY LANE } \\
\text { PINE RIDGE DRIVE }\end{array}$ & $\begin{array}{l}\text { MAYLIN COURT } \\
\text { RAYNIER PLACE } \\
\text { WOOD ROAD } \\
\text { MOBREY LANE } \\
\text { GRANDVIEW LANE }\end{array}$ & $\begin{array}{l}4051560731341 \\
4051010730821 \\
4050140731536 \\
4050180731520 \\
4 \varnothing 50080731506\end{array}$ & $\begin{array}{l}630225 \\
651130 \\
6301 \\
6301 \\
6301\end{array}$ & $\begin{array}{l}182 \\
177 \\
169 \\
169 \\
169\end{array}$ & $\begin{array}{l}139000 \\
224200 \\
342000 \\
455000 \\
477600\end{array}$ & $\begin{array}{l}152000 \\
235000 \\
388000 \\
455000 \\
477000\end{array}$ \\
\hline $\begin{array}{l}S \\
S \\
S \\
S \\
S\end{array}$ & $\begin{array}{l}203 \\
204 \\
205 \\
2 \varnothing 6 \\
207\end{array}$ & $\begin{array}{l}\text { PINE RIDGE DRIVE } \\
\text { LEDGEWOOD DRIVE } \\
\text { GRASSY POND DR. } \\
\text { GRASSY POND DR S } \\
\text { BREAD AND CHEESE }\end{array}$ & $\begin{array}{l}\text { LONE OAK PATH } \\
\text { MCARTHUR LANE } \\
\text { MCARTHUR LANE } \\
\text { BELMONT DRIVE } \\
\text { NORTH FIELD DR. }\end{array}$ & 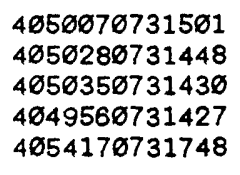 & $\begin{array}{l}6301 \\
6301 \\
6301 \\
6301 \\
610621\end{array}$ & $\begin{array}{l}169 \\
169 \\
169 \\
169 \\
171\end{array}$ & $\begin{array}{l}352060 \\
32 \varnothing 06 \varnothing \\
266 \varnothing 6 \varnothing \\
275 \varnothing \varnothing \varnothing \\
-\end{array}$ & $\begin{array}{l}470060 \\
380600 \\
330600 \\
350600 \\
150000\end{array}$ \\
\hline $\begin{array}{l}S \\
S \\
S \\
S \\
S\end{array}$ & $\begin{array}{l}208 \\
299 \\
210 \\
211 \\
212\end{array}$ & $\begin{array}{l}\text { JEFFERSON AVENUE } \\
\text { FAIRVIEW STREET } \\
\text { LINDNER PLACE } \\
\text { HOWELL DRIVE } \\
\text { DOGWOOD DRIVE }\end{array}$ & $\begin{array}{l}\text { WOODLAWN AVENUE } \\
\text { HILLCREST DR. W. } \\
\text { BROWNING STREET } \\
\text { BLACKMAN STREET } \\
\text { WHITE BIRCH CT. }\end{array}$ & $\begin{array}{l}4 \varnothing 5224 \varnothing 73694 \varnothing \\
4 \varnothing 5114 \varnothing 731 \varnothing 18 \\
4 \varnothing 5134 \varnothing 731019 \\
4 \varnothing 5134 \varnothing 731 \varnothing 25 \\
4 \varnothing 5137 \varnothing 731 \varnothing 39\end{array}$ & $\begin{array}{l}- \\
\overline{-} \\
\overline{-}\end{array}$ & $\begin{array}{l}180 \\
177 \\
180 \\
180 \\
185\end{array}$ & $\begin{array}{l}- \\
\overline{-} \\
\overline{-}\end{array}$ & $\begin{array}{l}- \\
\overline{-} \\
\overline{-}\end{array}$ \\
\hline $\begin{array}{l}\text { S } \\
\text { S } \\
\text { SD } \\
\text { SD } \\
\text { SD }\end{array}$ & $\begin{array}{r}213 \\
214 \\
4 \\
5 \\
6\end{array}$ & $\begin{array}{l}\text { MEADOW ROAD } \\
\text { CLOISTER DRIVE } \\
\text { BRIDGE LANE } \\
\text { ELIJAH'S LANE } \\
\text { ELIJAH'S LANE }\end{array}$ & $\begin{array}{l}\text { RIDGE ROAD } \\
\text { VETERANS HIGHWAY } \\
\text { NORTH ROAD } \\
\text { NORTH ROAD } \\
\text { L.I.R.R. }\end{array}$ & $\begin{array}{l}4 \varnothing 51350731319 \\
4 \varnothing 3934 \varnothing 731437 \\
410215 \varnothing 722854 \\
410633 \varnothing 723123 \\
41 \varnothing 032 \varnothing 7231 \varnothing 1\end{array}$ & $\begin{array}{l}- \\
- \\
-\end{array}$ & $\begin{array}{l}182 \\
196 \\
275 \\
281 \\
281\end{array}$ & $\begin{array}{l}- \\
\overline{-} \\
-\end{array}$ & $\begin{array}{l}\overline{-} \\
\overline{-} \\
\overline{-}\end{array}$ \\
\hline $\begin{array}{l}\text { SD } \\
\text { SD } \\
\text { SN } \\
\text { SN } \\
\text { SN }\end{array}$ & $\begin{array}{l}7 \\
8 \\
1 \\
2 \\
3\end{array}$ & $\begin{array}{l}\text { ALVAH'S LANE } \\
\text { OEPOT LANE } \\
\text { BAY VIEW DRIVE } \\
\text { RIDGE ROAD } \\
\text { STONY HILL ROAD }\end{array}$ & $\begin{array}{l}\text { NORTH ROAD } \\
\text { NORTH ROAD } \\
\text { LONG BEACH LANE } \\
\text { VALLEY ROAD } \\
\text { WICKATUCK LANE }\end{array}$ & $\begin{array}{l}416058 \varnothing 723 \varnothing 35 \\
410125 \varnothing 723 \varnothing 6 \varnothing \\
4 \varnothing 5928 \varnothing 721956 \\
4 \varnothing 5919 \varnothing 721952 \\
4 \varnothing 5986 \varnothing 721933\end{array}$ & $\begin{array}{l}- \\
- \\
-\end{array}$ & $\begin{array}{l}281 \\
275 \\
299 \\
299 \\
299\end{array}$ & $\begin{array}{l}- \\
- \\
- \\
-\end{array}$ & $\begin{array}{l}- \\
\overline{-} \\
\overline{-}\end{array}$ \\
\hline $\begin{array}{l}\text { SN } \\
\text { SN } \\
\text { SN } \\
\text { SN } \\
\text { SN }\end{array}$ & $\begin{array}{l}4 \\
5 \\
6 \\
7 \\
8\end{array}$ & $\begin{array}{l}\text { GREENFIELD ROAD } \\
\text { TUCKAHOE LANE } \\
\text { DAVID WHITES LA. } \\
\text { COUNTY RD. NO. } 39 \\
\text { PONQUJGUE AVENUE }\end{array}$ & $\begin{array}{l}\text { SHINNECOCK HILLS } \\
\text { COUNTY RD. NO. } 39 \\
\text { COUNTY RD. NO.39 } \\
\text { L.I.R.R. } \\
\text { BAY AVENUE }\end{array}$ & $\begin{array}{l}4853320722720 \\
4 \varnothing 5337 \varnothing 722523 \\
4654 \varnothing 5 \varnothing 722257 \\
4654616722246 \\
46515 \varnothing 6723 \varnothing 46\end{array}$ & $\bar{z}$ & $\begin{array}{l}368 \\
312 \\
312 \\
312 \\
293\end{array}$ & $\begin{array}{l}\overline{-} \\
\overline{-} \\
-\end{array}$ & $\begin{array}{l}\overline{-} \\
\overline{-} \\
\overline{-}\end{array}$ \\
\hline
\end{tabular}


N.Y. [Dash indicates no data. Altitudes are in feet above sea level.] (cont.)

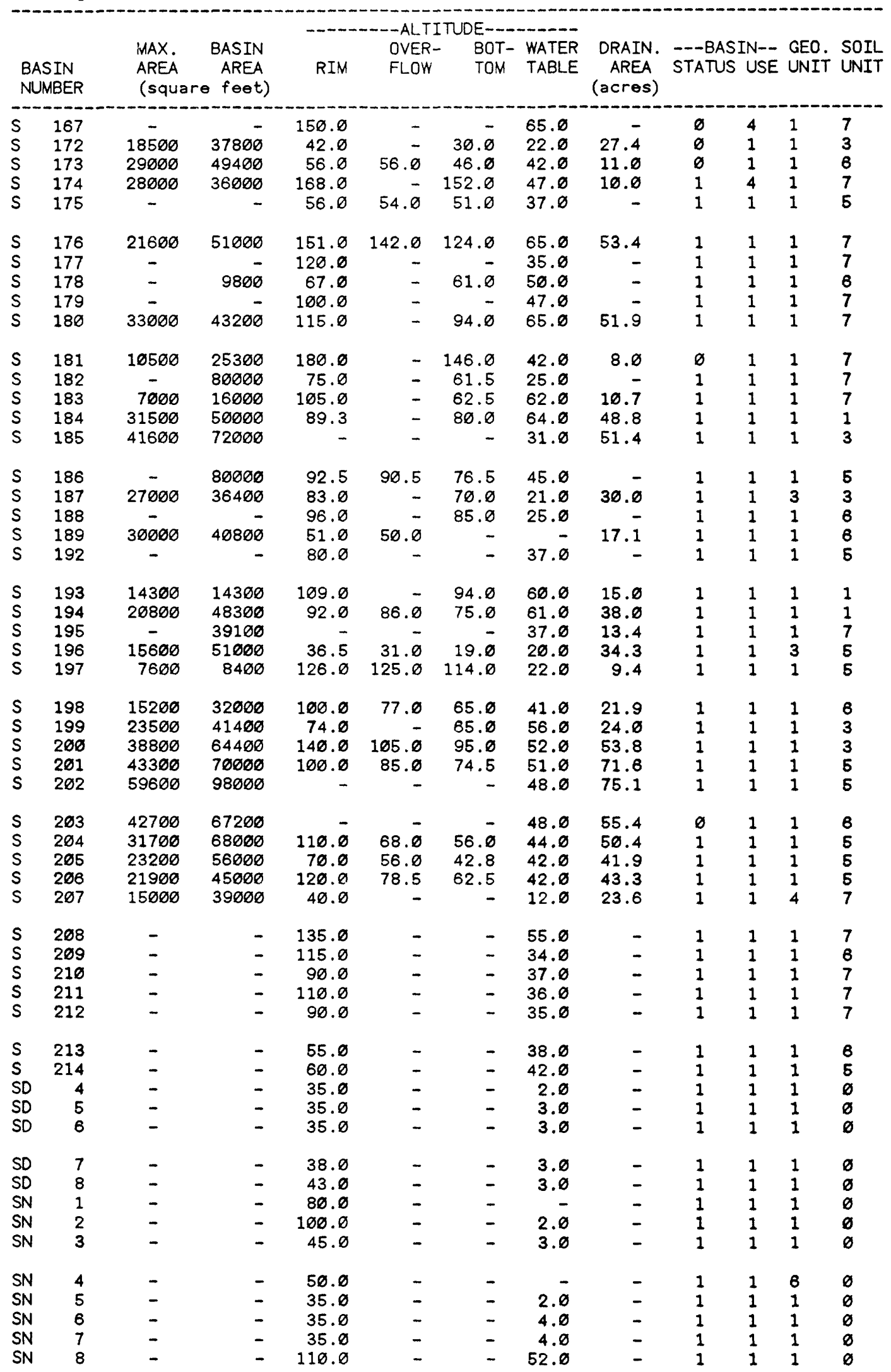


Appendix.--Location and basic physical features of recharge basins on Long Island,

\begin{tabular}{|c|c|c|c|c|c|c|c|c|}
\hline \multicolumn{2}{|c|}{$\begin{array}{l}\text { BASIN } \\
\text { NUMBER }\end{array}$} & \multicolumn{2}{|c|}{ NEAREST INTERSECTION } & $\begin{array}{l}\text { LATITUDE } \\
\text { LONGITUDE, } \\
\circ " \text { " }\end{array}$ & $\begin{array}{l}\text { DATE } \\
\text { BUILT } \\
\text { YrMOD }\end{array}$ & COMMUNITY & $\begin{array}{r}\text { DESIGN } \\
\text { CAPACITY } \\
\text { (cubic }\end{array}$ & $\begin{array}{l}\text { ACTUAL } \\
\text { CAPACITY } \\
\text { feet) }\end{array}$ \\
\hline $\begin{array}{l}\text { SN } \\
\text { SN } \\
\text { SN } \\
\text { SN } \\
\text { SN }\end{array}$ & $\begin{array}{r}9 \\
10 \\
11 \\
12 \\
13\end{array}$ & $\begin{array}{l}\text { MORICHES ROAD } \\
\text { U.S. SUFF.CO.AFB } \\
\text { U.S. SUFF.CO.AFB } \\
\text { U.S. SUFF.CO.AFB } \\
\text { OLD SQUIRETOWN }\end{array}$ & $\begin{array}{l}\text { TOWN LINE } \\
\text { ABERDEEN DRIVE }\end{array}$ & $\begin{array}{l}4 ø 51550724302 \\
4 \varnothing 49580723843 \\
4 \varnothing 5 \emptyset 59 \emptyset 7237 \varnothing 6 \\
4 ø 510107237 \varnothing 2 \\
4 \varnothing 5317 \varnothing 723134\end{array}$ & $\begin{array}{l}\overline{-} \\
\bar{z}\end{array}$ & $\begin{array}{l}264 \\
314 \\
314 \\
314 \\
310\end{array}$ & $\begin{array}{l}- \\
\overline{-} \\
-\end{array}$ & $\begin{array}{l}- \\
- \\
-\end{array}$ \\
\hline $\begin{array}{l}\text { SN } \\
\text { SN } \\
\text { SN } \\
\text { SN } \\
\text { SP }\end{array}$ & $\begin{array}{r}14 \\
15 \\
16 \\
17 \\
1\end{array}$ & $\begin{array}{l}\text { WILDWOOD LAKE GC } \\
\text { WILDWOOD LAKE GC } \\
\text { WILDWOOD LAKE GC } \\
\text { PHILLIPS AVENUE } \\
\text { CENTRAL AVENUE }\end{array}$ & $\begin{array}{l}\text { LUDLAM AVENUE } \\
\text { SOUTHERN ST. PKY }\end{array}$ & $\begin{array}{l}4 \varnothing 53140724122 \\
4 \varnothing 53110724048 \\
4 \varnothing 53 \emptyset 3 \emptyset 724 \varnothing 51 \\
4 \varnothing 5426 \varnothing 723906 \\
4 \varnothing 4107 \varnothing 734241\end{array}$ & $\begin{array}{l}- \\
\overline{-} \\
\overline{-}\end{array}$ & $\begin{array}{r}269 \\
269 \\
269 \\
269 \\
10\end{array}$ & $\begin{array}{l}- \\
- \\
-\end{array}$ & $\begin{array}{l}\bar{z} \\
\overline{-}\end{array}$ \\
\hline $\begin{array}{l}\text { SP } \\
\text { SP } \\
\text { SP } \\
\text { SP } \\
\text { SP }\end{array}$ & $\begin{array}{r}4 \\
9 \\
21 \\
25 \\
28\end{array}$ & $\begin{array}{l}\text { COES NECK ROAD } \\
\text { HICKSVILLE ROAD } \\
\text { MANETTO HILL RD. } \\
\text { S. OYSTER BAY RD } \\
\text { BROADWAY }\end{array}$ & $\begin{array}{ll}\text { SOUTHERN ST. PKY } \\
\text { SOUTHERN ST. PKY } \\
\text { NORTHERN ST. PKY } \\
\text { NORTHERN ST. PKY } \\
\text { NORTHERN ST. PKY }\end{array}$ & $\begin{array}{l}4 \varnothing 41 \varnothing 1 \varnothing 733614 \\
4 \varnothing 42 \varnothing 8 \varnothing 732902 \\
4 \varnothing 47390732906 \\
4 \varnothing 4735 \varnothing 733 \varnothing 18 \\
4 \varnothing 47 \varnothing 6 \varnothing 733146\end{array}$ & $\begin{array}{l}- \\
- \\
-\end{array}$ & $\begin{array}{r}40 \\
110 \\
122 \\
122 \\
104\end{array}$ & $\begin{array}{l}- \\
\overline{-} \\
\overline{-}\end{array}$ & $\begin{array}{l}- \\
\overline{-} \\
-\end{array}$ \\
\hline $\begin{array}{l}S P \\
S P \\
S P \\
S P \\
S P\end{array}$ & $\begin{array}{l}36 \\
37 \\
41 \\
46 \\
50\end{array}$ & $\begin{array}{l}\text { GLEN COVE ROAD } \\
\text { NORTHERN ST. PKY } \\
\text { SHELTER ROCK RD. } \\
\text { WANTAGH ST. PKWY } \\
\text { WANTAGH ST. PKWY }\end{array}$ & $\begin{array}{l}\text { NORTHERN ST. PKY } \\
\text { HILLSIDE AVENUE } \\
\text { NORTHERN ST. PKY } \\
\text { HEMPSTEAD TPKE. } \\
\text { PROSPECT AVENUE }\end{array}$ & $\begin{array}{l}4 \varnothing 452 \varnothing 0733728 \\
4 \varnothing 45380733724 \\
4 \varnothing 462 \varnothing 0734 \varnothing 25 \\
4 \varnothing 433207332 \varnothing 8 \\
4 \varnothing 4553 \varnothing 733322\end{array}$ & $\begin{array}{l}- \\
- \\
-\end{array}$ & $\begin{array}{r}52 \\
118 \\
87 \\
25 \\
7 \varnothing\end{array}$ & $\begin{array}{l}- \\
\overline{-} \\
\overline{-}\end{array}$ & $\begin{array}{l}- \\
- \\
-\end{array}$ \\
\hline $\begin{array}{l}S P \\
S P \\
S P \\
S P \\
S P\end{array}$ & $\begin{array}{l}54 \\
55 \\
56 \\
57 \\
58\end{array}$ & $\begin{array}{l}\text { FARMINGDALE ROAD } \\
\text { STRAIGHT PATH RD } \\
\text { EAST FORKS ROAD } \\
\text { HECKSCHER ST.PKY } \\
\text { ROBERT MOSES PKY }\end{array}$ & $\begin{array}{l}\text { SOUTHERN ST. PKY } \\
\text { SOUTHERN ST. PKY } \\
\text { SOUTHERN ST. PKY } \\
\text { MONTAUK HIGHWAY } \\
\text { MUNCEY ROAD }\end{array}$ & $\begin{array}{l}4 \varnothing 423 \varnothing \varnothing 732526 \\
4 \varnothing 4336 \varnothing 732233 \\
4 \emptyset 4455 \varnothing 73145 \emptyset \\
4 \varnothing 44 \varnothing 3 \varnothing 731 \varnothing \varnothing 9 \\
4 \emptyset 4341 \varnothing 7317 \varnothing 9\end{array}$ & $\begin{array}{l}- \\
- \\
-\end{array}$ & $\begin{array}{r}99 \\
165 \\
292 \\
193 \\
198\end{array}$ & $\begin{array}{l}- \\
- \\
-\end{array}$ & $\begin{array}{l}- \\
- \\
-\end{array}$ \\
\hline $\begin{array}{l}S P \\
\text { SP } \\
\text { SP } \\
\text { SP } \\
\text { SP }\end{array}$ & $\begin{array}{l}59 \\
60 \\
61 \\
62 \\
63\end{array}$ & $\begin{array}{l}\text { SAGTIKOS PARKWAY } \\
\text { GATEWOOD DRIVE } \\
\text { NEW HIGHWAY } \\
\text { NEW HIGHWAY } \\
\text { SAMBI LANE }\end{array}$ & $\begin{array}{l}\text { PILGRIM ST. HOSP } \\
\text { VETERANS MEM.HWY } \\
\text { VETERANS MEM. HWY } \\
\text { VETERANS MEM. HWY } \\
\text { FLORIDA AVENUE }\end{array}$ & $\begin{array}{l}4 \varnothing 473 \varnothing \varnothing 731641 \\
4 \varnothing 4944 \varnothing 73151 \emptyset \\
4 \varnothing 4936 \varnothing 731445 \\
4 \varnothing 4919 \varnothing 731543 \\
4 \varnothing 4855 \varnothing 731639\end{array}$ & $\begin{array}{l}- \\
- \\
- \\
-\end{array}$ & $\begin{array}{l}189 \\
169 \\
169 \\
169 \\
169\end{array}$ & $\begin{array}{l}- \\
\overline{-} \\
-\end{array}$ & $\begin{array}{l}\overline{-} \\
\overline{-} \\
\overline{-}\end{array}$ \\
\hline $\begin{array}{l}S P \\
S P \\
S P \\
S P \\
S P\end{array}$ & $\begin{array}{l}64 \\
65 \\
66 \\
67 \\
68\end{array}$ & $\begin{array}{l}\text { COMMACK ROAD } \\
\text { DALY ROAD } \\
\text { DALY ROAD } \\
\text { NORTHERN ST. PKY } \\
\text { NORTHERN ST. PKY }\end{array}$ & $\begin{array}{l}\text { NORTHERN ST. PKY } \\
\text { BLACKSMITH LANE } \\
\text { BLACKSMITH LANE } \\
\text { DALY ROAD } \\
\text { VILLANOVA LANE }\end{array}$ & $\begin{array}{l}4 \emptyset 4901 \emptyset 731733 \\
4 \varnothing 4916 \varnothing 731816 \\
4 \emptyset 4916 \varnothing 731816 \\
4 \varnothing 4938 \emptyset 731854 \\
4 \varnothing 4943 \varnothing 73194 \emptyset\end{array}$ & $\begin{array}{l}- \\
- \\
-\end{array}$ & $\begin{array}{l}169 \\
169 \\
169 \\
169 \\
169\end{array}$ & $\begin{array}{l}- \\
\overline{-} \\
-\end{array}$ & $\begin{array}{l}- \\
- \\
-\end{array}$ \\
\hline $\begin{array}{l}\text { SP } \\
\text { SP } \\
\text { SP } \\
\text { SP } \\
\text { SP }\end{array}$ & $\begin{array}{l}69 \\
70 \\
71 \\
72 \\
73\end{array}$ & $\begin{array}{l}\text { DIX HILLS ROAD } \\
\text { WOLF HILL ROAD } \\
\text { OLD COUNTRY ROAD } \\
\text { ROUND SWAMP ROAD } \\
\text { SUNKEN MEADOW }\end{array}$ & $\begin{array}{l}\text { NORTHERN ST. PKY } \\
\text { OLD COUNTRY ROAD } \\
\text { NORTHERN ST. PKY } \\
\text { NORTHERN ST. PKY } \\
\text { NEW HIGHWAY }\end{array}$ & $\begin{array}{l}4 \varnothing 49230732216 \\
4 \varnothing 48420732245 \\
4 \varnothing 475307324 \varnothing 6 \\
4 \varnothing 48100732634 \\
4 \varnothing 4927 \varnothing 731722\end{array}$ & $\begin{array}{l}- \\
- \\
-\end{array}$ & $\begin{array}{l}133 \\
133 \\
148 \\
148 \\
169\end{array}$ & $\begin{array}{l}- \\
- \\
-\end{array}$ & $\begin{array}{l}- \\
- \\
-\end{array}$ \\
\hline $\begin{array}{l}S P \\
S P \\
S P \\
S P \\
S P\end{array}$ & $\begin{array}{l}74 \\
75 \\
76 \\
77 \\
78\end{array}$ & $\begin{array}{l}\text { SUNKEN MEADOW } \\
\text { SUNKEN MEADOW } \\
\text { INDIAN HEAD ROAD } \\
\text { INDIAN HEAD ROAD } \\
\text { SUNKEN MEADOW }\end{array}$ & $\begin{array}{l}\text { CUTCHOGUE LANE } \\
\text { VETERANS MEM. HWY } \\
\text { JERICHO TURNPIKE } \\
\text { JERICHO TURNPIKE } \\
\text { HAYRICK LANE }\end{array}$ & $\begin{array}{l}4 \varnothing 50110731649 \\
4 \varnothing 5 \varnothing 280731652 \\
4 \varnothing 5 \varnothing 53 \varnothing 731647 \\
4 \varnothing 5 \varnothing 43 \varnothing 731651 \\
4 \varnothing 5117 \varnothing 731647\end{array}$ & $\begin{array}{l}- \\
- \\
-\end{array}$ & $\begin{array}{l}169 \\
169 \\
169 \\
169 \\
169\end{array}$ & $\begin{array}{l}- \\
- \\
-\end{array}$ & $\begin{array}{l}- \\
- \\
-\end{array}$ \\
\hline $\begin{array}{l}\text { SP } \\
\text { SP } \\
\text { SP } \\
\text { SP } \\
\text { SP }\end{array}$ & $\begin{array}{l}79 \\
80 \\
81 \\
82 \\
83\end{array}$ & $\begin{array}{l}\text { SUNKEN MEADOW } \\
\text { SUNKEN MEADOW } \\
\text { SUNKEN MEADOW } \\
\text { SUNKEN MEADOW } \\
\text { SUNKEN MEADOW }\end{array}$ & $\begin{array}{l}\text { OLD NORTHPORT RD } \\
\text { SCHOLAR LANE } \\
\text { NORTHPORT ROAD } \\
\text { NORTHPORT ROAD } \\
\text { NORTHPORT ROAD }\end{array}$ & 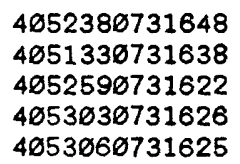 & $\begin{array}{l}- \\
- \\
- \\
-\end{array}$ & $\begin{array}{l}174 \\
169 \\
174 \\
174 \\
174\end{array}$ & $\begin{array}{l}- \\
- \\
-\end{array}$ & $\begin{array}{l}- \\
- \\
-\end{array}$ \\
\hline $\begin{array}{l}\text { ST } \\
\text { ST } \\
\text { ST } \\
\text { ST } \\
\text { ST }\end{array}$ & $\begin{array}{l}1 \\
2 \\
3 \\
4 \\
5\end{array}$ & $\begin{array}{l}\text { COMMUNITY DRIVE } \\
\text { SHELTER ROCK RD } \\
\text { SEARINGTOWN ROAD } \\
\text { L.I.E. } \\
\text { GLEN COVE ROAD }\end{array}$ & $\begin{array}{l}\text { L.I.E. } \\
\text { NORTHERN ST.PKY. } \\
\text { L.I.E. } \\
\text { L.I.R.R. } \\
\text { L.I.E. }\end{array}$ & $\begin{array}{l}4 \varnothing 46520734228 \\
4 \varnothing 4624 \varnothing 734 \varnothing \varnothing 9 \\
4 \varnothing 4647 \varnothing 73394 \varnothing \\
4 \varnothing 47 \varnothing 6 \varnothing 733835 \\
4 \varnothing 4644 \varnothing 733733\end{array}$ & $\begin{array}{l}- \\
\overline{-} \\
-\end{array}$ & $\begin{array}{r}- \\
72 \\
87 \\
83 \\
118\end{array}$ & $\begin{array}{l}- \\
\bar{z} \\
-\end{array}$ & $\begin{array}{l}- \\
- \\
-\end{array}$ \\
\hline
\end{tabular}


N.Y. [Dash indicates no data. Altitudes are in feet above sea level.] (cont.)

\begin{tabular}{|c|c|c|c|c|c|c|c|c|c|c|c|c|}
\hline & & $\begin{array}{l}\text { MAX. } \\
\text { AREA } \\
\text { (square }\end{array}$ & $\begin{array}{c}\text { BASIN } \\
\text { AREA } \\
\text { feet) }\end{array}$ & RIM & $\begin{array}{l}\text { OVER- } \\
\text { FLOW }\end{array}$ & $\begin{array}{l}\text { BOT- } \\
\text { TOM }\end{array}$ & $\begin{array}{l}\text { WATER } \\
\text { TABLE }\end{array}$ & $\begin{array}{c}\text { DRAIN. } \\
\text { AREA } \\
\text { (acres) }\end{array}$ & $\begin{array}{l}-- \text { BASI } \\
\text { STATUS }\end{array}$ & $\begin{array}{l}\text { IN-- } \\
\text { USSE }\end{array}$ & $\begin{array}{l}\text { GEO. } \\
\text { UNIT }\end{array}$ & $\begin{array}{l}\text { SOIL } \\
\text { UNIT }\end{array}$ \\
\hline $\begin{array}{l}\text { SN } \\
\text { SN } \\
\text { SN } \\
\text { SN } \\
\text { SN }\end{array}$ & $\begin{array}{l}9 \\
10 \\
11 \\
12 \\
13\end{array}$ & $\begin{array}{l}- \\
- \\
-\end{array}$ & $\begin{array}{l}- \\
- \\
- \\
-\end{array}$ & $\begin{array}{r}122.0 \\
52 . \varnothing \\
- \\
30 . \varnothing \\
40 . \varnothing\end{array}$ & $\begin{array}{l}- \\
- \\
- \\
-\end{array}$ & $\begin{array}{l}- \\
- \\
- \\
-\end{array}$ & $\begin{array}{r}3.0 \\
17.0 \\
17 . \overline{0} \\
2.0\end{array}$ & $\begin{array}{l}- \\
- \\
- \\
-\end{array}$ & $\begin{array}{l}1 \\
1 \\
1 \\
1 \\
1\end{array}$ & $\begin{array}{l}1 \\
1 \\
1 \\
1 \\
1\end{array}$ & $\begin{array}{l}1 \\
1 \\
1 \\
1 \\
6\end{array}$ & $\begin{array}{l}0 \\
8 \\
8 \\
8 \\
6\end{array}$ \\
\hline $\begin{array}{l}\text { SN } \\
\text { SN } \\
\text { SN } \\
\text { SN } \\
\text { SP }\end{array}$ & $\begin{array}{r}14 \\
15 \\
16 \\
17 \\
1\end{array}$ & $\begin{array}{l}- \\
- \\
- \\
-\end{array}$ & $\begin{array}{l}- \\
- \\
- \\
-\end{array}$ & $\begin{array}{r}140 . \varnothing \\
140 . \varnothing \\
150 . \varnothing \\
20 . \varnothing \\
46 . \varnothing\end{array}$ & $\begin{array}{l}- \\
- \\
- \\
-\end{array}$ & $\begin{array}{l}- \\
- \\
- \\
-\end{array}$ & $\begin{array}{l}48.0 \\
47.0 \\
51.0 \\
13.0 \\
13.0\end{array}$ & $\begin{array}{l}- \\
- \\
- \\
-\end{array}$ & $\begin{array}{l}1 \\
1 \\
1 \\
0 \\
1\end{array}$ & $\begin{array}{l}1 \\
1 \\
1 \\
1 \\
3\end{array}$ & $\begin{array}{l}6 \\
6 \\
6 \\
1 \\
1\end{array}$ & $\begin{array}{l}6 \\
5 \\
5 \\
8 \\
3\end{array}$ \\
\hline $\begin{array}{l}\text { SP } \\
\text { SP } \\
\text { SP } \\
\text { SP } \\
\text { SP }\end{array}$ & $\begin{array}{r}4 \\
9 \\
21 \\
25 \\
28\end{array}$ & $\begin{array}{l}- \\
- \\
-\end{array}$ & $\begin{array}{l}- \\
- \\
-\end{array}$ & $\begin{array}{r}40 . \varnothing \\
47 . \varnothing \\
180.0 \\
190 . \varnothing \\
165 . \varnothing\end{array}$ & $\begin{array}{l}- \\
- \\
- \\
-\end{array}$ & $\begin{array}{l}- \\
- \\
- \\
-\end{array}$ & $\begin{array}{l}27.0 \\
36.0 \\
80.0 \\
82.0 \\
78.0\end{array}$ & $\begin{array}{r}- \\
45.1 \\
- \\
-\end{array}$ & $\begin{array}{l}1 \\
1 \\
1 \\
1 \\
1\end{array}$ & $\begin{array}{l}3 \\
3 \\
3 \\
3 \\
3\end{array}$ & $\begin{array}{l}1 \\
1 \\
1 \\
1 \\
1\end{array}$ & $\begin{array}{l}1 \\
1 \\
2 \\
2 \\
1\end{array}$ \\
\hline $\begin{array}{l}\text { SP } \\
\text { SP } \\
\text { SP } \\
\text { SP } \\
\text { SP }\end{array}$ & $\begin{array}{l}36 \\
37 \\
41 \\
46 \\
50\end{array}$ & $\begin{array}{l}- \\
- \\
-\end{array}$ & $\begin{array}{l}\text { - } \\
\text { - } \\
\text { - }\end{array}$ & $\begin{array}{r}100 . \emptyset \\
95 . \varnothing \\
160.0 \\
100 . \varnothing \\
135 . \varnothing\end{array}$ & $\begin{array}{l}- \\
- \\
- \\
-\end{array}$ & $\begin{array}{l}- \\
- \\
- \\
-\end{array}$ & $\begin{array}{l}60.0 \\
62.0 \\
42.0 \\
55.0 \\
75.0\end{array}$ & $\begin{array}{r}100 . \overline{0} \\
19 . \varnothing \\
13 . \overline{0}\end{array}$ & $\begin{array}{l}1 \\
1 \\
0 \\
1 \\
1\end{array}$ & $\begin{array}{l}3 \\
3 \\
3 \\
3 \\
3\end{array}$ & $\begin{array}{l}1 \\
1 \\
1 \\
1 \\
1\end{array}$ & $\begin{array}{l}2 \\
1 \\
1 \\
2 \\
1\end{array}$ \\
\hline $\begin{array}{l}\text { SP } \\
\text { SP } \\
\text { SP } \\
\text { SP } \\
\text { SP }\end{array}$ & $\begin{array}{l}54 \\
55 \\
56 \\
57 \\
58\end{array}$ & $\begin{array}{l}- \\
\overline{-} \\
\bar{z}\end{array}$ & $\begin{array}{l}- \\
- \\
- \\
-\end{array}$ & $\begin{array}{l}45.0 \\
50.0 \\
40.0 \\
22.0 \\
40.0\end{array}$ & $\begin{array}{l}- \\
\overline{-} \\
-\end{array}$ & $\begin{array}{l}- \\
- \\
-\end{array}$ & $\begin{array}{l}39 . \varnothing \\
42 . \varnothing \\
30 . \varnothing \\
15 . \varnothing \\
26 . \varnothing\end{array}$ & $\begin{array}{l}- \\
- \\
- \\
-\end{array}$ & $\begin{array}{l}1 \\
1 \\
1 \\
1 \\
1\end{array}$ & $\begin{array}{l}3 \\
3 \\
3 \\
3 \\
3\end{array}$ & $\begin{array}{l}1 \\
1 \\
1 \\
1 \\
1\end{array}$ & $\begin{array}{l}1 \\
1 \\
3 \\
6 \\
7\end{array}$ \\
\hline $\begin{array}{l}S P \\
S P \\
S P \\
S P \\
S P\end{array}$ & $\begin{array}{l}59 \\
69 \\
61 \\
62 \\
63\end{array}$ & $\begin{array}{l}- \\
- \\
- \\
-\end{array}$ & $\begin{array}{l}- \\
- \\
- \\
-\end{array}$ & $\begin{array}{r}120 . \varnothing \\
100 . \varnothing \\
60.0 \\
155 . \varnothing \\
180 . \varnothing\end{array}$ & $\begin{array}{l}- \\
- \\
- \\
-\end{array}$ & $\begin{array}{l}- \\
- \\
-\end{array}$ & $\begin{array}{l}49 . \varnothing \\
49 . \varnothing \\
44 . \varnothing \\
50 . \varnothing \\
57 . \varnothing\end{array}$ & $\begin{array}{l}- \\
- \\
- \\
-\end{array}$ & $\begin{array}{l}1 \\
0 \\
1 \\
1 \\
1\end{array}$ & $\begin{array}{l}3 \\
3 \\
3 \\
3 \\
3\end{array}$ & $\begin{array}{l}1 \\
1 \\
1 \\
1 \\
1\end{array}$ & $\begin{array}{l}1 \\
8 \\
5 \\
3 \\
3\end{array}$ \\
\hline $\begin{array}{l}S P \\
S P \\
S P \\
S P \\
S P\end{array}$ & $\begin{array}{l}64 \\
65 \\
66 \\
67 \\
68\end{array}$ & $\begin{array}{l}- \\
- \\
- \\
-\end{array}$ & $\begin{array}{l}- \\
- \\
- \\
-\end{array}$ & $\begin{array}{l}115 . \varnothing \\
14 \varnothing . \varnothing \\
14 \varnothing . \varnothing \\
18 \varnothing . \varnothing \\
2 \varnothing \varnothing . \varnothing\end{array}$ & $\begin{array}{l}- \\
- \\
- \\
-\end{array}$ & $\begin{array}{l}- \\
- \\
- \\
-\end{array}$ & $\begin{array}{l}68.0 \\
71.0 \\
71.0 \\
74.0 \\
73.0\end{array}$ & $\begin{array}{l}- \\
- \\
- \\
-\end{array}$ & $\begin{array}{l}1 \\
1 \\
1 \\
1 \\
1\end{array}$ & $\begin{array}{l}3 \\
3 \\
3 \\
3 \\
3\end{array}$ & $\begin{array}{l}1 \\
1 \\
1 \\
5 \\
5\end{array}$ & $\begin{array}{l}5 \\
3 \\
3 \\
5 \\
5\end{array}$ \\
\hline $\begin{array}{l}\text { SP } \\
\text { SP } \\
\text { SP } \\
\text { SP } \\
\text { SP }\end{array}$ & $\begin{array}{l}69 \\
70 \\
71 \\
72 \\
73\end{array}$ & $\begin{array}{l}- \\
- \\
- \\
-\end{array}$ & $\begin{array}{l}- \\
- \\
- \\
-\end{array}$ & $\begin{array}{l}170.0 \\
170.0 \\
140.0 \\
230.0 \\
120.0\end{array}$ & $\begin{array}{l}- \\
- \\
- \\
-\end{array}$ & $\begin{array}{l}- \\
- \\
- \\
-\end{array}$ & $\begin{array}{l}73.0 \\
73.0 \\
77.0 \\
76 . \varnothing \\
65.0\end{array}$ & $\begin{array}{l}- \\
- \\
- \\
-\end{array}$ & $\begin{array}{l}1 \\
1 \\
1 \\
1 \\
0\end{array}$ & $\begin{array}{l}3 \\
3 \\
3 \\
3 \\
0\end{array}$ & $\begin{array}{l}1 \\
1 \\
1 \\
1 \\
0\end{array}$ & $\begin{array}{l}3 \\
3 \\
1 \\
7 \\
0\end{array}$ \\
\hline $\begin{array}{l}\text { SP } \\
\text { SF } \\
\text { SP } \\
\text { SP } \\
\text { SP }\end{array}$ & $\begin{array}{l}74 \\
75 \\
76 \\
77 \\
78\end{array}$ & $\begin{array}{l}- \\
- \\
- \\
-\end{array}$ & $\begin{array}{l}- \\
- \\
- \\
-\end{array}$ & $\begin{array}{l}155.0 \\
150.0 \\
145.0 \\
145.0 \\
155 . \varnothing\end{array}$ & $\begin{array}{l}- \\
- \\
- \\
-\end{array}$ & $\begin{array}{l}- \\
- \\
- \\
-\end{array}$ & $\begin{array}{l}63 . \varnothing \\
64 . \varnothing \\
64 . \varnothing \\
64 . \varnothing \\
62 . \varnothing\end{array}$ & $\begin{array}{l}- \\
- \\
- \\
-\end{array}$ & $\begin{array}{l}1 \\
1 \\
1 \\
1 \\
1\end{array}$ & $\begin{array}{l}3 \\
3 \\
3 \\
3 \\
3\end{array}$ & $\begin{array}{l}1 \\
1 \\
1 \\
1 \\
1\end{array}$ & $\begin{array}{l}3 \\
3 \\
7 \\
1 \\
1\end{array}$ \\
\hline $\begin{array}{l}\text { SF } \\
\text { SF } \\
\text { SF } \\
\text { SF } \\
\text { SF }\end{array}$ & $\begin{array}{l}79 \\
80 \\
81 \\
82 \\
83\end{array}$ & $\begin{array}{l}- \\
- \\
- \\
-\end{array}$ & $\begin{array}{l}- \\
- \\
- \\
-\end{array}$ & $\begin{array}{l}150.0 \\
16 \varnothing . \varnothing \\
175 . \emptyset \\
175 . \varnothing \\
175 . \varnothing\end{array}$ & $\begin{array}{l}- \\
- \\
- \\
-\end{array}$ & $\begin{array}{l}- \\
- \\
- \\
-\end{array}$ & $\begin{array}{l}50 . \varnothing \\
60 . \varnothing \\
46.0 \\
47 . \varnothing \\
45 . \varnothing\end{array}$ & $\begin{array}{l}- \\
\overline{-} \\
\overline{-}\end{array}$ & $\begin{array}{l}1 \\
1 \\
1 \\
1 \\
1\end{array}$ & $\begin{array}{l}3 \\
3 \\
3 \\
3 \\
3\end{array}$ & $\begin{array}{l}1 \\
1 \\
1 \\
1 \\
1\end{array}$ & $\begin{array}{l}1 \\
1 \\
1 \\
1 \\
1\end{array}$ \\
\hline $\begin{array}{l}\text { ST } \\
\text { ST } \\
\text { ST } \\
\text { ST } \\
\text { ST }\end{array}$ & $\begin{array}{l}1 \\
2 \\
3 \\
4 \\
5\end{array}$ & $\begin{array}{l}- \\
- \\
- \\
-\end{array}$ & $\begin{array}{l}25200 \\
392400 \\
289400 \\
155200 \\
480000\end{array}$ & $\begin{array}{l}140.0 \\
170.0 \\
140.0 \\
140.0 \\
140.0\end{array}$ & - & $\begin{array}{l}- \\
\overline{-} \\
-\end{array}$ & $\begin{array}{l}32 . \varnothing \\
44 . \varnothing \\
45 . \varnothing \\
50 . \varnothing \\
61.0\end{array}$ & $\begin{array}{r}21.3 \\
- \\
94.3 \\
60.3 \\
85.1\end{array}$ & $\begin{array}{l}1 \\
1 \\
1 \\
1 \\
1\end{array}$ & $\begin{array}{l}3 \\
3 \\
3 \\
3 \\
3\end{array}$ & $\begin{array}{l}3 \\
1 \\
1 \\
1 \\
1\end{array}$ & $\begin{array}{l}3 \\
1 \\
1 \\
1 \\
1\end{array}$ \\
\hline
\end{tabular}


Appendix.--Location and basic physical features of recharge basins on Long Island,

\begin{tabular}{|c|c|c|c|c|c|c|c|c|}
\hline \multicolumn{2}{|c|}{$\begin{array}{l}\text { BASIN } \\
\text { NUMBER }\end{array}$} & \multirow{2}{*}{\multicolumn{2}{|c|}{ NEAREST INTERSECTION }} & \multirow{2}{*}{$\begin{array}{l}\text { LATITUDE } \\
\text { LONGITUDE } \\
\circ, " \text { " " }\end{array}$} & \multirow{2}{*}{$\begin{array}{l}\text { DATE } \\
\text { BUILT } \\
\text { YrMOD }\end{array}$} & \multirow{2}{*}{ COMMUNITY } & \multirow{2}{*}{$\begin{array}{r}\text { DESIGN } \\
\text { CAPACITY } \\
\text { (cubic }\end{array}$} & \multirow{2}{*}{$\begin{array}{l}\text { ACTUAL } \\
\text { CAPACITY } \\
\text { feet) }\end{array}$} \\
\hline & & & & & & & & \\
\hline $\begin{array}{l}\text { ST } \\
\text { ST } \\
\text { ST } \\
\text { ST } \\
\text { ST }\end{array}$ & $\begin{array}{r}6 \\
7 \\
8 \\
9 \\
10\end{array}$ & $\begin{array}{l}\text { WHEATLEY ROAD } \\
\text { L.I.E. } \\
\text { POST ROAD } \\
\text { L.I.E. } \\
\text { L.I.E. }\end{array}$ & $\begin{array}{l}\text { L.I.E. } \\
\text { LEWIS PATH } \\
\text { L.I.E. } \\
\text { JERICHO TURNPIKE } \\
\text { JERICHO TURNPIKE }\end{array}$ & $\begin{array}{l}4 \emptyset 46460733627 \\
4 \varnothing 47 \varnothing 90733621 \\
4 \varnothing 4656 \varnothing 733539 \\
4 \varnothing 46530733331 \\
4 \varnothing 4657 \varnothing 733330\end{array}$ & $\begin{array}{l}- \\
- \\
-\end{array}$ & $\begin{array}{l}118 \\
118 \\
118 \\
105 \\
105\end{array}$ & $\begin{array}{l}- \\
- \\
- \\
-\end{array}$ & $\begin{array}{l}- \\
- \\
- \\
-\end{array}$ \\
\hline $\begin{array}{l}\text { ST } \\
\text { ST } \\
\text { ST } \\
\text { ST } \\
\text { ST }\end{array}$ & $\begin{array}{l}11 \\
12 \\
13 \\
14 \\
15\end{array}$ & $\begin{array}{l}\text { L.I.E. } \\
\text { CEDAR SWAMP ROAD } \\
\text { EAST NORWICH RD. } \\
\text { SUGAR TOMS ROAD } \\
\text { BERRY HILL ROAD }\end{array}$ & $\begin{array}{l}\text { JERICHO TURNPIKE } \\
\text { JERICHO TURNPIKE } \\
\text { BROOKVILLE ROAD } \\
\text { HIGHWOOD ROAD } \\
\text { MCCOUNS LANE }\end{array}$ & 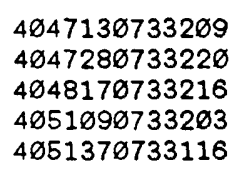 & $\begin{array}{l}- \\
- \\
- \\
-\end{array}$ & $\begin{array}{r}105 \\
105 \\
93 \\
98 \\
119\end{array}$ & $\begin{array}{l}- \\
- \\
-\end{array}$ & $\begin{array}{l}- \\
- \\
-\end{array}$ \\
\hline $\begin{array}{l}\text { ST } \\
\text { ST } \\
\text { ST } \\
\text { ST } \\
\text { ST }\end{array}$ & $\begin{array}{l}16 \\
17 \\
18 \\
19 \\
20\end{array}$ & $\begin{array}{l}\text { L.I.R.R. } \\
\text { WILLETS DRIVE } \\
\text { COLD SPRNG.HILLS } \\
\text { WEST HILLS ROAO } \\
\text { DETROIT PLACE }\end{array}$ & $\begin{array}{l}\text { JERICHO TURNPIKE } \\
\text { DORCAS AVENUE } \\
\text { JERICHO TURNPIKE } \\
\text { JERICHO TURNPIKE } \\
\text { AMITYVILLE ROAD }\end{array}$ & $\begin{array}{l}4 \emptyset 483307331 \varnothing 7 \\
4 \varnothing 4845 \emptyset 73302 \emptyset \\
4 \varnothing 4939 \emptyset 732621 \\
4 \varnothing 4939 \emptyset 73252 \emptyset \\
4 \varnothing 4933 \varnothing 732448\end{array}$ & $\begin{array}{l}- \\
- \\
- \\
-\end{array}$ & $\begin{array}{l}108 \\
108 \\
151 \\
151 \\
105\end{array}$ & $\begin{array}{l}- \\
- \\
- \\
-\end{array}$ & $\begin{array}{l}- \\
- \\
-\end{array}$ \\
\hline $\begin{array}{l}\text { ST } \\
\text { ST } \\
\text { ST } \\
\text { ST } \\
\text { ST }\end{array}$ & $\begin{array}{l}21 \\
22 \\
23 \\
24 \\
25\end{array}$ & $\begin{array}{l}\text { WALT WHITMAN RD. } \\
\text { HOLLAND STREET } \\
\text { NORTHERN ST. PKY } \\
\text { EMERALD LANE } \\
\text { ELWOOD ROAD }\end{array}$ & $\begin{array}{l}\text { WALT WHITMAN SC } \\
\text { FLANDERS AVENUE } \\
\text { WALT WHITMAN RD. } \\
\text { SAPPHIRE PLACE } \\
\text { JERICHO TURNPIKE }\end{array}$ & $\begin{array}{l}4 \varnothing 49090732441 \\
4 \varnothing 48380732432 \\
4 \varnothing 475 \varnothing 0732445 \\
4 \emptyset 49580732250 \\
4 \varnothing 50180732007\end{array}$ & $\begin{array}{l}- \\
- \\
-\end{array}$ & $\begin{array}{l}105 \\
151 \\
148 \\
151 \\
139\end{array}$ & $\begin{array}{l}- \\
- \\
- \\
-\end{array}$ & $\begin{array}{l}- \\
- \\
-\end{array}$ \\
\hline $\begin{array}{l}\text { ST } \\
\text { ST } \\
\text { ST } \\
\text { ST } \\
\text { ST }\end{array}$ & $\begin{array}{l}26 \\
28 \\
29 \\
30 \\
31\end{array}$ & $\begin{array}{l}\text { VETERANS MEM HWY } \\
\text { LEDGEWOOD DRIVE } \\
\text { HAUPPAUGE ROAD } \\
\text { TERRY ROAD } \\
\text { LYMAN COURT }\end{array}$ & $\begin{array}{l}\text { JERICHO TURNPIKE } \\
\text { JERICHO TURNPIKE } \\
\text { JERICHO TURNPIKE } \\
\text { JERICHO TURNPIKE } \\
\text { NORTH COUNTRY RD }\end{array}$ & $\begin{array}{l}4 ø 5 \emptyset 369731723 \\
465051073144 \emptyset \\
4 ø 51179731112 \\
4 ø 51190731042 \\
4 \varnothing 52000731038\end{array}$ & $\begin{array}{l}- \\
- \\
- \\
-\end{array}$ & $\begin{array}{l}169 \\
169 \\
185 \\
158 \\
180\end{array}$ & $\begin{array}{l}- \\
- \\
- \\
-\end{array}$ & $\begin{array}{l}- \\
- \\
-\end{array}$ \\
\hline $\begin{array}{l}\text { ST } \\
\text { ST } \\
\text { ST } \\
\text { ST } \\
\text { ST }\end{array}$ & $\begin{array}{l}32 \\
33 \\
34 \\
35 \\
36\end{array}$ & $\begin{array}{l}\text { NICHOLS ROAD } \\
\text { SMITHTOWN BLVD. } \\
\text { SMITH LANE } \\
\text { DARE ROAD } \\
\text { RIDGE ROAD }\end{array}$ & $\begin{array}{l}\text { MORICHES ROAD } \\
\text { MIDDLE COUNTRY } R \\
\text { MIDDLE COUNTRY } R \\
\text { MIDDLE COUNTRY } R \\
\text { RAYNOR ROAD }\end{array}$ & 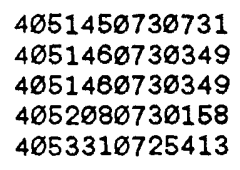 & $\begin{array}{l}- \\
- \\
-\end{array}$ & $\begin{array}{l}227 \\
253 \\
216 \\
244 \\
250\end{array}$ & $\begin{array}{l}- \\
- \\
-\end{array}$ & $\begin{array}{l}- \\
- \\
-\end{array}$ \\
\hline $\begin{array}{l}\text { ST } \\
\text { ST } \\
\text { ST } \\
\text { ST } \\
\text { ST }\end{array}$ & $\begin{array}{l}37 \\
38 \\
39 \\
40 \\
41\end{array}$ & $\begin{array}{l}\text { PONQUOGUE AVENUE } \\
\text { GRAVEL ROAD } \\
\text { OLD MAIN ROAD } \\
\text { TABOR ROAD } \\
\text { HAMPTON BAYS RD. }\end{array}$ & $\begin{array}{l}\text { KING STREET } \\
\text { L.I.R.R. BRIDGE } \\
\text { MAIN ROAD } \\
\text { MONTAUK HIGHWAY }\end{array}$ & $\begin{array}{l}4 ø 5206 \varnothing 723251 \\
4 ø 57 \varnothing 4 \varnothing 723426 \\
4 ø 59090723225 \\
41084 \varnothing \emptyset 721759 \\
4 \varnothing 52380723296\end{array}$ & $\begin{array}{l}- \\
- \\
-\end{array}$ & $\begin{array}{l}293 \\
267 \\
281 \\
286 \\
293\end{array}$ & $\begin{array}{l}- \\
-\end{array}$ & $\begin{array}{l}- \\
- \\
- \\
-\end{array}$ \\
\hline $\begin{array}{l}\text { ST } \\
\text { ST } \\
\text { ST } \\
\text { ST } \\
\text { ST }\end{array}$ & $\begin{array}{l}42 \\
43 \\
44 \\
45 \\
46\end{array}$ & $\begin{array}{l}\text { WINDMILL LANE } \\
\text { BELLPORT AVENUE } \\
\text { PATCHOGUE-YAP.RD } \\
\text { SIPP AVENUE } \\
\text { ROBINSON AVENUE }\end{array}$ & $\begin{array}{l}\text { MONTAUK HIGHWAY } \\
\text { SUNRISE HIGHWAY } \\
\text { SUNRISE HIGHWAY } \\
\text { SUNRISE HIGHWAY } \\
\text { SUNRISE HIGHWAY }\end{array}$ & 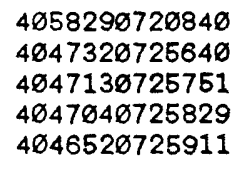 & $\begin{array}{l}- \\
- \\
-\end{array}$ & $\begin{array}{l}317 \\
236 \\
236 \\
236 \\
221\end{array}$ & $\begin{array}{l}- \\
- \\
-\end{array}$ & $\begin{array}{l}- \\
- \\
-\end{array}$ \\
\hline $\begin{array}{l}\text { ST } \\
\text { ST } \\
\text { ST } \\
\text { ST } \\
\text { ST }\end{array}$ & $\begin{array}{l}47 \\
48 \\
49 \\
50 \\
51\end{array}$ & $\begin{array}{l}\text { LAKELAND AVENUE } \\
\text { FARMINGDALE ROAD } \\
\text { FARMINGDALE ROAD } \\
\text { FARMINGDALE ROAD } \\
\text { FARMINGDALE ROAD }\end{array}$ & $\begin{array}{l}\text { SUNRISE HIGHWAY } \\
\text { STH AVENUE } \\
\text { WELLWODD AVENUE } \\
\text { NEW HIGHWAY } \\
\text { SOUTHERN ST. PKY }\end{array}$ & $\begin{array}{l}4 \emptyset 4532 \emptyset 73 ø 557 \\
4 \emptyset 4249 \emptyset 7323 \emptyset 2 \\
4 \emptyset 4256 \varnothing 732353 \\
404259 \emptyset 732418 \\
404308 \emptyset 732434\end{array}$ & $\begin{array}{l}- \\
- \\
-\end{array}$ & $\begin{array}{r}188 \\
165 \\
165 \\
99 \\
99\end{array}$ & $\begin{array}{l}- \\
- \\
- \\
-\end{array}$ & $\begin{array}{l}- \\
- \\
- \\
-\end{array}$ \\
\hline $\begin{array}{l}\text { ST } \\
\text { ST } \\
\text { ST } \\
\text { ST } \\
\text { ST }\end{array}$ & $\begin{array}{l}52 \\
53 \\
54 \\
55 \\
56\end{array}$ & $\begin{array}{l}\text { FARMINGDALE ROAD } \\
\text { FARMINGDALE ROAD } \\
\text { BROAD HOLLOW RD. } \\
\text { STEWART AVENUE } \\
\text { NEWBRIDGE ROAD }\end{array}$ & $\begin{array}{l}\text { BROAD HOLLOW RD. } \\
\text { CARMANS ROAD } \\
\text { CONKLIN STREET } \\
\text { HEMPSTEAD TPKE. } \\
\text { HEMPSTEAD TPKE. }\end{array}$ & $\begin{array}{l}4043210732536 \\
4 \varnothing 4332 \varnothing 732558 \\
4 \varnothing 44 \varnothing 5 \varnothing 732531 \\
4 \varnothing 4346 \varnothing 732857 \\
4 \varnothing 4321 \varnothing 73324 \varnothing\end{array}$ & $\begin{array}{l}- \\
- \\
-\end{array}$ & $\begin{array}{r}99 \\
99 \\
99 \\
121 \\
8\end{array}$ & - & - \\
\hline $\begin{array}{l}\text { ST } \\
\text { ST } \\
\text { ST } \\
\text { ST } \\
\text { ST }\end{array}$ & $\begin{array}{l}57 \\
58 \\
59 \\
60 \\
61\end{array}$ & $\begin{array}{l}\text { BROADWAY } \\
\text { COUNTY LINE RCAD } \\
\text { BROADWAY } \\
\text { BROADWAY } \\
\text { L.I.R.R. }\end{array}$ & $\begin{array}{l}\text { SUNRISE HIGHWAY } \\
\text { FRANCINE DRIVE } \\
\text { BREFNI STREET } \\
\text { L.I.E. } \\
\text { L.I.E. }\end{array}$ & $\begin{array}{l}4 \varnothing 4117 \varnothing 7325 \varnothing 7 \\
4 \varnothing 4144 \varnothing 732537 \\
4 \varnothing 4219 \emptyset 732537 \\
4 \varnothing 47 \varnothing 9 \varnothing 73315 \emptyset \\
4 \varnothing 4742 \varnothing 733048\end{array}$ & $\begin{array}{l}- \\
- \\
-\end{array}$ & $\begin{array}{l}156 \\
163 \\
163 \\
105 \\
108\end{array}$ & $\begin{array}{l}- \\
- \\
-\end{array}$ & - \\
\hline
\end{tabular}


N.Y. [Dash indicates no data. Altitudes are in feet above sea level.] (cont.)

\begin{tabular}{|c|c|c|c|c|c|c|c|c|c|c|c|c|}
\hline \multicolumn{2}{|c|}{$\begin{array}{l}\text { BASIN } \\
\text { NUMBER }\end{array}$} & $\begin{array}{l}\text { MAX. } \\
\text { AREA } \\
\text { (square }\end{array}$ & $\begin{array}{c}\text { BASIN } \\
\text { AREA } \\
\text { fe }\end{array}$ & RIM & $\begin{array}{l}--A L T] \\
\text { OVER } \\
\text { FLOW }\end{array}$ & $\begin{array}{l}\text { UDE--- } \\
\text { BOT- } \\
\text { TOM }\end{array}$ & $\begin{array}{l}\text { WATER } \\
\text { TABLE }\end{array}$ & $\begin{array}{c}\text { DRAIN. } \\
\text { AREA } \\
\text { (acres) }\end{array}$ & \multicolumn{2}{|c|}{$\begin{array}{l}---B A S I N-- \\
\text { STATUS USE }\end{array}$} & $\begin{array}{l}\text { GEO. } \\
\text { UNIT }\end{array}$ & $\begin{array}{l}\text { SOIL } \\
\text { UNIT }\end{array}$ \\
\hline $\begin{array}{l}\text { ST } \\
\text { ST } \\
\text { ST } \\
\text { ST } \\
\text { ST }\end{array}$ & $\begin{array}{r}6 \\
7 \\
8 \\
9 \\
10\end{array}$ & $\begin{array}{l}- \\
- \\
-\end{array}$ & $\begin{array}{r}250100 \\
400000 \\
- \\
- \\
-\end{array}$ & $\begin{array}{l}133.0 \\
150.0 \\
147.0 \\
155.0 \\
170.0\end{array}$ & $\begin{array}{l}- \\
- \\
- \\
-\end{array}$ & $\begin{array}{l}- \\
- \\
- \\
-\end{array}$ & $\begin{array}{l}66.0 \\
67.0 \\
70.0 \\
75.0 \\
75.0\end{array}$ & $\begin{array}{r}30.3 \\
- \\
- \\
101.7 \\
48.9\end{array}$ & $\begin{array}{l}1 \\
1 \\
0 \\
0 \\
1\end{array}$ & $\begin{array}{l}3 \\
3 \\
3 \\
3 \\
3\end{array}$ & $\begin{array}{l}1 \\
1 \\
1 \\
1 \\
6\end{array}$ & $\begin{array}{l}1 \\
1 \\
1 \\
1 \\
1\end{array}$ \\
\hline $\begin{array}{l}\text { ST } \\
\text { ST } \\
\text { ST } \\
\text { ST } \\
\text { ST }\end{array}$ & $\begin{array}{l}11 \\
12 \\
13 \\
14 \\
15\end{array}$ & $\begin{array}{l}- \\
- \\
- \\
-\end{array}$ & $\begin{array}{r}55300 \\
85500 \\
72900 \\
48800\end{array}$ & $\begin{array}{l}170.0 \\
190.0 \\
230.0 \\
120.0 \\
120.0\end{array}$ & $\begin{array}{l}- \\
- \\
- \\
-\end{array}$ & $\begin{array}{l}- \\
- \\
- \\
-\end{array}$ & $\begin{array}{l}77.0 \\
77.0 \\
76.0 \\
52.0 \\
43.0\end{array}$ & $\begin{array}{r}42.5 \\
13.9 \\
102.3 \\
-\end{array}$ & $\begin{array}{l}1 \\
1 \\
0 \\
1 \\
1\end{array}$ & $\begin{array}{l}3 \\
3 \\
3 \\
3 \\
3\end{array}$ & $\begin{array}{l}1 \\
1 \\
6 \\
3 \\
3\end{array}$ & $\begin{array}{l}1 \\
1 \\
1 \\
3 \\
3\end{array}$ \\
\hline $\begin{array}{l}\text { ST } \\
\text { ST } \\
\text { ST } \\
\text { ST } \\
\text { ST }\end{array}$ & $\begin{array}{l}16 \\
17 \\
18 \\
19 \\
20\end{array}$ & $\begin{array}{l}- \\
- \\
- \\
-\end{array}$ & $\begin{array}{r}37100 \\
218300 \\
72800 \\
70400 \\
69200\end{array}$ & $\begin{array}{l}175.0 \\
200.0 \\
160.0 \\
170.0 \\
150.0\end{array}$ & $\begin{array}{l}- \\
- \\
-\end{array}$ & $\begin{array}{r}- \\
150 . \overline{0} \\
160.0 \\
140.0\end{array}$ & $\begin{array}{l}75.0 \\
73.0 \\
63.0 \\
65.0 \\
67.0\end{array}$ & $\begin{array}{r}38.7 \\
25.5 \\
- \\
- \\
-\end{array}$ & $\begin{array}{l}1 \\
1 \\
1 \\
1 \\
1\end{array}$ & $\begin{array}{l}3 \\
3 \\
3 \\
3 \\
3\end{array}$ & $\begin{array}{l}1 \\
6 \\
1 \\
1 \\
1\end{array}$ & $\begin{array}{l}1 \\
1 \\
8 \\
8 \\
7\end{array}$ \\
\hline $\begin{array}{l}\text { ST } \\
\text { ST } \\
\text { ST } \\
\text { ST } \\
\text { ST }\end{array}$ & $\begin{array}{l}21 \\
22 \\
23 \\
24 \\
25\end{array}$ & $\begin{array}{l}- \\
\overline{-} \\
-\end{array}$ & $\begin{array}{r}68400 \\
42100 \\
74700 \\
71200 \\
199600\end{array}$ & $\begin{array}{r}150.0 \\
150 . \varnothing \\
135.0 \\
205.0 \\
-\end{array}$ & $\begin{array}{r}\overline{-} \\
\overline{-} \\
170.0\end{array}$ & $\begin{array}{r}140 . \bar{\emptyset} \\
195 . \bar{\varnothing} \\
-\end{array}$ & $\begin{array}{l}69.0 \\
74.0 \\
76.0 \\
68.0 \\
70.0\end{array}$ & $\begin{array}{l}\text { - } \\
\text { - } \\
\text { - }\end{array}$ & $\begin{array}{l}1 \\
1 \\
1 \\
1 \\
1\end{array}$ & $\begin{array}{l}3 \\
3 \\
3 \\
3 \\
3\end{array}$ & $\begin{array}{l}1 \\
1 \\
1 \\
1 \\
1\end{array}$ & $\begin{array}{l}0 \\
7 \\
1 \\
9 \\
1\end{array}$ \\
\hline $\begin{array}{l}\text { ST } \\
\text { ST } \\
\text { ST } \\
\text { ST } \\
\text { ST }\end{array}$ & $\begin{array}{l}26 \\
28 \\
29 \\
30 \\
31\end{array}$ & $\begin{array}{l}- \\
- \\
- \\
-\end{array}$ & $\begin{array}{r}154700 \\
71600 \\
79400 \\
76700 \\
59600\end{array}$ & $\begin{array}{r}140.0 \\
50.0 \\
60.0 \\
60.0 \\
130.0\end{array}$ & $\begin{array}{l}- \\
\overline{-} \\
\overline{-}\end{array}$ & $\begin{array}{r}\overline{-} \\
36 . \bar{g} \\
-\end{array}$ & $\begin{array}{r}67.0 \\
30 . \varnothing \\
35 . \varnothing \\
34 . \bar{\varnothing}\end{array}$ & $\begin{array}{r}3.8 \\
- \\
- \\
-\end{array}$ & $\begin{array}{l}1 \\
1 \\
1 \\
1 \\
1\end{array}$ & $\begin{array}{l}3 \\
3 \\
3 \\
3 \\
3\end{array}$ & $\begin{array}{l}1 \\
1 \\
1 \\
1 \\
1\end{array}$ & $\begin{array}{l}7 \\
5 \\
7 \\
1 \\
5\end{array}$ \\
\hline $\begin{array}{l}\text { ST } \\
\text { ST } \\
\text { ST } \\
\text { ST } \\
\text { ST }\end{array}$ & $\begin{array}{l}32 \\
33 \\
34 \\
35 \\
36\end{array}$ & $\begin{array}{l}- \\
- \\
-\end{array}$ & $\begin{array}{l}81900 \\
61900 \\
44000 \\
85000 \\
71600\end{array}$ & $\begin{array}{r}85.0 \\
95.0 \\
95.0 \\
85.0 \\
105.0\end{array}$ & $\begin{array}{l}- \\
- \\
-\end{array}$ & $\begin{array}{l}- \\
- \\
-\end{array}$ & $\begin{array}{l}58.0 \\
55.0 \\
54 . \varnothing \\
58 . \varnothing \\
50 . \varnothing\end{array}$ & $\begin{array}{l}- \\
\overline{-} \\
\overline{-}\end{array}$ & $\begin{array}{l}1 \\
1 \\
1 \\
1 \\
1\end{array}$ & $\begin{array}{l}3 \\
3 \\
3 \\
3 \\
3\end{array}$ & $\begin{array}{l}1 \\
1 \\
1 \\
1 \\
1\end{array}$ & $\begin{array}{l}3 \\
8 \\
6 \\
8 \\
3\end{array}$ \\
\hline $\begin{array}{l}\text { ST } \\
\text { ST } \\
\text { ST } \\
\text { ST } \\
\text { ST }\end{array}$ & $\begin{array}{l}37 \\
38 \\
39 \\
46 \\
41\end{array}$ & $\begin{array}{l}- \\
- \\
- \\
-\end{array}$ & $\begin{array}{r}82300 \\
133000 \\
78400 \\
26300\end{array}$ & $\begin{array}{l}25.0 \\
17.0 \\
20.0 \\
20.0 \\
48.0\end{array}$ & $\begin{array}{l}- \\
- \\
-\end{array}$ & $\begin{array}{l}- \\
\overline{-} \\
- \\
-\end{array}$ & $\begin{array}{r}18.0 \\
2.0 \\
3.0 \\
2.0 \\
8.0\end{array}$ & $\begin{array}{l}\text { - } \\
\overline{-} \\
\text { - }\end{array}$ & $\begin{array}{l}1 \\
1 \\
1 \\
1 \\
1\end{array}$ & $\begin{array}{l}3 \\
3 \\
3 \\
3 \\
3\end{array}$ & $\begin{array}{l}1 \\
1 \\
1 \\
1 \\
6\end{array}$ & $\begin{array}{l}5 \\
1 \\
0 \\
0 \\
5\end{array}$ \\
\hline $\begin{array}{l}\text { ST } \\
\text { ST } \\
\text { ST } \\
\text { ST } \\
\text { ST }\end{array}$ & $\begin{array}{l}42 \\
43 \\
44 \\
45 \\
46\end{array}$ & $\begin{array}{l}- \\
\overline{-} \\
-\end{array}$ & $\begin{array}{r}71400 \\
136000 \\
403200 \\
146500 \\
250800\end{array}$ & $\begin{array}{l}25.0 \\
69.0 \\
45.0 \\
45.0 \\
30.0\end{array}$ & $\begin{array}{l}\overline{-} \\
\bar{z} \\
\overline{-}\end{array}$ & $\begin{array}{l}- \\
\overline{-} \\
-\end{array}$ & $\begin{array}{r}2.0 \\
28.0 \\
29.0 \\
35.0 \\
28 . \varnothing\end{array}$ & $\begin{array}{l}- \\
\overline{-} \\
-\end{array}$ & $\begin{array}{l}1 \\
1 \\
1 \\
1 \\
1\end{array}$ & $\begin{array}{l}3 \\
3 \\
3 \\
3 \\
3\end{array}$ & $\begin{array}{l}1 \\
1 \\
1 \\
1 \\
1\end{array}$ & $\begin{array}{l}0 \\
6 \\
3 \\
3 \\
3\end{array}$ \\
\hline $\begin{array}{l}\text { ST } \\
\text { ST } \\
\text { ST } \\
\text { ST } \\
\text { ST }\end{array}$ & $\begin{array}{l}47 \\
48 \\
49 \\
50 \\
51\end{array}$ & $\begin{array}{l}- \\
- \\
- \\
-\end{array}$ & $\begin{array}{l}43500 \\
30000 \\
23000 \\
28900 \\
26500\end{array}$ & $\begin{array}{l}35.0 \\
45.0 \\
50.0 \\
50.0 \\
55.0\end{array}$ & $\begin{array}{l}- \\
\overline{-} \\
-\end{array}$ & $\begin{array}{l}- \\
- \\
-\end{array}$ & $\begin{array}{l}26.0 \\
36 . \emptyset \\
41 . \emptyset \\
42 . \emptyset \\
44 . \emptyset\end{array}$ & $\begin{array}{l}- \\
- \\
- \\
-\end{array}$ & $\begin{array}{l}1 \\
1 \\
1 \\
1 \\
1\end{array}$ & $\begin{array}{l}3 \\
3 \\
3 \\
3 \\
3\end{array}$ & $\begin{array}{l}1 \\
1 \\
1 \\
1 \\
1\end{array}$ & $\begin{array}{l}5 \\
7 \\
1 \\
1 \\
2\end{array}$ \\
\hline $\begin{array}{l}\text { ST } \\
\text { ST } \\
\text { ST } \\
\text { ST } \\
\text { ST }\end{array}$ & $\begin{array}{l}52 \\
53 \\
54 \\
55 \\
56\end{array}$ & $\begin{array}{l}- \\
\overline{-} \\
-\end{array}$ & $\begin{array}{r}9350 \overline{0} \\
6460 \overline{0} \\
85500\end{array}$ & $\begin{array}{l}65.0 \\
60.0 \\
75.0 \\
78.0 \\
85.0\end{array}$ & $\begin{array}{l}- \\
\overline{-} \\
-\end{array}$ & $\begin{array}{l}- \\
- \\
-\end{array}$ & $\begin{array}{l}48 . \varnothing \\
51.0 \\
52 . \varnothing \\
51.0 \\
55.0\end{array}$ & $\begin{array}{r}\overline{-} \\
70 . \overline{1} \\
-\end{array}$ & $\begin{array}{l}1 \\
1 \\
1 \\
1 \\
1\end{array}$ & $\begin{array}{l}3 \\
3 \\
3 \\
3 \\
3\end{array}$ & $\begin{array}{l}1 \\
1 \\
1 \\
1 \\
1\end{array}$ & $\begin{array}{l}1 \\
7 \\
8 \\
2 \\
2\end{array}$ \\
\hline $\begin{array}{l}\text { ST } \\
\text { ST } \\
\text { ST } \\
\text { ST } \\
\text { ST }\end{array}$ & $\begin{array}{l}57 \\
58 \\
59 \\
60 \\
61\end{array}$ & $\begin{array}{l}- \\
- \\
-\end{array}$ & $\begin{array}{r}48000 \\
88400 \\
28600 \\
- \\
451800\end{array}$ & $\begin{array}{r}30 . \emptyset \\
35 . \varnothing \\
45 . \varnothing \\
165 . \varnothing \\
175 . \varnothing\end{array}$ & $\begin{array}{l}- \\
\overline{-} \\
-\end{array}$ & $\begin{array}{l}- \\
\overline{-} \\
- \\
-\end{array}$ & $\begin{array}{l}21 . \emptyset \\
28 . \emptyset \\
39 . \emptyset \\
78 . \emptyset \\
79 . \emptyset\end{array}$ & $\begin{array}{r}\overline{-} \\
\overline{-} \\
70 . \overline{3}\end{array}$ & $\begin{array}{l}1 \\
1 \\
1 \\
1 \\
1\end{array}$ & $\begin{array}{l}3 \\
3 \\
3 \\
3 \\
3\end{array}$ & $\begin{array}{l}1 \\
1 \\
1 \\
1 \\
1\end{array}$ & $\begin{array}{l}7 \\
7 \\
1 \\
2 \\
2\end{array}$ \\
\hline
\end{tabular}


Appendix.--Location and basic physical features of recharge basins on Long Island,

\begin{tabular}{|c|c|c|c|c|c|c|c|c|}
\hline \multicolumn{2}{|c|}{$\begin{array}{l}\text { BASIN } \\
\text { NUMBER }\end{array}$} & \multicolumn{2}{|c|}{ NEAREST INTERSECTION } & $\begin{array}{l}\text { LATITUDE } \\
\text { LONGITUDE } \\
0, " 0, \text { " }\end{array}$ & $\begin{array}{l}\text { DATE } \\
\text { BUILT } \\
\text { YrMOD }\end{array}$ & COMMUNITY & $\begin{array}{r}\text { DESIGN } \\
\text { CAPACITY } \\
\text { (cubic }\end{array}$ & $\begin{array}{l}\text { ACTUAL } \\
\text { CAPACITY } \\
\text { feet) }\end{array}$ \\
\hline $\begin{array}{l}\text { ST } \\
\text { ST } \\
\text { ST } \\
\text { ST } \\
\text { ST }\end{array}$ & $\begin{array}{l}63 \\
64 \\
65 \\
86 \\
67\end{array}$ & $\begin{array}{l}\text { WOODBURY ROAD } \\
\text { PLAINVIEW ROAD } \\
\text { SUNNYSIDE BLVD. } \\
\text { WASHINGTON AVE. } \\
\text { ROUND SWAMP ROAD }\end{array}$ & $\begin{array}{l}\text { L.I.E. } \\
\text { L.I.E. } \\
\text { L.I.E. } \\
\text { L.I.E. } \\
\text { L.I.E. }\end{array}$ & $\begin{array}{l}4 \varnothing 4753 \emptyset 732852 \\
4 \emptyset 4749 \varnothing 732849 \\
4 \varnothing 4735 \emptyset 7328 \emptyset 1 \\
4 \emptyset 4714 \varnothing 732734 \\
4 \emptyset 4714 \varnothing 732644\end{array}$ & $\begin{array}{l}- \\
- \\
- \\
-\end{array}$ & $\begin{array}{l}128 \\
122 \\
122 \\
122 \\
148\end{array}$ & $\begin{array}{l}- \\
\overline{-} \\
\overline{-}\end{array}$ & $\begin{array}{l}- \\
- \\
-\end{array}$ \\
\hline $\begin{array}{l}\text { ST } \\
\text { ST } \\
\text { ST } \\
\text { ST } \\
\text { ST }\end{array}$ & $\begin{array}{l}68 \\
69 \\
70 \\
71 \\
72\end{array}$ & $\begin{array}{l}\text { BROADWAY } \\
\text { EAST NORWICH RD. } \\
\text { BROOKVILLE ROAD } \\
\text { CHAPIN ROAD } \\
\text { CENTRAL PARK RD. }\end{array}$ & $\begin{array}{l}\text { JERICHO TURNPIKE } \\
\text { JERICHO TURNPIKE } \\
\text { JERICHO-O.B. RD. } \\
\text { SEAFORD O.B.EXP. } \\
\text { SYDNEY STREET }\end{array}$ & 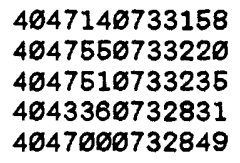 & $\begin{array}{l}- \\
\overline{-} \\
\overline{-}\end{array}$ & $\begin{array}{r}105 \\
105 \\
115 \\
92 \\
122\end{array}$ & $\begin{array}{l}- \\
- \\
- \\
-\end{array}$ & $\begin{array}{l}- \\
- \\
- \\
-\end{array}$ \\
\hline $\begin{array}{l}\text { ST } \\
\text { ST } \\
\text { ST } \\
\text { ST } \\
\text { ST }\end{array}$ & $\begin{array}{l}73 \\
74 \\
75 \\
76 \\
78\end{array}$ & $\begin{array}{l}\text { SEAFORD } 0 . B . \text { EXP. } \\
\text { SEAFORD } 0 . B \text {. EXP. } \\
\text { SEAFORD } 0 . B \text {. EXP. } \\
\text { CARNATION AVENUE } \\
\text { WALT WHITMAN RD. }\end{array}$ & $\begin{array}{l}\text { SOUTHERN ST. PKY } \\
\text { PHIPPS LANE } \\
\text { L.I.E. } \\
\text { LILY STREET } \\
\text { L.I.E. }\end{array}$ & $\begin{array}{l}4 \varnothing 472 \varnothing \varnothing 732913 \\
4 \varnothing 4651 \varnothing 732904 \\
4 \varnothing 4812 \varnothing 732912 \\
4 \varnothing 4328 \varnothing 734237 \\
4 \varnothing 465 \varnothing \varnothing 732533\end{array}$ & $\begin{array}{l}- \\
\overline{-} \\
\overline{-}\end{array}$ & $\begin{array}{r}46 \\
122 \\
128 \\
55 \\
148\end{array}$ & $\begin{array}{l}- \\
- \\
- \\
-\end{array}$ & $\begin{array}{l}- \\
- \\
- \\
-\end{array}$ \\
\hline $\begin{array}{l}\text { ST } \\
\text { ST } \\
\text { ST } \\
\text { ST } \\
\text { ST }\end{array}$ & $\begin{array}{l}79 \\
80 \\
81 \\
82 \\
83\end{array}$ & $\begin{array}{l}\text { OLD EAST NECK RD } \\
\text { UPPER HALF HOL. } \\
\text { WOODSEND ROAD } \\
\text { DEER PARK AVENUE } \\
\text { CARL'S STRAIGHT }\end{array}$ & $\begin{array}{l}\text { L.I.E. } \\
\text { L.I.E. } \\
\text { L.I.E. } \\
\text { L.I.E. } \\
\text { L.I.E. }\end{array}$ & $\begin{array}{l}4 \varnothing 47 \varnothing \varnothing \varnothing 732358 \\
4 \varnothing 4738 \varnothing 73222 \varnothing \\
4 \varnothing 4728 \varnothing 732129 \\
4 \varnothing 4737 \varnothing 732 \varnothing 39 \\
4 \varnothing 4752 \varnothing 731918\end{array}$ & $\begin{array}{l}- \\
\overline{-} \\
\overline{-}\end{array}$ & $\begin{array}{l}148 \\
143 \\
133 \\
133 \\
133\end{array}$ & $\begin{array}{l}- \\
- \\
-\end{array}$ & $\begin{array}{l}- \\
- \\
-\end{array}$ \\
\hline $\begin{array}{l}\text { ST } \\
\text { ST } \\
\text { ST } \\
\text { ST } \\
\text { ST }\end{array}$ & $\begin{array}{l}84 \\
87 \\
88 \\
89 \\
99\end{array}$ & $\begin{array}{l}\text { CARL'S STRAIGHT } \\
\text { WILLIAM FLOYD PK } \\
\text { LAMBERT AVENUE } \\
\text { MORICHES-YAP.RD. } \\
\text { JERUSLAEM HOLLOW }\end{array}$ & $\begin{array}{l}\text { L.I.E. } \\
\text { SUNRISE HIGHWAY } \\
\text { SUNRISE HIGHWAY } \\
\text { SUNRISE HIGHWAY } \\
\text { SUNRISE HIGHWAY }\end{array}$ & $\begin{array}{l}4 \varnothing 4752 \varnothing 731911 \\
4 \varnothing 4866 \varnothing 725217 \\
4 \varnothing 4843 \varnothing 7251 \varnothing 1 \\
4 \varnothing 4918 \varnothing 724928 \\
4 \varnothing 4921 \varnothing 724845\end{array}$ & $\begin{array}{l}- \\
- \\
-\end{array}$ & $\begin{array}{l}133 \\
230 \\
230 \\
217 \\
217\end{array}$ & $\begin{array}{l}- \\
- \\
-\end{array}$ & $\begin{array}{l}- \\
- \\
- \\
-\end{array}$ \\
\hline $\begin{array}{l}\text { ST } \\
\text { ST } \\
\text { ST } \\
\text { ST } \\
\text { ST }\end{array}$ & $\begin{array}{l}91 \\
92 \\
93 \\
94 \\
95\end{array}$ & $\begin{array}{l}\text { COZINE (WINES) RD. } \\
\text { E.MORICHES-MANOR } \\
\text { E.MORICHES-RIVER } \\
\text { RIVERHEAD-HAMP.R } \\
\text { WASHINGTON HTS. }\end{array}$ & $\begin{array}{l}\text { SUNRISE HIGHWAY } \\
\text { SUNRISE HIGHWAY } \\
\text { SUNRISE HIGHWAY } \\
\text { MONTAUK HIGHWAY } \\
\text { RIVERHEAD ROAD }\end{array}$ & $\begin{array}{l}4 \varnothing 492 \varnothing \varnothing 724749 \\
4 \varnothing 4928 \varnothing 72464 \varnothing \\
4 \varnothing 4956 \varnothing 7245 \varnothing \emptyset \\
4 \varnothing 5231 \varnothing 723136 \\
4 \varnothing 53 \varnothing 8 \emptyset 7231 \varnothing 1\end{array}$ & $\begin{array}{l}- \\
- \\
-\end{array}$ & $\begin{array}{l}217 \\
220 \\
220 \\
293 \\
293\end{array}$ & $\begin{array}{l}- \\
\overline{-} \\
-\end{array}$ & $\begin{array}{l}- \\
- \\
- \\
-\end{array}$ \\
\hline $\begin{array}{l}\text { ST } \\
\text { ST } \\
\text { ST } \\
\text { ST } \\
\text { ST }\end{array}$ & $\begin{array}{r}96 \\
97 \\
98 \\
99 \\
180\end{array}$ & $\begin{array}{l}\text { BENNETTS ROAD } \\
\text { BENNETTS ROAD } \\
\text { RIDGEWAY AVENUE } \\
\text { CROOKED HILL RD. } \\
\text { WICKS ROAD }\end{array}$ & $\begin{array}{l}\text { NORTH COUNTRY RD } \\
\text { NORTH COUNTRY RD } \\
\text { MAIN STREET } \\
\text { L.I.E. } \\
\text { L.I.E. }\end{array}$ & 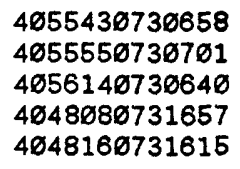 & $\begin{array}{l}- \\
- \\
-\end{array}$ & $\begin{array}{l}254 \\
254 \\
223 \\
189 \\
189\end{array}$ & $\begin{array}{l}- \\
- \\
- \\
-\end{array}$ & $\begin{array}{l}- \\
- \\
-\end{array}$ \\
\hline $\begin{array}{l}\text { ST } \\
\text { ST } \\
\text { ST } \\
\text { ST } \\
\text { ST }\end{array}$ & $\begin{array}{l}101 \\
103 \\
104 \\
106 \\
107\end{array}$ & $\begin{array}{l}\text { WICKS ROAD } \\
\text { WINFIELD AVENUE } \\
\text { OLD WILLETS PATH } \\
\text { MOTOR PARKWAY } \\
\text { VETERANS MEM. HWY }\end{array}$ & $\begin{array}{l}\text { L.I.E. } \\
\text { L.I.E. } \\
\text { L.I.E. } \\
\text { L.I.E. } \\
\text { L.I.E. }\end{array}$ & $\begin{array}{l}4048160731541 \\
4048170731441 \\
40483 \varnothing 0731422 \\
4 \varnothing 48290731134 \\
404836 \varnothing 731108\end{array}$ & $\begin{array}{l}- \\
- \\
- \\
-\end{array}$ & $\begin{array}{l}189 \\
189 \\
189 \\
196 \\
196\end{array}$ & $\begin{array}{l}- \\
- \\
- \\
-\end{array}$ & $\begin{array}{l}- \\
\overline{-} \\
\overline{-}\end{array}$ \\
\hline $\begin{array}{l}\text { ST } \\
\text { ST } \\
\text { ST } \\
\text { ST } \\
\text { ST }\end{array}$ & $\begin{array}{l}108 \\
109 \\
110 \\
111 \\
112\end{array}$ & $\begin{array}{l}\text { VETERANS MEM.HWY } \\
\text { SEAFORD } 0 . B \text {.EXP. } \\
\text { SEAFORD } 0 . B \text {.EXP. } \\
\text { SEAFORD } 0 . B \text {.EXP. } \\
\text { SEAFORD } 0 . B \text {.EXP. }\end{array}$ & $\begin{array}{l}\text { L.I.E. } \\
\text { HEMPSTEAD TPKE. } \\
\text { CENTRAL AVENUE } \\
\text { CENTRAL AVENUE } \\
\text { L.I.R.R. }\end{array}$ & $\begin{array}{l}4 \varnothing 4828 \varnothing 731 \emptyset 47 \\
4 \varnothing 4319 \varnothing 732829 \\
4 \varnothing 441 \varnothing 6732828 \\
4 \varnothing 4418 \varnothing 732825 \\
4 \varnothing 4416 \varnothing 732832\end{array}$ & $\begin{array}{l}- \\
- \\
-\end{array}$ & $\begin{array}{r}196 \\
121 \\
92 \\
92 \\
92\end{array}$ & $\begin{array}{l}- \\
- \\
-\end{array}$ & $\begin{array}{l}- \\
- \\
- \\
-\end{array}$ \\
\hline $\begin{array}{l}\text { ST } \\
\text { ST } \\
\text { ST } \\
\text { ST } \\
\text { ST }\end{array}$ & $\begin{array}{l}113 \\
114 \\
115 \\
116 \\
117\end{array}$ & $\begin{array}{l}\text { SEAFORD } 0 . B \text {. EXP. } \\
\text { SEAFORD } 0 . B \text {. EXP. } \\
\text { SEAFORD } 0 . B \text {. EXP. } \\
\text { NEWBRIDGE ROAD } \\
\text { DALY ROAD }\end{array}$ & $\begin{array}{l}\text { GILDO PLACE } \\
\text { HAY PATH ROAD } \\
\text { PLAINVIEW ROAD } \\
\text { SOUTHERN ST. PKY } \\
\text { JERICHO TURNPIKE }\end{array}$ & $\begin{array}{l}4 \emptyset 45 \varnothing 1 \varnothing 732833 \\
4 \emptyset 4534 \varnothing 732836 \\
4 \varnothing 4538 \varnothing 732832 \\
4 \varnothing 4146 \varnothing 733245 \\
4 \varnothing 5 \varnothing 17 \varnothing 731929\end{array}$ & $\begin{array}{l}- \\
- \\
-\end{array}$ & $\begin{array}{r}92 \\
122 \\
92 \\
32 \\
139\end{array}$ & $\begin{array}{l}- \\
- \\
-\end{array}$ & $\begin{array}{l}- \\
\overline{-} \\
\overline{-}\end{array}$ \\
\hline $\begin{array}{l}\text { ST } \\
\text { ST } \\
\text { ST } \\
\text { ST } \\
\text { ST }\end{array}$ & $\begin{array}{l}118 \\
119 \\
128 \\
121 \\
122\end{array}$ & $\begin{array}{l}\text { MAGNOLIA DRIVE } \\
\text { DEER PARK AVENUE } \\
\text { BROAD HOLLOW RD. } \\
\text { FULTON ROAD } \\
\text { BURR'S LANE }\end{array}$ & $\begin{array}{l}\text { JERICHO TURNPIKE } \\
\text { EASTON AVENUE } \\
\text { CARMANS ROAD } \\
\text { CARMAN ROAD } \\
\text { L.I.E. }\end{array}$ & $\begin{array}{l}4 \varnothing 5147 \varnothing 73 \varnothing 245 \\
4 \varnothing 4426 \varnothing 731921 \\
4 \varnothing 4354 \varnothing 732532 \\
4 \varnothing 4332 \varnothing 732552 \\
4 \varnothing 4729 \varnothing 7322 \varnothing 3\end{array}$ & $\begin{array}{l}- \\
- \\
- \\
-\end{array}$ & $\begin{array}{r}244 \\
159 \\
99 \\
99 \\
143\end{array}$ & $\begin{array}{l}- \\
- \\
- \\
-\end{array}$ & $\begin{array}{l}- \\
\overline{-} \\
-\end{array}$ \\
\hline
\end{tabular}


N.Y. [Dash indicates no data. Altitudes are in feet above sea level.] (cont.)

\begin{tabular}{|c|c|c|c|c|c|c|c|c|c|c|c|c|}
\hline \multicolumn{2}{|c|}{$\begin{array}{l}\text { BASIN } \\
\text { NUMBER }\end{array}$} & $\begin{array}{l}\text { MAX. } \\
\text { AREA } \\
\text { (squar }\end{array}$ & $\begin{array}{c}\text { BASIN } \\
\text { AREA } \\
\text { feet) }\end{array}$ & RIM & $\begin{array}{l}--A L T I \\
\text { OVER- } \\
\text { FLOW }\end{array}$ & $\begin{array}{l}\text { BOT-- } \\
\text { TOM }\end{array}$ & $\begin{array}{l}\text { WATER } \\
\text { TABLE }\end{array}$ & $\begin{array}{c}\text { DRAIN. } \\
\text { AREA } \\
\text { (acres) }\end{array}$ & STATL & $\begin{array}{l}\text { IN-- } \\
\text { USE }\end{array}$ & $\begin{array}{l}\text { GEO. } \\
\text { UNIT }\end{array}$ & $\begin{array}{l}\text { SOIL } \\
\text { UNIT }\end{array}$ \\
\hline $\begin{array}{l}\text { ST } \\
\text { ST } \\
\text { ST } \\
\text { ST } \\
\text { ST }\end{array}$ & $\begin{array}{l}63 \\
64 \\
65 \\
66 \\
67\end{array}$ & $\begin{array}{l}- \\
- \\
-\end{array}$ & $\begin{array}{r}224800 \\
87500 \\
- \\
243000 \\
390000\end{array}$ & $\begin{array}{l}185.0 \\
190.0 \\
195.0 \\
190.0 \\
205.0\end{array}$ & $\begin{array}{l}- \\
- \\
- \\
-\end{array}$ & $\begin{array}{r}79.0 \\
- \\
= \\
-\end{array}$ & $\begin{array}{l}80.0 \\
77.0 \\
76.0 \\
74.0\end{array}$ & $\begin{array}{r}112.5 \\
-\overline{8} \\
46.1 \\
-\end{array}$ & $\begin{array}{l}1 \\
1 \\
1 \\
1 \\
1\end{array}$ & $\begin{array}{l}3 \\
3 \\
3 \\
3 \\
3\end{array}$ & $\begin{array}{l}1 \\
1 \\
8 \\
8 \\
1\end{array}$ & $\begin{array}{l}1 \\
2 \\
1 \\
1 \\
1\end{array}$ \\
\hline $\begin{array}{l}\text { ST } \\
\text { ST } \\
\text { ST } \\
\text { ST } \\
\text { ST }\end{array}$ & $\begin{array}{l}68 \\
69 \\
70 \\
71 \\
72\end{array}$ & $\begin{array}{l}- \\
\overline{-} \\
\overline{-}\end{array}$ & $\begin{array}{r}6 \varnothing 300 \\
55200 \\
79900 \\
445300 \\
78000\end{array}$ & $\begin{array}{r}200.0 \\
210.0 \\
220.0 \\
82.0 \\
160.0\end{array}$ & $\begin{array}{l}- \\
- \\
- \\
-\end{array}$ & $\begin{array}{l}- \\
- \\
- \\
-\end{array}$ & $\begin{array}{l}76.0 \\
75.0 \\
75.0 \\
60.0 \\
81.0\end{array}$ & $\begin{array}{r}- \\
\overline{-} \\
\overline{-} \\
-\end{array}$ & $\begin{array}{l}1 \\
1 \\
1 \\
1 \\
1\end{array}$ & $\begin{array}{l}3 \\
3 \\
3 \\
3 \\
3\end{array}$ & $\begin{array}{l}1 \\
6 \\
6 \\
1 \\
1\end{array}$ & $\begin{array}{l}1 \\
1 \\
1 \\
1 \\
2\end{array}$ \\
\hline $\begin{array}{l}\text { ST } \\
\text { ST } \\
\text { ST } \\
\text { ST } \\
\text { ST }\end{array}$ & $\begin{array}{l}73 \\
74 \\
75 \\
76 \\
78\end{array}$ & $\begin{array}{l}- \\
- \\
- \\
-\end{array}$ & $\begin{array}{r}102000 \\
68900 \\
125100 \\
50000 \\
124800\end{array}$ & $\begin{array}{r}175.0 \\
160.0 \\
200.0 \\
91.0 \\
115.0\end{array}$ & $\begin{array}{l}- \\
- \\
- \\
-\end{array}$ & $\begin{array}{l}- \\
- \\
- \\
-\end{array}$ & $\begin{array}{l}82.0 \\
82.0 \\
77.0 \\
25.0 \\
70.0\end{array}$ & $\begin{array}{r}25.9 \\
61.4 \\
- \\
-\end{array}$ & $\begin{array}{l}1 \\
1 \\
1 \\
1 \\
0\end{array}$ & $\begin{array}{l}3 \\
3 \\
3 \\
3 \\
3\end{array}$ & $\begin{array}{l}1 \\
1 \\
1 \\
1 \\
1\end{array}$ & $\begin{array}{l}2 \\
2 \\
2 \\
1 \\
3\end{array}$ \\
\hline $\begin{array}{l}\text { ST } \\
\text { ST } \\
\text { ST } \\
\text { ST } \\
\text { ST }\end{array}$ & $\begin{array}{l}79 \\
80 \\
81 \\
82 \\
83\end{array}$ & $\begin{array}{l}- \\
\overline{-} \\
\overline{-}\end{array}$ & $\begin{array}{r}80000 \\
223100 \\
99000 \\
130000 \\
-\end{array}$ & $\begin{array}{l}125.0 \\
200.0 \\
180.0 \\
125.0 \\
130.0\end{array}$ & $\begin{array}{l}- \\
- \\
- \\
-\end{array}$ & $\begin{array}{l}- \\
- \\
- \\
-\end{array}$ & $\begin{array}{l}68 . \varnothing \\
62 . \varnothing \\
62 . \varnothing \\
60.0 \\
59 . \varnothing\end{array}$ & $\begin{array}{l}- \\
- \\
- \\
-\end{array}$ & $\begin{array}{l}1 \\
1 \\
1 \\
1 \\
1\end{array}$ & $\begin{array}{l}3 \\
3 \\
3 \\
3 \\
3\end{array}$ & $\begin{array}{l}1 \\
1 \\
8 \\
1 \\
1\end{array}$ & $\begin{array}{l}1 \\
1 \\
5 \\
1 \\
1\end{array}$ \\
\hline $\begin{array}{l}\text { ST } \\
\text { ST } \\
\text { ST } \\
\text { ST } \\
\text { ST }\end{array}$ & $\begin{array}{l}84 \\
87 \\
88 \\
89 \\
90\end{array}$ & $\begin{array}{l}- \\
- \\
- \\
-\end{array}$ & $\begin{array}{r}- \\
160500 \\
92100 \\
222000 \\
226200\end{array}$ & $\begin{array}{r}130.0 \\
55.0 \\
40.0 \\
55.0 \\
40.0\end{array}$ & $\begin{array}{l}- \\
- \\
- \\
-\end{array}$ & $\begin{array}{l}- \\
- \\
- \\
-\end{array}$ & $\begin{array}{l}59.0 \\
16.0 \\
23.0 \\
20.0 \\
20.0\end{array}$ & $\begin{array}{l}- \\
- \\
- \\
-\end{array}$ & $\begin{array}{l}1 \\
1 \\
1 \\
1 \\
1\end{array}$ & $\begin{array}{l}3 \\
3 \\
3 \\
3 \\
3\end{array}$ & $\begin{array}{l}1 \\
1 \\
1 \\
1 \\
1\end{array}$ & $\begin{array}{l}1 \\
3 \\
3 \\
5 \\
5\end{array}$ \\
\hline $\begin{array}{l}\text { ST } \\
\text { ST } \\
\text { ST } \\
\text { ST } \\
\text { ST }\end{array}$ & $\begin{array}{l}91 \\
92 \\
93 \\
94 \\
95\end{array}$ & $\begin{array}{l}- \\
- \\
- \\
-\end{array}$ & $\begin{array}{r}129000 \\
120000 \\
311300 \\
57600 \\
72400\end{array}$ & $\begin{array}{l}60.0 \\
50.0 \\
45.0 \\
40.0 \\
95.0\end{array}$ & $\begin{array}{l}- \\
- \\
- \\
-\end{array}$ & $\begin{array}{l}- \\
- \\
- \\
-\end{array}$ & $\begin{array}{r}22.0 \\
26.0 \\
31.0 \\
3.0 \\
2.0\end{array}$ & $\begin{array}{l}- \\
- \\
- \\
-\end{array}$ & $\begin{array}{l}1 \\
1 \\
1 \\
1 \\
1\end{array}$ & $\begin{array}{l}3 \\
3 \\
3 \\
3 \\
3\end{array}$ & $\begin{array}{l}1 \\
1 \\
1 \\
6 \\
6\end{array}$ & $\begin{array}{l}6 \\
3 \\
1 \\
5 \\
5\end{array}$ \\
\hline $\begin{array}{l}\text { ST } \\
\text { ST } \\
\text { ST } \\
\text { ST } \\
\text { ST }\end{array}$ & $\begin{array}{r}96 \\
97 \\
98 \\
99 \\
100\end{array}$ & $\begin{array}{l}- \\
- \\
- \\
-\end{array}$ & $\begin{array}{r}136000 \\
105000 \\
96000 \\
400000 \\
100000\end{array}$ & $\begin{array}{r}50.0 \\
40.0 \\
80.0 \\
140.0 \\
135.0\end{array}$ & $\begin{array}{l}- \\
- \\
- \\
-\end{array}$ & $\begin{array}{l}- \\
- \\
- \\
-\end{array}$ & $\begin{array}{l}30.0 \\
30.0 \\
25.0 \\
50.0 \\
41.0\end{array}$ & $\begin{array}{l}- \\
- \\
- \\
-\end{array}$ & $\begin{array}{l}0 \\
1 \\
1 \\
1 \\
1\end{array}$ & $\begin{array}{l}3 \\
3 \\
3 \\
3 \\
3\end{array}$ & $\begin{array}{l}3 \\
3 \\
3 \\
1 \\
1\end{array}$ & $\begin{array}{l}7 \\
7 \\
3 \\
1 \\
7\end{array}$ \\
\hline $\begin{array}{l}\text { ST } \\
\text { ST } \\
\text { ST } \\
\text { ST } \\
\text { ST }\end{array}$ & $\begin{array}{l}101 \\
103 \\
104 \\
106 \\
107\end{array}$ & $\begin{array}{l}- \\
- \\
- \\
-\end{array}$ & $\begin{array}{r}100000 \\
111800 \\
192000 \\
126000 \\
-\end{array}$ & $\begin{array}{l}145.0 \\
130.0 \\
140.0 \\
120.0 \\
125.0\end{array}$ & $\begin{array}{l}- \\
- \\
- \\
-\end{array}$ & $\begin{array}{l}- \\
- \\
- \\
-\end{array}$ & $\begin{array}{l}48.0 \\
46.0 \\
45.0 \\
39.0 \\
40.0\end{array}$ & $\begin{array}{l}- \\
- \\
- \\
-\end{array}$ & $\begin{array}{l}1 \\
1 \\
1 \\
1 \\
1\end{array}$ & $\begin{array}{l}3 \\
3 \\
3 \\
3 \\
3\end{array}$ & $\begin{array}{l}1 \\
1 \\
1 \\
6 \\
1\end{array}$ & $\begin{array}{l}7 \\
7 \\
3 \\
3 \\
1\end{array}$ \\
\hline $\begin{array}{l}\text { ST } \\
\text { ST } \\
\text { ST } \\
\text { ST } \\
\text { ST }\end{array}$ & $\begin{array}{l}108 \\
109 \\
110 \\
111 \\
112\end{array}$ & $\begin{array}{l}- \\
- \\
- \\
-\end{array}$ & $\begin{array}{l}114800 \\
116400 \\
176200 \\
226000 \\
161200\end{array}$ & $\begin{array}{l}85.0 \\
80.0 \\
80.0 \\
85.0 \\
90.0\end{array}$ & $\begin{array}{l}- \\
- \\
- \\
-\end{array}$ & $\begin{array}{l}- \\
- \\
- \\
-\end{array}$ & $\begin{array}{l}38.0 \\
53.0 \\
60.0 \\
60.0 \\
60.0\end{array}$ & $\begin{array}{r}-\overline{3} \\
7.8 \\
76.4 \\
72.1\end{array}$ & $\begin{array}{l}1 \\
1 \\
0 \\
0 \\
0\end{array}$ & $\begin{array}{l}3 \\
3 \\
3 \\
3 \\
3\end{array}$ & $\begin{array}{l}1 \\
1 \\
1 \\
1 \\
1\end{array}$ & $\begin{array}{l}1 \\
2 \\
6 \\
6 \\
1\end{array}$ \\
\hline $\begin{array}{l}\text { ST } \\
\text { ST } \\
\text { ST } \\
\text { ST } \\
\text { ST }\end{array}$ & $\begin{array}{l}113 \\
114 \\
115 \\
116 \\
117\end{array}$ & $\begin{array}{l}- \\
- \\
- \\
-\end{array}$ & $\begin{array}{r}422400 \\
162000 \\
235000 \\
80900 \\
65800\end{array}$ & $\begin{array}{r}95.0 \\
120.0 \\
115.0 \\
50.0 \\
155.0\end{array}$ & $\begin{array}{l}- \\
- \\
- \\
-\end{array}$ & $\begin{array}{l}- \\
- \\
- \\
-\end{array}$ & $\begin{array}{l}68 . \varnothing \\
73 . \varnothing \\
73.0 \\
32 . \varnothing \\
71.0\end{array}$ & $\begin{array}{r}269.5 \\
112.9 \\
81.0 \\
132.8 \\
-\end{array}$ & $\begin{array}{l}1 \\
1 \\
0 \\
1 \\
1\end{array}$ & $\begin{array}{l}3 \\
3 \\
3 \\
3 \\
3\end{array}$ & $\begin{array}{l}1 \\
1 \\
1 \\
1 \\
1\end{array}$ & $\begin{array}{l}1 \\
1 \\
1 \\
6 \\
7\end{array}$ \\
\hline $\begin{array}{l}\text { ST } \\
\text { ST } \\
\text { ST } \\
\text { ST } \\
\text { ST }\end{array}$ & $\begin{array}{l}118 \\
119 \\
120 \\
121 \\
122\end{array}$ & $\begin{array}{l}- \\
\overline{-} \\
\overline{-}\end{array}$ & $\begin{array}{r}90200 \\
53200 \\
153000 \\
90000 \\
107300\end{array}$ & $\begin{array}{r}105.0 \\
43.0 \\
70.0 \\
60.0 \\
210.0\end{array}$ & $\begin{array}{l}- \\
\overline{-} \\
\overline{-}\end{array}$ & $\begin{array}{l}- \\
- \\
- \\
-\end{array}$ & $\begin{array}{l}58 . \emptyset \\
36 . \varnothing \\
52 . \varnothing \\
51 . \varnothing \\
61 . \varnothing\end{array}$ & $\begin{array}{l}- \\
- \\
- \\
-\end{array}$ & $\begin{array}{l}1 \\
1 \\
1 \\
1 \\
1\end{array}$ & $\begin{array}{l}3 \\
3 \\
3 \\
3 \\
3\end{array}$ & $\begin{array}{l}1 \\
1 \\
1 \\
1 \\
6\end{array}$ & $\begin{array}{l}5 \\
7 \\
8 \\
1 \\
1\end{array}$ \\
\hline
\end{tabular}


Appendix.--Location and basic physical features of recharge basins on Long Island,

\begin{tabular}{|c|c|c|c|c|c|c|c|c|}
\hline \multicolumn{2}{|c|}{$\begin{array}{l}\text { BASIN } \\
\text { NUMBER }\end{array}$} & \multicolumn{2}{|c|}{ NEAREST INTERSECTION } & $\begin{array}{l}\text { LATITUDE } \\
\text { LONGITUDE } \\
\circ, " 0, n\end{array}$ & $\begin{array}{l}\text { DATE } \\
\text { BUILT } \\
\text { YrMOD }\end{array}$ & COMMUNITY & $\begin{array}{l}\text { DESIGN } \\
\text { CAPACITY } \\
\text { (cubic }\end{array}$ & $\begin{array}{l}\text { ACTUAL } \\
\text { CAPACITY } \\
\text { feet) }\end{array}$ \\
\hline $\begin{array}{l}\text { ST } \\
\text { ST } \\
\text { ST } \\
\text { ST } \\
\text { ST }\end{array}$ & $\begin{array}{l}123 \\
124 \\
125 \\
126 \\
127\end{array}$ & $\begin{array}{l}\text { E.MORICHES-RIVER } \\
\text { SEATUCK AVENUE } \\
\text { SEATUCK AVENUE } \\
\text { HAWKTON PLACE } \\
\text { PARK STREET }\end{array}$ & $\begin{array}{l}\text { EASTPORT MANOR R } \\
\text { OLD COUNTRY ROAD } \\
\text { L.I.R.R. } \\
\text { JERICHO TURNPIKE } \\
\text { KING STREET }\end{array}$ & 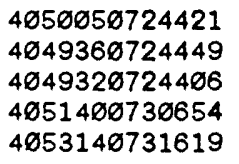 & $\begin{array}{l}- \\
- \\
- \\
-\end{array}$ & $\begin{array}{l}219 \\
219 \\
219 \\
216 \\
174\end{array}$ & $\begin{array}{l}- \\
\overline{-} \\
\overline{-}\end{array}$ & $\begin{array}{l}- \\
- \\
- \\
-\end{array}$ \\
\hline $\begin{array}{l}\text { ST } \\
\text { ST } \\
\text { ST } \\
\text { ST } \\
\text { ST }\end{array}$ & $\begin{array}{l}128 \\
129 \\
130 \\
131 \\
132\end{array}$ & $\begin{array}{l}\text { PARK AVENUE } \\
\text { HALES ROAD } \\
\text { MILL RIVER ROAD } \\
\text { OYSTER BAY ROAD } \\
\text { SPLIT ROCK ROAD }\end{array}$ & $\begin{array}{l}\text { JERICHO TURNPIKE } \\
\text { MAIN ROAD } \\
\text { REMSEN LANE } \\
\text { N.HEMPSTEAD TPK. } \\
\text { N.HEMPSTEAD TPK. }\end{array}$ & 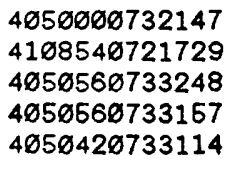 & $\begin{array}{l}- \\
\overline{-} \\
-\end{array}$ & $\begin{array}{r}133 \\
286 \\
98 \\
98 \\
98\end{array}$ & $\begin{array}{l}- \\
- \\
-\end{array}$ & $\begin{array}{l}- \\
- \\
- \\
-\end{array}$ \\
\hline $\begin{array}{l}\text { ST } \\
\text { ST } \\
\text { ST } \\
\text { ST } \\
\text { ST }\end{array}$ & $\begin{array}{l}133 \\
134 \\
135 \\
136 \\
137\end{array}$ & $\begin{array}{l}\text { SEAFORD } 0 . B . \text { EXP. } \\
\text { SEAFORD } 0 . B . \text { EXP. } \\
\text { SEAFORD } 0 . B . \text { EXP. } \\
\text { SEAFORD } 0 . B . \text { EXP. } \\
\text { SEAFORD } 0 . B . \text { EXP. }\end{array}$ & $\begin{array}{l}\text { JERUSALEM AVENUE } \\
\text { SOUTHERN ST. PKY } \\
\text { ARLINGTON DRIVE } \\
\text { KILDARE CRESCENT } \\
\text { KILDARE CRESCENT }\end{array}$ & $\begin{array}{l}4041300732944 \\
4 \varnothing 42130732920 \\
4042300732906 \\
4042400732904 \\
4042400732901\end{array}$ & $\begin{array}{l}- \\
\overline{-} \\
-\end{array}$ & $\begin{array}{r}38 \\
38 \\
110 \\
121 \\
121\end{array}$ & $\begin{array}{l}- \\
- \\
-\end{array}$ & $\begin{array}{l}- \\
- \\
- \\
-\end{array}$ \\
\hline $\begin{array}{l}\text { ST } \\
\text { ST } \\
\text { ST } \\
\text { ST } \\
\text { ST }\end{array}$ & $\begin{array}{l}138 \\
139 \\
14 \varnothing \\
141 \\
142\end{array}$ & $\begin{array}{l}\text { HALF HOLLOW ROAD } \\
\text { NICHOLS ROAD } \\
\text { NICHOLS ROAD } \\
\text { BREEZE AVENUE } \\
\text { OCEAN AVENUE }\end{array}$ & $\begin{array}{l}\text { L.I.E. } \\
\text { L.I.E. } \\
\text { L.I.E. } \\
\text { L.I.E. } \\
\text { L.I.E. }\end{array}$ & 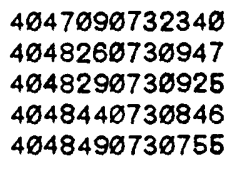 & - & $\begin{array}{l}143 \\
2 \varnothing \emptyset \\
2 ø 0 \\
252 \\
252\end{array}$ & $\begin{array}{l}- \\
- \\
- \\
-\end{array}$ & $\begin{array}{l}- \\
- \\
- \\
-\end{array}$ \\
\hline $\begin{array}{l}\text { ST } \\
\text { ST } \\
\text { ST } \\
\text { ST } \\
\text { ST }\end{array}$ & $\begin{array}{l}143 \\
144 \\
145 \\
14 \varepsilon \\
147\end{array}$ & $\begin{array}{l}\text { POND ROAD } \\
\text { RONKONKOMA AVE. } \\
\text { CARROLL AVENUE } \\
\text { PATCHOGUE-HOL.RD } \\
\text { HOLBROOK ROAD }\end{array}$ & $\begin{array}{l}\text { L.I.E. } \\
\text { L.I.E. } \\
\text { L.I.E. } \\
\text { L.I.E. } \\
\text { L.I.E. }\end{array}$ & 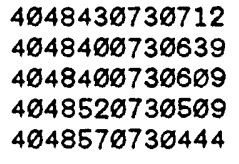 & $\begin{array}{l}- \\
- \\
- \\
-\end{array}$ & $\begin{array}{l}252 \\
252 \\
252 \\
252 \\
252\end{array}$ & $\begin{array}{l}\overline{-} \\
\overline{-} \\
-\end{array}$ & $\begin{array}{l}- \\
- \\
- \\
-\end{array}$ \\
\hline $\begin{array}{l}\text { ST } \\
\text { ST } \\
\text { ST } \\
\text { ST } \\
\text { ST }\end{array}$ & $\begin{array}{l}148 \\
149 \\
150 \\
151 \\
152\end{array}$ & $\begin{array}{l}\text { SAMPSON AVENUE } \\
\text { JERICHO-O.B. RD. } \\
\text { JERICHO-0.B. RD. } \\
\text { JERICHO-O.B. RD. } \\
\text { PORT JEFF-PATCH. }\end{array}$ & $\begin{array}{l}\text { L.I.E. } \\
\text { IRONWDOD ROAD } \\
\text { MUTTONTOWN ROAD } \\
\text { FARM HILL LANE } \\
\text { MIDDLE COUNTRY R }\end{array}$ & $\begin{array}{l}4 \varnothing 4823 \emptyset 730931 \\
4 \varnothing 4844 \varnothing 7332 \varnothing 1 \\
4 \varnothing 4946 \varnothing 733148 \\
4 \varnothing 5 \emptyset 14 \varnothing 73315 \emptyset \\
4 \varnothing 5214 \varnothing 730 \varnothing 15\end{array}$ & $\begin{array}{l}- \\
- \\
-\end{array}$ & $\begin{array}{l}290 \\
115 \\
115 \\
115 \\
218\end{array}$ & $\begin{array}{l}- \\
- \\
-\end{array}$ & $\begin{array}{l}- \\
- \\
-\end{array}$ \\
\hline $\begin{array}{l}\text { ST } \\
\text { ST } \\
\text { ST } \\
\text { ST } \\
\text { ST }\end{array}$ & $\begin{array}{l}153 \\
154 \\
155 \\
156 \\
157\end{array}$ & $\begin{array}{l}\text { SHIPYARD LANE } \\
\text { BROAD HOLLOW RD. } \\
\text { MAKAMAH ROAD } \\
\text { MEDFORD FORD } \\
\text { DEER PARK AVENUE }\end{array}$ & $\begin{array}{l}\text { MAIN ROAD } \\
\text { SMITH STREET } \\
\text { FORT SALONGA RD. } \\
\text { BARTON AVENUE } \\
\text { LONG ISLAND AVE. }\end{array}$ & $\begin{array}{l}4107100722057 \\
4 \varnothing 4527 \varnothing 732524 \\
4 \varnothing 54330731854 \\
4 \varnothing 47 \varnothing 8 \varnothing 73 \varnothing 831 \\
4 \varnothing 4542 \varnothing 731953\end{array}$ & $\begin{array}{l}- \\
\overline{-}\end{array}$ & $\begin{array}{r}278 \\
99 \\
171 \\
242 \\
159\end{array}$ & $\begin{array}{l}- \\
- \\
-\end{array}$ & $\begin{array}{l}- \\
- \\
-\end{array}$ \\
\hline $\begin{array}{l}\text { ST } \\
\text { ST } \\
\text { ST } \\
\text { ST } \\
\text { ST }\end{array}$ & $\begin{array}{l}168 \\
160 \\
161 \\
162 \\
163\end{array}$ & $\begin{array}{l}\text { DEER PARK AVENUE } \\
\text { HILLSIDE AVENUE } \\
\text { POST ROAD } \\
\text { HITCHCOCK LANE } \\
\text { POWELL LANE }\end{array}$ & $\begin{array}{l}\text { L.I.E. } \\
\text { JERICHO TURNPIKE } \\
\text { JERICHO TURNPIKE } \\
\text { JERICHO TURNPIKE } \\
\text { JERICHO TURNPIKE }\end{array}$ & $\begin{array}{l}4 \varnothing 47520732038 \\
4 \varnothing 4534 \varnothing 7337 \varnothing 1 \\
4 \varnothing 46 \varnothing \varnothing \varnothing 733531 \\
4 \varnothing 46 \varnothing 7 \varnothing 7335 \varnothing \emptyset \\
4 \varnothing 4622 \varnothing 733429\end{array}$ & $\begin{array}{l}- \\
- \\
-\end{array}$ & $\begin{array}{l}143 \\
118 \\
118 \\
118 \\
118\end{array}$ & $\begin{array}{l}- \\
- \\
-\end{array}$ & $\begin{array}{l}- \\
- \\
-\end{array}$ \\
\hline $\begin{array}{l}\text { ST } \\
\text { ST } \\
\text { ST } \\
\text { ST } \\
\text { ST }\end{array}$ & $\begin{array}{l}164 \\
165 \\
166 \\
167 \\
168\end{array}$ & $\begin{array}{l}\text { MAIN STREET } \\
\text { MAIN STREET } \\
\text { OLD TOWN ROAD } \\
\text { BENNETTS ROAD } \\
\text { MAIN STREET }\end{array}$ & $\begin{array}{l}\text { L.I.R.R. } \\
\text { L.I.R.R. } \\
\text { MAIN STREET } \\
\text { DETMER ROAD } \\
\text { HALLOCK AVENUE }\end{array}$ & 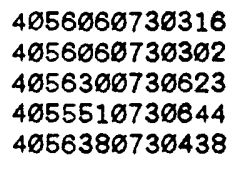 & $\begin{array}{l}- \\
\overline{-} \\
\overline{-}\end{array}$ & $\begin{array}{l}249 \\
218 \\
223 \\
223 \\
249\end{array}$ & $\begin{array}{l}- \\
- \\
-\end{array}$ & $\begin{array}{l}- \\
- \\
- \\
-\end{array}$ \\
\hline $\begin{array}{l}\text { ST } \\
\text { ST } \\
\text { ST } \\
\text { ST } \\
\text { ST }\end{array}$ & $\begin{array}{l}169 \\
170 \\
171 \\
172 \\
173\end{array}$ & $\begin{array}{l}\text { HORSE BLOCK ROAD } \\
\text { HORSE BLOCK ROAD } \\
\text { PATCHOGUE-YAP.RD } \\
\text { YAPHANK ROAD } \\
\text { MIDDLE ISLAND RD }\end{array}$ & $\begin{array}{l}\text { L.I.E. } \\
\text { L.I.E. } \\
\text { L.I.E. } \\
\text { L.I.E. } \\
\text { L.I.E. }\end{array}$ & $\begin{array}{l}4 \emptyset 4924 \emptyset 725851 \\
4 \emptyset 49140725812 \\
4 \emptyset 49420725602 \\
4 \varnothing 49530725502 \\
4 \varnothing 50110725416\end{array}$ & - & $\begin{array}{l}232 \\
232 \\
261 \\
261 \\
255\end{array}$ & $\begin{array}{l}- \\
- \\
- \\
-\end{array}$ & $\begin{array}{l}- \\
\overline{-} \\
\bar{z}\end{array}$ \\
\hline $\begin{array}{l}\text { ST } \\
\text { ST } \\
\text { ST } \\
\text { ST } \\
\text { ST }\end{array}$ & $\begin{array}{l}174 \\
175 \\
176 \\
177 \\
178\end{array}$ & $\begin{array}{l}\text { WILLIAM FLOYD PK } \\
\text { WILLIAM FLOYD PK } \\
\text { WILLIAM FLOYD PK } \\
\text { L.I.R.R. } \\
\text { L.I.R.R. }\end{array}$ & $\begin{array}{l}\text { L.I.E. } \\
\text { L.I.E. } \\
\text { L.I.E. } \\
\text { L.I.E. } \\
\text { L.I.E. }\end{array}$ & $\begin{array}{l}4050310725307 \\
4 \varnothing 50380725305 \\
4050420725214 \\
4 \varnothing 51080725143 \\
4 \varnothing 51110725117\end{array}$ & $\begin{array}{l}- \\
- \\
-\end{array}$ & $\begin{array}{l}255 \\
215 \\
255 \\
255 \\
215\end{array}$ & $\begin{array}{l}- \\
- \\
- \\
-\end{array}$ & $\begin{array}{l}- \\
\overline{-} \\
-\end{array}$ \\
\hline
\end{tabular}


N.Y. [Dash indicates no data. Altitudes are in feet above sea level.] (cont.)

\begin{tabular}{|c|c|c|c|c|c|c|c|c|c|c|c|c|}
\hline \multicolumn{2}{|c|}{$\begin{array}{l}\text { BASIN } \\
\text { NUMBER }\end{array}$} & $\begin{array}{l}\text { MAX. } \\
\text { AREA } \\
\text { (square }\end{array}$ & $\begin{array}{c}\text { BASIN } \\
\text { AREA } \\
\text { feet) }\end{array}$ & RIM & $\begin{array}{l}\text { - ALTI } \\
\text { OVER- } \\
\text { FLOW }\end{array}$ & $\begin{array}{l}\text { BOT- } \\
\text { TOM }\end{array}$ & $\begin{array}{l}\text { WATER } \\
\text { TABLE }\end{array}$ & $\begin{array}{c}\text { DRAIN. } \\
\text { AREA } \\
\text { (acres) }\end{array}$ & \multicolumn{2}{|c|}{$\begin{array}{l}---B A S I N-- \\
\text { STATUS USE }\end{array}$} & $\begin{array}{l}\text { GEO. } \\
\text { UNIT }\end{array}$ & $\begin{array}{l}\text { SOIL } \\
\text { UNIT }\end{array}$ \\
\hline $\begin{array}{l}\text { ST } \\
\text { ST } \\
\text { ST } \\
\text { ST } \\
\text { ST }\end{array}$ & $\begin{array}{l}123 \\
124 \\
125 \\
126 \\
127\end{array}$ & $\begin{array}{l}- \\
\bar{z} \\
\overline{-}\end{array}$ & $\begin{array}{r}58800 \\
82900 \\
60100 \\
69400 \\
174700\end{array}$ & $\begin{array}{r}30.0 \\
40.0 \\
30.0 \\
100.0 \\
185.0\end{array}$ & $\begin{array}{l}- \\
- \\
- \\
-\end{array}$ & $\begin{array}{l}- \\
- \\
-\end{array}$ & $\begin{array}{l}25.0 \\
24.0 \\
15.0 \\
54.0 \\
42 . \varnothing\end{array}$ & $\begin{array}{l}- \\
- \\
- \\
-\end{array}$ & $\begin{array}{l}1 \\
0 \\
1 \\
1 \\
1\end{array}$ & $\begin{array}{l}3 \\
3 \\
3 \\
3 \\
3\end{array}$ & $\begin{array}{l}1 \\
1 \\
1 \\
1 \\
1\end{array}$ & $\begin{array}{l}5 \\
6 \\
3 \\
3 \\
3\end{array}$ \\
\hline $\begin{array}{l}\text { ST } \\
\text { ST } \\
\text { ST } \\
\text { ST } \\
\text { ST }\end{array}$ & $\begin{array}{l}128 \\
129 \\
130 \\
131 \\
132\end{array}$ & $\begin{array}{l}- \\
- \\
-\end{array}$ & $\begin{array}{r}44800 \\
52500 \\
116400 \\
80000 \\
122100\end{array}$ & $\begin{array}{r}175.0 \\
17.0 \\
110.0 \\
115.0 \\
140.0\end{array}$ & $\begin{array}{l}- \\
- \\
- \\
-\end{array}$ & $\begin{array}{l}- \\
- \\
- \\
-\end{array}$ & $\begin{array}{r}70.0 \\
2.0 \\
53.0 \\
55.0 \\
56.0\end{array}$ & $\begin{array}{r}\overline{-} \\
\overline{-} \\
33 . \overline{1} \\
26.7\end{array}$ & $\begin{array}{l}0 \\
1 \\
0 \\
1 \\
0\end{array}$ & $\begin{array}{l}3 \\
3 \\
3 \\
3 \\
3\end{array}$ & $\begin{array}{l}1 \\
1 \\
3 \\
3 \\
3\end{array}$ & $\begin{array}{l}1 \\
9 \\
3 \\
3 \\
1\end{array}$ \\
\hline $\begin{array}{l}\text { ST } \\
\text { ST } \\
\text { ST } \\
\text { ST } \\
\text { ST }\end{array}$ & $\begin{array}{l}133 \\
134 \\
135 \\
136 \\
137\end{array}$ & $\begin{array}{l}- \\
- \\
-\end{array}$ & $\begin{array}{r}230000 \\
65000 \\
62500 \\
23100 \\
15000\end{array}$ & $\begin{array}{r}115.0 \\
55.0 \\
60.0 \\
60.0 \\
60.0\end{array}$ & $\begin{array}{l}- \\
- \\
- \\
-\end{array}$ & $\begin{array}{l}- \\
\bar{z} \\
\overline{-}\end{array}$ & $\begin{array}{l}52.0 \\
37.0 \\
45.0 \\
44.0 \\
44.0\end{array}$ & $\begin{array}{r}29.9 \\
9.7 \\
12.4 \\
49.3 \\
21.4\end{array}$ & $\begin{array}{l}0 \\
1 \\
1 \\
1 \\
1\end{array}$ & $\begin{array}{l}3 \\
3 \\
3 \\
3 \\
3\end{array}$ & $\begin{array}{l}1 \\
1 \\
1 \\
1 \\
1\end{array}$ & $\begin{array}{l}0 \\
1 \\
3 \\
3 \\
3\end{array}$ \\
\hline $\begin{array}{l}\text { ST } \\
\text { ST } \\
\text { ST } \\
\text { ST } \\
\text { ST }\end{array}$ & $\begin{array}{l}138 \\
139 \\
140 \\
141 \\
142\end{array}$ & $\begin{array}{l}- \\
z \\
-\end{array}$ & $\begin{array}{r}140000 \\
100000 \\
-\end{array}$ & $\begin{array}{r}135.0 \\
75.0 \\
70.0 \\
55.0 \\
140 . \varnothing\end{array}$ & $\begin{array}{l}- \\
- \\
- \\
-\end{array}$ & $\begin{array}{l}- \\
z \\
-\end{array}$ & $\begin{array}{l}68 . \varnothing \\
41 . \varnothing \\
41.0 \\
44 . \varnothing \\
48 . \varnothing\end{array}$ & $\begin{array}{l}- \\
- \\
-\end{array}$ & $\begin{array}{l}1 \\
1 \\
1 \\
1 \\
1\end{array}$ & $\begin{array}{l}3 \\
3 \\
3 \\
3 \\
3\end{array}$ & $\begin{array}{l}1 \\
1 \\
1 \\
1 \\
6\end{array}$ & $\begin{array}{l}1 \\
3 \\
3 \\
6 \\
6\end{array}$ \\
\hline $\begin{array}{l}\text { ST } \\
\text { ST } \\
\text { ST } \\
\text { ST } \\
\text { ST }\end{array}$ & $\begin{array}{l}143 \\
144 \\
145 \\
146 \\
147\end{array}$ & $\begin{array}{l}z \\
z \\
-\end{array}$ & $\begin{array}{r}173300 \\
122500 \\
154000\end{array}$ & $\begin{array}{r}105.0 \\
110.0 \\
95.0 \\
120.0 \\
110.0\end{array}$ & $\begin{array}{l}- \\
- \\
-\end{array}$ & $\begin{array}{l}- \\
\overline{-} \\
-\end{array}$ & $\begin{array}{l}47.0 \\
47.0 \\
47.0 \\
47.0 \\
47.0\end{array}$ & $\begin{array}{l}- \\
\overline{-} \\
-\end{array}$ & $\begin{array}{l}1 \\
1 \\
1 \\
1 \\
1\end{array}$ & $\begin{array}{l}3 \\
3 \\
3 \\
3 \\
3\end{array}$ & $\begin{array}{l}1 \\
1 \\
1 \\
1 \\
1\end{array}$ & $\begin{array}{l}7 \\
7 \\
7 \\
8 \\
6\end{array}$ \\
\hline $\begin{array}{l}\text { ST } \\
\text { ST } \\
\text { ST } \\
\text { ST } \\
\text { ST }\end{array}$ & $\begin{array}{l}148 \\
149 \\
150 \\
151 \\
152\end{array}$ & $\begin{array}{l}- \\
- \\
-\end{array}$ & $\begin{array}{r}672000 \\
114000 \\
96 \varnothing 00 \\
213000 \\
136000\end{array}$ & $\begin{array}{r}70.0 \\
245.0 \\
215.0 \\
200.0 \\
90.0\end{array}$ & $\begin{array}{l}- \\
- \\
-\end{array}$ & $\begin{array}{l}- \\
\bar{z}\end{array}$ & $\begin{array}{l}40.0 \\
72.0 \\
70.0 \\
62.0 \\
60.0\end{array}$ & $\begin{array}{r}- \\
\overline{-} \\
17.3 \\
-\end{array}$ & $\begin{array}{l}1 \\
\varnothing \\
0 \\
0 \\
\varnothing\end{array}$ & $\begin{array}{l}3 \\
3 \\
3 \\
3 \\
3\end{array}$ & $\begin{array}{l}1 \\
6 \\
1 \\
4 \\
1\end{array}$ & $\begin{array}{l}3 \\
1 \\
1 \\
1 \\
6\end{array}$ \\
\hline $\begin{array}{l}\text { ST } \\
\text { ST } \\
\text { ST } \\
\text { ST } \\
\text { ST }\end{array}$ & $\begin{array}{l}153 \\
154 \\
155 \\
156 \\
157\end{array}$ & $\begin{array}{l}z \\
z \\
z\end{array}$ & $\begin{array}{r}90000 \\
103100 \\
45000 \\
112500 \\
102400\end{array}$ & $\begin{array}{l}20.0 \\
90.0 \\
40.0 \\
50.0 \\
70.0\end{array}$ & $\begin{array}{l}- \\
z \\
z\end{array}$ & $\begin{array}{l}- \\
\bar{z} \\
-\end{array}$ & $\begin{array}{l}60 . \overline{ } \\
27 . \varnothing \\
3 \varnothing . \varnothing \\
44 . \varnothing\end{array}$ & $\begin{array}{l}- \\
\overline{-} \\
\overline{-}\end{array}$ & $\begin{array}{l}1 \\
1 \\
1 \\
1 \\
1\end{array}$ & $\begin{array}{l}3 \\
3 \\
3 \\
3 \\
3\end{array}$ & $\begin{array}{l}1 \\
1 \\
3 \\
1 \\
1\end{array}$ & $\begin{array}{l}0 \\
1 \\
5 \\
6 \\
7\end{array}$ \\
\hline $\begin{array}{l}\text { ST } \\
\text { ST } \\
\text { ST } \\
\text { ST } \\
\text { ST }\end{array}$ & $\begin{array}{l}158 \\
160 \\
161 \\
162 \\
163\end{array}$ & $\begin{array}{l}- \\
z \\
-\end{array}$ & $\begin{array}{l}93900 \\
89700 \\
60000 \\
63000 \\
71400\end{array}$ & $\begin{array}{l}150.0 \\
100 . \varnothing \\
125 . \varnothing \\
125 . \varnothing \\
135 . \varnothing\end{array}$ & $\begin{array}{l}- \\
z \\
-\end{array}$ & $\begin{array}{l}- \\
z \\
-\end{array}$ & $\begin{array}{l}61 . \varnothing \\
64 . \varnothing \\
71.0 \\
72 . \varnothing \\
73 . \varnothing\end{array}$ & $\begin{array}{r}\overline{-} \\
\overline{-} \\
\overline{-}\end{array}$ & $\begin{array}{l}1 \\
1 \\
1 \\
1 \\
1\end{array}$ & $\begin{array}{l}3 \\
3 \\
3 \\
3 \\
3\end{array}$ & $\begin{array}{l}1 \\
1 \\
1 \\
1 \\
1\end{array}$ & $\begin{array}{l}1 \\
1 \\
1 \\
1 \\
1\end{array}$ \\
\hline $\begin{array}{l}\text { ST } \\
\text { ST } \\
\text { ST } \\
\text { ST } \\
\text { ST }\end{array}$ & $\begin{array}{l}164 \\
165 \\
166 \\
167 \\
168\end{array}$ & $\begin{array}{l}- \\
- \\
-\end{array}$ & $\begin{array}{l}- \\
- \\
-\end{array}$ & $\begin{array}{r}190.0 \\
180.0 \\
60.0 \\
105.0 \\
110.0\end{array}$ & $\begin{array}{l}- \\
- \\
- \\
-\end{array}$ & $\begin{array}{l}- \\
- \\
-\end{array}$ & $\begin{array}{l}37.0 \\
37.0 \\
15.8 \\
32.8 \\
20.0\end{array}$ & $\begin{array}{l}- \\
- \\
-\end{array}$ & $\begin{array}{l}1 \\
1 \\
1 \\
1 \\
1\end{array}$ & $\begin{array}{l}3 \\
3 \\
3 \\
3 \\
3\end{array}$ & $\begin{array}{l}1 \\
4 \\
3 \\
3 \\
3\end{array}$ & $\begin{array}{l}1 \\
5 \\
1 \\
3 \\
3\end{array}$ \\
\hline $\begin{array}{l}\text { ST } \\
\text { ST } \\
\text { ST } \\
\text { ST } \\
\text { ST }\end{array}$ & $\begin{array}{l}169 \\
170 \\
171 \\
172 \\
173\end{array}$ & $\begin{array}{l}- \\
- \\
- \\
-\end{array}$ & $\begin{array}{r}343000 \\
116300 \\
87500 \\
-\end{array}$ & $\begin{array}{r}90.0 \\
100.0 \\
57.0 \\
50.0 \\
50.0\end{array}$ & $\begin{array}{l}- \\
\bar{z} \\
\overline{-}\end{array}$ & $\begin{array}{l}- \\
- \\
-\end{array}$ & $\begin{array}{l}41 . \varnothing \\
41 . \varnothing \\
36 . \varnothing \\
24 . \varnothing \\
27 . \varnothing\end{array}$ & $\begin{array}{l}- \\
- \\
- \\
-\end{array}$ & $\begin{array}{l}1 \\
1 \\
1 \\
1 \\
1\end{array}$ & $\begin{array}{l}3 \\
3 \\
3 \\
3 \\
3\end{array}$ & $\begin{array}{l}1 \\
1 \\
1 \\
1 \\
1\end{array}$ & $\begin{array}{l}7 \\
3 \\
3 \\
3 \\
3\end{array}$ \\
\hline $\begin{array}{l}\text { ST } \\
\text { ST } \\
\text { ST } \\
\text { ST } \\
\text { ST }\end{array}$ & $\begin{array}{l}174 \\
175 \\
176 \\
177 \\
178\end{array}$ & $\begin{array}{l}- \\
- \\
-\end{array}$ & $\begin{array}{r}- \\
- \\
16 \varnothing \varnothing \varnothing \varnothing \\
451 \varnothing \varnothing \\
7 \varnothing 5 \varnothing \varnothing\end{array}$ & $\begin{array}{l}95.0 \\
75.0 \\
70.0 \\
50.0 \\
65.0\end{array}$ & $\begin{array}{l}- \\
\bar{z} \\
-\end{array}$ & $\begin{array}{l}- \\
- \\
- \\
-\end{array}$ & $\begin{array}{l}34 . \varnothing \\
34 . \varnothing \\
35 . \varnothing \\
36 . \varnothing \\
36 . \varnothing\end{array}$ & $\begin{array}{l}- \\
- \\
-\end{array}$ & $\begin{array}{l}1 \\
1 \\
1 \\
1 \\
1\end{array}$ & $\begin{array}{l}3 \\
3 \\
3 \\
3 \\
3\end{array}$ & $\begin{array}{l}8 \\
1 \\
1 \\
1 \\
1\end{array}$ & $\begin{array}{l}6 \\
3 \\
6 \\
3 \\
3\end{array}$ \\
\hline
\end{tabular}


Appendix.--Location and basic physical features of recharge basins on Long Island,

\begin{tabular}{|c|c|c|c|c|c|c|c|c|}
\hline \multicolumn{2}{|c|}{$\begin{array}{l}\text { BASIN } \\
\text { NUMBER }\end{array}$} & \multicolumn{2}{|c|}{ NEAREST INTERSECTION } & $\begin{array}{l}\text { LATITUDE } \\
\text { LONGITUDE } \\
0^{\prime}, *\end{array}$ & $\begin{array}{l}\text { DATE } \\
\text { BUILT } \\
\text { YrMOD }\end{array}$ & COMMUNITY & $\begin{array}{r}\text { DESIGN } \\
\text { CAPACITY } \\
\text { (cubic }\end{array}$ & $\begin{array}{l}\text { ACTUAL } \\
\text { CAPACITY } \\
\text { feet) }\end{array}$ \\
\hline $\begin{array}{l}\text { ST } \\
\text { ST } \\
\text { ST } \\
\text { ST } \\
\text { ST }\end{array}$ & $\begin{array}{l}179 \\
180 \\
181 \\
182 \\
183\end{array}$ & $\begin{array}{l}\text { WEEKS AVENUE } \\
\text { WEEKS AVENUE } \\
\text { WEEKS AVENUE } \\
\text { WADING RIVER RD. } \\
\text { WADING RIVER RD. }\end{array}$ & $\begin{array}{l}\text { L.I.E. } \\
\text { L.I.E. } \\
\text { L.I.E. } \\
\text { L.I.E. } \\
\text { L.I.E. }\end{array}$ & $\begin{array}{l}4 \varnothing 5117 \varnothing 725039 \\
4 \varnothing 51250725037 \\
4 \varnothing 51320725001 \\
4 \varnothing 5157 \varnothing 724934 \\
4 \varnothing 51530724920\end{array}$ & $\begin{array}{l}- \\
- \\
-\end{array}$ & $\begin{array}{l}215 \\
215 \\
215 \\
215 \\
215\end{array}$ & $\begin{array}{l}- \\
- \\
-\end{array}$ & $\begin{array}{l}- \\
- \\
-\end{array}$ \\
\hline $\begin{array}{l}\text { ST } \\
\text { ST } \\
\text { ST } \\
\text { ST } \\
\text { ST }\end{array}$ & $\begin{array}{l}184 \\
185 \\
186 \\
187 \\
188\end{array}$ & $\begin{array}{l}\text { RYERSON AVENUE } \\
\text { RYERSON AVENUE } \\
\text { PORT JEF-W.HAM.R } \\
\text { GREENLAWN-BDWY.R } \\
\text { DEER PARK AVENUE }\end{array}$ & $\begin{array}{l}\text { L.I.E. } \\
\text { L.I.E. } \\
\text { L.I.E. } \\
\text { DIX HILLS ROAD } \\
\text { STRAIGHT PATH }\end{array}$ & 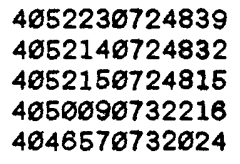 & $\begin{array}{l}- \\
- \\
- \\
-\end{array}$ & $\begin{array}{l}215 \\
215 \\
215 \\
141 \\
143\end{array}$ & $\begin{array}{l}- \\
- \\
- \\
-\end{array}$ & $\begin{array}{l}- \\
- \\
- \\
-\end{array}$ \\
\hline $\begin{array}{l}\text { ST } \\
\text { ST } \\
\text { ST } \\
\text { ST } \\
\text { ST }\end{array}$ & $\begin{array}{l}196 \\
191 \\
192 \\
193 \\
194\end{array}$ & $\begin{array}{l}\text { WASHINGTON AVE. } \\
\text { NICOLLS ROAD } \\
\text { BLUE POINT ROAD } \\
\text { OCEAN AVENUE } \\
\text { MEDFORD AVENUE }\end{array}$ & $\begin{array}{l}\text { L.I.E. } \\
\text { L.I.E. } \\
\text { L.I.E. } \\
\text { L.I.E. } \\
\text { L.I.E. }\end{array}$ & $\begin{array}{l}4049110730257 \\
4049110730311 \\
4049100730225 \\
4049140730120 \\
4049180730053\end{array}$ & $\begin{array}{l}- \\
- \\
- \\
-\end{array}$ & $\begin{array}{l}226 \\
226 \\
226 \\
224 \\
232\end{array}$ & $\begin{array}{l}- \\
- \\
- \\
-\end{array}$ & $\begin{array}{l}- \\
- \\
-\end{array}$ \\
\hline $\begin{array}{l}\text { ST } \\
\text { ST } \\
\text { ST } \\
\text { ST } \\
\text { ST }\end{array}$ & $\begin{array}{l}195 \\
196 \\
197 \\
198 \\
199\end{array}$ & $\begin{array}{l}\text { OHIO AVENUE } \\
\text { MEDFORO ROAD } \\
\text { CALIFORNIA AVE. } \\
\text { HORSE BLOCK ROAD } \\
\text { HALSEY MANOR RD. }\end{array}$ & $\begin{array}{l}\text { L.I.E. } \\
\text { L.I.E. } \\
\text { L.I.E. } \\
\text { L.I.E. } \\
\text { L.I.E. }\end{array}$ & $\begin{array}{l}4 \varnothing 4932073 \varnothing 038 \\
4 \varnothing 49200725948 \\
4 \varnothing 49180725909 \\
4 \varnothing 49150725832 \\
4 \varnothing 52370724657\end{array}$ & $\begin{array}{l}- \\
- \\
- \\
-\end{array}$ & $\begin{array}{l}232 \\
232 \\
232 \\
232 \\
264\end{array}$ & $\begin{array}{l}- \\
- \\
- \\
-\end{array}$ & $\begin{array}{l}- \\
- \\
-\end{array}$ \\
\hline $\begin{array}{l}\text { ST } \\
\text { ST } \\
\text { ST } \\
\text { ST } \\
\text { ST }\end{array}$ & $\begin{array}{l}290 \\
291 \\
202 \\
293 \\
204\end{array}$ & $\begin{array}{l}\text { HALSEY MANOR RD. } \\
\text { EDWARDS AVENUE } \\
\text { EDWARDS AVENUE } \\
\text { WILLIAM FLOYD PK } \\
\text { NEWBRIDGE ROAD }\end{array}$ & $\begin{array}{l}\text { L.I.E. } \\
\text { L.I.E. } \\
\text { L.I.E. } \\
\text { MIDDLE COUNTRY R } \\
\text { HEMPSTEAD TPKE. }\end{array}$ & $\begin{array}{l}4 \varnothing 530107246 \varnothing 8 \\
4 \varnothing 5334 \varnothing 724524 \\
4 \varnothing 54110724429 \\
4 \varnothing 53480725257 \\
4 \varnothing 43398733214\end{array}$ & $\begin{array}{l}- \\
- \\
- \\
-\end{array}$ & $\begin{array}{r}264 \\
264 \\
264 \\
250 \\
8\end{array}$ & $\begin{array}{l}- \\
- \\
- \\
-\end{array}$ & $\begin{array}{l}- \\
- \\
-\end{array}$ \\
\hline $\begin{array}{l}\text { ST } \\
\text { ST } \\
\text { ST } \\
\text { ST } \\
\text { ST }\end{array}$ & $\begin{array}{l}205 \\
206 \\
207 \\
208 \\
209\end{array}$ & $\begin{array}{l}\text { LORING ROAD } \\
\text { NEWBRIDGE ROAD } \\
\text { NEWBRIDGE ROAD } \\
\text { NEWBRIDGE ROAD } \\
\text { BROWNS ROAD }\end{array}$ & $\begin{array}{l}\text { HEMPSTEAD TPKE. } \\
\text { SALISBURY PK.DR. } \\
\text { GREENBELT LANE } \\
\text { FORDHAM AVENUE } \\
\text { NESCONSET HWY. }\end{array}$ & $\begin{array}{l}4 \varnothing 4322 \varnothing 7332 ø 5 \\
4 \varnothing 43590733221 \\
4 \varnothing 44110733221 \\
4 \varnothing 45 \varnothing 2 \varnothing 733156 \\
4 \varnothing 5125 \varnothing 73 \varnothing 83 \emptyset\end{array}$ & $\begin{array}{l}- \\
- \\
- \\
-\end{array}$ & $\begin{array}{r}25 \\
25 \\
25 \\
194 \\
177\end{array}$ & $\begin{array}{l}- \\
- \\
- \\
-\end{array}$ & $\begin{array}{l}- \\
- \\
- \\
-\end{array}$ \\
\hline $\begin{array}{l}\text { ST } \\
\text { ST } \\
\text { ST } \\
\text { ST } \\
\text { ST }\end{array}$ & $\begin{array}{l}210 \\
211 \\
212 \\
213 \\
214\end{array}$ & $\begin{array}{l}\text { MEADOWBROOK PKY. } \\
\text { MEADOWBROOK PKY. } \\
\text { MEDFORD AVENUE } \\
\text { MEDFORD AVENUE } \\
\text { MANOR LANE }\end{array}$ & $\begin{array}{l}\text { HEMPSTEAD TPKE. } \\
\text { HEMPSTEAD TPKE. } \\
\text { COLUMBIA STREET } \\
\text { JAMES STREET } \\
\text { SUNRISE HIGHWAY }\end{array}$ & $\begin{array}{l}4 \varnothing 42690733658 \\
4 \varnothing 4312 \varnothing 733658 \\
4 \varnothing 46 \varnothing 4 \varnothing 730 \varnothing 17 \\
4 \varnothing 4627 \varnothing 73 \varnothing \varnothing 27 \\
4 \varnothing 4347 \varnothing 731647\end{array}$ & $\begin{array}{l}- \\
- \\
- \\
-\end{array}$ & $\begin{array}{r}14 \\
14 \\
221 \\
221 \\
202\end{array}$ & $\begin{array}{l}- \\
- \\
- \\
-\end{array}$ & $\begin{array}{l}- \\
- \\
- \\
-\end{array}$ \\
\hline $\begin{array}{l}\text { ST } \\
\text { ST }\end{array}$ & $\begin{array}{l}215 \\
216\end{array}$ & $\begin{array}{l}\text { MIDDLE COUNTRY R } \\
\text { OLD COUNTRY ROAD }\end{array}$ & $\begin{array}{l}\text { L.I.E. } \\
\text { L.I.E. }\end{array}$ & $\begin{array}{l}4055190724342 \\
4055230724333\end{array}$ & $\overline{-}$ & $\begin{array}{l}269 \\
269\end{array}$ & $\overline{-}$ & - \\
\hline
\end{tabular}


N.Y. [Dash indicates no data. Altitudes are in feet above sea level.] (cont.)

\begin{tabular}{|c|c|c|c|c|c|c|c|c|c|c|c|c|}
\hline \multicolumn{2}{|c|}{$\begin{array}{l}\text { BASIN } \\
\text { NUMBER }\end{array}$} & $\begin{array}{l}\text { MAX. } \\
\text { AREA } \\
\text { (squar }\end{array}$ & $\begin{array}{r}\text { BASIN } \\
\text { AREA } \\
\text { fe fet) }\end{array}$ & RIM & $\begin{array}{l}\text { OVER- } \\
\text { FLOW }\end{array}$ & $\begin{array}{l}\text { BOT- } \\
\text { TOM }\end{array}$ & $\begin{array}{l}\text { WATER } \\
\text { TABLE }\end{array}$ & $\begin{array}{c}\text { DRAIN. } \\
\text { AREA } \\
\text { (acres) }\end{array}$ & \multicolumn{2}{|c|}{$\begin{array}{l}--B A S I N-- \\
\text { STATUS USE }\end{array}$} & $\begin{array}{l}\text { GEO. } \\
\text { UNIT }\end{array}$ & $\begin{array}{l}\text { SOIL } \\
\text { UNIT }\end{array}$ \\
\hline $\begin{array}{l}\text { ST } \\
\text { ST } \\
\text { ST } \\
\text { ST } \\
\text { ST }\end{array}$ & $\begin{array}{l}179 \\
180 \\
181 \\
182 \\
183\end{array}$ & $\begin{array}{l}- \\
- \\
- \\
-\end{array}$ & $\begin{array}{r}- \\
127600 \\
-\end{array}$ & $\begin{array}{l}50.0 \\
52.0 \\
50.0 \\
40.0 \\
45.0\end{array}$ & $\begin{array}{l}- \\
- \\
- \\
-\end{array}$ & $\begin{array}{l}- \\
- \\
- \\
-\end{array}$ & $\begin{array}{l}35.0 \\
37.0 \\
35.0 \\
35.0 \\
35.0\end{array}$ & $\begin{array}{l}- \\
- \\
- \\
-\end{array}$ & $\begin{array}{l}1 \\
1 \\
1 \\
1 \\
1\end{array}$ & $\begin{array}{l}3 \\
3 \\
3 \\
3 \\
3\end{array}$ & $\begin{array}{l}1 \\
1 \\
1 \\
1 \\
1\end{array}$ & $\begin{array}{l}6 \\
6 \\
3 \\
6 \\
6\end{array}$ \\
\hline $\begin{array}{l}\text { ST } \\
\text { ST } \\
\text { ST } \\
\text { ST } \\
\text { ST }\end{array}$ & $\begin{array}{l}184 \\
185 \\
186 \\
187 \\
188\end{array}$ & $\begin{array}{l}- \\
- \\
- \\
-\end{array}$ & $\begin{array}{r}- \\
- \\
41200 \\
96000\end{array}$ & $\begin{array}{r}45.0 \\
45.0 \\
55.0 \\
180.0 \\
95.0\end{array}$ & $\begin{array}{l}- \\
- \\
- \\
-\end{array}$ & $\begin{array}{l}- \\
- \\
-\end{array}$ & $\begin{array}{l}36.0 \\
35.0 \\
35.0 \\
68.0 \\
56.0\end{array}$ & $\begin{array}{l}- \\
- \\
- \\
-\end{array}$ & $\begin{array}{l}1 \\
1 \\
1 \\
0 \\
0\end{array}$ & $\begin{array}{l}3 \\
3 \\
3 \\
3 \\
3\end{array}$ & $\begin{array}{l}1 \\
1 \\
1 \\
1 \\
1\end{array}$ & $\begin{array}{l}6 \\
6 \\
6 \\
7 \\
3\end{array}$ \\
\hline $\begin{array}{l}\text { ST } \\
\text { ST } \\
\text { ST } \\
\text { ST } \\
\text { ST }\end{array}$ & $\begin{array}{l}190 \\
191 \\
192 \\
193 \\
194\end{array}$ & $\begin{array}{l}- \\
- \\
- \\
-\end{array}$ & $\begin{array}{r}160000 \\
1500 \emptyset 0 \\
227500 \\
327000 \\
-\end{array}$ & $\begin{array}{r}117.0 \\
122.0 \\
110.0 \\
60.0 \\
90.0\end{array}$ & $\begin{array}{l}- \\
- \\
- \\
-\end{array}$ & $\begin{array}{l}- \\
- \\
-\end{array}$ & $\begin{array}{l}45.0 \\
45.0 \\
43.0 \\
44.0 \\
44.0\end{array}$ & $\begin{array}{l}- \\
- \\
-\end{array}$ & $\begin{array}{l}1 \\
1 \\
1 \\
1 \\
1\end{array}$ & $\begin{array}{l}3 \\
3 \\
3 \\
3 \\
3\end{array}$ & $\begin{array}{l}1 \\
1 \\
1 \\
1 \\
1\end{array}$ & $\begin{array}{l}6 \\
5 \\
6 \\
5 \\
3\end{array}$ \\
\hline $\begin{array}{l}\text { ST } \\
\text { ST } \\
\text { ST } \\
\text { ST } \\
\text { ST }\end{array}$ & $\begin{array}{l}195 \\
196 \\
197 \\
198 \\
199\end{array}$ & $\begin{array}{l}- \\
- \\
- \\
-\end{array}$ & $\begin{array}{r}67600 \\
151200 \\
93500 \\
180000 \\
-\end{array}$ & $\begin{array}{l}90.0 \\
85.0 \\
95.0 \\
85.0 \\
50.0\end{array}$ & $\begin{array}{l}- \\
- \\
-\end{array}$ & $\begin{array}{l}- \\
- \\
-\end{array}$ & $\begin{array}{l}45.0 \\
41.0 \\
40.0 \\
41.0 \\
34.0\end{array}$ & $\begin{array}{l}- \\
- \\
-\end{array}$ & $\begin{array}{l}1 \\
1 \\
1 \\
1 \\
1\end{array}$ & $\begin{array}{l}3 \\
3 \\
3 \\
3 \\
3\end{array}$ & $\begin{array}{l}1 \\
1 \\
1 \\
1 \\
1\end{array}$ & $\begin{array}{l}3 \\
6 \\
3 \\
5 \\
5\end{array}$ \\
\hline $\begin{array}{l}\text { ST } \\
\text { ST } \\
\text { ST } \\
\text { ST } \\
\text { ST }\end{array}$ & $\begin{array}{l}200 \\
201 \\
202 \\
203 \\
204\end{array}$ & $\begin{array}{l}- \\
- \\
- \\
-\end{array}$ & $\begin{array}{r}135700 \\
363080 \\
287000 \\
30500\end{array}$ & $\begin{array}{r}50.0 \\
105.0 \\
40.0 \\
80.0 \\
90.0\end{array}$ & $\begin{array}{l}\text { - } \\
\text { - } \\
\text { - }\end{array}$ & $\begin{array}{l}- \\
- \\
-\end{array}$ & $\begin{array}{l}35.0 \\
35.0 \\
30.0 \\
52.0 \\
60.0\end{array}$ & $\begin{array}{r}- \\
\overline{-} \\
24 . \overline{7}\end{array}$ & $\begin{array}{l}1 \\
1 \\
1 \\
1 \\
1\end{array}$ & $\begin{array}{l}3 \\
3 \\
3 \\
3 \\
3\end{array}$ & $\begin{array}{l}1 \\
1 \\
1 \\
1 \\
1\end{array}$ & $\begin{array}{l}6 \\
3 \\
6 \\
3 \\
2\end{array}$ \\
\hline $\begin{array}{l}\text { ST } \\
\text { ST } \\
\text { ST } \\
\text { ST } \\
\text { ST }\end{array}$ & $\begin{array}{l}205 \\
206 \\
207 \\
208 \\
209\end{array}$ & $\begin{array}{l}- \\
- \\
- \\
-\end{array}$ & $\begin{array}{r}239500 \\
35500 \\
64800 \\
48600 \\
60000\end{array}$ & $\begin{array}{r}75.0 \\
100.0 \\
105.0 \\
125.0 \\
100.0\end{array}$ & $\begin{array}{l}- \\
- \\
- \\
-\end{array}$ & $\begin{array}{l}- \\
- \\
-\end{array}$ & $\begin{array}{l}55.0 \\
63.0 \\
65.0 \\
71.0 \\
60.0\end{array}$ & $\begin{array}{r}112.0 \\
- \\
- \\
-\end{array}$ & $\begin{array}{l}1 \\
1 \\
1 \\
1 \\
1\end{array}$ & $\begin{array}{l}3 \\
3 \\
3 \\
3 \\
3\end{array}$ & $\begin{array}{l}1 \\
1 \\
1 \\
1 \\
1\end{array}$ & $\begin{array}{l}2 \\
2 \\
2 \\
2 \\
1\end{array}$ \\
\hline $\begin{array}{l}\text { ST } \\
\text { ST } \\
\text { ST } \\
\text { ST } \\
\text { ST }\end{array}$ & $\begin{array}{l}210 \\
211 \\
212 \\
213 \\
214\end{array}$ & $\begin{array}{l}- \\
- \\
- \\
-\end{array}$ & $\begin{array}{r}70300 \\
123200 \\
57000 \\
53000 \\
-\end{array}$ & $\begin{array}{l}75 . \bar{\varnothing} \\
20 . \varnothing \\
25 . \varnothing \\
4 \varnothing . \varnothing\end{array}$ & $\begin{array}{l}- \\
- \\
-\end{array}$ & $\begin{array}{l}- \\
- \\
-\end{array}$ & $\begin{array}{l}69.0 \\
60.0 \\
18.0 \\
18.0 \\
27.0\end{array}$ & I- & $\begin{array}{l}1 \\
1 \\
1 \\
1 \\
1\end{array}$ & $\begin{array}{l}3 \\
3 \\
3 \\
3 \\
3\end{array}$ & $\begin{array}{l}1 \\
1 \\
1 \\
1 \\
1\end{array}$ & $\begin{array}{l}2 \\
2 \\
6 \\
6 \\
1\end{array}$ \\
\hline $\begin{array}{l}\text { ST } \\
\text { ST }\end{array}$ & $\begin{array}{l}215 \\
216\end{array}$ & - & - & $\begin{array}{l}60.0 \\
60.0\end{array}$ & - & - & $\begin{array}{l}28.0 \\
28.0\end{array}$ & - & $\begin{array}{l}1 \\
1\end{array}$ & $\begin{array}{l}3 \\
3\end{array}$ & $\begin{array}{l}1 \\
1\end{array}$ & $\begin{array}{l}1 \\
1\end{array}$ \\
\hline
\end{tabular}

